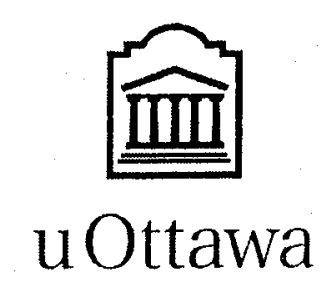

L'Université canadienne Canada's university 
FACULTÉ DES ÉTUDES SUPÉRIEURES

ET POSTOCTORALES
uOttawa

\section{I'Université canadienne}

Canade's university

\section{FACULTY OF GRADUATE AND POSDOCTORAL STUDIES}

Nabil Naas

AUTEUR DE LA THESE I AUTHOR OF THESIS

Ph.D. (Electrical Engineering)

GRADE /DEGREE

School of Information Technology and Engineering

FACULTE, ECOLE, DEPARTEMENT / FACULTY, SCHOOL, DEPARTMENT

Towards the Realistic Planning of the GMPLS-based Optical Transport Networks

TITRE DE LA THESE / TITLE OF THESIS

Hussein T. Mouftah

DIRECTEUR (DIRECTRICE) DE LA THESE / THESIS SUPERVISOR

CO-DIRECTEUR (CO-DIRECTRICE) DE LA THESE ITHESIS CO-SUPERVISOR

EXAMINATEURS (EXAMINATRICES) DE LA THĖSE / THESIS EXAMINERS

Abdelhakim Hafid

Ashrah Matrawy

\author{
Amiya Nayak
}

Shervin Shirmohammadi

Gary W. Slater

Le Doyen de la Facuite des études superieures et postdoctorales / Dean of the Faculty of Graduate and Postdoctoral Studies 


\title{
TOWARDS THE REALISTIC PLANNING OF THE GMPLS-BASED OPTICAL TRANSPORT NETWORKS
}

by

\author{
Nabil A. Naas \\ A thesis submitted to the \\ Faculty of Graduate and Postdoctoral Studies \\ in partial fulfillment of the requirements \\ for the Ph.D. degree in \\ Electrical and Computer Engineering
}

School of Information Technology and Engineering

Faculty of Engineering

University of Ottawa

(C) Nabil Naas, Ottawa, Canada, 2008 


$\begin{array}{ll}\begin{array}{l}\text { Library and } \\ \text { Archives Canada }\end{array} & \begin{array}{l}\text { Bibliothèque et } \\ \text { Archives Canada }\end{array} \\ \begin{array}{l}\text { Published Heritage } \\ \text { Branch }\end{array} & \begin{array}{l}\text { Direction du } \\ \text { Patrimoine de l'édition }\end{array} \\ \begin{array}{l}\text { 395 Wellington Street } \\ \text { Ottawa ON K1A 0N4 } \\ \text { Canada }\end{array} & \begin{array}{l}\text { 395, rue Wellington } \\ \text { Ottawa ON K1A 0N4 } \\ \text { Canada }\end{array}\end{array}$

Your file Votre référence ISBN: 978-0-494-50747-6 Our file Notre référence ISBN: 978-0-494-50747-6

NOTICE:

The author has granted a nonexclusive license allowing Library and Archives Canada to reproduce, publish, archive, preserve, conserve, communicate to the public by telecommunication or on the Internet, loan, distribute and sell theses worldwide, for commercial or noncommercial purposes, in microform, paper, electronic and/or any other formats.

The author retains copyright ownership and moral rights in this thesis. Neither the thesis nor substantial extracts from it may be printed or otherwise reproduced without the author's permission.
AVIS:

L'auteur a accordé une licence non exclusive permettant à la Bibliothèque et Archives Canada de reproduire, publier, archiver, sauvegarder, conserver, transmettre au public par télécommunication ou par l'Internet, prêter, distribuer et vendre des thèses partout dans le monde, à des fins commerciales ou autres, sur support microforme, papier, électronique et/ou autres formats.

L'auteur conserve la propriété du droit d'auteur et des droits moraux qui protège cette thèse. $\mathrm{Ni}$ la thèse ni des extraits substantiels de celle-ci ne doivent être imprimés ou autrement reproduits sans son autorisation.
In compliance with the Canadian Privacy Act some supporting forms may have been removed from this thesis.

While these forms may be included in the document page count, their removal does not represent any loss of content from the thesis.
Conformément à la loi canadienne sur la protection de la vie privée, quelques formulaires secondaires ont été enlevés de cette thèse.

Bien que ces formulaires aient inclus dans la pagination, il n'y aura aucun contenu manquant.

\section{Canada}




\section{Abstract}

The explosive growth of traffic in Wavelength Division Multiplexing (WDM)-based transport networks has necessitated an increase in the number of wavelengths per fiber. However, the continuation of this trend will definitely lead to both an explosion in the size of Optical Crossconnect (OXCs)/Reconfigurable Optical Add/Drop Multiplexers (ROADMs) and an increased difficulty in routing and managing wavelengths. With the introduction of Generalized Multiprotocol Label Switching (GMPLS), the multi-granular switching concept becomes essential to alleviating these serious scalability problems. However, in spite of the fact that this new switching concept brings many benefits, it also results in new network design and planning challenges.

In this thesis, a new problem in planning the GMPLS-based (or multi-granular) transport network, called the Routing and Multi-Granular Paths Assignment (RMGPA) problem, is addressed. The objective of the RMGPA problem is to minimize the total weighted port count in the transport network. The novelty of this problem lies in the incorporation of the following for the first time: (1) considering the whole traffic hierarchy defined in GMPLS; (2) optimizing multi-granularity traffic demands; (3) allowing the bifurcation of multi-granularity demands; (4) (de)grouping traffic flows at all granularity levels; (5) allowing wavelength, waveband, and fiber conversions; (6) imposing the optical-reach constraint on the length of all-optical paths; and (7) customizing the optical reach of all-optical paths. In addition, in order to realize the functionalities required by these newly addressed issues, a new GMPLS-based node architecture is proposed. 
Different versions of the RMGPA problem are formulated as Mixed Integer Linear Programming (MILP) and Non-linear Programming (NLP) models. Due to the computational complexity of the planning problem, only the MILP models of small-sized problems are solved. To satisfy the planning speed requirement, various efficient heuristics that are capable of solving large-sized problems in a reasonable amount of time are also proposed. In addition, the solutions to the MILP models are used to validate the proposed heuristics. Moreover, in order to achieve the best possible planning solution to different versions of the RMGPA problem in both fiber-scarce and plentiful situations, a comprehensive evaluation of different heuristic variations through illustrative examples and simulations is conducted. Furthermore, the results provide valuable insights into many issues that can contribute to further research and development in this area. 
"Success is peace of mind which is a direct result of self-satisfaction in knowing you did your best to become the best you are capable of becoming"John R. Wooden

To my FAMILY with love 


\section{Acknowledgements}

I wish to express my deepest gratitude to my supervisor, Professor Hussein T. Mouftah, for his guidance, valuable insights, support, and endless encouragement during the course of my study at the University of Ottawa. His commitment to innovation and excellence, hard work, and infinite patience have been and continue to be an inspiration to me.

I would also like to thank the University of Ottawa and School of Information Technology and Engineering for the financial support, kind assistance, and wonderful research resources they have provided. Special thanks go to the members of the Optical Network Research Lab for creating a flourishing environment for research and study.

Last but not least, words alone are not enough to fully express my love, appreciation, and gratitude to my dear parents, Abdulfattah and Lutfia; wife, Inas; sister, Nahla; brother, Nader; son, Yaseen; and daughter, Nour. I could not have reached this stage without their love, care and encouragement. May God always bless your hearts. 


\section{Contents}

$\begin{array}{ll}\text { Abstract } & \text { ii }\end{array}$

$\begin{array}{lll}\text { Acknowledgements } & \text { v }\end{array}$

List of Figures $\quad$ ix

List of Tables $\quad$ xiii

Abbreviations $\quad$ xviii

Chapter 1: Introduction 1

1.1 Background and Motivations................................................

1.2 Objectives..............................................................

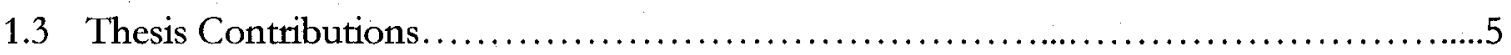

1.4 Thesis Outline ...................................................................

Chapter 2: Background and State-of-the-Art 9

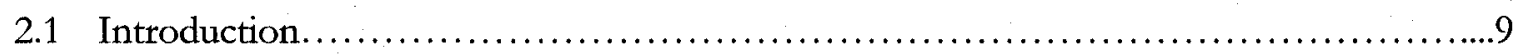

2.2 What is Network Planning? ........................................... 9

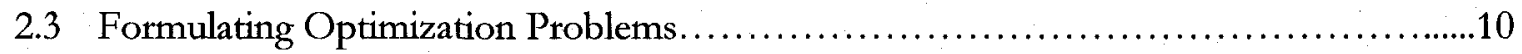

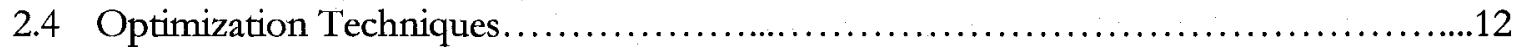

2.4.1 Exact Techniques.........................................................

2.4.2 Approximation Techniques.......................................... 14

2.5 Prior Related Work......................................................... 17

2.5.1 Traffic Grouping Strategies............................................. 17

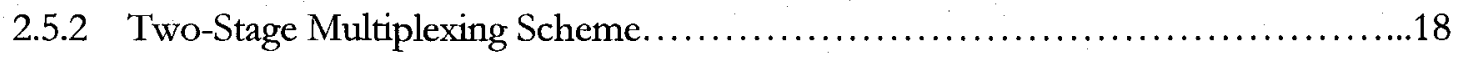

2.5.3 Comprehensive Study on Waveband Switching...............................21

2.5 .4 RWA with Tunnel Allocation........................................... 25

2.5.5 Non-Uniform versus Uniform Waveband Switching..........................28

2.5.6 Waveband Routing and Merging....................................... 30

2.5.7 Lagrangean Relaxation Approach....................................... 32

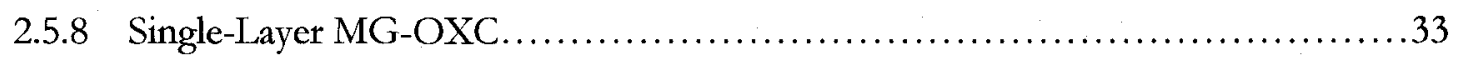

2.5.9 Disjoint Shared Waveband Path Protection ............................. 35

2.5.10 Dedicated and Shared Waveband Path Protection.............................. 36

2.5.11 Grouping Strategies with Joint Dedicated Path Protection.....................38 
2.5.12 Dedicated Waveband Sub-path Protection................................. 40

2.5 .13 Summary........................................................ 41

Chapter 3: Network Architecture and Framework 42

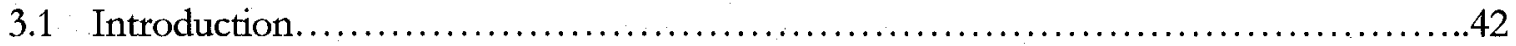

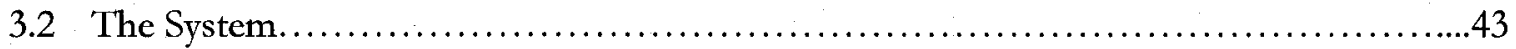

3.2.1 Node Architecture...................................................43

3.2.2 Path Types........................................................46

3.2.3 Grouping and Degrouping of Paths.................................... 52

3.3 Solution of the Planning Problem............................................... 54

3.3.1 Problem Statement...................................................... 54

3.3.2 Assumptions....................................................... 54

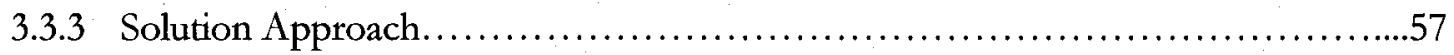

\section{Chapter 4: Planning of GMPLS-Based Transport Networks} without Conversion and Regeneration Capabilities $\quad 61$

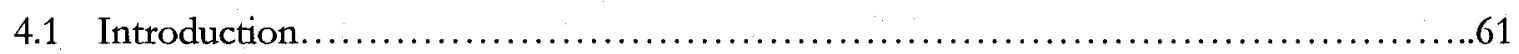

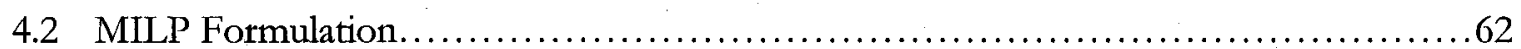

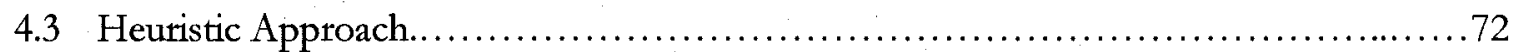

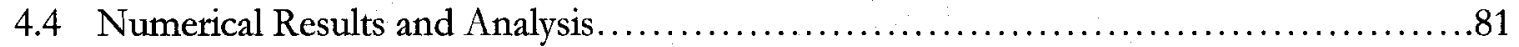

4.4.1 Verification of the Heuristic Approach.......................................... 81

4.4.2 Significance of Adjustment Phase........................................ 87

4.4.3 Impact of Increasing Fiber Resources.................................. 90

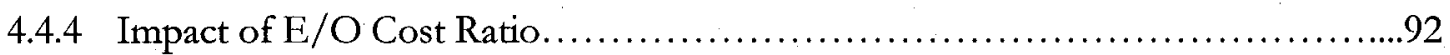

4.4.5 Why Bifurcating All Demands is an Expensive Choice.........................95

4.4.6 Effects of the $C-W-B$ Combination Selection...............................98

4.4.7 Comparison of Heuristics................................................. 103

\section{Chapter 5: Planning of GMPLS-Based Transport Networks} with Conversion and Regeneration Capabilities $\quad 121$

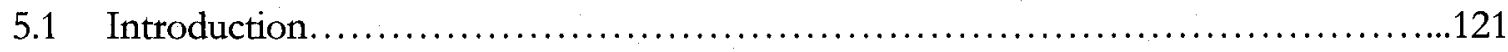

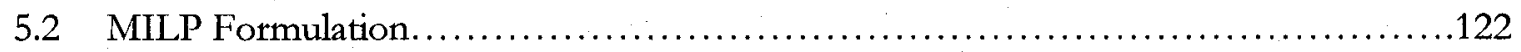

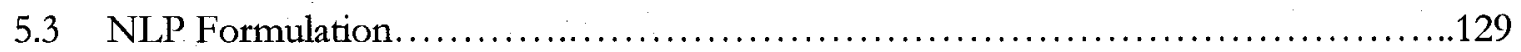

5.4 Heuristic Approach................................................ 131

5.5 Numerical Results and Analysis............................................. 140

5.5.1 Verification of the Heuristic Approach................................... 140

5.5.2 Impact of Color Conversion................................................. 143 
5.5.3 Impact of E/O Cost Ratio with Color-Continuity Constraints.................147

5.5.4 Effects of Maximum Optical Reach..................................... 150

5.5.5 Benefits of Customizing the Optical Reach................................. 153

5.5.6 Impact of E/O Cost Ratio with Optical-Reach Constraints......................156

5.5.7 Comparison of Heuristics with Color Conversion Capability....................157

5.5.8 Comparison of Heuristics with Signal Regeneration Capability .................168

Chapter 6: Conclusions and Future Research 180

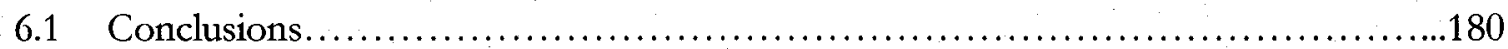

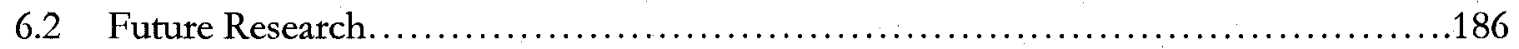

Appendix A: Results of Heuristics Comparison without Conversion and Regeneration Capabilities $\quad 189$

Appendix B: Results of Heuristics Comparison with Color Conversion Capability 226

Appendix C: Results of Heuristics Comparison with Signal Regeneration Capability 243

Appendix D: Confidence Interval 344

Bibliography 346

Publications $\quad 354$ 


\section{List of Figures}

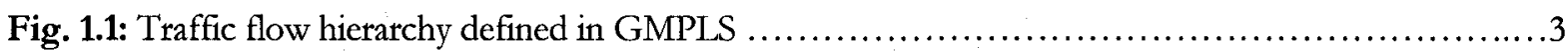

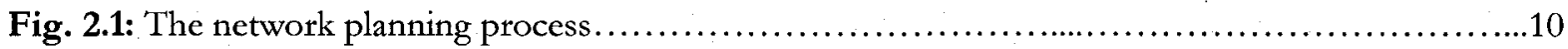

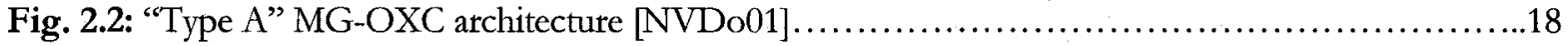

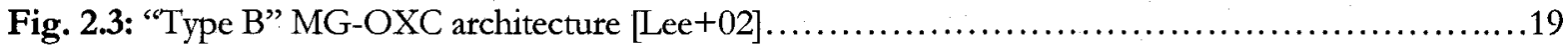

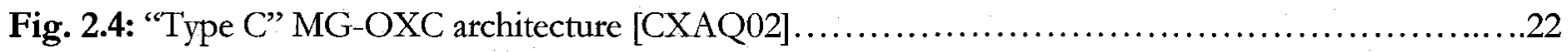

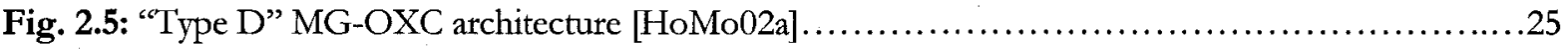

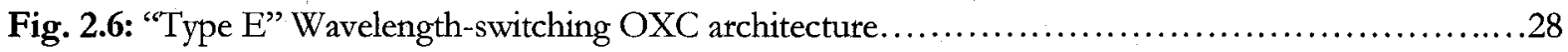

Fig. 2.7: "Type F" Fiber- \& wavelength-switching MG-OXC architecture [HMWu03] ..................28

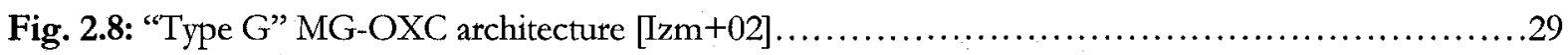

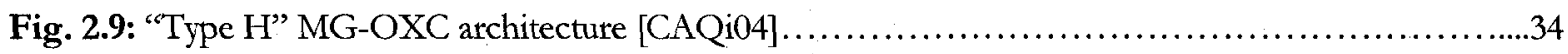

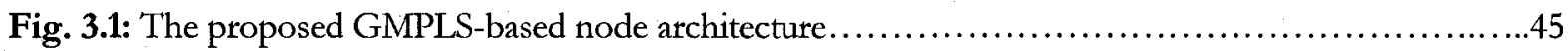

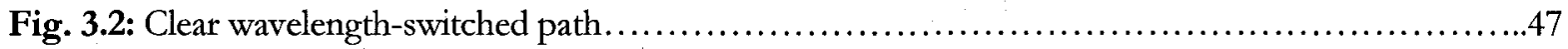

Fig. 3.3: Unclear wavelength-switched path, sub-wavelength flow (de)grouping scenario................48

Fig. 3.4: Unclear wavelength-switched path, optical signal regeneration scenario.....................48

Fig. 3.5: Unclear wavelength-switched path, wavelength conversion scenario $\ldots \ldots \ldots \ldots \ldots \ldots \ldots \ldots \ldots \ldots .49$

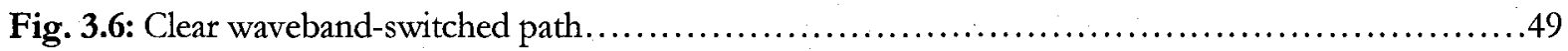

Fig. 3.7: Unclear waveband-switched path, optical signal regeneration scenario $\ldots \ldots \ldots \ldots \ldots \ldots \ldots \ldots \ldots \ldots$

Fig. 3.8: Unclear waveband-switched path, waveband conversion scenatio........................50

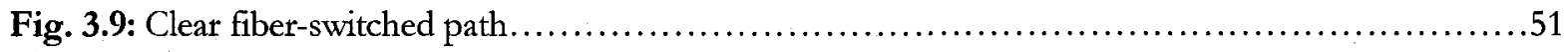

Fig. 3.10: Unclear fiber-switched path, optical signal regeneration scenario...........................51

Fig. 3.11: Unclear fiber-switched path, fiber conversion scenario.................................52

Fig. 3.12: Flowchart for the approach to be taken to solve the RMGPA problem optimally and sub-

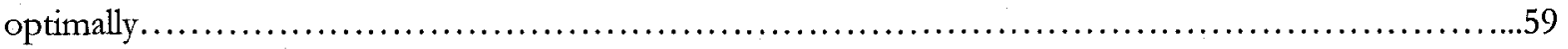

Fig. 4.1: Port configurations at all switching stages of the GMPLS-based transport node.................66 
Fig. 4.2: Switching options for the pass-through CWS/CBS-based virtual link.

Fig. 4.3: A high-level view of the heuristic approach proposed to solve the RMGPA problem with the color-continuity constraints and without the optical-reach constraints.................................

Fig. 4.4: 5-node sample network fiber layout with span distances in $\mathrm{km} \ldots \ldots \ldots \ldots \ldots \ldots \ldots \ldots \ldots \ldots . \ldots \ldots 2$

Fig. 4.5: The multi-granular virtual topology solution for the 5-node network example using the MILP formulation (left) and heuristic approach (right), given that $C=10 \mathrm{~Gb} / \mathrm{s}, W=3, B=3$, and $F=F_{\text {main }}=1$

Fig. 4.6: Port requirements at Node 0 of the 5-node sample network for the realization of the multigranular virtual topology solution using the MILP formulation (left) and heuristic approach (right).... .85

Fig. 4.7: Port requirements at Node 1 of the 5-node sample network for the implementation of the multigranular virtual topology solution using the MILP formulation (left) and heuristic approach (right).... .85

Fig. 4.8: Port requirements at Node 2 of the 5-node sample network for the realization of the multigranular virtual topology solution using the MILP formulation (left) and heuristic approach (right)........86

Fig. 4.9: Port requirements at Node 3 of the 5-node sample network for the implementation of the multigranular virtual topology solution using the MILP formulation (left) and heuristic approach (right) .......86

Fig. 4.10: Port requirements at Node 4 of the 5-node sample network for the implementation of the multigranular virtual topology solution using the MILP formulation (left) and heuristic approach (right).......86

Fig. 4.11: 8-node sample network fiber layout with span distances in $\mathrm{km} \ldots \ldots \ldots \ldots \ldots \ldots \ldots \ldots \ldots \ldots . \ldots 7$

Fig. 4.12: Minimum fiber requirements for all heuristics with/without the adjustment phase..............89

Fig. 4.13: Weighted port count at $F_{\text {min }}$ for all heuristics with/without the adjustment phase...............89

Fig. 4.14: Weighted port count vs. $F$, where $F \geq F_{\text {min }}$ for all sorting policies without bifurcating demands....91

Fig. 4.15: Weighted port count vs. $F$, where $F \geq F_{\text {min }}$ for all sorting policies by allowing the bifurcation of

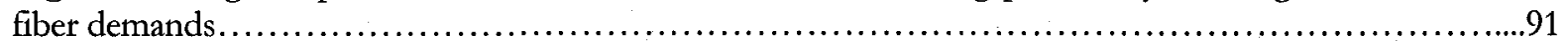

Fig. 4.16: Weighted port count vs. $F$, where $F \geq F_{\text {min }}$ for all sorting policies by allowing the bifurcation of all

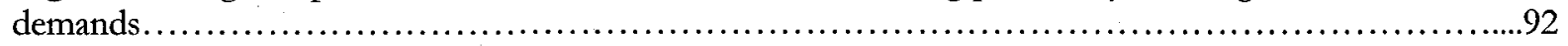

Fig. 4.17: The minimum fiber requirements for all heuristic variations for $\mathrm{E} / \mathrm{O}$ ratio $=2$ and $3 \ldots \ldots \ldots \ldots . . .93$

Fig. 4.18: The weighted port count at $F_{\text {min }}$ for all heuristic variations for $\mathrm{E} / \mathrm{O}$ ratio $=2$ and $3 \ldots \ldots \ldots \ldots \ldots . \ldots 4$

Fig. 4.19: 'The optimum fiber requirement for all heuristic variations for $\mathrm{E} / \mathrm{O}$ ratio $=2$ and $3 \ldots \ldots \ldots \ldots \ldots 9$

Fig. 4.20: The weighted port count at $F_{\text {opt }}$ for all heuristic variations for $\mathrm{E} / \mathrm{O}$ ratio $=2$ and $3 \ldots \ldots \ldots \ldots \ldots 9$

Fig. 4.21: The resultant "unweighted" port count breakdown at all nodes of the 8-node network at $F_{\text {op }}=22$

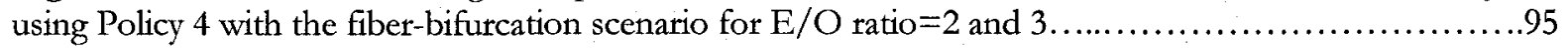

Fig. 4.22: The resultant "unweighted" port count breakdown at all nodes of the 8-node network at $F_{\text {opt }}=22$

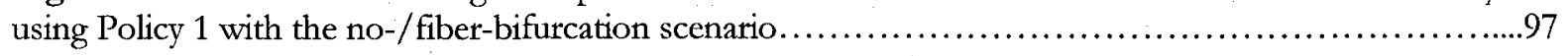

Fig. 4.23: The resultant "unweighted" port count breakdown at all nodes of the 8-node network at $F_{\text {opt }}=22$ using Policy 1 with the all-demand bifurcation scenario... 
Fig. 4.24: The required number of (de)multiplexers at all nodes of the 8-node network at $F_{o f t}=22$ using

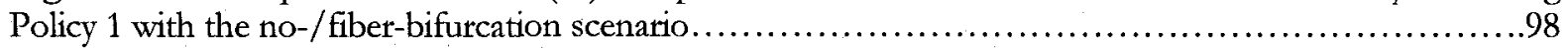

Fig. 4.25: The required number of (de)multiplexers at all nodes of the 8-node network at $F_{\text {opr }}=22$ using Policy 1 with the all-demand bifurcation scenario................................................ 98

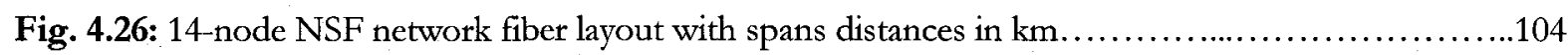

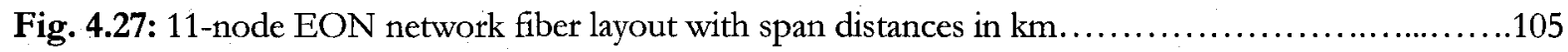

Fig. 5.1: A high-level view of the heuristic approach proposed to solve the RMGPA problem without the color-continuity constraints, with/without the optical-reach constraints, and with/without customizing the optical reach.

.131

Fig. 5.2: Multi-granular virtual topology solutions for the 5-node network example, given that $C=10 \mathrm{~Gb} / \mathrm{s}$,

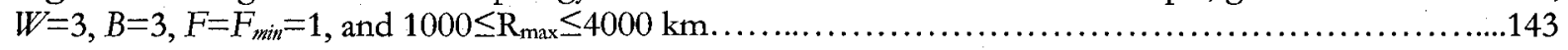

Fig. 5.3: Fiber requirements for the case of the 8-node network and $(0,1500) \mathrm{Gb} / \mathrm{s}$ range demands with $\mathrm{E} / \mathrm{O}$ ratio $=2$ using different heuristic variations with/without color conversions: (a) $F_{\text {minin }}$; (b) $F_{\text {opt }} \ldots \ldots \ldots \ldots 146$

Fig. 5.4: Weighted port count for the case of the 8-node network and $(0,1500) \mathrm{Gb} / \mathrm{s}$ range demands with $\mathrm{E} / \mathrm{O}$ ratio $=2$ using different heuristic variations with/without color conversions: (a) weighted port count at $F_{\text {min }}$; (b) weighted port count at $F_{o p}$ 146

Fig. 5.5: The number of OEO conversions for the case of the 8-node network and $(0,1500) \mathrm{Gb} / \mathrm{s}$ range demands with $\mathrm{E} / \mathrm{O}$ ratio $=2$ using different heuristic variations with color conversions................146

Fig. 5.6: Fiber requirements for the case of the 8-node network and $(0,1500) \mathrm{Gb} / \mathrm{s}$ range demands with $\mathrm{E} / \mathrm{O}$ ratio $=3$ using different heuristic variations with/without color conversions: (a) at $F_{m i n}$; (b) at

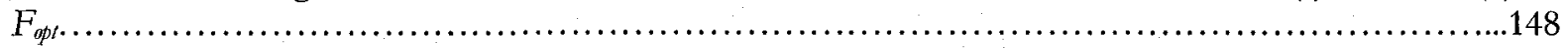

Fig. 5.7: Weighted port count for the case of the 8-node network and $(0,1500) \mathrm{Gb} / \mathrm{s}$ range demands with $\mathrm{E} / \mathrm{O}$ ratio=3 using different heuristic variations with/without color conversions: (a) at $F_{\text {minim }}$ (b) at

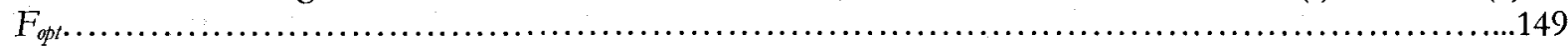

Fig. 5.8: The number of OEO conversions for the case of the 8-node network and $(0,1500) \mathrm{Gb} / \mathrm{s}$ range demands with $\mathrm{E} / \mathrm{O}$ ratio $=3$ using different heuristic variations with color conversions..................149

Fig. 5.9: The resultant "unweighted" port count breakdown at all nodes of the 8 -node network at $F_{\text {min }}=22$ using Policy 4 and the no-bifurcation scenario in the heuristic: (a) with $\mathrm{E} / \mathrm{O}$ ratio=2; (b) with $\mathrm{E} / \mathrm{O}$

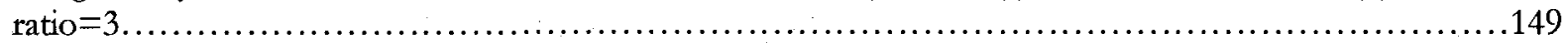

Fig. 5.10: Fiber requirements vs. maximum optical reach for the case of the 8-node network and $(0,1500)$ $\mathrm{Gb} / \mathrm{s}$ range demands using heuristics with Policies 1-6 and the no-bifurcation scenario and without

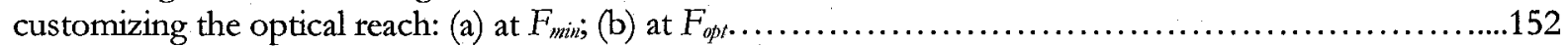

Fig. 5.11: Weighted port count vs. maximum optical reach for the case of the 8-node network and $(0,1500)$ $\mathrm{Gb} / \mathrm{s}$ range demands using heuristics with Policies 1-6 and the no-bifurcation scenario and without

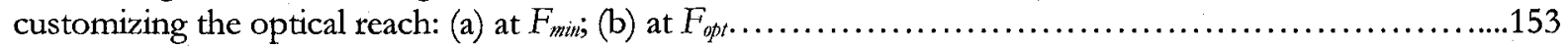

Fig. 5.12: Number of OEO conversions vs. maximum optical reach for the case of the 8-node network and $(0,1500) \mathrm{Gb} / \mathrm{s}$ range demands using heuristics with Policies 1-6 and the no-bifurcation scenario and without customizing the optical reach: (a) at $F_{m i n}$; (b) at $F_{o p t}$

Fig. 5.13: Fiber requirements vs. maximum optical reach for the case of the 8-node network and $(0,1500)$ $\mathrm{Gb} / \mathrm{s}$ range demands using heuristics with Policies $1-6$ and the no-bifurcation scenario and with

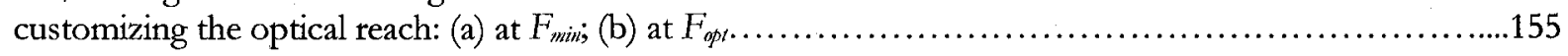


Fig. 5.14: Weighted port count vs. maximum optical reach for the case of the 8-node network and $(0,1500)$ $\mathrm{Gb} / \mathrm{s}$ range demands using heuristics with Policies 1-6 and the no-bifurcation scenario and with

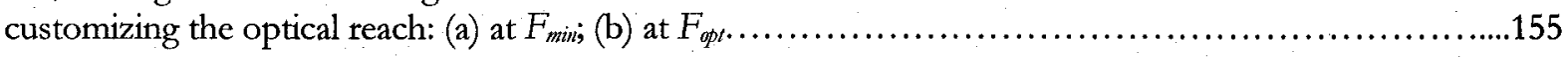

Fig. 5.15: Number of OEO conversions vs. maximum optical reach for the case of the 8-node network and $(0,1500) \mathrm{Gb} / \mathrm{s}$ range demands using heuristics with Policies 1-6 and the no-bifurcation scenario and with

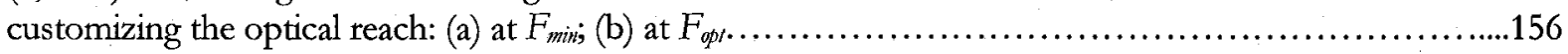




\section{List of Tables}

Table 2.1: Comparison of previous work based on various issues

Table 3.1: A demonstrative example to compare different MG-OXC architectures in terms of the weighted port count in a single node for different volumes of add traffic, given that all optical port types have the same weight, the EXC port weight is twice the optical port weight, and the number of

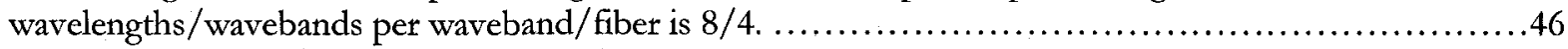

Table 3.2: A demonstrative example to compare different MG-OXC architectures in terms of the weighted port count in a single node for different volumes of pass-through traffic, given that all optical port types have the same weight, the EXC port weight is twice the optical port weight, and the number of wavelengths/wavebands per waveband/fiber is $8 / 4$.... . .46

Table 3.3: Comparison of different $s-d$ path types in terms of their involvement in the grouping process at all levels.

Table 4.1: Comparison between the results obtained using the MILP formulation and heuristic approach for the 5-node network example of solving the RMGPA problem with the color-continuity constraints and

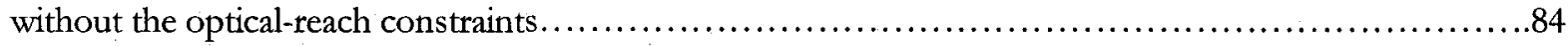

Table 4.2: The 8-node network raw traffic demands, which are chosen randomly from the range $(0,1000)$

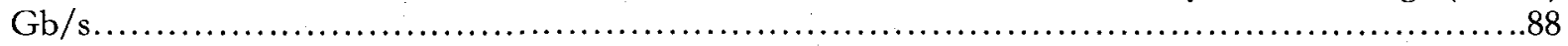

Table 4.3: The 8-node network multi-granularity demands, which are generated from the $(0,1000) \mathrm{Gb} / \mathrm{s}$ range raw demands along with the use of the $C=10 \mathrm{~Gb} / \mathrm{s}, W=4, \& \quad B=4$

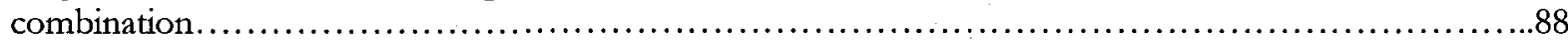

Table 4.4: The 8-node network optimized multi-granularity demands (which are generated from the raw traffic shown in Table 4.2) for different $C[\mathrm{~Gb} / \mathrm{s}]-W-B$ combinations, provided that all combinations give the same fiber capacity.

Table 4.5: Heuristics comparison under the fiber-scarce situation for different $C-B-W$ combinations, which lead to the same fiber capacity.

Table 4.6: Heuristics comparison under the fiber-plentiful situation for different $C-B-W$ combinations, which lead to the same fiber capacity....

Table 4.7: The 8-node network optimized multi-granularity demands (which are generated from the raw traffic shown in Table 4.2) for different fiber capacities, given that the fiber capacity $=C \times B \times$ $W \ldots$

Table 4.8: Heuristics comparison under the fiber-scarce situation for different $C-B-W$ combinations, which lead to different fiber capacities.

Table 4.9: Heuristics comparison under the fiber-plentiful situation for different $C-B-I W$ combinations, which lead to different fiber capacities.... . .103

Table 4.10: Comparison of heuristic variations for different traffic patterns in the 8-node network using the 10-4-4 combination under the fiber-scarce situation.

Table 4.11: Comparison of heuristic variations for different traffic patterns in the 8-node network using the 10-8-8 combination under the fiber-scarce situation. .

Table 4.12: Comparison of heuristic variations for different traffic patterns in the 8-node network using the 40-8-8 combination under the fiber-scarce situation. 
Table 4.13: Comparison of heuristic variations for different traffic patterns in the NSF network using the 10-4-4 combination under the fiber-scarce situation.

Table 4.14: Comparison of heuristic variations for different traffic patterns in the NSF network using the 10-8-8 combination under the fiber-scarce situation.

Table 4.15: Comparison of heuristic variations for different traffic patterns in the NSF network using the 40-8-8 combination under the fiber-scarce situation.

Table 4.16: Comparison of heuristic variations for different traffic patterns in the EON network using the 10-4-4 combination under the fiber-scarce situation. ...112

Table 4.17: Comparison of heuristic variations for different traffic patterns in the EON network using the 10-8-8 combination under the fiber-scarce situation.

Table 4.18: Comparison of heuristic variations for different traffic patterns in the EON network using the 40-8-8 combination under the fiber-scarce situation.

Table 4.19: Comparison of heuristic variations for different traffic patterns in the 8-node network using the 10-4-4 combination under the fiber-plentiful situation. .116

Table 4.20: Comparison of heuristic variations for different traffic patterns in the 8-node network using the 10-8-8 combination under the fiber-plentiful situation. .116

Table 4.21: Comparison of heuristic variations for different traffic patterns in the 8-node network using the 40-8-8 combination under the fiber-plentiful situation.

Table 4.22: Comparison of heuristic variations for different traffic patterns in the NSF network using the 10-4-4 combination under the fiber-plentiful situation.

Table 4.23: Comparison of heuristic variations for different traffic patterns in the NSF network using the 10-8-8 combination under the fiber-plentiful situation. .118

Table 4.24: Comparison of heuristic variations for different traffic patterns in the NSF network using the 40-8-8 combination under the fiber-plentiful situation. .118

Table 4.25: Comparison of heuristic variations for different traffic patterns in the EON network using the 10-4-4 combination under the fiber-plentiful situation. . .119

Table 4.26; Comparison of heuristic variations for different traffic patterns in the EON network using the 10-8-8 combination under the fiber-plentiful situation. .119

Table 4.27: Comparison of heuristic variations for different traffic patterns in the EON network using the 40-8-8 combination under the fiber-plentiful situation.

Table 4.28: The winning $C-W-B$ combination that leads to the minimal weighted port count in the fiberplentiful situation as the traffic pattern and topology change.

Table 5.1: Comparison between the results obtained using the MILP formulation and heuristic approach for the 5-node network example of solving the RMGPA problem without the color-continuity constraints, with the optical-reach constraints, and without the optical-reach customization.

Table 5.2: The 8-node network raw traffic demands, which are chosen randomly from the range $(0,1500)$ $\mathrm{Gb} / \mathrm{s}$.

Table 5.3: The 8-node network multi-granularity demands, which are generated from the $(0,1500) \mathrm{Gb} / \mathrm{s}$ range raw demands along with the use of the $C=10 \mathrm{~Gb} / \mathrm{s}, W=4, \& B=4$ combination.

Table 5.4: Summary of the heuristics comparison in the fiber-scarce situation with and without the opticalreach customization for the case of the 8-node network and $(0,1500) \mathrm{Gb} / \mathrm{s}$ range demands using different $\mathrm{E} / \mathrm{O}$ cost ratios. 
Table 5.5: Summary of the heuristics comparison in the fiber-plentiful situation with and without the optical-reach customization for the case of the 8-node network and $(0,1500) \mathrm{Gb} / \mathrm{s}$ range demands using different $\mathrm{E} / \mathrm{O}$ cost ratios.

Table 5.6: Results of the best heuristic variation with the color conversion capability for different traffic patterns in the 8-node network using the 10-4-4 combination under the fiber-scarce situation. The difference in results is relative to the best results obtained by keeping the color-continuity constraints.......161

Table 5.7: Results of the best heuristic variation with the color conversion capability for different traffic patterns in the 8-node network using the 10-8-8 combination under the fiber-scarce situation. The difference in results is relative to the best results obtained by keeping the color-continuity constraints......161

Table 5.8: Results of the best heuristic variation with the color conversion capability for different traffic patterns in the 8-node network using the 40-8-8 combination under the fiber-scarce situation. The difference in results is relative to the best results obtained by keeping the color-continuity constraints......162

Table 5.9: Results of the best heuristic variation with the color conversion capability for different traffic patterns in the NSF network using the 10-8-8 combination under the fiber-scarce situation. The difference in results is relative to the best results obtained by keeping the color-continuity constraints................162

Table 5.10: Results of the best heuristic variation with the color conversion capability for different traffic patterns in the EON network using the 10-8-8 combination under the fiber-scarce situation. The difference in results is relative to the best results obtained by keeping the color-continuity constraints.... 163

Table 5.11: Results of the best heuristic variation with the color conversion capability for different traffic patterns in the 8-node network using the 10-4-4 combination under the fiber-plentiful situation. The difference in results is relative to the best results obtained by keeping the color-continuity constraints......166

Table 5.12: Results of the best heuristic variation with the color conversion capability for different traffic patterns in the 8-node network using the 10-8-8 combination under the fiber-plentiful situation. The difference in results is relative to the best results obtained by keeping the color-continuity constraints......166

Table 5.13: Results of the best heuristic variation with the color conversion capability for different traffic patterns in the 8-node network using the 40-8-8 combination under the fiber-plentiful situation. The difference in results is relative to the best results obtained by keeping the color-continuity constraints......167

Table 5.14: Results of the best heuristic variation with the color conversion capability for different traffic patterns in the NSF network using the 10-8-8 combination under the fiber-plentiful situation. The difference in results is relative to the best results obtained by keeping the color-continuity constraints.....167

Table 5.15: Results of the best heuristic variation with the color conversion capability for different traffic patterns in the EON network using the 10-8-8 combination under the fiber-plentiful situation. The difference in results is relative to the best results obtained by keeping the color-continuity constraints.....168

Table 5.16: Results of the best heuristic variation with the color conversion and signal regeneration capabilities with/without the optical-reach customization for different traffic patterns in the 8-node network

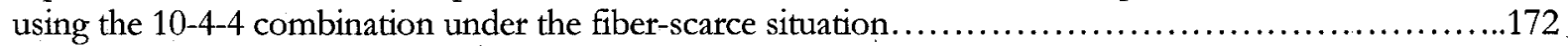

Table 5.17: Results of the best heuristic variation with the color conversion and signal regeneration capabilities with/without the optical-reach customization for different traffic patterns in the 8-node network using the 10-8-8 combination under the fiber-scarce situation.

Table 5.18: Results of the best heuristic variation with the color conversion and signal regeneration capabilities with/without the optical-reach customization for different traffic patterns in the 8-node network using the 40-8-8 combination under the fiber-scarce situation. 
Table 5.19: Results of the best heuristic variation with the color conversion and signal regeneration capabilities with/without the optical-reach customization for different traffic patterns in the NSF network using the 10-8-8 combination under the fiber-scarce situation.

Table 5.20: Results of the best heuristic variation with the color conversion and signal regeneration capabilities with/without the optical-reach customization for different traffic patterns in the EON network using the 10-8-8 combination under the fiber-scarce situation.

Table 5.21: Results of the best heuristic variation with the color conversion and signal regeneration capabilities with/without the optical-reach customization for different traffic patterns in the 8-node network using the 10-4-4 combination under the fiber-plentiful situation.

Table 5.22: Results of the best heuristic variation with the color conversion and signal regeneration capabilities with/without the optical-reach customization for different traffic patterns in the 8-node network using the 10-8-8 combination under the fiber-plentiful situation.

Table 5.23: Results of the best heuristic variation with the color conversion and signal regeneration capabilities with/without the optical-reach customization for different traffic patterns in the 8-node network using the 40-8-8 combination under the fiber-plentiful situation.

Table 5.24: Results of the best heuristic variation with the color conversion and signal regeneration capabilities with/without the optical-reach customization for different traffic patterns in the NSF network using the 10-8-8 combination under the fiber-plentiful situation.

Table 5.25: Results of the best heuristic variation with the color conversion and signal regeneration capabilities with/without the optical-reach customization for different traffic patterns with the EON network using the 10-8-8 combination under the fiber-plentiful situation...

Table A.1: Comparison of no-bifurcation-based heuristics without the color conversion and signal regeneration capabilities for the 8-node network.

Table A.2: Comparison of fiber-bifurcation-based heuristics without the color conversion and signal regeneration capabilities for the 8-node network.

Table A.3: Comparison of all-bifurcation-based heuristics without the color conversion and signal regeneration capabilities for the 8-node network

Table A.4: Comparison of no-bifurcation-based heuristics without the color conversion and signal regeneration capabilities for the NSF network.

Table A.5: Comparison of fiber-bifurcation-based heuristics without the color conversion and signal regeneration capabilities for the NSF network.

Table A.6: Comparison of all-bifurcation-based heuristics without the color conversion and signal

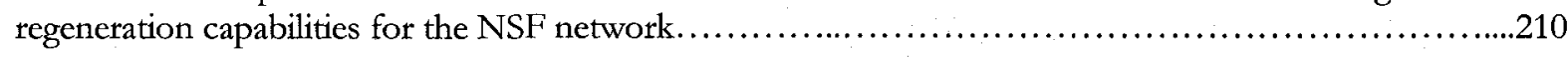

Table A.7: Comparison of no-bifurcation-based heuristics without the color conversion and signal regeneration capabilities for the EON network

Table A.8: Comparison of fiber-bifurcation-based heuristics without the color conversion and signal

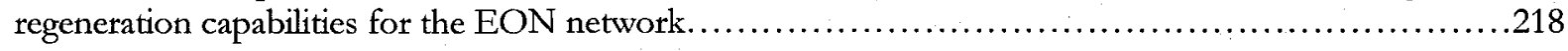

Table A.9: Comparison of all-bifurcation-based heuristics without the color conversion and signal regeneration capabilities for the EON network

Table B.1: Comparison of no-bifurcation-based heuristics with the color conversion capability for the 8node network. ....

Table B.2: Comparison of fiber-bifurcation-based heuristics with the color conversion capability for the 8node network.... 
Table B.3: Comparison of no-bifurcation-based heuristics with the color conversion capability for the NSF network.

Table B.4: Comparison of fiber-bifurcation-based heuristics with the color conversion capability for the NSF network.

Table B.5: Comparison of no-bifurcation-based heuristics with the color conversion capability for the EON network.

Table B.6: Comparison of fiber-bifurcation-based heuristics with the color conversion capability for the EON network.

Table C.1: Comparison of no-bifurcation-based heuristics without the optical-reach customization for the 8-node network.

Table C.2: Comparison of no-bifurcation-based heuristics with the optical-reach customization for the 8node network.

Table C.3: Comparison of fiber-bifurcation-based heuristics without the optical-reach customization for the 8-node network.

Table C.4: Comparison of fiber-bifurcation-based heuristics with the optical-reach customization for the 8node network.

Table C.5: Comparison of no-bifurcation-based heuristics without the reach customization for the NSF network.

Table C.6: Comparison of no-bifurcation-based heuristics with the optical-reach customization for the NSF network.

Table C.7: Comparison of fiber-bifurcation-based heuristics without the optical-reach customization for the NSF network.

Table C.8: Comparison of fiber-bifurcation-based heuristics with the optical-reach customization for the NSF network.

Table C.9: Comparison of no-bifurcation-based heuristics without the optical-reach customization for the EON network. ....

Table C.10: Comparison of no-bifurcation-based heuristics with the optical-reach customization for the EON network.

Table C.11: Comparison of fiber-bifurcation-based heuristics without the optical-reach customization for the EON network.

Table C.12: Comparison of fiber-bifurcation-based heuristics with the optical-reach customization for the EON network. 


\section{Abbreviations}

Re-amplification, Reshaping, and Re-timing

BLP

Binary Linear Programming

BPHT Balanced Path Routing with Heavy-Traffic first waveband assignment

BSC

Band Switch Capable

BTMH

Balanced Traffic routing with Maximum-Hop first waveband assignment

BXC

Band Crossconnect

CBS

Clear Band-Switched

CD

Chromatic Dispersion

CFS

Clear Fiber-Switched

CI

Confidence Interval

CWS

Clear Wavelength-Switched

EDFA

Erbium-Doped Fiber Amplifier

EON

European Optical Network

EXC

Electronic Crossconnect

FEM

Full OEO-based Merging

FF

First-Fit

F-LSP

Fiber-Switched Path

FSC

Fiber Switch Capable

FXC

Fiber Crossconnect

GMPLS Generalized Multiprotocol Label Switching

HXC Hierarchical Crossconnect

IETF Internet Engineering Task Force

ILP Integer Linear Programming

LCBF Least Cost Branch First

LP Linear Programming

LRH Lagrangean Relaxation with Heuristics

LSC Lambda Switch Capable

$\lambda$-ISP Wavelength-Switched Path 
MEMS Micro-Electro-Mechanical Systems

MG-OXC Multi-Granular Optical Crossconnect

MG-XC Multi-Granular Crossconnect

MILP _ Mixed Integer Linear Programming

MOR Maximum Overlap Ratio

MPLS Multiprotocol Label Switching

NLP Non-Linear Programming

NP Nondeterministic Polynomial

NSF National Science Foundation

OEO Optical-Electrical-Optical

OOO All-Optical

OXC Optical crossconnect

PEM Partial OEO-based Merging

PMD Polarization Mode Dispersion

RMGPA Routing Multi-Granular Paths Allocation

RNG Random Number Generator

ROADM Reconfigurable Optical Add/Drop Multiplexers

RWA Routing and Wavelength Assignment

$\mathrm{RWA}^{+} \quad \mathrm{RWA}$ in the multi-granular network

RWAT Routing and Wavelength Assignment with Tunnel allocation

TDM Time-Division-Multiplexing

UBS Unclear Band-Switched

UFS Unclear Fiber-Switched

UWS Unclear Wavelength-Switched

WB-LSP Waveband-Switched Path

WBO Waveband Oblivious Optimal

WBS Waveband Switching

WCP Wavelength Convergence Point

WDM Wavelength Division Multiplexing

WDP Wavelength Divergence Point

WXC Wavelength Crossconnect 


\section{Chapter 1}

\section{Introduction}

\subsection{Background and Motivations}

In order to satisfy the continuous growth in Internet traffic along with the dramatic increase in both broadband and wireless applications, more efficient and cost-effective transport network technologies are required. Optical networking using Wavelength Division Multiplexing (WDM) has become a key technology for accommodating these rapidly expanding demands [Gree93][VGIz03]. WDM divides the enormous fiber bandwidth into a large number of wavelengths (or $\lambda \mathrm{s}$ ), each now operating at a rate of $40 \mathrm{~Gb} / \mathrm{s}$ [FULL06]. With advances in the WDM technology, WDM networks using wavelength-routing by Optical Crossconnets (OXCs)/Reconfigurable Optical Add/Drop Multiplexers (ROADMs) have been widely recognized as the most feasible architectural solutions for transport networks [Lee+02][LYTi04]. In addition, OXCs/ROADMs allow transport networks to be reconfigurable to adapt to changes in traffic patterns as well as restore these networks after failures [HSKO99][Ciar00].

To meet the demands of ever-increasing traffic, a large number of wavelengths will ultimately be required. However, increasing the number of wavelengths per fiber will result in (1) an increased number of OXC ports, (2) increased footprint, (3) increased power consumption, (4) increased complexity of OXCs, (5) increased difficulty in implementing and maintaining OXCs, (6) increased complexity associated with the control and management of the network, and (7) more severe cross-talk requirements for WDM demultiplexers in OXCs [Acam94][HSKO99][Ciar00][Lee+02] [CAXQ03][VaJu04][ZZFZ04]. In order to overcome these drawbacks while maintaining high 
scalability, as well as to keep network complexity at a reasonable level and flexibility at a low cost, the number of ports in the switching fabric can be reduced by routing a group of wavelengths, called a waveband, together through a single port. Further port reduction is possible if these wavebands are grouped again into one fiber and routed through a single port. Reducing the port count is one of the main factors contributing to the cost reduction of OXC [NVDo01]. An OXC that uses this multi-granular switching concept is referred to as a Multi-Granular OXC (MG-OXC) [HSKO99][Ciar00][NVDo00][NVDo01][CAXQ03][LYTi04][VaJu04].

On the other hand, the idea of multi-granularity traffic flows, as shown in Fig. 1.1, has led to Generalized Multiprotocol Label Switching (GMPLS) since the early 2000s [Man+01]. GMPLS was extended from Multiprotocol Label Switching (MPLS) by the Internet Engineering Task Force (IETF) to support not only sub-wavelength switching but also wavelength, waveband, and fiber switching. This means that, with GMPLS, the same control plane can be used by both IP routers and other equipment such as OXCs [AwRe01]. GMPLS-based routers that use the multigranular switching concept have already been developed [Sat+02]. Moreover, for existing MPLS networks, the IETF is working on developing an appropriate strategy for the migration from MPLS to GMPLS. The strategy can be based on a service provider's network deployment plan, customer demand, available network equipment, etc [SPL+06].

Although a Label-Switched Path (LSP) in MPLS and a Wavelength-Switched Path $(\lambda$-LSP) in wavelength-routing networks are fundamentally different, GMPLS is equipped with the ability to provision multi-granularity flows and adds a suite of mechanisms that assigns a generalized label to these flows as follows and as illustrated in Fig. 1.1. First, a sub- $\lambda$-LSP is labeled with its frame/cell/packet header or the serial number of the TDM time slot. Second, a $\lambda$-LSP is labeled 
and switched according to its wavelength number. Next, with the MG-OXC, the switching types and traffic granularities are no longer limited to sub- $\lambda$ - and $\lambda$-LSPs, but consecutive wavelengths can be grouped as a waveband LSP (WB-LSP) that is labeled and switched as a whole according to its waveband number. Moreover, consecutive wavebands can be grouped as a fiber LSP (F-LSP) that is labeled and switched as an entity based on its fiber code (or fiber label/ID) on the unidirectional physical link [Ban+01][HoMo02a] [MoHo03].

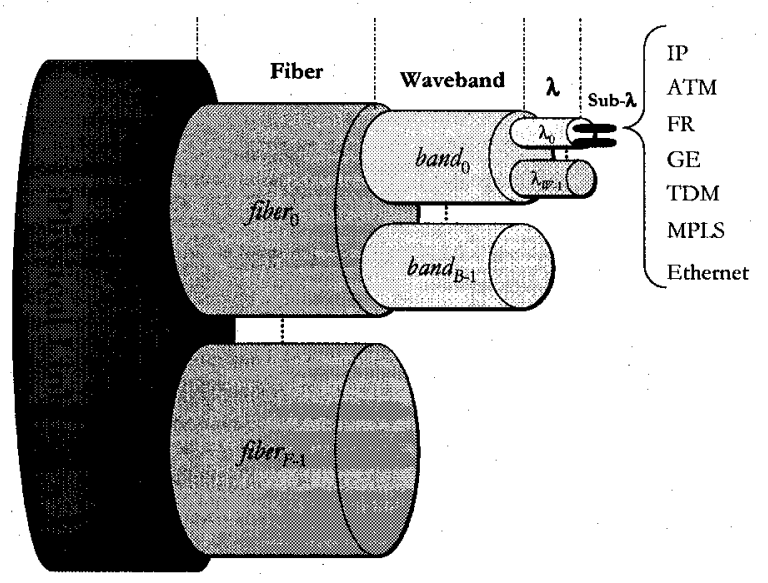

Fig. 1.1: Traffic flow hierarchy defined in GMPLS.

One major challenge in WDM networks has been to develop efficient methods for solving the Routing and Wavelength Assignment (RWA) problem. The RWA problem deals with (1) finding routes for traffic demands between source nodes to their respective destinations; and (2) assigning wavelengths to these routes, which is subject to the wavelength-continuity constraint if wavelength converters are absent. It has been shown that the RWA problem is an NP-complete problem [CGKa92]. Numerous approximation methods and heuristics have been extensively studied and proposed in [CGKa92][ZhAc95][RaSi96][LeLi96][MBRM96][GSMa97][Mukh97][RaSi98] [NaMo99a][NaMo99b][StBa99][DuRo00][LMMa00][ZJMu00][MoHo03]JMTh04][NaMo05] and their references with the aim of balancing the trade-off between accuracy and computational time complexity. However, once the multi-granular switching concept is taken into consideration, most 
of the existing RWA methods become functionally or economically infeasible [CAXQ03][LYTi04] [VaJu04][CAQi06][VaJu06]. Therefore, the efficient implementation of the GMPLS flow hierarchy has to be delivered by the appropriate routing, grouping, and label (or color) assignment of all flows [IGKV03]. Although a significant amount of open research has been carried out in this area, many unexplored issues still need to be addressed and investigated.

\subsection{Objectives}

The aim of our research is to contribute to the ongoing research and development in the area of GMPLS-based (or multi-granular) transport networks. Our focus is on developing new efficient methods for planning ${ }^{1}$ GMPLS-based transport mesh networks with and without the color conversion and optical signal regeneration capabilities. The broad objective of this thesis is to address many unexplored issues in this domain. Our objectives can be summarized as follows:

1. Consider the whole traffic hierarchy defined in GMPLS, namely sub-wavelength, wavelength, waveband, and fiber demands.

2. Create/adjust multi-granularity traffic demands in a way that maximizes the end-to-end grouping of demands of the same source and destination.

3. Perform (de)grouping of traffic flows at all granularity levels.

4. Incorporate the demand-bifurcation ${ }^{2}$ feature into the planning process and study its impact on resource utilization and port count.

5. Consider wavelength, waveband, and fiber conversion capability in the planning process and study its effect on resource utilization and port count. This means that the color-continuity constraint is not necessarily satisfied along the wavelength/waveband/fiber-switched path.

\footnotetext{
1 For the rest of this thesis, the terms planning, dimensioning, and designing refer to the same concept and will be used interchangeably.

${ }^{2}$ Bifurcation is the act of allowing the units of a node pair traffic demand of the same granularity to use different routes.
} 
6. Incorporate the optical signal regeneration capability into the planning process and study its impact on resource utilization and port count. This means that the optical-reach ${ }^{3}$ constraint needs to be used to ensure that the length of the all-optical path stays within the maximum optical reach value.

7. Consider the optical-reach customization in the planning process and study its significance on resource utilization and port count. Without the optical-reach customization, all network transceivers must have the same maximum optical reach, whereas customizing the reach means that the reach of the transceiver is decided based on the length of the all-optical path launched/terminated at the transceiver.

8. Develop a new GMPLS-based transport node architecture that is capable of (i) (de)grouping and switching the whole traffic flow hierarchy defined in GMPLS; and (ii) performing color conversion and optical signal regeneration functionalities on wavelength-, waveband-, and fiber-switched paths.

9. Develop Mixed Integer Linear Programming (MILP)/Non-Linear Programming (NLP) models, which address the afore-mentioned issues, to solve small-sized planning problems optimally and use their solutions as benchmarks for other optimization methods.

10. Develop fast and efficient planning heuristics, which also incorporate the afore-mentioned issues, to solve large-sized planning problems sub-optimally.

\subsection{Thesis Contributions}

The contributions of this thesis lie in the development of mathematical programming models and heuristics in conjunction with a newly developed GMPLS-based node architecture to address

\footnotetext{
3 Optical reach is the distance an optical signal can travel without the need for regeneration [Simm05].
} 
effectively the problem of planning a GMPLS-based transport network with and without the color conversion and signal regeneration capabilities. The contributions can be summarized as follows:

1. A new GMPLS-based node architecture that consists of a scalable MG-OXC segment and an Electronic Crossconnect (EXC) segment has been proposed. The hybrid architecture handles the entire traffic hierarchy defined in GMPLS. The MG-OXC segment provides both the optical-switching of wavelength-, waveband-, and fiber-switched paths and the grouping/degrouping of wavelengths into/from wavebands and wavebands into/from fibers. The EXC segment provides the electronic-switching of sub-wavelength flows, grouping/degrouping of sub-wavelength flows into/from wavelengths, and color conversion and regeneration of wavelength-, waveband-, and fiber-switched paths.

2. A method of generating optimized multi-granularity traffic demands has been devised to maximize the end-to-end grouping.

3. An MILP model has been developed to plan exactly a GMPLS-based transport network without color conversion and regeneration capabilities. This MILP model has been successfully solved for small-sized planning problems.

4. An MILP model has been developed to plan exactly a GMPLS-based transport network with color conversion and regeneration capabilities and without customizing the optical reach. This MILP model has been solved successfully for small-sized planning problems.

5. An NLP model has also been formulated to plan exactly a GMPLS-based transport network with color conversion and regeneration capabilities and with the customization of the optical reach. Although the model has not been solved due to its extreme complexity, it provides a foundation for finding the optimal solution for this problem in the future.

6. Various efficient heuristics have been proposed for solving large-sized planning problems (the afore-mentioned versions) in a reasonable amount of time. The proposed heuristics have been 
validated with the solutions to the MILP models and have been proven to be good approximations of the exact solutions.

7. The results, through many illustrative examples, have provided valuable insights into the effects of many parameters and the benefits of many heuristic enhancements on the planning solutions.

8. To obtain the best solutions to the planning problems in both fiber-scarce and plentiful situations, extensive simulations have been conducted. The results have allowed us to understand how different heuristic variations perform as the traffic pattern, physical topology, fiber capacity, and maximum optical reach value change as well as whether the color-continuity and optical-reach constraints are imposed and whether the optical reach is customized. Thus, instead of trying all possible heuristic variations, the planning time can be significantly reduced if we use only the promising heuristic variations, which have been identified in simulations. Moreover, through simulations, many important questions that can contribute to further research and development in this area have been answered. Among them are the following: (i) the value of bifurcating demands and at what level of granularity bifurcation makes sense, (ii) the significance of performing color conversion, (iii) the significance of customizing the optical reach, and (iv) what maximum optical reach the system vendors need to target.

\subsection{Thesis Outline}

The rest of this thesis is organized as follows. Chapter 2 presents the background and state-of-theart material on the planning of multi-granular optical transport networks. First, a brief overview is given of the network planning process and common optimization techniques used to solve the planning problem. Then, a survey of related work in the area of planning multi-granular transport networks as reported in the current literature is provided. Chapter 3 presents the proposed system 
of the GMPLS-based transport network to be considered in our work. First, it describes in detail the domain of the planning problem in terms of the proposed node architecture, path types, and (de)grouping strategies. Then, the new proposed planning problem that needs to be solved is defined. Afterword, the chapter presents our simplifying assumptions and the approach to be taken to solve the problem optimally and sub-optimally. Chapter 4 focuses on solving the planning problem without the color conversion and signal regeneration capabilities. It first presents the MILP formulation of the problem and then presents the heuristic approach. After that, it presents and discusses the numerical results obtained from the verification of the heuristic approach against the MILP formulation, many demonstration examples to elaborate on various issues, and the evaluation of different heuristic variations via simulation. Chapter 5 focuses on solving the planning problem with the color conversion and signal regeneration capabilities. First, it presents the MILP and NLP formulations of the problem without and with the optical-reach customization respectively. Then, it presents the heuristic approaches. Afterword, it presents and discusses the numerical results obtained from the verification of the heuristic approach against the MILP formulation. After that, for different versions of the planning problem, it presents and discusses the results obtained from both many demonstration examples to elaborate on a variety of issues and the evaluation of different heuristic variations via simulation. Finally, Chapter 6 presents our concluding remarks and proposes topics for future work. 


\section{Chapter 2}

\section{Background and State-of-the-Art}

\subsection{Introduction}

The multi-granular switching concept is expected to be the "future-proof" solution for mitigating the OXC/ROADM scalability problems associated with the continuing growth of traffic in optical transport networks. The new paradigm also brings new challenges, however, since the use of RWA methods with the MG-OXC may lead to more port requirements than the use of ordinary OXC/ROADM. The major challenges in multi-granular transport networks stem from the need to perform the following tasks efficiently: (1) determining the route and assigning colors to wavelength-, waveband-, and fiber-switched paths; and (2) grouping/degrouping sub-wavelengthswitched paths into/from wavelengths, wavelength-switched paths into/from wavebands, and waveband-switched paths into/from fibers. Since we will be dealing with the GMPLS-based transport network from the planning perspective throughout this thesis, our focus will be on solving the static (or off-line) case of this challenging problem. Accordingly, in the first part of this chapter, we present an overview of the most commonly used optimization techniques for planning transport networks. In the second part, we describe the relevant state-of-the art papers in the area of planning multi-granular optical transport networks.

\subsection{What is Network Planning?}

In transport network planning [Ari+00], forecasted traffic demands are given. The aim of the planning process is to come up with the best plan to fulfil these demands under a given physical topology, a set of parameters, and specified constraints and bounds. Fig. 2.1 depicts the general 
task of network planning. The planning process usually optimizes the network cost or port count; however, it may be quite normal to take into account other performance metrics such as the availability, capacity requirement, delay, number of hops, link utilization, etc. The optimum/sub-optimum network plan can include many details, such as the working and protection/restoration routes and the location and size of equipment.

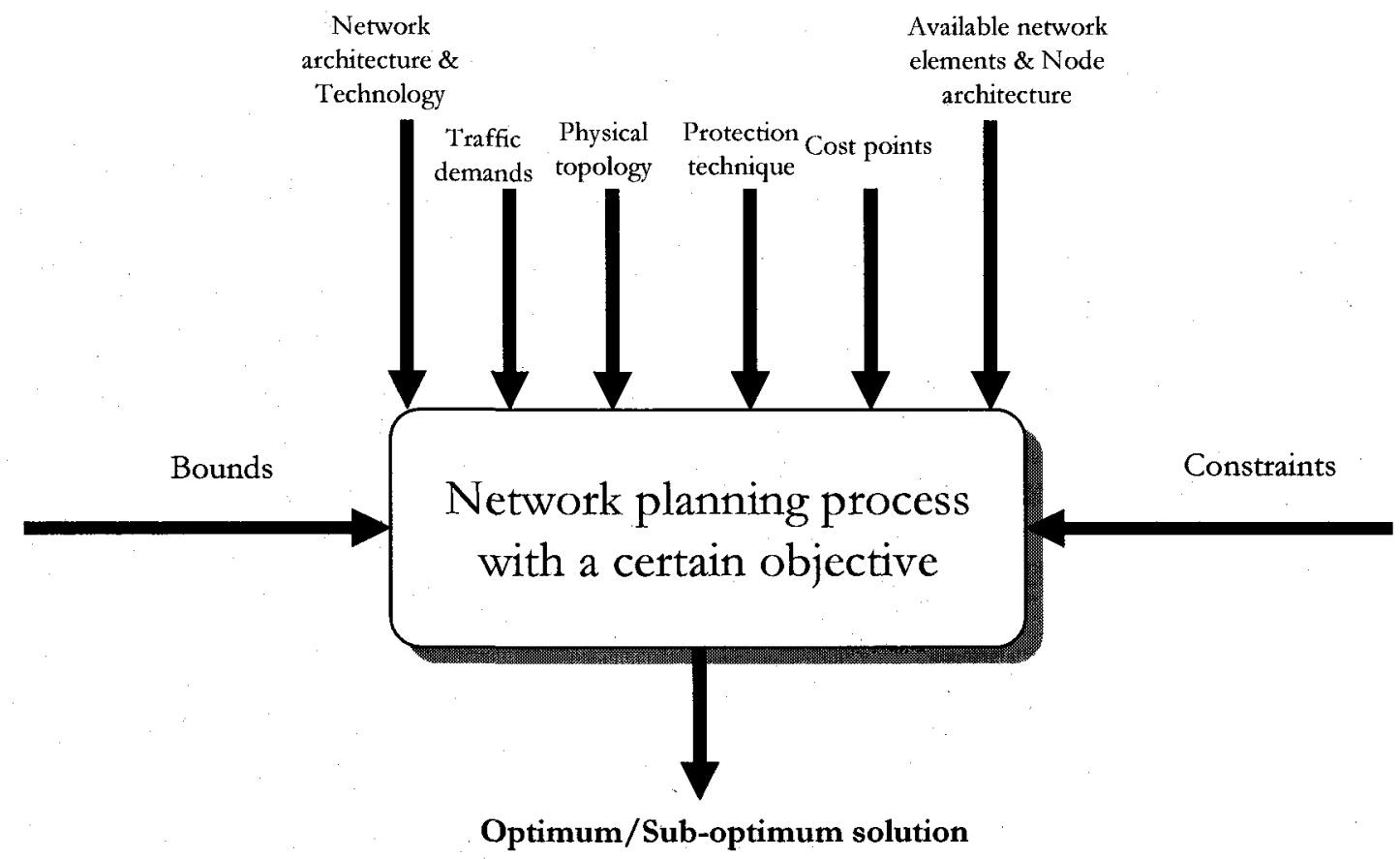

Fig. 2.1: The network planning process.

\subsection{Formulating Optimization Problems}

Generally, the optimization problem consists of four components [PTVF92][WiVe03][Chin04] [Grov04][Chino7]: the decision variables, objective function, constraints, and bounds. $A$ decision variable is the amount of each resource to be determined. Decision variables can be binary, integer, real, or mixed variables. The objective function is a function of the decision variables and can be either minimized or maximized. Also, it can be a linear or non-linear function. The constraints and bounds are sets of inequalities and/or equalities that restrict the decision 
variables to certain predetermined values. A constraint can be a linear or non-linear inequality/equality. Any solution (a set of decision variable values) that satisfies all of the constraints and bounds is said to be a feasible solution.

Most of the transport network planning problems can be formulated mathematically using the Linear Programming (LP) and Non-Linear Programming (NLP) models. The optimum solutions to these models are manageable for small-sized problems; however, as the problem size increases, the solve time increases exponentially and sometimes the solution cannot be reached. This issue necessitates the use of other optimization techniques that achieve sub-optimum solutions in a reasonable amount of time. The factors that determine the problem complexity are as follows: (1) the type and number of variables, (2) the type of the objective function, (3) the type and number of constraints, and (4) the number of bounds. Based on these factors, the optimization problems can be classified into the following categories:

- Linear Programming (LP) problem: variables are real and both the objective function and constraints are linear.

- Integer Linear Programming (ILP) problem: variables are integer and both the objective function and constraints are linear.

- Binary Linear Programming (BLP) problem: a special class of the ILP problem in which variables are binary.

- Mixed Integer Linear Programming (MILP) problem: variables are mixed and both the objective function and constraints are linear.

- Non-Linear Programming (NLP) problem: the objective function or/and at least one of the constraints is non-linear. 
In general, the NLP problem is much more difficult to solve than the ILP/LP problem. On the other hand, the ILP problem is much more difficult to solve than the LP problem. Moreover, solving the ILP problem using an LP-based optimization technique by rounding any non-integer variable value to the closest integer value has proven to be wrong [Chin04][Chin07]. However, if the solution of the LP-based optimization technique yields a solution in which all required integer variables are integer, then this solution is considered an optimum solution to the ILP problem [WiVe03].

There are two formulation approaches that have been widely used in network optimization and they are explained as follows [SwSi02]:

- Link formulation: In this approach, the main variables of the optimization problem are the flows on each link relative to each source-destination pair demand. For each demand, a flowconservation constraint has to be created at every network node. Therefore, the number of constraints grows polynomially with the number of nodes.

- Path formulation: In this approach, a number of predefined possible paths is given for each demand. The main variables are the flows to be routed along each path. The number of paths between a node pair increases exponentially with the number of nodes. To reduce the problem complexity, the path formulation will have to restrict itself to a few paths per node pair.

\subsection{Optimization Techniques}

In this section, the focus will be on the optimization techniques used to solve linear programming models. The non-linear models will not be covered here because none of the previous related work has used these models. In addition, we will be solving only linear 
programming models in our approaches. The optimization techniques can be classified into two main categories: exact techniques and approximation techniques.

\subsubsection{Exact Techniques}

With exact techniques, the optimum solution is always guaranteed. The following are the most commonly used exact methods:

- Simplex method: This method [PTVF92][WiVe03][Chin04][Chin07] is a simple algorithmic engine for solving LP problems. It converts the objective function and the constraints into a tableau where the number of rows represents the number of constraints and the number of columns represents the number of variables. The new tableau is created from the old one through some manipulations until the optimum tableau is reached from which the optimum solution can be extracted. The speed of this method depends mainly on the number of constraints and not the number of variables.

- Dual Simplex method: This method [WiVe03][Grov04] is a modified version of the Simplex method in which the LP tableau is turned sideways; therefore, its speed depends on the number of variables. Accordingly, this method is quite useful when the number of constraints is more than the number of variables.

- Enumeration method: This method [Chin04][Chin07] involves simply enumerating all possible solutions, evaluating them, and then choosing the best one. Given that there could be a huge number of permitted solutions for a particular network planning problem, enumeration is only possible for very-small-sized ILP/BLP problems.

- Branch-and-Bound method: This method [WiVe03][Chin04][Chin07] is a powerful technique for accelerating enumeration. Its main idea is to avoid growing the whole enumeration tree as much as possible. Instead, it grows the tree in stages and only the most promising tree nodes 
at any stage. Essential for this technique is the estimation of a bound on the best value of the objective function that can be obtained by growing a bud node further. This method is commonly used to solve small- and medium-sized ILP/BLP problems.

- Balas' method: This method [WiVe03] is a special type of the branch-and-bound method and is used to solve small- and medium-sized BLP problems.

- Dakin's method: This method [WiVe03] is also a special class of the branch-and-bound method that relies heavily on the Simplex method or other LP methods. Dakin's method is an important method for solving small and medium-sized MILP problems. In this method, the MILP model is treated as an LP model, which means that all non-real variables are treated as real variables, and branching occurs only on variables that are required to be integers. Every time branching occurs, a new constraint is added to the L.P model.

- Dynamic Programming method: This method [WiVe03] is a recursive technique that can be used to solve small- and medium-sized ILP problems. Some resource-allocation problems can be best solved using this technique. This method obtains solutions by breaking up the problem into a series of smaller, more manageable problems. By working backward, the optimum solution to the smaller part of the problem is found first, then the problem is enlarged slightly and the optimum solution is found for the enlarged problem by using the previously found optimum solution.

\subsubsection{Approximation Techniques}

With approximation techniques ${ }^{4}$, a faster solve time is guaranteed for the optimization problems but at the expense of having sub-optimal solutions. The following are the most well-known approximation methods:

${ }^{4}$ Some of the approximation techniques can also be used to solve NLP problems. 
- Heuristic method: This method [WiVe03][Grov04] is based on practical rules that require a deep knowledge of the problem. Although these rules may not have a mathematical proof, they are based on good results obtained in practice. Due to their relative simplicity, heuristic approaches are widely used in the optimization world. The heuristics may be combined with other exact optimization techniques for reducing both the complexity of the problem and solve time.

- Relaxation method: In addition to its ability to provide faster solve time, relaxation [Bert99][Guig03][WiVe03] plays a role in providing bounds for the optimum solution of large-sized problems and can be used as a starting point for other optimization techniques. There are two types of relaxations that are used most frequently: L.P relaxation and Lagrangean relaxation. LP relaxation relaxes the integer variables of the ILP model, which can provide a lower bound for the minimization ILP problem and an upper bound for the maximization ILP problem. On the other hand, Lagrangean relaxation removes some difficult constraints or substitutes them with other looser constrains. This requires the introduction of new variables called Lagrangean multipliers as additional terms in the objective function.

- Simulated Annealing method: This method [PTVF92][WiVe03][Grov04] is a meta-heuristic ${ }^{5}$ technique that attempts to simulate the phenomenon of cooling metals to achieve certain crystal properties. Simulated annealing is an attractive method for solving large-sized optimization problems. The implementation of this method is relatively simple, but requires mastering the fundamental physics of the process or following a trial-error method.

\footnotetext{
5 "Meta-heuristic is a general master strategy to solve problems, and they are based on intelligent search techniques. 'These techniques are based on natural adaptive systems. They can be based on evolutionary principles such as the method of natural selection, physical systems such as annealing process, and human learning as in the brain's adaptive neural strategy and memory".[WiVe03]
} 
Simulated annealing employs a random search that accepts changes that not only decrease the objective function, but also that increase it in order to escape the local optimality. The method parameters, which are fewer than the parameters used in other meta-heuristics, can be controlled to converge in a given time with a "good" solution.

- Genetic Programming metbod: This method [WiVe03][Grov04] is a meta-heuristic technique that attempts to simulate the phenomenon of the natural evolution of species. Genetic programming is a popular method for solving large-sized optimization problems and has a faster convergence. Candidate solutions must be encoded by strings (or chromosomes). Then, an initial population of chromosomes is selected randomly. Within the genetic algorithm, the population selection in each generation is based on the principle of survival of the fittest through a formula that represents the nature of the problem. The genetic programming method handles the problem constraints via a penalty approach, and searches are usually terminated when the best/worst solution has not changed for several generations.

- Tabu Search method: This method [WiVe03][Grov04] is a meta-heuristic technique that makes an extensive use of memory to emulate the heuristic rules that people use to incorporate previous experiences into today's decision-making process. The tabu search method is also used in large-sized optimization problems. Similar to simulated annealing, the aim of the tabu search method is to escape the local optimality; however, it is much greedier than simulated annealing. This characteristic is why the tabu search is more complicated to implement than simulated annealing. Still, in spite of this difficulty, tabu search has a faster convergence and can achieve better sub-optimum solutions. The main idea of the tabu search method is to explore the search space of all possible solutions through a sequence of movements. Thus, the selection of one solution or another will be the best of those possible. 
A tabu list disallows certain moves from taking place. The list specifies restrictions on the direction of moves. After each move is made, the tabu list will be updated. Different tabu searches may use different strategies to manipulate the tabu list. The choice of which moves are forbidden is based on the short-term history (to avoid cycling around a local neighbourhood in the solution space) and long-term history (to conduct searches in the most promising neighbourhoods) of the sequence of moves.

\subsection{Prior Related Work}

Because of its importance and difficulty, the multi-granular switching concept has drawn a lot of attention in recent years. In this section, we take a close look at the related work that has been done from the planning perspective. The review of each study covers various aspects. Among them are the node architecture used, main assumptions, approach and optimization methods used in solving the planning problem, and key findings of the study. The goal of this section is to provide a better background and perspective on our work; however, the studies covered might not represent a complete survey of all of the work done in this area.

\subsubsection{Traffic Grouping Strategies}

In [NVDo01], Noirie et al demonstrated the impact of using different grouping policies for wavelengths and wavebands on the port count of the different switching stages of the MG-OXC. This study is a continuation of the work done in [NVDo00] by the same group, in which wavelengths and wavebands are grouped only if they share the same source and destination. This is what the authors defined as end-to-end grouping. The suggested MG-OXC architecture, Type A as shown in Fig. 2.2, consists of three switching stages: Fiber Crossconnect (FXC), Band Crossconnect (BXC), and Wavelength Crossconnect (WXC). Adding, dropping, and bypassing traffic can be done at any of these stages. The WXC and BXC stages are interconnected through 
wavelength-to-band multiplexers and band-to-wavelength demultiplexers. Similarly, the BXC and FXC stages are interconnected through band-to-fiber multiplexers and fiber-to-band demultiplexers. The authors assumed that all wavebands/fibers have the same number of wavelengths/wavebands. They also assumed that wavelength/waveband converters might be required at the outputs of the $\mathrm{WXC} / \mathrm{BXC}$ stage.

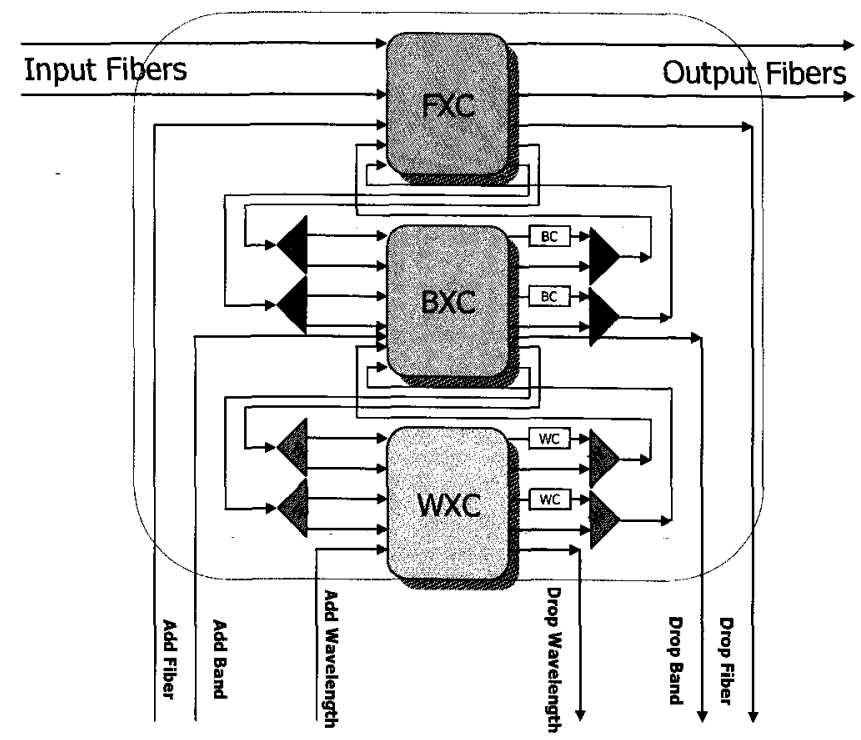

Fig. 2.2: "Type A" MG-OXC architecture [NVDo01].

The authors proposed a heuristic approach (although they did not present detailed steps) that is based on first achieving the end-to-end grouping of wavelengths into wavebands and fibers and then performing intermediate grouping on the remaining wavelengths and wavebands. The intermediate waveband/fiber grouping is based on the grouping of wavelengths/wavebands with different sources or different destinations along the longest common sub-path. The intermediate grouping does not include the grouping of traffic from different source nodes to different destination nodes. The shortest path is selected for each traffic demand.

The key simulation findings of this work are as follows. (1) OXCs that use the multi-granular switching concept can achieve a huge reduction (up to 97\%) in the port count with respect to the 
ordinary OXCs (see Fig. 2.6), particularly as the traffic volume increases. (2) Combining the intermediate grouping with the end-to-end grouping significantly reduces the size of the switching stages, especially WXCs and BXCs, compared to the case in which only the end-to-end grouping is used; also, the amount of reduction depends on the physical topology and traffic. The authors suggested that a further reduction could be achieved if intermediate grouping is also considered between traffic with different sources and destinations.

\subsubsection{Two-Stage Multiplexing Scheme}

Lee el al in [Lee+02] proposed a design scheme for multi-granular networks in which the waveband is formed by grouping wavelength paths that share the same destination. The authors used the term Hierarchical Crossconnect (HXC) as an alternative name for MG-OXC. The proposed MG-OXC architecture, Type B as shown in Fig. 2.3, consists of two switching stages only: WXC and BXC. Wavelength conversions are only allowed at the inputs of the WXC stage, and conversions cannot be performed at the BXC stage due to the technical difficulties of implementing waveband conversion. Wavelength demands are added only at the WXC stage and can be dropped at both the WXC and BXC stages.

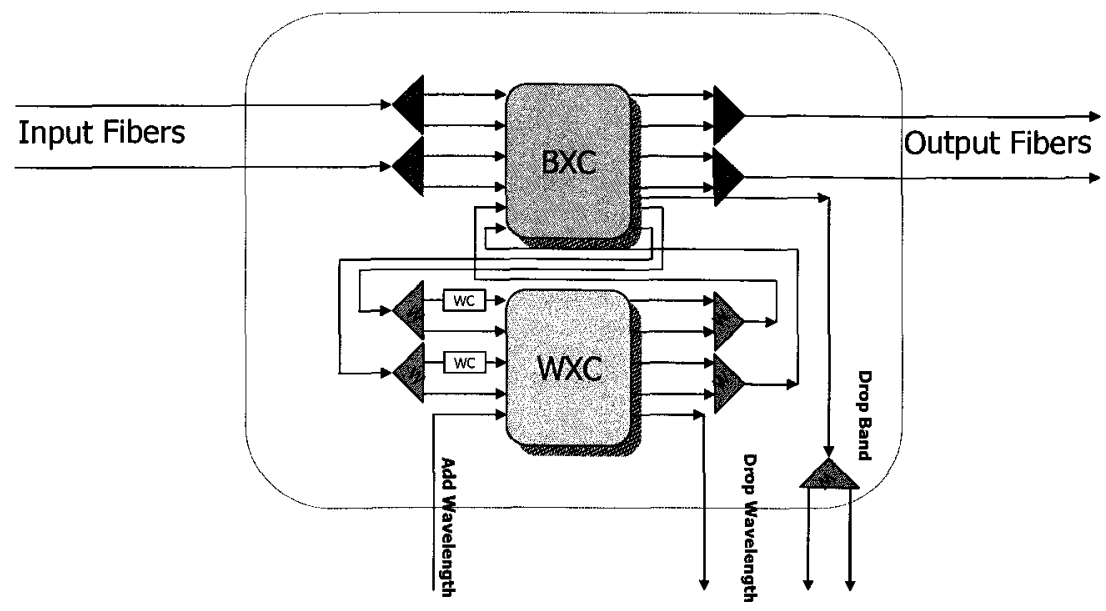

Fig. 2.3: "Type B" MG-OXC architecture [Lee+02]. 
The authors suggested three policies for waveband grouping: (1) grouping wavelength paths that share the same source and destination, (2) grouping wavelength paths that share the same destination but not the same source, and (3) grouping wavelength paths that share intermediate common physical links. The authors argued that the second policy could achieve a significant reduction in the WXC size in the sense that once wavelength paths are grouped into a waveband they do not need to be degrouped until they arrive at their destination. Moreover, a further reduction can be achieved if the whole waveband is dropped at the destination without entering the WXC stage, as illustrated in Fig. 2.3. The authors referred to the waveband path that uses the second policy as a packed-waveband path, and to the other waveband paths as unpackedwaveband paths.

The authors formulated the design problem (without solving it) as an ILP model based on the packed and unpacked path approach and using the path-formulation approach with the objective of minimizing the port count of MG-OXCs under the minimum number of wavelengths per link. To cope with the complexity of the problem, the authors developed a heuristic approach. The heuristic consists of three parts: routing, wavelength path grouping, and wavelength assignment. In the first part, the goal is to maximize the number of packed waveband paths. The number of physical hops is used as a cost metric for the shortest path calculation. The second part decides into which waveband each wavelength path will be grouped. Upon finishing this part, the size of the WXC and BXC in each network node can be calculated. In the third part, each wavelength path in packed waveband paths should have the same color for every link on its path since wavelength conversion is not allowed in the BXC. This leads to an increased wavelength requirement in the network. On the other hand, the wavelength assignment for a wavelength path in unpacked waveband paths is straightforward since wavelength conversion is possible in the 
WXC. The authors formulated the assignment problem for packed wavebands as a BLP problem, and pointed out that such a problem can be quite manageable since the number of constraints and number of variables are $2 \times$ band size and band size $e^{2}$ respectively.

As a benchmark, the MG-OXC-based network using the proposed heuristic is compared with the WXC-based network, which uses the ordinary OXC (Fig. 2.6) with wavelength converters placed at the inputs of the WXC. The key simulation findings of this work are as follows. (1) As the traffic volume increases, the use of MG-OXCs leads to significant reductions in both the size of crossconnects (up to $22 \%$ ) and number of $2 \times 2$ cross-points, which constitute the crossconnects, (up to 36\%). This agrees with the finding in [NVDo01]. (2) The optimum waveband size that yields a maximum reduction in both the port and cross-point count may exist, and its optimum value depends on the physical topology and traffic. (3) The reductions in the size of crossconnects at the optimum waveband come at the price of an increased wavelength requirement compared to the wavelength requirement in WXC-based networks, and this ratio tends to decrease as the traffic volume increases.

\subsubsection{Comprehensive Study on Waveband Switching}

In [CXAQ02][CAXQ03], Cao et al conducted a comprehensive study on waveband switching in multi-granular networks. The MG-OXC architecture considered in this study, Type $\mathrm{C}$ as shown in Fig. 2.4, is similar to the one used in [NVDo01] without employing wavelength and waveband converters since this technology is still immature and expensive. The authors developed a Waveband Switching (WBS) classification scheme, in which the number of wavebands/wavelengths per fiber/waveband can be either fixed or variable and the set of wavelengths in the waveband can be further classified as either pre-determined or adaptive. Accordingly, many variations of the WBS schemes are possible. However, the authors focused 
only on the simplest scheme, in which each fiber has a fixed number of wavebands and each waveband has both a fixed number and set of wavelengths, although they also mentioned that their ILP model and heuristics are applicable to other WBS schemes.

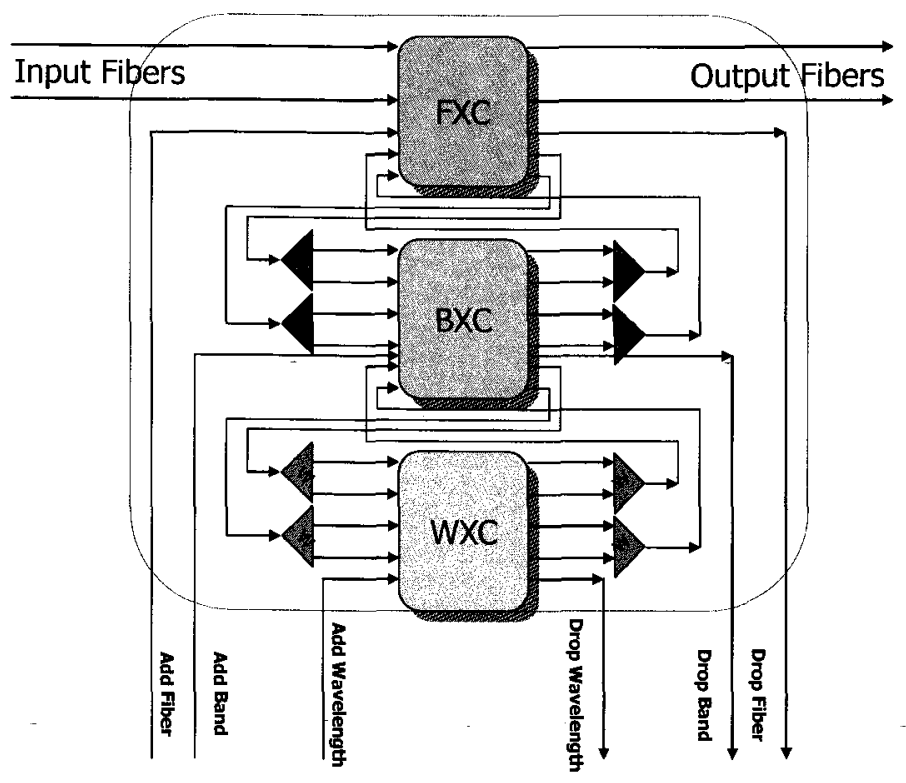

Fig. 2.4: "Type C" MG-OXC architecture [CXAQ02].

The ILP modeling of the WBS problem is quite different from the modeling that is done in [Lee+02]. The modeling in the latter is based mainly on grouping wavelength paths having the same destinations, whereas in this work a general policy is adopted in which wavelength paths of different sources and different destinations can be grouped. In addition, the new ILP model considers the case of multi-fiber networks, in which any link between two nodes can contain more than one fiber. Moreover, the new ILP model uses the link-formulation approach instead of the path-formulation approach. The objectives of the ILP model are the following: (1) to minimize the total cost associated with the MG-OXC ports in the network, where all ports are weighted by a factor depending on the type of the switching stage, and, for simplification, are assumed to have the same cost; and (2) to minimize the maximum size of the MG-OXC over all nodes. The 
authors argued that the solution to the ILP model is NP-complete since many constraints used in the problem are similar to the one used in the RWA problem, which is itself NP-complete.

To cope with the complexity of the problem, the authors proposed various heuristics. Many of these heuristics have been developed specifically for comparison purposes. The Waveband Oblivious Optimal RWA (WBO-RWA) heuristic is proposed to study the relationship between the WBS and traditional RWA. The heuristic first solves the ILP model used for the RWA in [MBRM96], in which the objective is to minimize the number of hops. Then, it groups the assigned wavelength paths into wavebands and fibers and calculates the port count accordingly.

The Balanced Path Routing with Heavy-Traffic first waveband assignment (BPHT) heuristic consists of three parts: balanced path routing, wavelength assignment, and waveband switching. The objective of the first part is to decide on the best physical route to be used by all wavelength paths of the source-destination $(s-d)$ pair that minimizes the maximum link load in the network. In the second part, the objective is to assign wavelengths to the pass-through wavelength paths first since they constitute $60-80 \%$ of the transport traffic. To maximize the benefit of wavebanding, these wavelength paths can also be grouped with other shorter overlapping wavelength paths. The wavelength paths with the smallest hop count are assigned wavelengths first. In the third part, the sizes of all switching stages in all network nodes are decided after switching fully formed fibers/wavebands using FXCs/BXCs and switching the remaining wavelength paths at WXCs separately.

The Balanced Traffic routing with Maximum-Hop first waveband assignment (BTMH) heuristic is a variation of the BPHT heuristic. In the BTMH heuristic, the first part of the BPHT heuristic is modified such that the physical routes for all $s$ - $d$ pairs are decided by balancing the load in each link 
in terms of the actual number of wavelength paths. Moreover, the second part is also modified such that the wavelength paths with the largest hop count are assigned wavelengths first. To test the performance of the BPHT heuristic, the authors proposed many analytical models in order to obtain the total number of ports in an MG-OXC-based network. One model is developed for the case in which the traffic demand per node pair is not a multiple of the waveband size. The other model is developed for the case in which the demand is a multiple of the waveband size. Moreover, models for generalized upper and lower bounds are also developed.

The main findings of this work are as follows. (1) For small-sized problems, the proposed BPHT heuristic can achieve good sub-optimum results in terms of the number of ports compared to the ILP model solution. (2) BPHT achieves a significant port count saving when compared with WBO-RWA. (3) In single/multi-fiber networks, the port count saving using the MG-OXC is around 50/70\% compared to the case in which the ordinary OXC is used, which is consistent with the results obtained in [NVDo01][Lee+02]. (4) On the other hand, the port count saving achieved with the ILP model and BPHT comes at the expense of an increased wavelength requirement in comparison with WBO-RWA, which agrees with the findings in [Lee+02], and the authors came to the conclusion that this is a problem of trade-off. (5) The performance evaluation shows that BPHT is even more beneficial in terms of reducing the port count and wavelength requirement in multi-fiber networks due to the natural wavelength conversion capability of these networks. (6) Comparing the developed analytical models with BPHT, the results show that heuristic results are within the limits and that the performance analysis is accurate. (7) The waveband size has an optimum value and has a large impact on the performance of multi-granular networks, which is again consistent with the finding in [Lee+02]. (8) BPHT has a better port count saving than 
B'TMH; however, it has a slightly higher wavelength requirement than BTMH, which in turn has a higher requirement than WBO-RW.

\subsubsection{RWA with Tunnel Allocation}

In [HMWu03], Ho et al proposed another approach for dimensioning multi-granular networks, which they called the problem of Routing and Wavelength Assignment with Tunnel allocation (RWAT). The authors used the same MG-OXC architecture, Type D as shown in Fig. 2.5, that they had proposed earlier in [HoMo02a], in which the architecture is used for developing algorithms for multi-granular networks under on-line traffic. There are many important observations to mention about this architecture. First, regardless of the volume of traffic, adding and dropping wavelength demands have to be done through the WXC stage and this definitely has a major impact on increasing the number of MG-OXC ports. Second, the architecture consists of two BXC/FXC stages, which also leads to the duplication of ports for pass-through wavebands and fibers. Third, the architecture has a fixed WXC size in all network nodes regardless of whether some nodes need a greater or smaller size.

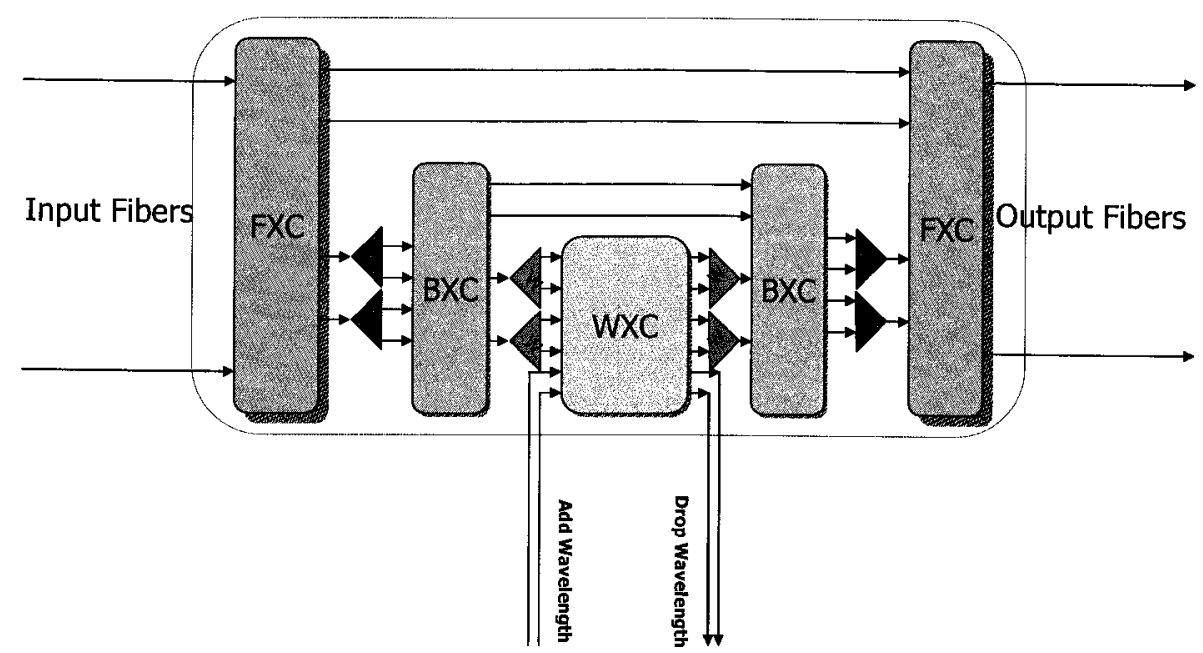

Fig. 2.5: "Type D" MG-OXC architecture [HoMo02a]. 
This work is motivated by the following. First, the ILP model in [CXAQ02] suffers from very high computational complexity since all of the input/output ports of all switching stages need to be numbered. Moreover, the weighting port parameters in the objective function of the ILP model have not been addressed. The proposed heuristic also does not consider increasing the chance of grouping wavelength paths, although in our opinion the main motive of [CXAQ02] is to improve grouping in comparison to the previous work in [NVDo01][Lee+02]. The authors' approach is based on the so-called tunneling principle, in which virtual connectivity is developed in the fiberand waveband-switching tiers to accommodate more traffic demands. A wavelength path is grouped with other wavelength paths having different wavelengths at the entry node and then separated at the exit node. Many assumptions are made about the tunnels. First, all tunnels have lengths in hops greater than or equal to the average distance in a network in order to reduce the resource fragmentation in the tunnel tiers. Second, a tunnel path is the shortest path between the entry and exit nodes. Third, all wavebands/fibers have the same number of wavelengths/wavebands.

The dimensioning problem is formulated as an ILP model using the path-formulation approach with the objective of minimizing the total number of ports in waveband- and fiber-switching tiers. Upon solving the ILP model, the total number of switching points in the network can be decided based on the resultant port count of each switching stage. Here, it is assumed that the twodimensional Micro-Electromechanical-System (MEMS) technology is used in the FXC, BXC, and WXC. To reduce the computational complexity of the ILP model, a heuristic is developed. The heuristic consists of three parts: the selection of entry-exit pairs, RWA, and tunnel allocation. The objective of the first part is to determine the entries and exits of all fiber and waveband tunnels. This can be achieved using a certain mathematical formulation that depends on a random 
manipulation of traffic demands and the likelihood of a node pair taking a tunnel. The second part performs the RWA process in order to route wavelength demands over the additional fiber and waveband tunnels, the endpoints of which are decided during the first part. The RWA problem is solved by formulating the problem as an ILP model with the objective of minimizing the number of wavelength demands that cannot be satisfied. Upon solving the ILP model, if some demands cannot be satisfied, then this means that more tunnels are required. Accordingly, the first part of the heuristic has to be repeated to create additional tunnels until all demands are satisfied by the RWA process. In the third part, physical routes are selected for all tunnels by formulating the problem as an ILP model. The objective function of the ILP model is to minimize the total cost of laying fibers for the fiber and waveband switching in each link. After finishing the third part, the number of ports for each switching tier should be easily determined; consequently, the total number of switching points, or mirrors, can be calculated.

The main findings of this study are as follows. (1) For a small-sized problem, the performance evaluation of the proposed RWAT heuristic, in terms of the port count of both FXCs and BXCs, is within $10 \%$ of the ILP model solution and can be accepted as a good sub-optimum solution. (2) The use of the suggested MG-OXC requires additional port count compared to the case in which a pure wavelength-switching (or ordinary) OXC, Type $\mathrm{E}$ as shown in Fig. 2.6, is used, which is contrary to the findings reported in previous work. However, this difference is expected because of the points we have mentioned earlier about this architecture. (3) The proposed MG-OXC achieves a lower port count than using the fiber- and wavelength-switching MG-OXCs, Type F as shown in Fig. 2.7. (4) The increase in the number of mirrors as the traffic demand grows can be significantly slowed with the proposed MG-OXC but at the expense of a moderate increase (5$15 \%$ ) in the port count with respect to the ordinary OXC. This means that in terms of the number 
of mirrors, the proposed MG-OXC has the best scalability and the ordinary OXC has the worst scalability. This is consistent with the finding in [Lee+02].

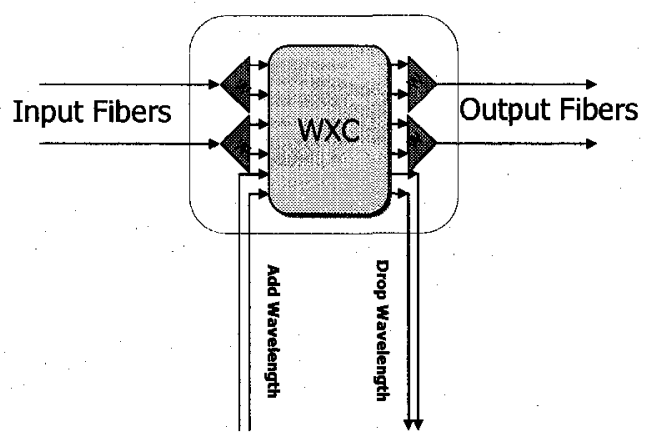

Fig. 2.6: "Type E" Wavelength-switching OXC architecture.

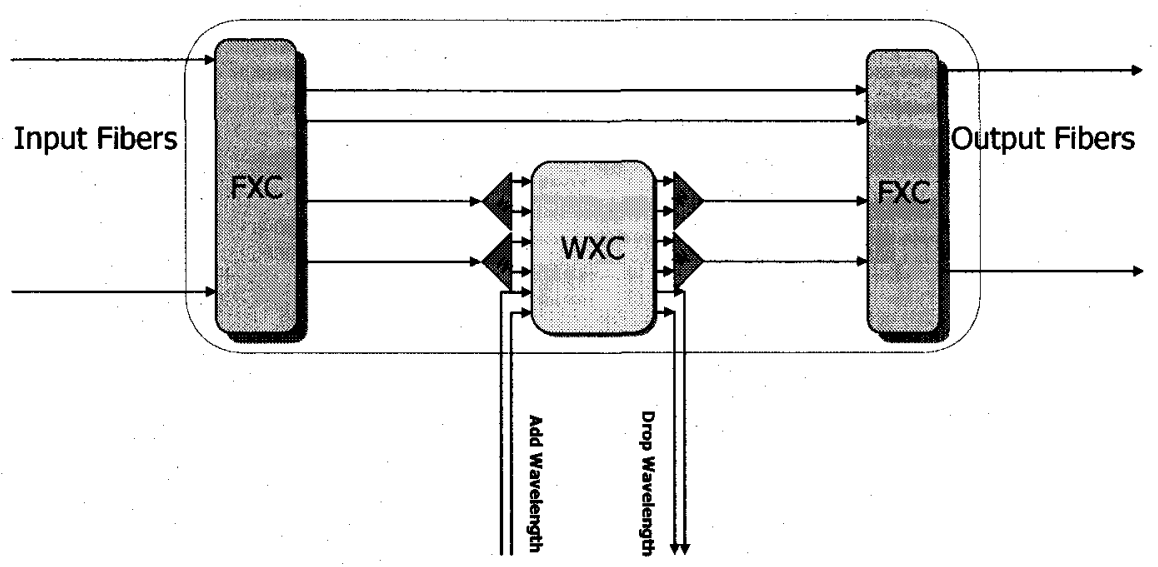

Fig. 2.7: "Type F" Fiber- \& wavelength-switching MG-OXC architecture [HMWu03].

\subsubsection{Non-Uniform versus Uniform Waveband Switching}

In [Izm+02][IGKV03], Izmailov et al investigated the performance of both the MG-OXC that uses uniform wavebands, in which the size of wavebands is fixed, and that using non-uniform wavebands, in which wavebands vary in size. The MG-OXC architecture used in this study is also called a hybrid hierarchical architecture. The hybrid architecture, Type $G$ as shown in Fig. 2.8, consists of an all-optical (OOO) crossconnect (or BXC) that switches wavebands as well as an Optical-Electrical-Optical (OEO)-based crossconnect (or EXC) that switches wavelengths and performs wavelength conversions. 


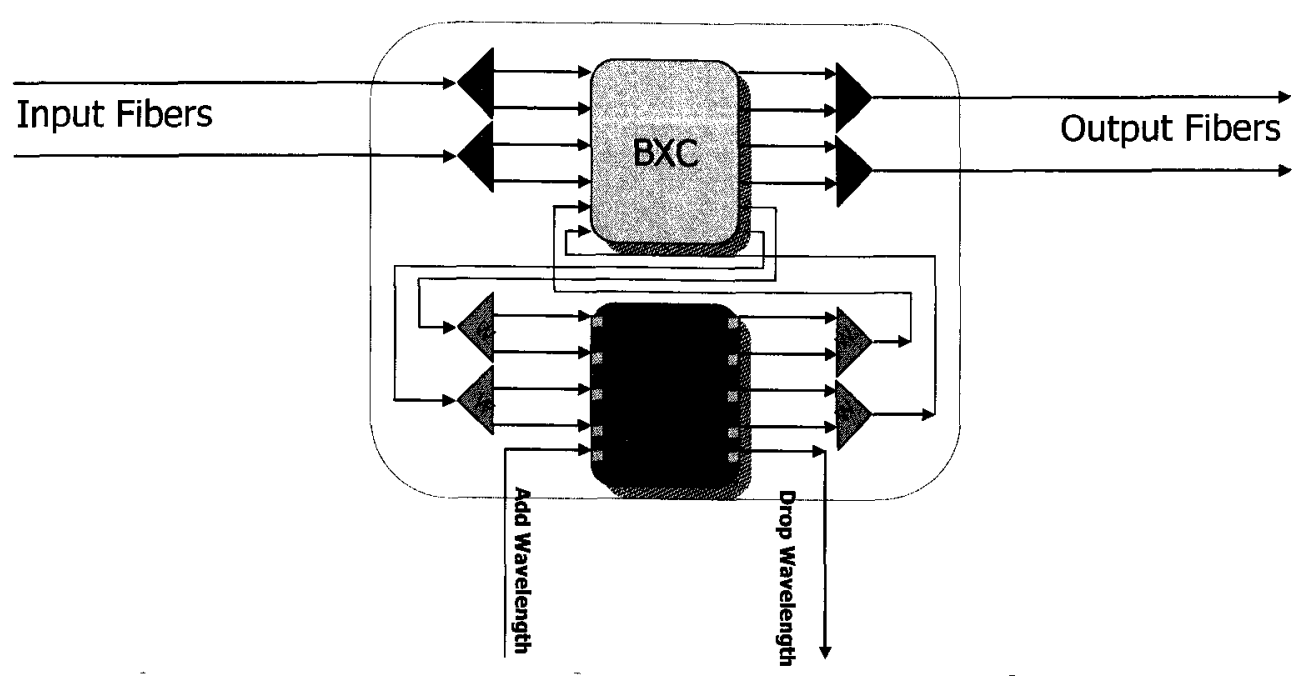

Fig. 2.8: "Type G" MG-OXC architecture [zzm+02].

The authors suggested three solutions for grouping pass-through wavelength paths. The first is the all-optical solution in which wavelength paths are grouped into uniform wavebands and switched optically by the BXC. These uniform wavebands can be partially filled. The second is the hybrid uniform wavebands solution in which the EXC is used to switch some of the coming wavelength paths to output fibers with either the same or a different color, and the remaining wavelength paths are grouped into fully filled uniform wavebands and switched optically by the BXC. The third is the non-uniform wavebands solution in which wavelength paths are grouped into fully filled non-uniform wavebands. The switching here can be either optical or hybrid if individual wavelength paths are left over. Determining the size of the non-uniform wavebands and the set of wavelengths to be used by each waveband requires solving the so-called waveband selection and waveband assignment problem. The authors formulated the problem as a dynamic programming problem with the objective of minimizing the weighted port count.

With the assumption that the cost of an EXC port is five times the cost of a BXC port, the authors showed through simulation that: (1) compared with the uniform wavebands solution, the 
use of the non-uniform wavebands solution can achieve a significant weighted port count reduction; and (2) the uniform waveband has an optimum size that leads to the highest weighted port count reduction, as has been shown by the previously reviewed work.

\subsubsection{Waveband Routing and Merging}

Ganguly et al in [GTIM04] extended the work done in [Izm+02][IGKV03]. The authors pointed out two essential problems associated with the hybrid hierarchical architecture. The first problem is how to route wavelengths and wavebands in the lowest cost manner (routing cost), while the second is how and where to merge wavelengths into wavebands in the lowest cost manner (merging cost). Decoupling both problems as in [Izm+02][IGKV03] limits the full benefits of waveband merging. Accordingly, the goal of this work is to combine both problems.

The authors considered the uniform waveband case and proposed two waveband merging architectures that are modified versions of the hybrid architecture proposed in [Izm+02] (Fig. 2.8): Full OEO-based Merging (FEM) architecture and Partial OEO-based Merging (PEM) architecture. In FEM architecture, two partially filled pass-through waveband paths arriving at two fiber ports are redirected to the EXC, where the two wavebands are merged into a new waveband path that is forwarded directly to the output port without the involvement of the BXC. The new waveband path can use a different color if there is a contention for the same color at the output fiber. This waveband conversion capability leads to better resource utilization. In PEM architecture, on the other hand, only one of the two partially filled pass-through waveband paths is redirected to the EXC and the other is switched optically by the BXC. The wavelengths of the OEO-switched waveband undergo wavelength conversions in such a way that the new wavelengths should be different from the wavelengths of the OOO-switched waveband. The OEO-switched wavelengths are merged with the OOO-switched waveband path through a 
coupler at the output port. The authors proposed two routing-and-merging heuristics to minimize the cost of routing and merging wavelengths and wavebands for both architectures.

In the FEM-based heuristic, the source-to-destination routing process of wavelength demands consists of three stages. In the first stage, wavelength demands in groups of the waveband size are routed along the shortest available routes from their source nodes to a node referred to as the Wavelength Convergence Point (WCP) that is designated for this set of demands. In the second stage, the WCP node merges these wavelength paths into wavebands and routes the resulting wavebands to a node called the Wavelength Divergence Point (WDP) via the available route with the smallest hop count (to reduce the number of OOOs or BXC ports). In the third stage, the grouped wavelengths are routed from the WDP to their destinations using the shortest available routes. The proper selections of WCP and WDP are essential to reducing the network cost since the merging and diverging cost (in terms of the number of OEOs or EXC ports) dominates the overall network cost. The WCP/WDP selection process is done through matrix manipulations to find the right node pair, namely one that serves a certain set of wavelength demands and at the same time minimizes the total merging and diverging cost.

The PEM-based heuristic, which the authors called Least Cost Branch First (LCBF), is based on the waveband-merging tree concept in which an unfilled waveband path can originate from multiple different sources and terminate at a filled waveband at a single node (the tree root). The authors resorted to the heuristic approach due to the fact that the optimal solution to the tree construction problem is known to be NP-complete. The LCBF heuristic constructs a merging tree for a set of single wavelength demands with the same destination by extending a branch each time a wavelength demand is served. The tree grows until all demands are served. While constructing waveband-merging trees, the heuristic tries to minimize the cost associated with each tree. The 
total cost for a given tree is the summation of the following: the total cost of OEOs, total cost of OOOs, and merging cost (coupler cost) at nodes where two or more wavebands are merged.

The key simulation findings are as follows. (1) The proposed heuristics outperform the heuristic used in [Izm+02][IGKV03] in terms of the network cost. (2) As has been shown by the previously reviewed work, the waveband size has an optimum value, and this value varies depending on the physical topology and traffic. (3) The PEM architecture and its corresponding merging heuristic provide the best cost reduction for medium traffic volumes; however, the topology and traffic conditions should decide which merging architecture should be chosen. It is worth mentioning here that the FEM and PEM comparison is similar to the comparison between the earlier mentioned waveband grouping policies, namely the shared intermediate sub-path policy and shared destination policy.

\subsubsection{Lagrangean Relaxation Approach}

In [LYTi04], Lee et al proposed an approximation approach to solve the ILP model of the RWA problem in the multi-granular network, which the authors named RWA ${ }^{+}$. Unlike in previously reported work, the authors assumed here that the network consists of different types of nodes (with no optical conversion capability in any type). The node can be Fiber Switch Capable (FSC), Lambda Switch Capable (ISC), or both. Due to the existence of these different types of nodes, the authors used a graph transformation method to replace each node by a bipartite subgraph that consists of phantom nodes. The number of these phantom nodes and how they are interconnected are functions of the number of wavelengths per fiber and physical nodal degree.

The RWA ${ }^{+}$problem is formulated as an ILP model using the path-formulation approach with the objective of minimizing the highest link utilization. The exact ILP problem is approximated using 
Lagrangean Relaxation with Heuristics (LRH), in which the inequality constraints are relaxed and the objective function is translated into a new objective function to reflect the constraints relaxation using Lagrangean multipliers. This type of relaxation, as we have mentioned in Subsection 2.4.2, is unlike the traditional LP approach that relaxes integers into non-integers. The LR approach here leaves integer constraints in the constraints sets while relaxing complex constraints such that the relaxed problem can be decomposed into independent manageable subproblems. Each sub-problem deals with part of the objective function of the relaxed problem, and the sub-problem is solved heuristically.

The authors did not solve the exact ILP problem. However, they showed through simulation, with the assumption that the network consists of only LSC nodes, that the LR approach provides tighter upper bounds and shorter solve time on the optimal solutions to the RWA ${ }^{+}$problem than those obtained by using the LP approximation approach in [BaMu96].

\subsubsection{Single-Layer MG-OXC}

Cao et al in [CAQi04][CAQi06] extended their previous work done in [CXAQ02][CAXQ03]. They compared their earlier proposed MG-OXC architecture (Type $\mathrm{C}$ as shown in Fig. 2.4), which they called the multi-layer MG-OXC, with a new proposed architecture that they called the single-layer MG-OXC, Type $\mathrm{H}$ as shown in Fig. 2.9. In the single-layer MG-OXC architecture, 'wavelengthto-band'/ 'band-to-fiber' multiplexers and 'band-to-wavelength'/'fiber-to-band' demultiplexers that are used to interconnect the 'WXC and $\mathrm{BXC} /{ }^{\circ} \mathrm{BXC}$ and $\mathrm{FXC}$ ' stages in the multi-layer architecture are removed. As a result, some input fibers are switched at the FXC. Other fibers are demultiplexed to wavebands. Some of these wavebands are switched at the BXC and others are demultiplexed to wavelengths and switched at the WXC. Because of these differences, the singlelayer architecture is expected to have a lower port requirement. Moreover, the optical signal quality 
is better than that of the multi-layer architecture, because all optical flows go through only one switching stage.

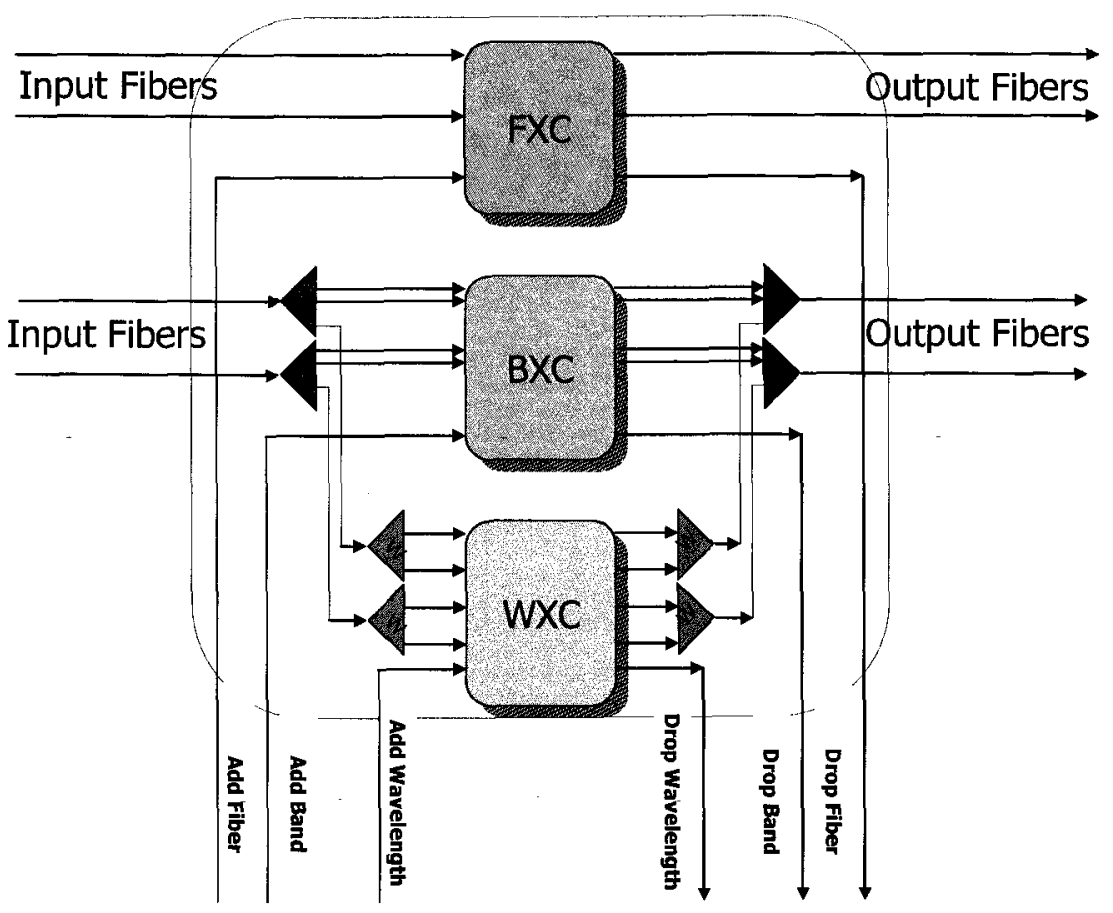

Fig. 2.9: "Type H" MG-OXC architecture [CAQi04].

The authors compared the performance of both architectures under off-line and on-line traffic. For the off-line case, they used the BPHT heuristic proposed in [CXAQ02][CAXQ03]. For the on-line case, they proposed and used an algorithm called Maximum Overlap Ratio (MOR), the objective of which is to minimize the request blocking probability.

The comparison via simulation shows that in the off-line case, the single-layer architecture can use up to $15 \%$ fewer ports than the multi-layer one. However, in the on-line case, the multi-layer architecture is better than the single-layer one since it achieves a lower blocking probability. 


\subsubsection{Disjoint Shared Waveband Path Protection}

In [VGIz03], Varsou et al is the first group to our knowledge to address the protection problem in multi-granular networks. The authors adopted the MG-OXC architecture proposed in [Izm+02] (Fig. 2.8). They assumed that the waveband paths (the working capacity) have already been decided for all $s-d$ pair wavelength demands, and the problem is how to protect a waveband path that carries wavelengths between an $s-d$ pair. This is what we call disjoint protection, as opposed to joint protection in which both the working and protection capacity are decided simultaneously. The authors suggested that this can be done by finding either a protection waveband path or several protection wavelength paths. These protection wavebands and wavelengths (the spare capacity) can be shared by many $s-d$ pairs. Therefore, the objective of the problem is to decide on which method to use for any $s-d$ pair such that it minimizes the total spare capacity. If both methods yield the same spare capacity requirement, then the one with the minimum number of ports will be chosen.

The proposed problem is formulated as an ILP model with two objective functions: the first is to minimize the spare capacity and the second is to minimize the number of ports. The authors used the link-formulation approach to develop the ILP model. However, they did not solve the ILP model and alternatively suggested software packages that can solve dual-objective ILP problems. To ease the complexity of the ILP model, the authors developed a heuristic that they referred to as the waveband/wavelength protection tree algorithm. The heuristic relies on the working capacity, which is obtained using the waveband routing and aggregation algorithm presented in [Ara+02]. In this waveband routing and aggregation algorithm, all wavelength demands are routed first along the shortest available route. Then, wavelength paths sharing the longest common links are grouped together into one waveband along the common sub-route. These steps are repeated until no 
further grouping is possible. In the waveband/wavelength protection tree algorithms, trees are constructed in a way that takes into account the number of waveband paths that can be protected as well as the number of available wavebands. $A$ link (or branch) in the tree is weighted by how many waveband paths it can protect either completely or partially and how many wavebands are available in its link. If a path is completely protected by the tree, it is removed from the list of paths that a link can protect. Trees continue to be constructed as long as there are still unprotected paths.

The key simulation finding of this work is that the proposed waveband/wavelength protection tree scheme (the mixed approach) outperforms the wavelength approach, in which waveband paths are protected by only shared wavelength paths.

\subsubsection{Dedicated and Shared Waveband Path Protection}

Varma and Jue in [VaJu04][VaJu06] considered the problem of establishing working and dedicated/shared protection waveband paths for wavelength demands in multi-granular networks. The authors adopted the single-layer MG-OXC architecture proposed in [CAXQ03] (Fig. 2.9) and assumed that all wavebands/fibers have the same number of wavelengths/wavebands.

The proposed problem is formulated as an ILP model using the link-formulation approach with the objective of minimizing the port count in the network. In the formulation, the joint dedicated/shared waveband path protection approach is used to allocate the protection capacity for each working waveband path. In the dedicated protection approach, to avoid non-linear constraints, the authors assumed that the shared protection wavelengths on a link cannot be grouped into wavebands. 
To cope with the complexity of the problem, the authors proposed a graph-based heuristic. The heuristic is based on creating auxiliary graphs from the given network topology. The number of these graphs is equal to the number of wavebands per fiber. The single node in the original network topology is transformed into many nodes if there is add/drop traffic at that node. The weights of the external links, which interconnect the node with other nodes, are the same as those in the original topology. The weights of the internal links between the generated nodes are updated by the heuristic every time an $s-d$ pair is served. Finding a path in the auxiliary graph is equivalent to finding the physical route and waveband color. The minimum cost working waveband path is chosen among paths obtained from all graphs. The coloring of wavelengths in the chosen waveband is done using the First-Fit (FF) color assignment in a way that satisfies the wavelengthcontinuity constraint. If one waveband path is not enough to serve the $s-d$ pair demands, then the second minimum cost path is selected and so on until all demands are served. The protection disjoint path is assigned sequentially to each working waveband path. In the dedicated protection problem, the protection path is found in the auxiliary graphs in the same way as the working path by modifying the internal links in a way that ensures that the working and protection paths are disjoint. In the shared protection problem, the auxiliary graphs are modified in such a way that the working paths having a protection path that shares wavelengths on the same link are link disjoint themselves since the problem is dealing with a single point of failure.

To benchmark the proposed heuristic, the authors used a heuristic based on the traditional RWA heuristic. In this heuristic, the working and protection paths are decided jointly by using the least combined cost path pair. In the dedicated protection approach, the coloring of wavelengths is done by the FF color assignment. In the shared protection approach, on the other hand, coloring is done in a way that increases the sharing among the protection paths. 
The authors were unable to solve the ILP problem even for small-sized problems. Alternatively, they relaxed the integer variables of the ILP model and solved the resultant LP problem to provide lower bounds on the solutions to small-sized ILP problems. The key findings of this work are the following. (1) Graph-based heuristics achieve significant savings in the port count (up to $25 \%$ ) with respect to traditional RWA heuristics, which agrees with the findings in [CXAQ02] [CAXQ03]. (2) Although dedicated protection consumes more wavelengths, it outperforms shared protection in terms of the port count for both heuristics and as well as the lower bound of the ILP problem. (3) Choosing the right waveband size is an important factor in maximizing the savings in the port count, which again agrees with the findings of the previously reviewed work.

\subsubsection{Grouping Strategies with Joint Dedicated Path Protection}

In [STPa07], Secci et al compared various grouping strategies in multi-granular networks with dedicated path protection. The authors adopted the single-layer MG-OXC architecture proposed in [CAQi04] (Fig. 2.9). They assumed that all wavebands/fibers have the same number of wavelengths/wavebands. Also, in the traffic model, they assumed that the bit-rate (or raw) traffic demand between any $s-d$ pair can be mapped over one or more of the following types of demand granularities: wavelength, waveband, and multi-waveband (or fiber) demands. The authors used the best rounding-up method to reduce the gap between the volume of the required raw demand and the capacity (in bit/sec) of the allocated multi-granularity demand.

The authors classified grouping strategies into three levels: the wavelength, waveband, and fiber levels. The wavelength grouping level consists of only an end-to-end wavelength, which carries the wavelength demand between an $s$ - $d$ pair. The waveband grouping level consists of (1) an end-toend waveband, which carries the waveband demand between an $s$ - $d$ pair; and (2) a local waveband, which is the sub-path/intermediate grouping of end-to-end wavelength paths. The fiber grouping 
level consists of (1) an end-to-end fiber, which carries the fiber demand between an $s-d$ pair; (2) a local fiber, which is the sub-path grouping of end-to-end waveband paths; and (3) a heterogeneous fiber, which is the sub-path grouping of end-to-end waveband and wavelength paths. Based on this grouping hierarchy, the authors proposed four network architecture cases: (1) the ordinary OXC (Fig. 2.6) without grouping, (2) MG-OXC with end-to-end wavebands and fibers, (3) MGOXC with end-to-end wavebands and fibers as well as local wavebands and fibers, and (4) MGOXC with all grouping strategies (Case 3 with heterogeneous fibers).

The authors formulated the network architecture cases mentioned above as ILP models using the link-formulation approach with the objective of minimizing the network cost. The network cost is expressed in terms of the port cost, fiber cost, and propagation delay cost. In the ILP formulation, they also used the joint dedicated link-disjoint path protection approach to allocate the spare capacity for each working wavelength/waveband/fiber path.

The ILP models are solved for different network topologies and traffic volumes. The authors were able to get the optimum solutions for these models for low traffic volumes, especially in network architecture cases that do not use sub-path grouping. Otherwise, they fixed a time limit for the ILP solver and considered the most feasible solutions available. The main findings of this study are as follows. (1) Adding grouping capabilities to network nodes through the use of the MG-OXC leads to a significant reduction in the network cost (up to 22\%) and port count (up to 88\%) with respect to the use of the ordinary OXC. In addition, greater reductions are expected as the traffic volume increases. These results are consistent with most of the findings in the previously reviewed work. (2) Incorporating sub-path grouping capabilities leads to additional slight savings in the network cost and port count with respect to the case in which only end-to-end groupings are used, which agrees with the finding in [NVDo01]. Moreover, the highest savings are more likely to occur for 
low traffic volumes and networks with high network physical connectivity. Nevertheless, these savings come at the expense of a significantly increased complexity of the ILP model and, accordingly, the solve time. (3) Although combining the sub-path grouping with heterogeneous grouping leads to the best results, it does not achieve considerable savings compared to the case without heterogeneous grouping given the fact the ILP model of this network architecture case becomes extremely difficult to solve.

\subsubsection{Dedicated Waveband Sub-path Protection}

In [YHSa07], Yamada et al considered the problem of jointly establishing working waveband paths and dedicated protection waveband sub-paths for wavelength demands in multi-granular networks. The authors used an MG-OXC architecture similar to the one proposed in [Lee+02] (Fig. 2.3), but without either the use of wavelength converters or dropping wavelength demands from the BXC stage. They assumed that all wavebands have the same number of wavelengths.

The authors proposed a heuristic approach to tackle this problem with the objective of minimizing the network cost, which is expressed in terms of port and fiber requirements. The approach is based on the idea that each working waveband path can be divided into many sub-paths, and protection link-and-node disjoint waveband paths ate obtained for these working sub-paths. Accordingly, a pair of working and protection waveband sub-paths forms a loop. The working/protection waveband path for any $s-d$ pair consists of at least three loops: the source, intermediate, and destination loops. The source/destination loop includes the sources/destinations of other $s-d$ pairs, which can use the spare capacity of the existing intermediate waveband loops. The heuristic consists of two parts. In the first part, source-destination loop pairs and intermediate loops are developed iteratively to accommodate as many demands as possible. The disjoint path pair is obtained using Suurballe's algorithm [Bhan97] using the distance as a cost metric. In the 
second part, the original physical topology, established working/protection wavebands, and available wavelengths in wavebands are converted into the so-called multi-layered modified dual graph. The remaining demands are accommodated by this graph using Dijkstra's Algorithm [Dijk59].

The authors compared the dedicated waveband sub-path protection with the dedicated waveband path protection. The key simulation finding is that the former can achieve a $10-50 \%$ network cost savings over the latter.

\subsubsection{Summary}

Table 2.1 summarizes the related work by comparing the studies based on various issues considered in the design problem. The objectives of this summary are (1) to demonstrate the novelty of these studies, and (2) to identify potential opportunities for further research and development in this area.

Table 2.1: Comparison of previous work based on various issues.

\begin{tabular}{|c|c|c|c|c|c|c|c|c|}
\hline Work & $\begin{array}{c}\text { Demand } \\
\text { granularity }\end{array}$ & $\begin{array}{c}\text { Grouping } \\
\text { level }\end{array}$ & $\begin{array}{l}\text { WBS } \\
\text { scheme }\end{array}$ & $\begin{array}{l}\text { Node } \\
\text { architecture }\end{array}$ & $\begin{array}{c}\text { Demand } \\
\text { bifurcation }\end{array}$ & $\begin{array}{c}\text { Color } \\
\text { conversion }\end{array}$ & $\begin{array}{l}\text { Optical } \\
\text { reach }\end{array}$ & $\begin{array}{l}\text { Protection } \\
\text { technique }\end{array}$ \\
\hline [NVDo01] & $\begin{array}{l}\lambda, \text { band } \\
\text { fiber }\end{array}$ & band, fiber & $\begin{array}{l}\text { uniform bands } \\
\text { \& fibers }\end{array}$ & Type A & No & $\lambda$, band & No & No \\
\hline [Lee+02] & $\lambda$ & band & uniform bands & Type 13 & No & $\lambda$ & No & No \\
\hline$\left[\begin{array}{l}\mathrm{CXAQ02} \\
{[\mathrm{CAXQ03}}\end{array}\right]$ & $\lambda$ & band, fiber & $\begin{array}{l}\text { uniform bands } \\
\text { \& fibers }\end{array}$ & Type C & No & No & No & No \\
\hline [HMWu03] & $\lambda$ & band, fiber & $\begin{array}{l}\text { uniform bands } \\
\text { \& fibers }\end{array}$ & Types D \& $\Gamma$ & No & No & No & No \\
\hline$\left[\begin{array}{l}\text { Izm+02 } \\
\text { IGKV03 }\end{array}\right]$ & $\lambda$ & band & $\begin{array}{l}\text { non-uniform } \\
\text { bands }\end{array}$ & 'Туре G & No & $\lambda$ & No & No \\
\hline [GTIM04] & $\lambda$ & band & uniform bands & $\begin{array}{l}\text { Type } G \text { with some } \\
\text { modifications }\end{array}$ & No & $\lambda$ & No & No. \\
\hline [LY'Ti04] & $\lambda$ & fiber & uniform fibers & $\mathrm{WXC}$ and/or FXC & No & No & No & No \\
\hline$\left[\begin{array}{l}\text { CAQi04 } \\
\text { CAQi06] }\end{array}\right.$ & $\lambda$ & band, fiber & $\begin{array}{l}\text { uniform bands } \\
\text { \& fibers }\end{array}$ & Type H & No & No & No & No \\
\hline [VGIz03] & $\lambda$ & band & uniform bands & '1ype G & No & No & No & $\begin{array}{c}\text { shared band } \\
\text { path protection }\end{array}$ \\
\hline $\begin{array}{l}\text { [VaJu04] } \\
\text { [VaJu06] }\end{array}$ & $\lambda$ & band, fiber & $\begin{array}{l}\text { uniform bands } \\
\& \text { fibers }\end{array}$ & Type $\mathrm{H}$ & No & No & No & $\begin{array}{c}\text { shared/dedicated } \\
\text { band path } \\
\text { protection }\end{array}$ \\
\hline [STPa07] & $\begin{array}{l}\lambda, \text { band, } \\
\text { fiber }\end{array}$ & band, fiber & $\begin{array}{l}\text { uniform bands } \\
\text { \& fibers }\end{array}$ & Type H & No & No & No & $\begin{array}{c}\text { dedicated } \\
\lambda / \text { band } / \text { ftber } \\
\text { path protection }\end{array}$ \\
\hline [YHSa07] & $\lambda$ & band & uniform bands & $\begin{array}{l}\text { Type B with some } \\
\text { modifications }\end{array}$ & No & No & No & $\begin{array}{l}\text { dedicated band } \\
\text { sub-path } \\
\text { protection }\end{array}$ \\
\hline
\end{tabular}




\section{Chapter 3}

\section{Network Architecture and Framework}

\subsection{Introduction}

Although extensive research work has been done in the area of multi-granular transport network planning, there are still some open issues that need to be considered and investigated. To the best of our knowledge, the following issues have not been mentioned or addressed in the literature: (1) considering the whole traffic hierarchy defined in GMPLS, (2) bifurcating multi-granularity traffic demands among distinct physical routes, (3) performing color conversions of the optical signal at all granularity levels, (4) imposing a constraint on the length of the all-optical path in order to cope with the optical-reach limitation, and (5) protecting the working multi-granularity traffic flows using the link and $\mathrm{p}$-cycle protection techniques. Addressing these issues will certainly pave the road to an efficient implementation of the GMPLS-based transport network. The focus of this thesis will be on the consideration of the first four points, and the last point will be left as a further extension to our work.

In this chapter, we explain in detail the domain of the new planning problem we are going to address. First, we proceed with a full description of the proposed GMPLS-based node architecture as well as the path types involved in the system. Then, the new planning problem is stated and the assumptions are explained. After that, the optimization approaches to be taken in order to solve the problem optimally and sub-optimally are described. 


\subsection{The System}

\subsubsection{Node Architecture}

A GMPLS-based transport mesh network consists of a group of nodes interconnected by fiber links. The proposed GMPLS-based node ${ }^{6}$ architecture [NaMo06a] consists of an MG-OXC segment and an EXC segment, as shown in Fig. 3.1. The MG-OXC segment is responsible for the grouping/degrouping and switching of optical flows (or lightpaths) ${ }^{7}$. In this thesis, we adopt the multi-layer MG-OXC architecture. Although it has been shown in [CAQi04][CAQi06] (Subsection 2.5.8) that the single-layer MG-OXC architecture can lead to a greater reduction in the port count under unprotected static traffic demands, it lacks the flexibility to (1) implement proper network provisioning, (2) cope with node/link failure, or (3) adapt to changes in the traffic pattern. Type C architecture [CXAQ02] (Fig. 2.4) or Type A architecture [NVDo01] (Fig. 2.2) without optical converters is chosen for the MG-OXC segment. Type $C$ architecture is the best choice for the optical segment for the following reasons. First, adding/dropping different optical traffic granularities directly at the designated crossconnect leads to a significant reduction in the port requirement in comparison to other MG-OXC architectures. This fact is demonstrated through the example shown in Table 3.1, where it is clear that Type $\mathrm{C}$ architecture has the best scalability as traffic demands increase. Second, Type $C$ also requires the lowest port requirement for passthrough traffic and scales better than other architectures as the volume of pass-through traffic flows increases, as is clearly demonstrated in Table 3.2. The FXC can be implemented using a space switch. Both the BXC and WXC can be implemented by various technologies such as liquid crystal and MEMS.

\footnotetext{
${ }^{6}$ For the rest of this thesis, the terms GMPLS-based node and Multi-Granular Crossconnect (MG-XC) refer to the same concept and will be used interchangeably.

7 'The terms lightpath, virtual link, clear path, and all-optical path have the same meaning and will be used interchangeably.
} 
To perform the switching of sub-wavelength flows and grouping/degrouping of sub-wavelength flows into/from wavelengths, it is necessary to combine the EXC with the MG-OXC segment. The EXC consists of three parts: the switching matrix, line-side interfaces, and tributary-side interfaces. The switching matrix can be a GMPLS core router. A line-side interface consists of an optical transceiver with a certain optical reach capability. Line-side interfaces are interconnected with the MG-OXC segment through different types of GMPLS interfaces [Ban+01]. A FiberSwitch-Capable (FSC) interface is used to launch/terminate a fiber-switched path, while a BandSwitch-Capable (BSC) interface is used to launch/terminate a waveband-switched path and a Lambda-Switch-Capable (LSC) interface is used to launch/terminate a wavelength-switched path. On the other hand, tributary-side interfaces are used to interconnect the $\mathrm{EXC}$ with metro core (or regional), metro edge, or excess networks, which are the sources and sinks of the transport network traffic. Tributary-side interfaces can be any of the interfaces mentioned previously as well as any of the following sub-wavelength type interfaces [Ban+01]: TDM-capable interfaces, PacketSwitch-Capable (PSC) interfaces, or Layer 2-Switch-Capable (L2SC) interfaces. However, tributary-side interfaces will not be included in our cost model.

Since the EXC allows for OEO domain conversion with the use of back-to-back transceivers, there are two added-value benefits associated with its deployment: the 3R (Re-amplification, Reshaping, and Re-timing) regeneration and color conversion of optical signals at all granularities. The regeneration functionality of the EXC is needed to reduce impairments that accumulate along the all-optical path. With the EXC, the use of expensive dispersion compensators along network spans can be avoided. The color conversion functionality removes the color-continuity constraint, improves the resource utilization of the network, and ultimately decreases the fiber requirements, which consequently reduces the computational complexity of the GMPLS control plane. It is 
worth mentioning here that when the signal regeneration is needed it is also an opportunity to perform color conversion and vice versa. The cost-effective selection of OEO locations for performing the afore-mentioned functionalities will no doubt be part of the optimization process.

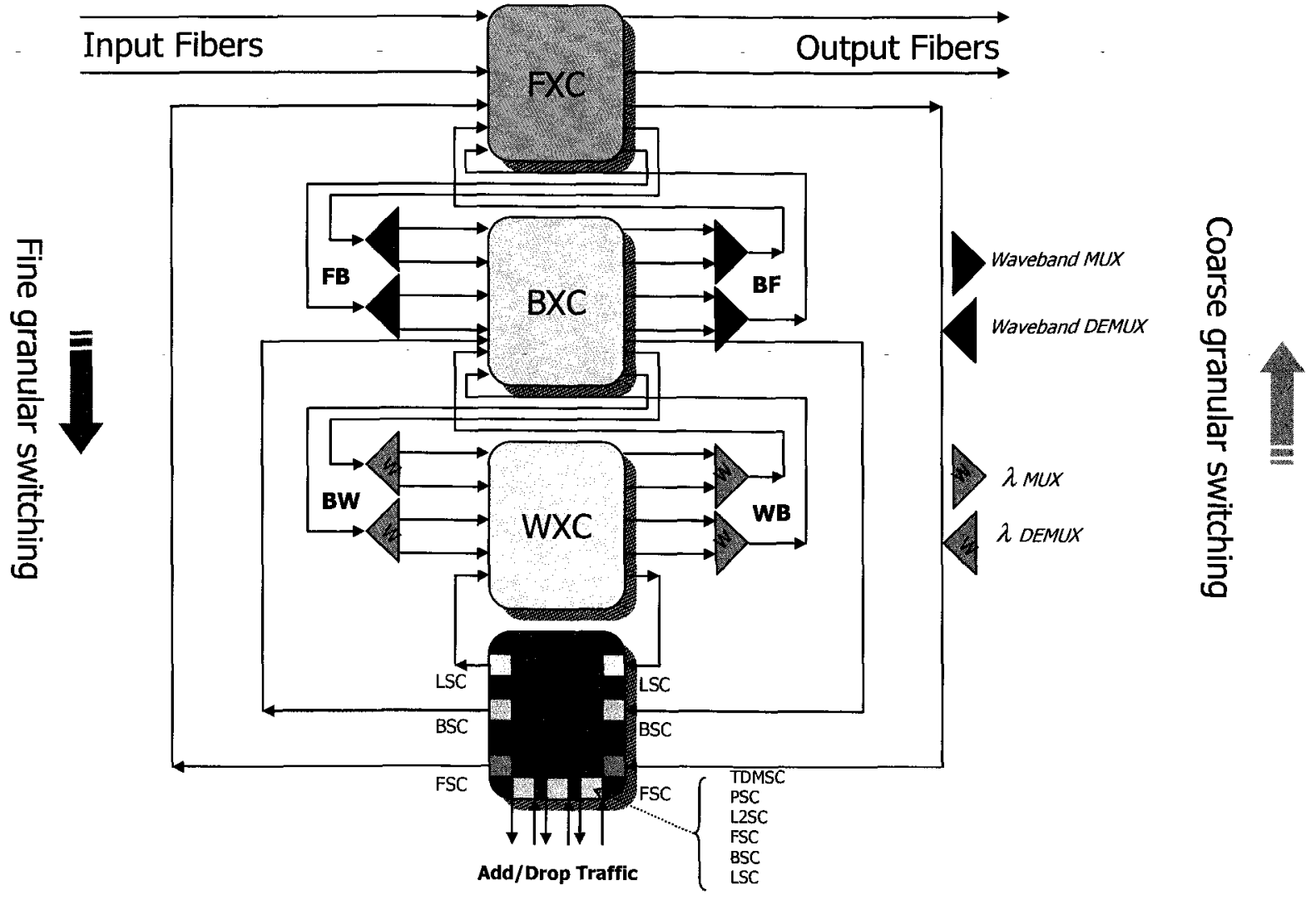

Fig. 3.1: The proposed GMPLS-based node architecture. 
Table 3.1: A demonstrative example to compare different MG-OXC architectures in terms of the weighted port count in a single node for different volumes of add traffic, given that all optical port types have the same weight, the EXC port weight is twice the optical port weight, and the number of wavelengths/wavebands per waveband/fiber is $8 / 4$.

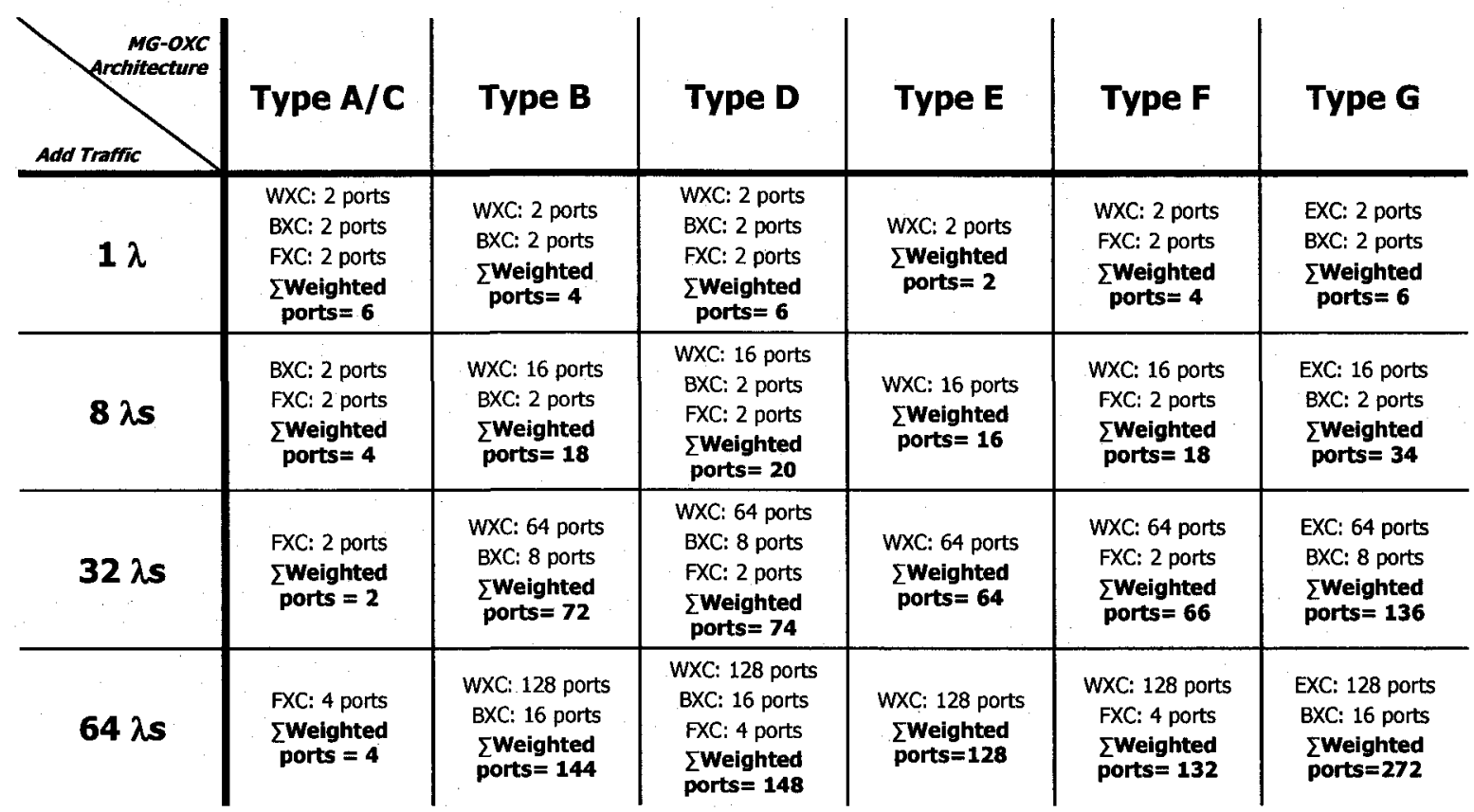

Table 3.2: A demonstrative example to compare different MG-OXC architectures in terms of the weighted port count in a single node for different volumes of pass-through traffic, given that all optical port types have the same weight, the EXC port weight is twice the optical port weight, and the number of wavelengths/wavebands per waveband/fiber is $8 / 4$.

\begin{tabular}{|c|c|c|c|c|c|c|}
\hline $\begin{array}{l}\text { Pass-Thr } \\
\text { Traffic }\end{array}$ & Type A/C & Type B & Type D & Type E & Type F & Type G \\
\hline 1 Fiber & $\begin{array}{l}\text { FXC: } 2 \text { ports } \\
\sum \text { Weighted } \\
\text { ports }=\mathbf{2}\end{array}$ & $\begin{array}{l}\text { BXC: } 8 \text { ports } \\
\text { ¿Weighted } \\
\text { ports }=8\end{array}$ & $\begin{array}{c}\text { FXC: } 4 \text { ports } \\
\sum \text { Weighted } \\
\text { ports }=4\end{array}$ & $\begin{array}{c}\text { WXC: } 64 \text { ports } \\
\sum \text { Weighted } \\
\text { ports }=64\end{array}$ & $\begin{array}{l}\text { FXC: } 4 \text { ports } \\
\sum \text { Weighted } \\
\text { ports }=4\end{array}$ & $\begin{array}{l}\text { BXC: } 8 \text { ports } \\
\text { ¿Weighted } \\
\text { ports }=8\end{array}$ \\
\hline 2 Fibers & $\begin{array}{l}\text { FXC: } 4 \text { ports } \\
\sum \text { Weighted } \\
\text { ports }=4\end{array}$ & $\begin{array}{l}\text { BXC: } 16 \text { ports } \\
\text { IWeighted } \\
\text { ports }=16\end{array}$ & $\begin{array}{c}\text { FXC: } 8 \text { ports } \\
\text { ¿Weighted } \\
\text { ports }=8\end{array}$ & $\begin{array}{l}\text { WXC: } 128 \text { ports } \\
\sum \text { Weighted } \\
\text { ports }=128\end{array}$ & $\begin{array}{c}\text { FXC: } 8 \text { ports } \\
\sum \text { Weighted } \\
\text { ports }=8\end{array}$ & $\begin{array}{c}\text { BXC: } 16 \text { ports } \\
\sum \text { Weighted } \\
\text { ports }=16\end{array}$ \\
\hline
\end{tabular}

\subsubsection{Path Types}

In this work, we consider the following path types between any source-destination $(s-d)$ pair in the GMPLS-based transport network [NaMo06a][NaMo07a]: 
- Clear Wavelength-Switched (CWS) Path: This path can carry either a wavelength demand or subwavelength demand(s) that share the same source and destination. The CWS path must have enough optical reach to use the same wavelength on the same waveband within the same fiber along the entire path without undergoing OEO domain conversion, as shown in Fig. 3.2. The launch/termination of this path is at the LSC interface. The CWS path can enter the intermediate WXC or BXC stages for grouping/degrouping the path segment with/from other lightpaths that share the same waveband or fiber, as illustrated in Fig. 3.2.

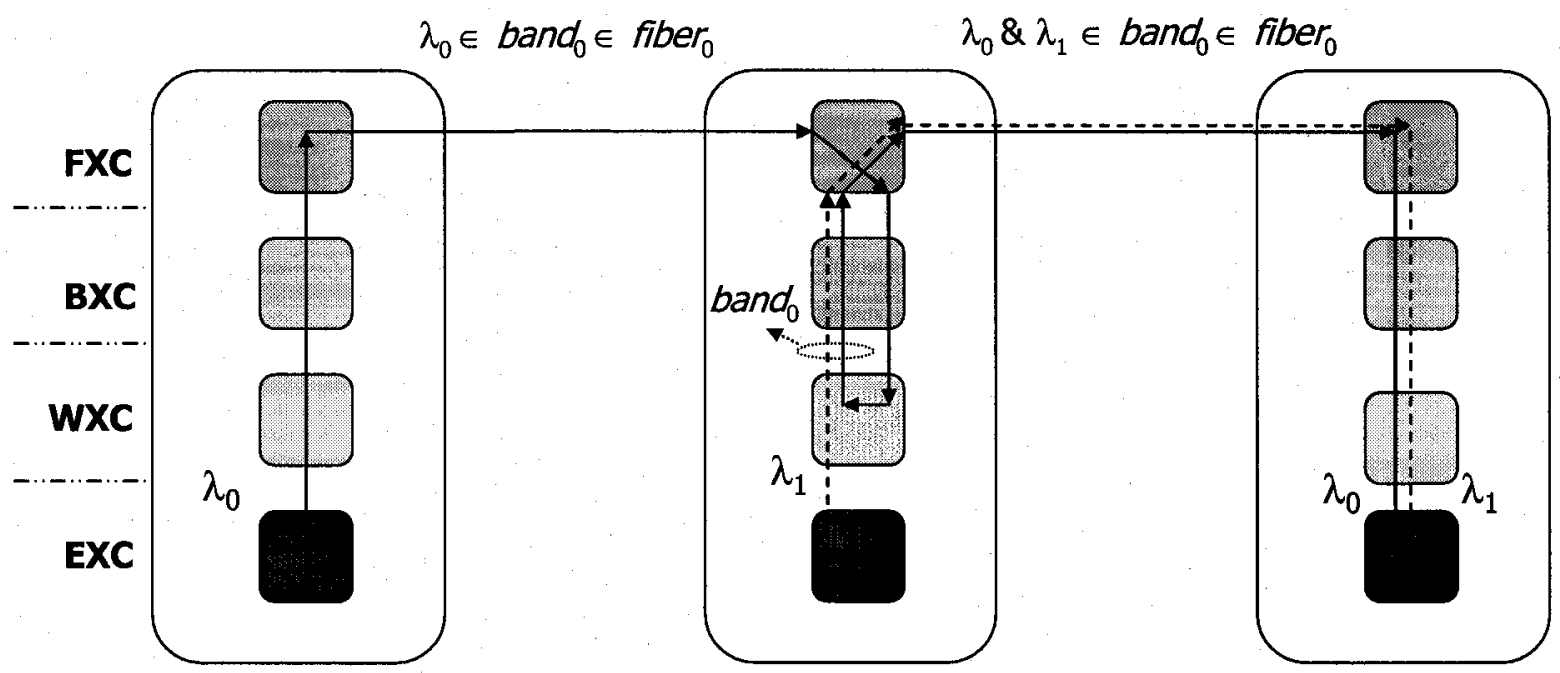

Fig. 3.2: Clear wavelength-switched path.

- Unclear Wavelength-Switched (UWS) Path: This path is used to carry either a wavelength demand or sub-wavelength demand(s). The UWS path is a concatenation of CWS paths. This path undergoes OEO conversion at least once for the purpose of (1) sharing the capacities of its CWS paths with other sub-wavelength demands (note that CWS paths here are to be used only by sub-wavelength demands), as depicted in Fig. 3.3, and we call this level of grouping the wavelength-level; (2) optical signal regeneration, if the length of all-optical path segments exceeds the optical reach, as 
shown in Fig. 3.4; and/or (3) wavelength color conversion to another wavelength color on the same waveband, a different waveband, or a different fiber, as shown in Fig. 3.5.

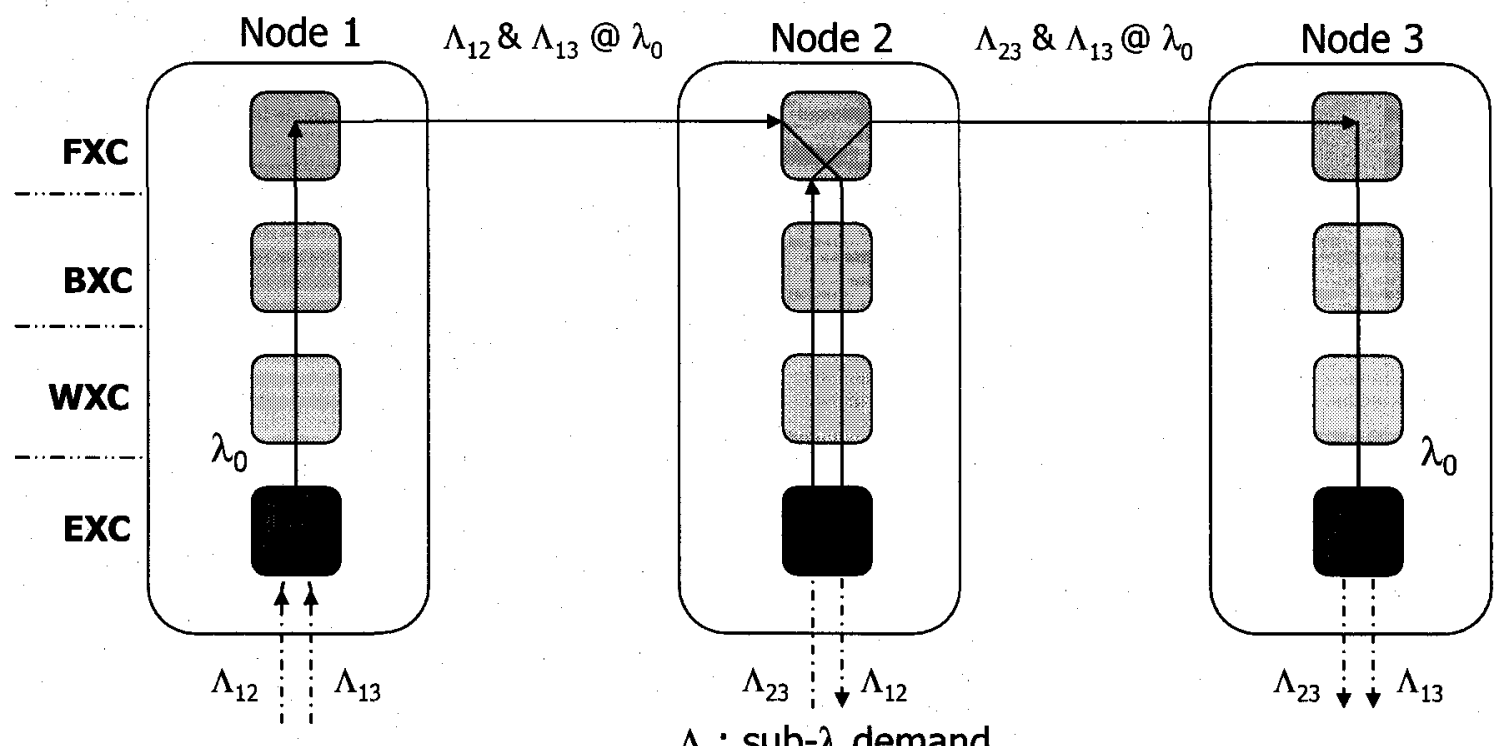

Fig. 3.3: Unclear wavelength-switched path, sub-wavelength flow (de)grouping scenario.

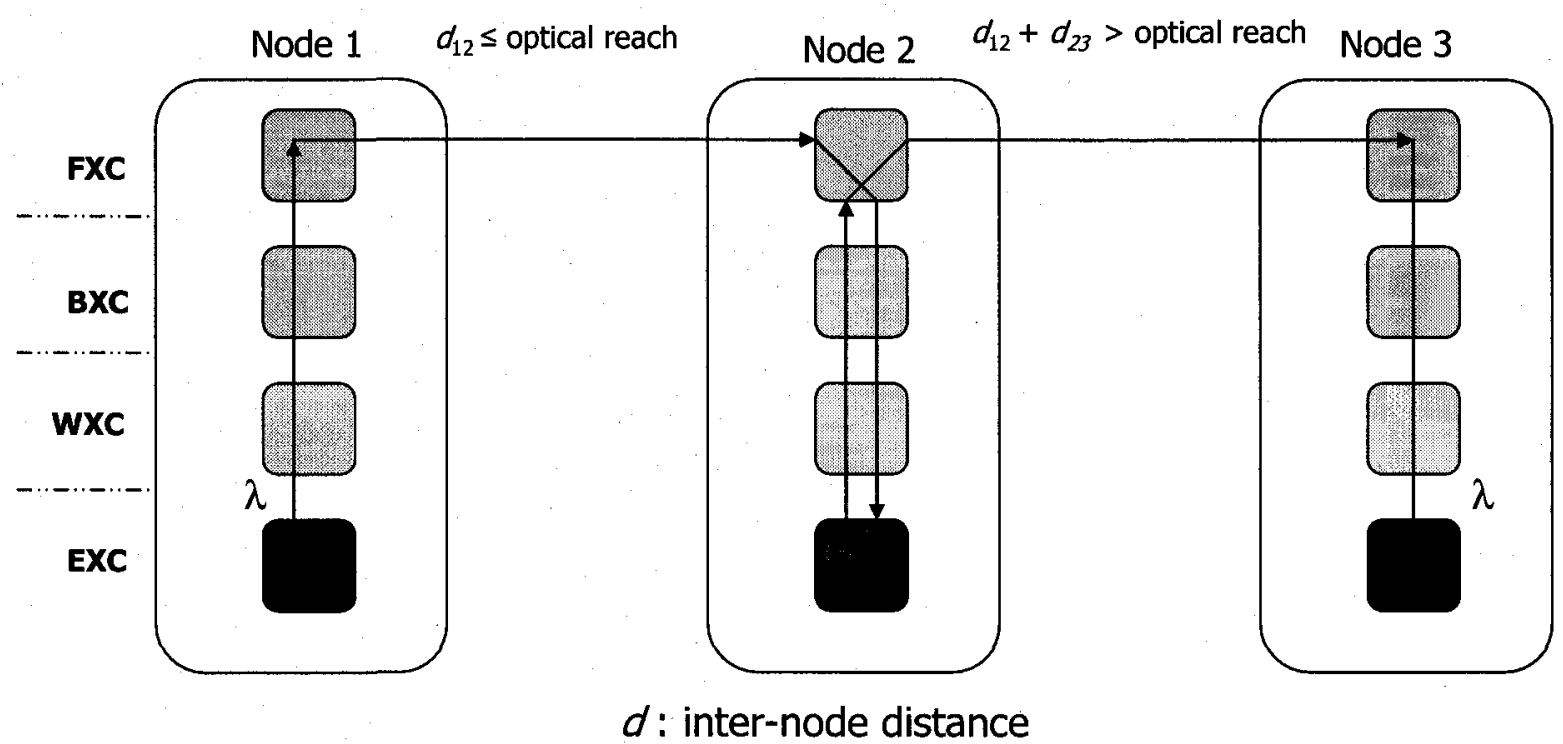

Fig. 3.4: Unclear wavelength-switched path, optical signal regeneration scenario. 


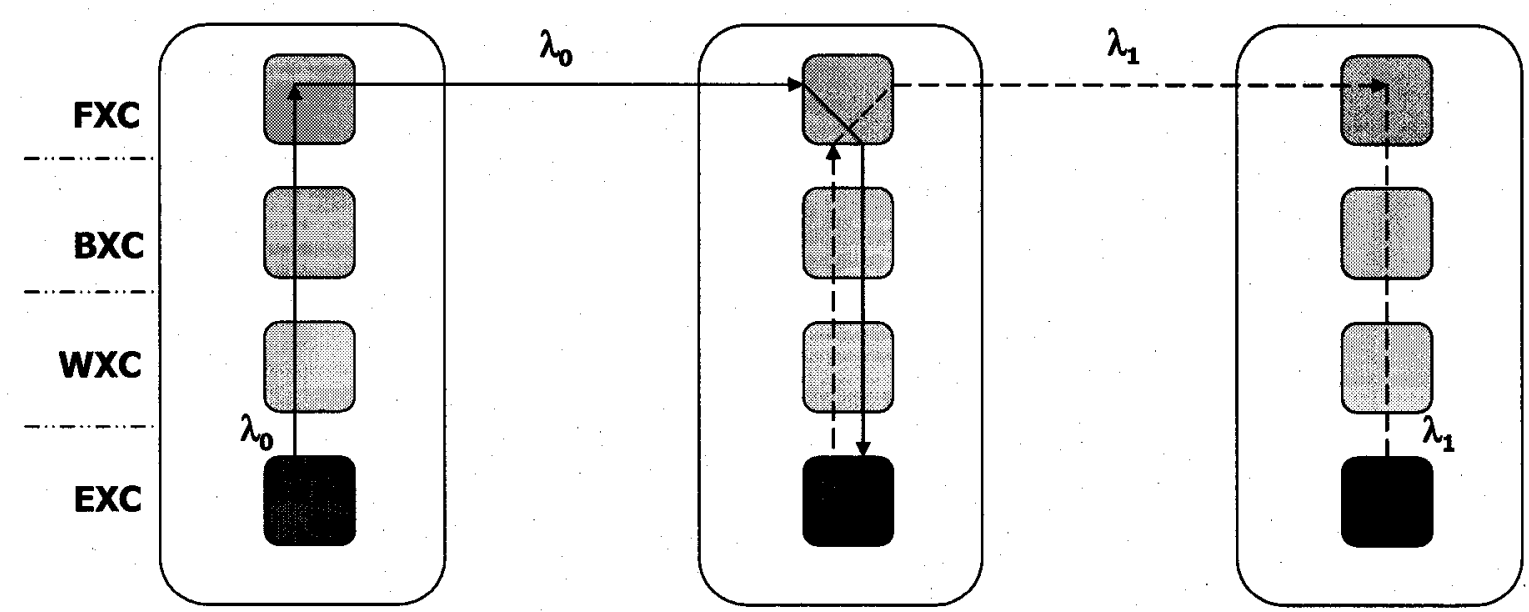

Fig. 3.5: Unclear wavelength-switched path, wavelength conversion scenario.

- Clear Band-Switched (CBS) Patb: The CBS path is used to carry a waveband demand. This type must have a sufficient optical reach to use the same waveband on the same fiber along the entire path without undergoing OEO conversion, as shown in Fig. 3.6. The launch/termination of this path is at the BSC interface. The CBS path can enter the intermediate BXC stages for grouping/degrouping the path segment with/from other lightpaths that share the same fiber, as illustrated in Fig. 3.6.

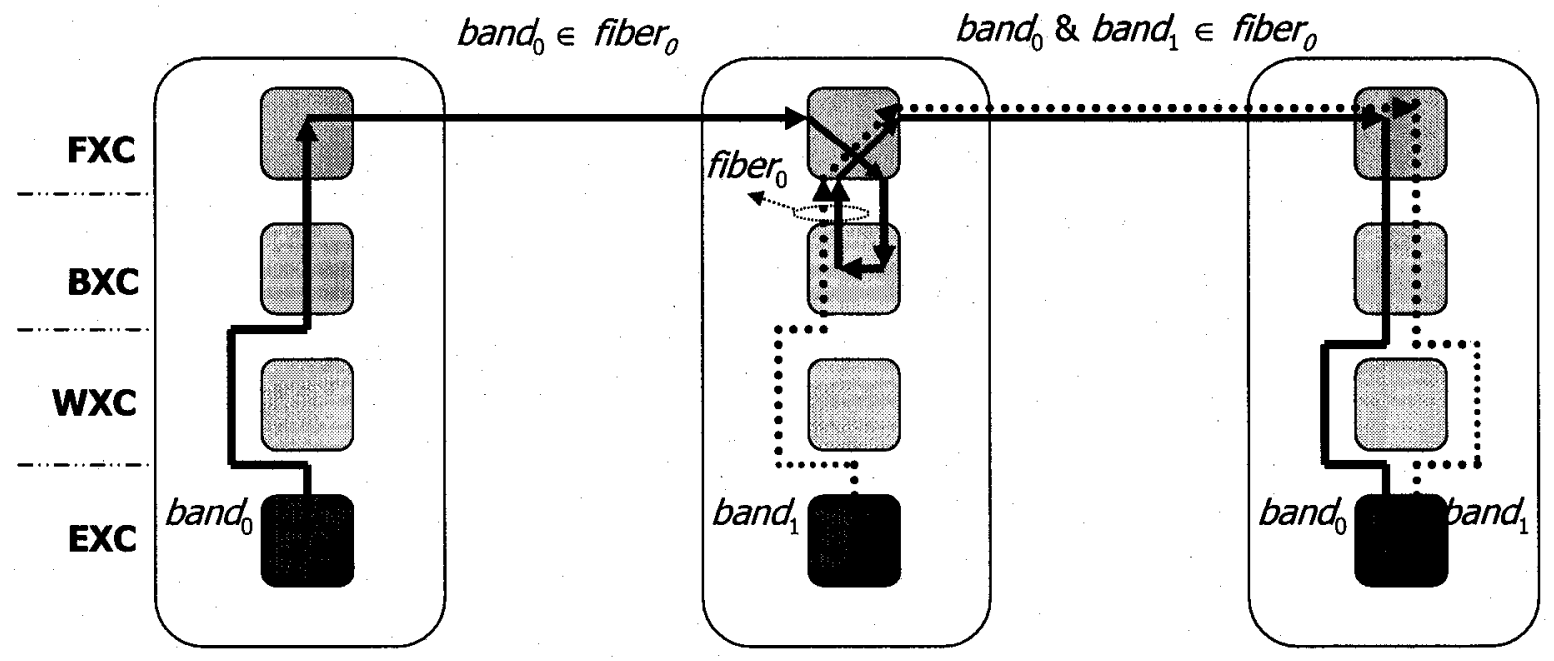

Fig. 3.6: Clear waveband-switched path. 
- Unclear Band-Switched (UBS) Path: This path is used to carry a waveband demand. The UBS path is a concatenation of $\mathrm{CBS}$ paths. This path undergoes $\mathrm{OEO}$ conversion at least once for the purpose of optical signal regeneration, as shown in Fig. 3.7, and/or waveband color conversion to another waveband color on either the same or a different fiber, as shown in Fig. 3.8.

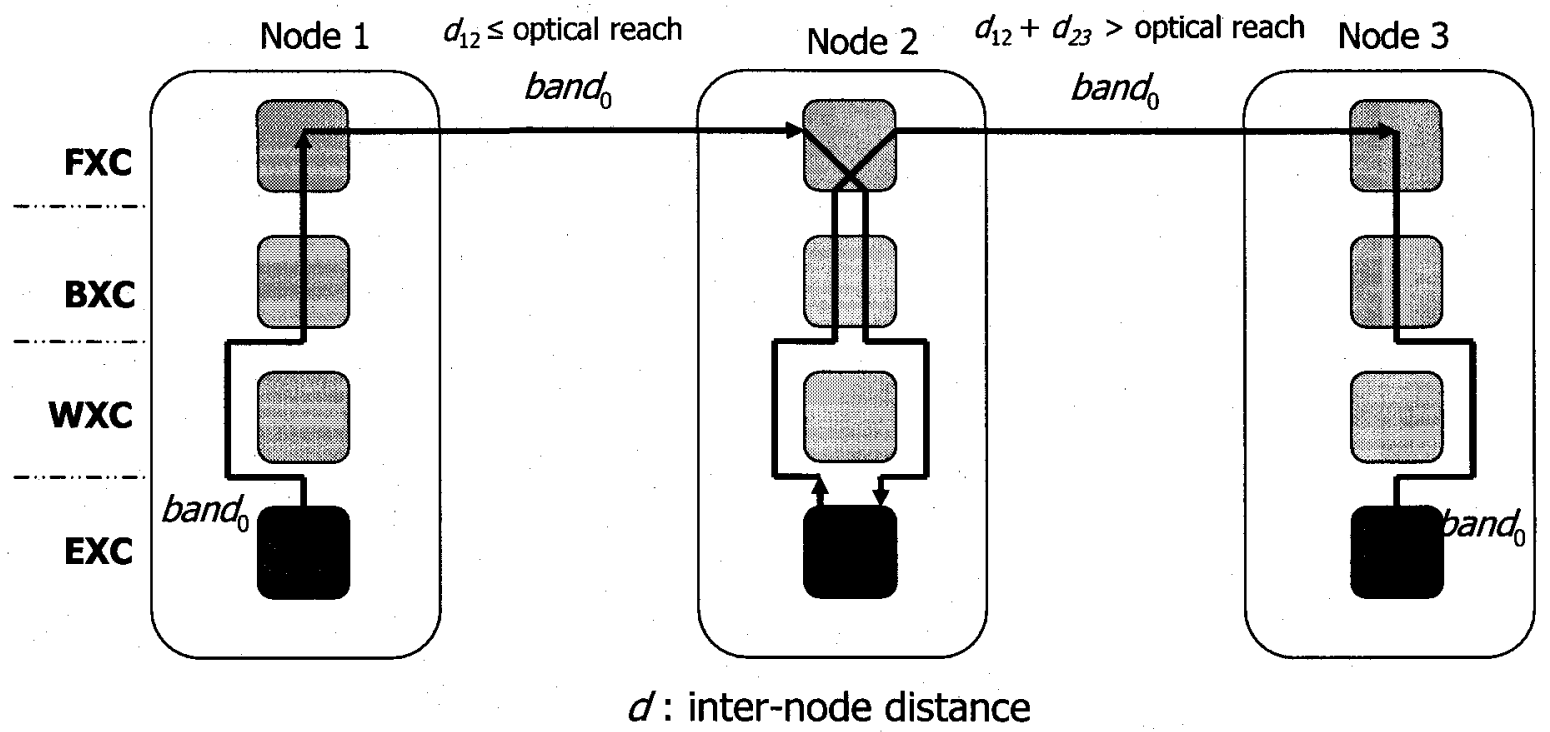

Fig. 3.7: Unclear waveband-switched path, optical signal regeneration scenario.

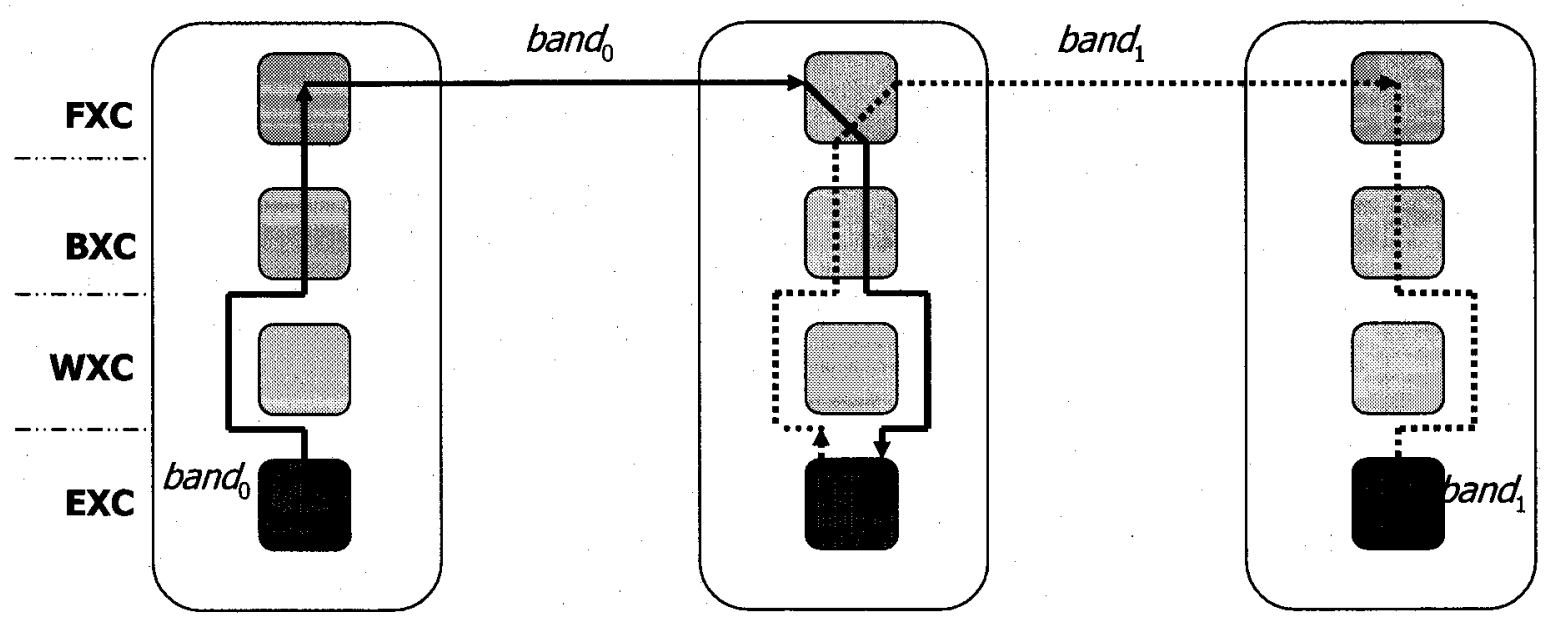

Fig. 3.8: Unclear waveband-switched path, waveband conversion scenario. 
- Clear Fiber-Switched (CFS) Patb: This path is used to carry a fiber demand. The path must have enough optical reach to use the same fiber along the entire path without undergoing OEO conversion. The launch/termination of this path is at the FSC interface, as shown in Fig. 3.9.

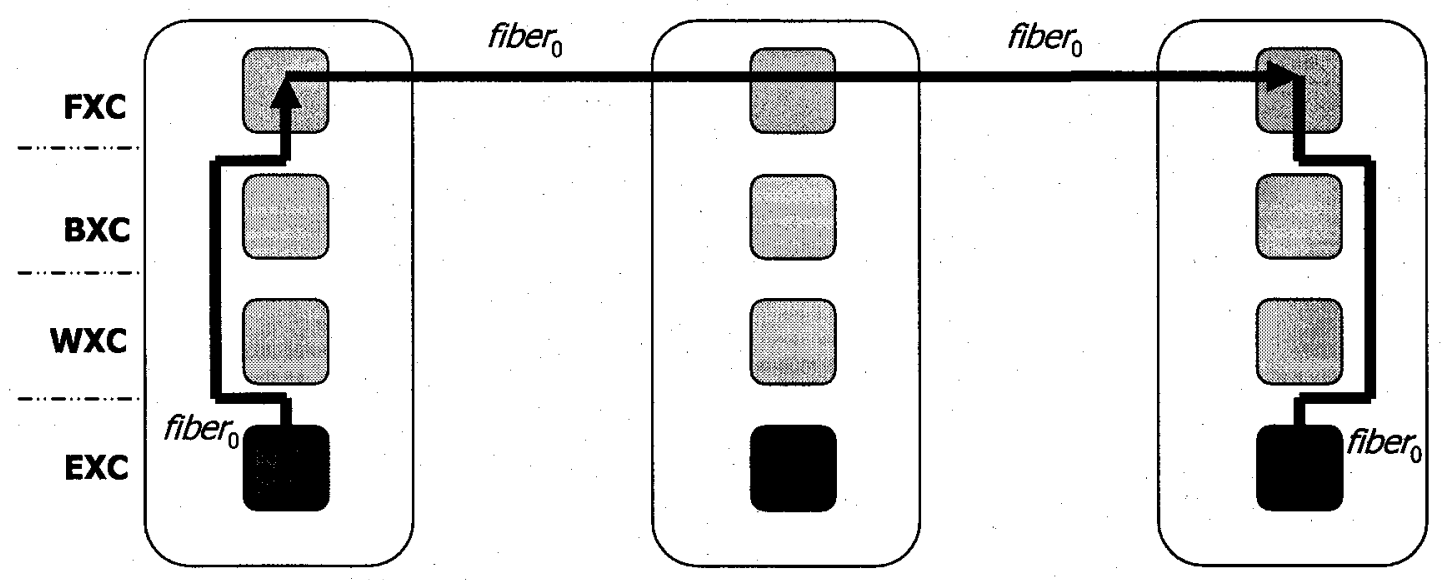

Fig. 3.9: Clear fiber-switched path.

- Unclear Fiber-Switched (UFS) Patb: The UFS path is used to carry a fiber demand. It is a concatenation of CFS paths and undergoes OEO conversion at least once for the purpose of optical signal regeneration and/or fiber-code conversion, as shown in Figs. 3.10 and 3.11 respectively.

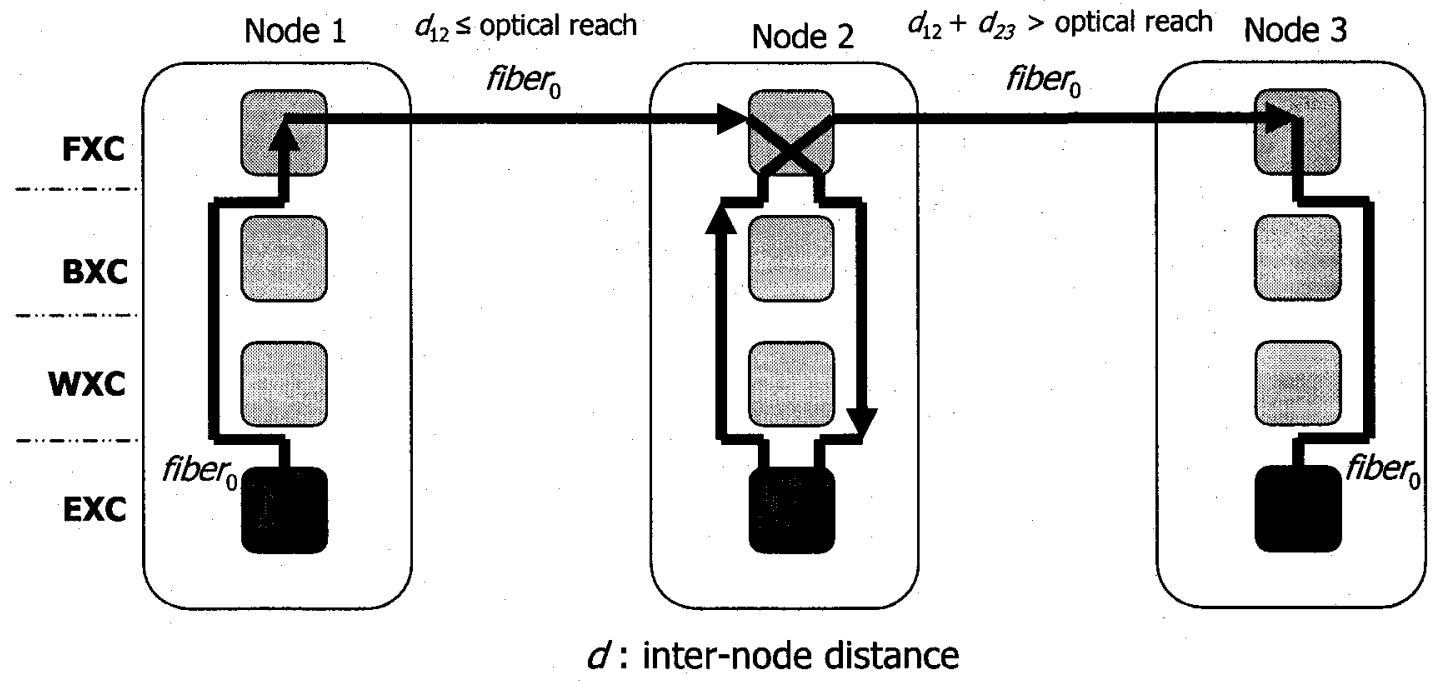

Fig. 3.10: Unclear fiber-switched path, optical signal regeneration scenario. 


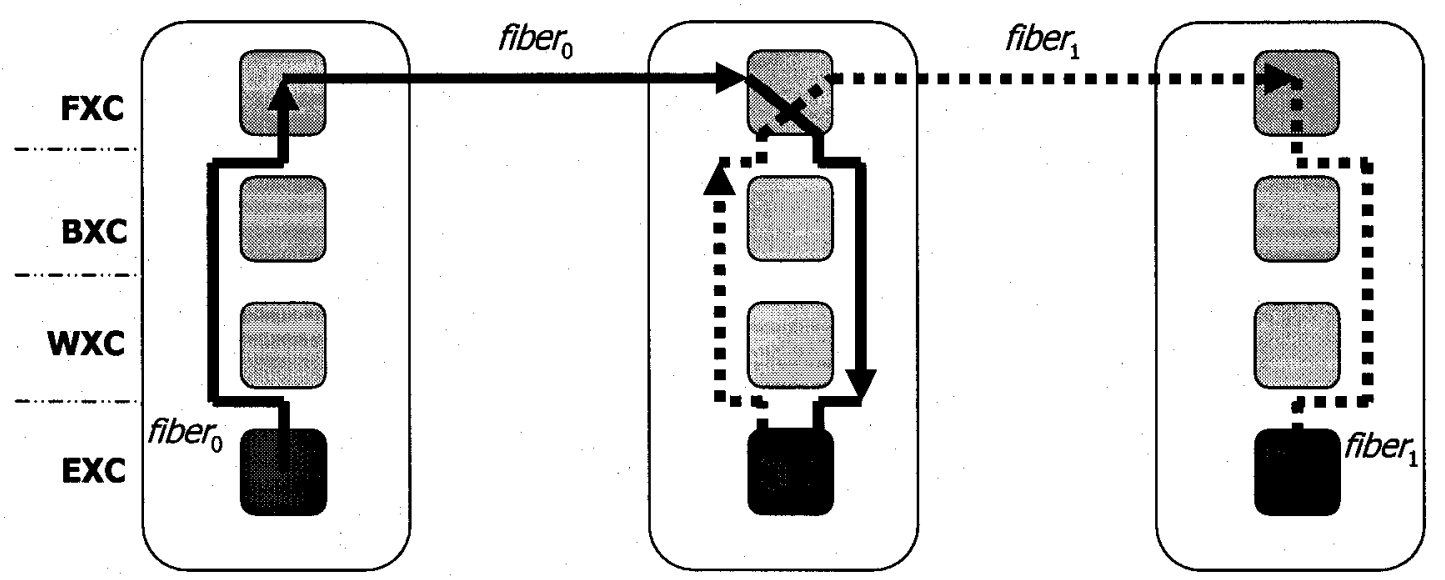

Fig. 3.11: Unclear fiber-switched path, fiber conversion scenario.

The CWS, CBS, and CFS paths whether they are used between $s-d$ pairs or as a result of using UWS, UBS, and UFS paths are the virtual links of the multi-granular virtual topology, which decides on the port requirements of all MG-XCs in the transport network.

\subsubsection{Grouping and Degrouping of Paths}

One of the main factors that play a major role in minimizing the port count in multi-granular transport networks is how the different types of paths are grouped and degrouped. There are three levels of grouping, and they are explained as follows. First, the fiber-level grouping (studied in the literature) groups waveband flows at a certain fiber. Second, the waveband-level grouping (studied extensively in the literature) groups wavelength flows at a certain waveband. Third, the wavelength-level grouping (a new contribution in the context of multi-granular networks) groups sub-wavelength flows at a certain wavelength. The consideration of all these levels and the interactions between them are part of the novelty of this thesis.

In each grouping level, paths can be grouped in many different ways, such as the following. (1) First is the grouping of paths that have the same source and destination (or the end-to-end grouping). The fiber/waveband/wavelength-switched path is a typical scenario of an end-to-end 
grouping of waveband/wavelength/sub-wavelength demands at the fiber/waveband/wavelengthlevel. Other forms of grouping include (2) the grouping of paths that have same source but different destinations, (3) the grouping of paths that have the same destination but different sources, and (4) the grouping of paths that have different sources and destinations (i.e. intermediate grouping). Table 3.3 summarizes how each path type can be involved in the grouping process at all levels.

Table 3.3: Comparison of different $s-d$ path types in terms of their involvement in the grouping process at all levels.

\begin{tabular}{|c|c|c|c|c|c|c|c|c|c|c|c|c|}
\hline $\begin{array}{c}\text { Grouping } \\
\text { involvement }\end{array}$ & & ele & th-L & & & veb & d-Le & & & ibe & evel & \\
\hline & 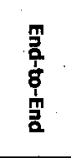 & 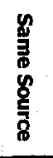 & 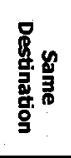 & 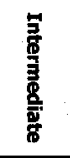 & $\begin{array}{l}\text { 帝 } \\
\text { 旁 }\end{array}$ & 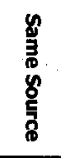 & 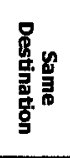 & 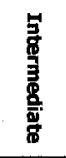 & 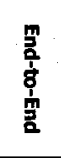 & 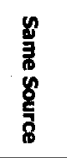 & 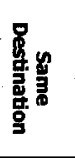 & 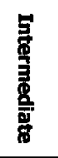 \\
\hline CWS & $\sqrt{ }$ & & & & & $\sqrt{ }$ & $\sqrt{ }$ & $\sqrt{ }$ & & $\sqrt{ }$ & $\sqrt{ }$ & $\sqrt{ }$ \\
\hline UWS & $\sqrt{ }$ & $\sqrt{ }$ & $\sqrt{ }$ & $\sqrt{ }$ & & $\sqrt{ }$ & $\sqrt{ }$ & $\sqrt{ }$ & & $\sqrt{ }$ & $\sqrt{ }$ & $\sqrt{ }$ \\
\hline CBS & & & & & $\sqrt{ }$ & & & & & $\sqrt{ }$ & $\sqrt{ }$ & $\sqrt{ }$ \\
\hline UBS & & & & & $\sqrt{ }$ & & & & & $\sqrt{ }$ & $\sqrt{ }$ & $\sqrt{ }$ \\
\hline CFS & & & & & & & & & $\sqrt{ }$ & & & \\
\hline UFS & & & & & & & & & $\sqrt{ }$ & & & \\
\hline
\end{tabular}

On the other hand, the degrouping of a sub-wavelength/wavelength/waveband-switched path from other path(s) at any transport node requires that all path types using the same incoming fiber enter finer switching stages. Although this process is necessary, it results in extra ports for passthrough paths that do not have to be degrouped at this node. Therefore, the questions of how and where paths should be degrouped are essential parts of the optimization process. Degrouping is needed for many reasons, and these are explained as follows: (1) to perform color conversion, 
because it is not possible for a particular path to continue grouping in the next route segment by using the same color utilized in the previous route segment; (2) to perform signal regeneration; (3) to drop a demand; and (4) to switch a path to a different route segment.

\subsection{Solution of the Planning Problem}

\subsubsection{Problem Statement}

The problem of planning the GMPLS-based transport network involves creating/adjusting multigranularity demands, selecting their path types and the corresponding physical routes and color assignments, and (de)grouping paths. All of these elements have to be combined in ways that minimize the total weighted port count of the transport nodes and satisfy all demands at the same time. The problem is solved given the following: the network fiber layout, the raw/'multigranularity' traffic demands between all node pairs, the wavelength capacity $(C)$, the waveband size or the number of wavelengths per waveband $(W)$, the number of wavebands per fiber $(B)$, the number of fibers per unidirectional physical link $(F)$, the maximum optical reach $\left(R_{\text {max }}\right)$, and the cost weights of all port types. We call this problem Routing and Multi-Granular Paths Assignment (RMGPA) [NaMo06a]. The RMGPA problem is NP-complete since it contains instances of the RWA problem, which is known to be an NP-complete. In this thesis, we will solve different versions of the RMGPA problem that depend on whether the color conversion and signal regeneration capabilities are considered.

\subsubsection{Assumptions}

To facilitate solving the RMGPA problem and make the problem more focused and tractable, our assumptions are explained as follows: 
- Network Model: The network fiber-conduit layout already exists in which each conduit between a node pair consists of two unidirectional physical links (or spans) working at opposite directions. Each unidirectional physical link, as shown in Fig. 1.1, is composed of a number of low-desperation dark fibers that can be lighted up as required. Each fiber is coded by its ID on a link and consists of uniform or fixed size wavebands, which in turn consist of a number of wavelengths and all wavelengths have the same capacity (i.e. they operate at the same bit rate). In this thesis, we assume that the network model is homogenous in the sense that $F, B, W$, and $C$ are the same throughout the network. Moreover, all transport nodes have the same architecture shown in Fig. 3.1.

- Traffic Model: The traffic demand between any node pair represents the long-term average traffic demand. The $s-d$ pair demand is asymmetric. The demand can be of either a raw traffic or multi-granularity traffic type that can be expressed as a multi-dimensional demand in which each dimension represents a different traffic granularity. The sub-wavelength demand is always expressed in bit/s regardless of its origin (i.e. whether it is L2, TDM, or ATM traffic, etc.). The traffic demand of the same granularity can also be bifurcated among distinct physical routes. The significance of bifurcating demands is one of the issues that will be investigated in this thesis.

- Coping with power losses and impairments. An optical signal may have to pass through a number of MG-OXCs and fiber segments. Therefore, while propagating through the network, the signal degrades in quality as it loses power due to such primary causes as fiber attenuations and insertion losses at the MG-OXC. To compensate for these losses, we assume that each transport node is already equipped with automatic-gain-control postamplifiers and preamplifiers. Moreover, each unidirectional physical link is already equipped with automaticgain-control Erbium-Doped Fiber Amplifiers (EDFAs). In addition to these losses, the optical 
signal is also affected by various serious physical impairments, such as Polarization Mode Dispersion (PMD) and Chromatic Dispersion (CD). These impairments accumulate over links within an all-optical path (or clear path). How far an all-optical path can be routed optically without the need for signal regeneration using the EXC depends on how the impairments accumulate without exceeding predefined thresholds. This is the definition of the so-called optical reach. The use of optical duobinary modulation techniques [HARD06] in the EXC line-side interfaces promises improved optical reach with the elimination of dispersion compensators along network spans [MASO07]. Moreover, the proper choice of the duobinary technique and the fiber type is key to extending the optical reach to thousands of kms [Tan+07]. Throughout this thesis, we assume that all EXC interfaces use the duobinary technique. Also, we assume that the maximum optical reach is given as an input parameter to the problem, and all-optical paths are chosen in such a way that the length of all-optical paths must not exceed the given optical reach value.

- Cost Model: The MG-OXC ports and EXC line-side interfaces (or ports) are included in our cost model. We assume that the input port and output port of the MG-OXC/EXC have the same cost weight. The cost of the EXC port depends on the type of interface used and the reach it can provide. Long-reach interfaces are more expensive than short-reach ones [Simm05]. In addition, EXC ports are more expensive than MG-OXC ports [Grub01] [Izm+02]. The cost ratio of an EXC port to an MG-OXC port (E/O ratio) as a function of the optical reach is decided using the following equation [Simm05]:

$$
\frac{\text { EXC port weight }}{\text { MG -OXC port weight }}=\text { RATIO }_{1000} \times(1.25)^{\log _{2}(R / 1000)}
$$

where $R$ is the optical reach in $\mathrm{km}$ and $\mathrm{RATIO}_{1000}$ is the $\mathrm{E} / \mathrm{O}$ ratio at $\mathrm{R}=1000 \mathrm{~km}$. These differences in cost will be reflected in the weighting factors of all port types when the port 
count of MG-XCs is minimized. Throughout this thesis, we assume that the 40G-based EXC port costs two-and-a-half times what a 10G-based EXC port would cost [FULL06].

- Label conversion: A wavelength/waveband/fiber-switched path can be assigned different labels (or colors) along the entire path. The label in a wavelength-switched path corresponds to the wavelength used at a certain waveband of a certain fiber ID. The label in a waveband-switched path corresponds to the waveband used at a certain fiber ID. The label in a fiber-switched path corresponds to the fiber ID used. Changing labels along the path is done electronically by switching the whole signal (data+label) into the EXC, where the label conversion is performed.

- Protection: Protection techniques regarding the possible failure of fiber links or nodes are not considered. In other words, our focus is only on the efficient allocation of the working traffic.

\subsubsection{Solution Approach}

In Chapters 4 and 5, different versions of the RMGPA problem will be tackled. A high-level description of the methodology to be used to solve the RMGPA problem is shown in Fig. 3.12. Regardless of the problem version and the optimization method to be used, the first subtask of the problem solution is to create optimized multi-granularity traffic demands from the given raw demands or adjust the given multi-granularity demands. The goal of this subtask is to increase the end-to-end grouping at the wavelength-, waveband-, and fiber-levels. In the sense that the volume of sub-wavelength demands that is equal to $C$ is treated as one unit of the wavelength demand and has to follow the same physical route. Similarly, the number of wavelength demands that is equal to $W$ is considered to be one unit of the waveband demand. Moreover, the number of waveband demands that is equal to $B$ is considered to be one unit of the fiber demand. Although there are some similarities between our approach [NaMo06a] and the approach presented in [STPa07] 
(Subsection 2.5.11), our approach (1) ensures that there is no gap between the volume of the required raw demand and the capacity of the allocated multi-granularity demand, (2) considers all of the traffic granularities defined in GMPLS, and (3) not only creates optimized multi-granularity demands from raw demands but also adjusts the given multi-granularity demands. The Demand Adjustment algorithm works as follows [NaMo06a]:

\section{Demand Adjustment Algorithm;}

Input: Raw/'multi-granularity' traffic demands, C, W, B

Output: Optimized/adjusted multi-granularity demands

\section{Begin}

\section{Initialization:}

If the given demands are raw demands

\{

- Set sub-wavelength demands = raw demands

- Set wavelength/waveband/fiber demands = zero

\}

2. For all sub-wavelength demands, do the following: If sub-wavelength demand $\geq \mathrm{C}$

\{

- Set wavelength demand (of the same $s-d$ pair) $=$ wavelength demand $+\lfloor$ sub-wavelength demand/C」

- Set sub-wavelength demand $=$ sub-wavelength demand $-($ Lsub-wavelength demand $/ C\rfloor) \times C$

\}

Else sub-wavelength demand remains unchanged

\section{For all wavelength demands, do the following:}

If wavelength demand $\geq W$

\{

- Set waveband demand = waveband demand $+\lfloor$ wavelength demand $/ W\rfloor$

- Set wavelength demand $=$ wavelength demand $-(\lfloor$ wavelength demand $/ W\rfloor) \times W$

\}

Else wavelength demand remains unchanged 


\section{For all waveband demands, do the following:}

If fiber demand $\geq B$

\{

- Set fiber demand = fiber demand $+\lfloor$ waveband demand $/ B\rfloor$

- Set waveband demand $=$ waveband demand $-(\lfloor$ waveband demand $/ B\rfloor) \times B$

\}

Else waveband demand remains unchanged

End.

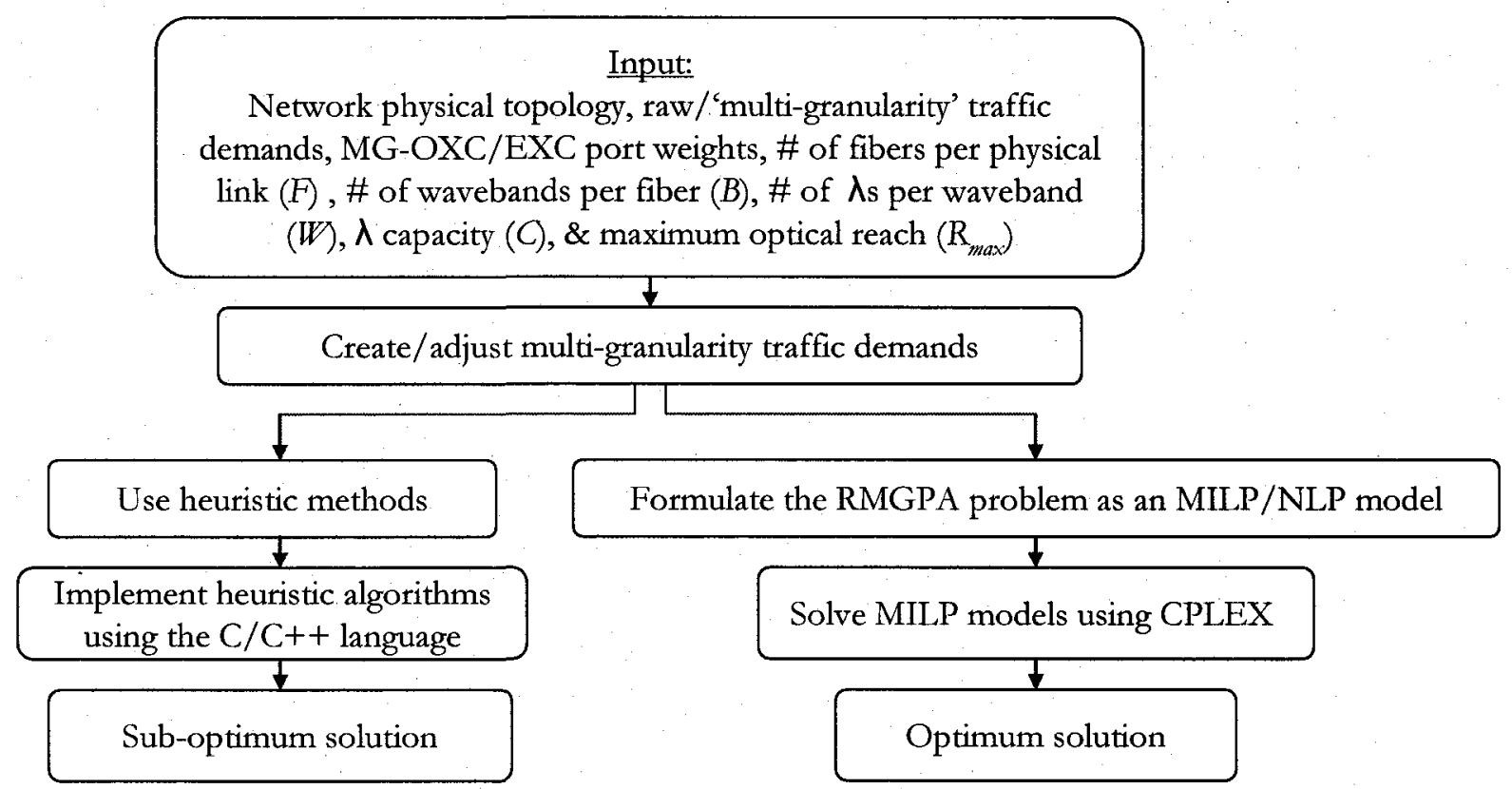

Fig. 3.12: Flowchart for the approach to be taken to solve the RMGPA problem optimally and sub-optimally.

After this point, the methodology proceeds in two directions. The aim of the first direction is to obtain an exact solution to the RMGPA problem. This can be achieved by formulating the problem as an MILP/NLP model using the path-formulation approach. The optimization variables consist of binary, integer, and real variables. Sub-wavelength flows are the main reason behind the existence of real variables. However, due to the extreme complexity of the problem, only MILP models will be solved for small-sized planning problems. The solutions of the MILP 
models will serve as valuable quality references for the sub-optimum methods developed [Grov04]. MILP models are solved using AMPL CPLEX version 7.0 software [CPLEX] [FGKe03], which uses Dakin's method (refer to Subsection 2.4.1).

The aim of the second direction is to solve large-sized planning problems and satisfy the planning speed requirement, which should be completed in a matter of minutes [Simm06]. However, this will be at the expense of a trade-off in the accuracy of the solution for a decrease in the complexity of the computation. This can be achieved by using heuristic methods. To determine the best suboptimum solution, many heuristics are proposed in this thesis. Heuristic algorithms will be implemented using the $\mathrm{C} / \mathrm{C}++$ language. The implementation platforms of MILP models and heuristics run on an Intel Pentium 4, 2.4G CPU, with $1 \mathrm{~GB}$ of physical memory. 


\section{Chapter 4}

\section{Planning of GMPLS-Based Transport Networks without Conversion and Regeneration Capabilities}

\subsection{Introduction}

In this chapter, we focus on planning the GMPLS-based transport network without considering the color conversion and optical signal regeneration capabilities. This means that the RMGPA problem here has to include the color-continuity constraints and exclude the optical-reach constraints. Accordingly, for fiber, waveband, and wavelength demands, only clear paths are to be used. However, for sub-wavelength demands, both clear and unclear paths can be involved. Unclear paths are needed in this case for the purpose of sharing the capacities of clear paths between many sub-wavelength demands. Thus, an unclear path can consist of clear paths with different colors. This version of the RMGPA problem is the right fit for the planning problem in metro core ${ }^{8}$ (or regional) transport networks in which the control plane requires that the colorcontinuity constraints be kept in order to reduce the resource fragmentation ${ }^{9}$ [HoMo02b].

This chapter proceeds with the MILP formulation of the RMGPA problem. Then, algorithms of the proposed heuristic approach are explained. Afterword, numerical results are presented by including the following: (1) the verification of the heuristic approach, (2) demonstrations of the

\footnotetext{
${ }^{8}$ In metro core networks, fiber links are generally short enough that optical signal regeneration is not generally required.

${ }^{9}$ Resource fragmentation means that unclear paths are used for the purpose of color conversions, and each clear path in the unclear path is represented as a link in the link-state database of the control plane. As a result, the computational complexity of the path selection in the control plane will be increased and more computational resources will be needed.
} 
impact of input parameters and some solution enhancements, and (3) a comparison of heuristics via simulation.

\subsection{MILP Formulation}

To achieve the optimum solution to the RMGPA problem, the problem is formulated as an MLLP model. The formulation proceeds as follows [NaMo06a] $[\mathrm{NaMo06b}][\mathrm{NaMo08c}]$.

\section{Parameters:}

- $N$ is the number of network nodes.

- $C, W, B$, and $F$.

- $P$ is the number of physical routes allowable between a node pair.

- $c f / c b / c w$ is the relative cost of the $\mathrm{FXC} / \mathrm{BXC} / \mathrm{WXC}$ port.

- $c g f / c g b / c g w$ is the relative cost of the FSC/BSC/LSC-based EXC port.

- $\Lambda_{s d}^{g}$ is the $s-d$ pair "adjusted" demand of the $g^{\text {th }}$ granularity obtained using the Demand Adjustment algorithm (refer to Subsection 3.3.3); $g=0 / 1 / 2 / 3$ for sub-wavelength/wavelength/ waveband/fiber demand.

- $\delta_{i j}=1$ if there is a fiber connectivity between node pair $i-j$; otherwise it is zero.

- $Q^{s d}$ is the set of $P$ distinct routes between the $s-d$ pair computed using the All Distinct Routes algorithm [Grov04].

- $\zeta_{i j}^{g, s d, p}=1$ if the $s-d$ demand of the $g^{\text {h }}$ granularity (note that $g \neq 0$ here) using the $p^{\text {th }}$ distinct route crosses physical link $i-j$; otherwise it is zero. This parameter is derived from $Q^{s d}, \Lambda_{s d}^{g}$ and $\delta_{i j}$.

- $\gamma_{i j}^{s t, p}=1$ if the node pair $s-d$ using the $p^{\text {th }}$ distinct route crosses physical link $i-j$; otherwise it is zero. This parameter is derived from $Q^{s d}$ and $\delta_{i j}$. 
Sets:

- Node indices: $s, d, i, j, m$, and $n \in\{0,1, . ., N-1\}$.

- Route index: $p \in\{0,1, \ldots, P-1\}$.

- Wavelength index: $k \in\{0,1, . ., W-1\}$.

- Waveband index: $b \in\{0,1, \ldots, B-1\}$.

- Fiber index: $f \in\{0,1, \ldots, F-1\}$.

Optimization Variables:

- $F X C_{i} / B X C_{i} / W X C_{i}$ is an integer variable that represents the number of $\mathrm{FXC} / \mathrm{BXC} / \mathrm{WXC}$ ports at node $i$.

- $\mathrm{FSCO}_{i} / \mathrm{BSCO}_{i} / \mathrm{WSCO}_{i}$ is an integer variable that represents the number of FSC/BSC/LSC-based GMPLS transmitters at node $i$.

- $\mathrm{FSCl}_{i} / \mathrm{BSCI}_{i} / \mathrm{WSCI}_{i}$ is an integer variable that represents the number of FSC/BSC/LSC-based GMPLS receivers at node $i$.

- $F A_{i} / B A_{i} / W A_{i}$ is an integer variable that represents the number of $F X C / B X C / W X C$ add ports at node $i$.

- $F D_{i} / B D_{i} / W D_{i}$ is an integer variable that represents the number of $F X C / B X C / W X C$ drop ports at node $i$.

- $F I_{i} / B I_{i} / W I_{i}$ is an integer variable that represents the number of $\mathrm{FXC} / \mathrm{BXC} / \mathrm{WXC}$ input ports at node $i$.

- $\mathrm{FO}_{i} / \mathrm{BO}_{i} / W \mathrm{WO}_{i}$ is an integer variable that represents the number of $\mathrm{FXC} / \mathrm{BXC} / \mathrm{WXC}$ output ports at node $i$. 
- $f i_{i}^{j, f} / f o_{i}^{j, f}=1$ if the FXC input/output port of node $i$ that is operating at fiber $f$ is used by the traffic 'arriving from'/'going to' the fiber-connected node $j$; otherwise it is zero.

- $b i_{i}^{j, f, b} / b o_{i}^{j, f, b}=1$ if the BXC input/output port of node $i$ that is operating at waveband $b$ of fiber $f$ is used by the traffic 'arriving from'/'going to' the fiber-connected node $j$; otherwise it is zero.

- $w i_{i}^{j, f, b, k} / w o_{i}^{j, f, b, k}=1$ if the WXC input/output port of node $i$ that is operating at wavelength $k$ of waveband $b$ and fiber $f$ is used by the traffic 'arriving from'/'going to' the fiber-connected node $j$; otherwise it is zero.

- $F B_{i} / B F_{i}$ is an integer variable that represents the number of FXC output/input ports that are connected to the $\mathrm{BXC}$ stage at node $i$.

- $B W_{i} / W B_{i}$ is an integer variable that represents the number of $\mathrm{BXC}$ output/input ports that are connected to the WXC stage at node $i$.

- $f b_{i}^{j, f} / b f_{i}^{j, f}=1$ if the FXC output/input port of node $i$ that is connected to the BXC stage and operating at fiber $f$ is used by the traffic 'arriving from'/'going to' the fiber-connected node $j$; otherwise it is zero.

- $b w_{i}^{j, f, b} / w b_{i}^{j, f, b}=1$ if the BXC output/input port of node $i$ that is connected to the WXC stage and operating at waveband $b$ of fiber $f$ is used by the traffic 'arriving from'/'going to' the fiberconnected node $j$; otherwise it is zero.

- $V F_{f}^{s d, p}=1$ if there is a CFS path between an $s-d$ pair that has a fiber demand, uses the $p^{\text {th }}$ distinct route, and utilizes fiber $f$ along the entire path; otherwise it is zero.

- $V B_{f, b}^{s d, p}=1$ if there is a CBS path between an $s-d$ pair that has a waveband demand, uses the $p^{\text {th }}$ distinct route, and utilizes waveband $b$ of fiber $f$ along the entire path; otherwise it is zero. 
- $V W_{f, b, k}^{s d, p}=1$ if there is a CWS path between an $s-d$ pair that has a wavelength demand, uses the $p^{\text {th }}$ distinct route, and is carried on wavelength $k$ of waveband $b$ and fiber $f$ along the entire path; otherwise it is zero.

- $V S_{f, b, k}^{s d, p}=1$ if there is a CWS path between an $s-d$ pair that uses the $p^{\text {th }}$ distinct route and is carried on wavelength $k$ of waveband $b$ and fiber $f$ along the entire path; otherwise it is zero. This CWS path is used solely by sub-wavelength demands.

- $B F P T_{f, b}^{s d, p, i, m n}=1 / W F P T_{f, b, k}^{s d, p, i, m n}=1 / S F P T_{f, b, k}^{s d, p, i, m n}=1$ if the portion of the virtual link $V B_{f, b}^{s d, p} / V W_{f, b, k}^{s d, p} /$ $V S_{f, b, k}^{s d, p}$ that passes through node $i$, arrives from node $m$, and goes to node $n$ is only fiber-switched; otherwise it is zero.

- $B P T_{f, b}^{s d, p, i, m n}=1 / W B P T_{f, b, k}^{s d, p, i, m n}=1 / S B P T_{f, b, k}^{s d, p, i, m n}=1$ if the portion of the virtual link $V B_{f, b}^{s d, p} / V W_{f, b, k}^{s d, p} / V S_{f, b, k}^{s d, p}$ that passes through node $i$, arrives from node $m$, and goes to node $n$ is waveband-switched; otherwise it is zero.

- $W P T_{f, b, k}^{s d, p, i, m n}=1 / S P T_{f, b, k}^{s d, p, i, m n}=1$ if the portion of the virtual link $V W_{f, b, k}^{s d, p} / V S_{f, b, k}^{s d, p}$ that passes through node $i$, arrives from node $m$, and goes to node $n$ is wavelength-switched; otherwise it is zero.

- $\varphi s_{p, i, b, f}^{s d, i j}$ is a real variable that represents the amount of the sub-wavelength flow of the $s-d$ demand that is carried on the virtual link $V S_{f, b, k}^{i j, p}$.

\section{Objective:}

Minimize the total weighted port count in the GMPLS-based transport network:

$$
\min \left(\begin{array}{c}
c f \cdot \sum_{i} F X C_{i}+c b \cdot \sum_{i} B X C_{i}+c w \cdot \sum_{i} W X C_{i}+ \\
c g f \cdot \sum_{i}\left(F S C O_{i}+F S C I_{i}\right)+c g b \cdot \sum_{i}\left(B S C O_{i}+B S C I_{i}\right)+c g w \cdot \sum_{i}\left(W S C O_{i}+W S C I_{i}\right)
\end{array}\right)
$$

\section{Constraints:}

Minimizing the weighted port count is subject to the following constraints: 
1) Port configurations at all switching stages of the GMPLS-based transport node, as illustrated in Fig. 4.1:

$$
\begin{gathered}
W X C_{i}=W D_{i}+W A_{i}+W O_{i}+W I_{i} \quad \forall i \\
B X C_{i}=B D_{i}+B A_{i}+W B_{i}+B W_{i}+B O_{i}+B I_{i} \quad \forall i \\
F X C_{i}=F D_{i}+F A_{i}+F B_{i}+B F_{i}+F O_{i}+F I_{i} \quad \forall i \\
W S C O_{i}=W A_{i} \quad \forall i \\
W S C I_{i}=W D_{i} \quad \forall i \\
B S C I_{i}=B D_{i} \quad \forall i \\
B S C O_{i}=B A_{i} \quad \forall i \\
F S C I_{i}=F D_{i} \quad \forall i \\
F S C O_{i}=F A_{i} \quad \forall i
\end{gathered}
$$

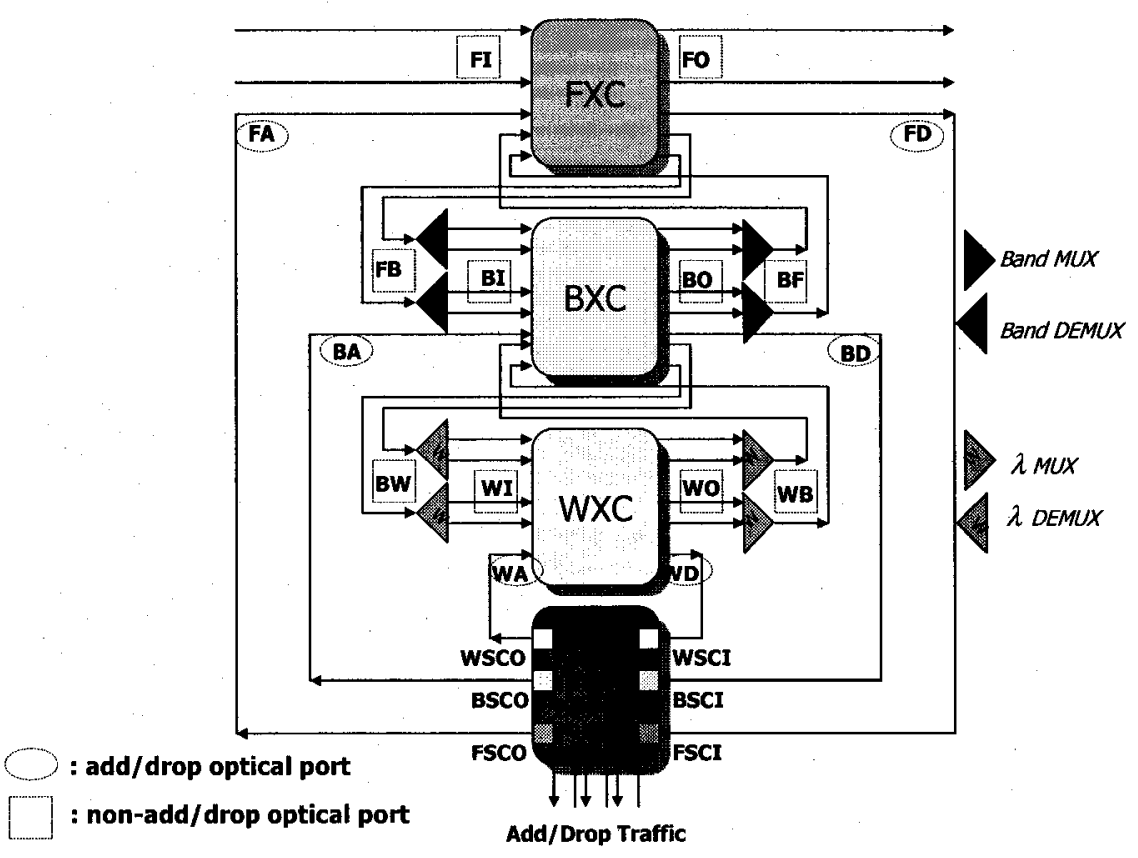

Fig. 4.1: Port configurations at all switching stages of the GMPLS-based transport node. 
2) WXC add and drop ports (refer to Fig. 4.1) are decided by the endpoints of the CWS-based virtual links that are needed to handle both wavelength and sub-wavelength demands; $\mathrm{BXC} / \mathrm{FXC}$ add and drop ports are decided by the endpoints of the CBS/CFS-based virtual links as well as waveband/fiber demands:

$$
\begin{aligned}
& W A_{s}=\sum_{j}^{j \neq s} \sum_{m}^{m \neq s} \sum_{p} \sum_{f} \sum_{b} \sum_{k}\left(\gamma_{s m}^{s, p} \cdot V S_{f, b, k}^{s, p}+\zeta_{s m}^{1, s, p, p} \cdot V W_{f, b, k}^{s, p}\right) \forall s \\
& W A_{s}=\sum_{j}^{j \neq s} \sum_{p} \sum_{f} \sum_{b} \sum_{k}\left(V S_{f, b, k}^{s j, p}+V W_{f, b, k}^{s j, p}\right) \forall s \\
& W D_{d}=\sum_{i}^{i \neq d} \sum_{n}^{n \neq d} \sum_{p} \sum_{f} \sum_{b} \sum_{k}\left(\gamma_{n d}^{i d, p} \cdot V S_{f, b, k}^{i d, p}+\zeta_{n d}^{1, i d, p} \cdot V W_{f, b, k}^{i d, p}\right) \forall d \\
& W D_{d}=\sum_{i}^{i \neq d} \sum_{p} \sum_{f} \sum_{b} \sum_{k}\left(V S_{f, b, k}^{i d, p}+V W_{f, b, k}^{i d, p}\right) \forall d \\
& B A_{s}=\sum_{j}^{j \neq s} \sum_{m}^{m \neq s} \sum_{p} \sum_{f} \sum_{b}\left(\zeta_{s m}^{2, s j, p} \cdot V B_{f, b}^{s, p}\right) \forall s \\
& B A_{s}=\sum_{d}^{d \neq s} \Lambda_{s d}^{2} \quad \forall s \\
& B D_{d}=\sum_{i}^{i \neq d} \sum_{n}^{n \neq d} \sum_{p} \sum_{f} \sum_{b}\left(\zeta_{n d}^{2, i d, p} \cdot V B_{f, b}^{i d, p}\right) \forall d \\
& B D_{d}=\sum_{s}^{s \neq d} \Lambda_{s d}^{2} \quad \forall d \\
& F A_{s}=\sum_{j}^{j \neq s} \sum_{m}^{m \neq s} \sum_{p} \sum_{f}\left(\zeta_{s m}^{3, s j, p} \cdot V F_{f}^{s, p}\right) \forall s \\
& F A_{s}=\sum_{d}^{d \neq s} \Lambda_{s d}^{3} \forall s \\
& F D_{d}=\sum_{i}^{i \neq d} \sum_{n}^{n \neq d} \sum_{p} \sum_{f}\left(\zeta_{n d}^{3, i d, p} \cdot V F_{f}^{i d, p}\right) \forall d
\end{aligned}
$$




$$
F D_{d}=\sum_{s}^{s \neq d} \Lambda_{s d}^{3} \quad \forall d
$$

3) The multi-granular virtual topology must accommodate all multi-granularity traffic demands:

$$
\begin{gathered}
\sum_{j}^{j \neq s} \sum_{p} \sum_{f} \sum_{b} \sum_{k} \varphi s_{p, k, b, f}^{s d, s j}=\Lambda_{s d}^{0} \forall s, d \mid s \neq d \\
\sum_{i}^{i \neq d} \sum_{p} \sum_{f} \sum_{b} \sum_{k} \varphi s_{p, k, b, f}^{s d, i d}=\Lambda_{s d}^{0} \forall s, d \mid s \neq d \\
\sum_{p} \sum_{f} \sum_{b} \sum_{k} V W_{f, b, k}^{s d, p}=\Lambda_{s d}^{1} \quad \forall s, d \mid s \neq d \\
\sum_{p} \sum_{f} \sum_{b} V B_{f, b}^{s d, p}=\Lambda_{s d}^{2} \quad \forall s, d \mid s \neq d \\
\sum_{p} \sum_{f} V F_{f}^{s d, p}=\Lambda_{s d}^{3} \quad \forall s, d \mid s \neq d
\end{gathered}
$$

4) Sub-wavelength flows must satisfy the wavelength capacity constraint:

$$
\sum_{s} \sum_{d}^{d \neq s} \varphi s_{p, k, k, f, f}^{s d, j} \leq V S_{f, b, k}^{i j, p} \cdot C \quad \forall p, f, b, k, i, j \mid i \neq j
$$

5) A pass-through CWS/CBS-based virtual link at each intermediate node can be switched at the 'BXC or WXC'/BXC, as depicted in Fig. 4.2, for degrouping and grouping purposes; otherwise, it is switched at the FXC:

$$
\begin{aligned}
& S P T_{f, b, k}^{s d, p, i, m n}+S B P T_{f, b, k}^{s d, p, i, m n}+S F P T_{f, b, k}^{s d, p, i, m n}=V S_{f, b, k}^{s d, p} \\
& \forall k, b, f, p, i, j, m, n, s, d \mid s \neq d \neq i, m \neq d, n \neq s, m \neq n, \gamma_{i n}^{s d, p}=1, \gamma_{m i}^{s d, p}=1 \\
& W P T_{f, b, k}^{s d, p, i, m n}+W B P T_{f, b, k}^{s d, p, i, m n}+W F P T_{f, b, k}^{s d, p, i, m n}=V W_{f, b, k}^{s d, p} \\
& \forall k, b, f, p, i, j, m, n, s, d \mid \Lambda_{s d}^{1}>0, s \neq d \neq i, m \neq d, n \neq s, m \neq n, \zeta_{i n}^{1, s d, p}=1, \zeta_{m i}^{1, s d, p}=1
\end{aligned}
$$




$$
\begin{aligned}
& B P T_{f, b}^{s d, p, i, m n}+B F P T_{f, b}^{s d, p, i, m n}=V B_{f, b}^{s d, p} \\
& \forall b, f, p, i, j, m, n, s, d \mid \Lambda_{s d}^{2}>0, s \neq d \neq i, m \neq d, n \neq s, m \neq n, \zeta_{i n}^{2, s d, p}=1, \zeta_{m i}^{2, s d, p}=1
\end{aligned}
$$

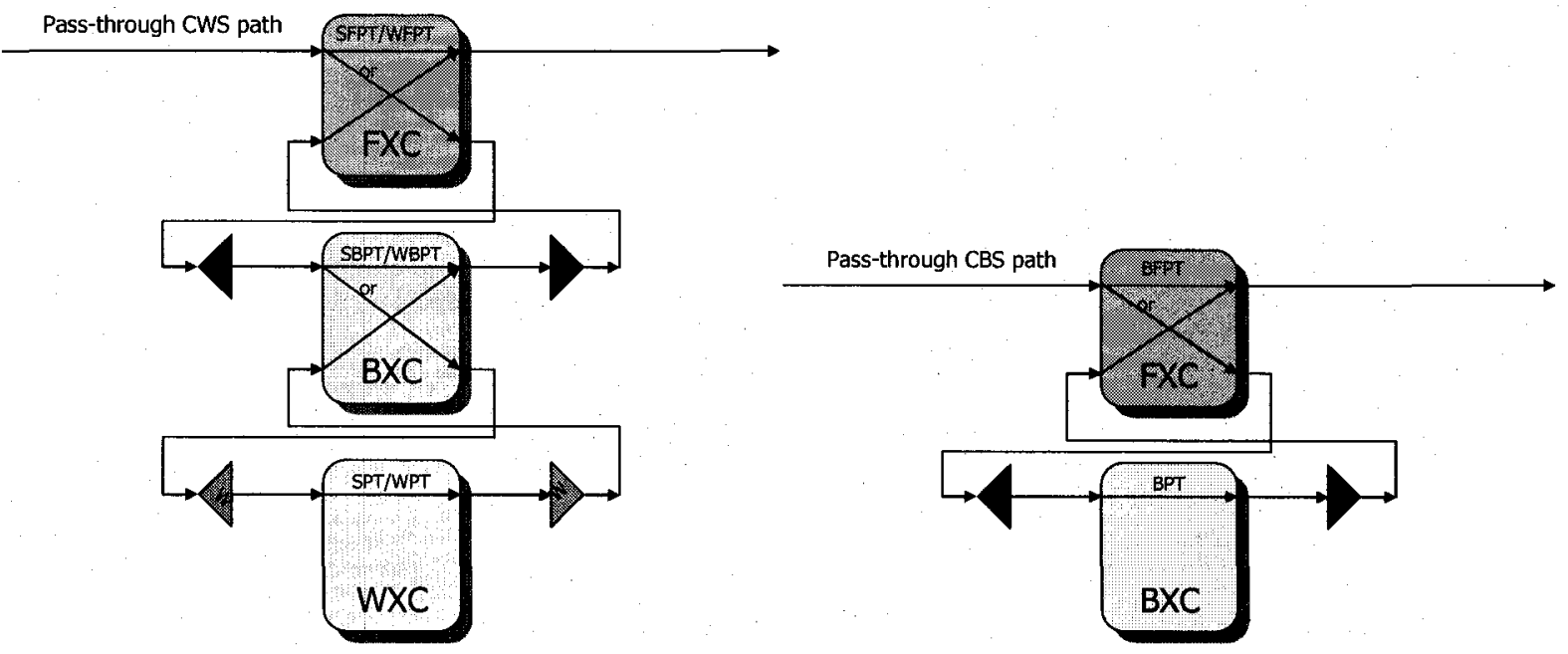

Fig. 4.2: Switching options for the pass-through CWS/CBS-based virtual link.

6) Pass-through sub-wavelength flow conservation must be ensured at each node in the CWSbased virtual topology:

$$
\sum_{i}^{i \neq m} \sum_{j}^{j \neq m} \sum_{p} \sum_{f} \sum_{b} \sum_{k}\left(\varphi s_{p, k, b, f}^{s d, i m}-\varphi s_{p, k, b, f}^{s d, m j}\right)=0 \quad \forall m, s, d \mid \Lambda_{s d}^{0}>0, m \neq s \neq d
$$

7) WXC/BXC/FXC non-add and non-drop ports (refer to Fig. 4.1) are decided based on add, drop, and pass-through virtual links in a way that ensures a non-blocking architecture at all optical switching stages:

$$
\begin{gathered}
w o_{i}^{n, f, b, k}=\sum_{d}^{d \neq i} \sum_{p}\left(\zeta_{i n}^{1, i d, p} \cdot V W_{f, b, k}^{i d, p}+\gamma_{i n}^{i d, p} \cdot V S_{f, b, k}^{i d, p}\right)+ \\
\sum_{s}^{s \neq n} \sum_{d}^{d \neq s \neq i} \sum_{m}^{d \neq n \neq d \neq i} \sum_{p}\left(\zeta_{m i}^{1, s d, p} \cdot \zeta_{i n}^{1, s d, p} \cdot W P T_{f, b, k}^{s d, p, i, m n}+\gamma_{m i}^{s d, p} \cdot \gamma_{i n}^{s d, p} \cdot S P T_{f, b, k}^{s d, p, i, m n}\right) \\
\forall i, n, f, b, k \mid i \neq n, \delta_{i n}>0 \\
W O_{i}=\sum_{n}^{n \neq i} \sum_{f} \sum_{b} \sum_{k}\left(\delta_{i n} \cdot w o_{i}^{n, f, b, k}\right) \forall i
\end{gathered}
$$




$$
\begin{gathered}
w b_{i}^{n, f, b} \geq \sum_{k}\left(\frac{w o_{i}^{n, f, b, k}}{W}\right) \forall i, n, f, b \mid i \neq n, \delta_{i n}>0 \\
W B_{i}=\sum_{n}^{n \neq i} \sum_{f} \sum_{b}\left(\delta_{i n} \cdot w b_{i}^{n, f, b}\right) \forall i
\end{gathered}
$$

$$
\begin{gathered}
w i_{i}^{m, f, b, k}=\sum_{s}^{s \neq i} \sum_{p}\left(\zeta_{m i}^{1, s i, p} \cdot V W_{f, b, k}^{s i, p}+\gamma_{m i}^{s i, p} \cdot V S_{f, b, k}^{s i, p}\right)+ \\
\sum_{s}^{s \neq d \neq i d \neq m \neq i} \sum_{d} \sum_{n}^{\neq \neq m \neq s} \sum_{p}\left(\zeta_{m i}^{1, s d, p} \cdot \zeta_{i n}^{1, s d, p} \cdot W P T_{f, b, k}^{s d, p, i, m n}+\gamma_{m i}^{s d, p} \cdot \gamma_{i n}^{s d, p} \cdot S P T_{f, b, k}^{s d, p, i, m n}\right) \\
\forall i, m, f, b, k \mid i \neq m, \delta_{m i}>0 \\
W I_{i}=\sum_{m}^{m \neq i} \sum_{f} \sum_{b} \sum_{k}\left(\delta_{m i} \cdot w i_{i}^{m, f, b, k}\right) \forall i
\end{gathered}
$$

$$
\begin{gathered}
b w_{i}^{m, f, b} \geq \sum_{k}\left(\frac{w i_{i}^{m, f, b, k}}{W}\right) \forall i, m, f, b \mid i \neq m, \delta_{m i}>0 \\
B W_{i}=\sum_{m}^{m \neq i} \sum_{f} \sum_{b}\left(\delta_{m i} \cdot b w_{i}^{m, f, b}\right) \forall i
\end{gathered}
$$

$$
\begin{gathered}
b o_{i}^{n, f, b} \geq \sum_{d}^{d \neq i} \sum_{p}\left(\zeta_{i n}^{2, i d, p} \cdot V B_{f, b}^{i d, p}\right)+W b_{i}^{n, f, b}+\sum_{s}^{s \neq n} \sum_{d}^{d \neq s \neq i m \neq n \neq d \neq i} \sum_{m} \sum_{p}\left(\zeta_{m i}^{2, s d, p} \cdot \zeta_{i n}^{2, s d, p} \cdot B P T_{f, b}^{s d, p, i, m n}\right)+ \\
\sum_{s} \sum_{d}^{s \neq n} \sum_{m}^{d \neq s \neq i \neq m \neq n \neq d \neq i} \sum_{p} \frac{\left(\zeta_{m i}^{1, s d, p} \cdot \zeta_{i n}^{1, s d, p} \cdot W B P T_{f, b, k}^{s d, p, m n}+\gamma_{m i}^{s d, p} \cdot \gamma_{i n}^{s d, p} \cdot S B P T_{f, b, k}^{s d, p, m n}\right)}{W} \\
B O_{i}=\sum_{n}^{n \neq i} \sum_{f} \sum_{b}\left(\delta_{i n} \cdot b o_{i}^{n, f, b}\right) \forall i \\
b f_{i}^{n, f} \geq \sum_{b}\left(\frac{b o_{i}^{n, f, b}}{B}\right) \forall i, n, f \mid i \neq n, \delta_{i n}>0 \\
B F_{i}=\sum_{n}^{n \neq i} \sum_{f}\left(\delta_{i n} \cdot b f_{i}^{n, f}\right) \forall i
\end{gathered}
$$




$$
\begin{aligned}
& b i_{i}^{m, f, b}=\sum_{s}^{s \neq i} \sum_{p}\left(\zeta_{m i}^{2, s i, p} \cdot V B_{f, b}^{s i, p}\right)+b w_{i}^{m, f, b}+\sum_{s}^{s \neq d \neq i} \sum_{d}^{d \neq m \neq i} \sum_{n}^{n \neq m \neq s} \sum_{p}\left(\zeta_{m i}^{2, s d, p} \cdot \zeta_{i n}^{2, s d, p} \cdot B P T_{f, b}^{s d, p, i, m n}\right)+ \\
& \sum_{s} \sum_{d}^{s \neq d \neq i d \neq m \neq i=i} \sum_{n}^{s \neq m \neq s} \sum_{p} \frac{\left(\zeta_{m i}^{1, s d, p} \cdot \zeta_{i n}^{1, s d, p} \cdot W B P T_{f, b, k}^{s d, p, i, m n}+\gamma_{m i}^{s d, p} \cdot \gamma_{i n}^{s d, p} \cdot S B P T_{f, b, k}^{s d, p, i, m n}\right)}{W}
\end{aligned}
$$

$\forall i, m, f, b \mid i \neq m, \delta_{m i}>0$

$$
B I_{i}=\sum_{m}^{m \neq i} \sum_{f} \sum_{b}\left(\delta_{m i} \cdot b i_{i}^{m, f, b}\right) \forall i
$$

$$
\begin{gathered}
f b_{i}^{m, f} \geq \sum_{b}\left(\frac{b i_{i}^{m, f, b}}{B}\right) \forall i, m, f \mid i \neq m, \delta_{m i}>0 \\
F B_{i}=\sum_{m}^{m \neq i} \sum_{f}\left(\delta_{m i} \cdot f b_{i}^{m, f}\right) \forall i
\end{gathered}
$$

$$
\begin{aligned}
& f o_{i}^{n, f} \geq \sum_{d}^{d \neq i} \sum_{p}\left(\zeta_{i n}^{3, i d, p} \cdot V F_{f}^{i d, p}\right)+b f_{i}^{n, f}+\sum_{s}^{s \neq n} \sum_{d}^{d \neq s \neq i m \neq n \neq d \neq i} \sum_{m} \sum_{p}\left(\zeta_{m i}^{3, s d, p} \cdot \zeta_{i n}^{3, s d, p} \cdot V F_{f}^{s d, p}\right)+ \\
& \sum_{s}^{s \neq n} \sum_{d}^{s \neq s \neq i m \neq n \neq n \neq d \neq i} \sum_{m}^{\substack{d \\
m}} \frac{\left(\zeta_{m i}^{2, s d, p} \cdot \zeta_{i n}^{2, s d, p} \cdot B F P T_{f, b}^{s d, p, i, m n}\right)^{m}}{B}+ \\
& \sum_{s} \sum_{d}^{s \neq n} \sum_{m}^{\substack{d \neq s \neq i m \neq n \neq d \neq i \\
m}} \sum_{p} \frac{\left(\zeta_{m i}^{1, s d, p} \cdot \zeta_{i n}^{1, s d, p} \cdot W F P T_{f, b, k}^{s d, p, i, m n}+\gamma_{m i}^{s d, p} \cdot \gamma_{i n}^{s d, p} \cdot S F P T_{f, b, k}^{s d, p, m n}\right)}{W \cdot B}
\end{aligned}
$$

$\forall i, n, f \mid i \neq n, \delta_{\text {in }}>0$

$$
F O_{i}=\sum_{n}^{n \neq i} \sum_{f}\left(\delta_{i n} \cdot f o_{i}^{n, f}\right) \forall i
$$

$$
\begin{aligned}
& f_{i}^{m, f} \geq \sum_{s}^{s \neq i} \sum_{p}\left(\zeta_{m i}^{3, s i, p} \cdot V F_{f}^{s i, p}\right)+f b_{i}^{m, f}+\sum_{s}^{s \neq d \neq i d \neq m \neq i n \neq m \neq s} \sum_{d} \sum_{n} \sum_{p}\left(\zeta_{m i}^{3, s d, p} \cdot \zeta_{i n}^{3, s d, p} \cdot V F_{f}^{s d, p}\right)+ \\
& \sum_{s}^{s \neq d \neq i d \neq \neq m \neq i} \sum_{d} \sum_{n}^{p \neq m \neq s} \sum_{p} \frac{\left(\zeta_{m i}^{2, s d, p} \cdot \zeta_{i n}^{2, s d, p} \cdot B F P T_{f, b}^{s d, p, i, m n}\right)}{B}+ \\
& \sum_{s} \sum_{d}^{s \neq d \neq i d \neq m \neq i=i \neq m \neq s} \sum_{n}^{\substack{n \\
s \neq m \neq s}} \frac{\left(\zeta_{m i}^{1, s d, p} \cdot \zeta_{i n}^{1, s d, p} \cdot W F P T_{f, b, k}^{s d, p, i, m n}+\gamma_{m i}^{d d, p} \cdot \gamma_{i n}^{s d, p} \cdot S F P T_{f, b, k}^{s d, p, i, m n}\right)}{W \cdot B}
\end{aligned}
$$

$\forall i, m, f \mid i \neq m, \delta_{m i}>0$

$$
F I_{i}=\sum_{m}^{m \neq i} \sum_{f}\left(\delta_{m i} \cdot f i_{i}^{m, f}\right) \forall i
$$

8) Port count conservation must be ensured at all optical switching stages:

$$
W O_{i}+W D_{i}=\dot{W I}_{i}+W A_{i} \quad \forall i
$$




$$
\begin{aligned}
& B O_{i}+B W_{i}+B D_{i}=B I_{i}+W B_{i}+B A_{i} \quad \forall i \\
& F O_{i}+F B_{i}+F D_{i}=F I_{i}+B F_{i}+F A_{i} \quad \forall i
\end{aligned}
$$

\subsection{Heuristic Approach}

The computational complexity of the MILP model calls for the use of heuristics to solve the problem sub-optimally in a reasonable amount of time. A high-level view of the approach to be taken to solve the RMGPA problem heuristically is shown in Fig. 4.3. The heuristic approach consists of two phases. The task of the first phase is to build the multi-granular virtual topology. The task of the second phase is to decrease the port requirement of the pass-through segments of the built virtual links. Both phases are explained individually as follows.

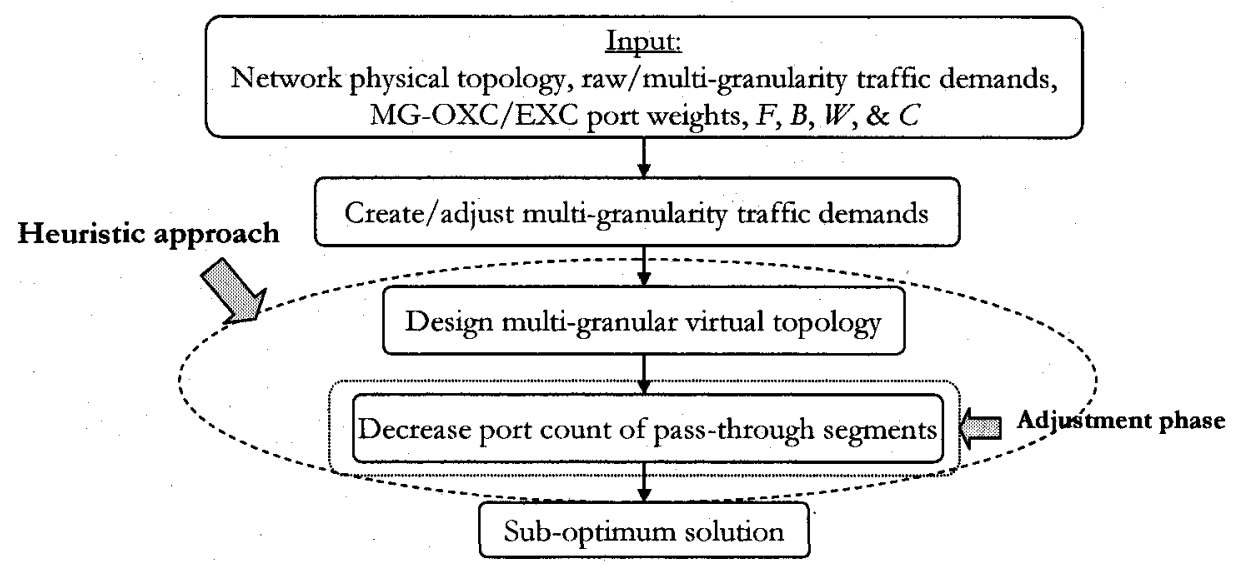

Fig. 4.3: A high-level view of the heuristic approach proposed to solve the RMGPA problem with the color-continuity constraints and without the optical-reach constraints.

The objective of the first phase is to build a multi-granular virtual topology that can accommodate all traffic demands with the lowest weighted port count. In this phase, clear paths will be assigned to fiber, waveband, and wavelength demands, while both clear and unclear paths will be assigned to sub-wavelength demands. The idea of the heuristic is to perform in an integrated manner the routing and color assignment for the largest granularity traffic (fiber demands) first, and then to 
work on those having smaller granularity traffic by using as much of the remaining resources as possible and so on until all demands are served. The sequence in which $s$ - $d$ pairs are served needs to follow a certain policy. Four sorting policies are considered in this version of the RMGPA problem, and they are explained as follows. In Policy $1 / 2, s-d$ pairs are sorted in the decreasing/increasing order of their traffic demands. In Policy 3/4, s- $d$ pairs are sorted in the decreasing/increasing order of their lowest physical hop requirement, which is obtained via Dijkstra's algorithm [Dijk59][Stal00] using the number of physical hops as a cost metric. The multi-granular virtual topology is constructed using the following algorithm [NaMo07a] [NaMo07d]:

\section{Multi-Granular Virtual Topology Algorithm;}

Input: Network physical topology, adjusted multi-granularity traffic demands, $C, W, B, F, M G-O X C / E X C$ port weights

Output: Sub-optimum multi-granular virtual topology

\section{Begin}

\section{Initialization:}

- For all nodes, mark all WXC/BXC/FXC add, drop, input, and output ports as unused; a WXC add/output port is designated as <wavelength $k$, waveband $b$, fiber $f$, next destination node>; $a$ $W X C$ drop/input port is designated as <wavelength $k$, waveband $b$, fiber $f$, arriving from node>; a BXC add/output port is designated as <waveband $b$, fiber $f$, next destination node>; a BXC drop/input port is designated as < waveband $b$, fiber $f$, arriving from node>; a FXC add/output port is designated as <fiber $f$, next destination node>; a FXC drop/input port is designated as $<$ fiber $f$, arriving from node>

- For all nodes, mark all EXC output and input ports as unused; a LSC output/input port is designated as <wavelength $k$, waveband $b$, fiber $f$, 'next destination'/'arriving from' node>; $a$ BSC output/input port is designated as <waveband $b$, fiber $f$, 'next destination'/"arriving from' node>; a FSC output/input port is designated as <fiber $f$, 'next destination'/'arriving from' node>

\section{Fiber-Switched Path Assignment:}

- Let $M=\left\{m_{0}, m_{1}, \ldots . ..\right\}$ be a set of unserved fiber demands whose traffic $\neq 0$

- While $(M \neq \varnothing)$ 
- Order unserved fiber demands in $M$ according to the sorting policy used

- Set served demand = fiber demand, $\Lambda_{s d}^{3}$, at the top of the sorted list, which is the element mo of $M$

- Set $D=1$, if fiber demand bifurcation is allowed; otherwise, set $D=$ served demand

- While (served demand $\neq 0$ )

\{

- Evaluate the cost of all physical links according to the availability of fibers along the physical link; link cost $=1$ if the remaining (or residual) unused fibers $\geq D$, otherwise link cost $=\infty$

- Find all possible physical routes for the served demand using the All Distinct Routes algorithm [Grov04] and choose the routes that satisfy the color (fiber index)-continuity constraint using the First-Fit color assignment scheme [ZJMUOO], in which a lower index color is considered before a higher index color

- If there is no feasible route, then end the algorithm

- Evaluate the incremental port count requirement for all feasible routes; the incremental port count for a feasible eligible route is the summation of all unused FXC and EXC ports needed along the route, but these ports will not be marked as used at this step

- Choose the candidate route that incurs the lowest incremental port count

- Along the best route, update residual fiber resources and mark required FXC and EXC ports as used

- Create CFS-based virtual link(s) between the $s$-d pair served; the virtual link attributes are the best route and the color used along the path

- Set served demand = served demand $-D$

\}

- Remove mo from $M$

\}

\section{Waveband-Switched Path Assignment:}

- Let $M=\left\{m_{0}, m_{1}, \ldots . ..\right\}$ be a set of unserved waveband demands whose traffic $\neq 0$

- While $(M \neq \varnothing)$

\{

- Order unserved waveband demands in $M$ according to the sorting policy used

- Set served demand = waveband demand, $\Lambda_{s d}^{2}$, at the top of the sorted list

- Set $D=1$, if waveband demand bifurcation is allowed; otherwise, set $D=$ served demand 
- While (served demand $\neq 0$ )

\{

- Evaluate the cost of all physical links according to the availability of wavebands along the physical link; link cost $=1$ if the remaining unused wavebands $\geq D$, otherwise link cost $=\infty$

- Find all possible physical routes for the served demand using the All Distinct Routes algorithm and choose the routes that satisfy the color (waveband index of the same fiber index)- continuity constraint using the First-Fit color assignment scheme

- If there is no feasible route, then end the algorithm

- Evaluate the worst incremental port count requirement for all feasible routes; the incremental port count for a feasible eligible route is the summation of all unused FXC, $B X C$, and EXC ports needed along the route. The worst scenario for deciding the unused ports is based on the fact that a waveband-switched path at an intermediate node can be (1) switched at the FXC if the fiber utilization at both the incoming and outgoing links is zero and there are no further add/drop waveband/wavelength/subwavelength demands at this intermediate node, or (2) switched at the BXC otherwise (refer to the right part of Fig. 4.2)

- Choose the candidate route that incurs the lowest incremental port count

- Along the best route, update the residual waveband resources and mark the required FXC, BXC, and EXC ports as used

- Create CBS-based virtual link(s) between the $s-d$ pair served

- Set served demand $=$ served demand $-D$

\}

- Remove mo from $M$

\}

\section{Wavelength-Switched Path Assignment:}

- Let $M=\left\{m_{0}, m_{1}, \ldots . ..\right\}$ be a set of unserved wavelength demands whose traffic $\neq 0$

- While $(M \neq \varnothing)$

\{

- Order unserved wavelength demands in $M$ according to the sorting policy used

- Set served demand = wavelength demand, $\Lambda_{s d}^{1}$, at the top of the sorted list

- Set $D=1$, if wavelength demand bifurcation is allowed; otherwise, set $D=$ served demand

- While (served demand $\neq 0$ ) 
- Evaluate the cost of all physical links according to the availability of wavelengths along the physical link; link cost $=1$ if the remaining unused wavelengths $\geq D$, otherwise link cost $=\infty$

- Find all possible physical routes for the served demand using the All Distinct Routes algorithm and choose the routes that satisfy the color (wavelength index of the same waveband index at the same fiber index)-continuity constraint using the First-Fit color assignment scheme

- If there is no feasible route, then end the algorithm

- Evaluate the worst incremental port count requirement for all feasible routes; the incremental port count for a feasible eligible route is the summation of all unused FXC, BXC, WXC, and EXC ports needed along the route. The worst scenario for deciding the unused ports is based on the fact that a wavelength-switched path at an intermediate node can be (1) switched at the FXC if the fiber and waveband utilization at both the incoming and outgoing links is zero and there are no further add/drop wavelength/sub-wavelength demands at this intermediate node, (2) switched at the BXC if the waveband utilization at both the incoming and outgoing links is zero and there are no further add/drop wavelength/sub-wavelength demands at this intermediate node, or (3) switched at the WXC otherwise (refer to the left part of Fig.

- Choose the candidate route that incurs the lowest incremental port count

- Along the best route, update the residual wavelength resources and mark the required FXC, BXC, WXC, and EXC ports as used

- Create CWS-based virtual link(s) between the s-d pair served

- Set served demand $=$ served demand $-D$

\}

- Remove mo from $M$

\}

\section{Sub-Wavelength-Switched Path Assignment:}

- Let $M=\left\{m_{0}, m_{1}, \ldots . ..\right\}$ be a set of unserved sub-wavelength demands whose traffic $\neq 0$

- While $(M \neq \varnothing)$

\{

- Order unserved sub-wavelength demands in $M$ according to the sorting policy used

- Set served demand = sub-wavelength demand, $\Lambda_{s d}^{0}$, on the top of the sorted list

- Set $D=1$, if sub-wavelength demand bifurcation is allowed; otherwise, set $D=$ served demand 
- While (served demand $\neq 0$ ) \{

- Evaluate the cost of all CWS-based virtual links according to the availability of wavelength capacities along the physical link; link cost $=1$ if the remaining wavelength capacity $\geq D$, otherwise link cost $=\infty$

- Find the shortest virtual route over the existing CWS-based virtual topology using Dijkstra's algorithm, which uses the number of virtual hops (a virtual hop represents an already-built virtual link) as a cost metric. The reason behind using this cost metric is to reduce the duplication of the traffic demand among virtual links as much as possible in order to save the existing capacity resources for the remaining demands.

- If a virtual route exists (which means that there is an UWS path and no new ports are required)

\{

O Update the residual wavelength-capacity resources along the best path

- Set served demand $=$ served demand $-D$

\}

- Else (which means that there is a need to build a CWS path and new ports are required)

\{

- Evaluate the cost of all physical links according to the availability of wavelengths along the physical link; link cost $=1$ if the remaining unused wavelengths $\geq 1$, otherwise link cost $=\infty$

- Find all possible physical routes for the served demand using the All Distinct Routes algorithm and choose the routes that satisfy the color (wavelength index of a certain waveband index at a certain fiber index)-continuity constraint using the First-Fit color assignment scheme

O If there is no feasible route, then end the algorithm

- Evaluate the worst incremental port count requirement for all feasible routes; the incremental port count for a feasible eligible route is the summation of all unused FXC, BXC, WXC, and EXC ports needed along the route. The worst scenario for deciding the unused ports is based on the fact that a wavelengthswitched path at an intermediate node can be (1) switched at the FXC if the fiber and waveband utilization at both the incoming and outgoing links is zero and there are no further add/drop sub-wavelength demands at this intermediate node, (2) switched at the BXC if the waveband utilization at both 
the incoming and outgoing links is zero and there are no further add/drop subwavelength demands at this intermediate node, or (3) switched at the WXC otherwise

- Choose the candidate route that incurs the lowest incremental port count

- Along the best route, update the residual wavelength and wavelength-capacity resources (new residual wavelengths/'wavelength capacity' = old residual wavelengths/"wavelength capacity' - 1/D) and mark the required FXC, BXC, WXC, and EXC ports as used

o. Create a CWS-based virtual link between the $s$-d pair served

- Set served demand $=$ served demand $-D$

\}

\}

- Remove mo from $M$

\}

6. If the resultant multi-granular virtual topology can handle all demands, then this virtual topology is both feasible and sub-optimum

End.

Running the previous heuristic phase alone can result in situations where, at some nodes, many waveband-switched paths would have to be switched at the BXC stage instead of the FXC stage. Similarly, wavelength-switched paths would have to be switched either at the BXC stage instead of the FXC stage or at the WXC stage rather than the BXC or FXC stage. These can occur because the worst (de)grouping scenarios are assumed when these paths are originally allocated. Therefore, an adjustment phase is needed to minimize the port count of pass-through segments at all nodes and, accordingly, to decide on the final weighted port count. The adjustment phase works as follows $[\mathrm{NaMo07a][NaMo07d]:}$

\section{Pass-Through Adjustment Algorithm;}

Input: Feasible multi-granular virtual topology, W, B, F, MG-OXC/EXC port weights 
Output: Sub-optimum weighted port count

\section{Begin}

\section{Initialization:}

- For all nodes, create separate FXC, BXC and WXC-based planes

- For all nodes, assume that $\mathrm{FXC} / \mathrm{BXC} / \mathrm{WXC}$ ports are integer variables and mark them as unused; $X C$ port types with their attributes are denoted as follows: INPUT <current node (i), arriving from node $(m)$, color $>$; OUTPUT $<i$, next destination node $(n)$, color $>$; ADD $<i, n$, color $>$; DROP $<i, m$, color>

- For all nodes, assign all $F X C / B X C / W X C$ ports with the value of their next destination node based on their involvement with the CFS/CBS/CWS-based virtual links

\section{For all assigned WXC output ports at all nodes, do the following:}

If the WXC output port has a CWS-based pass-through segment (i.e. INPUT $<i, m$, fwavelength $k$, waveband $b$, fiber $f\}>=$ OUTPUT $<i, n,\{$ wavelength $k$, waveband $b$, fiber $f\}>n$ )

If 'other CWS/CBS-based pass-through segments arriving from different nodes', 'WXC/BXC add ports', or ' $W X C / B X C$ drop ports' are sharing the same waveband $b$ and/or fiber $f$ that are used by the WXC output port

\{

If sharing exists only at fiber $f$ (i.e. the CWS path needs to be switched at the BXC stage) \{

- Set BXC OUTPUT $<i, n,\{b, f\}>=$ WXC OUTPUT $<i, n,\{k, b, f\}>$

- Set BXC INPUT $<i, m,\{b, f\}>=W X C$ INPUT $<i, m,\{k, b, f\}>$

- Mark WXC OUTPUT $<i, n,\{k, b, f\}>$ as unused

- Mark WXC INPUT $<i, m,\{k, b, f\}>$ as unused

\}

Else (i.e. the sharing exists at waveband $b$; therefore, the CWS path needs to be switched at the WXC stage)

\{

Port assignment remains unchanged

\}

\}

Else (i.e. there is no sharing; therefore, the CWS path needs to be switched only at the FXC stage) \{

- Set FXC OUTPUT $<i, n, f>=W X C$ OUTPUT $<i, n,\{k, b, f\}>$ 
- Set FXC INPUT $<i, m, f>=W X C$ INPUT $<i, m,\{k, b, f\}>$

- Mark WXC OUTPUT $<i, n,\{k, b, f\}>$ as unused

- Mark WXC INPUT $<i, m,\{k, b, f\}>$ as unused

\}

\}

\section{For all assigned BXC output ports at all nodes, do the following:}

If the BXC output port has a CBS-based pass-through segment (i.e. INPUT $<i, m,\{$ waveband $b$, fiber $f\}>=$ OUTPUT $<i, n$, $\{$ waveband $b$, fiber $f\}>=n$ )

If 'other CWS/CBS-based pass-through segments arriving from different nodes', 'WXC/BXC add ports', or ' $W X C / B X C$ drop ports' are sharing the same fiber $f$ that is used by the BXC output port, then the port assignment remains unchanged

Else (i.e. there is no sharing; therefore, the CBS path needs to be switched only at the FXC stage) \{

- Set FXC OUTPUT $<i, n, f>=$ BXC OUTPUT $<i, n,\{b, f\}>$

- Set FXC INPUT $<i, m, f>=B X C$ INPUT $<i, m,\{b, f\}>$

- Mark BXC OUTPUT $<i, n,\{b, f\}>$ as unused

- Mark BXC INPUT $<i, m,\{b, f\}>$ as unused \}

\}

\section{For all nodes, calculate the WB, BW, FB and BF ports:}

The ports needed to interconnect the three $X C$ planes at node $i$ (refer to Fig. 4.1) are calculated as follows

- $W B_{i}=\sum_{n=0}^{N-1} \sum_{f=0}^{F-1} \sum_{b=0}^{B-1}\left[\frac{\sum_{k=0}^{W-1} \text { count assigned WXC OUTPUT }<i, n,\{k, b, f\}>}{W}\right]$

- $B W_{i}=\sum_{m=0}^{N-1} \sum_{f=0}^{F-1} \sum_{b=0}^{B-1}\left[\frac{\sum_{k=0}^{W-1} \text { count assigned WXC INPUT }<i, m,\{k, b, f\}>}{W}\right]$ 
- $B F_{i}=\sum_{n=0}^{N-1} \sum_{f=0}^{F-1}\left[\frac{\sum_{b=0}^{B-1} \text { count assigned BXC OUTPUT }<i, n,\{b, f\}>}{B}\right\rceil$

- $F B_{i}=\sum_{m=0}^{N-1} \sum_{f=0}^{F-1}\left[\frac{\sum_{b=0}^{B-1} \text { count assigned BXC INPUT }<i, m,\{b, f\}>}{B}\right\rceil$

5. The total port count is the weighted summation of all assigned ports in addition to the ports calculated in Step 4.

End.

\subsection{Numerical Results and Analysis ${ }^{10}$}

\subsubsection{Verification of the Heuristic Approach}

To verify the heuristic approach, we compare the heuristic results with the results obtained from the MILP formulation. Due to the computational complexity of the MILP model, which would involve a large amount of computational time and high computational intensity in order to obtain an optimal solution, the optimization is conducted on a small-sized planning problem. In this subsection, we consider a 5 -node sample network as a physical topology ${ }^{11}$, as shown in Fig. 4.4. Since some of the $s-d$ pairs can have up to four distinct eligible routes, the parameter $P$ is set to 4 . To keep the problem computationally manageable, we use a set of a few adjusted traffic demands, as illustrated in Table 4.1. The rest of the input parameters used in this subsection are as follows: $C=10 \mathrm{~Gb} / \mathrm{s}, W=3, B=3, F \geq 1$, all MG-OXC/EXC ports have the same weight $($ i.e. $c f=c b=c w /$ $c g f=c g b=c g w)$, and the EXC port weight is twice the MG-OXC port weight (i.e. $\frac{c g f}{c f}=\frac{c g b}{c b}=\frac{c g w}{c w}=2$ ).

\footnotetext{
${ }^{10}$ For the sake of simplicity, for the rest of the thesis, the term "optimum" always refers to the term "sub-optimum" when applied to the results obtained using heuristics.

11 The distance information shown in all topologies in this chapter will be used only in Chapter 5 when the signal regeneration capability is considered.
} 


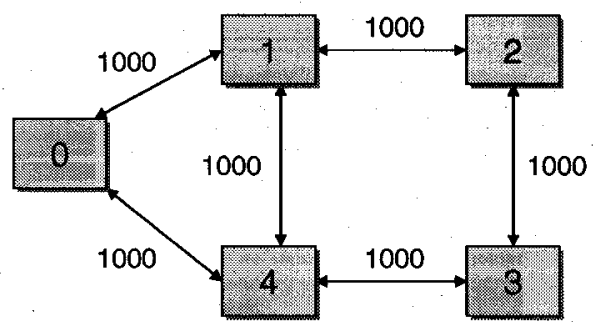

Fig. 4.4: 5-node sample network fiber layout with span distances in $\mathrm{km}$.

Table 4.1 illustrates the solution to the RMGPA problem using both optimization methods for various traffic demand instances in terms of the weighted port count, the solve time, and the minimum parameter $F$ needed to obtain a feasible solution, which is denoted as $F_{\text {min }}$. Throughout this thesis, the RMGPA problem will be solved for different values of the parameter $F$, and $F_{m i n}$ will be the measure of the most efficient utilization of network resources. Heuristic variations are based on the sorting policy and demand-bifurcation scenario used. Three demand-bifurcation scenarios will be considered throughout this thesis: (1) bifurcation is not allowed at any traffic granularity, (2) bifurcation is allowed only at the fiber-traffic granularity, and (3) bifurcation is allowed at all traffic granularities. It is important to note here that in a certain bifurcation scenario, the suggested four policies have led to the same solution since few demands per granularity-level have been considered. The best bifurcation scenario, and accordingly the best heuristic variation, is defined as the one that achieves the lowest $F_{m i n}$ with the lowest weighted port count.

From Table 4.1, it is obvious that increasing the problem size in terms of the number of constraints and variables by imposing more traffic on the network increases exponentially the solve time needed by CPLEX until it becomes hard to obtain an optimum solution in a reasonable amount of time with the given computing capability. This increase in the problem size stems mainly from the increase in $F_{m i n}$ required to build a multi-granular virtual topology that can handle all demands. This increase is expected since the number of optimization variables and constraints 
of the MILP formulation, as explained in Section 4.2, depend heavily on the parameter $F$. For instance, Table 4.1 clearly shows the considerable difference between the problem size at $F_{\text {min }}=1$ and $F_{\text {min }}=2$.

On the other hand, solving the RMGPA problem with the heuristic approach takes less than one second, which can be considered a significant saving in the solve time. This finding also indicates that the heuristic approach scales well with respect to the increase in the problem size. Most importantly, the solutions produced by the heuristic approach are within $\approx 0-11 \%$ of the MILP formulation solutions and can be accepted as good approximations of the optimum solutions. It is worth mentioning here that allowing the bifurcation of fiber demands in the heuristic approach yields better solutions for some traffic instances. This result can occur when the fiber demands are greater than one; otherwise, there is no point in performing the bifurcation.

Now, we verify the feasibility of the MILP formulation and heuristic approach solutions and, at the same time, demonstrate how the resultant multi-granular virtual topology decides the network port count. Fig. 4.5 illustrates the multi-granular virtual topology solution of both the MILP formulation and heuristic approach for the traffic demand instance that leads to the $11 \%$ difference in weighted port count (the underlined port counts in Table 4.1). From Fig. 4.5, it is clear that both virtual topologies are feasible due to the following: (1) all CFS/CBS/CWS-based virtual links sharing a common unidirectional physical link are assigned distinct colors; (2) all flows along a common unidirectional physical link are within the span, fiber, waveband, and wavelength capacity; (3) all virtual links satisfy the color-continuity constraint; and (4) all demands are handled by the virtual topology. 
Figs. 4.6-4.10 depict in detail how the different types of clear paths, which constitute the virtual topology shown in Fig. 4.5, are 'grouped with'/ degrouped from' other clear paths within all network nodes. From Figs. 4.6-4.10, it is apparent that the realization of the multi-granular virtual topology dictates the port requirements at all nodes. Moreover, both realizations are feasible. Furthermore, in comparing the port requirement breakdown of the MILP formulation solution with that of the heuristic approach solution at all nodes (by comparing the left part with the right part of Figs. 4.6-4.10), it is quite clear that choosing the right virtual topology has an impact on reducing the total weighted port count.

Table 4.1: Comparison between the results obtained using the MILP formulation and heuristic approach for the 5node network example of solving the RMGPA problem with the color-continuity constraints and without the opticalreach constraints.

\begin{tabular}{|c|c|c|c|c|c|c|c|c|c|}
\hline \multirow[b]{2}{*}{$\begin{array}{c}\text { \# of traffic } \\
\text { elements } \\
\text { per } \\
\text { granularity } \\
\text { matrix }\end{array}$} & \multirow[b]{2}{*}{$\begin{array}{c}\text { Adjusted } \\
\text { traffic demands }\end{array}$} & \multirow[b]{2}{*}{$F_{m i n}$} & \multirow[b]{2}{*}{$\begin{array}{c}\text { \# of } \\
\text { constraints }\end{array}$} & \multicolumn{2}{|c|}{ MILP } & \multirow[b]{2}{*}{$\begin{array}{l}\text { Weighted } \\
\text { Port } \\
\text { count }\end{array}$} & \multirow[b]{2}{*}{$\begin{array}{l}\text { Total } \\
\text { solve } \\
\text { time } \\
\text { [sec] }\end{array}$} & \multicolumn{2}{|c|}{ Heuristic } \\
\hline & & & & $\begin{array}{c}\text { \# of } \\
\text { variables }\end{array}$ & $\begin{array}{l}\text { Total } \\
\text { solve } \\
\text { time } \\
\text { [sec] }\end{array}$ & & & $\begin{array}{c}\text { Weighted } \\
\text { port } \\
\text { count }\end{array}$ & $\begin{array}{c}\text { Best } \\
\text { bifurcation } \\
\text { scenario }\end{array}$ \\
\hline 1 & $\begin{array}{l}\Lambda^{0}{ }^{20}=1 \\
\Lambda^{102}=1 \\
\Lambda^{20}=2 \\
\Lambda^{203}=1\end{array}$ & 1 & 2234 & 13817 & 651.703 & 66 & 0.03 & 66 & $\begin{array}{c}\text { No } \\
\text { bifurcation }\end{array}$ \\
\hline 1 & $\begin{array}{l}\Lambda^{0}{ }^{10}=2 \\
\Lambda^{134}=2 \\
\Lambda^{2^{34}}=2 \\
\Lambda_{43}^{3^{10}}=2\end{array}$ & 1 & 2207 & 13736 & 56.8438 & 82 & 0.03 & 82 & $\begin{array}{c}\text { Bifurcating fiber } \\
\text { demands }\end{array}$ \\
\hline 1 & $\begin{array}{l}\Lambda^{0}=1 \\
\Lambda^{132}=2 \\
\Lambda^{2^{42}}=1 \\
\Lambda_{14}^{30}=2\end{array}$ & 1 & 2210 & 13733 & 1007.94 & 74 & 0.04 & 74 & $\begin{array}{c}\text { No } \\
\text { bifurcation }\end{array}$ \\
\hline 1 & $\begin{array}{l}\Lambda_{1}^{0}=2 \\
\Lambda^{131}=2 \\
\Lambda^{24}=2 \\
\Lambda_{02}^{30}=2 \\
3^{30}=2\end{array}$ & 1 & 2210 & 13733 & 193.984 & $\underline{90}$ & 0.03 & 100 & $\begin{array}{c}\text { Bifurcating fiber } \\
\text { demands }\end{array}$ \\
\hline 2 & $\begin{array}{l}\Lambda_{201}^{0}=\Lambda_{34}^{0}=2 \\
\Lambda_{01}^{10}=\Lambda^{13}=2 \\
\Lambda_{30}^{2}=\Lambda_{43}^{2}=1 \\
\Lambda_{01}^{3}=\Lambda_{13}^{3}=1\end{array}$ & 1 & 2282 & 14202 & 25131.7 & 124 & 0.01 & 124 & $\begin{array}{c}\text { No } \\
\text { bifurcation }\end{array}$ \\
\hline 2 & $\begin{array}{l}\Lambda_{31}^{0}=\Lambda^{0}{ }^{124}=2 \\
\Lambda_{30}^{1}=\Lambda_{02}^{1}=2 \\
\Lambda_{04}^{2}=\Lambda_{40}^{2}=1 \\
\Lambda_{41}^{3}=\Lambda_{42}^{30}=1\end{array}$ & 1 & 2329 & 14361 & 70249.2 & 130 & 0.03 & 130 & $\begin{array}{c}\text { No } \\
\text { bifurcation }\end{array}$ \\
\hline 3 & $\begin{array}{l}\Lambda_{30}^{0}=\Lambda_{31}^{0}=\Lambda_{24}^{0}=1 \\
\Lambda_{02}^{1}=\Lambda_{40}^{1}=\Lambda_{42}^{1}=1 \\
\Lambda_{01}^{2}=\Lambda_{14}^{2}=\Lambda_{41}^{2}=1 \\
\Lambda_{24}^{3}=\Lambda_{31}^{3}=\Lambda_{34}^{3}=1\end{array}$ & 2 & 4553 & 29281 & $\begin{array}{c}>766545 \\
\text { (9 days) }\end{array}$ & \begin{tabular}{|c|} 
N/A \\
(PC man out \\
of memory)
\end{tabular} & 0.05 & 178 & $\begin{array}{c}\text { No } \\
\text { bifurcation }\end{array}$ \\
\hline 3 & $\begin{array}{l}\Lambda_{24}^{0}=\Lambda_{30}^{0}=\Lambda_{31}^{0}=2 \\
\Lambda_{04}^{1}=\Lambda_{40}^{1}=1, \Lambda_{02}^{1}=2 \\
\Lambda_{01}^{2}=2, \Lambda_{41}^{2}=\Lambda_{44}^{2}=1 \\
\Lambda_{14}^{3}=\Lambda_{24}^{3}=2, \Lambda_{31}^{3}=1\end{array}$ & 2 & 4547 & 29269 & $\begin{array}{l}>1385120 \\
\text { (16 days) }\end{array}$ & $\begin{array}{c}\mathrm{N} / \mathrm{A} \\
\text { (PC ran out } \\
\text { of memory) }\end{array}$ & 0.04 & 212 & $\begin{array}{c}\text { No } \\
\text { bifurcation }\end{array}$ \\
\hline
\end{tabular}



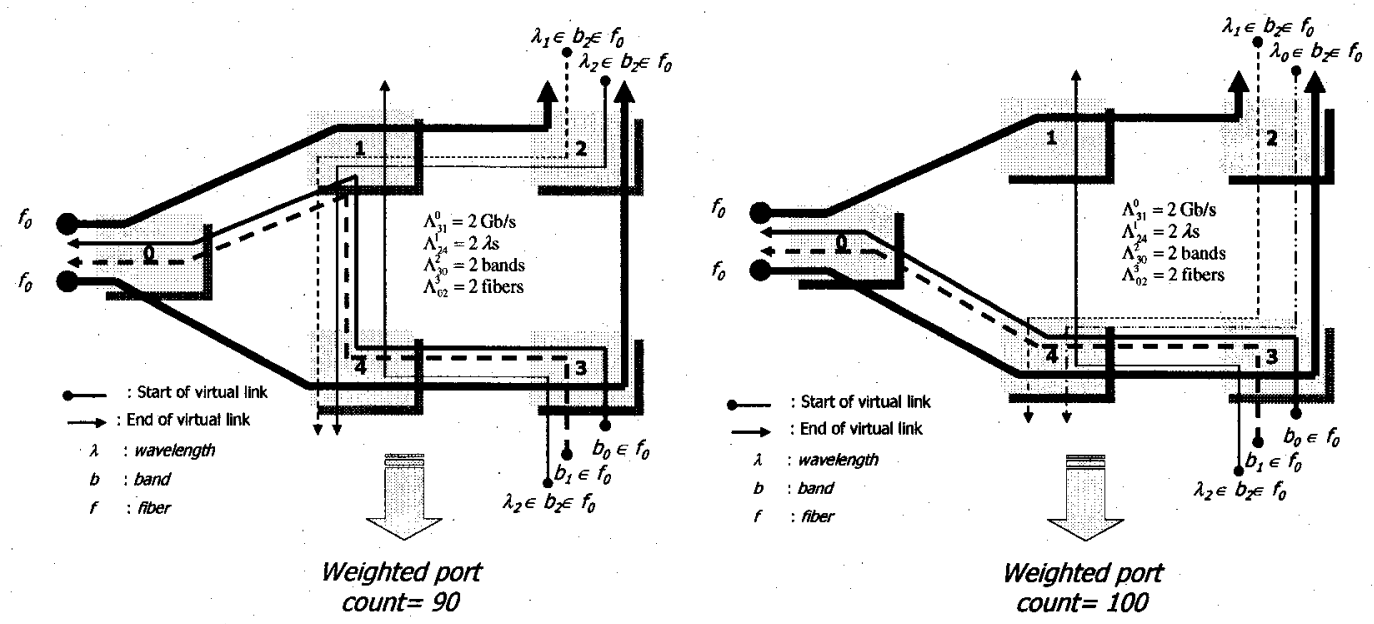

Fig. 4.5: The multi-granular virtual topology solution for the 5-node network example using the MILP formulation (left) and heuristic approach (right), given that $C=10 \mathrm{~Gb} / \mathrm{s}, W=3, B=3$, and $F=F_{\text {min }}=1$.
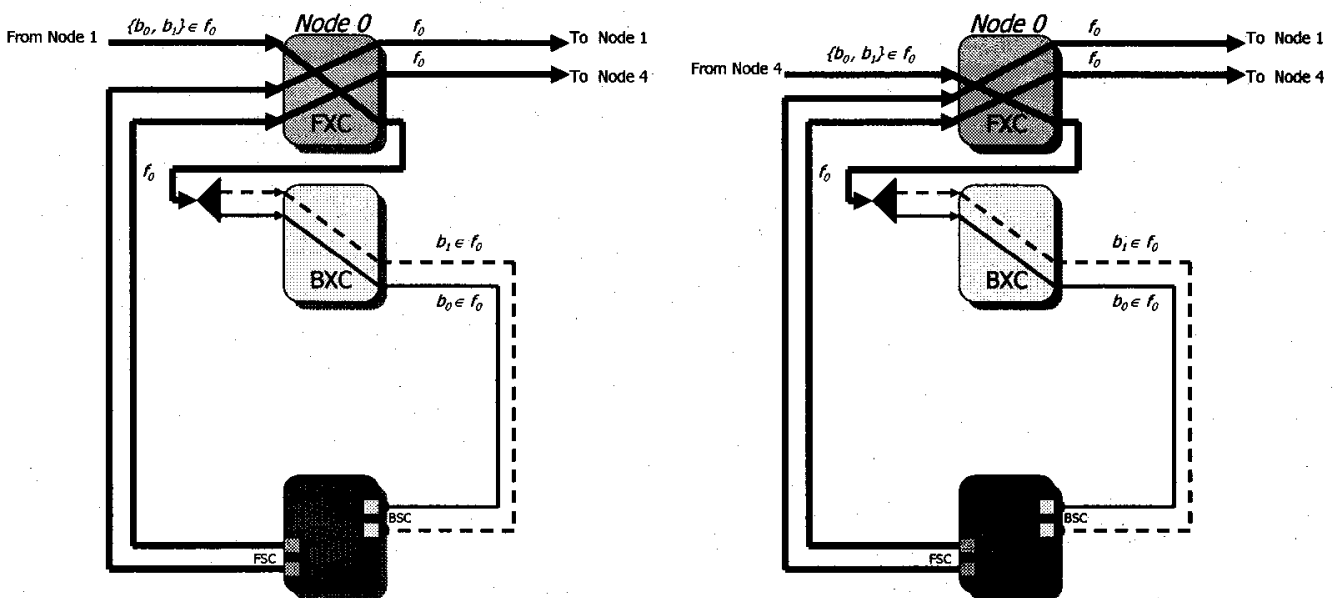

Fig. 4.6: Port requirements at Node 0 of the 5 -node sample network for the realization of the multi-granular virtual topology solution using the MILP formulation (left) and heuristic approach (right).
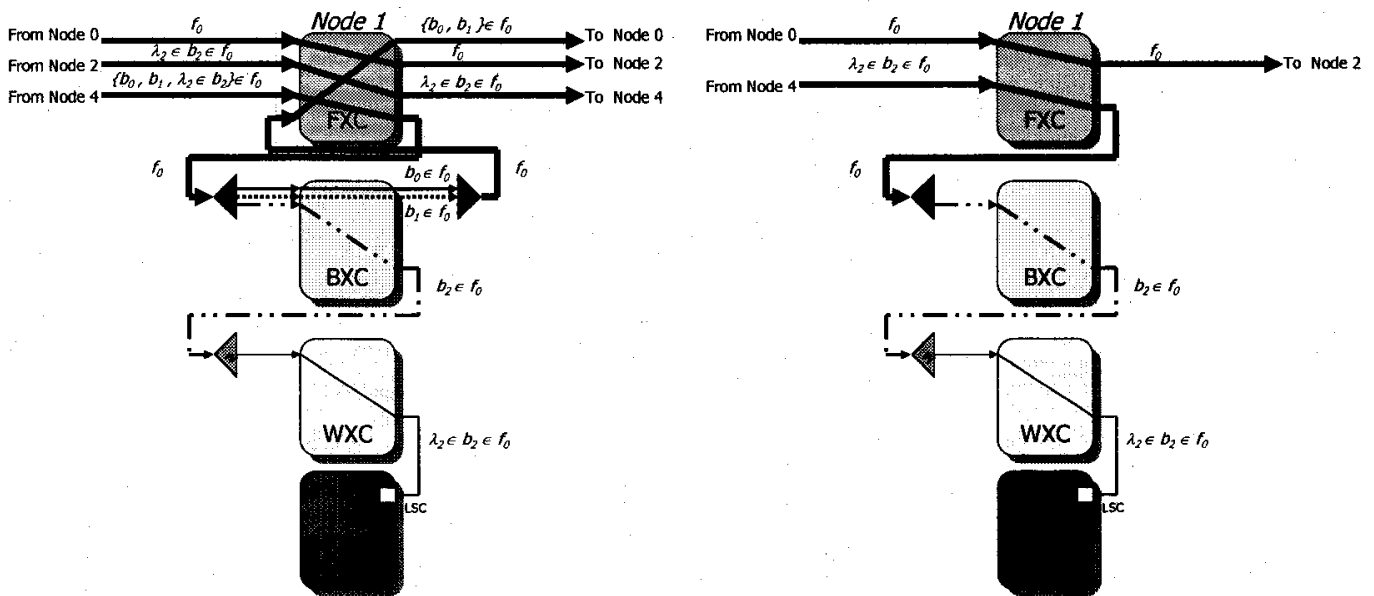

Fig. 4.7: Port requirements at Node 1 of the 5-node sample network for the implementation of the multi-granular virtual topology solution using the MILP formulation (left) and heuristic approach (right). 

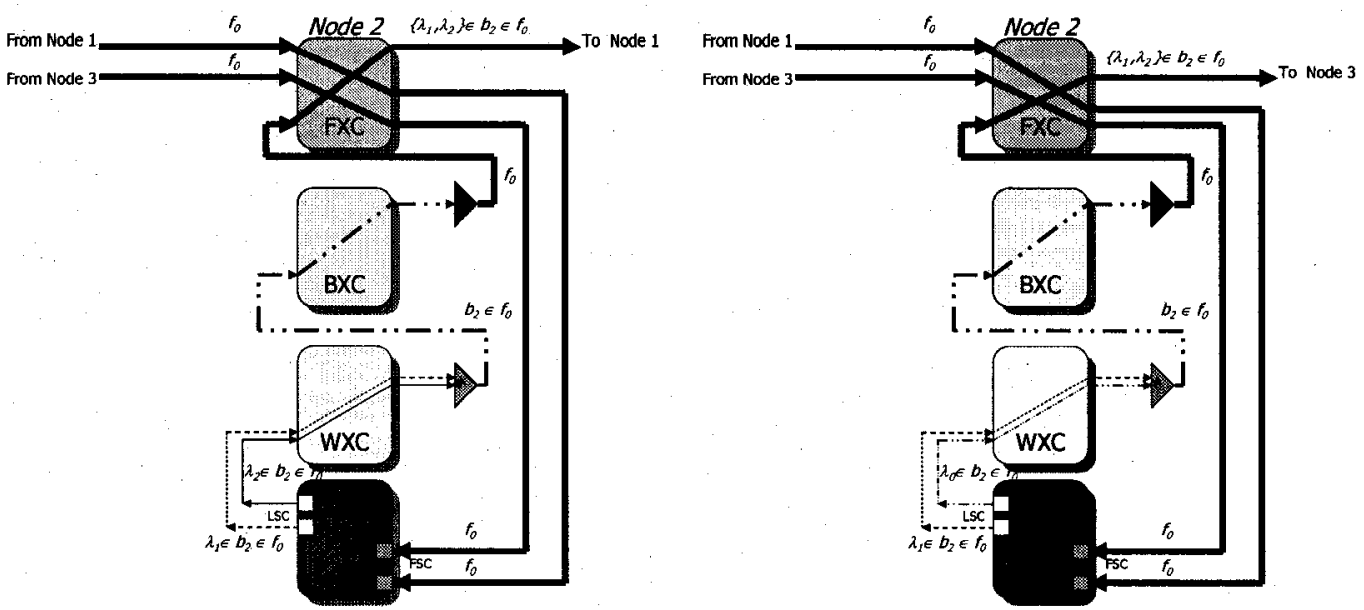

Fig. 4.8: Port requirements at Node 2 of the 5-node sample network for the implementation of the multi-granular virtual topology solution using the MILP formulation (left) and heuristic approach (right).
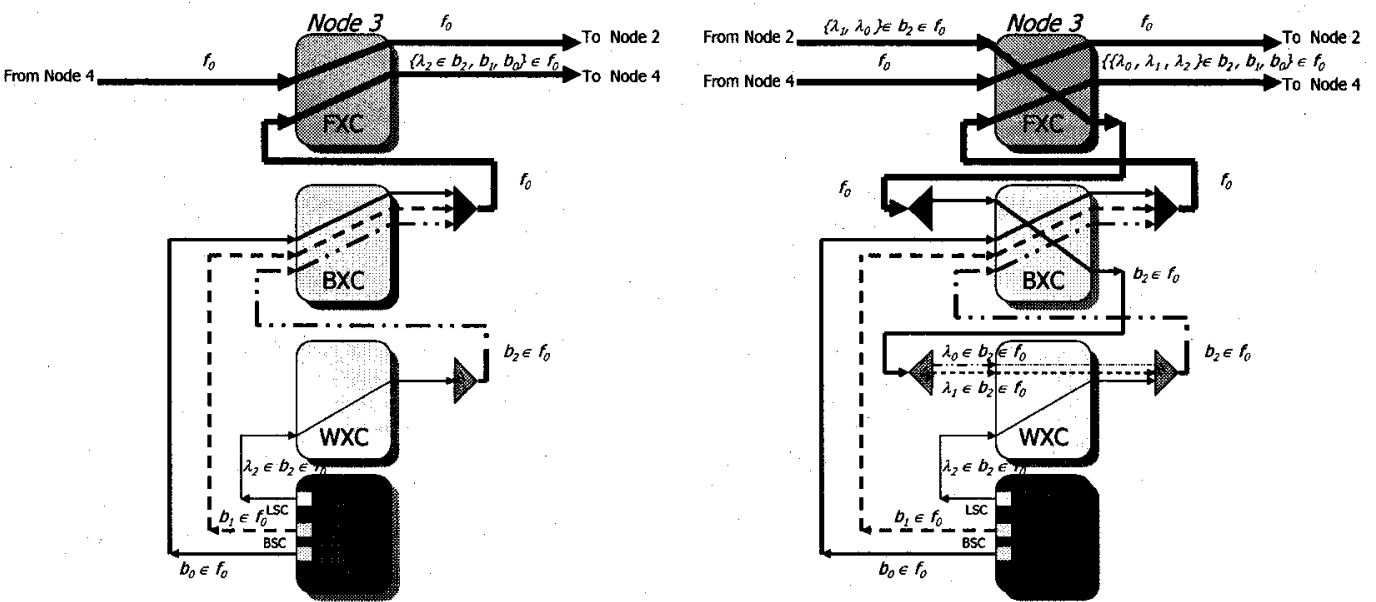

Fig. 4.9: Port requirements at Node 3 of the 5 -node sample network for the implementation of the multi-granular virtual topology solution using the MILP formulation (left) and heuristic approach (right).
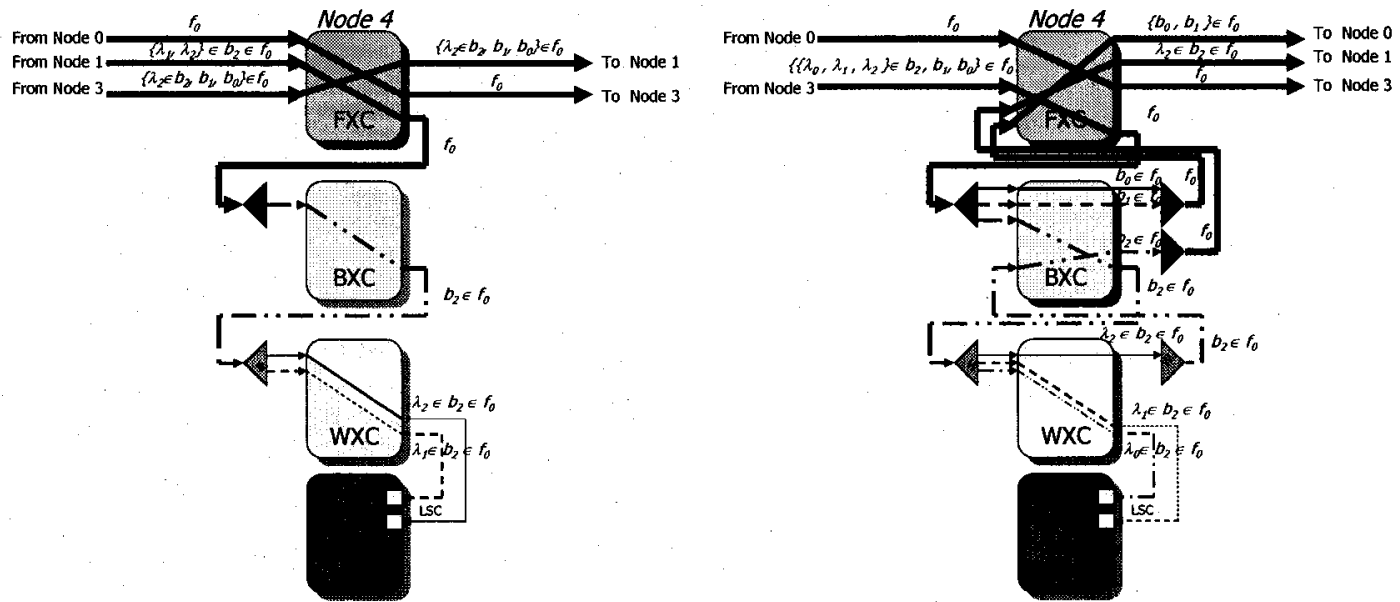

Fig. 4.10: Port requirements at Node 4 of the 5-node sample network for the implementation of the multi-granular virtual topology solution using the MILP formulation (left) and heuristic approach (right). 


\subsubsection{Significance of Adjustment Phase}

In this subsection, we demonstrate a proof-of-concept of performing the adjustment phase upon designing the multi-granular virtual topology. An 8-node sample network shown in Fig. 4.11 is used in this demonstration. In this topology, the number of distinct routes between a node pair can be as high as 29 routes. The used raw traffic demands are randomly chosen from the range $(0,1000) \mathrm{Gb} / \mathrm{s}$, as shown in Table 4.2 . The rest of the input parameters are as follows: $C=10 \mathrm{~Gb} / \mathrm{s}$, $W=4, B=4, F=F_{\text {min }}$, all $\mathrm{MG}-\mathrm{OXC} / \mathrm{EXC}$ ports have the same weight, and the $\mathrm{EXC}$ port weight is twice the MG-OXC port weight. The optimized multi-granularity traffic demands are shown in Table 4.3, which is the result of running the Demand Adjustment algorithm (refer to Subsection 3.3.3). The solutions to the planning problem using different heuristic variations with and without the adjustment phase are shown in Figs. 4.12 and 4.13.

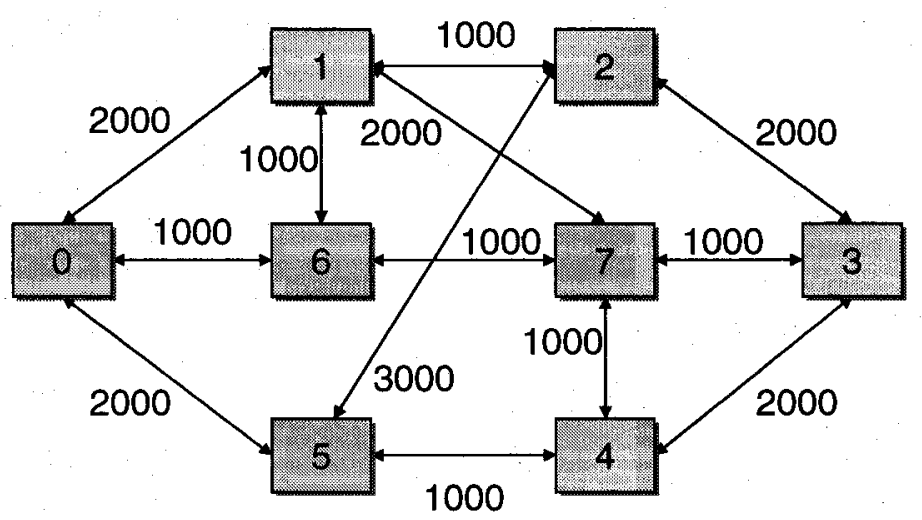

Fig. 4.11: 8-node sample network fiber layout with span distances in $\mathrm{km}$. 
Table 4.2: The 8-node network raw traffic demands, which are chosen randomly from the range $(0,1000) \mathrm{Gb} / \mathrm{s}$.

\section{Destination}

\begin{tabular}{|c|c|c|c|c|c|c|c|c|c|}
\hline & & 0 & 1 & 2 & 3 & 4 & 5 & 6 & 7 \\
\hline & 0 & 0 & 416 & 92 & 757 & 530 & 931 & 383 & 654 \\
\hline & 1 & 66 & 0 & 723 & 671 & 383 & 632 & 885 & 519 \\
\hline & 2 & 652 & 238 & 0 & 262 & 762 & 754 & 910 & 72 \\
\hline & 3 & 272 & 898 & 275 & 0 & 516 & 359 & 247 & 487 \\
\hline & 4 & 847 & 831 & 34 & 992 & 0 & 679 & 767 & 60 \\
\hline & 5 & 384 & 478 & 948 & 935 & 905 & 0 & 319 & 701 \\
\hline & 6 & 7 & 365 & 266 & 987 & 277 & 630 & 0 & 166 \\
\hline & 7 & 736 & 436 & 983 & 736 & 233 & 90 & 505 & 0 \\
\hline
\end{tabular}

Table 4.3: The 8-node network multi-granularity demands, which are generated from the $(0,1000) \mathrm{Gb} / \mathrm{s}$ range raw demands along with the use of the $C=10 \mathrm{~Gb} / \mathrm{s}, W=4, \& B=4$ combination.

\section{Destination}

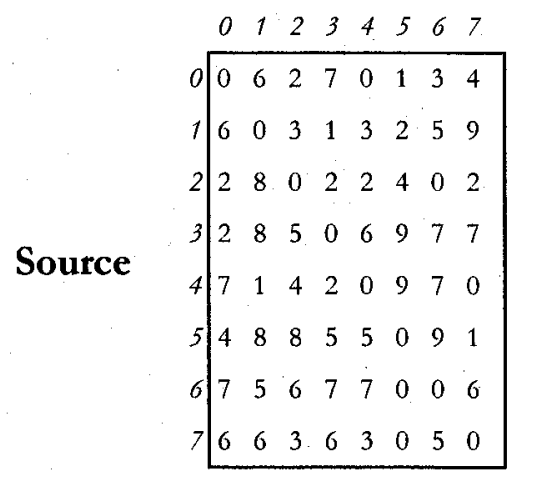

Sub- $\lambda[\mathbf{G b} / \mathbf{s}]$

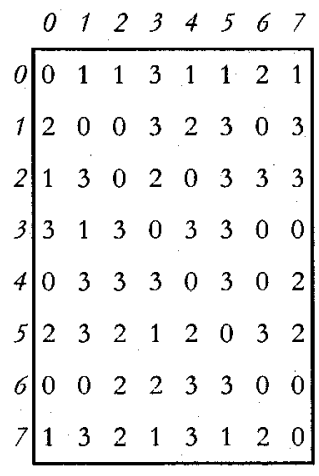

$\lambda$

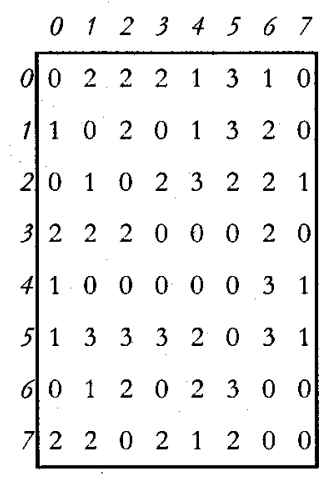

Waveband

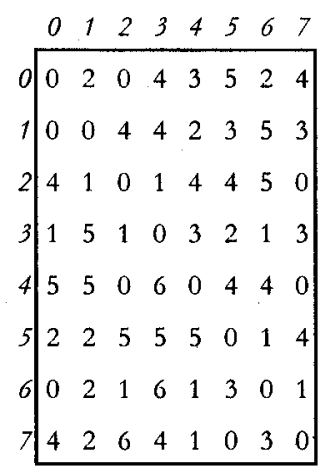

Fiber

Figure 4.12 shows that adding the adjustment phase does not affect the fiber requirement since the multi-granular virtual topology remains the same. However, Fig. 4.13 clearly illustrates that there is a consistent port saving when the adjustment phase is added to all heuristic variations. This saving is due to the decrease in the number of MG-OXC ports needed by pass-through segments by avoiding going into unnecessarily finer granular switching stages. 


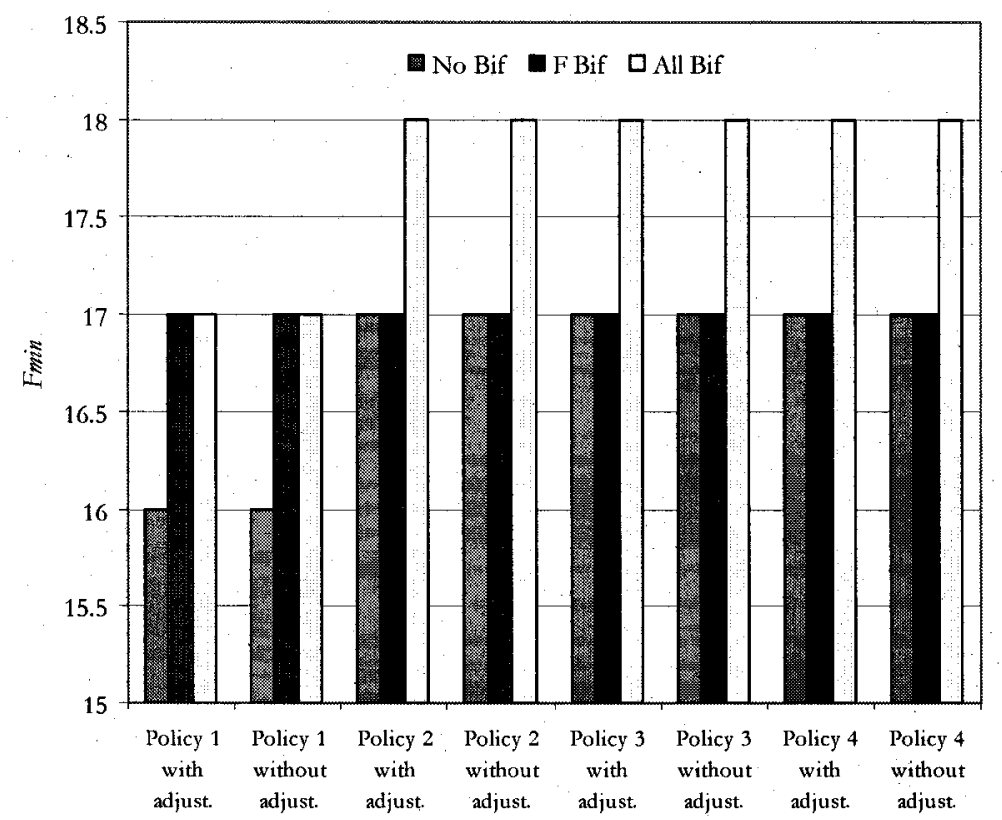

Fig. 4.12: Minimum fiber requirements for all heuristics with/without the adjustment phase.

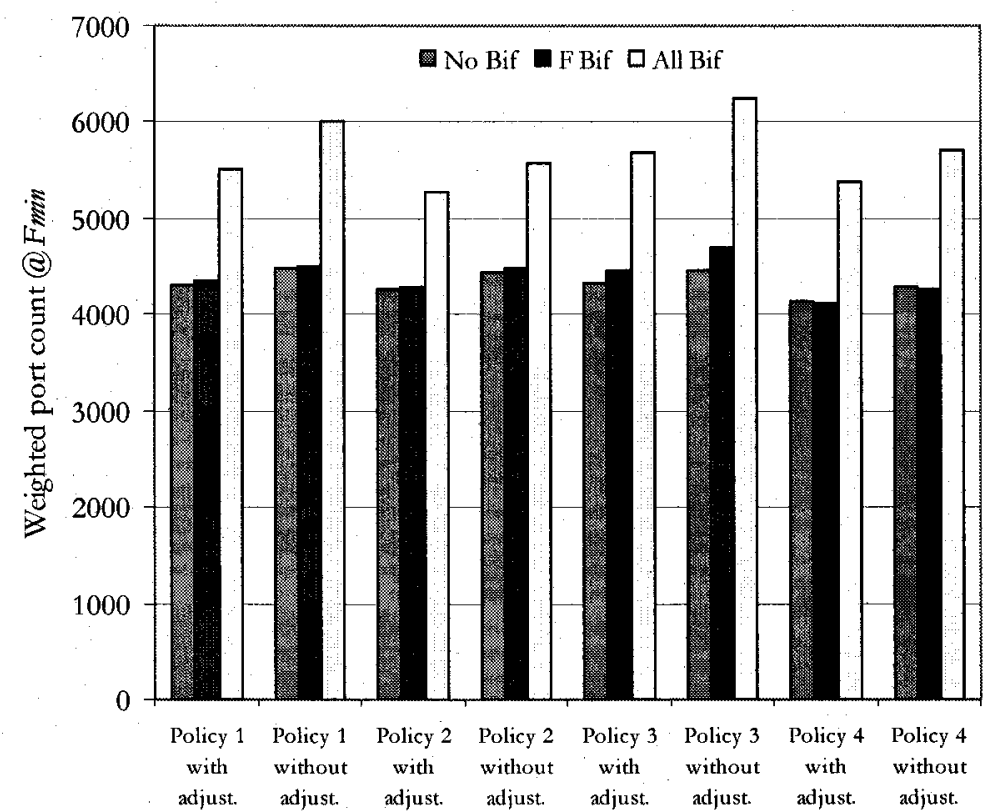

Fig. 4.13: Weighted port count at $F_{m i n}$ for all heuristics with/without the adjustment phase. 


\subsubsection{Impact of Increasing Fiber Resources}

If fiber resources are plentiful, increasing the parameter $F$ beyond $F_{\text {min }}$ can bring a further reduction in the port count. In this subsection, we demonstrate the impact of increasing fiber resources on the planning problem solution. Using the same input data and parameters considered in Subsection 4.4.2, the results of heuristic variations for $F \geq F_{\text {min }}$ are shown in Figs. 4.14-4.16.

From the figures, it is quite clear that regardless of the bifurcation scenario and sorting policy employed, the weighted port count changes after $F_{\text {min }}$ and remains unchanged or becomes saturated beyond a certain $F$, which we call $F_{s a t}$ Within the $\left[F_{m i n}, F_{s a l}\right]$ range, the weighted port count can reach the minimal point. The corresponding $F$ to the minimal weighted port count is denoted as $F_{\text {opt }}$ where $F_{\text {min }} \leq F_{\text {opt }} \leq F_{\text {sal }}$. The weighted port count tends to decrease as the parameter $F$ increases, since selecting shorter physical routes for (un)clear paths will be more likely. However, sometimes using short routes for some (un)clear paths will lead to an increase in the weighted port count instead, as shown in Figs. 4.14-4.16. In the optimum situation, these (un)clear paths would have used longer routes in order to increase grouping, which can lead to more savings than the savings achieved by using short routes. Because of the way the heuristics work while serving demand, it is hard to foresee the grouping opportunity with other unserved demands. In addition, the results indicate that the best heuristic variation at $F_{m i n}$ is not necessarily also the best one at $F_{o p r}$ Furthermore, this case study shows that the use of Policy 1 without demand bifurcation, as illustrated in Fig. 4.14, can achieve the lowest fiber requirement $\left(F_{m i n}=16\right)$ and will be beneficial if fiber resources are scarce. In contrast, if fiber resources are plentiful, then using Policy 4 with the fiber-bifurcation scenario, as depicted in Fig. 4.15, will lead to the lowest weighted port count (at $F_{o p t}=22$ ). It is worth mentioning here that the savings in the port count at $F_{\text {opt }}$ will come at the expense of an increased total solve time of the planning problem. This result is expected since in 
the fiber-plentiful situation the RMGPA problem will be solved several times from $F=1$ to $F=F_{\text {sat }}$ whereas in the fiber-scarce situation the RMGPA problem will be solved from $F=1$ to $F=F_{m i n}$.

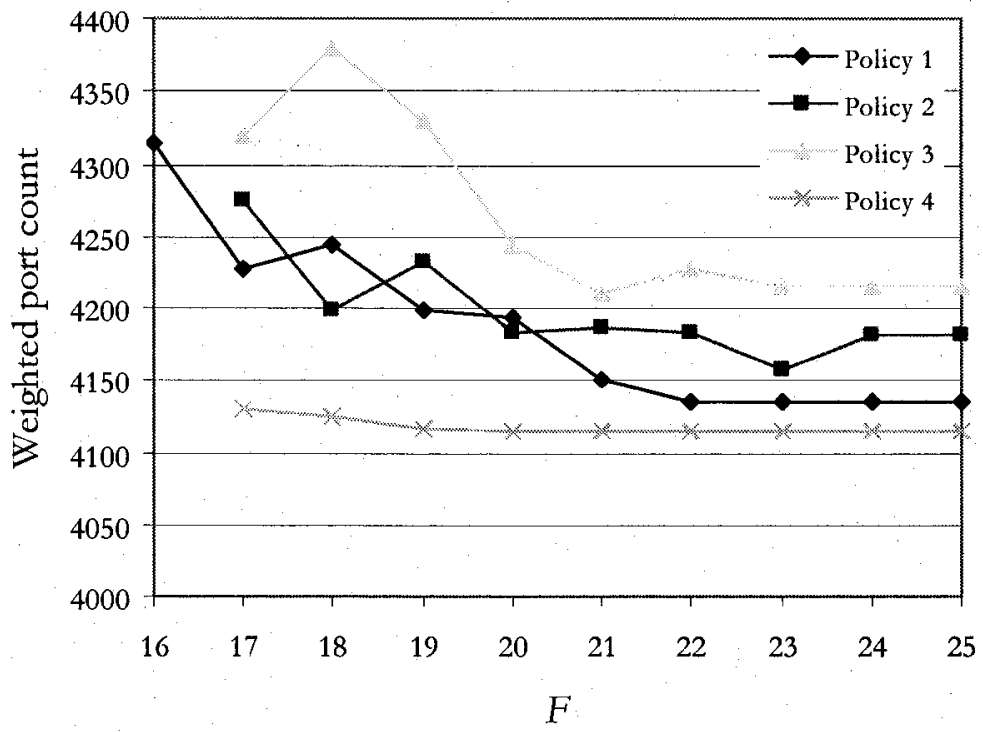

Fig. 4.14: Weighted port count vs. $F$, where $F \geq F_{m i n}$ for all sorting policies without bifurcating demands.

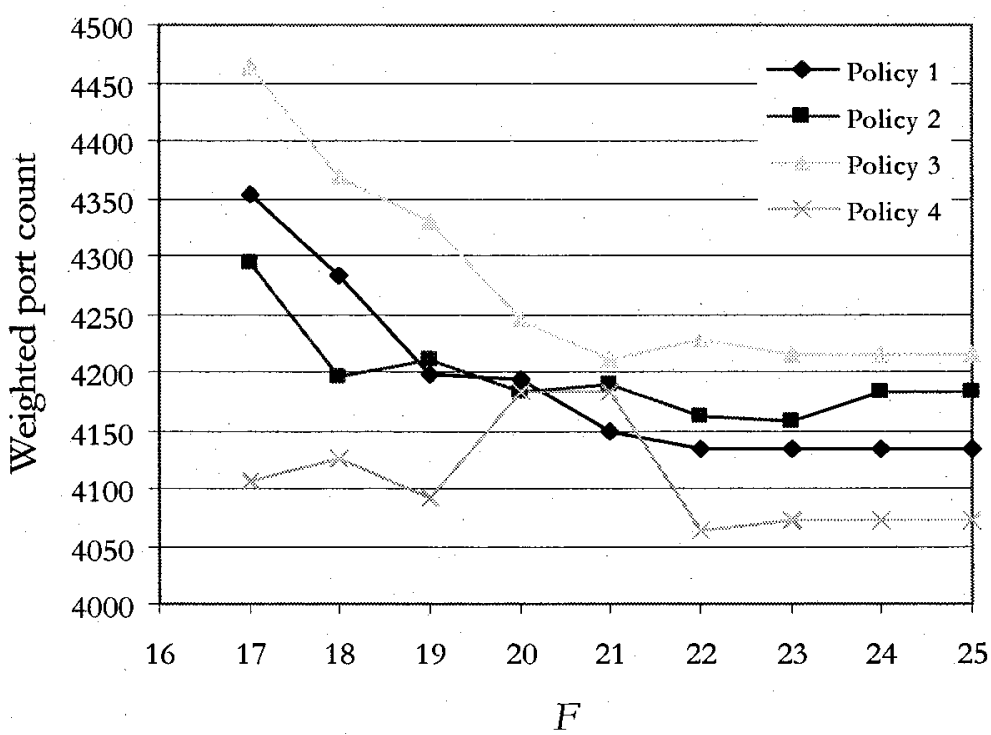

Fig. 4.15: Weighted port count vs. $F$, where $F \geq F_{\text {min }}$ for all sorting policies by allowing the bifurcation of fiber demands. 


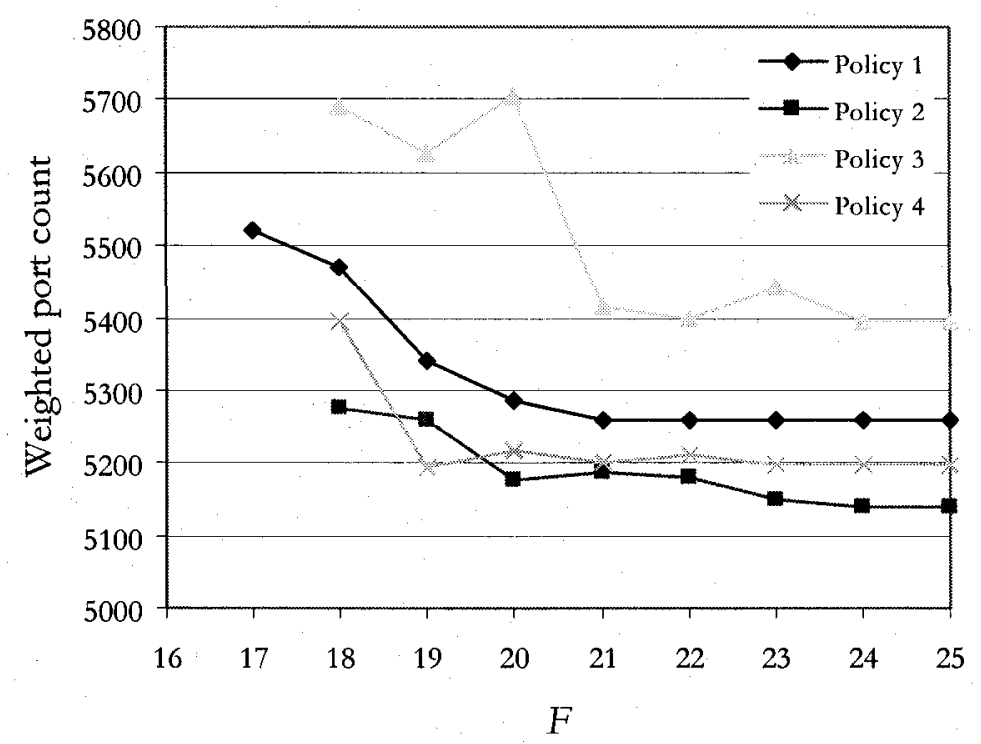

Fig. 4.16: Weighted port count vs. $F$, where $F \geq F_{\min }$ for all sorting policies by allowing the bifurcation of all demands.

\subsubsection{Impact of E/O Cost Ratio}

This subsection captures the impact of changing the $\mathrm{E} / \mathrm{O}$ cost ratio on the solution of the planning problem. Using the same input data and parameters considered in Subsection 4.4.2, except that the $\mathrm{E} / \mathrm{O}$ cost ratio $=2$ and 3 , the results of heuristic variations at $F=F_{m i n}$ and $F_{o p t}$ are shown in Figs. 4.17-4.20.

From the figures, it is obvious that in spite of the fact that increasing the $\mathrm{E} / \mathrm{O}$ ratio increases the weighted port count, this increase does not change the fiber requirements, the best sorting policy, or the best bifurcation scenario. This result stems from the fact that the resultant multi-granular virtual topology remains unchanged as the $\mathrm{E} / \mathrm{O}$ ratio changes. This can be implied from the unchanged "unweighted" port count breakdown shown in Fig. 4.21 as the E/O ratio changes. The multi-granular virtual topology is insensitive to the change in the $\mathrm{E} / \mathrm{O}$ ratio because of the following. (1) 'Some sub-wavelength and wavelength'/waveband/fiber demands use CWS/CBS/CFS paths (or CWS/CBS/CFS-based virtual links), which means that no OEO 
domain conversions are performed at intermediate nodes. As a result, the number of virtual links will remain the same, and changing the $\mathrm{E} / \mathrm{O}$ ratio will not affect the selection of the physical route and the color to be assigned to the clear path. (2) Although sub-wavelength demands can use UWS paths, which involve the use of intermediate EXCs to perform the grouping/degrouping of subwavelength flows into/from existing CWS-based virtual links, minimizing the number of CWSbased virtual hops is the selection criteria for physical routes in order to utilize efficiently the remaining capacities of CWS paths regardless of the $\mathrm{E} / \mathrm{O}$ ratio used.

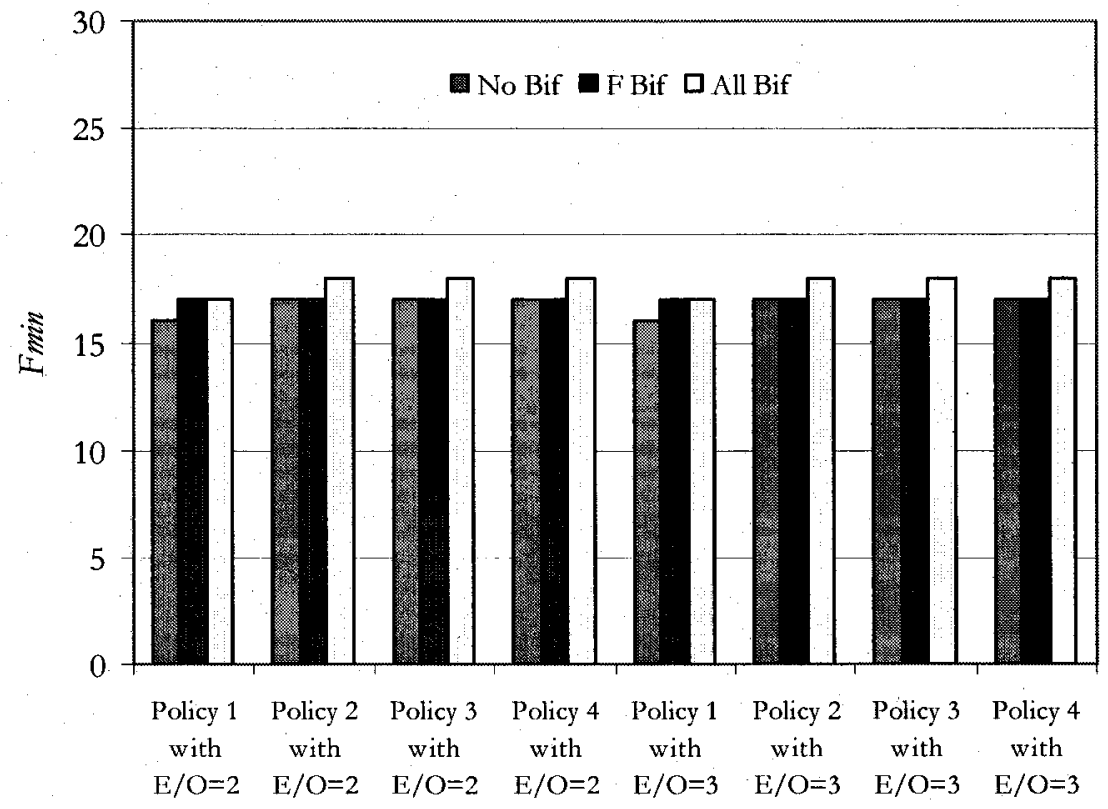

Fig. 4.17: The minimum fiber requirements for all heuristic variations for $E / O$ ratio $=2$ and 3 . 


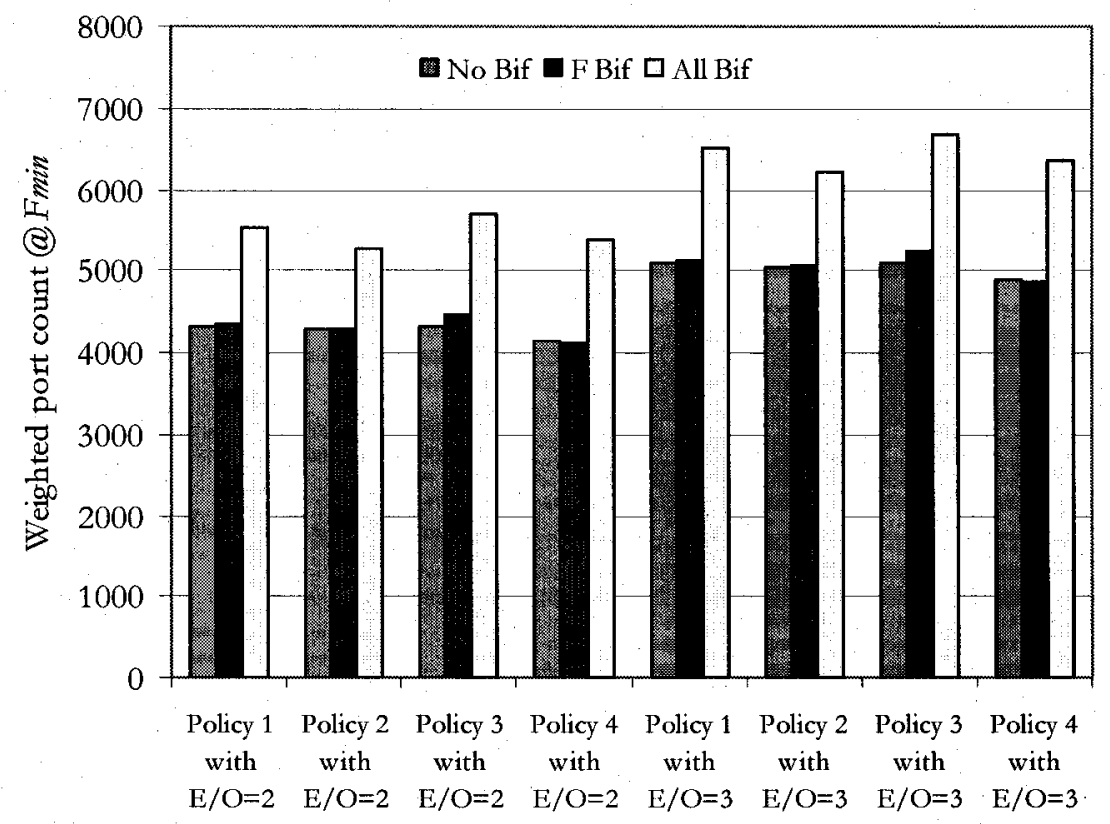

Fig. 4.18: The weighted port count at $F_{\text {min }}$ for all heuristic variations for $\mathrm{E} / \mathrm{O}$ ratio $=2$ and 3 .

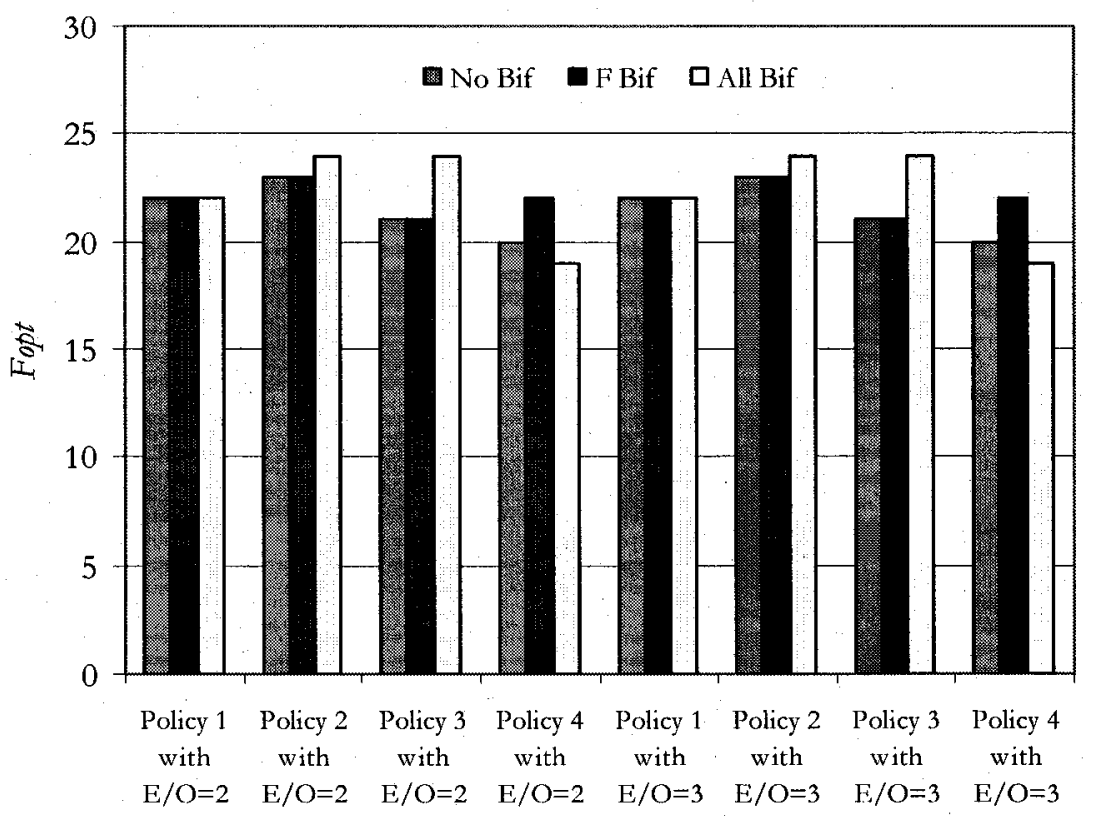

Fig. 4.19: The optimum fiber requirement for all heuristic variations for $E / O$ ratio $=2$ and 3 . 


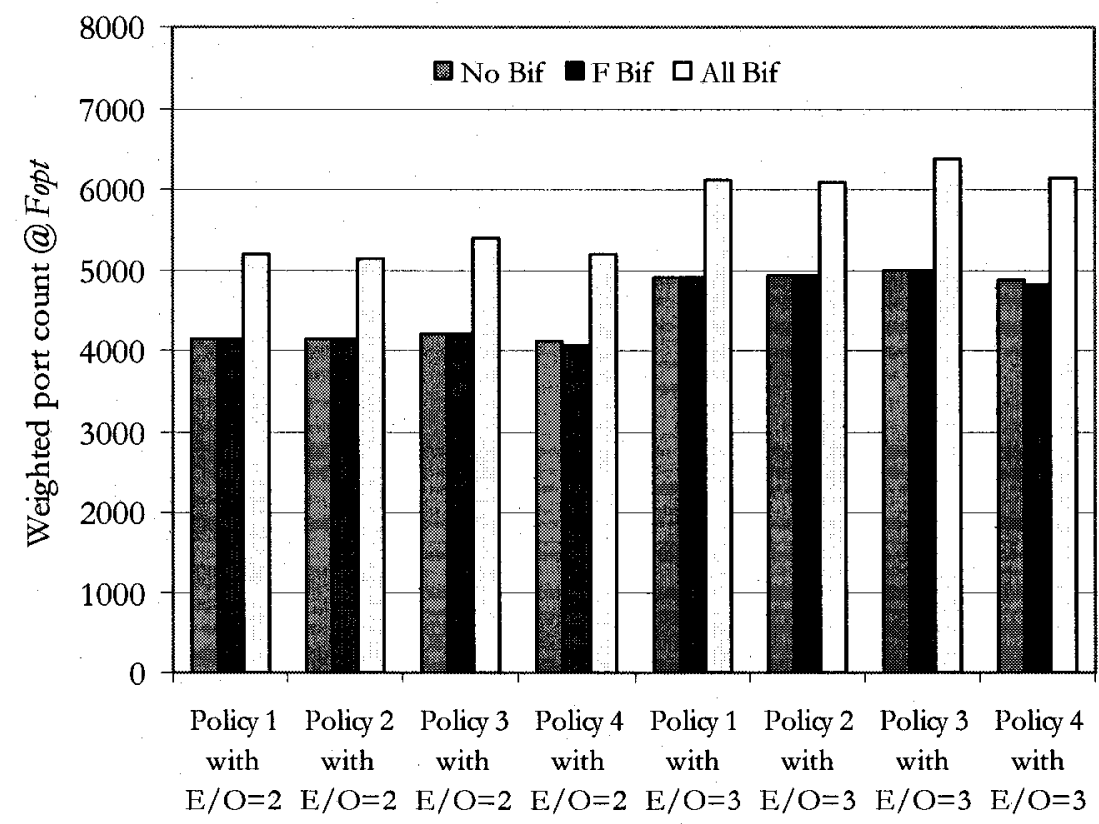

Fig. 4.20: The weighted port count at $F_{\text {opt }}$ for all heuristic variations for $\mathrm{E} / \mathrm{O}$ ratio $=2$ and 3.

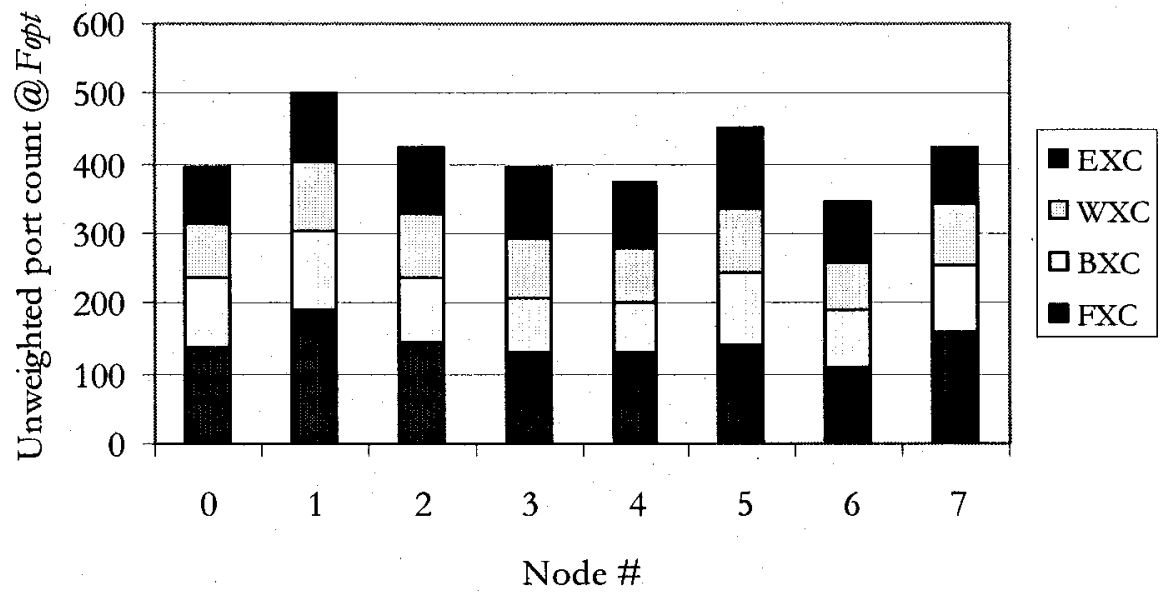

Fig. 4.21: The resultant "unweighted" port count breakdown at all nodes of the 8 -node network at $F_{\text {opp }}=22$ using Policy 4 with the fiber-bifurcation scenario for $\mathrm{E} / \mathrm{O}$ ratio $=2$ and 3 .

\subsubsection{Why Bifurcating All Demands is an Expensive Choice}

In this subsection, we illustrate why allowing demand bifurcation at all traffic granularities achieves the highest weighted port count. We use the input data and parameters used in Subsection 4.4.2. For the purpose of comparing bifurcation scenarios, only one sorting policy is considered in 
heuristic variations. With Policy 1 , all bifurcation scenarios achieve the same $F_{o f t}=22$, as shown in Figs. 4.14-4.16. The port and (de)multiplexer count breakdown at all nodes at $F_{o p t}$ using all bifurcation scenarios is shown in Figs. 4.22 and 4.23 and Figs. 4.24 and 4.25 respectively.

By comparing Fig. 4.22 with Fig. 4.23 , it is clear that allowing demand bifurcation at all traffic granularities will lead to an increase in the port count at all switching stages. This surge in port count is due to the following. (1) The first cause is the increase in the number of CWS paths needed to handle sub-wavelength demands, since every bifurcated unit of the sub-wavelength demand will be treated separately (this increase can be inferred from the increase in the number of EXC ports in the sense that bifurcating other traffic granularities will not affect the number of EXC ports). (2) The second factor is the increase in the $\mathrm{WB} / \mathrm{BW}$ and $\mathrm{BF} / \mathrm{FB}$ ports (refer to Fig. 4.1) needed to interconnect the $\mathrm{WXC}$ stage with the $\mathrm{BXC}$ stage and the $\mathrm{BXC}$ stage with the FXC stage, since treating each bifurcated unit of the sub-wavelength, wavelength, and waveband demand separately increases the chance that these units will use separate wavelength and waveband (de)multiplexers (this port increase can be implied from the significant increase in the number of (de)multiplexers, as can be observed clearly by comparing Fig. 4.24 with Fig. 4.25). (3) Lastly, the increase in the number of both EXC ports and (de)multiplexers has a ripple effect that leads to a further duplication of ports in the next coarse granular switching stages. Because of the ripple effect, it is expected that more wavelength, waveband, and fiber resources will be consumed in order to achieve the minimum fiber requirement, as we shall see in the heuristics evaluation subsection. It is also interesting to note from Fig. 4.22 that allowing the bifurcation of fiber demands can be a promising bifurcation scenario, since the finer granular switching stages are not involved when fiber demands are bifurcated. 


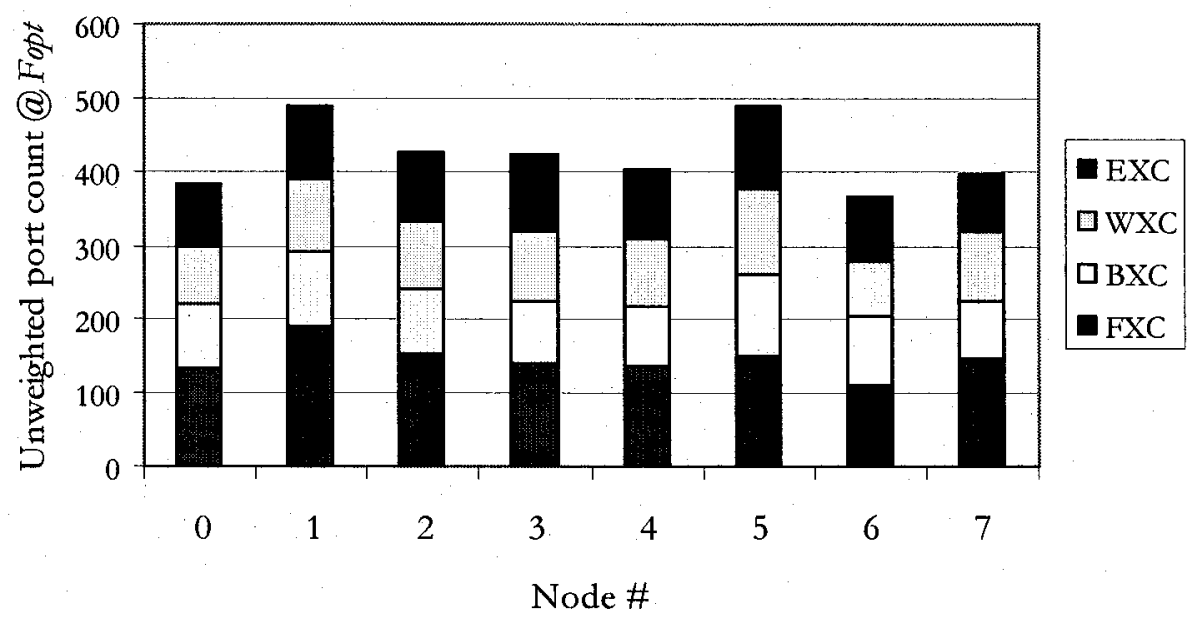

Fig. 4.22: The resultant "unweighted" port count breakdown at all nodes of the 8 -node network at $F_{\text {op }}=22$ using Policy 1 with the no-/fiber-bifurcation scenario.

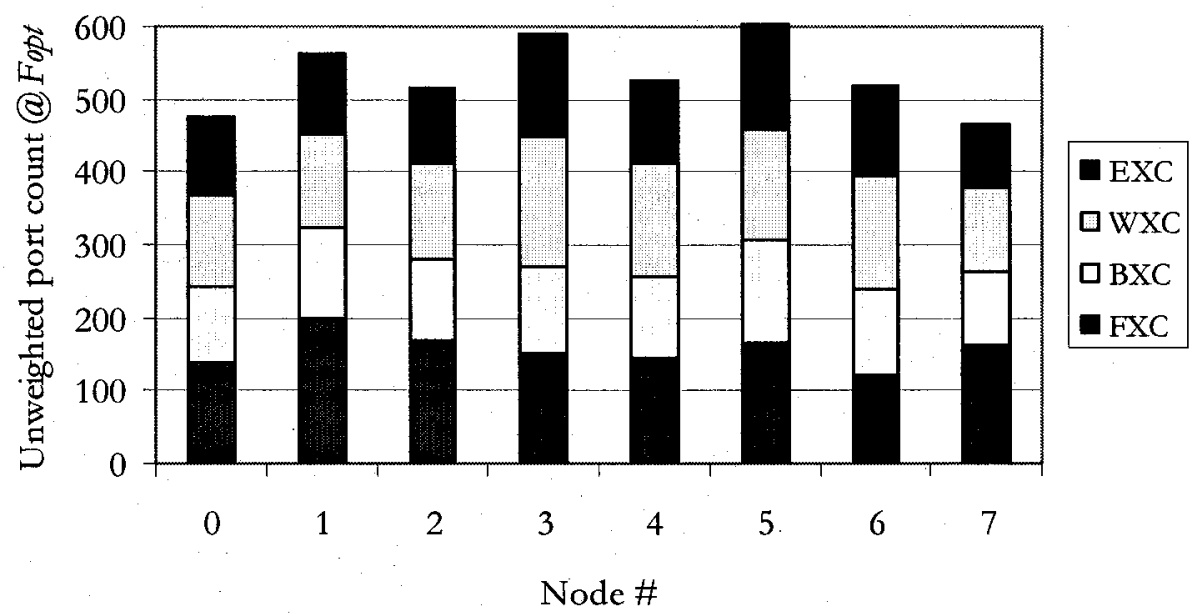

Fig. 4.23: The resultant "unweighted" port count breakdown at all nodes of the 8-node network at $F_{\text {opi }}=22$ using Policy 1 with the all-demand bifurcation scenario. 


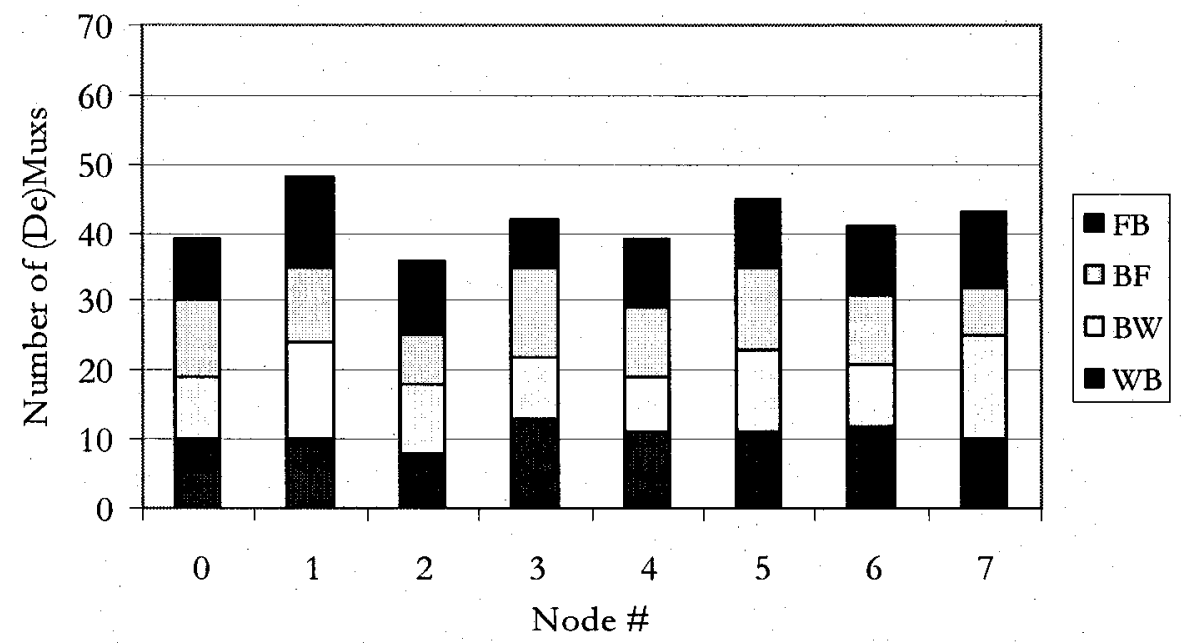

Fig. 4.24: The required number of (de)multiplexers at all nodes of the 8-node network at $F_{\text {opt }}=22$ using Policy 1 with the no-/fiber-bifurcation scenario.

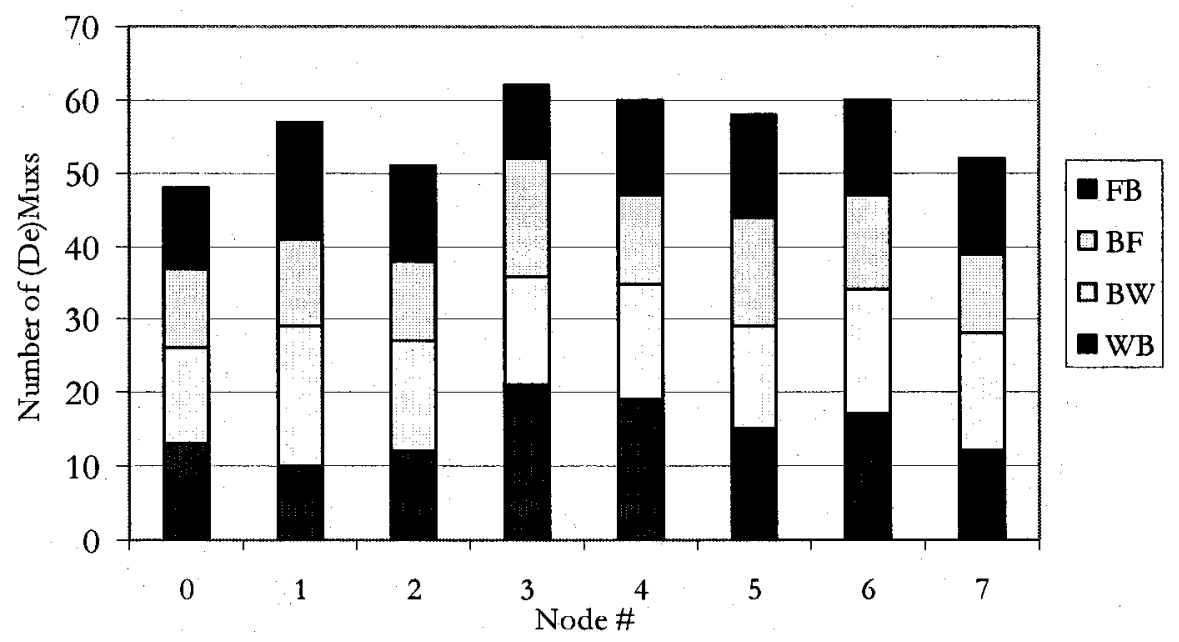

Fig. 4.25: The required number of (de)multiplexers at all nodes of the 8 -node network at $F_{\text {opt }}=22$ using Policy 1 with the all-demand bifurcation scenario.

\subsubsection{Effects of the $C-W-B$ Combination Selection}

This subsection examines the impact of having different combinations of parameters $C, W$, and $B$ that lead to the same fiber capacity, where the fiber capacity $=C \times W \times B$, or to different fiber capacities. The input data and parameters used in this subsection are as follows: the physical topology of the 8-node network shown in Fig. 4.11; the raw traffic demands shown in Table 4.2; 
$C=10$ and $40 \mathrm{~Gb} / \mathrm{s} ; W=2,4$, and $8 ; B=2,4$, and $8 ; F \geq F_{\text {min }}$; all MG-OXC/EXC ports have the same weight; and the 10/40G-based EXC port weight is $2 / 5$ times the MG-OXC port weight.

In the first part of this subsection, we look at the $C-W-B$ combinations that lead to the same fiber capacity. Table 4.4 shows the optimized multi-granularity demands for several combinations.

Table 4.4: The 8-node network optimized multi-granularity demands (which are generated from the raw traffic shown in Table 4.2) for different $C[\mathrm{~Gb} / \mathrm{s}]-W-B$ combinations, provided that all combinations give the same fiber capacity.

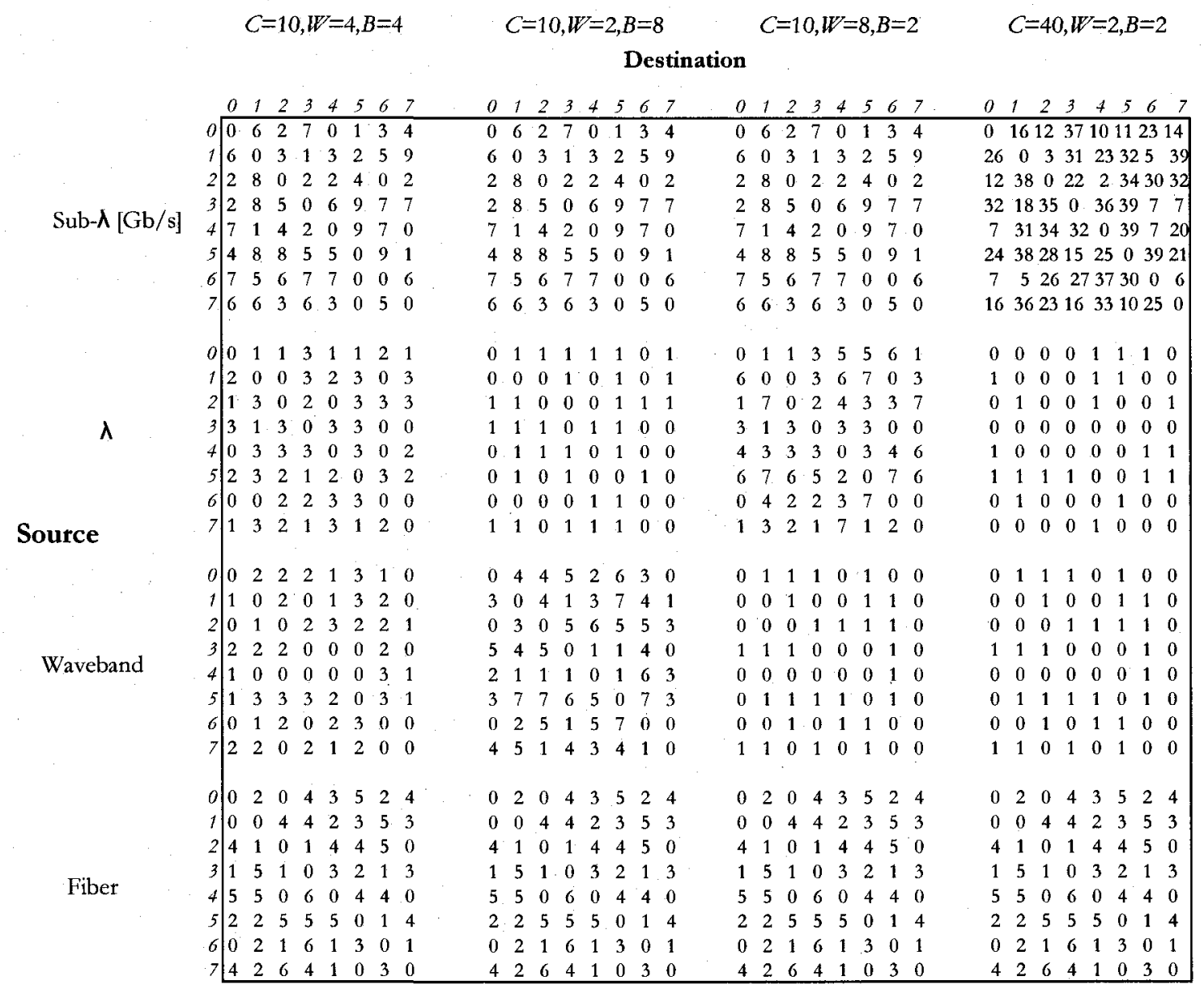

The comparison of heuristic variations for different $C-W-B$ combinations is performed for two fiber-resource situations. In the first fiber situation, it is assumed that fiber resources are scarce. Results in terms of $F_{m i n}$ and the weighted port count at $F_{m i n}$ are shown in Table 4.5. The best heuristic variation is the one that achieves the lowest $F_{\text {min }}$. If it happens that more than one 
variation has the same $F_{m i n}$ then the best heuristic variation will be the one that achieves the lowest weighted port count. In the second fiber situation, it is assumed that fiber resources are plentiful. Results in terms of $F_{o p t}$ and the weighted port count at $F_{o p t}$ are shown in Table 4.6. The best heuristic variation in this situation is the one that achieves the lowest weighted port count at $F_{\text {opr }}$ If it happens that more than one variation has the same weighted port count, then the best heuristic variation will be the one that achieves the lowest $F_{\text {opr }}$

From Tables 4.5 and 4.6, the following observations can be made. First, with the 10-4-4 combination, the best planning solutions, which are shown as the highlighted dark parts in the tables, can be obtained for both fiber situations. This means that the $C-W-B$ combination has an optimum combination and, accordingly, selecting the right combination can lead to substantial savings in the fiber requirement and weighted port count. Second, at a certain $C-W-B$ combination, the winning bifurcation scenario, which is shown as the highlighted part in the tables, alternates between the no-bifurcation and fiber-bifurcation scenarios. Furthermore, the winning bifurcation scenario in the fiber-scarce situation is not necessarily the winner in the fiber-plentiful situation. Moreover, the best sorting policy used along the bifurcation scenario is changing, and the best sorting policy in the fiber-scarce situation is different from the best policy in the fiber-plentiful situation. Therefore, the $C-W-B$ combination decides the best heuristic variation. Third, allowing demand bifurcation at all traffic granularities increases the fiber requirement and weighted port count (for the reasons explained in Subsection 4.4.5), most notably with the 40-2-2 combination due to the dramatic increase in sub-wavelength demands, as illustrated in Table 4.4. 
Table 4.5: Heuristics comparison under the fiber-scarce situation for different $C-B-W$ combinations, which lead to the same fiber capacity.

\begin{tabular}{|c|c|c|c|c|c|c|c|c|c|}
\hline \multirow[b]{2}{*}{$\begin{array}{l}C[\mathrm{~Gb} / \mathrm{s}]-\mathrm{W}-B \\
\text { combination }\end{array}$} & \multicolumn{3}{|c|}{ No bifurcation } & \multicolumn{3}{|c|}{ Fiber bifurcation } & \multicolumn{3}{|c|}{ All bifurcation } \\
\hline & $\begin{array}{c}\text { Best } \\
\text { policy }\end{array}$ & $F_{\min }$ & $\begin{array}{l}\text { Weighted } \\
\text { port count } \\
\text { @ } F_{\min }\end{array}$ & $\begin{array}{c}\text { Best } \\
\text { policy }\end{array}$ & $F_{\min }$ & $\begin{array}{l}\text { Weighted } \\
\text { port count } \\
\text { @ } F_{\min }\end{array}$ & $\begin{array}{c}\text { Best } \\
\text { policy }\end{array}$ & $F_{\min }$ & $\begin{array}{l}\text { Weighted } \\
\text { port count } \\
\text { (a) } F_{\min }\end{array}$ \\
\hline $10-4-4$ & 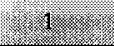 & - 16 & $19314=$ & 4 & 17 & 4106 & 1 & 17 & 5520 \\
\hline $10-8-2$ & 1 & 16 & 4600 & 4 & 16 & 4610 & 4 & 17 & 5738 \\
\hline $10-2-8$ & 1 & 16 & 4586 & 4 & 16 & 4555 & 2 & 18 & 5651 \\
\hline $40-2-2$ & .1 & 16 & 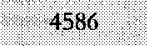 & 4 & 17 & 4544 & 2 & 34 & 15379 \\
\hline
\end{tabular}

Table 4.6: Heuristics comparison under the fiber-plentiful situation for different $C-B-W$ combinations, which lead to the same fiber capacity.

\begin{tabular}{|c|c|c|c|c|c|c|c|c|c|}
\hline \multirow[b]{2}{*}{$\begin{array}{c}C[\mathrm{~Gb} / \mathrm{s}]-W-B \\
\text { combination }\end{array}$} & \multicolumn{3}{|c|}{ No bifurcation } & \multicolumn{3}{|c|}{ Fiber bifurcation } & \multicolumn{3}{|c|}{ All bifurcation } \\
\hline & $\begin{array}{l}\text { Best } \\
\text { policy }\end{array}$ & $F_{o p t}$ & $\begin{array}{l}\text { Weighted } \\
\text { port count } \\
\text { (a) } F_{\text {opt }}\end{array}$ & $\begin{array}{c}\text { Best } \\
\text { policy }\end{array}$ & $F_{o p t}$ & $\begin{array}{l}\text { Weighted } \\
\text { port count } \\
\text { (a) } F_{\text {opt }}\end{array}$ & $\begin{array}{c}\text { Best } \\
\text { policy }\end{array}$ & $F_{o p r}$ & $\begin{array}{c}\text { Weighted } \\
\text { port count } \\
\text { (a) } F_{\text {opt }}\end{array}$ \\
\hline $10-4-4$ & 4 & 20 & 4114 & $4 \sqrt{3}$ & 22 & 4063 & 2 & 24 & 5139 \\
\hline $10-8-2$ & 4 & 18 & 4357 & 4 & 18 & 4357 & 4 & 23 & 5491 \\
\hline $10-2-8$ & 4 & 10 & 4423 & 4 & 22 & 4432 & 1 & 22 & 5518 \\
\hline $40-2-2$ & 4 & 18. & $7+486$ & 4 & 18 & 4486 & 4 & 41 & 15298 \\
\hline
\end{tabular}

In the second part of this subsection, we look at the effect of changing the $C-W-B$ combination in a way that leads to the increase in the fiber capacity. Table 4.7 shows the optimized multigranularity demands for different fiber capacities.

The results of comparing heuristic variations using different fiber capacities for both fiber situations are shown in Tables 4.8 and 4.9. In the fiber-scarce situation, it is clear that using the 408-8 combination, which has the highest fiber capacity, requires the lowest fiber requirement. This means that in the fiber-scarce situation, increasing the fiber capacity usually leads to the best planning solution. However, if increasing the fiber capacity does not lead to a further reduction in $F_{m i n}$ then we need to look at the fiber capacity that achieves the lowest weighted port count. In the fiber-plentiful situation, using the 10-4-4 combination leads to the lowest weighted port count. Again, this result has to do with the fact the $C-W-B$ combination has an optimum combination. 
Therefore, this finding suggests that, especially in the fiber-plentiful situation, choosing the proper fiber capacity has a major impact on improving the planning solution. It is important to note here that as the fiber capacity increases (as is the case with the $10-8-8$ and $40-8-8$ combinations), the bifurcation of fiber demands will not occur since the fiber demand between any $s-d$ pair is $\leq 1$, as shown in Table 4.7. Finally, similar to the observations made in the first part of this subsection, the $C-W-B$ combination along with the fiber situation decides the best heuristic variation.

Table 4.7: The 8-node network optimized multi-granularity demands (which are generated from the raw traffic shown in Table 4.2) for different fiber capacities, given that the fiber capacity $=C \times B \times W$.

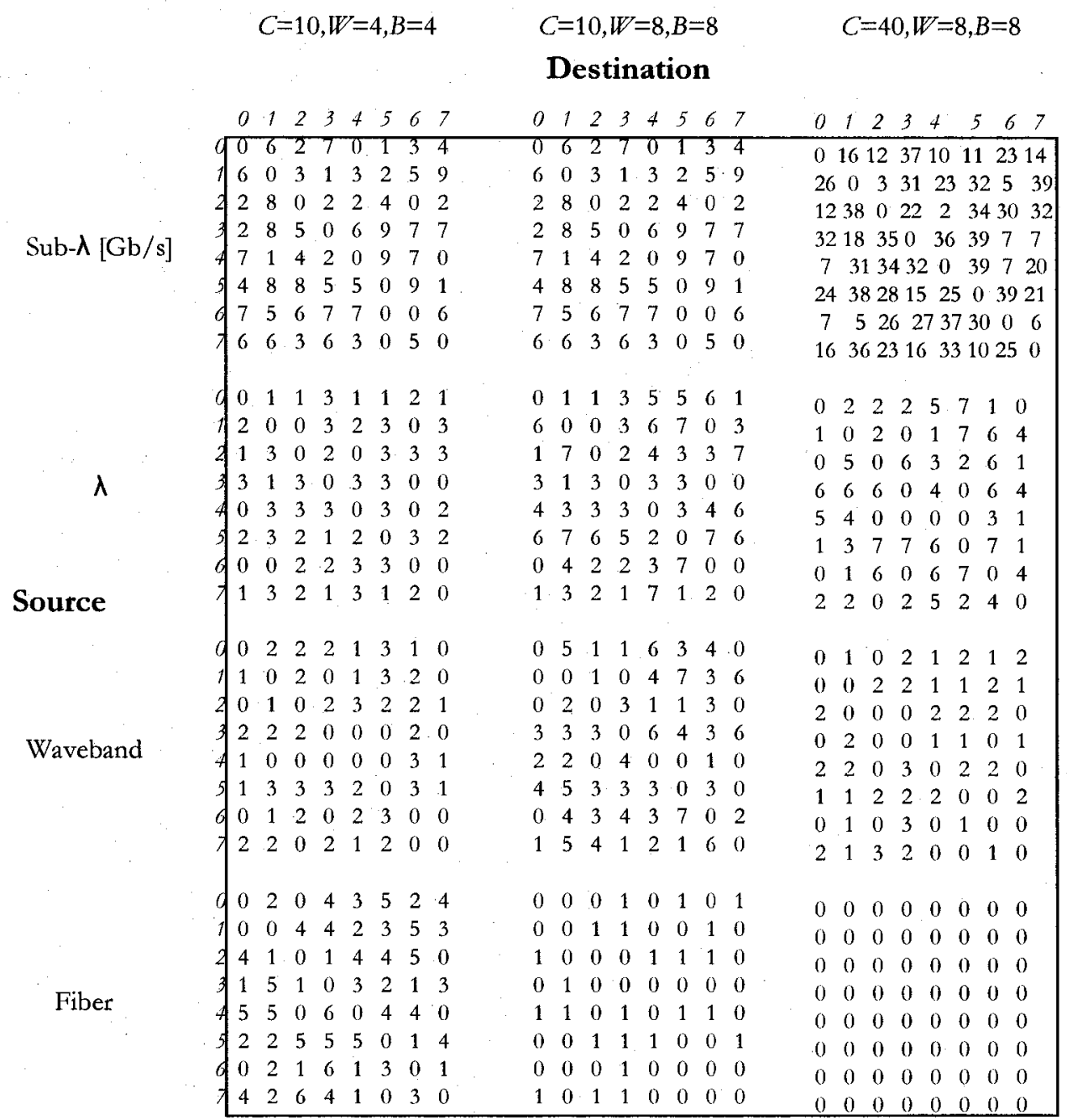


Table 4.8: Heuristics comparison under the fiber-scarce situation for different $C-B-W$ combinations, which lead to different fiber capacities.

\begin{tabular}{|c|c|c|c|c|c|c|c|c|c|}
\hline \multirow{2}{*}{$\begin{array}{l}C[\mathrm{~Gb} / \mathrm{s}]-\mathbb{W}-B \\
\text { combination }\end{array}$} & \multicolumn{3}{|c|}{ No bifurcation } & \multicolumn{3}{|c|}{ Fiber bifurcation } & \multicolumn{3}{|c|}{ All bifurcation } \\
\hline & $\begin{array}{c}\text { Best } \\
\text { policy }\end{array}$ & $F_{\min }$ & $\begin{array}{c}\text { Weighted } \\
\text { port count } \\
@ F_{\min }\end{array}$ & $\begin{array}{c}\text { Best } \\
\text { policy }\end{array}$ & $\boldsymbol{F}_{\min }$ & $\begin{array}{l}\text { Weighted } \\
\text { port count } \\
\text { @ } F_{\min }\end{array}$ & $\begin{array}{c}\text { Best } \\
\text { policy }\end{array}$ & $F_{\min }$ & $\begin{array}{l}\text { Weighted } \\
\text { port count } \\
\text { (a) } F_{\min }\end{array}$ \\
\hline $10-4-4$ & 1 & 10 & 4314 & 4 & 17 & 4106 & 1 & 17 & 5520 \\
\hline $10-8-8$ & 4 & 5 & 4220 & 4 & 5 & 4220 & 4 & 5 & 5371 \\
\hline $40-8-8$ & $=3$ & 2 & 5008 & 1 & 2 & 5008 & 2 & 3 & 14596 \\
\hline
\end{tabular}

Table 4.9: Heuristics comparison under the fiber-plentiful situation for different $C-B-W$ combinations, which lead to different fiber capacities.

\begin{tabular}{|c|c|c|c|c|c|c|c|c|c|}
\hline \multirow[b]{2}{*}{$\begin{array}{l}C[\mathrm{~Gb} / \mathrm{s}]-W-B \\
\text { combination }\end{array}$} & \multicolumn{3}{|c|}{ No bifurcation } & \multicolumn{3}{|c|}{ Fiber bifurcation } & \multicolumn{3}{|c|}{ All bifurcation } \\
\hline & $\begin{array}{l}\text { Best } \\
\text { policy }\end{array}$ & $F_{\text {opt }}$ & $\begin{array}{c}\text { Weighted } \\
\text { port count } \\
\text { (a) } F_{\text {opt }} \\
\end{array}$ & $\begin{array}{c}\text { Best } \\
\text { policy }\end{array}$ & $F_{\text {opt }}$ & $\begin{array}{c}\text { Weighted } \\
\text { port count } \\
@ F_{\text {opt }} \\
\end{array}$ & $\begin{array}{l}\text { Best } \\
\text { policy }\end{array}$ & $F_{o p t}$ & $\begin{array}{c}\text { Weighted } \\
\text { port count } \\
\text { (a) } F_{\text {opt }} \\
\end{array}$ \\
\hline $10-4-4$ & 4 & 20 & 4114 & 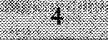 & 22. & 4063 & 2 & 24 & 5139 \\
\hline $10-8-8$ & 4 & 7. & 4193 & 4 & 7. & 4193 & 4 & 6 & 5254 \\
\hline $40-8-8$ & 1 & 2 & 5008 & 1 & 2 & 5008 & 2 & 3 & 14596 \\
\hline
\end{tabular}

\subsubsection{Comparison of Heuristics}

In this subsection, we evaluate through simulation different heuristic variations under different traffic patterns, network physical topologies, and fiber capacities. Heuristic variations are based on the combination of various bifurcation scenarios, namely the no-bifurcation, fiber-bifurcation, and all-bifurcation scenarios, with different sorting policies, namely Policies 1-4.

Traffic patterns are generated as follows [RaSi95b][RaSi96][BaMu97][BYCh97][Mukh97]:

- $(100-F R) \%$ of the raw traffic matrix elements is randomly and uniformly distributed over the range $(0, I N)$, where $I N$ is the maximum offered traffic intensity per $s-d$ pair

- $F R \%$ of elements is randomly and uniformly distributed over the range $(0, I N / 100)$. $F R$ is a measure of the density of the traffic pattern; increasing FR means that the traffic density is decreasing and the traffic thus becomes more concentrated among a few $s-d$ pairs, while 
decreasing FR means that the traffic density is increasing and the traffic thus becomes more evenly distributed among $s-d$ pairs.

- Traffic patterns are generated based on the combination of the following parameters: $F R=0$, 20,50 , and $80 \% ; I N=500,1000$, and $1500 \mathrm{~Gb} / \mathrm{s}$. If $F R=0 \%$, then a uniform traffic pattern is generated; otherwise, a non-uniform traffic pattern is generated.

The physical topologies considered in our simulation are the 8-node sample network, the 14-node NSF (National Science Foundation) network [Mukh97], and the 11-node EON (European Optical Network) network [Grov04], as shown in Figs. 4.11, 4.26, and 4.27 respectively. These topologies are characterized as follows. The 8-node/NSF/EON network has an average network physical connectivity (or nodal degree) of $3.25 / 3 / 4.73$ and, accordingly, the number of distinct routes between a network node pair can go up to $29 / 120 / 1720$ routes.

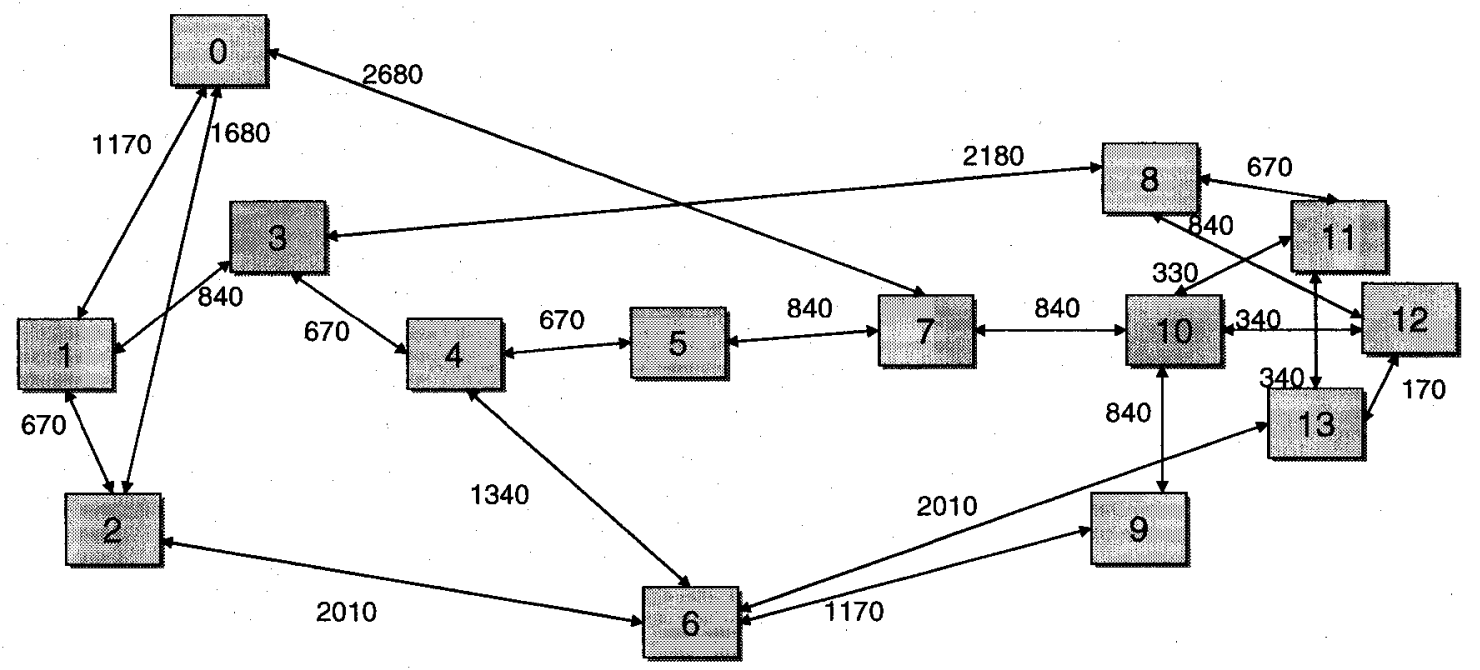

Fig. 4.26: 14-node NSF network fiber layout with span distances in $\mathrm{km}$. 


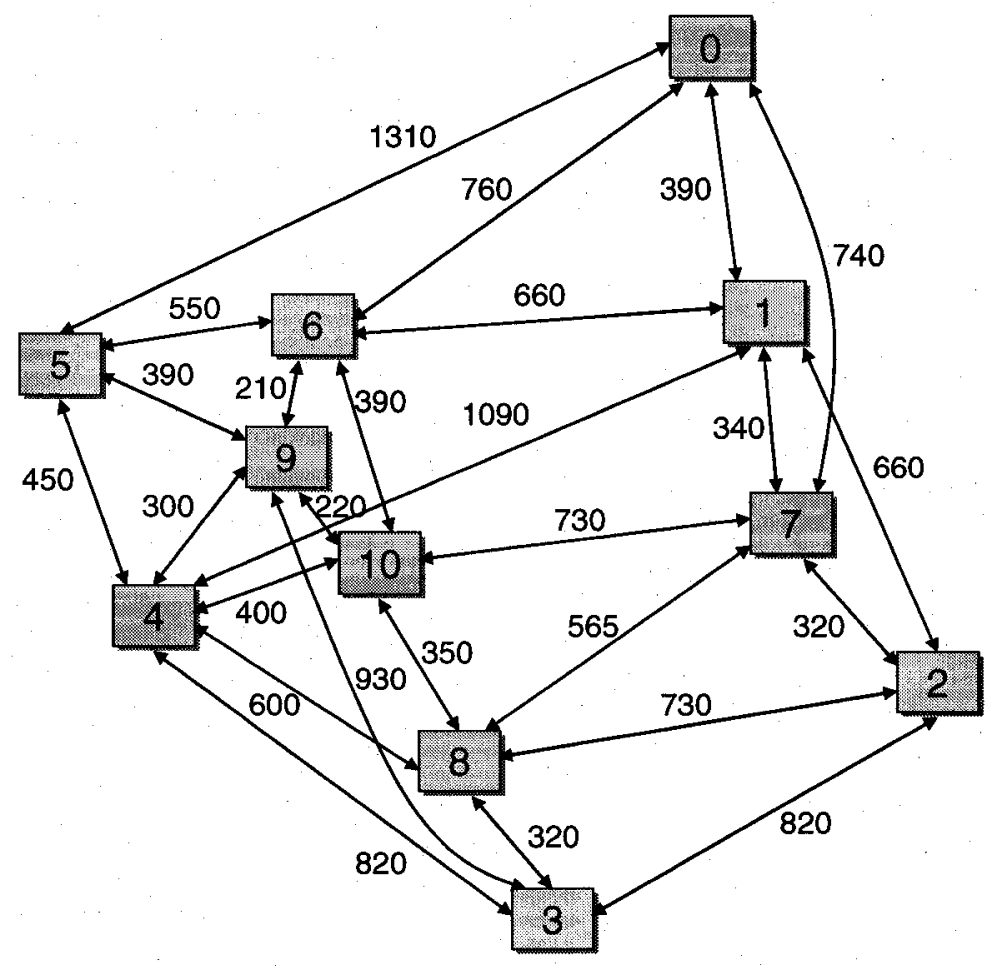

Fig. 4.27: 11-node EON network fiber layout with span distances in $\mathrm{km}$.

The fiber capacities used here are based on using the following $C[\mathrm{~Gb} / \mathrm{s}]-W-B$ combinations: $10-4-$ 4, 10-8-8, and 40-8-8. The rest of the input parameters employed in the simulation are as follows: $1 \leq F \leq F_{\text {sat }}$ all MG-OXC/EXC ports have the same weight, and the $10 / 40 \mathrm{~Gb} / \mathrm{s}$-based EXC port weight is $2 / 5$ times the MG-OXC port weight.

The results of the heuristic variations are compared in terms of the fiber requirement, weighted port count, and solve time under the fiber-scarce and fiber-plentiful situations. These results are based on averaging 10 simulation runs. In each simulation run, different seeds are used for Random Number Generators (RNGs) [PTVF92] that generate both $(0, I N)$ and $(0, I N / 100)$ traffic ranges. Also, a different RNG with a different seed is used to decide which of the $s-d$ pairs will be considered in the $(0, I N / 100)$ traffic range. Detailed results with $95 \%$ confidence intervals are 
provided in Appendix A. The mathematical derivation of the confidence interval can be found in Appendix D.

Since the total solve time for carrying out the network planning process is still essential, it is important to understand the factors that contribute to the increase in the solve time of the RMGPA problem at $F_{m i n}$ and $F_{o p r}$ From Tables A.1-A.9, the following observations can be made. First, increasing the amount of traffic demands imposed on the network by either increasing $I N$ and fixing $F R$ or decreasing FR and fixing $I N$ increases the solve time. Second, increasing both the network size and connectivity increases the solve time due to the increase in the number of distinct routes between an $s-d$ pair that needs to be evaluated by the heuristic. This factor explains clearly (1) why the EON network has the longest solve time in comparison with other topologies, and (2) why the NSF network has a longer solve time than the 8-node network. Third, bifurcating demands also increases the solve time, since each unit of the same demand will be treated separately by the heuristic. This factor explains (1) why heuristics in which demand bifurcation is allowed at all granularity levels have the longest solve time, and (2) why heuristics in which demand bifurcation is not performed achieve the shortest solve time.

Heuristic variations using the same bifurcation scenario are compared under the fiber-scarce and fiber-plentiful situations using the same criteria explained in Subsection 4.4.6. Accordingly, Tables 4.10-4.27 summarize the results in Tables A.1-A.9 for both fiber situations. Likewise, deciding the best bifurcation scenario in Tables $4.10-4.27$ is based on the same rules used in deciding the best policy. Accordingly, the best heuristic variations are highlighted in the tables.

Overall, as shown in Tables 4.10-4.27, it is relatively clear that regardless of the heuristic variation employed, increasing the traffic amount imposed on the network by either increasing $I N$ and 
fixing FR or decreasing FR and fixing $I N$ results in an increase in both the weighted port count and fiber requirement. This increase occurs because more clear paths are needed to accommodate all demands.

We first evaluate heuristics under the fiber-scarce situation. From Tables 4.10-4.18, in comparing heuristics based on the bifurcation scenario used, it is apparent that for the majority of the traffic patterns, topologies, and fiber capacities tested, the heuristics based on the no- and fiberbifurcation scenarios outperform those based on the all-bifurcation scenario. With the 8node/NSF/EON network, compared to the winning heuristic variation, the increase in $F_{\text {min }}$ using the all-bifurcation-based heuristic can go up to $\approx 14 / 19 / 35 \%, \approx 58 / 21 / 43 \%$, and $\approx$ $100 / 200 / 133 \%$ when employing the $10-4-4,10-8-8$, and 40-8-8 combinations respectively. Even with traffic patterns where the same $F_{\text {min }}$ is achieved using all heuristic variations, as shown in Table 4.12 , the increase in the weighted port count at $F_{m i n}$ can go up to $\approx 199 \%$ with the use of the allbifurcation-based heuristic in comparison to the weighted port count of the winning heuristic variation. This happens for the reasons we have already explained in Subsection 4.4.5. However, at a certain physical topology and fiber capacity, as $I N$ increases and/or FR decreases, we can note that the difference in $F_{\text {min }}$ drops. This drop stems from the increased grouping of unclear and clear paths at all levels, which increases the utilization of network resources. As a result of this trend, we can observe from Table 4.17 that in the case of the EON network (which has the highest physical connectivity) along with the use of the $10-8-8$ combination, when $I N=1500 \mathrm{~Gb} / \mathrm{s}$ and $F R=0 \%$ (which is the highest intensity uniform traffic pattern), allowing the bifurcation of all demands turns out to be more fiber-efficient since it achieves $\approx 8 \%$ less $F_{\text {min }}$ compared to the nobifurcation-based heuristic. A possible explanation for this unique situation is as follows. When the traffic pattern is uniform and the traffic intensity is high, wavelength capacities, wavebands, and 
fibers become fully utilized. Consequently, the (de)multiplexers used by the bifurcated units of a certain $s-d$ demand, as we have explained in Subsection 4.4 .5 , become increasingly shared among the bifurcated units of other demands. Otherwise, this situation can lead to an inefficient use of network resources. Besides, when the network physical connectivity is high, the chance that all bifurcated units are using physical routes with the smallest (or close to the smallest) hop count increases, since using longer routes significantly duplicates resources and, accordingly increases $F_{m i n}$

Moreover, with traffic patterns in which fiber demands exist, which happens at $I N={ }^{c} 1000$ and $1500^{\prime} / 1500 \mathrm{~Gb} / \mathrm{s}$ with the $10-4-4 / 10-8-8$ combination, we can note that there is no clear winner between choosing not to bifurcate demands or to bifurcate fiber demands only. However, with the 8-node network and EON network, in which the physical connectivity is higher than the NSF network, the fiber-bifurcation-based heuristic tends to be more on the winning side, as is clearly illustrated through the comparison of Tables $4.10,4.11,4.13,4.14,4.16$, and 4.17 . This is because increasing the physical connectivity increases the chance that the alternative routes to be used by the bifurcated units of the same fiber demand can have the same physical hop count. As a result, the situation in which some of the bifurcated units of the same fiber demand use long routes (which leads to the duplication of resources) while the rest use short routes, as is the case with the NSF network, will be avoided.

In comparing heuristic variations based on the sorting policy used, results show that there is no clear winning sorting policy as the traffic pattern, physical topology, and fiber capacity change. Moreover, the set of winning policies, which consists of the winning policies of all traffic patterns, changes according to the physical topology and fiber capacity. 
In addition, in looking for the $C-W-B$ combination that can achieve the lowest $F_{m i n}$, it is intuitive that the combination that increases the fiber capacity will be the best choice. However, this is not always the case. For example, with the 8 -node network at $I N=1000 \mathrm{~Gb} / \mathrm{s}$ and $F R=80 \%$, as shown in Tables 4.11 and 4.12 , we can notice that $F_{\text {min }}$ is the same using the $10-8-8$ and $40-8-8$ combinations. Nevertheless, with the $10-8-8$ combination, a lower weighted port count is realized in spite of the fact that the $10-8-8$ combination leads to a lower fiber capacity than the $40-8-8$ combination. Therefore, this indicates that choosing the best $C-W-B$ combination in the fiberscarce situation can be a function of the traffic pattern and the physical topology.

Table 4.10: Comparison of heuristic variations for different traffic patterns in the 8-node network using the 10-4-4 combination under the fiber-scarce situation.

\begin{tabular}{|c|c|c|c|c|c|c|c|c|c|}
\hline \multirow[b]{2}{*}{$\begin{array}{l}\text { Traffic pattern: } \\
(I N G \mathrm{~s} / \mathrm{s}, F R \%)\end{array}$} & \multicolumn{3}{|c|}{ No bifurcation } & \multicolumn{3}{|c|}{ Fiber bifurcation } & \multicolumn{3}{|c|}{ All bifurcation } \\
\hline & $\begin{array}{c}\text { Best } \\
\text { policy }\end{array}$ & $F_{\min }$ & $\begin{array}{l}\text { Weighted } \\
\text { port count } \\
\text { @ } F_{\min }\end{array}$ & $\begin{array}{l}\text { Best } \\
\text { policy }\end{array}$ & $F_{\min }$ & $\begin{array}{c}\text { Weighted } \\
\text { port count } \\
\text { (a) } F_{\min }\end{array}$ & $\begin{array}{l}\text { Best } \\
\text { policy }\end{array}$ & $F_{\min }$ & $\begin{array}{c}\text { Weighted } \\
\text { port count } \\
\text { @ } F_{\min }\end{array}$ \\
\hline$(500,0)$ & 4 & 8.4 & 31049 & 4 & 8.8 & 3015.6 & 4 & 9.6 & 4320.8 \\
\hline$(1000,0)$ & 3 & 164 & $4027 \mathrm{~s}$ & 3 & 16.4 & 4034.3 & 4 & 17.2 & 5260.7 \\
\hline$(1500,0)$ & 1 & 24.2 & 4737.6 & $1:-1 \%: 0$ & 23,8 & $=17705$ & 4 & 24.8 & 5939.2 \\
\hline$(500,20)$ & 4 & 7.6 & 2478.6 & 4 & 7.6 .12 .7 & 24729 & 4 & 8 & 3500.3 \\
\hline$(1000,20)$ & 4 & $=0.13 .4$ & 31597 & 1 & 13.4 & 3182.9 & 4 & 14.4 & 4333.3 \\
\hline$(1500,20)$ & 4 & 19.8 & 3800.6 & 4 & 19,4 & 3813.8 & 1 & 19.8 & 5112.7 \\
\hline$(500,50)$ & 4 & 5.8 & 1789.3 & 4 & 58 & 7778.4 & 4 & 6 & 2667 \\
\hline$(1000,50)$ & 4 & 10.6 & 2294.2 & 2 & $=102$ & $2403 \%$ & 4 & 10.8 & 3470.1 \\
\hline$(1500,50)$ & 1 & 15.4 & 2862.9 & 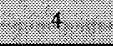 & 15.2 & 2855 & 4 & 16 & 3889 \\
\hline$(500,80)$ & 4 & 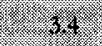 & $10006=$ & 4 & 3.4 & 1006.8 & 2 & 3.8 & 1647.5 \\
\hline$(1000,80)$ & 1 & 5.8 & 1311.9 & $j$ & 5.6 & 1292 & 2 & 6.2 & 2460 \\
\hline$(1500,80)$ & 1 & 8.8 & 1660.2 & 1 & 4.6 & $17=172$ & 1 & 8.4 & 2855 \\
\hline
\end{tabular}


Table 4.11: Comparison of heuristic variations for different traffic patterns in the 8-node network using the 10-8-8 combination under the fiber-scarce situation.

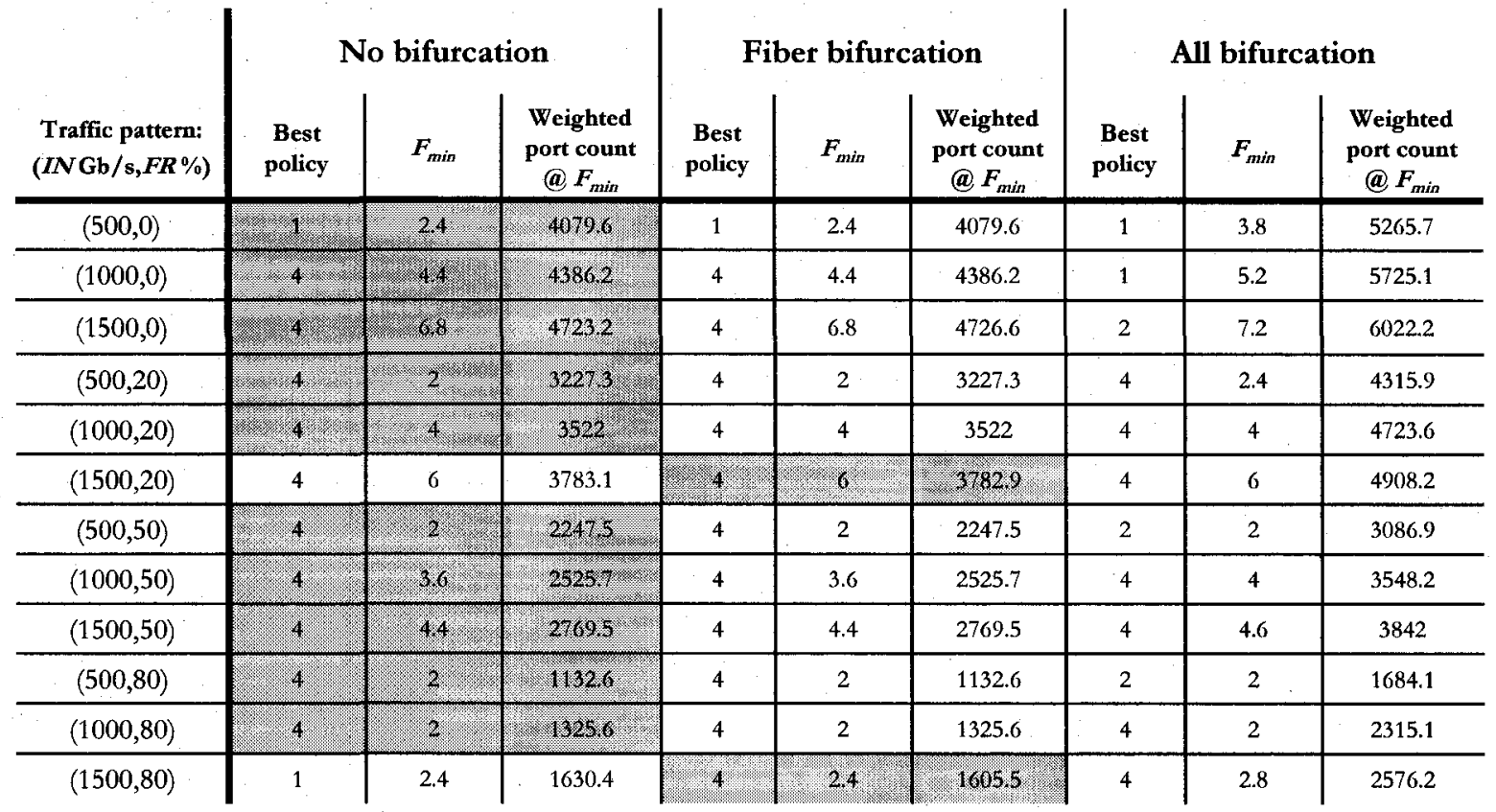

Table 4.12: Comparison of heuristic variations for different traffic patterns in the 8-node network using the 40-8-8 combination under the fiber-scarce situation.

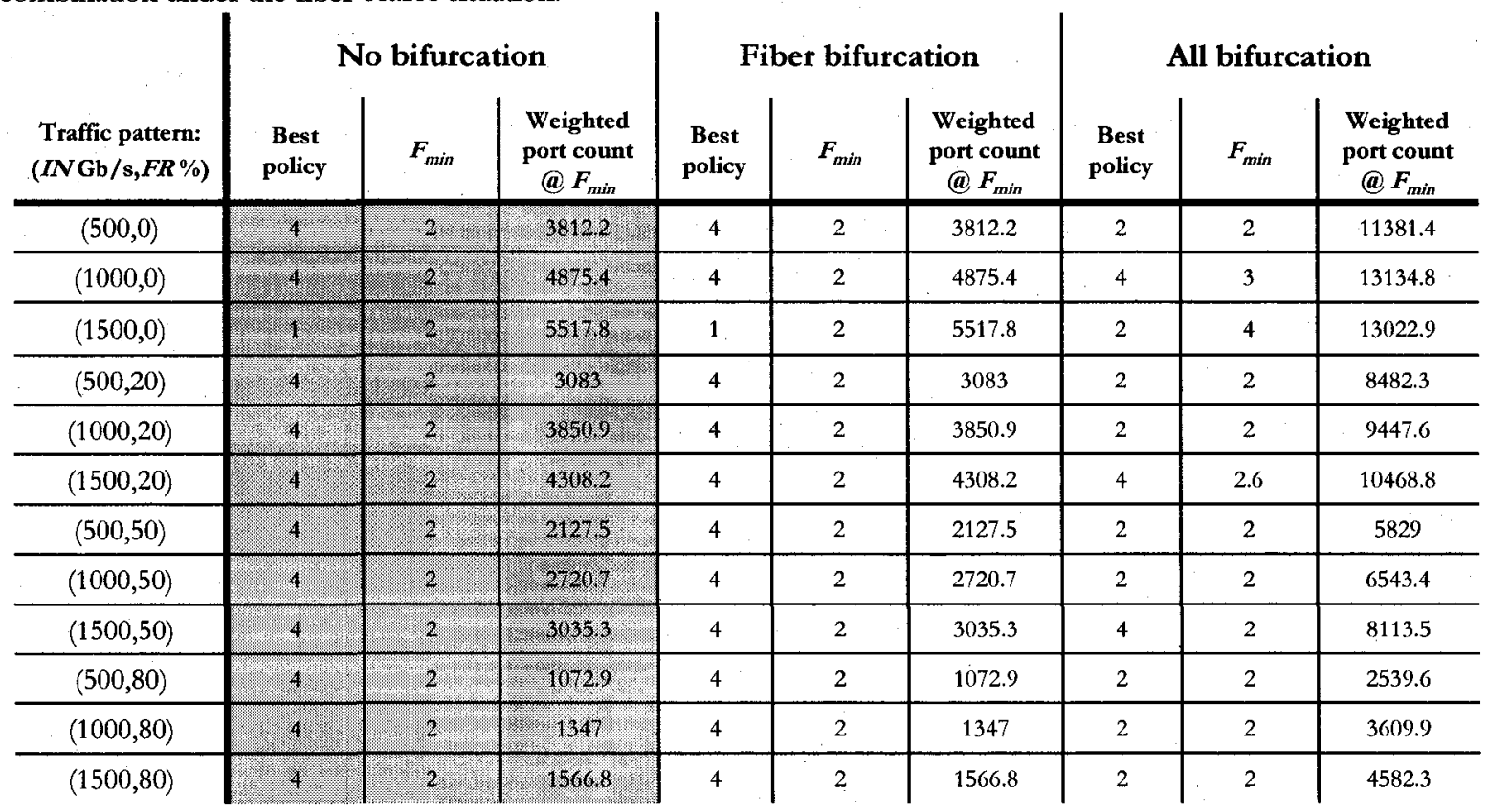


Table 4.13: Comparison of heuristic variations for different traffic patterns in the NSF network using the 10-4-4 combination under the fiber-scarce situation.

\begin{tabular}{|c|c|c|c|c|c|c|c|c|c|}
\hline \multirow[b]{2}{*}{$\begin{array}{l}\text { Traffic pattern: } \\
(I N G b / \mathrm{s}, F R \%)\end{array}$} & \multicolumn{3}{|c|}{ No bifurcation } & \multicolumn{3}{|c|}{ Fiber bifurcation } & \multicolumn{3}{|c|}{ All bifurcation } \\
\hline & $\begin{array}{c}\text { Best } \\
\text { policy }\end{array}$ & $F_{\min }$ & $\begin{array}{l}\text { Weighted } \\
\text { port count } \\
\quad @ F_{\min }\end{array}$ & $\begin{array}{c}\text { Best } \\
\text { policy }\end{array}$ & $F_{\min }$ & $\begin{array}{l}\text { Weighted } \\
\text { port count } \\
\text { @ } F_{\min }\end{array}$ & $\begin{array}{l}\text { Best } \\
\text { policy }\end{array}$ & $F_{\min }$ & $\begin{array}{l}\text { Weighted } \\
\text { port count } \\
\text { @ } F_{\min }\end{array}$ \\
\hline$(500,0)$ & 3. & 23.8 & 1.5532 & 3 & 23.8 & 11553.2 & 4 & 26.8 & 17173 \\
\hline$(1000,0)$ & 3. & 4.68 & 14752.6 & 3 & 46.8 & 14772.6 & 3 & 49.6 & 21061.4 \\
\hline$(1500,0)$ & 3 & 69.8 & 17708.2 & 3 & 692 & 17738.5 & 3 & 72.6 & 23760.9 \\
\hline$(500,20)$ & 1. & 204 & $=9573.2$ & 1 & 20.4 & 9573.2 & 2 & 23.2 & 15011.7 \\
\hline$(1000,20)$ & 3 & 39.8 & 12496.4 & $3: 8$ & 39 & 12521.1 & 3 & 42.6 & 18472.3 \\
\hline$(1500,20)$ & 3 & 58.4 & 15000 & 3. & 58.2 & 14985.5 & 3 & 61 & 20816.3 \\
\hline$(500,50)$ & 1 & 14.9 & 6854.6 & 4 & 14.9 & 6788.8 & 2 & 17 & 11096.8 \\
\hline$(1000,50)$ & 1 & 28.4 & 8757.9 & 3 & 28.2 & 89021 & 3 & 30.6 & 14624.5 \\
\hline$(1500,50)$ & 3 & 41.8 & 108064 & 3 & 42.4 & 10917.5 & 1 & 44.6 & 15898.6 \\
\hline$(500,80)$ & 1. & 1.74 & $42273^{3}$ & 1 & 7.4 & 4027.3 & 4 & 8.8 & 7334.9 \\
\hline$(1000,80)$ & 1 & 14.8 & 5076.8 & 1 & 14.6 & 5093.6. & 1 & 17 & 10425.8 \\
\hline$(1500,80)$ & 4 & 20.2 & 6169 & 2 & 20 & 6529.6 & 2 & 22.2 & 11112 \\
\hline
\end{tabular}

Table 4.14: Comparison of heuristic variations for different traffic pattems in the NSF network using the 10-8-8 combination under the fiber-scarce situation.

\begin{tabular}{|c|c|c|c|c|c|c|c|c|c|}
\hline \multirow[b]{2}{*}{$\begin{array}{l}\text { Traffic pattern: } \\
(I N G b / s, F R \%)\end{array}$} & \multicolumn{3}{|c|}{ No bifurcation } & \multicolumn{3}{|c|}{ Fiber bifurcation } & \multicolumn{3}{|c|}{ All bifurcation } \\
\hline & $\begin{array}{l}\text { Best } \\
\text { policy }\end{array}$ & $F_{\min }$ & $\begin{array}{l}\text { Weighted } \\
\text { port count } \\
\text { (a) } F_{\min }\end{array}$ & $\begin{array}{l}\text { Best } \\
\text { policy }\end{array}$ & $F_{\min }$ & $\begin{array}{c}\text { Weighted } \\
\text { port count } \\
\text { (a) } F_{\min }\end{array}$ & $\begin{array}{c}\text { Best } \\
\text { policy }\end{array}$ & $F_{\min }$ & $\begin{array}{c}\text { Weighted } \\
\text { port count } \\
\text { @ } F_{\min }\end{array}$ \\
\hline$(500,0)$ & 1 & 7 & $1+1,799$ & 1 & 7 & 14777.9 & 1 & 7.2 & 20865 \\
\hline$(1000,0)$ & 3 & 12.4 & 16298.6 & 3 & 12.4 & 16298.6 & 4 & 13.2 & 21721.5 \\
\hline$(1500,0)$ & 3 & 18.2 & $174+0.4$ & 3 & 18.2 & 17440.4 & 1 & 19 & 23079.3 \\
\hline$(500,20)$ & 2 & 5.6 & 12526,8 & 2 & 5.6 & 12526.8 & 4 & 6.8 & 17515.1 \\
\hline$(1000,20)$ & 3 & 1006 & 13556.1 & 3 & 10.6 & 13556.1 & 2 & 11.4 & 19015.6 \\
\hline$(1500,20)$ & 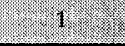 & $=15.4$ & 14336 & 1 & 15.4 & 14336 & 1 & 16 & 19870 \\
\hline$(500,50)$ & 2.1 .1$. & 5 & 8445.4 & 1 & 5 & 8445.4 & 2 & 5 & 12708.9 \\
\hline$(1000,50)$ & 3 & -1.6 & 9001.8 & 3 & 7.6 & 9600.4 & 4 & 8.8 & 14716.1 \\
\hline$(1500,50)$ & 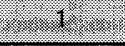 & 114 & 10258.1 & 1 & 11.4 & 10258.1 & 4 & 11.8 & 15262.2 \\
\hline$(500,80)$ & 1. & 3 & 44687 & 1 & 3 & 4468.7 & 1 & 3 & 7683.5 \\
\hline$(1000,80)$ & 2 & 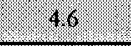 & 5330 & 2 & 4.6 & 5330 & 4 & 5 & 10177.9 \\
\hline$(1500,80)$ & .2 & 5.4 & 6248.6 & 2 & 5.6 & 6223.2 & 3 & 6.4 & 11238.3 \\
\hline
\end{tabular}


Table 4.15: Comparison of heuristic variations for different traffic patterns in the NSF network using the 40-8-8 combination under the fiber-scarce situation.

\begin{tabular}{|c|c|c|c|c|c|c|c|c|c|}
\hline \multirow[b]{2}{*}{$\begin{array}{l}\text { Traffic pattern: } \\
(I N G b / \mathrm{s}, F R \%)\end{array}$} & \multicolumn{3}{|c|}{ No bifurcation } & \multicolumn{3}{|c|}{ Fiber bifurcation } & \multicolumn{3}{|c|}{ All bifurcation } \\
\hline & $\begin{array}{c}\text { Best } \\
\text { policy }\end{array}$ & $F_{\min }$ & $\begin{array}{l}\text { Weighted } \\
\text { port count } \\
\text { @ } F_{\min }\end{array}$ & $\begin{array}{c}\text { Best } \\
\text { policy }\end{array}$ & $F_{m i n}$ & $\begin{array}{l}\text { Weighted } \\
\text { port count } \\
\text { @ } F_{\min }\end{array}$ & $\begin{array}{c}\text { Best } \\
\text { policy }\end{array}$ & $F_{\min }$ & $\begin{array}{l}\text { Weighted } \\
\text { port count } \\
\text { (a) } F_{\min }\end{array}$ \\
\hline$(500,0)$ & 4 & 3 & 135560 & 4 & 3 & 13566 & 4 & 6.4 & 53156.4 \\
\hline$(1000,0)$ & 1 & $=3.4$ & 3. 172485 & 1 & 3.4 & 17248.5 & 2 & 7.6 & 58077.8 \\
\hline$(1500,0)$ & 4 & P. & 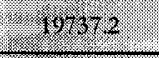 & 4 & 5 & 19737.2 & 2 & 9 & 59423 \\
\hline$(500,20)$ & 4 & 3 & 11293 & 4 & 3 & 11293 & 2 & 5 & 43028.2 \\
\hline$(1000,20)$ & 1. & 3 & 143224 & 1 & 3 & 14322.4 & 2 & 7 & 48467.4 \\
\hline$(1500,20)$ & 4 & 5 & -163172 & 4 & 5 & 16317.2 & 2 & 7.6 & 50105.5 \\
\hline$(500,50)$ & 4 & 1.6 & $=7901 \%$ & 4 & 1.6 & 7901.1 & 2 & 4.4 & 28493.7 \\
\hline$(1000,50)$ & 1 & $\sqrt{3}$ & 2. 9940.3 & 1 & 3 & 9940.3 & 2 & 5 & 33622.7 \\
\hline$(1500,50)$ & 2 & 2.3 & 117843 & 2 & 3 & 11784.3 & 2 & 6.2 & 37090 \\
\hline$(500,80)$ & 4 & 17 & 12918 & 4 & 1 & 4291.8 & 2 & 3 & 13562.1 \\
\hline$(1000,80)$ & 1 & 18 & 5520.8 & 1 & 1.8 & 5520.8 & 2 & 3 & 18487.8 \\
\hline$(1500,80)$ & 1 & 3 & (1) & 1 & 3 & 6014.6 & 2 & 3.8 & 23684 \\
\hline
\end{tabular}

Table 4.16: Comparison of heuristic variations for different traffic patterns in the EON network using the 10-4-4 combination under the fiber-scarce situation.

\begin{tabular}{|c|c|c|c|c|c|c|c|c|c|}
\hline \multirow[b]{2}{*}{$\begin{array}{l}\text { Traffic pattern: } \\
(I N G b / \mathrm{s}, F R \%)\end{array}$} & \multicolumn{3}{|c|}{ No bifurcation } & \multicolumn{3}{|c|}{ Fiber bifurcation } & \multicolumn{3}{|c|}{ All bifurcation } \\
\hline & $\begin{array}{l}\text { Best } \\
\text { policy }\end{array}$ & $F_{\min }$ & $\begin{array}{l}\text { Weighted } \\
\text { port count } \\
\text { (a) } F_{\min }\end{array}$ & $\begin{array}{c}\text { Best } \\
\text { policy }\end{array}$ & $F_{\min }$ & $\begin{array}{c}\text { Weighted } \\
\text { port count } \\
@ F_{\min }\end{array}$ & $\begin{array}{c}\text { Best } \\
\text { policy }\end{array}$ & $F_{\text {min }}$ & $\begin{array}{l}\text { Weighted } \\
\text { port count } \\
\text { (a) } F_{\min }\end{array}$ \\
\hline$(500,0)$ & 4 & 37.6 & 7204.4 & 4 & 37 & 7190.5 & 1 & 40.4 & 9872.4 \\
\hline$(1000,0)$ & 1 & 70.2 & 9310 & 1 & 70 & 9267 & 1 & 72.2 & 11909.9 \\
\hline$(1500,0)$ & 1 & 100.8 & 11177.6 & 3 & $93: 2$ & 11255.2 & 3 & 99.2 & 13958.5 \\
\hline$(500,20)$ & 3 & 30.6 & 6214.3 & 3 & 30.2 & 6217.5 & 1 & 32 & 8437.2 \\
\hline$(1000,20)$ & 1 & 61 & 7853 & 1 & 62.2 & 7910.2 & 2 & 66.2 & 10244 \\
\hline$(1500,20)$ & 1 & 90.8 & 9576.2 & 3 & 88.6 & $9387 \%$ & 1 & 96.8 & 11969.8 \\
\hline$(500,50)$ & 2 & 20.8 & 4513.5 & 3 & 20.4 & 4.417 .5 & 2 & 22.6 & 5953.3 \\
\hline$(1000,50)$ & 1. & 40.6 & 5469.6 & 4 & 40.8 & 5451.6 & 4 & 42.2 & 7774.6 \\
\hline$(1500,50)$ & 1 & 56.8 & 673.5 & 1 & 59.4 & 6736.5 & 1 & 63.6 & 8911 \\
\hline$(500,80)$ & 4 & 9 & 2434.7 & 4 & 8.8 & 24245 & 2 & 11 & 3800.6 \\
\hline$(1000,80)$ & 1 & 15.8 & 3130.5 & 4 & 158 & 3030.6 . & 1 & 20.8 & 5500.4 \\
\hline$(1500,80)$ & 1 & 216 & 40256 & 1 & 22.6 & 3981.3 & 1 & 29.2 & 6120.2 \\
\hline
\end{tabular}


Table 4.17: Comparison of heuristic variations for different traffic patterns in the EON network using the 10-8-8 combination under the fiber-scarce situation.

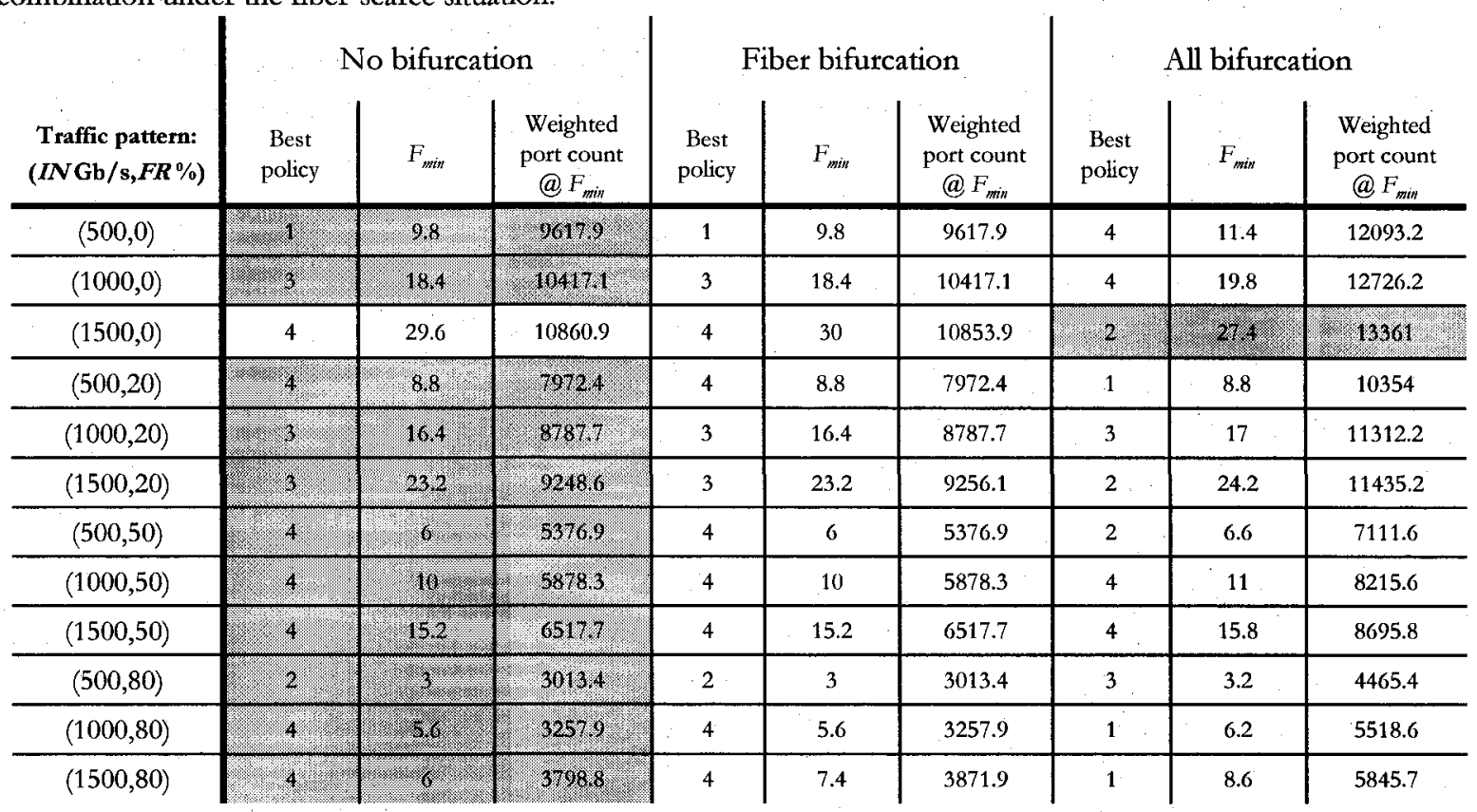

Table 4.18: Comparison of heuristic variations for different traffic patterns in the EON network using the 40-8-8 combination under the fiber-scarce situation.

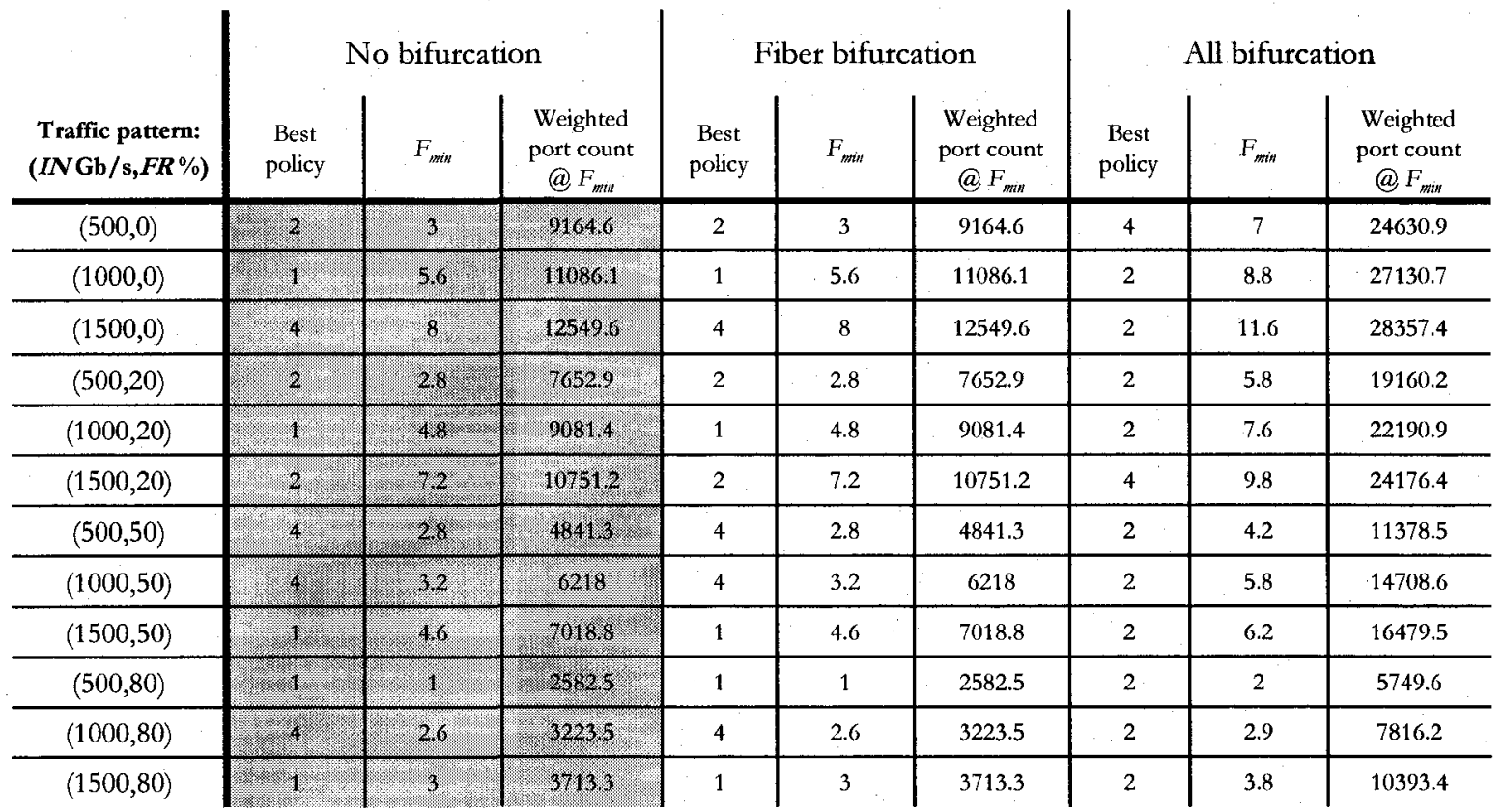


We now evaluate heuristics under the fiber-plentiful situation. As we mentioned earlier in Subsection 4.4.3, going beyond $F_{m i n}$ increases fiber resources and the choice of short physical routes for clear paths will be more likely; as a result, we expect to have a lower weighted port count. Depending on the traffic pattern, physical topology and fiber capacity, we expect up to an $\approx$ $88 \%$ increase in the fiber requirement needed to reach the minimal weighted port count, which we call the weighted port count at $F_{o p t}$ On the other hand, the saving achievable at the minimal weighted port count can go up to $\approx 20 \%$ compared to the weighted port count at $F_{\text {min }}$ which is a considerable saving. It is worth mentioning here that sometimes going beyond $F_{\text {min }}$ will not bring any further port count reduction, as is clearly demonstrated from the comparison of Table 4.12 with Table 4.21. This result usually occurs when the applied traffic volume is relatively low compared to the fiber capacity; as a result, the given resources will be sufficient to ensure that short routes are selected for all demands.

From Tables 4.19-4.27, in comparing heuristics based on the bifurcation scenario used, it is clear that for all traffic patterns, physical topologies, and fiber capacities tested, the heuristics based on bifurcating demands at all granularity levels drastically increases the weighted port count in comparison to the no- or fiber-bifurcation-based heuristics, which are highlighted in the tables. This observation is consistent with our finding in Subsection 4.4.5. With the 8-node/NSF/EON network, this increase can go up to $\approx 79 / 105 / 70 \%, \approx 73 / 98 / 64 \%$, and $\approx 198 / 293 / 175 \%$ using the $10-4-4,10-8-8$, and $40-8-8$ combinations respectively. It is interesting to note here that the use of the $40-8-8$ combination leads to the worst increase in the weighted port count. This increase occurs because more sub-wavelength demands are needed with the $40 \mathrm{~Gb} / \mathrm{s}$ wavelength capacity. This explanation is based on the fact that, as we mentioned in Subsection 4.4.5, treating each bifurcated unit of the sub-wavelength demand separately increases the chance that these units will 
use separate EXC ports and wavelength and waveband (de)multiplexers. Consequently, this increase in the number of EXC ports and (de)multiplexers will have a ripple effect in duplicating the ports of the next coarse-granular crossconnects.

Furthermore, regarding traffic patterns in which fiber demands exist, which happens at $I N=' 1000$ and $1500^{\prime} / 1500 \mathrm{~Gb} / \mathrm{s}$ with the $10-4-4 / 10-8-8$ combination, we can note that choosing to bifurcate fiber demands in the fiber-plentiful situation becomes a less attractive scenario than not bifurcating demands. This is expected to occur with the 8-node network and NSF network, both of which have a lower physical connectivity than the EON network, as is clearly illustrated through the comparison of Tables 4.19/4.20/4.22/4.23/4.25/4.26 with 4.10/4.11/4.13/4.14/4.16/4.17. This finding happens because increasing the fiber resources, especially in low-connectivity networks, means that the same best route can be used by most of the fiber demand units.

In comparing heuristic variations based on the sorting policy used, it is quite clear that Policies 1 and 4 are the only contenders as the traffic pattern, physical topology, and fiber capacity change. Moreover, the set of winning policies, which consists of the winning policies of all traffic patterns, changes according to the physical topology and fiber capacity. Furthermore, Policy 4 tends to be the most cost-effective policy for the NSF network, which has the lowest physical connectivity, along with the use of the 10-4-4 combination, which has the lowest fiber capacity, as shown in Table 4.22. In general, Policies 1 and 4 are the clear winners, because both policies try to avoid the duplication of resources as follows. Policy 1 ensures that the biggest portion of traffic demands is served first using short routes in terms of hop count, whereas Policy 4 ensures that short routes are granted to the demands with the lowest physical hop requirements. 
Finally, in looking for the $C-W-B$ combination/fiber capacity that leads to the minimal weighted port count, Table 4.28 summarizes the winning $C-W-B$ combination according to the traffic pattern and topology. Needless to say, the table clearly indicates that choosing the best/optimum $C-W-B$ combination depends on the traffic pattern and physical topology.

Table 4.19: Comparison of heuristic variations for different traffic patterns in the 8-node network using the 10-4-4 combination under the fiber-plentiful situation.

\begin{tabular}{|c|c|c|c|c|c|c|c|c|c|}
\hline \multirow[b]{2}{*}{$\begin{array}{l}\text { Traffic pattern: } \\
(I N G b / \mathrm{s}, F R \%)\end{array}$} & \multicolumn{3}{|c|}{ No bifurcation } & \multicolumn{3}{|c|}{ Fiber bifurcation } & \multicolumn{3}{|c|}{ All bifurcation } \\
\hline & $\begin{array}{l}\text { Best } \\
\text { policy }\end{array}$ & $F_{o p t}$ & $\begin{array}{l}\text { Weighted } \\
\text { port count } \\
\text { (a) } F_{\text {opt }}\end{array}$ & $\begin{array}{c}\text { Best } \\
\text { policy }\end{array}$ & $F_{o p t}$ & $\begin{array}{l}\text { Weighted } \\
\text { port count } \\
\text { (a) } F_{\text {opt }}\end{array}$ & $\begin{array}{c}\text { Best } \\
\text { policy }\end{array}$ & $F_{o p t}$ & $\begin{array}{c}\text { Weighted } \\
\text { port count } \\
\text { @ } F_{\text {opt }}\end{array}$ \\
\hline$(500,0)$ & 4 & 10.2 & 2982.4 & 4 & 10.6 & 2989.5 & 2 & 12.4 & 4179.9 \\
\hline$(1000,0)$ & 4 & $21 \%$ & 38519 & 4 & 21.8 & 3855.4 & 2 & 22.8 & 5072.5 \\
\hline$(1500,0)$ & 4 & 29 & 4621.7 & 4 & 31.8 & 46178 & 4 & 31.6 & 5673.2 \\
\hline$(500,20)$ & 4 & 10 & 2423.5 & 4 & 9.8 & $2422 \%$ & 2 & 10.4 & 3362.1 \\
\hline$(1000,20)$ & 1 & 17.8 & 3098 & 4 & 16.8 & 3096.6 & 2 & 19.6 & 4199.5 \\
\hline$(1500,20)$ & 4 & 264 & 1.37279 & 4 & 26 & 3733.3 & 2 & 27.8 & 4765.9 \\
\hline$(500,50)$ & 4. & 7.8. & $\because-17575$ & 4 & 7.8 & 1757.5 & 2 & 8.6 & 2584.8 \\
\hline$(1000,50)$ & 4 & 12.8 & .2238 .6 & 4 & 14.4 & 2239.6 & 4 & 14.4 & 3305.8 \\
\hline$(1500,50)$ & 4 & 10 & 2767.1 & 4 & 18.4 & 2776.1 & 4 & 19.8 & 3748.4 \\
\hline$(500,80)$ & 4 & 5 & 0.973 & 4 & 5 & 973 & 2 & 5.4 & 1558.4 \\
\hline$(1000,80)$ & 4. & 8.8 & 12315 & 4 & 8.8 & 1232.6 & 4 & 10 & 2198.8 \\
\hline$(1500,80)$ & 4 & 12.4 & 1584.8 & 4 & 128 & 1583.8 & 2 & 13.6 & 2487.1 \\
\hline
\end{tabular}

Table 4.20: Comparison of heuristic variations for different traffic patterns in the 8-node network using the 10-8-8 combination under the fiber-plentiful situation.

\begin{tabular}{|c|c|c|c|c|c|c|c|c|c|}
\hline \multirow[b]{2}{*}{$\begin{array}{l}\text { Traffic pattern: } \\
(I N G b / s, F R \%)\end{array}$} & \multicolumn{3}{|c|}{ No bifurcation } & \multicolumn{3}{|c|}{ Fiber bifurcation } & \multicolumn{3}{|c|}{ All bifurcation } \\
\hline & $\begin{array}{c}\text { Best } \\
\text { policy }\end{array}$ & $F_{o p t}$ & $\begin{array}{c}\text { Weighted } \\
\text { port count } \\
\text { (a. } F_{\text {opt }} \\
\end{array}$ & $\begin{array}{l}\text { Best } \\
\text { policy }\end{array}$ & $F_{o p t}$ & $\begin{array}{c}\text { Weighted } \\
\text { port count } \\
@ \text { (a. } F_{\text {opt }} \\
\end{array}$ & $\begin{array}{l}\text { Best } \\
\text { policy }\end{array}$ & $F_{o p t}$ & $\begin{array}{l}\text { Weighted } \\
\text { port count } \\
\text { (a) } F_{\text {opt }} \\
\end{array}$ \\
\hline$(500,0)$ & $4: 3$ & 34 & 4019 & 4 & 3.4 & 4019 & 2 & 4 & 5250.5 \\
\hline$(1000,0)$ & 4 & 13.8 & 4347.4 & 4 & 5.6 & 4347.4 & 2 & 6.6 & 5593.3 \\
\hline$(1500,0)$ & $14:$ & 1.78 & 4702.4 & 4 & 7.8 & 4702.4 & 1 & 9.4 & 5823.7 \\
\hline$(500,20)$ & 4. & 1.3 .3 & 32113 & 4. & 3 & 3211.3 & 2 & 3.8 & 4203.2 \\
\hline$(1000,20)$ & $\sqrt{1}=4.4$ & $=.9 .4$. & 3989.7 & 4 & 5.4 & 3489.7 & 4 & 5.8 & 4629.9 \\
\hline$(1500,20)$ & $\sqrt{1.0}$ & 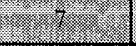 & 37660.6 & 4 & 7 & 3766.6 & 4 & 7.6 & 4820.5 \\
\hline$(500,50)$ & 4 & $=.2 .60$ & 22834 & 4 & 2.6 & 2243.4 & 2 & 2.4 & 3076.4 \\
\hline$(1000,50)$ & 4 & $=4.4$ & 24910 & 4 & 4.4 & 2491.9 & 4 & 4.4 & 3532 \\
\hline$(1500,50)$ & $\sqrt{4}$ & 5.6 & 2734 & 4 & 5.6 & 2734 & 4 & 6.4 & 3706.6 \\
\hline$(500,80)$ & $=4$ & 2 & $=1326$ & 4 & 2 & 1132.6 & 2 & 2 & 1681.1 \\
\hline$(1000,80)$ & $\sqrt{4}+2$ & (2) 32 & 13082 & 4 & 3.2 & 1308.2 & 4 & 3.2 & 2268.2 \\
\hline$(1500,80)$ & 4 & 3.8 & 1546.8 & 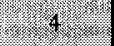 & 38 & 15452 & 2 & 3.8 & 2440.3 \\
\hline
\end{tabular}


Table 4.21: Comparison of heuristic variations for different traffic patterns in the 8-node network using the 40-8-8 combination under the fiber-plentiful situation.

\begin{tabular}{|c|c|c|c|c|c|c|c|c|c|}
\hline \multirow[b]{2}{*}{$\begin{array}{l}\text { Traffic pattern: } \\
(I N G b / \mathrm{s}, F R \%)\end{array}$} & \multicolumn{3}{|c|}{ No bifurcation } & \multicolumn{3}{|c|}{ Fiber bifurcation } & \multicolumn{3}{|c|}{ All bifurcation } \\
\hline & $\begin{array}{c}\text { Best } \\
\text { policy }\end{array}$ & $F_{o p t}$ & $\begin{array}{l}\text { Weighted } \\
\text { port count } \\
\text { (a) } F_{\text {opt }}\end{array}$ & $\begin{array}{c}\text { Best } \\
\text { policy }\end{array}$ & $F_{\text {opt }}$ & $\begin{array}{c}\text { Weighted } \\
\text { port count } \\
\text { (a) } F_{\text {opr }}\end{array}$ & $\begin{array}{c}\text { Best } \\
\text { policy }\end{array}$ & $F_{o p t}$ & $\begin{array}{l}\text { Weighted } \\
\text { port coun } \\
\text { @ } F_{\text {opt }}\end{array}$ \\
\hline$(500,0)$ & 4 & 2 & 3812.2 & 4 & 2 & 3812.2 & 2 & 2.8 & 11353.6 \\
\hline$(1000,0)$ & 1 & 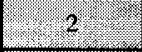 & 4875.4 & 4 & 2 & 4875.4 & 2 & 4 & 12267.6 \\
\hline$(1500,0)$ & 1 & 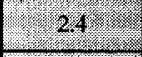 & 5513.2 . & 1 & 2.4 & 5513.2 & 2 & 4 & 13022.9 \\
\hline$(500,20)$ & 4 & $x^{2}$ & $3083=$ & 4 & 2 & 3083 & 2 & 2 & 8482.3 \\
\hline$(1000,20)$ & 4 & 2 & 3850.9 & 4 & 2 & 3850.9 & 2 & 3.2 & 9433.7 \\
\hline$(1500,20)$ & 4 & $x^{24}$ & 4053 & 4 & 2.4 & 4305.3 & 2 & 3.8 & 10301.1 \\
\hline$(500,50)$ & 4. & 2 & 6. $212 \pi \%$ & 4 & 2 & 2127.5 & 2 & 2 & 5829 \\
\hline$(1000,50)$ & 4 & 2 & 2720.7 & 4 & 2 & 2720.7 & 2 & 2 & 6543.4 \\
\hline$(1500,50)$ & 4 & 2 & 3035.3 & 4 & 2 & 3035.3 & 2 & 3 & 7783.3 \\
\hline$(500,80)$ & 4 & 10.2 & 1072.9 & 4 & 2 & 1072.9 & 2 & 2 & 2539.6 \\
\hline$(1000,80)$ & 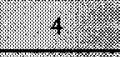 & 2 & 0.1347 & 4 & 2 & 1347 & 2 & 2 & 3609.9 \\
\hline$(1500,80)$ & 4 & II 2 & $\mid \begin{array}{ll}15668 \\
-10\end{array}$ & 4 & 2 & 1566.8 & 2 & 2 & 4582.3 \\
\hline
\end{tabular}

Table 4.22: Comparison of heuristic variations for different traffic patterns in the NSF network using the 10-4-4 combination under the fiber-plentiful situation.

\begin{tabular}{|c|c|c|c|c|c|c|c|c|c|}
\hline \multirow[b]{2}{*}{$\begin{array}{l}\text { Traffic pattern: } \\
(I N G b / s, F R \%)\end{array}$} & \multicolumn{3}{|c|}{ No bifurcation } & \multicolumn{3}{|c|}{ Fiber bifurcation } & \multicolumn{3}{|c|}{ All bifurcation } \\
\hline & $\begin{array}{l}\text { Best } \\
\text { policy }\end{array}$ & $F_{\text {opr }}$ & $\begin{array}{c}\text { Weighted } \\
\text { port count } \\
\text { (a) } F_{\text {opt }}\end{array}$ & $\begin{array}{c}\text { Best } \\
\text { policy }\end{array}$ & $F_{o p t}$ & $\begin{array}{c}\text { Weighted } \\
\text { port count } \\
\text { @ } F_{\text {opr }}\end{array}$ & $\begin{array}{c}\text { Best } \\
\text { policy }\end{array}$ & $F_{o p t}$ & $\begin{array}{c}\text { Weighted } \\
\text { port count } \\
\text { @ } F_{o p t}\end{array}$ \\
\hline$(500,0)$ & 4 & 272 & 11088.3 & 4 & 27.2 & 11088.3 & 4 & 30 & 16976.9 \\
\hline$(1000,0)$ & 4 & 54.6 & 141017 & 4 & 54.6 & 14101.7 & 4 & 56.8 & 19823.9 \\
\hline$(1500,0)$ & 4 & 812 & 169503 & 4 & 80.8 & 16951.6 & 4 & 83.6 & 22564.6 \\
\hline$(500,20)$ & 4 & 23.6 & 10.9335 & 4 & 23.6 & 9335 & 4 & 25.2 & 14620.7 \\
\hline$(1000,20)$ & 4 & 4.88 & 11555.2 & 4 & 46.8 & 11955.2 & 4 & 49.4 & 17433.2 \\
\hline$(1500,20)$ & 4 & .674 & 0.16322 .0 & 4 & 68 & 14326.7 & 4 & 69.2 & 19588.4 \\
\hline$(500,50)$ & 4 & 171 & .6694 .7 & 4 & 17.1 & 6694.7 & 2 & 19.4 & 10984.5 \\
\hline$(1000,50)$ & 4 & 35.4 & 8440.3 & 4. & 352 & 84362 & 4 & 37.8 & 13681.4 \\
\hline$(1500,50)$ & 4 & 49 & 10308.2 & 4. & 51.4 & 10297.6 & 2 & 54 & 15249.3 \\
\hline$(500,80)$ & 4 & 19.2 & 3910.6 & 4 & 10.2 & 3916.1 & 2 & 11.8 & 7127.7 \\
\hline$(1000,80)$ & 4 & 20.3 & 4807.7 & 4 & ?. 20.1 & . 4805.5 & 4 & 21.4 & 9864.4 \\
\hline$(1500,80)$ & 4 & 262 & 60575 & 4 & 26.2 & 6091.1 & 2 & 30.6 & 10738.2 \\
\hline
\end{tabular}


Table 4.23: Comparison of heuristic variations for different traffic patterns in the NSF network using the 10-8-8 combination under the fiber-plentiful situation.

\begin{tabular}{|c|c|c|c|c|c|c|c|c|c|}
\hline \multirow[b]{2}{*}{$\begin{array}{l}\text { Traffic pattern: } \\
(I N G b / s, F R \%)\end{array}$} & \multicolumn{3}{|c|}{ No bifurcation } & \multicolumn{3}{|c|}{ Fiber bifurcation } & \multicolumn{3}{|c|}{ All bifurcation } \\
\hline & $\begin{array}{l}\text { Best } \\
\text { policy }\end{array}$ & $F_{o p t}$ & $\begin{array}{l}\text { Weighted } \\
\text { port count } \\
\text { (a) } F_{\text {opt }}\end{array}$ & $\begin{array}{c}\text { Best } \\
\text { policy }\end{array}$ & $F_{o p t}$ & $\begin{array}{l}\text { Weighted } \\
\text { port count } \\
\text { @ } F_{\text {opt }}\end{array}$ & $\begin{array}{c}\text { Best } \\
\text { policy }\end{array}$ & $F_{\text {opt }}$ & $\begin{array}{l}\text { Weighted } \\
\text { port count } \\
\text { @ } F_{\text {opt }}\end{array}$ \\
\hline$(500,0)$ & 4 & 78 & 1.4682 .4 & 4 & 7.8 & 14682.4 & 4 & 8.2 & 20535.8 \\
\hline$(1000,0)$ & $\sqrt{1}$ & 14. & $.15869 ?$ & 1 & 14 & 15869.3 & 4 & 14.4 & 21642.7 \\
\hline$(1500,0)$ & 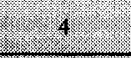 & 21 & 0.16959 .1 . & 4 & 21 & 16959.1 & 1 & 21.2 & 22745.5 \\
\hline$(500,20)$ & 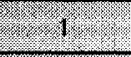 & 6.8 & 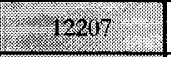 & 1 & 6.8 & 12207 & 4 & 7 & 17505.9 \\
\hline$(1000,20)$ & 11 & 12.2 & 1322 & 1 & 12.2 & 13227 & 4 & 12.4 & 18822 \\
\hline$(1500,20)$ & 1. & 17 & 14253.4 & 1 & 17 & 14253.4 & 4 & 17.6 & 19548.3 \\
\hline$(500,50)$ & 4 & 52. & $8485 \%$ & 4 & 5.2 & 8435.9 & 2 & 5.6 & 12693.9 \\
\hline$(1000,50)$ & 1. & $9.6 \%$ & $=.9252$ & 1 & 9.6 & 9252 & 4 & 9.6 & 14606.9 \\
\hline$(1500,50)$ & 1. & 13.8 & .10166 .2 & 1 & 13.8 & 10166.2 & 2 & 14.8 & 15031.5 \\
\hline$(500,80)$ & 17 & 3 & $=94687$ & 1 & 3 & 4468.7 & 2 & 3 & 7647.2 \\
\hline$(1000,80)$ & 4 & 5.6 & 50681 & 4 & 5.6 & 5068.1 & 4 & 5.6 & 10017.3 \\
\hline$(1500,80)$ & 4 & 7.6 & - 5873.9 & 4 & 7.6 & 5873.9 & 2 & 7.4 & 10471.6 \\
\hline
\end{tabular}

Table 4.24: Comparison of heuristic variations for different traffic patterns in the NSF network using the 40-8-8 combination under the fiber-plentiful situation.

\begin{tabular}{|c|c|c|c|c|c|c|c|c|c|}
\hline \multirow[b]{2}{*}{$\begin{array}{l}\text { Traffic pattern: } \\
(I N G b / \mathrm{s}, F R \%)\end{array}$} & \multicolumn{3}{|c|}{ No bifurcation } & \multicolumn{3}{|c|}{ Fiber bifurcation } & \multicolumn{3}{|c|}{ All bifurcation } \\
\hline & $\begin{array}{c}\text { Best } \\
\text { policy }\end{array}$ & $F_{o p t}$ & $\begin{array}{c}\text { Weighted } \\
\text { port count } \\
\text { (a) } F_{\text {opt }}\end{array}$ & $\begin{array}{c}\text { Best } \\
\text { policy }\end{array}$ & $F_{o p t}$ & $\begin{array}{c}\text { Weighted } \\
\text { port count } \\
\text { @ } F_{\text {opt }}\end{array}$ & $\begin{array}{c}\text { Best } \\
\text { policy }\end{array}$ & $F_{o p t}$ & $\begin{array}{l}\text { Weighted } \\
\text { port count } \\
\text { (a) } F_{\text {opt }}\end{array}$ \\
\hline$(500,0)$ & 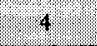 & 3 & 13566 & 4 & 3 & 13566 & 2 & 7.2 & 52486 \\
\hline$(1000,0)$ & 4 & 46. & 17133.8 & 4 & 4.6 & 17133.8 & 2 & 8.4 & 57963.6 \\
\hline$(1500,0)$ & 4 & 56 & 106948 & 4 & 5.6 & 19694.8 & 2 & 9.4 & 59402.5 \\
\hline$(500,20)$ & 4 & 3. & $1=1293$ & 4 & 3 & 11293 & 2 & 6.2 & 42990.2 \\
\hline$(1000,20)$ & 4 & 3.6 & $1420 \%$ & 4 & 3.6 & 14209 & 2 & 7.8 & 48383.2 \\
\hline$(1500,20)$ & 4 & ? & 103172 & 4 & 5 & 16317.2 & 2 & 8.8 & 50040 \\
\hline$(500,50)$ & 4 & 3. & $78083=$ & 4 & 3 & 7808.3 & 2 & 5 & 28450.4 \\
\hline$(1000,50)$ & ? & ? 3 & 9940.3 & 1 & 3 & 9940.3 & 2 & 5.6 & 33522.3 \\
\hline$(1500,50)$ & 1 & 4.4 & $112796=$ & 1 & 4.4 & 11279.6 & 2 & 7 & 37050.1 \\
\hline$(500,80)$ & 4 & 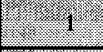 & 4291.8 & 4 & 1 & 4291.8 & 2 & 3 & 13562.1 \\
\hline$(1000,80)$ & $\sqrt{1}$ & 128 & 5390.9 & 4 & 2.8 & 5399.9 & 2 & 3.4 & 18482.6 \\
\hline$(1500,80)$ & 1 & 3 & 6014.6 & 1 & 3 & 6014.6 & 2 & 5 & 23641.1 \\
\hline
\end{tabular}


Table 4.25: Comparison of heuristic variations for different traffic patterns in the EON network using the 10-4-4 combination under the fiber-plentiful situation.

\begin{tabular}{|c|c|c|c|c|c|c|c|c|c|}
\hline \multirow[b]{2}{*}{$\begin{array}{l}\text { Traffic pattern: } \\
(I N G b / s, F R \%)\end{array}$} & \multicolumn{3}{|c|}{ No bifurcation } & \multicolumn{3}{|c|}{ Fiber bifurcation } & \multicolumn{3}{|c|}{ All bifurcation } \\
\hline & $\begin{array}{c}\text { Best } \\
\text { policy }\end{array}$ & $F_{o p t}$ & $\begin{array}{l}\text { Weighted } \\
\text { port count } \\
\text { @ } F_{o p t}\end{array}$ & $\begin{array}{l}\text { Best } \\
\text { policy }\end{array}$ & $F_{o p t}$ & $\begin{array}{l}\text { Weighted } \\
\text { port count } \\
@ F_{a t t}\end{array}$ & $\begin{array}{l}\text { Best } \\
\text { policy }\end{array}$ & $F_{o p t}$ & $\begin{array}{c}\text { Weighted } \\
\text { port count } \\
\text { @ } F_{\text {att }}\end{array}$ \\
\hline$(500,0)$ & 4 & 40.6 & 7187.1 & 4 & 38.4 & 784.9 & 4 & 51.2 & 9621.3 \\
\hline$(1000,0)$ & 4 & 82.6 & 9249.4 & 1 & 7,7 & $=0.922 .7$ & 2 & 91.2 & 11667.9 \\
\hline$(1500,0)$ & 1 & 19 & 11135.9 & 1 & 115.6 & 11164.3 & 2 & 138.4 & 13535.4 \\
\hline$(500,20)$ & 4 & 36.4 & 6050.6 & 4 & 35.4 & .6050. & 2 & 41.4 & 8176.1 \\
\hline$(1000,20)$ & 1 & 62.4 & 78283 & 4 & 75.4 & 7830 & 2 & 77.2 & 10089.4 \\
\hline$(1500,20)$ & $=4$ & 100.4 & 946.6 & 4 & 113.4 & 9553.1 & 2 & 109.4 & 11583.3 \\
\hline$(500,50)$ & 4 & 23 & 4245.3 & 4 & 23 & I $=72299$ & 2 & 26.4 & 5848.5 \\
\hline$(1000,50)$ & 4 & 45.4 & 5454.2 & 4 & 44 & 5409.2 & 2 & 54 & 7686.9 \\
\hline$(1500,50)$ & 4 & 65.4 & 6701.2 & W & 624 & 6.6912 & 2 & 73.4 & 8634.5 \\
\hline$(500,80)$ & 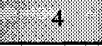 & 106 & 28897 & 4 & 10 & 2390.1 & 2 & 14 & 3703.9 \\
\hline$(1000,80)$ & 4 & 23.2 & 3063.9 & 4 & 18.6 & 9018 & 2 & 30 & 5137.5 \\
\hline$(1500,80)$ & 1 & 24.6 & 3970.1 & 1. & 28 & 39065 & 2 & 42.8 & 5863.1 \\
\hline
\end{tabular}

Table 4.26: Comparison of heuristic variations for different traffic patterns in the EON network using the 10-8-8 combination under the fiber-plentiful situation.

\begin{tabular}{|c|c|c|c|c|c|c|c|c|c|}
\hline \multirow[b]{2}{*}{$\begin{array}{l}\text { Traffic pattern: } \\
(I N G b / s, F R \%)\end{array}$} & \multicolumn{3}{|c|}{ No bifurcation } & \multicolumn{3}{|c|}{ Fiber bifurcation } & \multicolumn{3}{|c|}{ All bifurcation } \\
\hline & $\begin{array}{l}\text { Best } \\
\text { policy }\end{array}$ & $F_{o p r}$ & $\begin{array}{l}\text { Weighted } \\
\text { port count } \\
\text { @ } F_{o b t}\end{array}$ & $\begin{array}{l}\text { Best } \\
\text { policy }\end{array}$ & $F_{o p t}$ & $\begin{array}{l}\text { Weighted } \\
\text { port count } \\
\text { @ } F_{o p t}\end{array}$ & $\begin{array}{c}\text { Best } \\
\text { policy }\end{array}$ & $F_{o p t}$ & $\begin{array}{l}\text { Weighted } \\
\text { port count } \\
\text { @ } F_{\text {opt }}\end{array}$ \\
\hline$(500,0)$ & 24 & 11.6 & 9399.4 & 4 & 11.6 & 9399.4 & 4 & 14.4 & 11937.2 \\
\hline$(1000,0)$ & 24 & 22,4 & 10131 & 4 & 22.4 & 10131 & 4 & 26.4 & 12489.4 \\
\hline$(1500,0)$ & 4 & 35 & 10744.4 & 4 & 35 & 10744.4 & 4 & 39.2 & 13109.1 \\
\hline$(500,20)$ & 4 & 102 & 7913.6 & 4 & 10.2 & 7913.6 & 2 & 11.8 & 10091.7 \\
\hline$(1000,20)$ & 1 & 17.6 & 85246 & 1 & 17.6 & 8524.3 & 4 & 22.2 & 10781.7 \\
\hline$(1500,20)$ & 1 & 25.4 & 9063.1 & 1 & 25.4 & 9063.1 & 4 & 29.8 & 11291.8 \\
\hline$(500,50)$ & 4 & 10.6 & 5362.8 & 4 & 6.6 & 5362.8 & 2 & 7.6 & 7086.6 \\
\hline$(1000,50)$ & 4 & 1.14 & 5829.9 & 4 & 11.4 & 5829.9 & 2 & 14.8 & 8115.8 \\
\hline$(1500,50)$ & $\left.1\right|^{12}$ & 126 & 0.6132 & 1 & 17.6 & 6432 & 4 & 19.6 & 8502.2 \\
\hline$(500,80)$ & 1. & 3.6 & 2805.4 & 1 & 3.6 & 2805.4 & 2 & 3.2 & 4053.3 \\
\hline$(1000,80)$ & 4. & 0.4 & $1239 ?$ & 4 & 6.4 & 3239.7 & 2 & 6 & 5324.8 \\
\hline$(1500,80)$ & 4 & 76 & 3754.4 & 4 & 9.2 & 3825.2 & 2 & 9.8 & 5662.6 \\
\hline
\end{tabular}


Table 4.27: Comparison of heuristic variations for different traffic patterns in the EON network using the 40-8-8 combination under the fiber-plentiful situation.

\begin{tabular}{|c|c|c|c|c|c|c|c|c|c|}
\hline \multirow[b]{2}{*}{$\begin{array}{l}\text { Traffic pattern: } \\
(I N G b / \mathrm{s}, F R \%)\end{array}$} & \multicolumn{3}{|c|}{ No bifurcation } & \multicolumn{3}{|c|}{ Fiber bifurcation } & \multicolumn{3}{|c|}{ All bifurcation } \\
\hline & $\begin{array}{c}\text { Best } \\
\text { policy }\end{array}$ & $F_{o p t}$ & $\begin{array}{l}\text { Weighted } \\
\text { port count } \\
\text { (@) } F_{o p t}\end{array}$ & $\begin{array}{l}\text { Best } \\
\text { policy }\end{array}$ & $F_{o p t}$ & $\begin{array}{l}\text { Weighted } \\
\text { port count } \\
\text { @. } F_{o p t}\end{array}$ & $\begin{array}{c}\text { Best } \\
\text { policy }\end{array}$ & $F_{o p t}$ & $\begin{array}{l}\text { Weighted } \\
\text { port count } \\
@ F_{\theta g t}\end{array}$ \\
\hline$(500,0)$ & 4 & 4 & 1.87 .31 .1 & 4 & 4 & 8731.1 & 2 & 8.6 & 23211.8 \\
\hline$(1000,0)$ & 4 & 6. & 10999 & 4 & 6.4 & 10999 & 2 & 12 & 26302.7 \\
\hline$(1500,0)$ & 1 & 9.6 & 12495.4 & 1 & 9.6 & 12495.4 & 2 & 14.8 & 27574.3 \\
\hline$(500,20)$ & 4 & 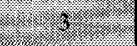 & 17.71697 & 4 & 3 & 7169.7 & 2 & 6.8 & 18803.8 \\
\hline$(1000,20)$ & .1 & $=\sqrt{2}, 3$ & $9078 \%$ & 1 & 5 & 9078.7 & 2 & 10.6 & 21683.3 \\
\hline$(1500,20)$ & 1. & 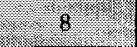 & 103583 & 4 & 8 & 10358.3 & 2 & 12.4 & 22723.9 \\
\hline$(500,50)$ & 4 & 28 & 48413 & 4 & 2.8 & 4841.3 & 2 & 5.2 & 11315.3 \\
\hline$(1000,50)$ & 4 & 42 & 61693 & 4 & 4.2 & 6169.3 & 2 & 7.2 & 14424 \\
\hline$(1500,50)$ & $1 ?$ & 5.4 & $6997 ?$ & 1 & 5.4 & 6997.7 & 2 & 8.2 & 16071.9 \\
\hline$(500,80)$ & 4 & 12 & 2533 & 4 & 1.2 & 2553 & 2 & 2.6 & 5735.3 \\
\hline$(1000,80)$ & 4 & 2.6 & 32235 & 4 & 2.6 & 3223.5 & 2 & 3.4 & 7652.8 \\
\hline$(1500,80)$ & 1 & $3+4$ & 37104 & 1 & 3.4 & 3710.4 & 2 & 5.1 & 10186.3 \\
\hline
\end{tabular}

Table 4.28: The winning $C-W-B$ combination that leads to the minimal weighted port count in the fiber-plentiful situation as the traffic pattern and topology change.

\begin{tabular}{c|c|c|c}
$\begin{array}{c}\text { Traffic pattern: } \\
(\boldsymbol{I N G b} / \mathbf{s}, \boldsymbol{F R} \%)\end{array}$ & 8-node network & NSF network & EON network \\
\hline$(500,0)$ & $10-4-4$ & $10-4-4$ & $10-4-4$ \\
\hline$(1000,0)$ & $10-4-4$ & $10-4-4$ & $10-4-4$ \\
\hline$(1500,0)$ & $10-4-4$ & $10-4-4$ & $\mathbf{1 0 - 8 - 8}$ \\
\hline$(500,20)$ & $10-4-4$ & $10-4-4$ & $10-4-4$ \\
\hline$(1000,20)$ & $10-4-4$ & $10-4-4$ & $10-4-4$ \\
\hline$(1500,20)$ & $10-4-4$ & $\mathbf{1 0 - 8 - 8}$ & $\mathbf{1 0 - 8 - 8}$ \\
\hline$(500,50)$ & $10-4-4$ & $10-4-4$ & $10-4-4$ \\
\hline$(1000,50)$ & $10-4-4$ & $10-4-4$ & $10-4-4$ \\
\hline$(1500,50)$ & $\mathbf{1 0 - 8 - 8}$ & $\mathbf{1 0 - 8 - 8}$ & $\mathbf{1 0 - 8 - 8}$ \\
\hline$(500,80)$ & $10-4-4$ & $10-4-4$ & $10-4-4$ \\
\hline$(1000,80)$ & $10-4-4$ & $10-4-4$ & $10-4-4$ \\
\hline$(1500,80)$ & $\mathbf{1 0 - 8 - 8}$ & $\mathbf{1 0 - 8 - 8}$ & $\mathbf{4 0 - 8 - 8}$
\end{tabular}


Chapter 5

\section{Planning of GMPLS-Based Transport Networks with Conversion and Regeneration Capabilities}

\subsection{Introduction}

The focus of this chapter is on planning the GMPLS-based transport network with the consideration of the color conversion capability and both with and without the optical signal regeneration capability. This means that the RMGPA problem here has to exclude the colorcontinuity constraints and either include or exclude the optical-reach constraints. Therefore, either clear or unclear paths are required for fiber, waveband, wavelength, and sub-wavelength demands. In this chapter, we will deal with three versions of the RMGPA problem that are explained as follows:

- RMGPA problem without color-continuity or optical-reach constraints. This version deals with the planning of the GMPLS-based transport network with the color conversion capability and without the optical signal regeneration capability. This version fits the planning problem in metro core transport networks, especially when fiber resources are scarce. The exact solution to this version of the problem can be obtained by formulating the problem as an MILP model.

- RMGPA problem without color-continuity constraints, with optical-reach constraints, and without customizing the optical reach of clear paths: This version deals with the planning of GMPLS-based transport networks with both the color conversion and optical signal regeneration capabilities. In addition, all EXC ports, which launch and terminate clear paths, have the same optical reach, and the lengths of all clear paths must be within a given maximum optical reach. In other words, the $\mathrm{E} / \mathrm{O}$ ratio here is the same for all EXC ports of the identical GMPLS interface type, and the 
ratio will be independent of the actual length of its clear path. This version fits the planning problem in long-haul transport networks. The exact solution to this version of the problem requires formulating the problem as an MILP model.

- RMGPA problem without color-continuity constraints, with optical-reach constraints, and with customizing the optical reach of clear paths. This version deals with the planning of GMPLS-based transport networks with both the color conversion and optical signal regeneration capabilities. In addition, the optical reach of each EXC port is customized based on the actual length of its clear path, although the lengths of all clear paths must still be within a given maximum optical reach value. In other words, the cost of the EXC port here will be a function of the actual length of its clear path. This version fits the planning problem in long-haul transport networks in which the selection of the transponders at the transport node is possible based on a variety of fine optical reach values. Using the reach-customization notion, the exact solution to this version of the problem can be obtained by formulating the problem as an NLP model.

This chapter proceeds with the MILP and NLP formulations of the afore-mentioned versions of the RMGPA problem. Then, the algorithms of the proposed heuristic approaches used to solve the three versions of the RMGPA problem are explained. Afterword, numerical results are presented in terms of the following: (1) the verification of heuristics, (2) demonstrations of the impact of input parameters and certain solution enhancements, and (3) comparisons of heuristics via simulation.

\subsection{MILP Formulation}

To achieve the optimum solution to the RMGPA problem without the color-continuity constraints, with/without the optical-reach constraints, and without customizing the optical reach 
of clear paths, the problem will be formulated as an MILP model. The formulation proceeds as follows [NaMo08a][NaMo08b].

\section{Parameters and Sets:}

In addition to the parameters and sets defined in Chapter 4, other new parameters that we need in order to formulate the new versions of the RMGPA problem are the following:

- $d_{i j}$ is the distance between node $i$ and node $j$.

- $R_{\max }$ is the maximum optical reach.

\section{Optimization Variables:}

In addition to the optimization variables defined in Chapter 4, the following new variables are introduced:

- $\varphi w_{p, k, b, f}^{s d, i j} / \varphi b_{p, b, f}^{s d, i j} / \varphi f_{p, f}^{s d, i j}$ is a binary variable that represents the amount of wavelength/ waveband/fiber flow of the $s-d$ pair that is carried on the virtual link $V W_{f, b, k}^{i j, p} / V B_{f, b}^{i, p} / V F_{f}^{i, p}$.

\section{Objective:}

Minimize the total weighted port count in the GMPLS-based transport network:

\section{Objective function in Equation 4.1}

If the RMGPA problem considers the optical-reach constraints, then the E/O ratio will be a function of the maximum optical reach by using Equation 3.1. Accordingly, substituting Equation 3.1 into Equation 4.1 yields:

$$
\min \left(\begin{array}{c}
c f \cdot \sum_{i} F X C_{i}+c b \cdot \sum_{i} B X C_{i}+c w \cdot \sum_{i} W X C_{i}+ \\
\operatorname{RATIO}_{1000} \times 1.25^{\log _{2}\left(\frac{R_{\max }}{1000}\right)^{\prime}} \cdot\left(c f \cdot \sum_{i}\left(F S C O_{i}+F S C I_{i}\right)+c b \cdot \sum_{i}\left(B S C O_{i}+B S C I_{i}\right)+c w \cdot \sum_{i}\left(W S C O_{i}+W S C I_{i}\right)\right)
\end{array}\right)
$$




\section{Constraints:}

Subject to:

1) Port configurations at all switching stages of the GMPLS-based transport node:

Inequalities in Equations 4.2-4.10

2) WXC add and drop ports (refer to Fig. 4.1) are decided by the endpoints of the CWS-based virtual links needed to handle both wavelength and sub-wavelength demands; BXC/FXC add and drop ports are decided by the endpoints of the CBS/CFS-based virtual links. Adding and dropping virtual links here stem not only from add/drop demands, but also from color conversion and/or signal regeneration:

$$
\begin{gathered}
W A_{s}=\sum_{j}^{j \neq s} \sum_{m}^{m \neq s} \sum_{p} \sum_{f} \sum_{b} \sum_{k}\left(\gamma_{s m}^{s, p} \cdot V S_{f, b, k}^{s j, p}+\gamma_{s m}^{s, p} \cdot V W_{f, b, k}^{s, p}\right) \forall s \\
W A_{s}=\sum_{j}^{j \neq s} \sum_{p} \sum_{f} \sum_{b} \sum_{k}\left(V S_{f, b, k}^{s j, p}+V W_{f, b, k}^{s, p}\right) \forall s \\
W D_{d}=\sum_{i}^{i \neq d} \sum_{n}^{n \neq d} \sum_{p} \sum_{f} \sum_{b} \sum_{k}\left(\gamma_{n d}^{i d, p} \cdot V S_{f, b, k}^{i d, p}+\gamma_{n d}^{i d, p} \cdot V W_{f, b, k}^{i d, p}\right) \forall d \\
W D_{d}=\sum_{i}^{i \neq d} \sum_{p} \sum_{f} \sum_{b} \sum_{k}\left(V S_{f, b, k}^{i d, p}+V W_{f, b, k}^{i d, p}\right) \forall d \\
B A_{s}=\sum_{j}^{j \neq s} \sum_{m}^{m \neq s} \sum_{p} \sum_{f} \sum_{b}\left(\gamma_{s m}^{s j, p} \cdot V B_{f, b}^{s, p}\right) \forall s \\
B A_{s} \geq \sum_{d}^{d \neq s} \Lambda_{s d}^{2} \quad \forall s \\
B D_{d}=\sum_{i}^{i \neq d} \sum_{n}^{n \neq d} \sum_{p} \sum_{f} \sum_{b}\left(\gamma_{n d}^{i d, p} \cdot V B_{f, b}^{i d, p}\right) \forall d \\
B \sum_{s}^{s \neq d} \Lambda_{s d}^{2} \quad \forall d
\end{gathered}
$$




$$
\begin{gathered}
F A_{s}=\sum_{j}^{j \neq s} \sum_{m}^{m \neq s} \sum_{p} \sum_{f}\left(\gamma_{s m}^{j^{j, p}} \cdot V F_{f}^{s j, p}\right) \forall s \\
F A_{s} \geq \sum_{d}^{d \neq s} \Lambda_{s d}^{3} \forall s \\
F D_{d}=\sum_{i}^{i \neq d} \sum_{n}^{n \neq d} \sum_{p} \sum_{f}\left(\gamma_{n d}^{i d, p} \cdot V F_{f}^{i d, p}\right) \forall d \\
F D_{d} \geq \sum_{s}^{s \neq d} \Lambda_{s d}^{3} \forall d
\end{gathered}
$$

3) The multi-granular virtual topology must accommodate all multi-granularity traffic demands:

$$
\begin{aligned}
& \sum_{j}^{j \neq s} \sum_{p} \sum_{f} \sum_{b} \sum_{k} \varphi s_{p, k, b, f}^{s d, s j}=\Lambda_{s d}^{0} \forall s, d \mid s \neq d \\
& \sum_{i}^{i \neq d} \sum_{p} \sum_{f} \sum_{b} \sum_{k} \varphi s_{p, k, b, f}^{s d, i d}=\Lambda_{s d}^{0} \forall s, d \mid s \neq d \\
& \sum_{j}^{j \neq s} \sum_{p} \sum_{f} \sum_{b} \sum_{k} \varphi w_{p, k, b, f}^{s d, s s}=\Lambda_{s d}^{1} \forall s, d \mid s \neq d \\
& \sum_{i}^{i \neq d} \sum_{p} \sum_{f} \sum_{b} \sum_{k} \varphi w_{p, k, b, f,}^{s d i d d}=\Lambda_{s d}^{1} \forall s, d \mid s \neq d \\
& \sum_{j}^{j \neq s} \sum_{p} \sum_{f} \sum_{b} \varphi b_{p, b, f}^{s d d s j}=\Lambda_{s d}^{2} \quad \forall s, d \mid s \neq d \\
& \sum_{i}^{i \neq d} \sum_{p} \sum_{f} \sum_{b} \varphi b_{p, b, f}^{s d, i d}=\Lambda_{s d}^{2} \forall s, d \mid s \neq d \\
& \sum_{j}^{j \neq s} \sum_{p} \sum_{f} \varphi f_{p, f}^{s d, s j}=\Lambda_{s d}^{3} \forall s, d \mid s \neq d \\
& \sum_{i}^{i \neq d} \sum_{p} \sum_{f} \varphi f_{p, f}^{s t, i d}=\Lambda_{s d}^{3} \forall s, d \mid s \neq d
\end{aligned}
$$


4) The summation of flows at any virtual link must not exceed its capacity:

$$
\begin{gathered}
\sum_{s} \sum_{d}^{d \neq s} \varphi s_{p, k, b, f}^{s d, i j} \leq V S_{f, b, k}^{i j, p} \cdot C \quad \forall p, f, b, k, i, j \mid i \neq j \\
\sum_{s} \sum_{d}^{d \neq s} \varphi w_{p, k, b, f}^{s d, i j}=V W_{f, b, k}^{i j, p} \quad \forall p, f, b, k, i, j \mid i \neq j \\
\sum_{s} \sum_{d}^{d \neq s} \varphi b_{p, b, f}^{s d, i j}=V B_{f, b}^{i, p} \quad \forall p, f, b, i, j \mid i \neq j \\
\sum_{s} \sum_{d}^{d \neq s} \varphi \oint_{p, f}^{s d, i j}=V F_{f}^{i, p,} \quad \forall p, f, i, j \mid i \neq j
\end{gathered}
$$

5) A pass-through CWS/CBS-based virtual link at each intermediate node can be switched at the BXC or WXC'/BXC for degrouping and grouping purposes; otherwise, it is switched at the FXC:

$$
\begin{aligned}
& S P T_{f, b, k}^{s d, p, i, m n}+S B P T_{f, b, k}^{s d, p, i, m n}+S F P T_{f, b, k}^{s d, p, i, m n}=V S_{f, b, k}^{s d, p} \\
& \forall k, b, f, p, i, j, m, n, s, d \mid s \neq d \neq i, m \neq d, n \neq s, m \neq n, \gamma_{i n}^{s d, p}=1, \gamma_{m i}^{s d, p}=1 \\
& W P T_{f, b, k}^{s d, p, i m n}+W B P T_{f, b, k}^{s d, p, i, m n}+W F P T_{f, b, k}^{s d, p, i, m n}=V W_{f, b, k}^{s d, p} \\
& \forall k, b, f, p, i, j, m, n, s, d \mid s \neq d \neq i, m \neq d, n \neq s, m \neq n, \gamma_{i n}^{s d, p}=1, \gamma_{m i}^{s d, p}=1 \\
& B P T_{f, b}^{s d, p, i, m n}+B F P T_{f, b}^{s d, p, i, m n}=V B_{f, b}^{s d, p} \\
& \forall b, f, p, i, j, m, n, s, d \mid s \neq d \neq i, m \neq d, n \neq s, m \neq n, \gamma_{i n}^{s d, p}=1, \gamma_{m i}^{s d, p}=1
\end{aligned}
$$

6) Pass-through flow conservation must be ensured at each node in the multi-granular virtual topology:

$$
\begin{gathered}
\sum_{i}^{i \neq m} \sum_{j}^{j \neq m} \sum_{p} \sum_{f} \sum_{b} \sum_{k}\left(\varphi s_{p, k, b, f}^{s d, i m m}-\varphi s_{p, k, b, f}^{s d, m j}\right)=0 \quad \forall m, s, d \mid \Lambda_{s d}^{0}>0, m \neq s \neq d \\
\sum_{i}^{i \neq m} \sum_{j}^{j \neq n} \sum_{p} \sum_{f} \sum_{b} \sum_{k}\left(\varphi w_{p, k, b, f}^{s d, i m}-\varphi w_{p, k, b, f}^{s d, m j}\right)=0 \quad \forall m, s, d \mid \Lambda_{s d}^{1}>0, m \neq s \neq d \\
\sum_{i}^{i \neq m} \sum_{j}^{j \neq m} \sum_{p} \sum_{f} \sum_{b}\left(\varphi b_{p, b, f}^{s d, i m}-\varphi b_{p, b, f}^{s d, m j}\right)=0 \quad \forall m, s, d \mid \Lambda_{s d}^{2}>0, m \neq s \neq d
\end{gathered}
$$




$$
\sum_{i}^{i \neq m} \sum_{j}^{i \neq m} \sum_{p} \sum_{f}\left(\varphi_{p, f}^{s d, i m}-\varphi f_{p, f}^{s d, m j}\right)=0 \quad \forall m, s, d \mid \Lambda_{s d}^{3}>0, m \neq s \neq d
$$

7) WXC/BXC/FXC non-add and non-drop ports (refer to Fig. 4.1) are decided based on add, drop, and pass-through virtual links in a way that ensures a non-blocking architecture at all optical switching stages:

$$
\begin{aligned}
& w o_{i}^{n, f, b, k}=\sum_{d}^{d \neq i} \sum_{p}\left(\gamma_{i n}^{i d, p} \cdot V W_{f, b, k}^{i d, p}+\gamma_{i n}^{i d, p} \cdot V S_{f, b, k}^{i d, p}\right)+ \\
& \sum_{s}^{s \neq n} \sum_{d}^{d \neq s \neq i m \neq n \neq d \neq \neq} \sum_{m} \sum_{p}\left(\gamma_{m i}^{s d, p} \cdot \gamma_{i n}^{s d, p} \cdot W P T_{f, b, k}^{s d, p, i, m n}+\gamma_{m i}^{s d, p} \cdot \gamma_{i n}^{s d, p} \cdot S P T_{f, b, k}^{s d, p, i, m n}\right) \\
& \forall i, n, f, b, k \mid i \neq n, \delta_{i n}>0 \\
& W O_{i}=\sum_{n}^{n \neq i} \sum_{f} \sum_{b} \sum_{k}\left(\delta_{i n} \cdot w o_{i}^{n, f, b, k}\right) \forall i \\
& w b_{i}^{n, f, b} \geq \sum_{k}\left(\frac{w o_{i}^{n, f, b, k}}{W}\right) \forall i, n, f, b \mid i \neq n, \delta_{i n}>0 \\
& W B_{i}=\sum_{n}^{n \neq i} \sum_{f} \sum_{b}\left(\delta_{i n} \cdot w b_{i}^{n, f, b}\right) \forall i \\
& w i_{i}^{m, f, b, k}=\sum_{s}^{s \neq i} \sum_{p}\left(\gamma_{m i}^{s i, p} \cdot V W_{f, b, k}^{s i, p}+\gamma_{m i}^{s i, p} \cdot V S_{f, b, k}^{s i, p}\right)+
\end{aligned}
$$

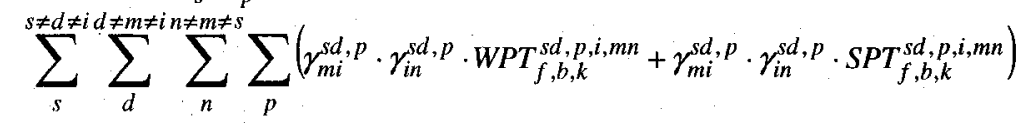

$$
\begin{aligned}
& \forall i, m, f, b, k \mid i \neq m, \delta_{m i}>0 \\
& W I_{i}=\sum_{m}^{m \neq i} \sum_{f} \sum_{b} \sum_{k}\left(\delta_{m i} \cdot w i_{i}^{m, f, b, k}\right) \forall i \\
& b w_{i}^{m, f, b} \geq \sum_{k}\left(\frac{w i_{i}^{m, f, b, k}}{W}\right) \forall i, m, f, b \mid i \neq m, \delta_{m i}>0 \\
& B W_{i}=\sum_{m}^{m \neq i} \sum_{f} \sum_{b}\left(\delta_{m i} \cdot b w_{i}^{m, f, b}\right) \forall i
\end{aligned}
$$




$$
\begin{aligned}
& b o_{i}^{n, f, b} \geq \sum_{d}^{d \neq i} \sum_{p}\left(\gamma_{i n}^{i d, p} \cdot V B_{f, b}^{i d, p}\right)+w b_{i}^{n, f, b}+\sum_{s}^{s \neq n} \sum_{d}^{d \neq s \neq i} \sum_{m}^{m \neq n \neq d \neq i} \sum_{p}\left(\gamma_{m i}^{s d, p} \cdot \gamma_{i n}^{s d, p} \cdot B P T_{f, b}^{s d, p, i, m n}\right)+ \\
& \sum_{s}^{s \neq n} \sum_{d}^{d \neq s \neq i} \sum_{m}^{m \neq n \neq d \neq i} \sum_{p} \frac{\left(\gamma_{m i}^{s d, p} \cdot \gamma_{i n}^{s d, p} \cdot W B P T_{f, b, k}^{s d, p, i, m n}+\gamma_{m i}^{s d, p} \cdot \gamma_{i n}^{s d, p} \cdot S B P T_{f, b, k}^{s d, p, i, m n}\right)}{W} \\
& \forall i, n, f, b \mid i \neq n, \delta_{i n}>0 \\
& B O_{i}=\sum_{n}^{n \neq i} \sum_{f} \sum_{b}\left(\delta_{i n} \cdot b o_{i}^{n, f, b}\right) \forall i \\
& b f_{i}^{n, f} \geq \sum_{b}\left(\frac{b o_{i}^{n, f, b}}{B}\right) \forall i, n, f \mid i \neq n, \delta_{i n}>0 \\
& B F_{i}=\sum_{n}^{n \neq i} \sum_{f}\left(\delta_{i n} \cdot b f_{i}^{n, f}\right) \forall i \\
& \sum_{s}^{s \neq d \neq i d \neq m \neq i=i} \sum_{d}^{\substack{p \\
d \neq m \neq s}} \sum_{n} \frac{\left(\gamma_{m i}^{s d, p} \cdot \gamma_{i n}^{s d, p} \cdot W B P T_{, b, k}^{s d, p, i, m n}+\gamma_{m i}^{s d, p} \cdot \gamma_{i n}^{p d, p} \cdot S B P T_{f, b, k}^{s d, p, i, m n}\right)}{W} \\
& \forall i, m, f, b \mid i \neq m, \delta_{m i}>0 \\
& B I_{i}=\sum_{m}^{m \neq i} \sum_{f} \sum_{b}\left(\delta_{m i} \cdot b i_{i}^{m, f, b}\right) \forall i \\
& f b_{i}^{m, f} \geq \sum_{b}\left(\frac{b i_{i}^{m, f, b}}{B}\right) \forall i, m, f \mid i \neq m, \delta_{m i}>0 \\
& F B_{i}=\sum_{m}^{m \neq i} \sum_{f}\left(\delta_{m i} \cdot f b_{i}^{m, f}\right) \forall i \\
& \begin{array}{l}
f o_{i}^{n, f} \geq \sum_{d}^{d \neq i} \sum_{p}\left(\gamma_{i n}^{i d, p} \cdot V F_{f}^{i d, p}\right)+b f_{i}^{n, f}+\sum_{s}^{s \neq n} \sum_{d}^{d \neq s \neq i} \sum_{m}^{m \neq n \neq d \neq i} \sum_{p}\left(\gamma_{m i}^{s d, p} \cdot \gamma_{i n}^{s d, p} \cdot V F_{f}^{s d, p}\right)+ \\
\sum_{s}^{s \neq n} \sum_{d}^{d \neq s \neq i} \sum_{m}^{s \neq n \neq d \neq i} \sum_{p} \frac{\left(\gamma_{m i}^{s d, p} \cdot \gamma_{i n}^{s d, p} \cdot B F P T_{f, b}^{s d, p, i, m n}\right)}{B}+ \\
\sum_{s}^{s \neq n} \sum_{d}^{d \neq s \neq i} \sum_{m}^{d \neq n \neq d \neq i} \sum_{p} \frac{\left(\gamma_{m i}^{s d, p} \cdot \gamma_{i n}^{s d, p} \cdot W F P T_{f, b, k}^{s d, p, i, m n}+\gamma_{m i}^{s d, p} \cdot \gamma_{i n}^{s d, p} \cdot S F P T_{f, b, k}^{s d, p, i, m n}\right)}{W \cdot B}
\end{array} \\
& \forall i, n, f \mid i \neq n, \delta_{i n}>0 \\
& F O_{i}=\sum_{n}^{n \neq i} \sum_{f}\left(\delta_{i n} \cdot f o_{i}^{n, f}\right) \forall i
\end{aligned}
$$




$$
\begin{aligned}
& f_{i}^{m, f} \geq \sum_{s}^{s \neq i} \sum_{p}\left(\gamma_{m i}^{s i, p} \cdot V F_{f}^{s i, p}\right)+f b_{i}^{m, f}+\sum_{s}^{s \neq d \neq i d \neq m \neq i} \sum_{d} \sum_{n}^{n \neq m \neq s} \sum_{p}\left(\gamma_{m i}^{s d, p} \cdot \gamma_{i n}^{s d, p} \cdot V F_{f}^{s d, p}\right)+ \\
& \sum_{s}^{s \neq d \neq i} \sum_{d}^{\substack{s \\
d \neq m \neq i}} \sum_{n}^{p \neq m \neq s} \sum_{p} \frac{\left(\gamma_{m i}^{s d, p} \cdot \gamma_{i n}^{s d, p} \cdot B F P T_{f, b}^{s d, p, i, m n}\right)}{B}+ \\
& \sum_{s} \sum_{d}^{s \neq d \neq i} \sum_{n}^{\substack{d \neq m \neq i \\
d \neq \neq m \neq s}} \sum_{p} \frac{\left(\gamma_{m i}^{s d, p} \cdot \gamma_{i n}^{s d, p} \cdot W F P T_{f, b, k}^{s d, p, i, m n}+\gamma_{m i}^{s d, p} \cdot \gamma_{i n}^{s d, p} \cdot S F P T_{f, b, k}^{s d, p, m n}\right)}{W \cdot B} \\
& \forall i, m, f \mid i \neq m, \delta_{m i}>0 \\
& F I_{i}=\sum_{m}^{m \neq i} \sum_{f}\left(\delta_{m i} \cdot f_{i}^{m, f}\right) \forall i
\end{aligned}
$$

8) Port count conservation must be ensured at all optical switching stages:

Inequalities in Equations 4.53-4.55

9) The length of the CWS/CBS/CFS-based virtual link must be within the maximum optical reach value (note that these constraints will be excluded from the MILP formulation if the optical signal regeneration capability is not considered):

$$
\begin{gathered}
\sum_{i} \sum_{j}^{j \neq i}\left(\gamma_{i j}^{s d, p} \cdot d_{i j} \cdot V S_{f, b, k}^{s d, p}\right) \leq R_{\max } \forall s, d, f, b, k, p \mid s \neq d \\
\sum_{i} \sum_{j}^{j \neq i}\left(\gamma_{i j}^{s d, p} \cdot d_{i j} \cdot V W_{f, b, k}^{s d, p}\right) \leq R_{\max } \forall s, d, f, b, k, p \mid s \neq d \\
\sum_{i} \sum_{j}^{j \neq i}\left(\gamma_{i j}^{s d, p} \cdot d_{i j} \cdot V B_{f, b}^{s d, p}\right) \leq R_{\max } \forall s, d, f, b, p \mid s \neq d \\
\sum_{i} \sum_{j}^{j \neq i}\left(\gamma_{i j}^{s d, p} \cdot d_{i j} \cdot V F_{f}^{s d, p}\right) \leq R_{\max } \forall s, d, f, p \mid s \neq d
\end{gathered}
$$

\subsection{NLP Formulation}

To achieve the optimum solution to the RMGPA problem without the color-continuity constraints, with the optical-reach constraints, and with the customization of the optical reach of 
clear paths, the problem will be formulated as an NLP model. The NLP model consists of a nonlinear objective function and linear constraints. The formulation proceeds as follows.

Parameters, Sets, and Optimization Variables:

Parameters, sets, and optimization variables are the same as those used in the MILP formulation.

Objective:

Minimize the total weighted port count in the GMPLS-based transport network. Here, Equation 3.1 is used to determine the weighted port count of the EXC segment, as follows. The cost of the FSC/BSC/LSC-based EXC port relative to the cost of the FXC/BXC/WXC-based MG-OXC port is a function of the CFS/CBS/CWS-based virtual link length:

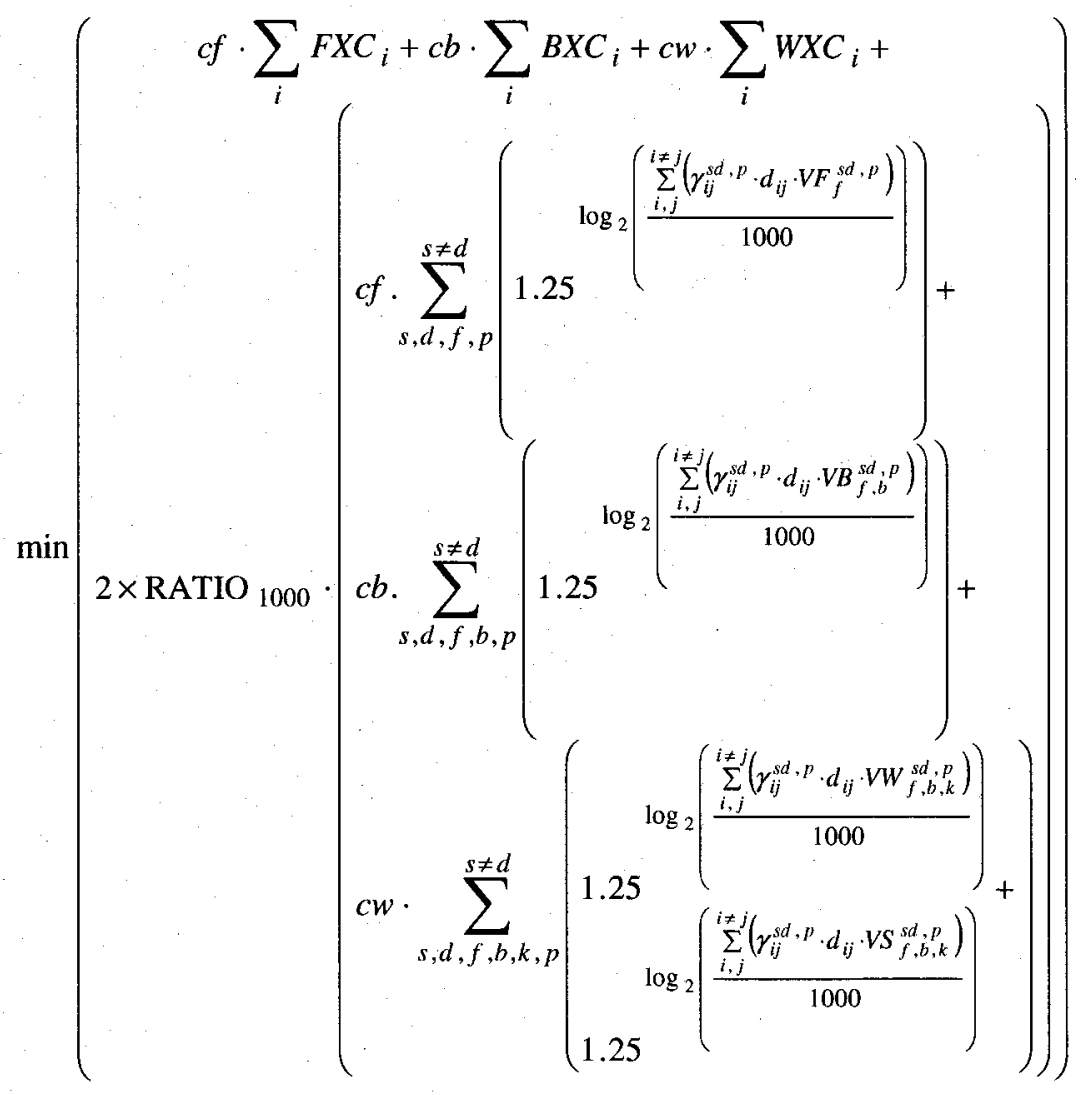

\section{Constraints:}

Subject to Constraints 1-9 used in the previous MILP formulation. 


\subsection{Heuristic Approach}

The computational complexity of the MILP and NLP models calls for the use of heuristics to solve the problem sub-optimally in a reasonable amount of time. A high-level view of the approach to be taken to solve the three versions of the RMGPA problem heuristically is shown in Fig. 5.1. The heuristic approach consists of two phases. The task of the first phase is to build the multi-granular virtual topology. The tasks of the second phase, which we call the adjustment phase, are (1) to decrease the port count of the pass-through segments of the built virtual links; and (2) to customize the optical reach of EXC ports based on the length of their virtual links, if the opticalreach customization is considered. Both phases are explained respectively as follows.

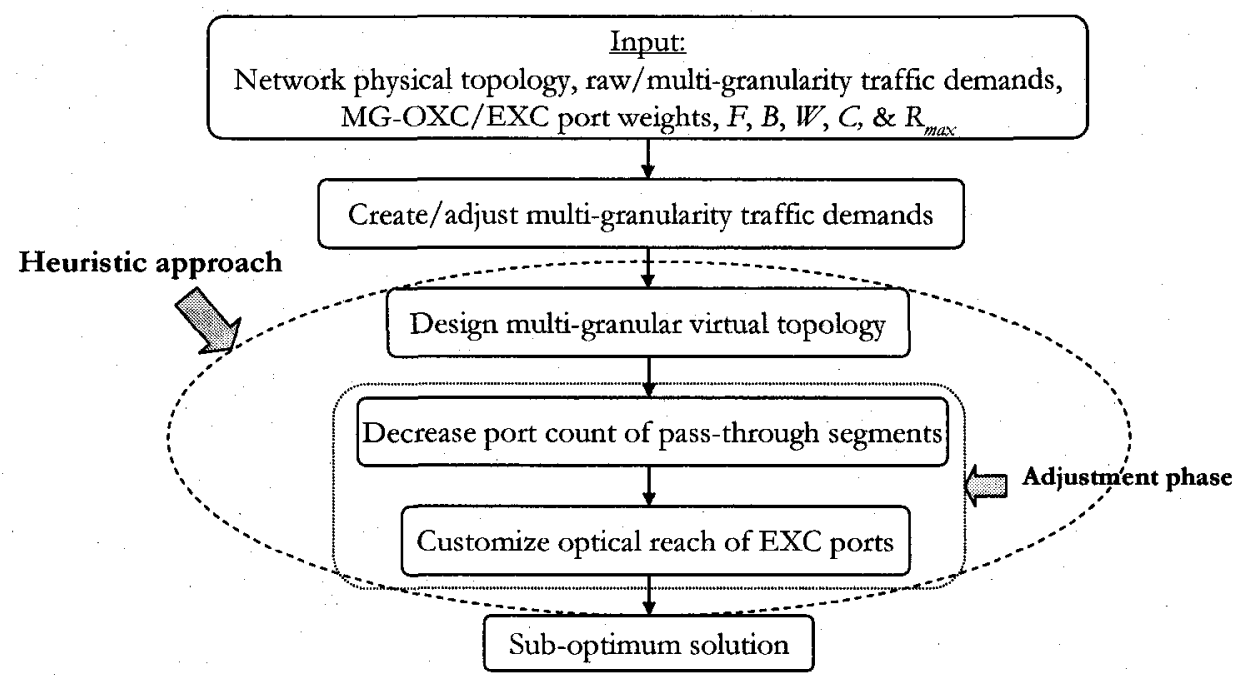

Fig. 5.1: A high-level view of the heuristic approach proposed to solve the RMGPA problem without the colorcontinuity constraints, with/without the optical-reach constraints, and with/without customizing the optical reach.

The objective of the first phase is to build a multi-granular virtual topology that can accommodate all traffic demands with the lowest weighted port count. In this phase, clear and unclear paths will be assigned to fiber, waveband, wavelength, and sub-wavelength demands. The sequence in which $s-d$ pairs are served needs to follow a certain policy. In addition to the four sorting policies adopted in Chapter 4, here we add two distance-based sorting polices that will be used if the optical-reach 
constraints are considered in the RMGPA problem, and they are explained as follows. In Policy $5 / 6, s-d$ pairs are sorted in the decreasing/increasing order of their shortest route lengths, which are obtained via Dijkstra's algorithm using the distance as a cost metric. The multi-granular virtual topology is constructed using the following algorithm [NaMo07b][NaMo07c][NaMo07e] [NaMo07f]:

\section{Multi-Granular Virtual Topology Algorithm;}

Input: Network physical topology, adjusted multi-granularity traffic demands, C, W, B, F, $R_{\max }, M G-O X C$ port weights, EXC port weights (obtained form Equation 3.1)

Output: Sub-optimum multi-granular virtual topology

\section{Begin}

\section{Initialization:}

- For all nodes, mark all WXC/BXC/FXC add, drop, input, and output ports as unused; a WXC add/output port is designated as <wavelength $k$, waveband $b$, fiber $f$, next destination node>; a $W X C$ drop/input port is designated as <wavelength $k$, waveband $b$, fiber $f$, arriving from node>; a BXC add/output port is designated as <waveband $b$, fiber $f$, next destination node>; $a$ BXC drop/input port is designated as < waveband $b$, fiber $f$, arriving from node>; a FXC add/output port is designated as <fiber $f$, next destination node>; a FXC drop/input port is designated as $<$ fiber $f$, arriving from node>

- For all nodes, mark all EXC output and input ports as unused; a LSC output/input port is designated as <wavelength $k$, waveband $b$, fiber $f$, 'next destination'/'arriving from' node>; a BSC output/input port is designated as <waveband $b$, fiber $f$, 'next destination'/'arriving from' node>; a FSC output/input port is designated as < fiber $f$, 'next destination'/"arriving from' node>

\section{Fiber-Switched Path Assignment:}

- Let $M=\left\{m_{0}, m_{1}, \ldots . ..\right\}$ be a set of unserved fiber demands whose traffic $\neq 0$

- While $(M \neq \varnothing)$

\{

- Order unserved fiber demands in $M$ according to the sorting policy used

- Set served demand = fiber demand, $\Lambda_{s d}^{3}$, at the top of the sorted list, which is the element mo of $M$

- Set $D=1$, if fiber demand bifurcation is allowed; otherwise, set $D=$ served demand

- While (served demand $\neq 0$ ) 
- Evaluate the cost of all physical links according to the availability of fibers along the physical link; link cost $=1$ if the remaining unused fibers $\geq D$, otherwise link cost $=\infty$

- Find all possible physical routes for the served demand using the All Distinct Routes algorithm

- For every eligible physical route, perform the following for every unit of the served demand:

- Try to choose the color (fiber index) that ensures color-continuity along the whole route using the First-Fit color assignment scheme. If a color that satisfies the colorcontinuity constraint exists and the optical-reach constraint is considered, then check whether signal regeneration is required along the route. Signal regeneration is needed if the distance summation of consecutive route spans $>$ $R_{\max }$. If regeneration is needed, then the unit of the served fiber demand will use an UFS path that consists of a concatenation of CFS paths; otherwise, the unit of the served demand will use a CFS path

- If it is not possible to satisfy the color-continuity constraint, then the color assignment will be done at each route span using the First-Fit color assignment scheme. The need for color conversion means that the unit of the served demand will use an UFS path that consists of a concatenation of CFS paths. If the opticalreach constraint is considered, then entering the OEO domain conversion process for the purpose of color conversion is an opportunity to refresh the reach of the optical signal. Also, check whether signal regeneration is required along the CFS paths that constitute the UFS path. If regeneration is required, then a CFS path will be broken into further CFS paths

\}

- If there is no feasible route, then end the algorithm

- Evaluate the incremental port count requirement for all feasible routes; the incremental port count for a feasible eligible route is the summation of all unused FXC and EXC ports needed by all units of the served demand along the route, but these ports will not be marked as used at this step

- Choose the candidate route that incurs the lowest incremental port count

- Along the best route, update the residual fiber resources and mark the required FXC and EXC ports as used 
- Create CFS-based virtual link(s) along the best route of the $s-d$ pair served; the virtual link attributes are the best sub-route/route and the color used along the subroute/route

- Set served demand $=$ served demand $-D$

\}

- Remove mo from $M$

\}

\section{Waveband-Switched Path Assignment:}

- Let $M=\left\{m_{0}, m_{1}, \ldots . ..\right\}$ be a set of unserved waveband demands whose traffic $\neq 0$

- While $(M \neq \varnothing)$

\{

- Order unserved waveband demands in $M$ according to the sorting policy used

- Set served demand = waveband demand, $\Lambda_{s d}^{2}$, at the top of the sorted list

- Set $D=1$, if waveband demand bifurcation is allowed; otherwise, set $D=$ served demand

- While (served demand $\neq 0$ )

\{

- Evaluate the cost of all physical links according to the availability of wavebands along the physical link; link cost $=1$ if the remaining unused wavebands $\geq D$, otherwise link $\operatorname{cost}=\infty$

- Find all possible physical routes for the served demand using the All Distinct Routes algorithm

- For every eligible physical route, perform the following for every unit of the served demand:

\{

- Try to choose the color (the combination of waveband and fiber indices) that ensures color-continuity along the whole route using the First-Fit color assignment scheme. If a color that satisfies the color-continuity constraint exists and the optical-reach constraint is considered, then check whether signal regeneration is required along the route. If regeneration is needed, then the unit of the served waveband demand will use an UBS path that consists of a concatenation of CBS paths; otherwise, the unit of the served demand will use a CBS path

- If it is not possible to satisfy the color-continuity constraint, then the color assignment will be done at each route span using the First-Fit color assignment scheme. The need for color conversion means that the unit of the served demand 
will use an UBS path that consists of a concatenation of CBS paths. If the opticalreach constraint is considered, then check whether signal regeneration is required along the CBS paths that constitute the UBS path. If regeneration is required, then a CBS path will be broken into further CBS paths

\}

- If there is no feasible route, then end the algorithm

- Evaluate the worst incremental port count requirement for all feasible routes; the incremental port count for a feasible eligible route is the summation of all unused FXC, $B X C$, and EXC ports needed by all units of the served demand along the route. The worst scenario for deciding the unused ports is based on the fact that a wavebandswitched path at an intermediate node can be (1) switched at the FXC if the fiber utilization at both the incoming and outgoing links is zero and there are no further add/drop waveband/wavelength/sub-wavelength demands at this intermediate node, or (2) switched at the BXC otherwise

- Choose the candidate route that incurs the lowest incremental port count

- Along the best route, update the residual waveband resources and mark the required FXC, BXC, and EXC ports as used

- Create CBS-based virtual link(s) along the best route of the $s-d$ pair served

- Set served demand = served demand $-D$

\}

- Remove mo from $M$

\}

\section{Wavelength-Switched Path Assignment:}

- Let $M=\left\{m_{0}, m_{1}, \ldots . ..\right\}$ be a set of unserved wavelength demands whose traffic $\neq 0$

- While $(M \neq \varnothing)$

\{

- Order unserved wavelength demands in $M$ according to the sorting policy used

- Set served demand = wavelength demand, $\Lambda_{s d}^{1}$, on the top of the sorted list

- Set $D=1$, if wavelength demand bifurcation is allowed; otherwise, set $D=$ served demand

- While (served demand $\neq 0$ ) \{

- Evaluate the cost of all physical links according to the availability of wavelengths along the physical link; link cost $=1$ if the remaining unused wavelengths $\geq D$, otherwise link cost $=\infty$ 
- Find all possible physical routes for the served demand using the All Distinct Routes algorithm

- For every eligible physical route, perform the following for every unit of the served demand:

\{

- Try to choose the color (the combination of wavelength, waveband, and fiber indices) that ensures color-continuity along the whole route using the First-Fit color assignment scheme. If a color that satisfies the color-continuity constraint exists and the optical-reach constraint is considered, then check whether signal regeneration is required along the route. If regeneration is needed, then the unit of the served wavelength demand will use an UWS path that consists of a concatenation of CWS paths; otherwise, the unit of the served demand will use a CWS path

- If it is not possible to satisfy the color-continuity constraint, then the color assignment will be done at each route span using the First-Fit color assignment scheme. The need for color conversion means that the unit of the served demand will use an UWS path that consists of a concatenation of CWS paths. If the optical-reach constraint is considered, then check whether signal regeneration is required along the CWS paths that constitute the UWS path. If regeneration is required, then a CWS path will be broken into further CWS paths

\}

- If there is no feasible route, then end the algorithm

- Evaluate the worst incremental port count requirement for all feasible routes; the incremental port count for a feasible eligible route is the summation of all unused $F X C$, $B X C, W X C$, and EXC ports needed by all units of the served demand along the route. The worst scenario for deciding the unused ports is based on the fact that a wavelength-switched path at an intermediate node can be (1) switched at the FXC if the fiber and waveband utilization at both the incoming and outgoing links is zero and there are no further add/drop wavelength/sub-wavelength demands at this intermediate node, (2) switched at the BXC if the waveband utilization at both the incoming and outgoing links is zero and there are no further add/drop wavelength/sub-wavelength demands at this intermediate node, or (3) switched at the WXC otherwise

- Choose the candidate route that incurs the lowest incremental port count 
- Along the best route, update the residual wavelength resources and mark the required FXC, BXC, WXC, and EXC ports as used

- Create CWS-based virtual link(s) along the best route of the $s$-d pair served

- Set served demand = served demand $-D$

\}

- Remove mo from $M$

\}

\section{Sub-Wavelength-Switched Path Assignment:}

- Let $M=\left\{m_{0}, m_{1}, \ldots . ..\right\}$ be a set of unserved sub-wavelength demands whose traffic $\neq 0$

- While $(M \neq \varnothing)$

\{

- Order unserved sub-wavelength demands in $M$ according to the sorting policy used

- Set served demand = sub-wavelength demand, $\Lambda_{s d}^{0}$, at the top of the sorted list

- Set $D=1$, if sub-wavelength demand bifurcation is allowed; otherwise, set $D=$ served demand

- While (served demand $\neq 0$ )

- Evaluate the cost of all CWS-based virtual links according to the availability of wavelength capacities along the physical link; link cost $=1$ if the remaining wavelength capacity $\geq D$, otherwise link cost $=\infty$

- Find the shortest virtual route over the existing CWS-based virtual topology via Dijkstra's algorithm using the number of virtual hops as a cost metric

- If a virtual route exists (which means that there is an UWS path and no new ports are required)

\{

- Update the residual wavelength-capacity resources along the best path

O Set served demand $=$ served demand $-D$

\}

- Else (which means that there is a need to build a CWS/UWS path and new ports are required)

\{

- Evaluate the cost of all physical links according to the availability of wavelengths along the physical link; link cost $=1$ if the remaining unused wavelengths $\geq 1$, otherwise link cost $=\infty$ 
- Find all possible physical routes for the served demand using the All Distinct Routes algorithm

- For every eligible physical route, perform the following:

\{

- Try to choose the color (the combination of wavelength, waveband, and fiber indices) that ensures color-continuity along the whole route using the First-Fit color assignment scheme. If a color that satisfies the color-continuity constraint exists and the optical-reach constraint is considered, then check whether signal regeneration is required along the route. If regeneration is needed, then the served demand will use an UWS path that consists of a concatenation of CWS paths; otherwise, the served demand will use a CWS path

- If it is not possible to satisfy the color-continuity constraint, then the color assignment will be done at each route span using the First-Fit color assignment scheme. The need for color conversion means that the served demand will use an UWS path that consists of a concatenation of CWS paths. If the optical-reach constraint is considered, then check whether signal regeneration is required along the CWS paths that constitute the UWS path. If regeneration is required, then a CWS path will be broken into further CWS paths

\}

- If there is no feasible route, then end the algorithm

- Evaluate the worst incremental port count requirement for all feasible routes; the incremental port count for a feasible eligible route is the summation of all unused FXC, BXC, WXC, and EXC ports needed along the route. The worst scenario for deciding the unused ports is based on the fact that a wavelengthswitched path at an intermediate node can be (1) switched at the FXC if the fiber and waveband utilization at both the incoming and outgoing links is zero and there are no further add/drop sub-wavelength demands at this intermediate node, (2) switched at the BXC if the waveband utilization at both the incoming and outgoing links is zero and there are no further add/drop subwavelength demands at this intermediate node, or (3) switched at the WXC otherwise

- Choose the candidate route that incurs the lowest incremental port count 


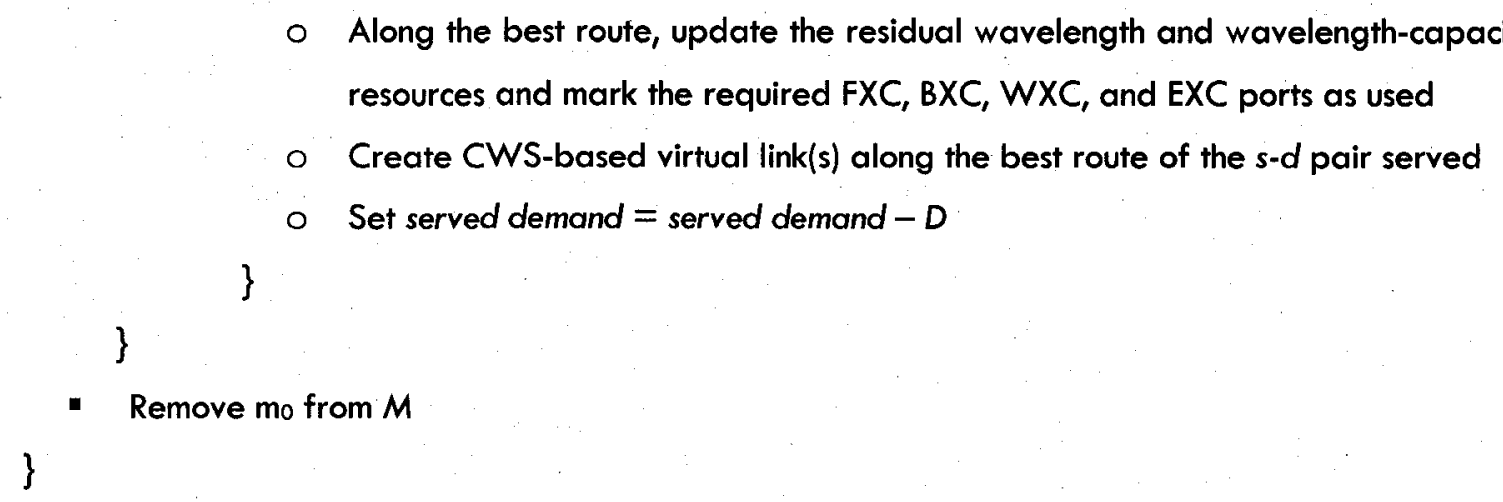

6. If the resultant multi-granular virtual topology can handle all demands, then this virtual topology is both feasible and sub-optimum

End.

In the second phase of the heuristic, the Pass-Through Adjustment algorithm explained in Section 4.3 is used to minimize the port count of the pass-through segments of the built virtual links. If the optical-reach customization is not considered, then the resultant weighted port count of the PassThrough Adjustment algorithm will be the final weighted port count. Otherwise, the output of the algorithm will decide the final weighted port count of all MG-OXCs, whereas the final weighted port count of all EXCs will be decided by customizing the optical reach of the EXC ports according to the length of their virtual links, which are launched and terminated at these EXC ports. The Optical Reach Customization algorithm works as follows [NaMo07c]:

\section{Optical Reach Customization Algorithm;}

Input: Feasible multi-granular virtual topology, W, B, F, MG-OXC port weights ( $c f, c b, \& c w$ ), weighted port count of all MG-OXCs

Output: Sub-optimum weighted port count

\section{Begin}

1. Initialization: Set sub-optimum weighted port count $=$ weighted port count of all MG-OXCs

\section{For all CWS/CBS/CFS-based virtual links, do the following:}

- Calculate the length, $l$, of the CFS/CBS/CWS-based virtual link 
- Using Equation 3.1, set the cost of the CFS/CBS/CWS-based EXC port (the cost of one of the virtual link ends $)=\operatorname{cgf}(l) / \mathrm{cgb}(l) / \mathrm{cgw}(l)=c f / c b / c w \times \mathrm{RATIO}_{1000} \times 1.25^{\log _{2}(l / 1000)}$

- Set sub-optimum weighted port count $=$ sub-optimum weighted port count $+2 \times \operatorname{cgf}(l) / \mathrm{cgb}(l) / \mathrm{cgw}(l)$

\section{End.}

\subsection{Numerical Results and Analysis}

\subsubsection{Verification of the Heuristic Approach}

Since the exact solution to the RMGPA problem with the consideration of the optical-reach customization requires solving a complicated NLP model that cannot be solved by the current optimization methods implemented in CPLEX software [CPLEX], here we focus only on solving the RMGPA problem without the optical-reach customization. To verify the heuristic approach, we compare its results with the results obtained from the MILP formulation. Due to the complexity of the MILP model, the optimization is conducted on a small-sized planning problem. In this subsection, we consider the 5-node sample network shown in Fig. 4.1 as a physical topology. To keep the problem computationally manageable, we use a set of a few adjusted traffic demands, as illustrated in Table 5.1. The rest of input parameters used in this subsection are as follows: $C=10 \mathrm{~Gb} / \mathrm{s}, W=3, B=3, F \geq 1, \mathrm{MG}-\mathrm{OXC} / \mathrm{EXC}$ ports have the same weight, the ratio of EXC port weight to MG-OXC port weight is decided by Equation 3.1 with RATIO $_{1000}=2$ (i.e. $\left.\frac{c g f\left(R_{\max }\right)}{c f}=\frac{c g b\left(R_{\max }\right)}{c b}=\frac{c g w\left(R_{\max }\right)}{c w}=2 \times(1.25)^{\log _{2}\left(R_{\max } / 1000\right)}\right)$, and $1000 \leq \mathrm{R}_{\max } \leq 4000 \mathrm{~km}$.

Table 5.1 illustrates the solution to the RMGPA problem using both optimization methods for various traffic demand instances at different maximum optical reach values. From the table, it is clear that imposing more traffic on the network exponentially increases the problem solve time needed by CPLEX until it becomes difficult to obtain an optimum solution in a reasonable 
amount of time. The solve time generally increases in proportion to the increase in the maximum optical reach value because (1) the size of the problem increases, and (2) the number of feasible solutions decreases. Accordingly, CPLEX exhaustively searches the branch-and-bound tree, which grows as the number of variables increases, in an effort to find a feasible solution. However, randomness plays a big role in speeding up the search process in finding the first feasible solution, because by finding the first feasible solution many tree nodes can be pruned. This randomness factor explains why in some higher reach values we actually obtained a shorter solve time.

In contrast, solving the RMGPA problem using the heuristic approach takes less than one second in all cases. Most importantly, the solutions produced by the heuristic approach are within $\approx 0$ $10 \%$ of the MILP formulation solutions and can be accepted as good approximations of the optimum solutions. In addition, results show that allowing the bifurcation of fiber demands can improve the planning solutions.

Moreover, it is quite clear from Table 5.1 that the maximum optical reach has an optimum value that leads to a minimal weighted port count. This stems from the fact that increasing the maximum optical reach increases the cost of $\mathrm{EXC}$ ports and reduces the number of OEO conversions, which offsets the increase in the weighted port count. As a result, this increase leads to a crossover situation in which the minimal weighted port count occurs. It is interesting to note here that the optimum (or best) optical reach changes according to the traffic demand set. Also, beyond the optimum maximum optical reach, the "weighted" port count is expected to increase. Fig. 5.2, which is based on the solutions obtained from using the first demand set in Table 5.1, demonstrates how changing the maximum optical reach can change the resultant multi-granular virtual topology. Figs. 5.2 (b), (c), and (d) illustrate that beyond a certain reach the virtual topology will not change, and the weighted port count will increase due to the increase in the cost of EXC 
ports. In other words, the "unweighted" port count remains unchanged when the problem solution yields the same virtual topology.

Table 5.1: Comparison between the results obtained using the MILP formulation and heuristic approach for the 5node network example of solving the RMGPA problem without the color-continuity constraints, with the opticalreach constraints, and without the optical-reach customization.

\begin{tabular}{|c|c|c|c|c|c|c|c|c|c|c|c|c|}
\hline \multirow{2}{*}{$\begin{array}{l}\text { \# of traffic } \\
\text { elements } \\
\text { per } \\
\text { granularity } \\
\text { matrix } \\
-\end{array}$} & \multirow{2}{*}{$\begin{array}{c}\text { Adjusted } \\
\text { traffic demands }\end{array}$} & \multirow{2}{*}{$\begin{array}{c}\text { Max. } \\
\text { optical } \\
\text { reach } \\
{[\mathrm{km}]}\end{array}$} & \multirow{2}{*}{$\begin{array}{l}\mathrm{E} / \mathrm{O} \\
\text { ratio }\end{array}$} & \multirow[b]{2}{*}{$F_{\min }$} & \multicolumn{4}{|c|}{ MILP } & \multicolumn{4}{|c|}{ Heuristic } \\
\hline & & & & & $\begin{array}{c}\text { \# of } \\
\text { constraints }\end{array}$ & $\begin{array}{c}\text { \# of } \\
\text { variables }\end{array}$ & $\begin{array}{l}\text { Total } \\
\text { solve } \\
\text { time } \\
\text { [sec] }\end{array}$ & $\begin{array}{c}\text { Weighted } \\
\text { Port } \\
\text { count }\end{array}$ & $\begin{array}{l}\text { Total } \\
\text { solve } \\
\text { time } \\
\text { [sec] } \\
\end{array}$ & $\begin{array}{c}\# \text { of } \\
\text { OEO } \\
\text { conversions }\end{array}$ & $\begin{array}{l}\text { Weighted } \\
\text { port } \\
\text { count }\end{array}$ & $\begin{array}{c}\text { Best } \\
\text { bifurcation } \\
\text { scenario }\end{array}$ \\
\hline \multirow{4}{*}{1} & \multirow{4}{*}{$\begin{array}{l}\Lambda^{0}{ }^{20}=1 \\
\Lambda^{1^{02}}=1 \\
\Lambda_{10}^{22}=2 \\
\Lambda_{03}^{3^{10}}=1\end{array}$} & 1000 & 2 & 1 & 1214 & 9355 & 10.0156 & 96 & 0.04 & 3 & 96 & \\
\hline & & 2000 & 2.5 & 1 & 1988 & 16144 & 47.7188 & 71 & 0.03 & 0 & 71 & No \\
\hline & & 3000 & 2.849 & 1 & 3268 & 24828 & 650.188 & 74.486 & 0.01 & 0 & 74.486 & Bifurcation \\
\hline & & 4000 & 3.125 & 1 & 4458 & 31789 & 563.312 & 77.25 & 0.04 & 0 & 77.25 & \\
\hline \multirow{4}{*}{1} & \multirow{4}{*}{$\begin{array}{l}\Lambda_{0}^{0}=2 \\
\Lambda_{34}^{1^{20}}=2 \\
\Lambda_{01}^{2^{30}}=2 \\
\Lambda_{43}^{3}=2\end{array}$} & 1000 & 2 & 1 & 1019 & 7663 & 0.625 & 108 & 0.07 & 3 & 108 & \\
\hline & & 2000 & 2.5 & 1 & 1988 & 16134 & 11.391 & 96 & 0.03 & 1 & 96 & Bifurcating \\
\hline & & 3000 & 2.849 & 1 & 3268 & 24848 & 80.359 & 93.880 & 0.07 & 0 & 93.880 & fiber demands \\
\hline & & 4000 & 3.125 & 1 & 4458 & 31789 & 115.5 & 97.75 & 0.031 & 0 & 97.75 & \\
\hline \multirow{4}{*}{1} & \multirow{4}{*}{$\begin{array}{l}\Lambda^{0}{ }^{0}=1 \\
\Lambda^{1^{22}}=2 \\
\Lambda^{{ }^{2}}=1 \\
\Lambda^{30}=1 \\
{ }_{14}^{3}=2\end{array}$} & 1000 & 2 & 1 & 1214 & 9367 & 0.766 & $\underline{104}$ & 0.06 & 4 & $\underline{112}$ & \\
\hline & & 2000 & 2.5 & 1 & 1988 & 16115 & 5.890 & 80 & 0.1 & 0 & 80 & Bifurcating \\
\hline & & 3000 & 2.849 & 1 & 3268 & 24845 & 693.453 & 84.183 & 0.03 & 0 & 84.183 & fiber demands \\
\hline & & 4000 & 3.125 & 1 & 4458 & 31789 & 284.844 & 87.5 & 0,03 & 0 & 87.5 & \\
\hline \multirow{4}{*}{1} & \multirow{4}{*}{$\begin{array}{l}\Lambda^{0}{ }^{31}=2 \\
\Lambda^{{ }^{24}}=2 \\
\Lambda^{30}=2 \\
\Lambda_{02}^{30}=2\end{array}$} & 1000 & 2 & 1 & 1214 & 9367 & 0.234 & 148 & 0.111 & 8 & 148 & \\
\hline & & 2000 & 25 & 1 & 1988 & 16115 & 29.766 & $\underline{108}$ & 0.09 & 1 & $\underline{114}$ & Bifurcating \\
\hline & & 3000 & 2.849 & 1 & 3268 & 24845 & 779.672 & $\underline{101.88}$ & 0.04 & 0 & $\underline{111.88}$ & fiber demands \\
\hline & & 4000 & 3.125 & 1 & 4458 & 31789 & 250.859 & 105.75 & 0.04 & 0 & $\lfloor 15.75$ & \\
\hline \multirow{4}{*}{2} & \multirow{4}{*}{$\begin{array}{l}\Lambda_{20}^{0}=\Lambda_{34}^{0}=2 \\
\Lambda_{01}^{1}=\Lambda_{43}^{1}=2 \\
\Lambda_{30}^{2}=\Lambda_{43}^{2}=1 \\
\Lambda_{01}^{3}=\Lambda_{13}^{3}=1\end{array}$} & 1000 & 2 & 1 & 1234 & 9576 & 35.922 & $\underline{156}$ & 0.03 & 5 & $\underline{172}$ & \\
\hline & & 2000 & 2.5 & 1 & 2008 & 16494 & 387.328 & 134 & 0.03 & 0 & 134 & $N_{0}$ \\
\hline & & 3000 & 2.849 & 1 & 3288 & 25362 & 24510.6 & 140.972 & 0.03 & 0 & 140.972 & Bifurcation \\
\hline & & 4000 & 3.125 & 1 & 4478 & 32405 & 47721.4 & 146.5 & 0.03 & 0 & 146.5 & \\
\hline \multirow{4}{*}{2} & \multirow{4}{*}{$\begin{array}{l}\Lambda_{31}^{0}=\Lambda_{24}^{0}=2 \\
\Lambda_{30}^{1}=\Lambda_{02}^{1}=2 \\
\Lambda_{04}^{2}=\Lambda_{40}^{2}=1 \\
\Lambda_{41}^{3}=\Lambda_{42}^{3}=1\end{array}$} & 1000 & 2 & 1 & 1234 & 9594 & 770 & $\underline{180}$ & 0.08 & 8 & $\underline{196}$ & \\
\hline & & 2000 & 2.5 & 1 & 2008 & 16470 & 4151.19 & 147 & 0.08 & 1 & 147 & No \\
\hline & & 3000 & 2.849 & 1 & 3288 & 25336 & 31852.7 & 146.972 & 0.04 & 0 & 146.972 & Bifurcation \\
\hline & & 4000 & 3.125 & 1 & 4478 & 32405 & 78265.8 & 152.5 & 0.03 & 0 & 152.5 & \\
\hline \multirow{4}{*}{3} & \multirow{4}{*}{$\begin{array}{l}\Lambda_{31}^{0}=\Lambda_{24}^{0}=\Lambda_{30}^{0}=2 \\
\Lambda_{04}^{1}=\Lambda_{40}^{1}=1, \Lambda_{02}^{1}=2 \\
\Lambda_{41}^{2}=\Lambda_{42}^{2}=1, \Lambda_{01}^{2}=2 \\
\Lambda_{31}^{3}=1, \Lambda_{14}^{3}=\Lambda_{24}^{3}=2\end{array}$} & 1000 & 2 & 2 & 2278 & 19493 & \multirow{4}{*}{$>32295.7$} & \multirow{4}{*}{$\begin{array}{c}\mathrm{N} / \mathrm{A} \\
\text { (PC man out } \\
\text { of memory) }\end{array}$} & 0.02 & 10 & 268 & \\
\hline & & 2000 & 2.5 & 2 & 3826 & 33537 & & & 0.02 & 2 & 233 & No \\
\hline & & 3000 & 2.849 & 2 & 6386 & 51565 & & & 0.02 & 0 & 222.394 & Bifurcation \\
\hline & & 4000 & 3.125 & 2 & 8766 & 65917 & & & 0.01 & 0 & 222.394 & \\
\hline \multirow{4}{*}{3} & $\Lambda_{30}^{0}=\Lambda_{31}^{0}=\Lambda_{24}^{0}=1$ & 1000 & 2 & 2 & 2278 & 19531 & \multirow{4}{*}{$>25125.4$} & \multirow{4}{*}{$\begin{array}{c}\mathrm{N} / \mathrm{A} \\
\text { (PC ran out } \\
\text { of memory) }\end{array}$} & 0.02 & 7 & 220 & \\
\hline & $\Lambda_{01}^{1}=\Lambda_{14}^{1}=\Lambda_{41}^{1}=1$ & 2000 & 2.5 & 2 & 3820 & 33551 & & & 0.01 & 0 & 185 & No \\
\hline & $\Lambda_{01}^{2}=\Lambda_{14}^{2}=\Lambda_{41}^{2}=1$ & 3000 & 2.849 & 2 & 6386 & 51571 & & & 0.02 & 0 & 185 & Bifurcation \\
\hline & $\Lambda_{24}^{3}=\Lambda_{31}^{3}=\Lambda_{34}^{3}=1$ & 4000 & 3.125 & 2 & 8766 & 65917 & & & 0.02 & 0 & 185 & \\
\hline
\end{tabular}




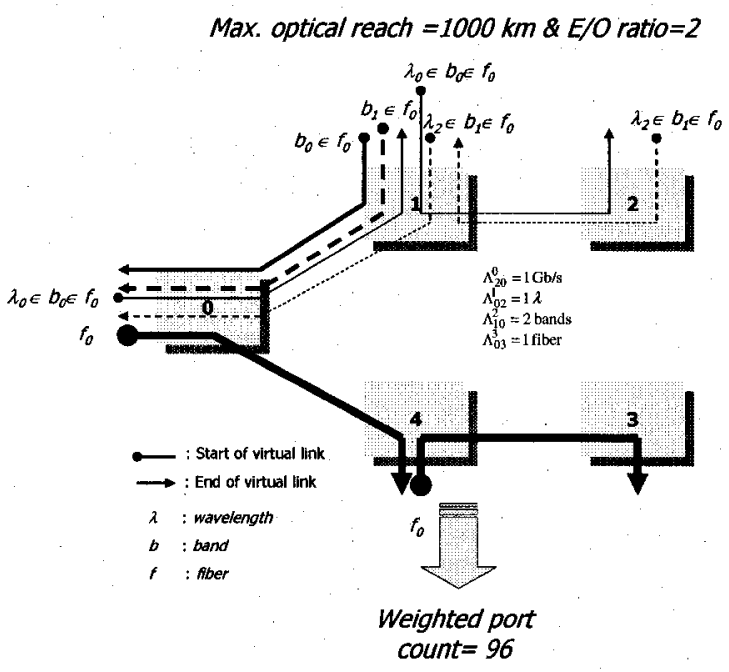

(a)

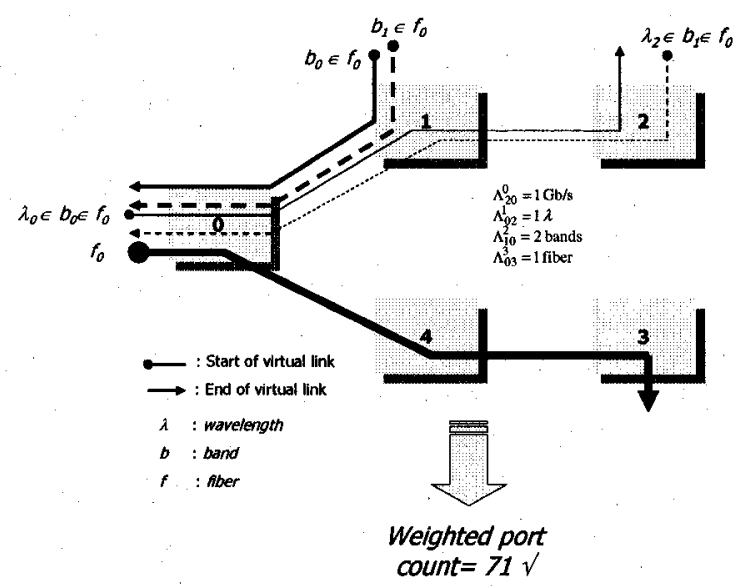

(b)

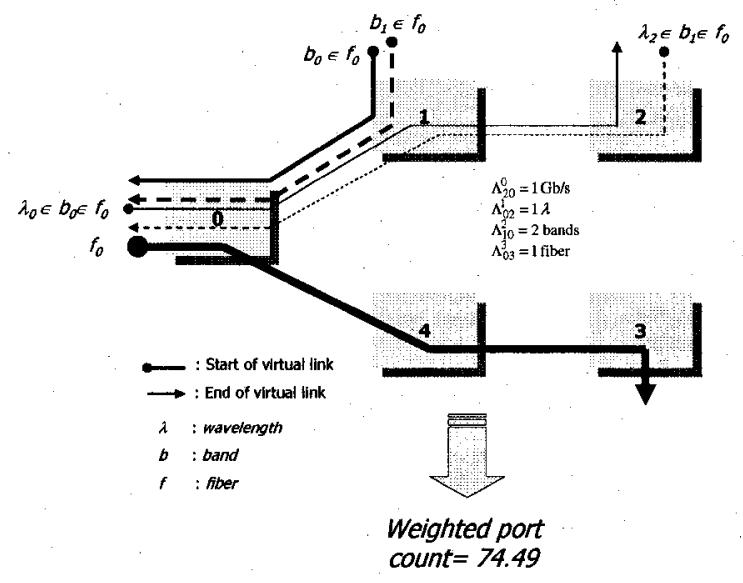

(c)

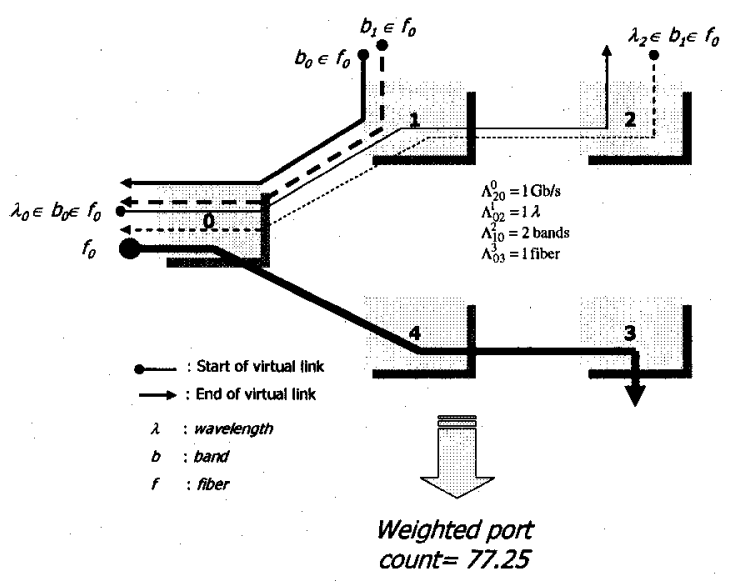

(d)

Fig. 5.2: Multi-granular virtual topology solutions for the 5-node network example, given that $C=10 \mathrm{~Gb} / \mathrm{s}, W=3, B=3$, $F=F_{m i h}=1$, and $1000 \leq \mathrm{R}_{\max } \leq 4000 \mathrm{~km}$.

\subsubsection{Impact of Color Conversion}

This subsection demonstrates the impact of performing color conversion at all granularity levels through the use of OEO domain conversion at the EXC segment. To conduct this demonstration, we solve the RMGPA problem with/without the color-continuity constraints and without the optical-reach constraints. The input data and parameters used here are as follows: the physical topology used is that of the 8-node network, as shown in Fig. 4.11; the raw traffic demands are 
chosen randomly from the range $(0,1500) \mathrm{Gb} / \mathrm{s}$, as shown in Table $5.2 ; C=10 \mathrm{~Gb} / \mathrm{s} ; W=4 ; B=4$; $F \geq F_{\text {min }}$; all MG-OXC/EXC ports have the same weight; and the EXC port weight is twice the MG-OXC port weight. The optimized multi-granularity traffic demands are shown in Table 5.3. The results of solving the planning problem using different heuristic variations with and without color conversions at $F=F_{m i n}$ and $F_{\text {opt }}$ are shown in Figs. 5.3-5.5.

Table 5.2: The 8-node network raw traffic demands, which are chosen randomly from the range $(0,1500) \mathrm{Gb} / \mathrm{s}$.

Destination

\begin{tabular}{cc|cccccccc}
\multicolumn{1}{c}{} & 0 & 0 & 1 & 2 & 3 & 4 & 5 & 6 & 7 \\
\cline { 3 - 9 } Source & 1 & 0 & 624 & 138 & 1135 & 795 & 1396 & 575 & 981 \\
& 2 & 100 & 0 & 1084 & 1007 & 575 & 948 & 1327 & 779 \\
& 3 & 477 & 356 & 0 & 393 & 1144 & 1130 & 1364 & 109 \\
& 4 & 1270 & 1247 & 51 & 1487 & 0 & 1019 & 1150 & 90 \\
& 5 & 576 & 717 & 1422 & 1402 & 1357 & 0 & 478 & 1052 \\
& 6 & 11 & 548 & 399 & 1480 & 415 & 944 & 0 & 249 \\
& 7 & 1104 & 655 & 1474 & 1105 & 350 & 136 & 757 & 0 \\
\hline
\end{tabular}

Table 5.3: The 8-node network multi-granularity demands, which are generated from the $(0,1500) \mathrm{Gb} / \mathrm{s}$ range raw demands along with the use of the $C=10 \mathrm{~Gb} / \mathrm{s}, W=4, \& B=4$ combination.

\section{Destination}

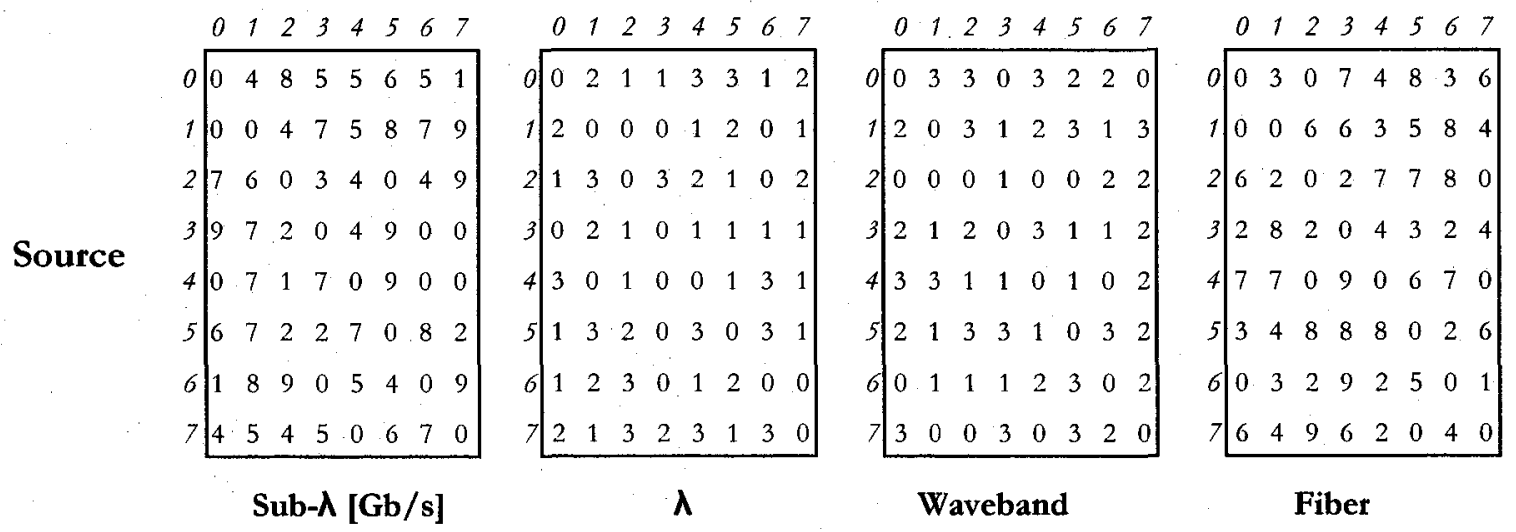

From Fig. 5.3 (a), it is clear that allowing color conversions by using UWS, UBS, and UFS paths, which utilize resources efficiently, decreases the minimum fiber requirement, which can be quite 
useful in the fiber-scarce situation. Also, the figure illustrates that the amount of reduction in $F_{m i n}$ obtained through the use of color conversions depends on both the bifurcation scenario and sorting policy used in the heuristic. However, the decrease in $F_{m i n}$ can come at the expense of an increased weighted port count, as shown in Fig. 5.4 (a). This trade-off is due to the need for OEO conversions, as is shown in Fig. 5.5. Moreover, the need for OEO conversions tends to vanish at $F_{o p h}$ as shown in Fig. 5.5. This occurs because, as the parameter $F$ increases, more resources will be available and the choice of a clear path rather than an unclear path will be more likely. It is worth mentioning here that, in some cases, OEO conversions can still occur at $F_{\text {ppo }}$ as shown in Fig. 5.5. This is because when path candidates are being evaluated for a certain $s-d$ pair while building the multi-granular virtual topology, a short unclear path will be selected rather than an expensive long clear path. Still, this path selection does not guarantee that the final weighted port count at $F_{\text {opt }}$ will be lower than the weighted port count achieved without color conversions, as is the case when the heuristic uses the fiber-bifurcation scenario with Policy 1 (this observation can be made from Figs. 5.4 (b) and 5.5). Furthermore, provided that OEO conversions are not needed at $F_{o p p}$ it is expected that heuristics using both the same bifurcation scenario and sorting policy will yield the same weighted port counts regardless of whether color conversions are allowed. However, on some occasions, as shown in Fig. 5.4 (b), a heuristic that considers color conversions will yield a solution with no OEO conversions, but its weighted port count will be slightly higher/lower than that of a heuristic that does not consider color conversions. These slight differences are expected because the heuristic with color conversions works differently than the heuristic without color conversions. Finally, Figs. 5.3 (a) and 5.4 (a) clearly show that in the fiber-scarce situation, the best heuristic variation with the color-continuity constraints is not necessarily the best without these constraints. 


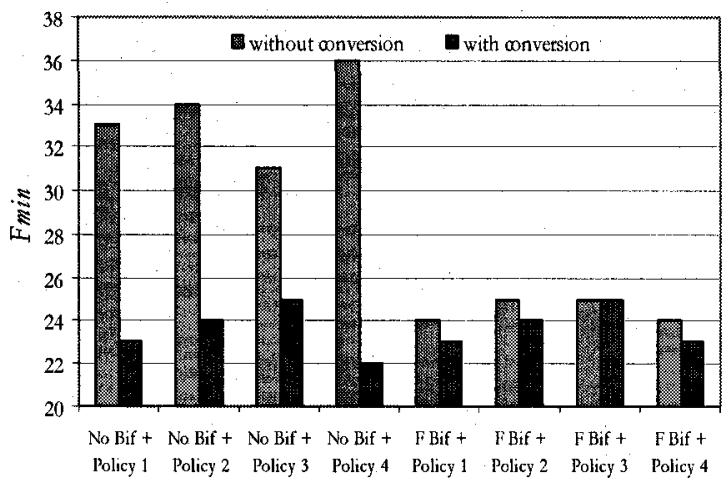

(a)

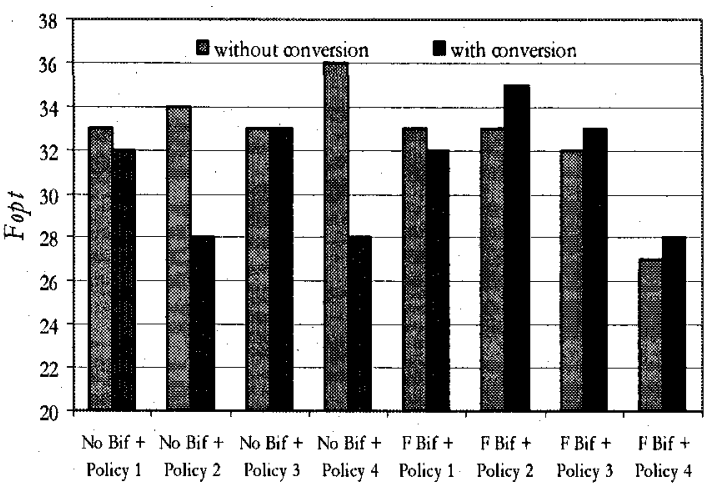

(b)

Fig. 5.3: Fiber requirements for the case of the 8 -node network and $(0,1500) \mathrm{Gb} / \mathrm{s}$ range demands with $\mathrm{E} / \mathrm{O}$ ratio $=2$ using different heuristic variations with/without color conversions: (a) $F_{m i n}$; (b) $F_{a p r}$

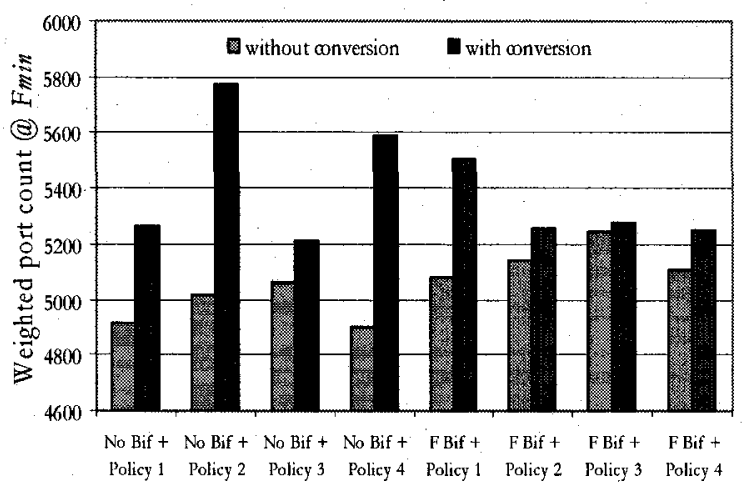

(a)

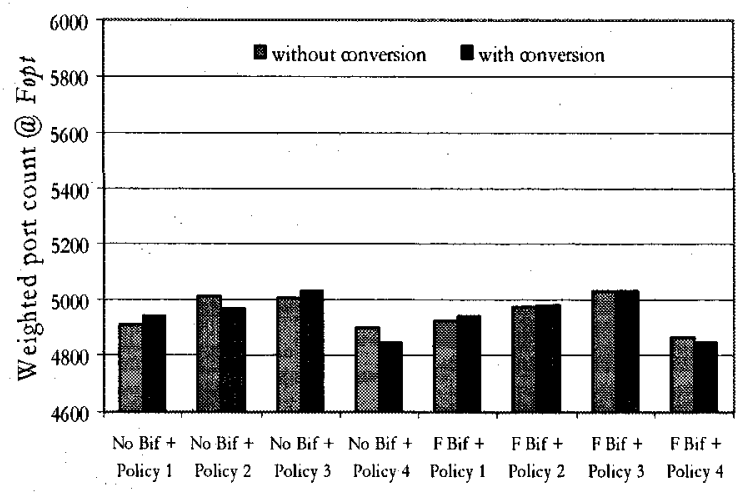

(b)

Fig. 5.4: Weighted port count for the case of the 8-node network and $(0,1500) \mathrm{Gb} / \mathrm{s}$ range demands with $\mathrm{E} / \mathrm{O}$ ratio $=2$ using different heuristic variations with/without color conversions: (a) weighted port count at $F_{m i n}$; (b) weighted port count at $F_{o p t}$

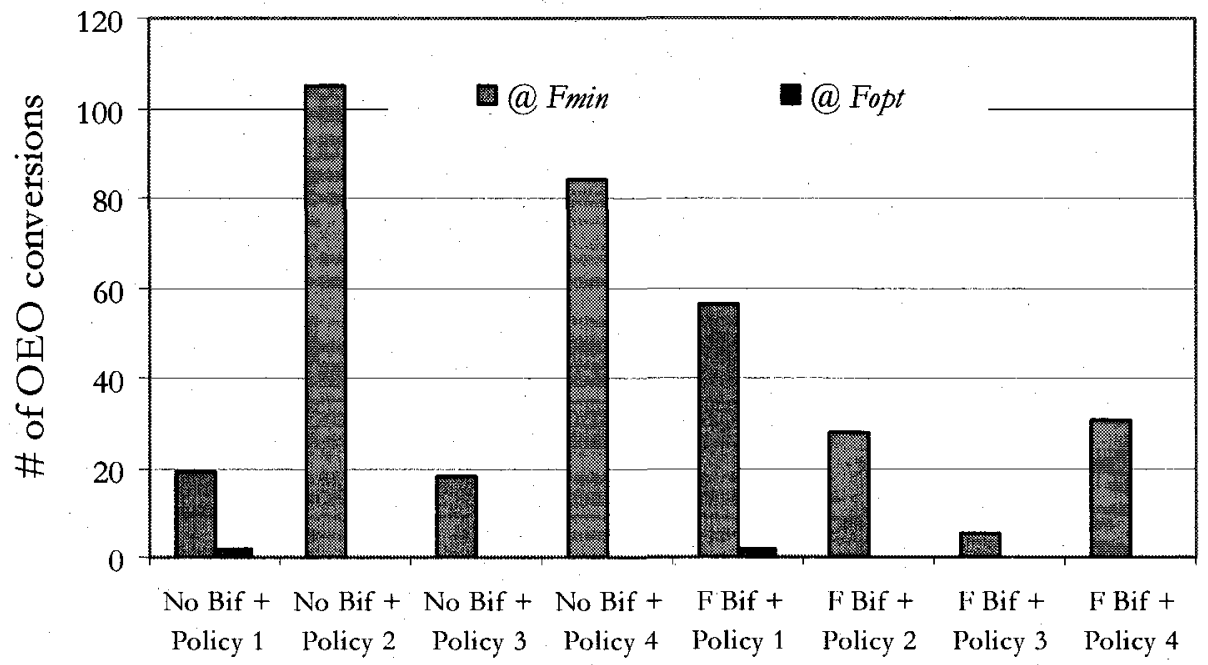

Fig. 5.5: The number of OEO conversions for the case of the 8-node network and $(0,1500) \mathrm{Gb} / \mathrm{s}$ range demands with $\mathrm{E} / \mathrm{O}$ ratio $=2$ using different heuristic variations with color conversions. 


\subsubsection{Impact of E/O Cost Ratio with Color-Continuity Constraints}

The goal of this subsection is to see how changing the $\mathrm{E} / \mathrm{O}$ cost ratio will affect the solution to the planning problem involving the color conversion capability. Using the same input data and parameters considered in Subsection 5.5.2, except that the $\mathrm{E} / \mathrm{O}$ cost ratio $=2$ and 3 , the results of different heuristic variations with and without color conversions at $F=F_{\text {min }}$ and $F_{p p t}$ are shown in Figs. 5.6-5.8.

In comparing Figs. 5.3-5.5 with Figs. 5.6-5.8, the following observations can be made. First, it is clear that increasing the $\mathrm{E} / \mathrm{O}$ ratio can change the fiber requirement. This change occurs because when path candidates are evaluated for a certain $s$ - $d$ pair, a long clear path will be selected rather than an expensive short unclear path. This also means that the long path will consume more resources. As a result, if fiber resources are scarce, other $s-d$ pairs might be forced to use unclear paths instead of clear paths or unclear paths with many virtual hops instead of those having a few virtual hops, either of which scenario means that more OEO conversions would be required. If fiber resources are plentiful, then other $s-d$ pairs can still use clear paths or unclear paths with a few virtual hops. These explain why, as illustrated by the comparison of Fig. 5.8 with Fig. $5.5,(1)$ in the fiber-scarce situation (at $F_{m i n}$ ) with some heuristic variations, the number of OEO conversions with the $\mathrm{E} / \mathrm{O}$ ratio $=3$ is more than the number achieved with the $\mathrm{E} / \mathrm{O}$ ratio $=2$; and (2) in the fiberplentiful situation $\left(\right.$ at $\left.F_{\text {opt }}\right)$, the number of $\mathrm{OEO}$ conversions vanishes as the $\mathrm{E} / \mathrm{O}$ ratio increases. Second, increasing the $\mathrm{E} / \mathrm{O}$ ratio changes the best heuristic variation. Figs. ' 5.3 (a) and 5.4 (a)'/‘5.6 (a) and 5.7 (a)' demonstrate that in the fiber-scarce situation, when the $\mathrm{E} / \mathrm{O}$ ratio $=2 / 3$, the best variation is the one that employs 'Policy 4 along with the no-bifurcation scenario'/'Policy 4 along with the fiber-bifurcation scenario'. On the other hand, Figs. '5.3 (b) and 5.4 (b)'/“5.6 (b) and 5.7 (b)' demonstrate that in the fiber-plentiful situation, when the $\mathrm{E} / \mathrm{O}$ ratio $=2 / 3$, the best heuristic 
variation is the one that utilizes 'Policy 4 along with the no- or fiber-bifurcation scenario'/'Policy 4 along with the no-bifurcation scenario'. Third, varying the $\mathrm{E} / \mathrm{O}$ cost ratio changes the resultant multi-granular virtual topology even in situations where $F_{\text {min }}$ is the same. For instance, the use of Policy 4 along with the no-bifurcation scenario in the heuristic leads to the same $F_{\text {min }}=22$ using both $\mathrm{E} / \mathrm{O}$ ratios. The first clue to the virtual topology alteration is the change in the number of OEO conversions by comparing Fig. 5.5 with Fig. 5.8 . The second clue is the difference in the port count breakdown at all nodes between both $\mathrm{E} / \mathrm{O}$ ratios, as shown in Fig. 5.9. In short, the design of the multi-granular virtual topology without considering the color-continuity constraints is sensitive to the $\mathrm{E} / \mathrm{O}$ cost ratio. This result is contrary to the finding made in Subsection 4.4.4, where it was demonstrated that the multi-granular virtual topology is insensitive to the $\mathrm{E} / \mathrm{O}$ cost ratio when the color-continuity constraints are satisfied.

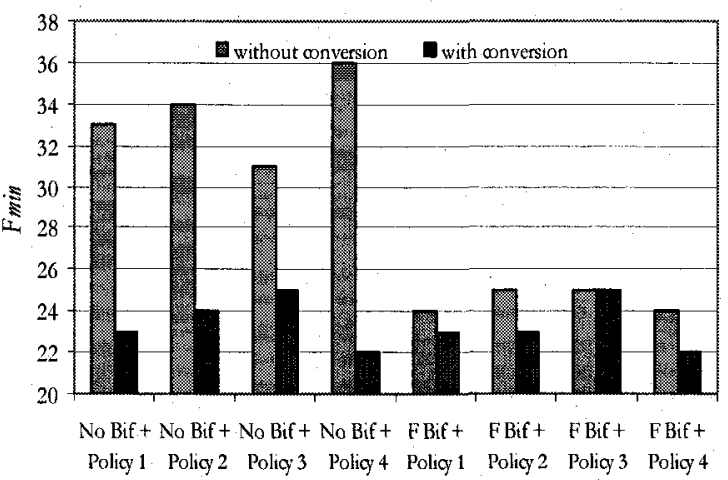

(a)

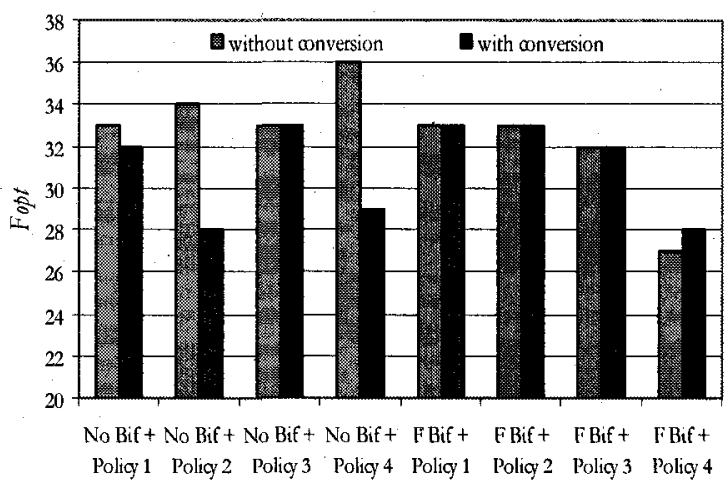

(b)

Fig. 5.6: Fiber requirements for the case of the 8-node network and $(0,1500) \mathrm{Gb} / \mathrm{s}$ range demands with $\mathrm{E} / \mathrm{O}$ ratio $=3$ using different heuristic variations with/without color conversions: (a) at $F_{\text {min }}$; (b) at $F_{\text {opr }}$ 


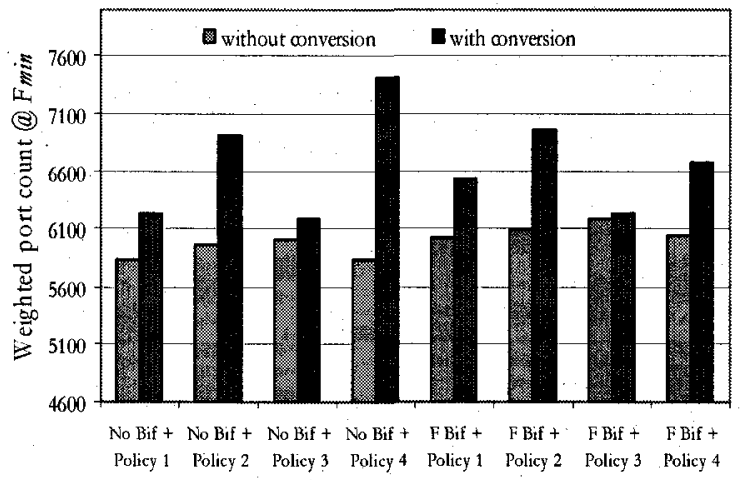

(a)

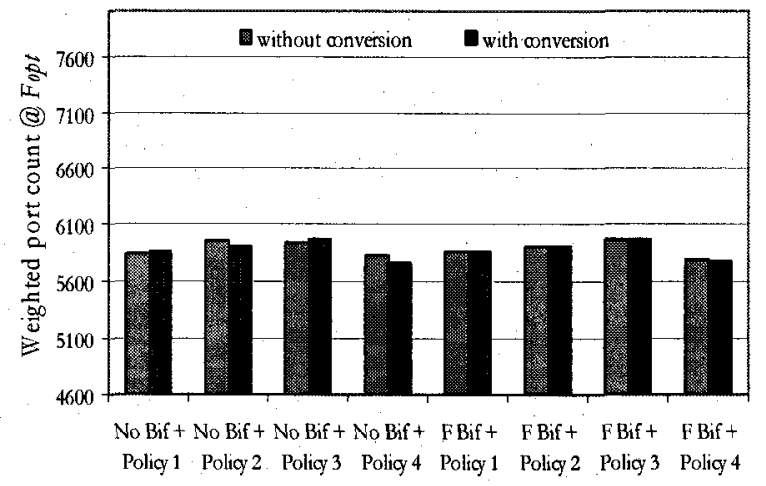

(b)

Fig. 5.7: Weighted port count for the case of the 8-node network and $(0,1500) \mathrm{Gb} / \mathrm{s}$ range demands with E/O ratio $=3$ using different heuristic variations with/without color conversions: (a) at $F_{\text {min }}$; (b) at $F_{\text {opp }}$

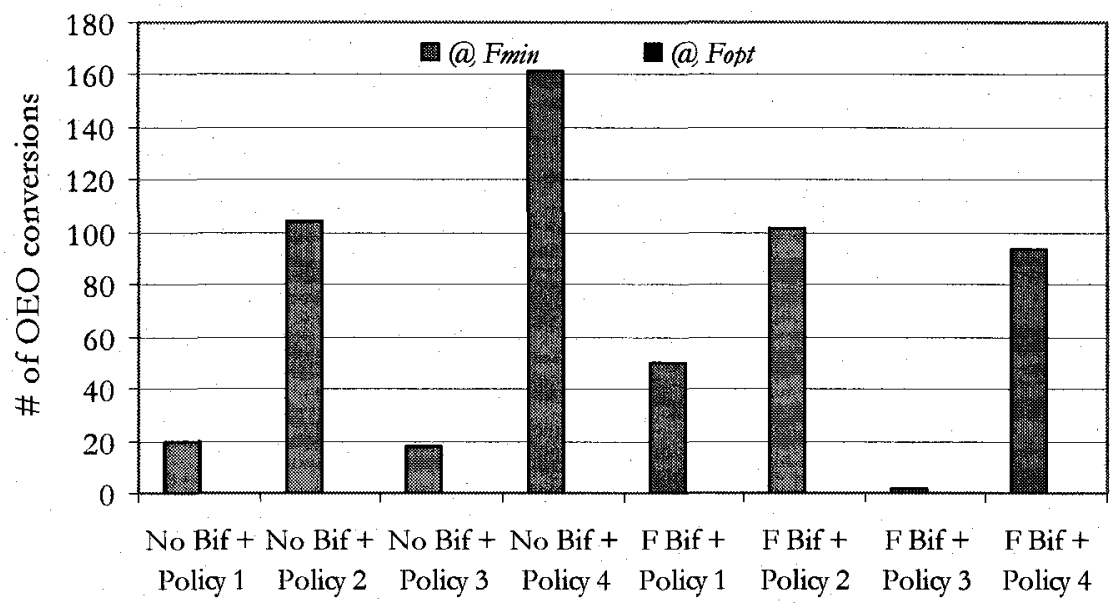

Fig. 5.8: The number of OEO conversions for the case of the 8-node network and $(0,1500) \mathrm{Gb} / \mathrm{s}$ range demands with $\mathrm{E} / \mathrm{O}$ ratio $=3$ using different heuristic variations with color conversions.

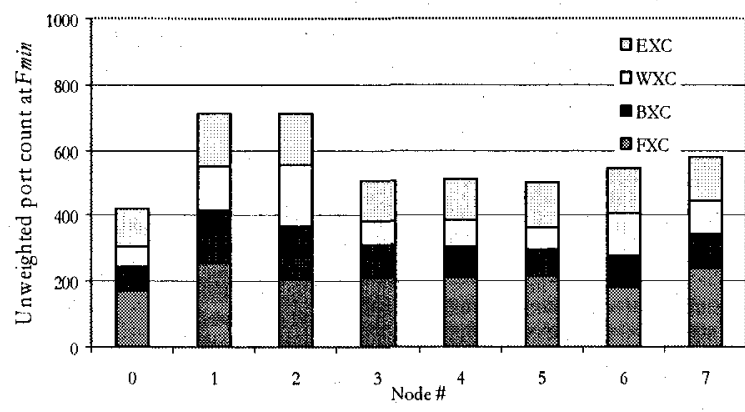

(a)

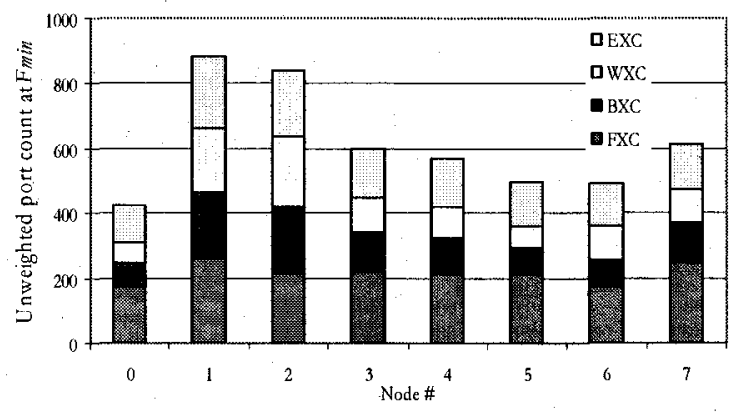

(b)

Fig. 5.9: The resultant "unweighted" port count breakdown at all nodes of the 8 -node network at $F_{\text {min }}=22$ using Policy 4 and the no-bifurcation scenario in the heuristic: (a) with $\mathrm{E} / \mathrm{O}$ ratio $=2$; (b) with $\mathrm{E} / \mathrm{O}$ ratio $=3$. 


\subsubsection{Effects of Maximum Optical Reach}

This subsection demonstrates the effect of changing the value of the maximum optical reach on the planning solution. Here, we solve the RMGPA problem without the color-continuity constraints, with the optical-reach constraints, and without customizing the optical reach. We use the same input data and parameters used in Subsection 5.5.2, except that the ratio of the EXC port weight to the MG-OXC port weight is decided by the relation given in Equation 3.1 with RATIO $_{1000}=2$ and $1000 \leq R_{\text {max }} \leq 10000 \mathrm{~km}$. Heuristic variations in this example are based on using Policies 1-6 and the no-bifurcation scenario. The results of solving the RMGPA problem at $F=F_{\text {min }}$ and $F_{\text {opt }}$ are shown in Figs. 5.10-5.12.

In comparing solutions at both $F_{\text {min }}$ and $F_{p p t}$ and at a certain maximum optical reach value, we observe that the weighted port count at $F_{\text {opt }}$ can achieve greater savings at the expense of having a higher fiber requirement, as shown in Figs. 5.10 and 5.11. This port count saving stems from the increase in the number of clear paths since the need for OEO conversions to perform color conversions vanishes as fiber resources becomes plentiful. The decrease in the number of color conversions can be implied from the decrease in the number of OEO conversions, as shown by the comparison of Figs. 5.12 (a) and 5.12 (b) (note that Fig. 5.12 shows the total number of OEO conversions regardless of whether they are originated from color conversions or signal regenerations).

Moreover, from Figs. 5.10-5.12, it is quite clear that when the maximum optical reach value is close to $1000 \mathrm{~km}$, the fiber requirement, weighted port count, and number of OEO conversions are extremely high. This can be explained as follows. When the maximum optical reach value is less than the length of some physical spans and the network connectivity is low (note that the average physical connectivity of the 8 -node network is 3.25 , which is considered to be low), $s-d$ 
pairs will have limited choices for route selection and some will be forced to use relatively long routes that duplicate resources unnecessarily. This situation leads to the continual usage of the same set of long routes; as a result, significantly more fiber resources; port count, and OEO conversions are required. In contrast, when the reach value exceeds the length of all physical spans, $s-d$ pairs will have more choices for route selection. As a result, shorter and less congested routes are expected to require less fiber resources, port count, and OEO conversions.

However, as we have mentioned in Subsection 5.5.1, increasing the maximum optical reach value raises the cost of the EXC port and reduces the number of OEO conversions. Accordingly, increasing the reach value leads to a crossover situation in which the minimal weighted port count occurs, as shown in Fig. 5.11. Fig. 5.11 (b) clearly shows that the maximum optical reach of 3000 $\mathrm{km}$ along with the use of Policy 6 is the most cost-effective combination for this case study, provided that fiber resources are plentiful. In addition, increasing the maximum optical reach decreases the fiber requirement until it becomes almost unchanged, as shown in Fig. 5.10. This occurs because the number of OEO conversions due to signal regeneration completely vanishes as the maximum optical reach increases. After they vanish, the remaining OEO conversions will be due to color conversions, which are quite noticeable at $F_{\text {min }}$ as depicted in Fig. 5.12 (a).

Now, with the consideration of the optical-reach constraints, deciding the best planning solution for a given range of the maximum optical reach values will be as follows. In the fiber-scarce situation, deciding the best combination in terms of the heuristic variation and maximum optical reach value used will be based on the following criteria. The best combination is the one that achieves the lowest $F_{m i n}$. If more than one combination has the same $F_{m i n}$, then the best combination will be the one that yields the lowest weighted port count at $F_{\text {min }}$. If we still have more than one winning combination, then the one with the lowest maximum optical reach value will be 
selected. Accordingly, Figs. 5.10 (a) and 5.11 (a) demonstrate that the use of Policy 4 with the maximum optical reach of $5000 \mathrm{~km}$ is the most fiber-efficient combination for this case study. On the other hand, in the fiber-plentiful situation, the best combination is the one that realizes the lowest weighted port count at $F_{\text {opr }}$ If more than one combination has the same weighted port count, then the best combination will be the one that achieves the lowest $F_{\text {opt }}$ If still we have more than one winning combination, which is unlikely to occur, we will then choose the one with the lowest maximum optical reach value. Thus, as we have mentioned earlier, the use of Policy 6 with the maximum optical reach of $3000 \mathrm{~km}$ is the most cost-efficient combination for this case study. This finding also shows that the best combination changes according to the fiber situation. For the rest of this thesis, we will refer to the maximum optical reach value that leads to the best solution, whether in fiber-plentiful or scarce situations, as the "best" maximum optical reach.

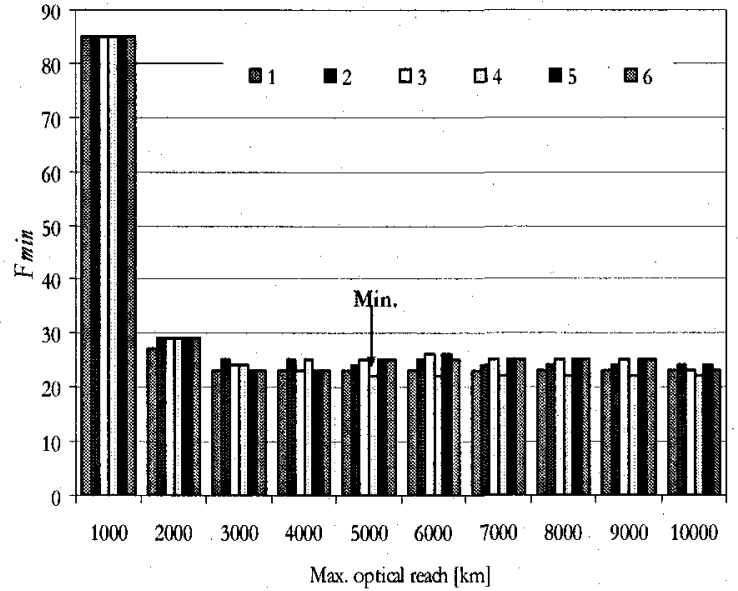

(a)

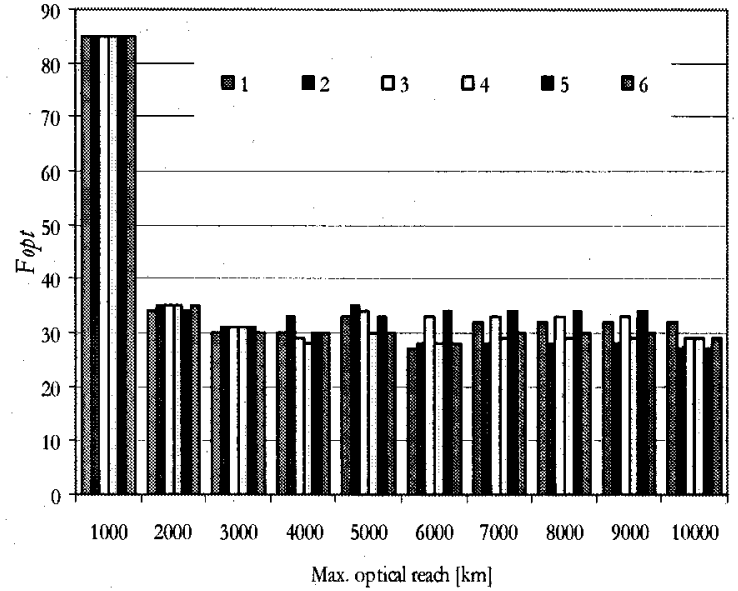

(b)

Fig. 5.10: Fiber requirements vs. maximum optical reach for the case of the 8 -node network and $(0,1500) \mathrm{Gb} / \mathrm{s}$ range demands using heuristics with Policies 1- 6 and the no-bifurcation scenario and without customizing the optical reach: (a) at $F_{p i n i}$; (b) at $F_{\text {opt }}$ 


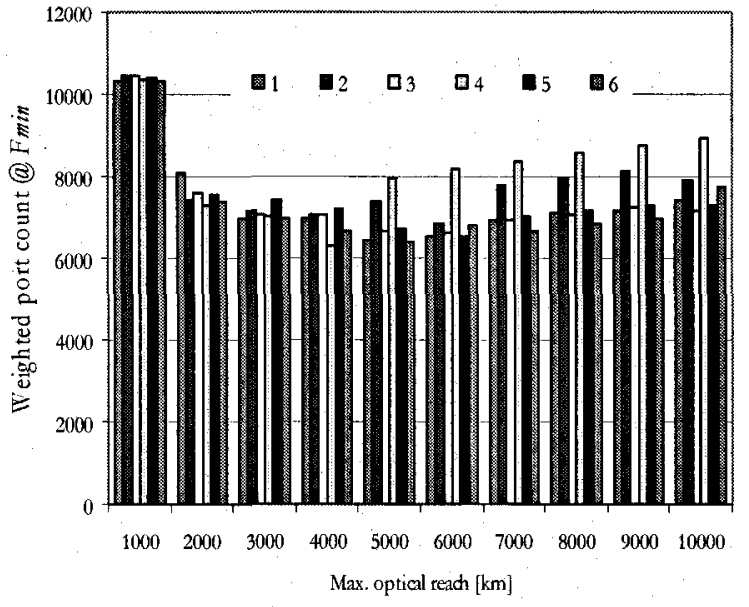

(a)

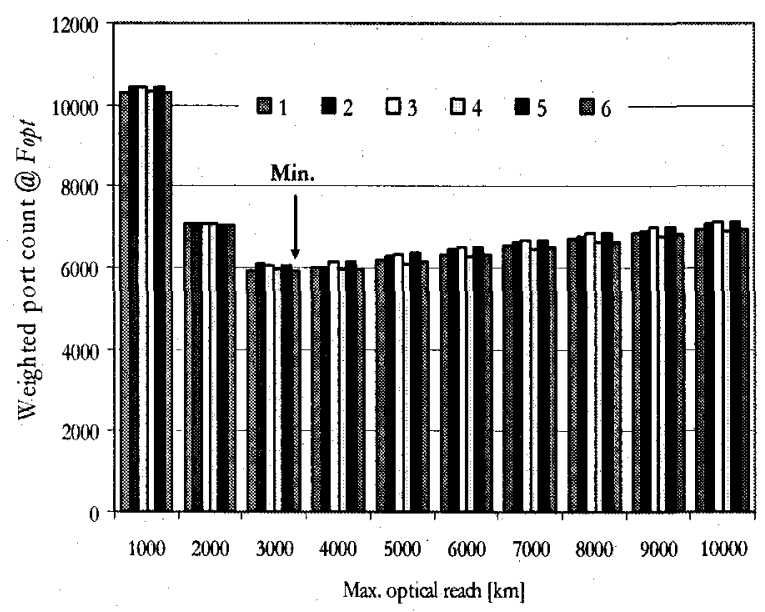

(b)

Fig. 5.11: Weighted port count vs. maximum optical reach for the case of the 8 -node network and $(0,1500) \mathrm{Gb} / \mathrm{s}$ range demands using heuristics with Policies 1-6 and the no-bifurcation scenario and without customizing the optical reach: (a) at $F_{m i n}$ (b) at $F_{\text {opt }}$

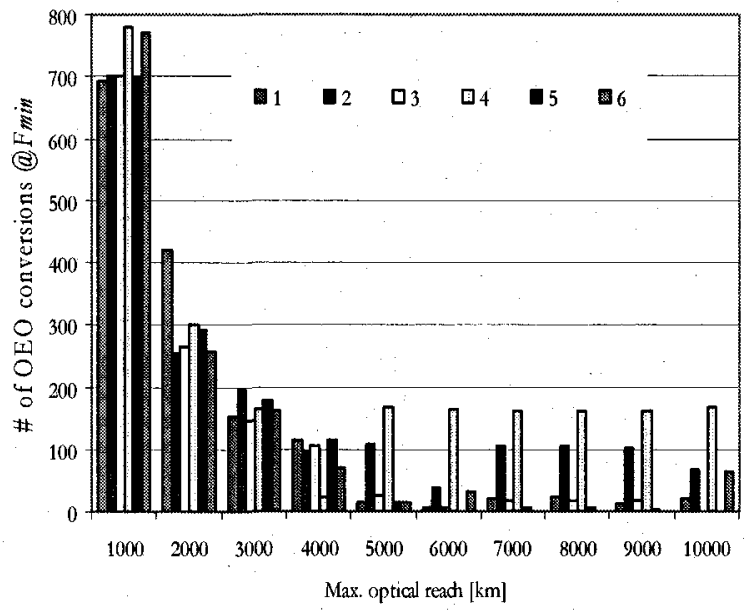

(a)

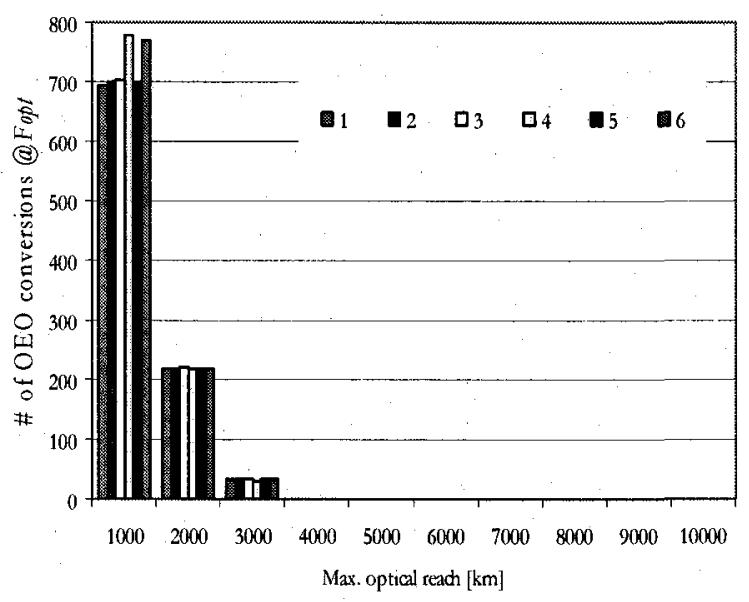

(b)

Fig. 5.12: Number of OEO conversions vs. maximum optical reach for the case of the 8 -node network and $(0,1500)$ $\mathrm{Gb} / \mathrm{s}$ range demands using heuristics with Policies 1-6 and the no-bifurcation scenario and without customizing the optical reach: (a) at $F_{m i n}$; (b) at $F_{\text {opp }}$

\subsubsection{Benefits of Customizing the Optical Reach}

The aim of this subsection is to demonstrate the significance of customizing the optical reach of all clear paths based on their actual lengths rather than utilizing the same maximum optical reach with all clear paths. Here, we solve the RMGPA problem without the color-continuity constraints, with the optical-reach constraints, and with customizing the optical reach. The same example and 
heuristic variations used in the previous subsection are used here. The results at $F=F_{\text {min }}$ and $F_{o p t}$ are shown in Figs. 5.13-5.15.

In the fiber-plentiful situation, even though the cost of the EXC port in the customized reach case is a function of the length of its clear path, varying the maximum optical reach value still leads to a minimal weighted port count as shown in Fig. 5.14 (b). Moreover, for all maximum optical reach values tested, customizing the optical reach for all clear paths leads to consistent savings in the weighted port count compared to the non-customized reach case, as illustrated by the comparison of Fig. 5.14 (b) with Fig. 5.11 (b). It is worth mentioning here that even though the $\approx 9 \%$ saving obtained with the optical-reach customization comes at the expense of an increase from $3000 \mathrm{~km}$ to $6000 \mathrm{~km}$ in the best maximum optical reach, customizing the reach at the maximum optical reach of $3000 \mathrm{~km}$ can still achieve a saving of $\approx 6 \%$.

In fiber-scarce situations, although the same $F_{\text {min }}$ is achieved in both the customized reach case and non-customized reach case, a lower weighted port count at $F_{\text {min }}$ is obtained with the customized reach case, as shown by the comparison of Figs. 5.13 (a) and 5.14 (a) with Figs. 5.10 (a) and 5.11 (a). Similar to the observation made in the fiber-plentiful situation, while the $\approx 14 \%$ saving in the weighted port count obtained with the optical-reach customization comes at the expense of an increase from $5000 \mathrm{~km}$ to $7000 \mathrm{~km}$ in the best maximum optical reach, customizing the reach at the maximum optical reach of $5000 \mathrm{~km}$ can still achieve a saving of $\approx 13.5 \%$.

Furthermore, in comparing the number of OEO conversions with and without customizing the optical reach for all maximum optical reach values tested, it is clear that the number of OEO conversions at $F_{\min }$ is identical, as shown in the comparison of Fig. 5.12 (a) with Fig. 5.15 (a). In addition, at $F_{o p t}$ there is a slight difference between the two cases, as illustrated by the comparison 
of Fig. 5.12 (b) with Fig. 5.15(b). As expected, these results imply that at a certain maximum optical reach value (1) customizing the optical reach does not change the resultant multi-granular virtual topology at $F_{\text {min }}$ since the customization part is performed upon deciding the virtual topology; and (2) customizing the optical reach may lead to a different multi-granular virtual topology at $F_{\text {opt }}$ because with both reach cases the optimum virtual topology has to be decided from the range $\left[F_{m i n}, F_{s a t}\right]$.

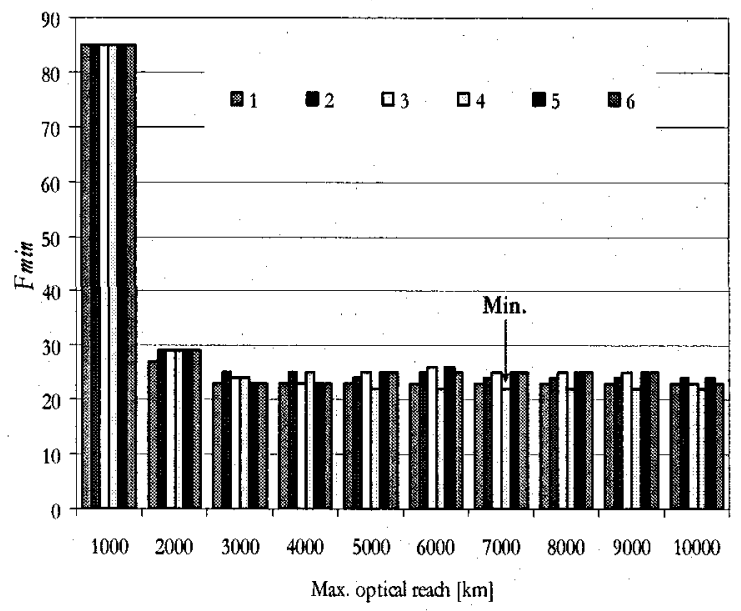

(a)

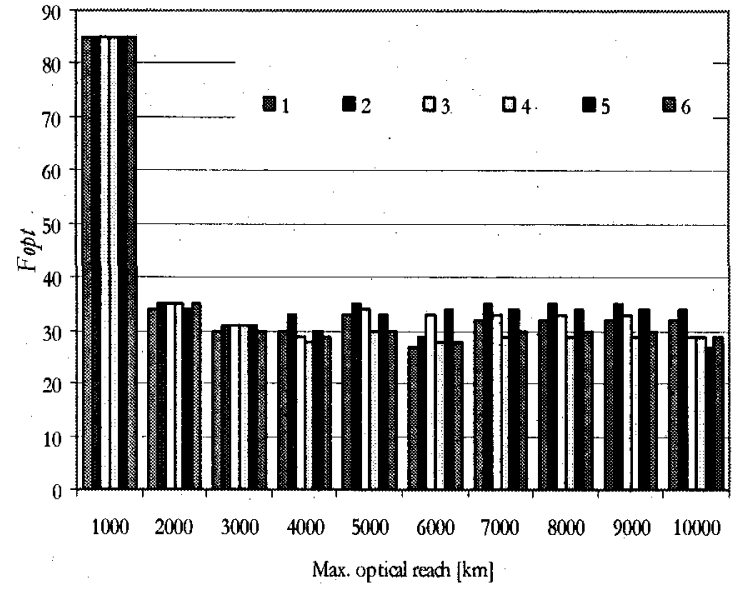

(b)

Fig. 5.13: Fiber requirements vs. maximum optical reach for the case of the 8 -node network and $(0,1500) \mathrm{Gb} / \mathrm{s}$ range demands using heuristics with Policies 1-6 and the no-bifurcation scenario and with customizing the optical reach: (a) at $F_{m i n}$ (b) at $F_{\text {opr }}$

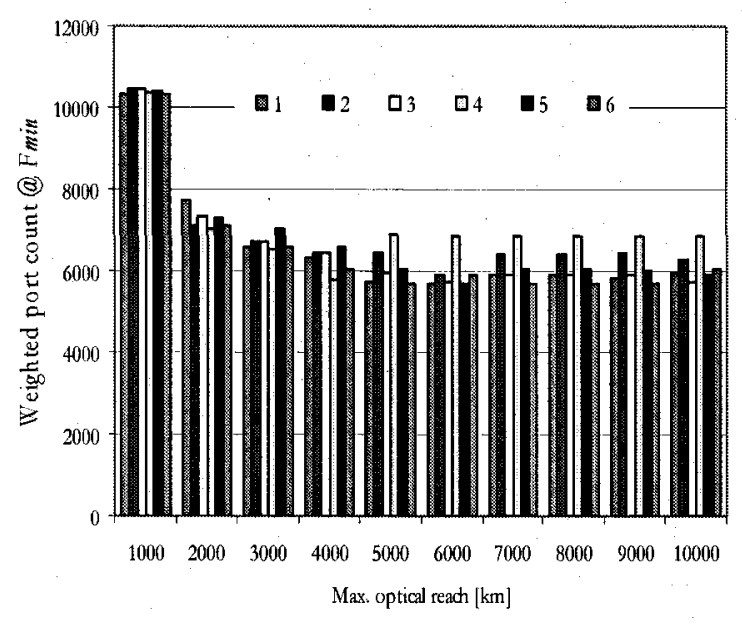

(a)

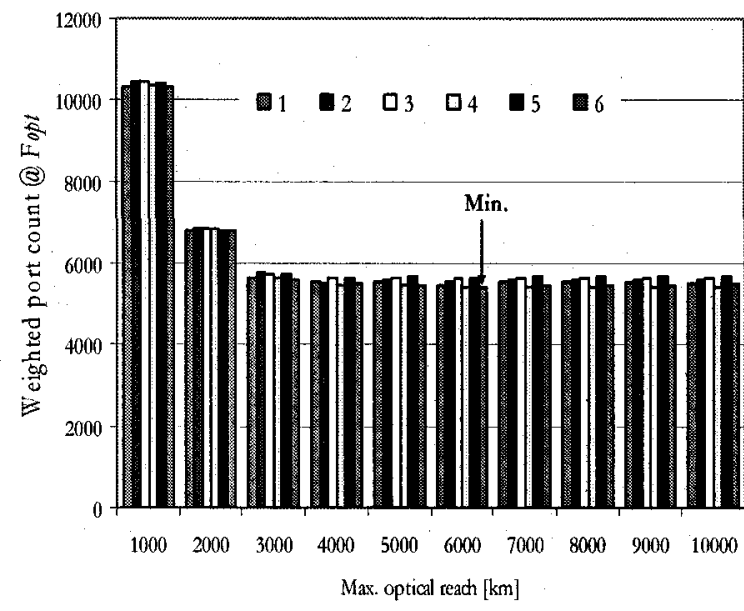

(b)

Fig. 5.14: Weighted port count vs. maximum optical reach for the case of the 8-node network and $(0,1500) \mathrm{Gb} / \mathrm{s}$ range demands using heuristics with Policies $1-6$ and the no-bifurcation scenario and with customizing the optical reach: (a) at $F_{m i n}$; (b) at $F_{o p r}$ 


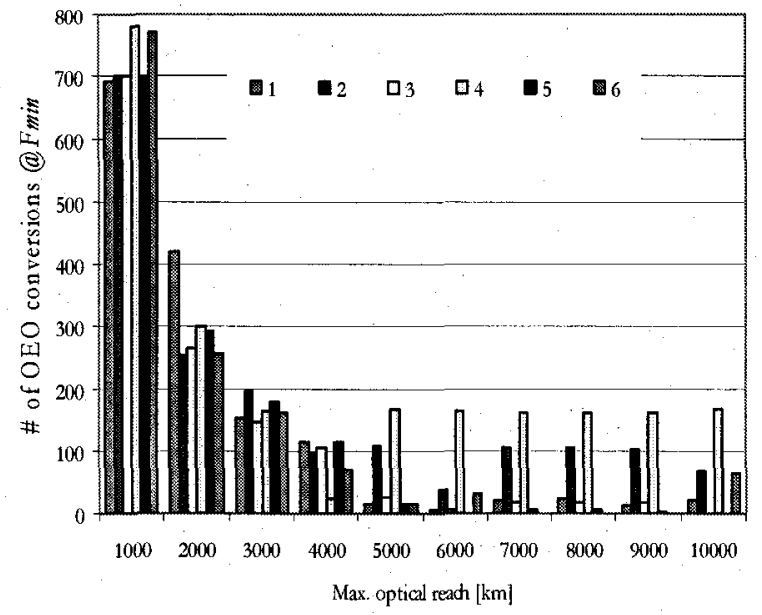

(a)

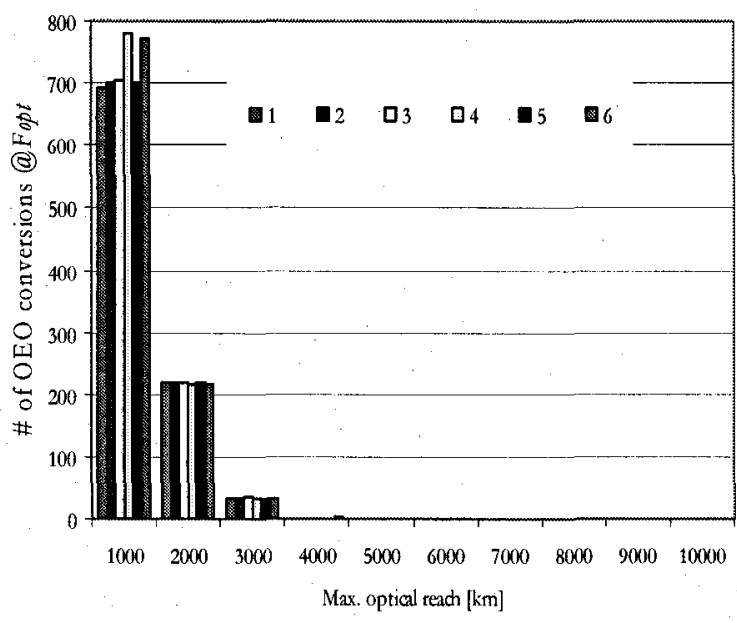

(b)

Fig. 5.15: Number of OEO conversions vs. maximum optical reach for the case of the 8-node network and $(0,1500)$ $\mathrm{Gb} / \mathrm{s}$ range demands using heuristics with Policies 1-6 and the no-bifurcation scenario and with customizing the optical reach: (a) at $F_{m i n}$; (b) at $F_{o p r}$

\subsubsection{Impact of E/O Cost Ratio with Optical-Reach Constraints}

The goal of this subsection is to demonstrate the sensitivity of the planning solution to the change in the $\mathrm{E} / \mathrm{O}$ cost ratio. Here we solve the RMGPA problem without the color-continuity constraints, with the optical-reach constraints, and with/without customizing the optical reach. We employ the same input data and parameters used in Subsection 5.5.2, except that the ratio of the EXC port weight to the MG-OXC weight is decided by the relation given in Equation 3.1 with RATIO $_{1000}=2$ and 3 and $1000 \leq R_{\max } \leq 10000 \mathrm{~km}$. Heuristic variations in this example are based on using Policies 1-6 and the no-bifurcation scenario. Results of the best combination in terms of the best maximum optical reach and best heuristic variation in both fiber situations using both reach cases are shown in Tables 5.4 and 5.5.

From the tables, it is clear that changing the $\mathrm{E} / \mathrm{O}$ cost ratio has an impact on changing the winning combination, especially in the fiber-plentiful situations. Overall, for similar reasons to those explained in Subsection 5.5.3, changing the cost ratio leads to the change in the optimum multi-granular virtual topology. Even when the winning combination does not change, still the 
change in the virtual topology can be implied from the change in the number of OEO conversions, as shown in Table 5.4. Besides, decreasing the $\mathrm{E} / \mathrm{O}$ cost ratio decreases the amount of savings in the weighted port count achieved with customizing the optical reach in both fiber situations. Tables 5.4 and 5.5 demonstrate that the saving using the $\mathrm{E} / \mathrm{O}$ cost ratio $=3$ is $\approx$ $14 / 20 \%$, whereas the saving using the $\mathrm{E} / \mathrm{O}$ ratio $=2$ is $\approx 9 / 10 \%$, in the fiber-scarce/fiber-plentiful situation.

Table 5.4: Summary of the heuristics comparison in the fiber-scarce situation with and without the optical-reach customization for the case of the 8-node network and $(0,1500) \mathrm{Gb} / \mathrm{s}$ range demands using different $\mathrm{E} / \mathrm{O}$ cost ratios.

\begin{tabular}{c|c|c|c|c|c|c|c|c|c|c} 
& \multicolumn{4}{|c|}{ Without Customized Reach } & \multicolumn{5}{c}{ With Customized Reach } \\
RATIO $_{1000}$ & $\begin{array}{c}\text { Best } R_{\max } \\
{[\mathrm{km}]}\end{array}$ & $\begin{array}{c}\text { Best } \\
\text { policy }\end{array}$ & $F_{\min }$ & $\begin{array}{c}\text { Weighted } \\
\text { port count } \\
\text { @ } F_{\min }\end{array}$ & $\begin{array}{c}\text { \# of OEO } \\
\text { Conversions } \\
@ F_{\min }\end{array}$ & $\begin{array}{c}\text { Best } R_{\max } \\
{[\mathrm{km}]}\end{array}$ & $\begin{array}{c}\text { Best } \\
\text { policy }\end{array}$ & $F_{\min }$ & $\begin{array}{c}\text { Weighted } \\
\text { port count } \\
@ F_{\text {min }}\end{array}$ & $\begin{array}{c}\text { \# of OEO } \\
\text { Conversions } \\
@ F_{\min }\end{array}$ \\
\hline 2 & 5000 & 4 & 22 & 7920.33 & 166 & 7000 & 4 & 22 & 6805.15 & 161 \\
\hline 3 & 5000 & 4 & 22 & 10018.92 & 165 & 9000 & 4 & 22 & 7972.35 & 130
\end{tabular}

Table 5.5: Summary of the heuristics comparison in the fiber-plentiful situation with and without the optical-reach customization for the case of the 8-node network and $(0,1500) \mathrm{Gb} / \mathrm{s}$ range demands using different $\mathrm{E} / \mathrm{O}$ cost ratios.

\begin{tabular}{|c|c|c|c|c|c|c|c|c|c|c|}
\hline \multirow[b]{2}{*}{ RATIOO $_{1000}$} & \multicolumn{5}{|c|}{ Without Customized Reach } & \multicolumn{5}{|c|}{ With Customized Reach } \\
\hline & $\begin{array}{c}\text { Best } R_{m a x} \\
{[\mathrm{~km}]}\end{array}$ & $\begin{array}{l}\text { Best } \\
\text { policy }\end{array}$ & $F_{p p t}$ & $\begin{array}{l}\text { Weighted } \\
\text { port count } \\
\text { (a) } F_{o b t}\end{array}$ & $\begin{array}{c}\text { \# of OEO } \\
\text { Conversions } \\
\text { @ } F_{o p t}\end{array}$ & $\begin{array}{c}\text { Best } R_{\max } \\
{[\mathrm{km}]}\end{array}$ & $\begin{array}{c}\text { Best } \\
\text { policy }\end{array}$ & $F_{o p t}$ & $\begin{array}{c}\text { Weighted } \\
\text { port count } \\
\text { @ } F_{\text {opt }}\end{array}$ & $\begin{array}{c}\text { \# of OEO } \\
\text { Conversions } \\
\text { @ } F_{o p t}\end{array}$ \\
\hline 2 & 3000 & 6 & 30 & 5930.19 & 33 & 6000 & 6 & 28 & 5409.11 & 0 \\
\hline 3 & 3000 & 1 & 29 & 7341.14 & 32 & 9000 & 4 & 30 & 6617.36 & 0 \\
\hline
\end{tabular}

\subsubsection{Comparison of Heuristics with Color Conversion Capability}

In this subsection, we evaluate heuristics via simulation without the consideration of either the color-continuity or optical-reach constraints. In addition, to investigate the impact of performing color conversion at all granularity levels, we compare the results of the heuristic variations with the results obtained in Subsection 4.4.7, in which the color-continuity constraints are considered. 
Heuristic variations that are based on the all-bifurcation scenario are excluded from our comparisons in this subsection and the following subsection. This is because we expect the performance of such heuristics to be worse when employing the color conversion and signal regeneration capabilities, since the use of separate unclear paths by the bifurcated units of traffic demands for the purposes of color conversion and signal regeneration will further duplicate resources and port requirements.

The procedure for generating traffic patterns used in Subsection 4.4 .7 is utilized again here. The physical topologies considered in the simulation are the 8-node, NSF, and EON networks, as shown in Figs. 4.11, 4.26, and 4.27 respectively. The fiber capacities used here are based on using the $10-4-4,10-8-8$, and $40-8-8$ combinations. However, due to the space limitation in this and the following subsection, the sensitivity analysis of the fiber capacity is carried out only on the 8-node network using the three combinations. On the other hand, the sensitivity analysis of the physical topology is carried out on all three topologies using only the $10-8-8$ combination. The rest of the input parameters employed in the simulation are as follows: $1 \leq F \leq F_{\text {sal }}$, all $\mathrm{MG}-\mathrm{OXC} / \mathrm{EXC}$ ports have the same weight, and the $10 / 40 \mathrm{~Gb} / \mathrm{s}$-based EXC port weight is $2 / 5$ times the MG-OXC port weight.

Detailed results based on averaging 10 simulation runs with $95 \%$ confidence intervals are shown in Appendix B. In addition to the fiber requirements and weighted port counts, the results of the heuristic variations are also compared in terms of the number of OEO conversions. Tables '5.6$5.10^{\prime} /{ }^{\prime} 5.11-5.15^{\prime}$ summarize the results in Tables B.1-B.6 and provide comparisons with the results in Tables '4.10-4.12, 4.14, and 4.17'/‘4.19-4.21, 4.23, and 4.26' under the fiber-scarce/plentiful situation. 
We first analyze the results obtained in the fiber-scarce situation. Overall, from Tables 5.6-5.10, the following observations can be made. First, allowing color conversions has an obvious affect on reducing the minimum fiber requirement (up to $\approx 67 \%$ reduction) compared to the case in which color conversions are not allowed. The amount of reduction varies according to the traffic pattern, network topology, and fiber capacity. This reduction is the result of using unclear paths when fiber resources are not sufficient to establish clear paths. Second, the reduction in the fiber requirement can be accompanied by either a decrease (up to $\approx 6 \%$ ) or an increase (up to $\approx 12 \%$ ) in the weighted port count compared to the case in which color conversions are not allowed. Third, even with traffic patterns in which there are no reductions in $F_{m i n}$, performing color conversions can still bring further slight weighted port count reductions (up to $\approx 4 \%$ ). It is interesting to note that this slight reduction can be realized either with or without OEO conversions. This observation can be made by first identifying these reduction situations in Tables 5.6-5.10. Then both the corresponding traffic pattern and the winning heuristic variation can be located in Appendix B, after which the corresponding number of OEO conversions at $F_{m i n}$ can be found. The reduction in the weighted port count with the existence of OEO conversions is due to the fact that some $s-d$ pairs prefer short unclear paths to long expensive clear paths. On the other hand, the reduction in the weighted port count with no OEO conversions occurs because the heuristic with color conversions works differently than the heuristic without color conversions.

In comparing the winning heuristic variations with and without maintaining the color-continuity constraints, as shown by the comparison of Tables $4.10-4.12,4.14$, and 4.17 with Tables 5.6-5.10, the following comments can be made. First, the winning sets of bifurcation scenarios/sorting policies for all traffic patterns tested, each at a certain physical topology and fiber capacity, are not the same. Second, it is interesting to see that Policy 3 is out of the contest in all traffic patterns 
tested with the 8-node and NSF networks, in which the physical connectivity is lower than in the EON network, compared to the case where color conversions are not allowed. Therefore, not only is choosing the best heuristic variation a function of the traffic pattern, physical topology and fiber capacity, but it is also a function of whether color conversions are allowed.

Furthermore, in looking for the $C-W-B$ combination/fiber capacity that can achieve the best results in the fiber-scarce situation by comparing Tables $5.6-5.8$, it is clear that the $40-8-8$ combination is the best choice for all traffic patterns. In contrast, without color conversions, as shown in Tables 4.10-4.12, although the best combination for the majority of traffic patterns is the $40-8-8$ combination, the best choice for the traffic pattern with $I N=1000 \mathrm{~Gb} / \mathrm{s}$ and $F R=80 \%$ is the 10-8-8 combination. Thus, this result indicates that choosing the best $C-W-B$ combination in the fiber-scarce situation is also a function of whether color conversions are allowed.

Tables 5.7, 5.9, and 5.10 indicate beyond doubt that the physical topology has an enormous impact on reducing the minimum fiber requirement. In the EON network, which has the highest physical connectivity in comparison to the 8-node and NSF networks, a significant reduction in $F_{\text {min }}$ in the range of $\approx 50-67 \%$ is achieved with all traffic patterns. These reductions can come with a weighted port count decrease/increase of up to $\approx 6.5 / 12 \%$. This dramatic reduction stems from the fact that the number of distinct routes between an $s$ - $d$ pair can be as high as 1720 routes in the EON network, which increases the availability of unclear paths at a certain $F$. On the other hand, in the 8-node and NSF networks the number of distinct routes between an $s-d$ pair is relatively low, which relatively decreases the availability of unclear paths at the same $F$. 
Table 5.6: Results of the best heuristic variation with the color conversion capability for different traffic patterns in the 8 -node network using the 10-4-4 combination under the fiber-scarce situation. The difference in results is relative to the best results obtained by keeping the color-continuity constraints.

\begin{tabular}{|c|c|c|c|c|c|c|}
\hline $\begin{array}{l}\text { Traffic pattern: } \\
(I N G b / \mathrm{s}, F R \%)\end{array}$ & $\begin{array}{l}\text { Best bifurcation } \\
\text { scenario }\end{array}$ & $\begin{array}{c}\text { Best } \\
\text { policy }\end{array}$ & $F_{\min }$ & $\begin{array}{l}\text { Weighted } \\
\text { port count } \\
\text { @ } F_{\min }\end{array}$ & $\begin{array}{c}\text { Difference in } \\
F_{\min }\end{array}$ & $\begin{array}{c}\text { Difference in } \\
\text { Weighted port } \\
\text { count } @ F_{\min }\end{array}$ \\
\hline$(500,0)$ & No bifurcation & 4 & 8.2 & 3004.70 & $-2.38 \%$ & $-0.01 \%$ \\
\hline$(1000,0)$ & No bifurcation & 4 & 15.4 & 3980.30 & $-6.10 \%$ & $-1.17 \%$ \\
\hline$(1500,0)$ & Fiber bifurcation & 4 & 22.4 & 4981.90 & $-5.88 \%$ & $4.43 \%$ \\
\hline$(500,20)$ & Fiber bifurcation & 4 & 6.6 & 2542.80 & $-13.16 \%$ & $2.83 \%$ \\
\hline$(1000,20)$ & Fiber bifurcation & 2 & 12.6 & 3319.50 & $-5.97 \%$ & $5.06 \%$ \\
\hline$(1500,20)$ & Fiber bifurcation & 4 & 18 & 4011.30 & $-7.22 \%$ & $5.18 \%$ \\
\hline$(500,50)$ & No bifurcation & 4 & 5.4 & 1813.00 & $-6.90 \%$ & $1.95 \%$ \\
\hline$(1000,50)$ & No bifurcation & 1 & 9.6 & 2376.00 & $-5.88 \%$ & $-1.16 \%$ \\
\hline$(1500,50)$ & Fiber bifurcation & 4 & 14.6 & 2978.70 & $-3.95 \%$ & $4.33 \%$ \\
\hline$(500,80)$ & No bifurcation & 4 & 3.4 & 998.80 & $0.00 \%$ & $-0.18 \%$ \\
\hline$(1000,80)$ & Fiber bifurcation & 4 & 5.6 & 1290.30 & $0.00 \%$ & $-0.18 \%$ \\
\hline$(1500,80)$ & Fiber bifurcation & 1 & 7.6 & 1725.60 & $0.00 \%$ & $-0.08 \%$ \\
\hline
\end{tabular}

Table 5.7: Results of the best heuristic variation with the color conversion capability for different traffic pattems in the 8 -node network using the $10-8-8$ combination under the fiber-scarce situation. The difference in results is relative to the best results obtained by keeping the color-continuity constraints.

\begin{tabular}{|c|c|c|c|c|c|c|}
\hline $\begin{array}{l}\text { Traffic pattern: } \\
\text { (INGb/s,FR\%) }\end{array}$ & $\begin{array}{c}\text { Best bifurcation } \\
\text { scenario }\end{array}$ & $\begin{array}{c}\text { Best } \\
\text { policy }\end{array}$ & $F_{\min }$ & $\begin{array}{l}\text { Weighted } \\
\text { port count } \\
\text { (a) } F_{\min }\end{array}$ & $\begin{array}{c}\text { Difference in } \\
F_{m i n}\end{array}$ & $\begin{array}{l}\text { Difference in } \\
\text { Weighted port } \\
\text { count } @ F_{m i n}\end{array}$ \\
\hline$(500,0)$ & No bifurcation & 4 & 2.2 & 4081.60 & $-8.33 \%$ & $0.05 \%$ \\
\hline$(1000,0)$ & No bifurcation & 4 & 4.4 & 4384.50 & $0.00 \%$ & $-0.04 \%$ \\
\hline$(1500,0)$ & No bifurcation & 4 & 6.2 & 4822.50 & $-8.82 \%$ & $2.10 \%$ \\
\hline$(500,20)$ & No bifurcation & 4 & 2 & 3212.50 & $0.00 \%$ & $-0.46 \%$ \\
\hline$(1000,20)$ & No bifurcation & 4 & 4 & 3499.60 & $0.00 \%$ & $-0.64 \%$ \\
\hline$(1500,20)$ & No bifurcation & 4 & 5.8 & 3823.60 & $-3.33 \%$ & $1.08 \%$ \\
\hline$(500,50)$ & No bifurcation & 4 & 1.9 & 2239.70 & $-5.00 \%$ & $-0.35 \%$ \\
\hline$(1000,50)$ & No bifurcation & 4 & 3.4 & 2521.70 & $-5.56 \%$ & $-0.16 \%$ \\
\hline$(1500,50)$ & No bifurcation & 4 & 4.4 & 2763.00 & $0.00 \%$ & $-0.23 \%$ \\
\hline$(500,80)$ & No bifurcation & 4 & 1 & 1136.20 & $-50.00 \%$ & $0.32 \%$ \\
\hline$(1000,80)$ & No bifurcation & 4 & 1.8 & 1335.30 & $-10.00 \%$ & $0.73 \%$ \\
\hline$(1500,80)$ & Fiber bifurcation & 4 & 2.3 & 1600.60 & $-4.17 \%$ & $-0.31 \%$ \\
\hline
\end{tabular}


Table 5.8: Results of the best heuristic variation with the color conversion capability for different traffic patterns in the 8-node network using the 40-8-8 combination under the fiber-scarce situation. The difference in results is relative to the best results obtained by keeping the color-continuity constraints.

\begin{tabular}{c|c|c|c|c|c|c}
$\begin{array}{c}\text { Traffic pattern: } \\
(I N G \text { G } / \mathbf{s}, \boldsymbol{R} \%)\end{array}$ & $\begin{array}{c}\text { Best bifurcation } \\
\text { scenario }\end{array}$ & $\begin{array}{c}\text { Best } \\
\text { policy }\end{array}$ & $\boldsymbol{F}_{\min }$ & $\begin{array}{c}\text { Weighted } \\
\text { port count } \\
\text { @ } \boldsymbol{F}_{\min }\end{array}$ & $\begin{array}{c}\text { Difference in } \\
\boldsymbol{F}_{\min }\end{array}$ & $\begin{array}{c}\text { Difference in } \\
\text { Weighted port } \\
\text { count @ } \boldsymbol{F}_{\text {min }}\end{array}$ \\
\hline$(500,0)$ & No bifurcation & 4 & 1 & 3798.00 & $-50.00 \%$ & $-0.37 \%$ \\
\hline$(1000,0)$ & No bifurcation & 4 & 1.5 & 4894.80 & $-25.00 \%$ & $0.40 \%$ \\
\hline$(1500,0)$ & No bifurcation & 4 & 2 & 5496.10 & $0.00 \%$ & $-0.39 \%$ \\
\hline$(500,20)$ & No bifurcation & 4 & 1 & 3082.80 & $-50.00 \%$ & $-0.01 \%$ \\
\hline$(1000,20)$ & No bifurcation & 4 & 1 & 3868.10 & $-50.00 \%$ & $0.45 \%$ \\
\hline$(1500,20)$ & No bifurcation & 1 & 1.9 & 4390.10 & $-5.00 \%$ & $1.90 \%$ \\
\hline$(500,50)$ & No bifurcation & 4 & 1 & 2124.90 & $-50.00 \%$ & $-0.12 \%$ \\
\hline$(1000,50)$ & No bifurcation & 4 & 1 & 2716.60 & $-50.00 \%$ & $-0.15 \%$ \\
\hline$(1500,50)$ & No bifurcation & 4 & 1.2 & 3055.00 & $-40.00 \%$ & $0.65 \%$ \\
\hline$(500,80)$ & No bifurcation & 4 & 1 & 1069.80 & $-50.00 \%$ & $-0.29 \%$ \\
\hline$(1000,80)$ & No bifurcation & 4 & 1 & 1337.50 & $-50.00 \%$ & $-0.71 \%$ \\
\hline$(1500,80)$ & No bifurcation & 4 & 1 & 1558.40 & $-50.00 \%$ & $-0.54 \%$
\end{tabular}

Table 5.9: Results of the best heuristic variation with the color conversion capability for different traffic patterns in the NSF network using the 10-8-8 combination under the fiber-scarce situation. The difference in results is relative to the best results obtained by keeping the color-continuity constraints.

\begin{tabular}{|c|c|c|c|c|c|c|}
\hline $\begin{array}{l}\text { Traffic pattern: } \\
(I N G b / s, F R \%)\end{array}$ & $\begin{array}{c}\text { Best bifurcation } \\
\text { scenario }\end{array}$ & $\begin{array}{c}\text { Best } \\
\text { policy }\end{array}$ & $F_{m i n}$ & $\begin{array}{l}\text { Weighted } \\
\text { port count } \\
\qquad @ F_{m i n}\end{array}$ & $\begin{array}{c}\text { Difference in } \\
F_{m i n}\end{array}$ & $\begin{array}{l}\text { Difference in } \\
\text { Weighted port } \\
\text { count } @ F_{\text {min }}\end{array}$ \\
\hline$(500,0)$ & No bifurcation & 4 & 7 & 14709.50 & $0.00 \%$ & $-0.46 \%$ \\
\hline$(1000,0)$ & No bifurcation & 1 & 11.8 & 16324.20 & $-4.84 \%$ & $0.16 \%$ \\
\hline$(1500,0)$ & No bifurcation & 4 & 17.6 & 17325.30 & $-3.30 \%$ & $-0.66 \%$ \\
\hline$(500,20)$ & No bifurcation & 1 . & 5.2 & 12338.90 & $-7.14 \%$ & $-1.50 \%$ \\
\hline$(1000,20)$ & No bifurcation & 2 & 10.2 & 13789.40 & $-3.77 \%$ & $1.72 \%$ \\
\hline$(1500,20)$ & No bifurcation & 1 & 14.4 & 15205.00 & $-6.49 \%$ & $6.06 \%$ \\
\hline$(500,50)$ & No bifurcation & 4 & 4.8 & 8498.40 & $-4.00 \%$ & $0.63 \%$ \\
\hline$(1000,50)$ & No bifurcation & 1 & 7 & 9483.80 & $-7.89 \%$ & $-1.21 \%$ \\
\hline$(1500,50)$ & Fiber bifurcation & 4 & 10.4 & 10724.60 & $-8.77 \%$ & $4.55 \%$ \\
\hline$(500,80)$ & No bifurcation & 1 & 3 & 4465.90 & $0.00 \%$ & $-3.54 \%$ \\
\hline$(1000,80)$ & No bifurcation & 4 & 4.4 & 5116.90 & $-4.35 \%$ & $-4.00 \%$ \\
\hline$(1500,80)$ & No bifurcation & 1 & 5.2 & 6080.70 & $-7.14 \%$ & $-2.29 \%$ \\
\hline
\end{tabular}


Table 5.10: Results of the best heuristic variation with the color conversion capability for different traffic patterns in the EON network using the 10-8-8 combination under the fiber-scarce situation. The difference in results is relative to the best results obtained by keeping the color-continuity constraints.

\begin{tabular}{|c|c|c|c|c|c|c|}
\hline $\begin{array}{l}\text { Traffic pattern: } \\
(I N G b / \mathrm{s}, F R \%)\end{array}$ & $\begin{array}{c}\text { Best bifurcation } \\
\text { scenario }\end{array}$ & $\begin{array}{c}\text { Best } \\
\text { policy }\end{array}$ & $F_{m i n}$ & $\begin{array}{l}\text { Weighted } \\
\text { port count } \\
@ F_{\min }\end{array}$ & $\begin{array}{c}\text { Difference in } \\
\qquad F_{m i n}\end{array}$ & $\begin{array}{l}\text { Difference in } \\
\text { Weighted port } \\
\text { count@ } F_{\min }\end{array}$ \\
\hline$(500,0)$ & No bifurcation & 3 & 4 & 9626.70 & $-59.18 \%$ & $0.09 \%$ \\
\hline$(1000,0)$ & No bifurcation & 1 & 7.4 & 10244.90 & $-59.78 \%$ & $-1.65 \%$ \\
\hline$(1500,0)$ & No bifurcation & 3 & 9.8 & 10968.70 & $-66.89 \% \%^{*}$ & $0.99 \%$ \\
\hline$(500,20)$ & No bifurcation & 1 & 3.2 & 8095.20 & $-63.64 \%$ & $1.54 \%$ \\
\hline$(1000,20)$ & No bifurcation & 2 & 6 & 8873.60 & $-63.41 \%$ & $0.98 \%$ \\
\hline$(1500,20)$ & No bifurcation & 3 & 8.6 & 9203.20 & $-62.93 \%$ & $-0.49 \%$ \\
\hline$(500,50)$ & No bifurcation & 1 & 3 & 5716.00 & $-50.00 \%$ & $6.31 \%$ \\
\hline$(1000,50)$ & No bifurcation & 1 & 4.2 & 5785.10 & $-58.00 \%$ & $-1.59 \%$ \\
\hline$(1500,50)$ & Fiber bifurcation & 3 & 5.8 & 6523.80 & $-61.84 \%$ & $0.09 \%$ \\
\hline$(500,80)$ & No bifurcation & 4 & 1 & 2818.60 & $-66.67 \%$ & $-6.46 \%$ \\
\hline$(1000,80)$ & No bifurcation & 2 & 2.8 & 3643.70 & $-50.00 \%$ & $11.84 \%$ \\
\hline$(1500,80)$ & Fiber bifurcation & 1 & 3 & 3719.10 & $-50.00 \%$ & $-2.10 \%$ \\
\hline
\end{tabular}

Now, we analyze the results obtained in the fiber-plentiful situation. In general, in comparing Tables 5.11-5.15 with Tables 5.6-5.10, it is clear that increasing the fiber resources leads to a reduction in the weighted port count. This is due to the decrease in the number of unclear paths and/or the number of clear paths that constitute the unclear path (or the decrease in the virtual hop count of the unclear path), as clear paths becomes favorable choices for $s-d$ pairs. This decrease can be implied from the fading of the number of OEO conversions at $F_{o p t}$ with respect to the equivalent number at $F_{\text {mith }}$ as shown in Appendix B.

Moreover, from Tables 5.11-5.15 and Tables B.1-B.6, for most traffic patterns the reduction/increase in the weighted port count at $F_{\text {opt }}$ compared to the case in which color conversions are not allowed is up to $\approx 3 / 1 \%$. This slight difference can be achieved both with and without the need for OEO conversions at $F_{\text {opp }}$ explained as follows. (1) The number of OEO 
conversions at $F_{o p t}$ can be zero, and there is a decrease in the weighted port count. (2) The number of OEO conversions at $F_{\text {opt }}$ is greater than zero, but there is a slight increase in the weighted port count. Both the former and latter results can occur because, as we mentioned earlier, the heuristic with color conversions works differently than the heuristic without color conversions. (3) OEO conversions exist at $F_{\text {opp }}$ and there is a slight decrease in the weighted port count (up to $\approx 3 \%$ ). This result stems from the fact that, for a certain $s-d$ pair, selecting a short unclear path rather than a long clear path is more cost-effective. It is worth mentioning here that this decrease in the weighted port count can be accompanied by a reduction in $F_{\text {opt }}$ as well (up to $\approx 57 \%$ ).

In comparing the winning heuristic variations with and without keeping the color-continuity constraints, as shown by the comparison of Tables 4.19-4.21, 4.23, and 4.26 with Tables 5.11-5.15, the following observations can be made. First, Policies 1 and 4 are still the only contenders. Second, in the 8-node and NSF networks, which have a lower connectivity than the EON network, the winning sets of bifurcation scenarios/sorting policies are slightly different for all traffic patterns tested at a certain topology and fiber capacity. In contrast, in the EON network, the winning sets are noticeably different. This result is expected because the achieved differences in the weighted port count are greater with the EON network than with the 8-node and NSF networks, as illustrated in Tables 5.12, 5.14, and 5.15. In short, in the fiber-plentiful situation, as in the fiber-scarce situation, the winning heuristic variation is still a function of whether color conversions are allowed.

In looking for the $C-W-B$ combination/fiber capacity that can achieve the best planning results by comparing Tables 5.11-5.13, it is clear that the best choices for all traffic patterns are similar to those obtained without color conversions, as shown in Table 4.28. Again, this similarity has to do with the insignificant difference in the weighted port count between heuristics with and without 
color conversions. Therefore, this finding indicates that choosing the best $C-W-B$ combination in the fiber-plentiful situation, as apposed to the fiber-scarce situation, is less sensitive to whether color conversions are allowed.

Finally, Tables 5.12, 5.14, and 5.15 capture the impact of color conversions as the physical topology changes. Although increasing both the connectivity and size of the topology with the use of color conversions has a slight impact on reducing the weighted port count at $F_{o p p}$ as is the case with the EON network (in which the reduction comes with the number of $O E O \neq 0$, as shown in Appendix B), the decrease in $F_{a p t}$ is still considerable. This dramatic decrease stems from the fact that the high number of distinct routes between an $s$ - $d$ pair increases the availability of short unclear paths, which avoids the duplication of resources. It is interesting to note that allowing the bifurcation of fiber demands in the EON network is quite beneficial when color conversions are considered, as shown by the comparison of Table 4.26 with Table 5.15 . This is because the availability of less expensive unclear paths for a bifurcated unit of the fiber demand is greater than when the fiber demand is treated as a whole entity. 
Table 5.11: Results of the best heuristic variation with the color conversion capability for different traffic patterns in the 8-node network using the 10-4-4 combination under the fiber-plentiful situation. The difference in results is relative to the best results obtained by keeping the color-continuity constraints.

\begin{tabular}{|c|c|c|c|c|c|c|}
\hline $\begin{array}{l}\text { Traffic pattern: } \\
(I N G b / s, F R \%)\end{array}$ & $\begin{array}{l}\text { Best bifurcation } \\
\text { scenario }\end{array}$ & $\begin{array}{c}\text { Best } \\
\text { policy }\end{array}$ & $F_{o p t}$ & $\begin{array}{l}\text { Weighted } \\
\text { port count } \\
\text { @ } F_{\text {opt }}\end{array}$ & $\begin{array}{c}\text { Difference in } \\
F_{o p t}\end{array}$ & $\begin{array}{c}\text { Difference in } \\
\text { Weighted port } \\
\text { count @ } F_{\text {opt }}\end{array}$ \\
\hline$(500,0)$ & No bifurcation & 4 & 9.40 & 2981.10 & $-7.84 \%$ & $-0.04 \%$ \\
\hline$(1000,0)$ & Fiber bifurcation & 4 & 21.20 & 3841.10 & $0.95 \%$ & $-0.28 \%$ \\
\hline$(1500,0)$ & No bifurcation & 4 & 27.40 & 4613.30 & $-13.84 \%$ & $-0.10 \%$ \\
\hline$(500,20)$ & Fiber bifurcation & 4 & 9.60 & 2417.20 & $-2.04 \%$ & $-0.24 \%$ \\
\hline$(1000,20)$ & Fiber bifurcation & 1 & 18.20 & 3089.40 & $8.33 \%$ & $-0.23 \%$ \\
\hline$(1500,20)$ & Fiber bifurcation & 4 & 25.00 & 3722.80 & $-5.30 \%$ & $-0.14 \%$ \\
\hline$(500,50)$ & Fiber bifurcation & 4 & 7.00 & 1750.40 & $-10.26 \%$ & $-0.40 \%$ \\
\hline$(1000,50)$ & No bifurcation & 4 & 13.20 & 2237.90 & $3.12 \%$ & $-0.03 \%$ \\
\hline$(1500,50)$ & No bifurcation & 4 & 19.60 & 2762.70 & $3.16 \%$ & $-0.16 \%$ \\
\hline$(500,80)$ & No bifurcation & 4 & 5.00 & 975.80 & $0.00 \%$ & $0.29 \%$ \\
\hline$(1000,80)$ & No bifurcation & 4 & 8.80 & 1224.80 & $0.00 \%$ & $-0.54 \%$ \\
\hline$(1500,80)$ & No bifurcation & 4 & 12.60 & 1573.70 & $-1.56 \%$ & $-0.64 \%$ \\
\hline
\end{tabular}

Table 5.12: Results of the best heuristic variation with the color conversion capability for different traffic patterns in the 8-node network using the 10-8-8 combination under the fiber-plentiful situation. The difference in results is relative to the best results obtained by keeping the color-continuity constraints.

\begin{tabular}{|c|c|c|c|c|c|c|}
\hline $\begin{array}{l}\text { Traffic pattern: } \\
(I N \mathrm{NG} / \mathrm{s}, F R \%)\end{array}$ & $\begin{array}{l}\text { Best bifurcation } \\
\text { scenario }\end{array}$ & $\begin{array}{c}\text { Best } \\
\text { policy }\end{array}$ & $F_{o p t}$ & $\begin{array}{l}\text { Weighted } \\
\text { port count } \\
\text { @ } F_{o p t}\end{array}$ & $\begin{array}{c}\text { Difference in } \\
F_{o p t}\end{array}$ & $\begin{array}{l}\text { Difference in } \\
\text { Weighted port } \\
\text { count } @ F_{o p t}\end{array}$ \\
\hline$(500,0)$ & No bifurcation & 1 & 4 & 4023.30 & $17.65 \%$ & $0.11 \%$ \\
\hline$(1000,0)$ & No bifurcation & 4 & 5.6 & 4345.70 & $0.00 \%$ & $-0.04 \%$ \\
\hline$(1500,0)$ & No bifurcation & 4 & 8 & 4717.90 & $2.56 \%$ & $0.33 \%$ \\
\hline$(500,20)$ & No bifurcation & 4 & 2.8 & 3197.40 & $-6.67 \%$ & $-0.43 \%$ \\
\hline$(1000,20)$ & No bifurcation & 4 & 4.8 & 3487.90 & $-11.11 \%$ & $-0.05 \%$ \\
\hline$(1500,20)$ & No bifurcation & 4 & 7.2 & 3776.80 & $2.86 \%$ & $0.27 \%$ \\
\hline$(500,50)$ & No bifurcation & 4 & 2.5 & 2236.20 & $-3.85 \%$ & $-0.32 \%$ \\
\hline$(1000,50)$ & No bifurcation & 4 & 4.2 & 2489.50 & $-4.55 \%$ & $-0.10 \%$ \\
\hline$(1500,50)$ & No bifurcation & 4 & 5.6 & 2730.80 & $0.00 \%$ & $-0.12 \%$ \\
\hline$(500,80)$ & No bifutcation & 4 & 1.8 & 1127.20 & $-10.00 \%$ & $-0.48 \%$ \\
\hline$(1000,80)$ & No bifurcation & 4 & 3.4 & 1307.20 & $6.25 \%$ & $-0.08 \%$ \\
\hline$(1500,80)$ & Fiber bifurcation & 4 & 3.7 & 1540.60 & $-2.63 \%$ & $-0.30 \%$ \\
\hline
\end{tabular}


Table 5.13: Results of the best heuristic variation with the color conversion capability for different traffic patterns in the 8-node network using the 40-8-8 combination under the fiber-plentiful situation. The difference in results is relative to the best results obtained by keeping the color-continuity constraints.

\begin{tabular}{c|c|c|c|c|c|c}
$\begin{array}{c}\text { Traffic pattern: } \\
(\boldsymbol{I N G} / \mathbf{s}, \boldsymbol{F R} \%)\end{array}$ & $\begin{array}{c}\text { Best bifurcation } \\
\text { scenario }\end{array}$ & $\begin{array}{c}\text { Best } \\
\text { policy }\end{array}$ & $\boldsymbol{F}_{\text {opt }}$ & $\begin{array}{c}\text { Weighted } \\
\text { port count } \\
\text { ( } \boldsymbol{F}_{\text {opt }}\end{array}$ & $\begin{array}{c}\text { Difference in } \\
\boldsymbol{F}_{\text {opt }}\end{array}$ & $\begin{array}{c}\text { Difference in } \\
\text { Weighted port } \\
\text { count @ } \boldsymbol{F}_{\text {opt }}\end{array}$ \\
\hline$(500,0)$ & No bifurcation & 4 & 1 & 3798.00 & $-50.00 \%$ & $-0.37 \%$ \\
\hline$(1000,0)$ & No bifurcation & 4 & 2.3 & 4849.90 & $15.00 \%$ & $-0.53 \%$ \\
\hline$(1500,0)$ & No bifurcation & 4 & 2.4 & 5488.40 & $0.00 \%$ & $-0.45 \%$ \\
\hline$(500,20)$ & No bifurcation & 4 & 1 & 3082.80 & $-50.00 \%$ & $-0.01 \%$ \\
\hline$(1000,20)$ & No bifurcation & 4 & 2.4 & 3831.10 & $20.00 \%$ & $-0.52 \%$ \\
\hline$(1500,20)$ & No bifurcation & 4 & 2 & 4292.40 & $-16.67 \%$ & $-0.30 \%$ \\
\hline$(1000,50)$ & No bifurcation & 4 & 1 & 2124.90 & $-50.00 \%$ & $-0.12 \%$ \\
\hline$(500,80)$ & No bifurcation & 4 & 1.4 & 2713.20 & $-30.00 \%$ & $-0.28 \%$ \\
\hline$(1000,80)$ & No bifurcation & 4 & 1 & 1069.80 & $-50.00 \%$ & $-0.29 \%$ \\
\hline$(1500,80)$ & No bifurcation & 4 & 1 & 1337.50 & $-50.00 \%$ & $-0.71 \%$ \\
\hline
\end{tabular}

Table 5.14: Results of the best heuristic variation with the color conversion capability for different traffic patterns in the NSF network using the 10-8-8 combination under the fiber-plentiful situation. The difference in results is relative to the best results obtained by keeping the color-continuity constraints.

\begin{tabular}{|c|c|c|c|c|c|c|}
\hline $\begin{array}{l}\text { Traffic pattern: } \\
(I N G b / s, F R \%)\end{array}$ & $\begin{array}{l}\text { Best bifurcation } \\
\text { scenario }\end{array}$ & $\begin{array}{l}\text { Best } \\
\text { policy }\end{array}$ & $F_{\text {opt }}$ & $\begin{array}{l}\text { Weighted } \\
\text { port count } \\
\quad @ F_{o p t}\end{array}$ & $\begin{array}{c}\text { Difference in } \\
F_{o p t}\end{array}$ & $\begin{array}{c}\text { Difference in } \\
\text { Weighted port } \\
\text { count @ } F_{\text {opt }}\end{array}$ \\
\hline$(500,0)$ & No bifurcation & 4 & 7.6 & 14673.30 & $-2.56 \%$ & $-0.06 \%$ \\
\hline$(1000,0)$ & No bifurcation & 1 & 14.2 & 15878.10 & $1.43 \%$ & $0.06 \%$ \\
\hline$(1500,0)$ & No bifurcation & 4 & 20.4 & 16974.10 & $-2.86 \%$ & $0.09 \%$ \\
\hline$(500,20)$ & No bifurcation & 1 & 6.4 & 12192.10 & $-5.88 \%$ & $-0.12 \%$ \\
\hline$(1000,20)$ & No bifurcation & 1 & 12.4 & 13216.80 & $1.64 \%$ & $-0.08 \%$ \\
\hline$(1500,20)$ & No bifurcation & 1 & 17.2 & 14274.10 & $1.18 \%$ & $0.15 \%$ \\
\hline$(500,50)$ & No bifurcation & 4 & 5 & 8422.30 & $-3.85 \%$ & $-0.16 \%$ \\
\hline$(1000,50)$ & No bifurcation & 1 & 9.6 & 9287.10 & $0.00 \%$ & $0.38 \%$ \\
\hline$(1500,50)$ & No bifurcation & 1 & 13.8 & 10162.50 & $0.00 \%$ & $-0.04 \%$ \\
\hline$(500,80)$ & No bifurcation & 1 & 3 & 4465.90 & $0.00 \%$ & $-0.06 \%$ \\
\hline$(1000,80)$ & No bifurcation & 4 & 5.4 & 5061.20 & $-3.57 \%$ & $-0.14 \%$ \\
\hline$(1500,80)$ & No bifurcation & 4 & 7.2 & 5880.80 & $-5.26 \%$ & $0.12 \%$ \\
\hline
\end{tabular}


Table 5.15: Results of the best heuristic variation with the color conversion capability for different traffic patterns in the EON network using the $10-8-8$ combination under the fiber-plentiful situation. The difference in results is relative to the best results obtained by keeping the color-continuity constraints.

\begin{tabular}{c|c|c|c|c|c|c}
$\begin{array}{c}\text { Traffic pattern: } \\
(I N G \text { G }, F R \%)\end{array}$ & $\begin{array}{c}\text { Best bifurcation } \\
\text { scenario }\end{array}$ & $\begin{array}{c}\text { Best } \\
\text { policy }\end{array}$ & $\boldsymbol{F}_{\text {opt }}$ & $\begin{array}{c}\text { Weighted } \\
\text { port count } \\
\text { @ } \boldsymbol{F}_{\text {opt }}\end{array}$ & $\begin{array}{c}\text { Difference in } \\
\boldsymbol{F}_{\text {opt }}\end{array}$ & $\begin{array}{c}\text { Difference in } \\
\text { Weighted port } \\
\text { count @ } \boldsymbol{F}_{\text {opt }}\end{array}$ \\
\hline$(500,0)$ & No bifurcation & 4 & 11 & 9375.40 & $-5.17 \%$ & $-0.26 \%$ \\
\hline$(1000,0)$ & No bifurcation & 1 & 9.8 & 9925.90 & $-56.25 \%$ & $-2.02 \%$ \\
\hline$(1500,0)$ & Fiber bifurcation & 1 & 15 & 10451.10 & $-57.14 \%$ & $-2.73 \%$ \\
\hline$(500,20)$ & No bifurcation & 1 & 5.2 & 7892.70 & $-49.02 \%$ & $-0.26 \%$ \\
\hline$(1000,20)$ & No bifurcation & 1 & 9.2 & 8315.50 & $-47.73 \%$ & $-2.45 \%$ \\
\hline$(1500,20)$ & Fiber bifurcation & 1 & 12.8 & 8818.40 & $-49.61 \%$ & $-2.70 \%$ \\
\hline$(500,50)$ & No bifurcation & 4 & 5.8 & 5361.10 & $-12.12 \%$ & $-0.03 \%$ \\
\hline$(1000,50)$ & No bifurcation & 1 & 5.4 & 5741.80 & $-52.63 \%$ & $-1.51 \%$ \\
\hline$(1500,50)$ & No bifurcation & 1 & 10.4 & 6291.30 & $-40.91 \%$ & $-2.19 \%$ \\
\hline$(500,80)$ & No bifurcation & 1 & 2.6 & 2759.10 & $-27.78 \%$ & $-1.65 \%$ \\
\hline
\end{tabular}

\subsubsection{Comparison of Heuristics with Signal Regeneration Capability}

This subsection compares different heuristic variations via simulation without the consideration of the color-continuity constraints and with the consideration of the optical-reach constraints. In this subsection, we also try to gain a greater understanding of the factors that lead to the change in both the best maximum optical reach and the amount of saving achieved in deciding between customizing the optical reach of all clear paths and using the same reach with all clear paths. Heuristic variations are based on combinations of bifurcation scenarios, namely the no-bifurcation and fiber-bifurcation scenarios, and sorting policies, namely Policies 1-6. Moreover, heuristic variations are evaluated both with and without the optical-reach customization. 
The input data and parameters used for the simulation are given as follows. (1) The procedure for generating traffic patterns used in Section 4.4.7 is utilized again here. Due to space limitations, the traffic patterns here will be generated based on the combination of only the following parameter values: $F R=0$ and $80 \%$; IN=500 and $1500 \mathrm{~Gb} / \mathrm{s}$. (2) The topologies considered in the simulation are again the 8-node, NSF, and EON networks, as shown in Figs. 4.11, 4.26, and 4.27 respectively. It is important to mention here that the average node-to-node distance, which represents the distance characterization of the topology, in the 8-node/NSF/EON network is 1061.91/1538.46/578.65 km. (3) All MG-OXC/EXC ports have the same weight, and the ratio of the 10/40G-based EXC port weight to the MG-OXC port weight is decided by the relation given in Equation 3.1 with $\mathrm{RATIO}_{1000}=2 / 5$. (4) Similar to Subsection 5.5.7, fiber capacities used with the 8-node network are based on using the 10-4-4, 10-8-8, and 40-8-8 combinations, whereas the fiber capacity used with the NSF and EON networks is based on the $10-8-8$ combination. (5) The maximum optical reach value, $R_{\text {max }}$ is tested over a range of $1000-10000 \mathrm{~km}$ for the 8 -node and EON networks, whereas for the NSF network, the tested range is $2000-10000 \mathrm{~km}$. The reason behind this difference in the optical reach range is explained as follows. The lengths of all physical spans originating from Nodes 0 and 6 of the NSF network are greater than $1000 \mathrm{~km}$, as shown in Fig. 4.26, which means that with the $1000 \mathrm{~km}$ optical reach, demands originating from/destined to these nodes cannot be served; as a result, the RMGPA problem cannot be solved for any value of the parameter $F$. (6) The RMGPA problem is solved for $1 \leq F \leq F_{\text {sat }}$

Detailed results based on averaging 10 simulation runs with $95 \%$ confidence intervals are shown in Appendix C. Tables 5.16-5.20 and Tables 5.21-5.25 summarize the results in Tables C.1-C.12 for the fiber-scarce and plentiful situations respectively, based on the scheme adopted in Subsection 5.5.4, which is used to decide both the best maximum optical reach and best heuristic variation. 
In the fiber-scarce situation, as shown in Tables 5.16-5.20, the following observations can be extracted. First, it is clear that the best maximum optical reach needed to achieve the lowest $F_{m i n}$ varies according to the physical topology, traffic pattern, and fiber capacity, as well as whether the optical reach of all clear paths is customized. Second, by comparing Tables 5.17, 5.19, and 5.20, it is quite clear that, at a certain traffic pattern, the best $R_{\max }$ is the largest in the NSF network and the smallest in the EON network. This finding is due to the fact that the average node-to-node distance is the largest in the NSF network and the smallest in the EON network. Third, even though customizing the optical reach leads to the same $F_{m i n}$ obtained without customizing the reach, it achieves a consistent saving in the weighted port count at $F_{m i n}$. The saving is in the range of $\approx 6-13 \%$, and it varies according to the traffic pattern, physical topology, and fiber capacity. Fourth, the best $R_{\max }$ with the optical-reach customization is always equal to or greater than the best $R_{\text {max }}$ achieved without the reach customization. This occurs because, with the optical-reach customization, increasing the optical reach further can be an opportunity for many $s$ - $d$ pairs to use (1) clear paths with a few long-reach expensive EXC ports rather than unclear paths with many short-reach less-expensive EXC ports, and/or (2) unclear paths with both a low virtual hop count and a few long-reach expensive EXC ports instead of unclear paths with both a greater virtual hop count and many short-reach less-expensive EXC ports. Both scenarios can be implied from the reduction in the number of OEO conversions at $F_{\text {min }}$ as shown by the comparison of Tables C.1, C.3, C.5, C.7, C.9, and C.11 with Tables C.2, C.4, C.6, C.8, C.10, and C.12. On the other hand, without the optical-reach customization, although increasing the reach reduces the number of unclear paths and decreases the virtual hop count of the remaining unclear paths (this can be implied from the reduction in the number of OEO conversions at $F_{m i n}$, as shown in Appendix C), it is the fact that all EXC ports are assigned the same cost that increases the weighted port count beyond the best $R_{\text {max }}$. 
In comparing the winning heuristic variations with and without the optical-reach customization, the following remarks can be made. First, as we have seen before, it is apparent that the winning bifurcation scenario/sorting policy changes according to the traffic pattern, physical topology, and fiber capacity. Second, the winning heuristic variation with the reach customization is not necessarily also the best without the reach customization, as is clearly shown in Table 5.18. Third, there is no clear sorting policy winner. However, Policy 6, which is a distance-based sorting policy, turns out to be fiber-efficient for some traffic patterns in both the 8-node and NSF networks, in which the 'node-to-node distance'/'physical connectivity' is greater/lower than in the EON network. In contrast, Policy 5, which is also a distance-based policy, is clearly out of the contest in all cases tested. Moreover, Policy 3 is out of the contest in all traffic patterns tested with the 8node and NSF networks, as is the case when both the optical-reach and color-continuity constraints are not imposed in the fiber-scarce situation. Fourth, the winning set of heuristic variations for all traffic patterns tested at a certain topology and fiber capacity is not the same set obtained by (1) keeping the color-continuity constraints without the consideration of the opticalreach constraints (this observation can be made by the comparison of Tables 5.16-5.20 with Tables $4.10,4.11,4.12,4.14$, and 4.17); and (2) not keeping the color-continuity constraints but retaining the consideration of the optical-reach constraints (this observation can be made by the comparison of Tables 5.16-5.20 with Tables 5.6-5.10). This finding clearly indicates that choosing the best heuristic variation in the fiber-scarce situation is also a function of whether optical-reach constraints are imposed.

Furthermore, in looking for the $C-W-B$ combination/fiber capacity that can achieve the best results in the fiber-scarce situation, the following observations can be made from Tables 5.16-5.18. First, the saving achieved by customizing the optical reach is the greatest with the $40-8-8$ 
combination due to its high $\mathrm{E} / \mathrm{O}$ cost ratio relative to the ratio used with the $10-4-4$ and $10-8-8$ combinations. Second, the best $C-W-B$ combination with the reach customization is not necessarily the best without the reach customization, as is the case with the $I N=500 \mathrm{~Gb} / \mathrm{s}$ and $F R=80 \%$ scenario, the best choice for which is the $10-8-8 / 40-8-8$ combination without/with the optical-reach customization. More to the point, the best combination set obtained here, which represents the winners of all traffic patterns, are not exactly the same sets obtained without considering optical-reach constraints, as shown in Tables 4.10-4.12 (without color conversions) and Tables 5.6-5.8 (with color conversions). Accordingly, one can conclude that choosing the best $C-W-B$ combination in the fiber-scarce situation is also a function of whether the optical-reach constraints are imposed and the optical reach is customized.

Table 5.16: Results of the best heuristic variation with the color conversion and signal regeneration capabilities with/without the optical-reach customization for different traffic patterns in the 8-node network using the 10-4-4 combination under the fiber-scarce situation.

\begin{tabular}{|c|c|c|c|c|c|c|}
\hline $\begin{array}{l}\text { Traffic pattern: } \\
(I N G b / s, F R \%)\end{array}$ & $\begin{array}{c}\text { Optical reach } \\
\text { customization } \\
\text { scenario }\end{array}$ & $\begin{array}{c}\text { Best } \\
\text { bifurcation } \\
\text { scenario }\end{array}$ & $\begin{array}{c}\text { Best } \\
\text { policy }\end{array}$ & $\begin{array}{c}\text { Best } \boldsymbol{R}_{\max } \\
@ F_{\min } \\
{[\mathbf{k m}]}\end{array}$ & $F_{\min }$ & $\begin{array}{c}\text { Weighted } \\
\text { port count } \\
\text { @ } F_{\min }\end{array}$ \\
\hline \multirow{2}{*}{$(500,0)$} & $\begin{array}{l}\text { Without } \\
\text { customization }\end{array}$ & $\begin{array}{c}\text { No } \\
\text { bifurcation }\end{array}$ & 1 & 4000 & 8 & 3712.3 \\
\hline & $\begin{array}{c}\text { With } \\
\text { customization }\end{array}$ & $\begin{array}{c}\text { No } \\
\text { bifurcation }\end{array}$ & 1 & 4000 & 8 & $\begin{array}{c}3409.02 \\
(8.17 \% \downarrow)\end{array}$ \\
\hline \multirow{2}{*}{$(500,80)$} & $\begin{array}{c}\text { Without } \\
\text { customization }\end{array}$ & $\begin{array}{c}\text { Fiber } \\
\text { bifurcation }\end{array}$ & 6 & 5000 & 3.2 & 1199.32 \\
\hline & $\begin{array}{c}\text { With } \\
\text { customization }\end{array}$ & $\begin{array}{c}\text { Fiber } \\
\text { bifurcation }\end{array}$ & 6 & 8000 & 3.2 & $\begin{array}{c}1074.46 \\
(10.41 \% \downarrow)\end{array}$ \\
\hline \multirow{2}{*}{$(1500,0)$} & $\begin{array}{c}\text { Without } \\
\text { customization }\end{array}$ & $\begin{array}{c}\text { No } \\
\text { bifurcation }\end{array}$ & 6 & 3000 & 22.2 & 6187.08 \\
\hline & $\begin{array}{l}\text { With } \\
\text { customization }\end{array}$ & $\begin{array}{c}\text { No } \\
\text { bifurcation }\end{array}$ & 6 & 3000 & 22.2 & $\begin{array}{c}5792.86 \\
(6.37 \% \downarrow)\end{array}$ \\
\hline \multirow{2}{*}{$(1500,80)$} & $\begin{array}{c}\text { Without } \\
\text { customization }\end{array}$ & $\begin{array}{c}\text { Fiber } \\
\text { bifurcation }\end{array}$ & 2 & 4000 & 7.4 & 2205.25 \\
\hline & $\begin{array}{c}\text { With } \\
\text { customization }\end{array}$ & $\begin{array}{c}\text { Fiber } \\
\text { bifurcation }\end{array}$ & 2 & 4000 & 7.4 & $\begin{array}{c}2051.17 \\
(6.99 \% \downarrow)\end{array}$ \\
\hline
\end{tabular}


Table 5.17: Results of the best heuristic variation with the color conversion and signal regeneration capabilities with/without the optical-reach customization for different traffic patterns in the 8-node network using the 10-8-8 combination under the fiber-scarce situation.

\begin{tabular}{|c|c|c|c|c|c|c|}
\hline $\begin{array}{l}\text { Traffic pattern: } \\
(I N G b / s, F R \%)\end{array}$ & $\begin{array}{c}\text { Optical reach } \\
\text { customization } \\
\text { scenario }\end{array}$ & $\begin{array}{c}\text { Best } \\
\text { bifurcation } \\
\text { scenario }\end{array}$ & $\begin{array}{l}\text { Best } \\
\text { policy }\end{array}$ & $\begin{array}{c}\text { Best } \boldsymbol{R}_{\max } \\
\left(\mathfrak{a} \boldsymbol{F}_{\min }\right. \\
{[\mathbf{k m}]}\end{array}$ & $F_{\min }$ & $\begin{array}{l}\text { Weighted } \\
\text { port count } \\
\text { (a) } F_{\min }\end{array}$ \\
\hline \multirow{2}{*}{$(500,0)$} & $\begin{array}{c}\text { Without } \\
\text { customization }\end{array}$ & $\begin{array}{c}\text { No } \\
\text { bifurcation }\end{array}$ & 4 & 4000 & 3 & 4864.15 \\
\hline & $\begin{array}{c}\text { With } \\
\text { customization }\end{array}$ & $\begin{array}{c}\text { No } \\
\text { bifurcation }\end{array}$ & 4 & 4000 & 3 & $\begin{array}{c}4439.47 \\
(8.73 \% \downarrow) \\
\end{array}$ \\
\hline \multirow{2}{*}{$(500,80)$} & $\begin{array}{c}\text { Without } \\
\text { customization }\end{array}$ & $\begin{array}{c}\text { No } \\
\text { bifurcation }\end{array}$ & 6 & 4000 & 1 & 1345.35 \\
\hline & $\begin{array}{c}\text { With } \\
\text { customization }\end{array}$ & $\begin{array}{c}\text { No } \\
\text { bifurcation }\end{array}$ & 6 & 6000 & 1 & $\begin{array}{c}1215.51 \\
(9.65 \% \downarrow)\end{array}$ \\
\hline \multirow{2}{*}{$(1500,0)$} & $\begin{array}{c}\text { Without } \\
\text { customization }\end{array}$ & $\begin{array}{c}\text { Fiber } \\
\text { bifurcation }\end{array}$ & 4 & 3000 & 6.4 & 6153.47 \\
\hline & $\begin{array}{c}\text { With } \\
\text { customization }\end{array}$ & $\begin{array}{c}\text { Fiber } \\
\text { bifurcation }\end{array}$ & 4 & 3000 & 6.4 & $\begin{array}{c}5772.54 \\
(6.19 \% \downarrow) \\
\end{array}$ \\
\hline \multirow{2}{*}{$(1500,80)$} & $\begin{array}{c}\text { Without } \\
\text { customization }\end{array}$ & $\begin{array}{c}\text { Fiber } \\
\text { bifurcation }\end{array}$ & 6 & 4000 & 2.8 & 1905.33 \\
\hline & $\begin{array}{c}\text { With } \\
\text { customization }\end{array}$ & $\begin{array}{c}\text { Fiber } \\
\text { bifurcation }\end{array}$ & 6 & 7000 & 2.8 & $\begin{array}{c}1744.03 \\
(8.47 \% \downarrow)\end{array}$ \\
\hline
\end{tabular}

Table 5.18: Results of the best heuristic variation with the color conversion and signal regeneration capabilities with/without the optical-reach customization for different traffic patterns in the 8-node network using the 40-8-8 combination under the fiber-scarce situation.

\begin{tabular}{|c|c|c|c|c|c|c|}
\hline $\begin{array}{l}\text { Traffic pattern: } \\
(I N G b / s, F R \%)\end{array}$ & $\begin{array}{c}\text { Optical reach } \\
\text { customization } \\
\text { scenario } \\
\end{array}$ & $\begin{array}{c}\text { Best } \\
\text { bifurcation } \\
\text { scenario } \\
\end{array}$ & $\begin{array}{c}\text { Best } \\
\text { policy }\end{array}$ & $\begin{array}{c}\text { Best } \boldsymbol{R}_{\max } \\
\text { (a) } \boldsymbol{F}_{\min } \\
{[\mathrm{km}]} \\
\end{array}$ & $F_{\min }$ & $\begin{array}{c}\text { Weighted } \\
\text { port count } \\
\text { (a) } F_{\min } \\
\end{array}$ \\
\hline \multirow{2}{*}{$(500,0)$} & $\begin{array}{c}\text { Without } \\
\text { customization }\end{array}$ & $\begin{array}{c}\text { No } \\
\text { bifurcation }\end{array}$ & 4 & 3000 & 1 & 4987.31 \\
\hline & $\begin{array}{c}\text { With } \\
\text { customization }\end{array}$ & $\begin{array}{c}\text { No } \\
\text { bifurcation } \\
\end{array}$ & 6 & 4000 & 1 & $\begin{array}{c}4421.99 \\
(11.34 \% \downarrow) \\
\end{array}$ \\
\hline \multirow{2}{*}{$(500,80)$} & $\begin{array}{c}\text { Without } \\
\text { customization }\end{array}$ & $\begin{array}{c}\text { No } \\
\text { bifurcation } \\
\end{array}$ & 6 & 3000 & 1 & 1351.79 \\
\hline & $\begin{array}{c}\text { With } \\
\text { customization } \\
\end{array}$ & $\begin{array}{c}\text { No } \\
\text { bifurcation } \\
\end{array}$ & 6 & 4000 & 1 & $\begin{array}{c}1189.21 \\
(12.03 \% \downarrow) \\
\end{array}$ \\
\hline \multirow{2}{*}{$(1500,0)$} & $\begin{array}{c}\text { Without } \\
\text { customization } \\
\end{array}$ & $\begin{array}{c}\text { No } \\
\text { bifurcation } \\
\end{array}$ & 1 & 3000 & 3 & 7257.57 \\
\hline & $\begin{array}{c}\text { With } \\
\text { customization } \\
\end{array}$ & $\begin{array}{c}\text { No } \\
\text { bifurcation }\end{array}$ & 6 & 4000 & 3 & $\begin{array}{c}6424.49 \\
(11.48 \% \downarrow) \\
\end{array}$ \\
\hline \multirow{2}{*}{$(1500,80)$} & $\begin{array}{c}\text { Without } \\
\text { customization }\end{array}$ & $\begin{array}{c}\text { No } \\
\text { bifurcation }\end{array}$ & 4 & 3000 & 1 & 1986.55 \\
\hline & $\begin{array}{c}\text { With } \\
\text { customization }\end{array}$ & $\begin{array}{c}\text { No } \\
\text { bifurcation }\end{array}$ & 6 & 4000 & 1 & $\begin{array}{c}1802.62 \\
(9.26 \% \downarrow)\end{array}$ \\
\hline
\end{tabular}


Table 5.19: Results of the best heuristic variation with the color conversion and signal regeneration capabilities with/without the optical-reach customization for different traffic patterns in the NSF network using the 10-8-8 combination under the fiber-scarce situation.

\begin{tabular}{|c|c|c|c|c|c|c|}
\hline $\begin{array}{l}\text { Traffic pattern: } \\
(I N G b / s, F R \%)\end{array}$ & $\begin{array}{c}\text { Optical reach } \\
\text { customization } \\
\text { scenario } \\
\end{array}$ & $\begin{array}{c}\text { Best } \\
\text { bifurcation } \\
\text { scenario } \\
\end{array}$ & $\begin{array}{l}\text { Best } \\
\text { policy }\end{array}$ & $\begin{array}{c}\text { Best } \boldsymbol{R}_{\max } \\
\text { (a) } \boldsymbol{F}_{\min } \\
{[\mathbf{k m}]}\end{array}$ & $F_{\text {min }}$ & $\begin{array}{c}\text { Weighted } \\
\text { port count } \\
\text { @ } F_{\min } \\
\end{array}$ \\
\hline \multirow{2}{*}{$(500,0)$} & $\begin{array}{c}\text { Without } \\
\text { customization }\end{array}$ & $\begin{array}{c}\text { No } \\
\text { bifurcation }\end{array}$ & 6 & 5000 & 7 & 18243.97 \\
\hline & $\begin{array}{c}\text { With } \\
\text { customization }\end{array}$ & $\begin{array}{c}\text { No } \\
\text { bifurcation }\end{array}$ & 6 & 8000 & 7 & $\begin{array}{c}16166.20 \\
(11.39 \% \downarrow)\end{array}$ \\
\hline \multirow{2}{*}{$(500,80)$} & $\begin{array}{c}\text { Without } \\
\text { customization }\end{array}$ & $\begin{array}{c}\text { No } \\
\text { bifurcation }\end{array}$ & 6 & 5000 & 3 & 5341.52 \\
\hline & $\begin{array}{c}\text { With } \\
\text { customization }\end{array}$ & $\begin{array}{c}\text { No } \\
\text { bifurcation }\end{array}$ & 6 & 7000 & 3 & $\begin{array}{c}4782 \\
(10.48 \% \downarrow)\end{array}$ \\
\hline \multirow{2}{*}{$(1500,0)$} & $\begin{array}{c}\text { Without } \\
\text { customization }\end{array}$ & $\begin{array}{c}\text { No } \\
\text { bifurcation }\end{array}$ & 4 & 4000 & 17.2 & 24012.28 \\
\hline & $\begin{array}{c}\text { With } \\
\text { customization }\end{array}$ & $\begin{array}{c}\text { No } \\
\text { bifurcation }\end{array}$ & 4 & 4000 & 17.2 & $\begin{array}{l}21646.31 \\
(9.85 \% \downarrow)\end{array}$ \\
\hline \multirow{2}{*}{$(1500,80)$} & $\begin{array}{c}\text { Without } \\
\text { customization }\end{array}$ & $\begin{array}{c}\text { No } \\
\text { bifurcation }\end{array}$ & 1 & 7000 & 5.2 & 7717.50 \\
\hline & $\begin{array}{c}\text { With } \\
\text { customization }\end{array}$ & $\begin{array}{c}\text { No } \\
\text { bifurcation }\end{array}$ & 1 & 8000 & 5.2 & $\begin{array}{c}6744.37 \\
(12.61 \% \downarrow)\end{array}$ \\
\hline
\end{tabular}

Table 5.20: Results of the best heuristic variation with the color conversion and signal regeneration capabilities with/without the optical-reach customization for different traffic patterns in the EON network using the 10-8-8 combination under the fiber-scarce situation.

\begin{tabular}{|c|c|c|c|c|c|c|}
\hline $\begin{array}{l}\text { Traffic pattern: } \\
(I N G b / s, F R \%)\end{array}$ & $\begin{array}{c}\text { Optical reach } \\
\text { customization } \\
\text { scenario }\end{array}$ & $\begin{array}{c}\text { Best } \\
\text { bifurcation } \\
\text { scenario }\end{array}$ & $\begin{array}{l}\text { Best } \\
\text { policy }\end{array}$ & $\begin{array}{c}\text { Best } R_{\max } \\
@ F_{\min } \\
{[\mathrm{km}]}\end{array}$ & $F_{\min }$ & $\begin{array}{l}\text { Weighted } \\
\text { port count } \\
\text { (a) } F_{\min }\end{array}$ \\
\hline \multirow{2}{*}{$(500,0)$} & $\begin{array}{l}\text { Without } \\
\text { customization }\end{array}$ & $\begin{array}{c}\text { No } \\
\text { bifurcation }\end{array}$ & 2 & 1000 & 3.4 & 13381.5 \\
\hline & $\begin{array}{c}\text { With } \\
\text { customization }\end{array}$ & $\begin{array}{c}\text { No } \\
\text { bifurcation }\end{array}$ & 2 & 1000 & 3.4 & $\begin{array}{c}12597.1 \\
(5.86 \% \downarrow)\end{array}$ \\
\hline \multirow{2}{*}{$(500,80)$} & $\begin{array}{c}\text { Without } \\
\text { customization }\end{array}$ & $\begin{array}{c}\text { No } \\
\text { bifurcation }\end{array}$ & 1. & 3000 & 1 & 3263.01 \\
\hline & $\begin{array}{l}\text { With } \\
\text { customization }\end{array}$ & $\begin{array}{l}\text { No } \\
\text { bifurcation }\end{array}$ & 1 & 5000 & 1 & $\begin{array}{c}2882.79 \\
(11.65 \% \downarrow)\end{array}$ \\
\hline \multirow{2}{*}{$(1500,0)$} & $\begin{array}{c}\text { Without } \\
\text { customization }\end{array}$ & $\begin{array}{c}\text { No } \\
\text { bifurcation }\end{array}$ & 3 & 1000 & 9 & 13819.90 \\
\hline & $\begin{array}{c}\text { With } \\
\text { customization }\end{array}$ & $\begin{array}{c}\text { No } \\
\text { bifurcation }\end{array}$ & 3 & 1000 & 9 & $\begin{array}{l}13000.58 \\
(5.93 \% \downarrow)\end{array}$ \\
\hline \multirow{2}{*}{$(1500,80)$} & $\begin{array}{c}\text { Without } \\
\text { customization }\end{array}$ & $\begin{array}{c}\text { Fiber } \\
\text { bifurcation }\end{array}$ & 1 & 4000 & 3 & 4363.53 \\
\hline & $\begin{array}{c}\text { With } \\
\text { customization }\end{array}$ & $\begin{array}{c}\text { Fiber } \\
\text { bifurcation }\end{array}$ & 1 & 6000 & 3 & $\begin{array}{c}3801.85 \\
(12.87 \% \downarrow)\end{array}$ \\
\hline
\end{tabular}


In the second part of this subsection, we analyze the results obtained in the fiber-plentiful situation. From Tables 5.21-5.25, the same observations that have been made in the fiber-scarce situation regarding the best $R_{\max }$ are generally applicable here. In addition, the following comments can be made. First, the best $R_{\max }$ with the optical-reach customization in the EON network, where $I N=1500 \mathrm{~Gb} / \mathrm{s}$ and $F R=0 \%$ (uniform traffic pattern with high intensity), surprisingly exceeds the corresponding best $R_{\text {max }}$ of other topologies. This exceptional scenario can be explained as follows. With the high physical connectivity of the EON network and the availability of fibers, some $s-d$ pairs tend to select significantly longer unclear paths rather than short clear paths in order to maximize intermediate grouping with other $s-d$ pairs. Here the benefit of grouping offsets the cost associated with the resource duplication resulting from using long routes instead of short routes. In other words, the choice of short clear paths here would be more expensive because grouping opportunities are limited. Now, increasing the optical reach reduces the virtual hop count of the unclear path, which means that fewer OEO conversions are needed along the unclear path. This observation can be implied from the slight decrease in the number of OEO conversions at $F_{o p t}$ as the reach value increases, as shown in Table C.10. However, the fact that at the best $R_{\max }=10000$ $\mathrm{km}$, the average number of $\mathrm{OEO}$ conversions $=39$, as well as the fact that fiber resources are plentiful and thus no OEO conversions are expected at this reach value for the purpose of color conversions, clearly indicates that the lengths of some clear path segments of unclear paths are in the range of $10000 \mathrm{~km}$. Second, the range of savings obtained with the optical-reach customization is $\approx 5-14 \%$, which is close to what has been achieved in the fiber-scarce situation.

In comparing the winning heuristic variations with and without customizing the optical reach, the first and second observations that have been made in this regard in the fiber-scarce situation are also valid here. Furthermore, the following comments can be made. First, in comparing Tables 
5.16-5.20 with Tables 5.21-5.25, it is quite clear that the winning sets of heuristic variations are different in both fiber situations. Second, it is interesting to see Policy 5, which is a distance-based sorting policy, as a cost-effective sorting policy for one of the traffic patterns in the EON network when the optical reach is customized. Third, Policy 1, which is a traffic-based sorting policy, becomes a major contender in the EON network, which has the highest physical connectivity and lowest node-to-node distance. Conversely, Policy 6, which is a distance-based sorting policy, becomes a major contender in the 8-node and NSF networks, which have a lower physical connectivity and higher node-to-node distance than the EON network. Fourth, Policies 2 and 3 are out of the contest in all cases tested, as is the case when the optical-reach constraints are not considered in the fiber-plentiful situation. Fifth, similar to the fiber-scarce situation, the winning set of heuristic variations for all traffic patterns tested at a certain topology and fiber capacity is not the same set attained by (1) keeping the color-continuity constraints without the consideration of the optical-reach constraints (this observation can be made through the comparison of Tables 5.21-5.25 with Tables 4.19, 4.20,4.21, 4.23, and 4.26); and (2) relaxing the color-continuity constraints without the consideration of the optical-reach constraints (this observation can be made through the comparison of Tables 5.21-5.25 with Tables 5.11-5.15). This finding clearly shows that in the fiber-plentiful situation, choosing the best heuristic variation is still a function of whether optical-reach constraints are imposed.

Finally, in searching for the $C-W-B$ combination/fiber capacity that can achieve the best results in the fiber-plentiful situation, the following observations can be made from Tables 5.21-5.23. First, similar to the fiber-scarce situation, the saving achieved with the optical-reach customization is the greatest with the $40-8-8$ combination due to its high $\mathrm{E} / \mathrm{O}$ cost ratio. Second, the best $C-W-B$ combinations with the reach customization for all traffic patterns are similar to the ones without 
the reach customization. It is interesting to note that the best $C-W-B$ combination set is similar to the set obtained by not considering the optical-reach constraints, as shown in Tables 4.19-4.21 (without color conversions) and Tables 5.11-5.13 (with color conversions). Therefore, one can conclude that, unlike in the fiber-scarce situation, choosing the best $C-W-B$ combination in the fiber-plentiful situation for a certain traffic pattern and physical topology is less sensitive to whether the optical-reach constraints are imposed and the optical reach is customized.

Table 5.21: Results of the best heuristic variation with the color conversion and signal regeneration capabilities with/without the optical-reach customization for different traffic patterns in the 8-node network using the 10-4-4 combination under the fiber-plentiful situation.

\begin{tabular}{|c|c|c|c|c|c|c|}
\hline $\begin{array}{l}\text { Traffic pattern: } \\
(I N G b / s, F R \%)\end{array}$ & $\begin{array}{c}\text { Optical reach } \\
\text { customization } \\
\text { scenario } \\
\end{array}$ & $\begin{array}{c}\text { Best } \\
\text { bifurcation } \\
\text { scenario }\end{array}$ & $\begin{array}{l}\text { Best } \\
\text { policy }\end{array}$ & $\begin{array}{c}\text { Best } \boldsymbol{R}_{\max } \\
@ \boldsymbol{F}_{o p t} \\
{[\mathrm{~km}]}\end{array}$ & $F_{o p t}$ & $\begin{array}{c}\text { Weighted } \\
\text { port count } \\
\text { @ } F_{\text {opt }} \\
\end{array}$ \\
\hline \multirow{2}{*}{$(500,0)$} & $\begin{array}{c}\text { Without } \\
\text { customization }\end{array}$ & $\begin{array}{c}\text { No } \\
\text { bifurcation } \\
\end{array}$ & 1 & 3000 & 11.6 & 3560.28 \\
\hline & $\begin{array}{c}\text { With } \\
\text { customization }\end{array}$ & $\begin{array}{c}\text { No } \\
\text { bifurcation }\end{array}$ & 4 & 4000 & 10.6 & $\begin{array}{c}3280.67 \\
(7.85 \% \downarrow) \\
\end{array}$ \\
\hline \multirow{2}{*}{$(500,80)$} & $\begin{array}{c}\text { Without } \\
\text { customization }\end{array}$ & $\begin{array}{c}\text { No } \\
\text { bifurcation }\end{array}$ & 6 & 4000 . & 4.8 & 1106 \\
\hline & $\begin{array}{c}\text { With } \\
\text { customization }\end{array}$ & $\begin{array}{c}\text { No } \\
\text { bifurcation }\end{array}$ & 6 & 5000 & 4.8 & $\begin{array}{c}1012.51 \\
(8.45 \% \downarrow)\end{array}$ \\
\hline \multirow{2}{*}{$(1500,0)$} & $\begin{array}{c}\text { Without } \\
\text { customization }\end{array}$ & \begin{tabular}{|c|} 
No \\
bifurcation \\
\end{tabular} & 4 & 3000 & 30.8 & 5580.31 \\
\hline & $\begin{array}{l}\text { With } \\
\text { customization }\end{array}$ & $\begin{array}{c}\text { No } \\
\text { bifurcation }\end{array}$ & 4 & 4000 & 29.2 & $\begin{array}{c}5094.01 \\
(8.71 \% \downarrow) \\
\end{array}$ \\
\hline \multirow{2}{*}{$(1500,80)$} & $\begin{array}{c}\text { Without } \\
\text { customization }\end{array}$ & $\begin{array}{c}\text { No } \\
\text { bifurcation }\end{array}$ & 1 & 3000 & 8.8 & 1988.55 \\
\hline & $\begin{array}{c}\text { With } \\
\text { customization }\end{array}$ & $\begin{array}{c}\text { No } \\
\text { bifurcation }\end{array}$ & 1 & 3000 & 8.8 & $\begin{array}{c}1886.39 \\
(5.14 \% \downarrow)\end{array}$ \\
\hline
\end{tabular}


Table 5.22: Results of the best heuristic variation with the color conversion and signal regeneration capabilities with/without the optical-reach customization for different traffic patterns in the 8-node netwotk using the 10-8-8 combination under the fiber-plentiful situation.

\begin{tabular}{|c|c|c|c|c|c|c|}
\hline $\begin{array}{l}\text { Traffic pattern: } \\
(I N G b / s, F R \%)\end{array}$ & $\begin{array}{c}\text { Optical reach } \\
\text { customization } \\
\text { scenario }\end{array}$ & $\begin{array}{c}\text { Best } \\
\text { bifurcation } \\
\text { scenario }\end{array}$ & $\begin{array}{c}\text { Best } \\
\text { policy }\end{array}$ & $\begin{array}{c}\text { Best } R_{\max } \\
@ F_{o p t} \\
{[\mathrm{~km}]}\end{array}$ & $F_{\text {opt }}$ & $\begin{array}{c}\text { Weighted } \\
\text { port count } \\
\text { @ } F_{o p t}\end{array}$ \\
\hline \multirow{2}{*}{$(500,0)$} & $\begin{array}{c}\text { Without } \\
\text { customization }\end{array}$ & $\begin{array}{c}\text { No } \\
\text { bifurcation }\end{array}$ & 4 & 3000 & 3.4 & 4853.11 \\
\hline & $\begin{array}{c}\text { With } \\
\text { customization }\end{array}$ & $\begin{array}{c}\text { No } \\
\text { bifurcation }\end{array}$ & 4 & 4000 & 3.2 & $\begin{array}{c}4437.64 \\
(8.56 \% \downarrow)\end{array}$ \\
\hline \multirow{2}{*}{$(500,80)$} & $\begin{array}{l}\text { Without } \\
\text { customization }\end{array}$ & $\begin{array}{c}\text { No } \\
\text { bifurcation }\end{array}$ & 6 & 3000 & 2 & 1307.89 \\
\hline & $\begin{array}{c}\text { With } \\
\text { customization }\end{array}$ & $\begin{array}{c}\text { No } \\
\text { bifurcation }\end{array}$ & 6 & 6000 & 2 & $\begin{array}{c}1200.13 \\
(8.24 \% \downarrow)\end{array}$ \\
\hline \multirow{2}{*}{$(1500,0)$} & $\begin{array}{c}\text { Without } \\
\text { customization }\end{array}$ & $\begin{array}{c}\text { No } \\
\text { bifurcation }\end{array}$ & 1 & 4000 & 8.6 & 5724.3 \\
\hline & $\begin{array}{c}\text { With } \\
\text { customization }\end{array}$ & $\begin{array}{c}\text { No } \\
\text { bifurcation }\end{array}$ & 1 & 4000 & 8.2 & $\begin{array}{c}5225.31 \\
(8.72 \% \downarrow)\end{array}$ \\
\hline \multirow{2}{*}{$(1500,80)$} & $\begin{array}{c}\text { Without } \\
\text { customization }\end{array}$ & $\begin{array}{c}\text { No } \\
\text { bifurcation } \\
\end{array}$ & 6 & 3000 & 4.4 & 1827.83 \\
\hline & $\begin{array}{c}\text { With } \\
\text { customization }\end{array}$ & $\begin{array}{c}\text { No } \\
\text { bifurcation }\end{array}$ & 6 & 3000 & 4.2 & $\begin{array}{c}1685.07 \\
(7.81 \% \downarrow)\end{array}$ \\
\hline
\end{tabular}

Table 5.23: Results of the best heuristic variation with the color conversion and signal regeneration capabilities with/without the optical-reach customization for different traffic patterns in the 8-node network using the 40-8-8 combination under the fiber-plentiful situation.

\begin{tabular}{|c|c|c|c|c|c|c|}
\hline $\begin{array}{l}\text { Traffic pattern: } \\
(\text { INGb/s,FR } \%)\end{array}$ & $\begin{array}{c}\begin{array}{c}\text { Optical reach } \\
\text { customization } \\
\text { scenario }\end{array} \\
\end{array}$ & $\begin{array}{c}\text { Best } \\
\text { bifurcation } \\
\text { scenario } \\
\end{array}$ & $\begin{array}{l}\text { Best } \\
\text { policy }\end{array}$ & $\begin{array}{c}\text { Best } \boldsymbol{R}_{\max } \\
\text { @ } \boldsymbol{F}_{\text {opt }} \\
{[\mathrm{km}]}\end{array}$ & $F_{o p t}$ & $\begin{array}{c}\text { Weighted } \\
\text { port count } \\
\text { @ } F_{\text {opt }} \\
\end{array}$ \\
\hline \multirow{2}{*}{$(500,0)$} & $\begin{array}{c}\text { Without } \\
\text { customization }\end{array}$ & $\begin{array}{c}\text { No } \\
\text { bifurcation }\end{array}$ & 4 & 3000 & 1 & 4987.31 \\
\hline & $\begin{array}{c}\text { With } \\
\text { customization }\end{array}$ & $\begin{array}{c}\text { No } \\
\text { bifurcation }\end{array}$ & 6 & 4000 & 1.2 & $\begin{array}{c}4415.82 \\
(11.46 \% \downarrow)\end{array}$ \\
\hline \multirow{2}{*}{$(500,80)$} & $\begin{array}{c}\text { Without } \\
\text { customization }\end{array}$ & $\begin{array}{c}\text { No } \\
\text { bifurcation }\end{array}$ & 6 & 3000 & 1 & 1351.79 \\
\hline & $\begin{array}{c}\text { With } \\
\text { customization }\end{array}$ & $\begin{array}{c}\text { No } \\
\text { bifurcation }\end{array}$ & 6 & 4000 & 1 & $\begin{array}{c}1189.21 \\
(12.03 \% \downarrow)\end{array}$ \\
\hline \multirow{2}{*}{$(1500,0)$} & $\begin{array}{c}\text { Without } \\
\text { customization }\end{array}$ & $\begin{array}{c}\text { No } \\
\text { bifurcation }\end{array}$ & 1 & 3000 & 3 & 7257.57 \\
\hline & $\begin{array}{c}\text { With } \\
\text { customization }\end{array}$ & $\begin{array}{c}\text { No } \\
\text { bifurcation }\end{array}$ & 6 & 4000 & 3 & $\begin{array}{c}6424.48 \\
(11.48 \% \downarrow) \\
\end{array}$ \\
\hline \multirow{2}{*}{$(1500,80)$} & $\begin{array}{c}\text { Without } \\
\text { customization }\end{array}$ & $\begin{array}{c}\text { No } \\
\text { bifurcation }\end{array}$ & 6 & 3000 & 1.4 & 1979.68 \\
\hline & $\begin{array}{c}\text { With } \\
\text { customization }\end{array}$ & $\begin{array}{c}\text { No } \\
\text { bifurcation }\end{array}$ & 6 & 4000 & 1.6 & $\begin{array}{c}1783.22 \\
(9.92 \% \downarrow)\end{array}$ \\
\hline
\end{tabular}


Table 5.24: Results of the best heuristic variation with the color conversion and signal regeneration capabilities with/without the optical-reach customization for different traffic patterns in the NSF network using the 10-8-8 combination under the fiber-plentiful situation.

\begin{tabular}{|c|c|c|c|c|c|c|}
\hline $\begin{array}{l}\text { Traffic pattern: } \\
(I N G b / s, F R \%)\end{array}$ & $\begin{array}{c}\text { Optical reach } \\
\text { customization } \\
\text { scenario }\end{array}$ & $\begin{array}{c}\text { Best } \\
\text { bifurcation } \\
\text { scenario } \\
\end{array}$ & $\begin{array}{c}\text { Best } \\
\text { policy }\end{array}$ & $\begin{array}{c}\text { Best } \boldsymbol{R}_{\max } \\
@ \boldsymbol{F}_{o p t} \\
{[\mathbf{k m}]}\end{array}$ & $F_{o p t}$ & $\begin{array}{c}\text { Weighted } \\
\text { port count } \\
\text { @a } F_{o p t} \\
\end{array}$ \\
\hline \multirow{2}{*}{$(500,0)$} & $\begin{array}{l}\text { Without } \\
\text { customization }\end{array}$ & $\begin{array}{c}\text { No } \\
\text { bifurcation }\end{array}$ & 6 & 5000 & 7.8 & 18224.9 \\
\hline & $\begin{array}{c}\text { With } \\
\text { customization }\end{array}$ & $\begin{array}{c}\text { No } \\
\text { bifurcation }\end{array}$ & 6 & 8000 & 7.6 & $\begin{array}{c}16127.67 \\
(11.51 \% \downarrow)\end{array}$ \\
\hline \multirow{2}{*}{$(500,80)$} & $\begin{array}{l}\text { Without } \\
\text { customization }\end{array}$ & $\begin{array}{c}\text { No } \\
\text { bifurcation }\end{array}$ & 6 & 5000 & 3 & 5341.52 \\
\hline & $\begin{array}{c}\text { With } \\
\text { customization }\end{array}$ & $\begin{array}{c}\text { No } \\
\text { bifurcation }\end{array}$ & 6 & 7000 & 3 & $\begin{array}{c}4782 \\
(10.48 \% \downarrow)\end{array}$ \\
\hline \multirow{2}{*}{$(1500,0)$} & $\begin{array}{l}\text { Without } \\
\text { customization }\end{array}$ & $\begin{array}{c}\text { No } \\
\text { bifurcation }\end{array}$ & 4 & 5000 & 20.2 & 21181.6 \\
\hline & $\begin{array}{c}\text { With } \\
\text { customization }\end{array}$ & $\begin{array}{c}\text { No } \\
\text { bifurcation }\end{array}$ & 6 & 6000 & 20.4 & $\begin{array}{c}18736.2 \\
(11.55 \% \downarrow)\end{array}$ \\
\hline \multirow{2}{*}{$(1500,80)$} & $\begin{array}{c}\text { Without } \\
\text { customization }\end{array}$ & $\begin{array}{c}\text { No } \\
\text { bifurcation }\end{array}$ & 6 & 5000 & 7.4 & 7147.14 \\
\hline & $\begin{array}{c}\text { With } \\
\text { customization }\end{array}$ & $\begin{array}{c}\text { Fiber } \\
\text { bifurcation }\end{array}$ & 6 & 9000 & 7.2 & $\begin{array}{c}6418.01 \\
(10.20 \% \downarrow)\end{array}$ \\
\hline
\end{tabular}

Table 5.25: Results of the best heuristic variation with the color conversion and signal regeneration capabilities with/without the optical-reach customization for different traffic patterns in the EON network using the 10-8-8 combination under the fiber-plentiful situation.

\begin{tabular}{|c|c|c|c|c|c|c|}
\hline $\begin{array}{l}\text { Traffic pattern: } \\
(I N G b / s, F R \%)\end{array}$ & $\begin{array}{c}\text { Optical reach } \\
\text { customization } \\
\text { scenario } \\
\end{array}$ & $\begin{array}{c}\text { Best } \\
\text { bifurcation } \\
\text { scenario } \\
\end{array}$ & $\begin{array}{l}\text { Best } \\
\text { policy }\end{array}$ & $\begin{array}{c}\text { Best } R_{\max } \\
\text { @ } \boldsymbol{F}_{\text {opr }} \\
{[\mathrm{km}]} \\
\end{array}$ & $F_{\text {opt }}$ & $\begin{array}{l}\text { Weighted } \\
\text { port count } \\
\text { (a) } F_{o p t} \\
\end{array}$ \\
\hline \multirow{2}{*}{$(500,0)$} & $\begin{array}{c}\text { Without } \\
\text { customization }\end{array}$ & $\begin{array}{c}\text { No } \\
\text { bifurcation }\end{array}$ & 4 & 4000 & 11.2 & 11157.35 \\
\hline & $\begin{array}{c}\text { With } \\
\text { customization }\end{array}$ & $\begin{array}{c}\text { No } \\
\text { bifurcation }\end{array}$ & 5 & 5000 & 4.2 & $\begin{array}{c}9810.38 \\
(12.07 \% \downarrow) \\
\end{array}$ \\
\hline \multirow{2}{*}{$(500,80)$} & $\begin{array}{c}\text { Without } \\
\text { customization }\end{array}$ & $\begin{array}{c}\text { No } \\
\text { bifurcation }\end{array}$ & 1 & 4000 & 2.6 & 3225.05 \\
\hline & $\begin{array}{c}\text { With } \\
\text { customization }\end{array}$ & $\begin{array}{c}\text { No } \\
\text { bifurcation }\end{array}$ & 1 & 5000 & 1.4 & $\begin{array}{c}2864.62 \\
(11.18 \% \downarrow)\end{array}$ \\
\hline \multirow{2}{*}{$(1500,0)$} & $\begin{array}{c}\text { Without } \\
\text { customization }\end{array}$ & $\begin{array}{c}\text { Fiber } \\
\text { bifurcation }\end{array}$ & 1 & 3000 & 13 & 12416.24 \\
\hline & $\begin{array}{c}\text { With } \\
\text { customization }\end{array}$ & $\begin{array}{c}\text { No } \\
\text { bifurcation }\end{array}$ & 1 & 10000 & 12.6 & $\begin{array}{c}10723.61 \\
(13.63 \% \downarrow)\end{array}$ \\
\hline \multirow{2}{*}{$(1500,80)$} & $\begin{array}{c}\text { Without } \\
\text { customization }\end{array}$ & $\begin{array}{c}\text { Fiber } \\
\text { bifurcation }\end{array}$ & 1 & 4000 & 5.4 & 4324.28 \\
\hline & $\begin{array}{c}\text { With } \\
\text { customization }\end{array}$ & $\begin{array}{c}\text { Fiber } \\
\text { bifurcation }\end{array}$ & 1 & 6000 & 3 & $\begin{array}{c}3801.85 \\
(12.08 \% \downarrow)\end{array}$ \\
\hline
\end{tabular}




\section{Chapter 6}

\section{Conclusions and Future Research}

\subsection{Conclusions}

Although the multi-granular optical network promises exciting opportunities, it also presents new design challenges that have been the focus of research and development in this area. Much work has been devoted to developing node architectures and efficient methods for planning the multigranular optical network. Extending these efforts in this thesis, important contributions have been made to the ongoing research in this area by covering many issues that have not yet been considered elsewhere.

First, a novel problem in planning the GMPLS-based transport network, called the RMGPA problem, has been defined. The problem addresses the following issues for the first time in the domain of multi-granular networks:

- Considering all traffic granularities defined in GMPLS;

- Creating/adjusting multi-granularity traffic demands;

- Grouping and degrouping traffic flows at all granularity levels;

- Allowing the bifurcation of multi-granularity traffic demands among different physical routes;

- Allowing wavelength, waveband, and fiber conversions;

- Considering the optical-reach limitation of optical signals;

- Considering the optical-reach customization of all-optical paths.

Moreover, in order to realize the issues addressed in the RMGPA problem, a new GMPIS-based node architecture that can handle the entire traffic hierarchy defined in GMPLS has been 
proposed. The new architecture is capable of performing switching, grouping/degrouping, color conversion and optical signal regeneration at all granularity levels.

In addition, four versions of the RMGPA problem with the same optimization objective, which is to minimize the total weighted port count, have been proposed. These versions are as follows:

1. RMGPA problem with the color-continuity constraints but without the optical-reach constraints. This version is the best fit for the planning problem in metro core (or regional) transport networks in which the control plane requires that the color-continuity constraints be kept in order to reduce resource fragmentation.

2. RMGPA problem without either the color-continuity or optical-reach constraints. This version fits the planning problem in metro core transport networks, especially when fiber resources are scarce.

3. RMGPA problem without the color-continuity constraints, with the optical-reach constraints, and without the optical-reach customization. This version fits the planning problem in longhaul transport networks.

4. RMGPA problem without the color-continuity constraints, with the optical-reach constraints, and with the optical-reach customization. This version fits the planning problem in long-haul transport networks in which it is possible to select the transponders based on a variety of fine optical reach values.

Furthermore, in order to obtain the exact solution to the planning problem, the first three versions of the RMGPA problem have been formulated as MLP models while the last version has been formulated as an NLP model. Due to the computational complexity of the problem, only the MILP models have been solved for small-sized planning problems. To satisfy the planning speed requirement, various heuristics have been proposed to solve different versions of the planning 
problem sub-optimally for large-sized problems. The solutions to the MILP models have been used as benchmarks in order to validate the heuristic approaches. Comparing the results of both optimization methods has shown that the proposed heuristics can achieve a good approximation of the exact solution to the planning problem. Additionally, the feasibility of the solutions obtained using both optimization methods have been validated.

Besides, many illustrative examples of all versions of the RMGPA problem have been shown to elaborate on the effects of input parameters, heuristic variations, and heuristic enhancements on the planning solution. The following effects have been demonstrated:

- Adding the adjustment phase upon designing the multi-granular virtual topology leads to a consistent saving in the weighted port count. This saving can be achieved by (1) decreasing the port requirement of the pass-through segments of virtual links, and (2) customizing the optical reach of virtual links instead of using the same reach with all virtual links.

- If fiber resources are plentiful, then solving the RMGPA problem beyond the minimum fiber requirement needed to obtain a feasible solution can lead to a minimal weighted port count.

- Heuristic variations based on the bifurcation of all demands always lead to the highest weighted port count in comparison to other bifurcation scenarios.

- Color (or label) conversions are quite beneficial in the fiber-scarce situation since they can significantly reduce the minimum fiber requirements. However, in the fiber-plentiful situation, color conversions can bring a slight decrease in the weighted port count.

- The maximum optical reach has an optimum value that leads to a minimal weighted port count. 
- The selection of the wavelength capacity, waveband size, and number of wavebands per fiber such that it leads to the same or different fiber capacity has an optimum combination that can further improve the planning solution.

- Changing the ratio of EXC port weight to the MG-OXC port weight does not change the resultant multi-granular virtual topology if the color-continuity constraints are kept and the optical-reach constraints are not imposed. Otherwise, changing the ratio can alter the resultant virtual topology, best maximum optical reach, and winning heuristic variation.

Finally, a thorough investigation of the heuristic variations proposed for different versions of the RMGPA problem has been conducted via simulation using different physical topologies, traffic patterns, fiber capacities, and maximum optical reach values. The analysis and simulation results have provided valuable insights into many issues in both fiber-scarce and fiber-plentiful situations. Among these issues are the definition of the best heuristic variation, fiber capacity $/ C-W-B$ combination, and maximum optical reach as well as the significance of color conversions, demand bifurcation, and customizing the optical reach. The findings of this investigation suggest the following:

- Heuristics based on the bifurcation of demands at all traffic granularity levels can only be beneficial in the fiber-scarce environment provided that the traffic pattern is uniform and both the traffic intensity and network physical connectivity are high. Otherwise, they offer no advantage over not bifurcating demands or bifurcating only fiber demands.

- With the existence of fiber demands, there is no clear winner between the heuristic based on the bifurcation of fiber demands and the one that does not bifurcate. However, the likelihood that the latter outperforms the former increases in the case when the network physical connectivity is low and fiber resources are plentiful. 
- In the fiber-scarce situation, there is no clear overall sorting policy winner. However, without/with the consideration of the color-continuity/optical-reach constraints, the heuristic variations based on Policy 3 are out of the contest when the network physical connectivity is low. Moreover, with the optical-reach constraints, Policy 5 is out of the contest. Accordingly, Policies 3 and 5 can be excluded from the planning process in these problem situations if the planning time is an issue.

- In the fiber-plentiful situation, the size of the contender set is smaller than in the fiber-scarce situation. Without the optical-reach constraints, Policies 1 and 4 are the main contenders, and Policy 4 tends to be more cost-effective in the case where both the network physical connectivity and fiber capacity are low. On the other hand, with the optical-reach constraints, Policies ' 1,4 , and 6 ' $/$ Policies $1,4,5$, and 6 ' are the main contenders without/with customizing the optical reach. However, Policy $6 / 1$ shows potential where the network physical connectivity is low/high and the node-to-node distance is high/low. Accordingly, to save on the planning time, the heuristics based on Policies 2 and 3 can be excluded from the planning process, and what should be included in terms of the sorting policies depends on the version of the RMGPA problem.

- The best heuristic variation is decided by the traffic pattern, physical topology, fiber capacity/C-W-B combination, ratio of EXC port weight to the MG-OXC port weight, whether the color-continuity and optical-reach constraints are imposed, whether the optical-reach customization is considered, and whether fiber resources are scarce or plentiful.

- Color conversions not only reduce the fiber requirement in fiber-scarce situation, but can also cause a marginal reduction in the weighted port count in the fiber-plentiful situation. Color conversions are beneficial when the network physical connectivity is high. However, the 
amount of savings achieved with color conversions is also a function of the traffic pattern and fiber capacity/C-W-B combination.

- The best maximum optical reach is a function of the physical topology, traffic pattern, fiber capacity/C-W-B combination, whether the optical-reach customization is considered, and whether fiber resources are scarce or plentiful.

- Customizing the optical reach always brings a consistent saving (up to $\approx 14 \%$ ) in the weighted port count. This saving varies according to the physical topology, traffic pattern, fiber capacity/C-W-B combination, and condition of fiber resources. Although at the same maximum optical reach the saving is always achieved by customizing the optical reach, further savings can be realized by increasing the maximum optical reach. Some of these savings are achieved by increasing the maximum optical reach value up to $10000 \mathrm{~km}$ compared with 5000 $\mathrm{km}$, which is feasible nowadays, if the reach is not customized. Now, assuming that the NSF and EON network cases are representative long-haul network references and given the fact that a lot of research is being conducted in order to extend the optical reach to thousands of $\mathrm{kms}$, this saving will justify the need for system vendors to target an optical reach around $10000 \mathrm{~km}$.

- The best fiber capacity/C-IV-B combination is a function of the physical topology and traffic pattern, whether the optical-reach customization is considered, and whether fiber resources are scarce or plentiful. However, the selection of the best fiber capacity/C-W-B combination is less sensitive to the consideration of both the color-continuity and optical-reach constraints in the fiber-plentiful situation than it is in the case where fiber resources are scarce. 


\subsection{Future Research}

As an extension to this work, we conclude this thesis with a list of open problems based on our current work:

- Our optimization approaches can be extended for use in other GMPLS-based node architectures. One possible architecture with which to start is to replace the multi-layer-based MG-OXC architecture used in the optical segment of our GMPLS-based node architecture with the single-layer-based MG-OXC proposed in [CAQi04][CAQi06].

- More efforts can be directed toward improving the optimization approach used to solve the RMGPA problem sub-optimally while satisfying the planning speed requirement at the same time. This improvement can be made by (1) developing new sorting policies and multigranular virtual topology design approaches, and (2) using other optimization techniques such as genetic programming and tabu search methods. Our heuristics can be used as a benchmark for the newly developed optimization approaches.

- Extending the size of the solvable MILP models to increase the precision of the validation procedure can be achieved as follows. (1) The computing resources can be increased through the use of super computers. (2) Since finding an initial feasible solution in the branch-andbound tree takes a considerable amount of time, reducing the size of the branch-and-bound tree in the MILP model can be done by feeding the feasible solution obtained through the heuristic approach to the tree. This means that a considerable number of tree nodes will be trimmed and the optimum solution can be obtained in a shorter amount of time. However, the challenge here is how to provide a feasible solution to CPLEX software. (3) The use of the latest version of CPLEX software (version 11.0) can significantly reduce the solve time. 
- Further research is still needed to conduct a sensitivity analysis of the cost weight of all MGOXC and EXC port types using up-to-date cost models from the industry. The objective of this investigation is to see how changing the cost points will alter our findings in this thesis.

- To improve the reliability of the GMPLS-based transport network, the RMGPA problem can include the availability calculations in the route selection criteria while building the multigranular virtual topology. In order to achieve this, availability parameters such as the mean time to failure of all MG-OXC and EXC elements are required. Good resources for this work can be found in [HWNM03][Grov04][TMPa05][KMOk08].

- Protection techniques can be incorporated into our proposed approaches. This will be also a good opportunity to examine both the single- and multi-layer-based MG-OXC architectures under protected traffic conditions. We recommend that the focus be on the p-cycle technique, since it is receiving more attention recently in both industry and academia because it offers a ring-like restoration speed and mesh-like capacity efficiency. Good references for beginning this work can be found in [Grov04][WaMo05].

- With the advances in optical signal processing, our approaches can also be extended to tackle the planning problem in which changing colors (or labels) can occur optically without the need of the EXC. Moreover, optical converters can be used in the MG-OXC architecture, meaning that the EXC will be required mainly for (de)grouping and switching sub-wavelength flows as well as optical signal regeneration.

- Our approaches can also be extended to solve the adaptive (also known as scheduled) planning problem in which the problem is solved for several traffic demand matrices, where each matrix represents the average traffic demands over a certain period (e.g. hours, days, months, or years). Useful references for such work can be found in [GADO01][ZZMo02][Kur+03]. 
- Minimizing the number of MG-XC cross-points instead of the number of MG-XC ports is the most accurate way to determine the node cost. Solving such a problem exactly requires formulating it as an NLP model. It will be interesting to incorporate this notion into our approaches and compare it with the approach in which the number of cross-points is decided upon solving the optimization problem, as is the case in [Lee+02][HMWu03].

- Incorporating the physical impairments, such as PMD and CD, in the RMGPA problem will lead to a more realistic planning of the GMPLS-based transport network. This requires developing impairment accumulation models, and satisfying the optical-reach constraint in this case means that the impairments accumulate without exceeding predefined thresholds. However, solving such a problem exactly requires formulating it as an NLP model. Good related papers on this subject can be found in [MVTo04][ZZFZ04][Lav+07][Li+07][PRMa07] [Tomk07].

- Our focus in this thesis has been on the solution to the off-line case of the RMGPA problem. Extending our approaches to solve the on-line case of the RMGPA will be very useful as a control-plane algorithm. Moreover, it can be useful in the post-planning evaluation phase, in which a more extensive evaluation of the designed network can be performed. Such evaluations can include the analysis of the network behavior for unexpected failure scenarios. and dynamic traffic conditions. Good papers with which to start this work can be found in [HoMo02a][CAQi04][ZXWu06][CAQi07].

- Although our approach has addressed a new problem by considering the whole GMPLS traffic hierarchy, it will be interesting to change the approach to accommodate different granularity options and compare the modified versions of the approach with other existing approaches that address the same type of problems. 
Appendix $A$

\section{Results of Heuristics Comparison without Conversion and Regeneration Capabilities}

In this appendix, we present the detailed results with $95 \%$ confidence intervals of the heuristics comparison without the consideration of the color conversion and optical signal regeneration capabilities. Tables A.1-A.3, A.4-A.6, and A.7-A.9 show the results for the 8-node network, 14node NSF network, and 11-node EON network respectively. 


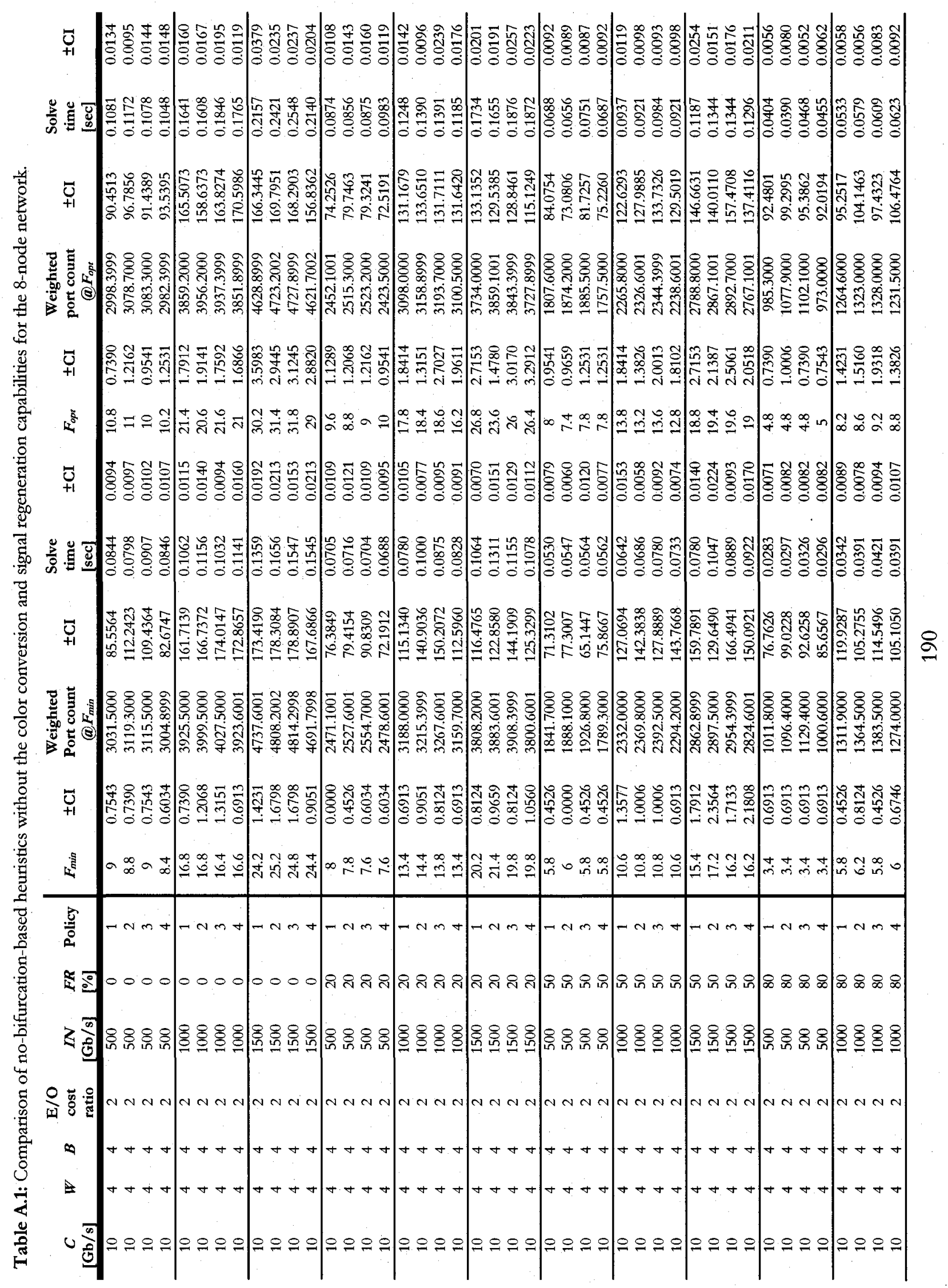




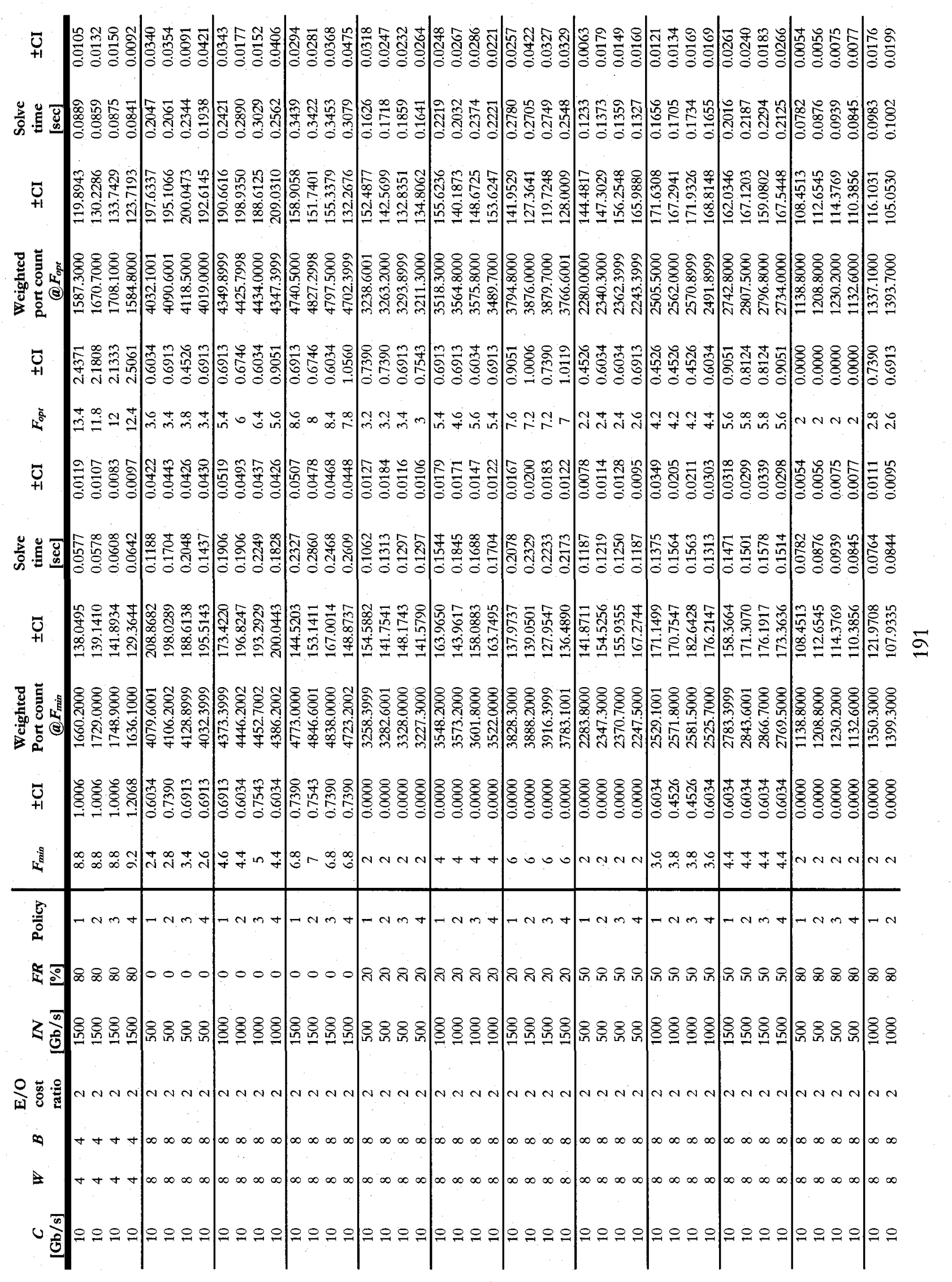




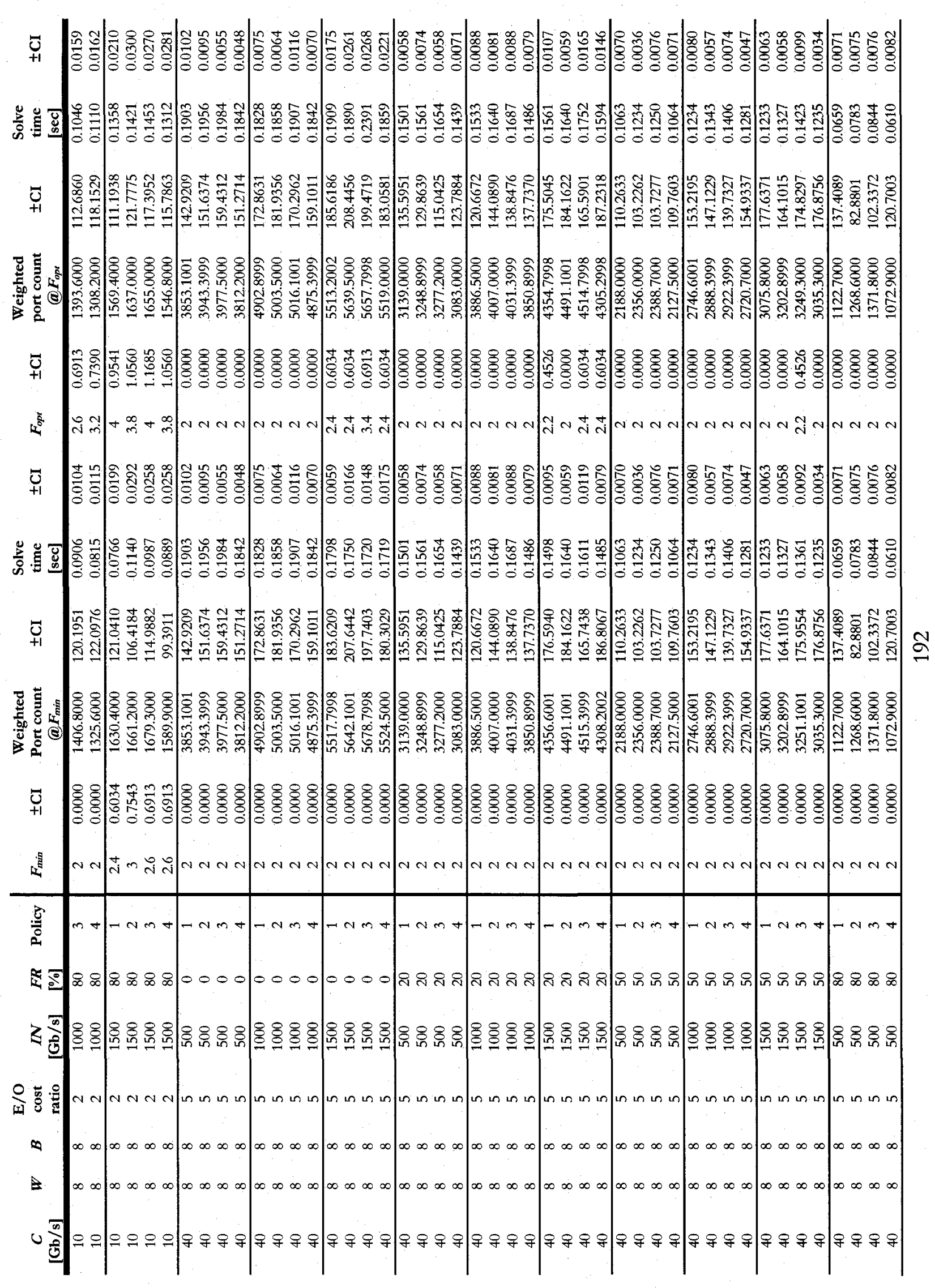




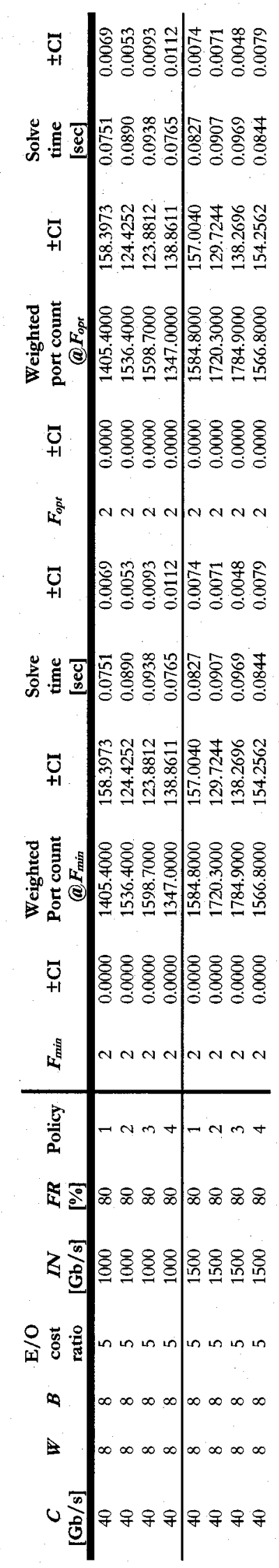

2 


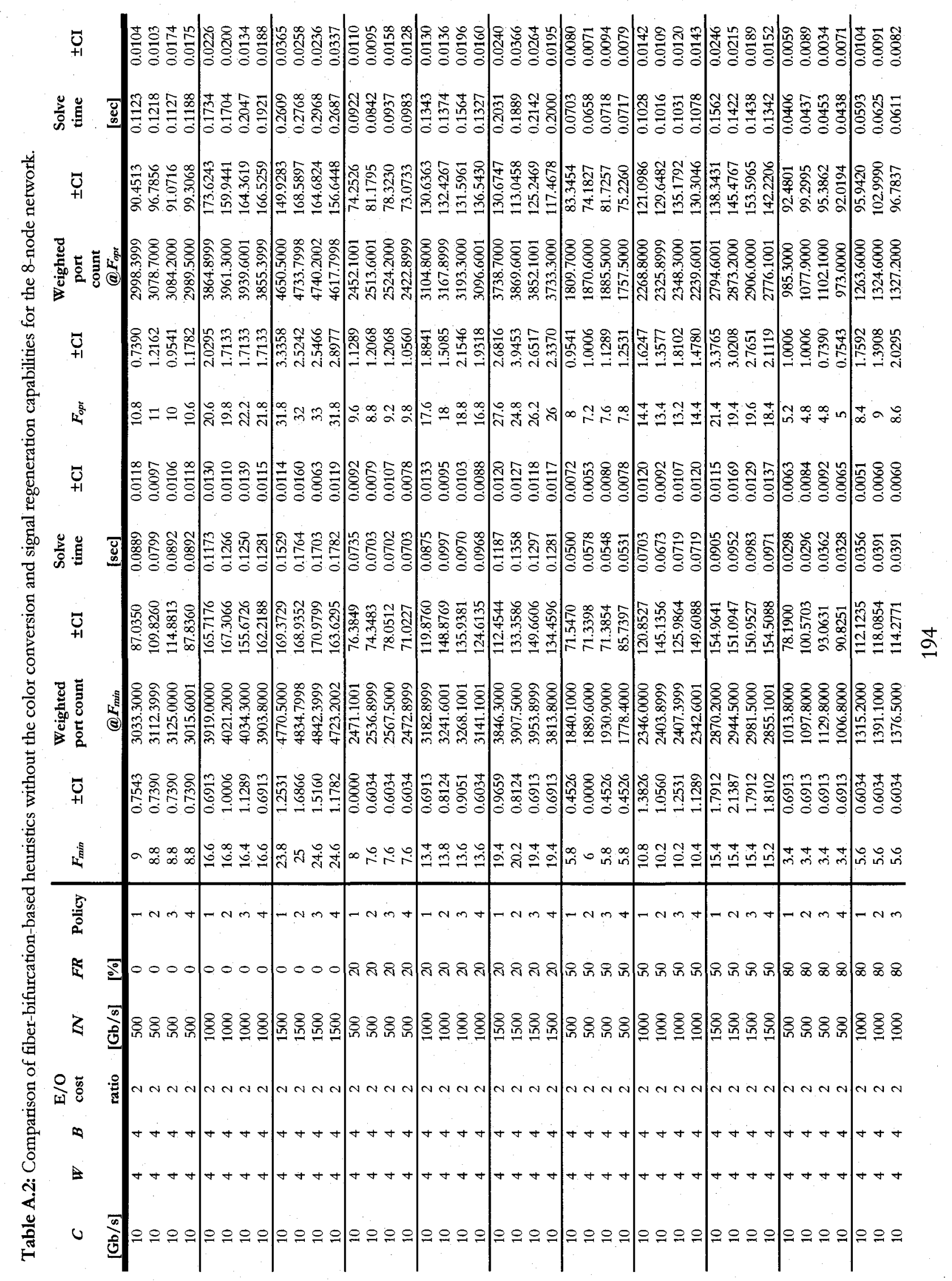




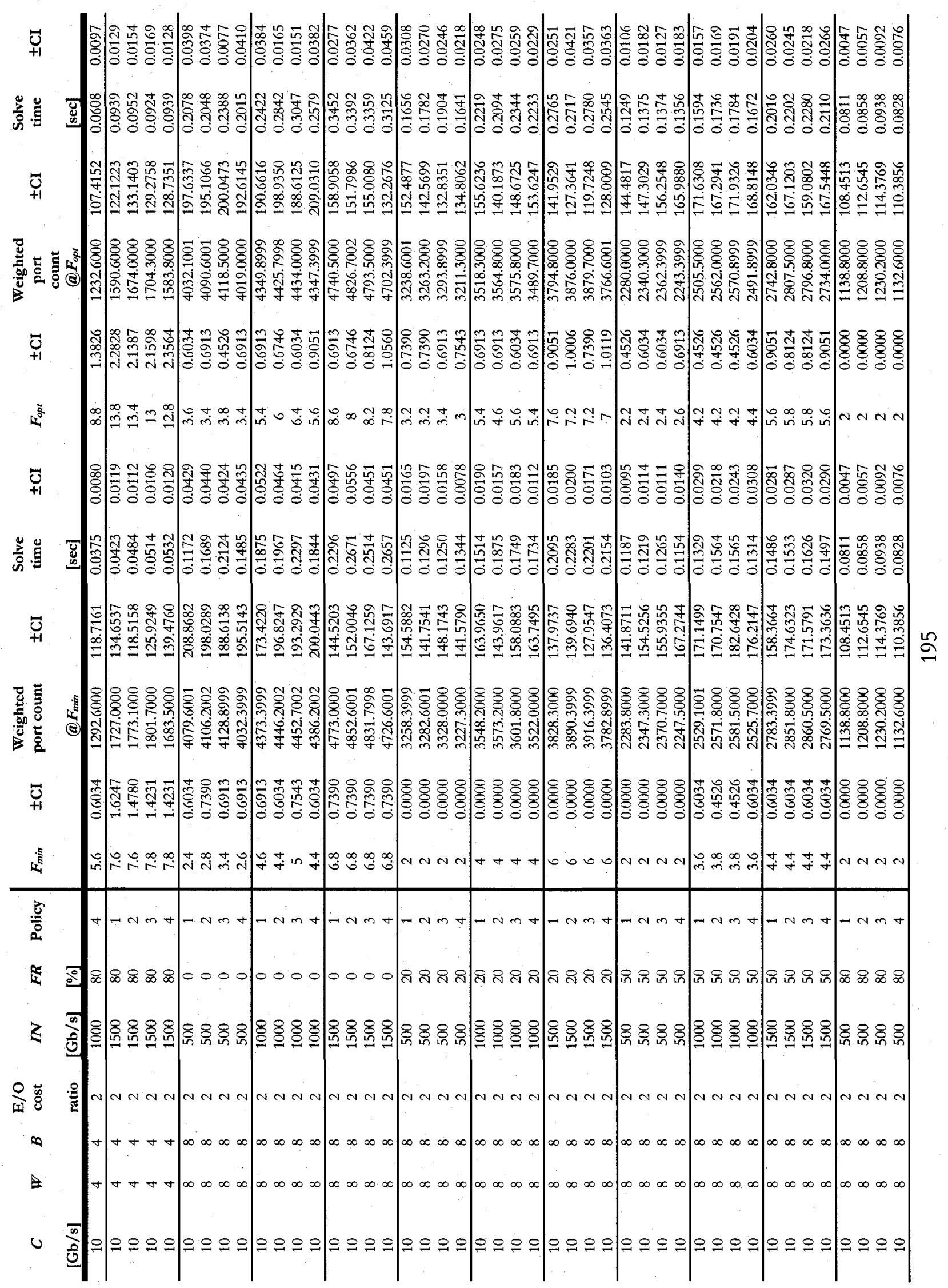




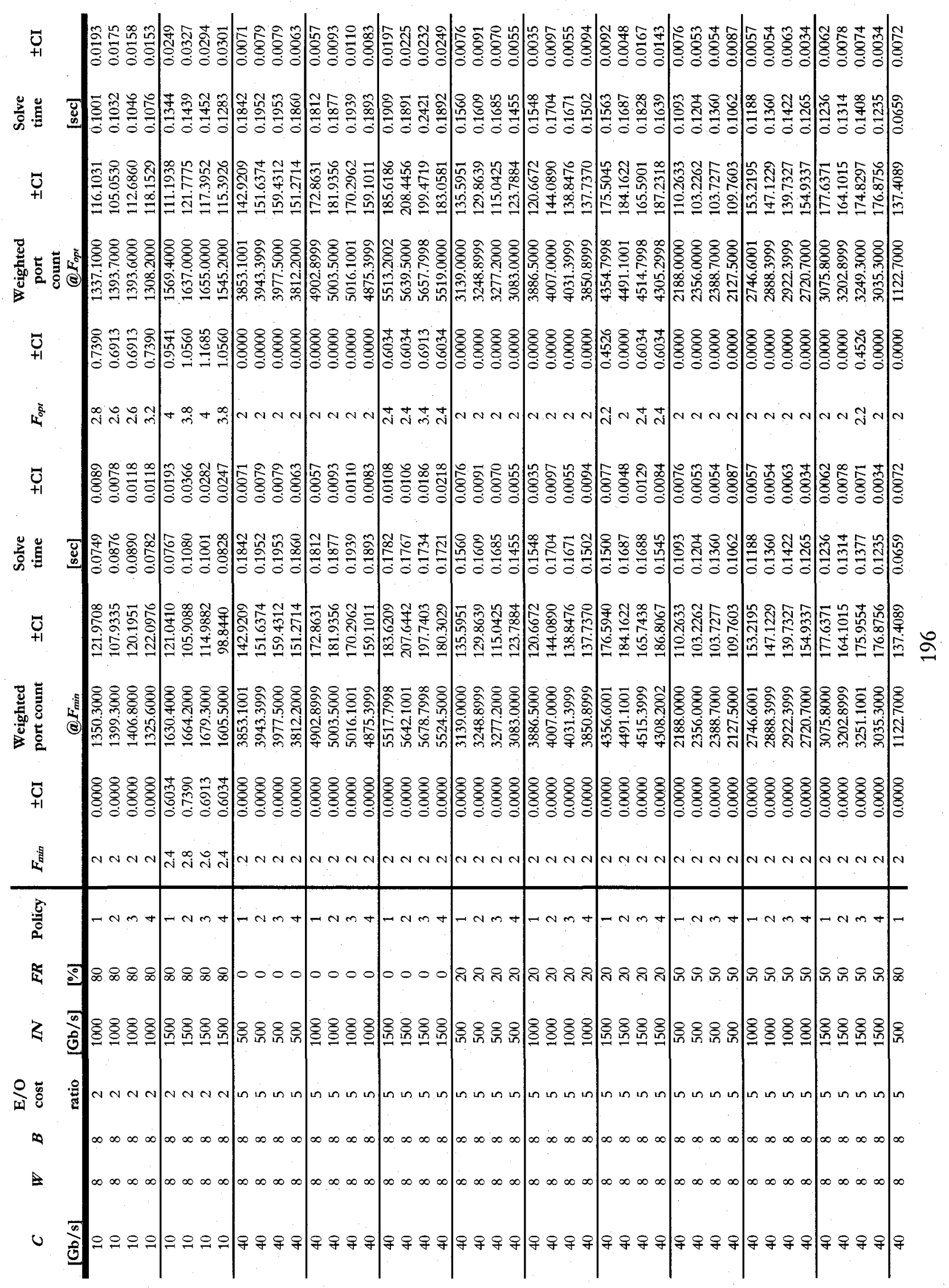




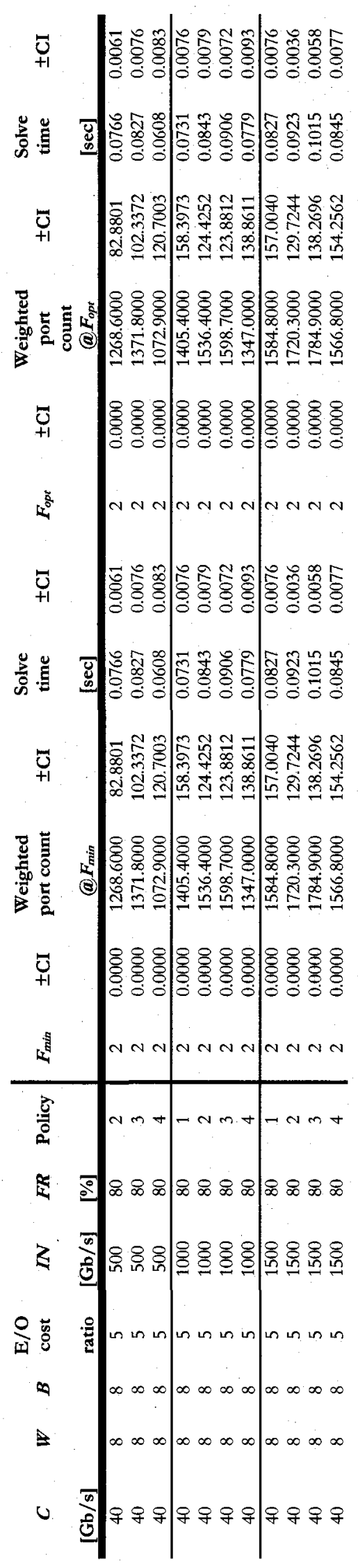




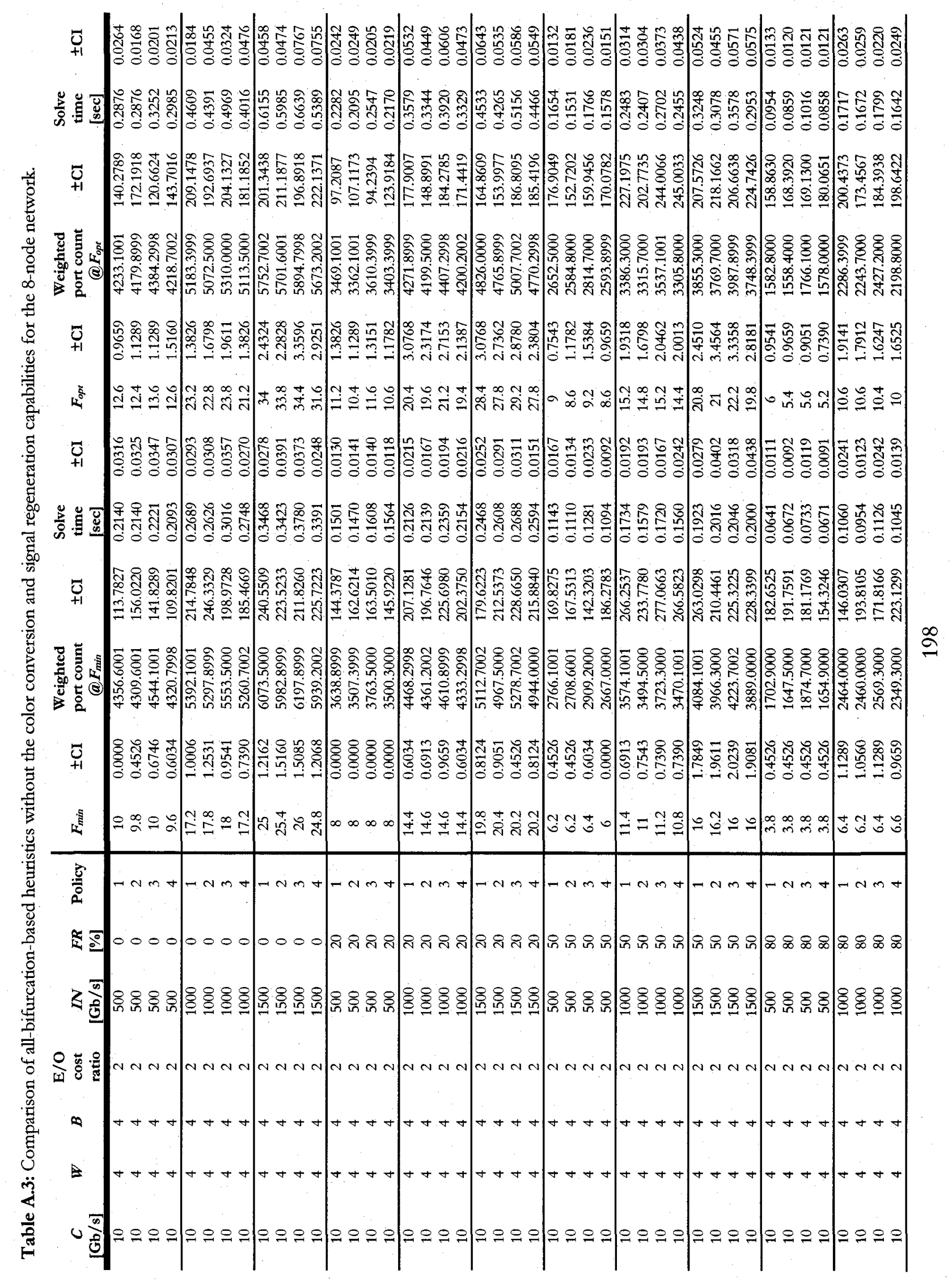




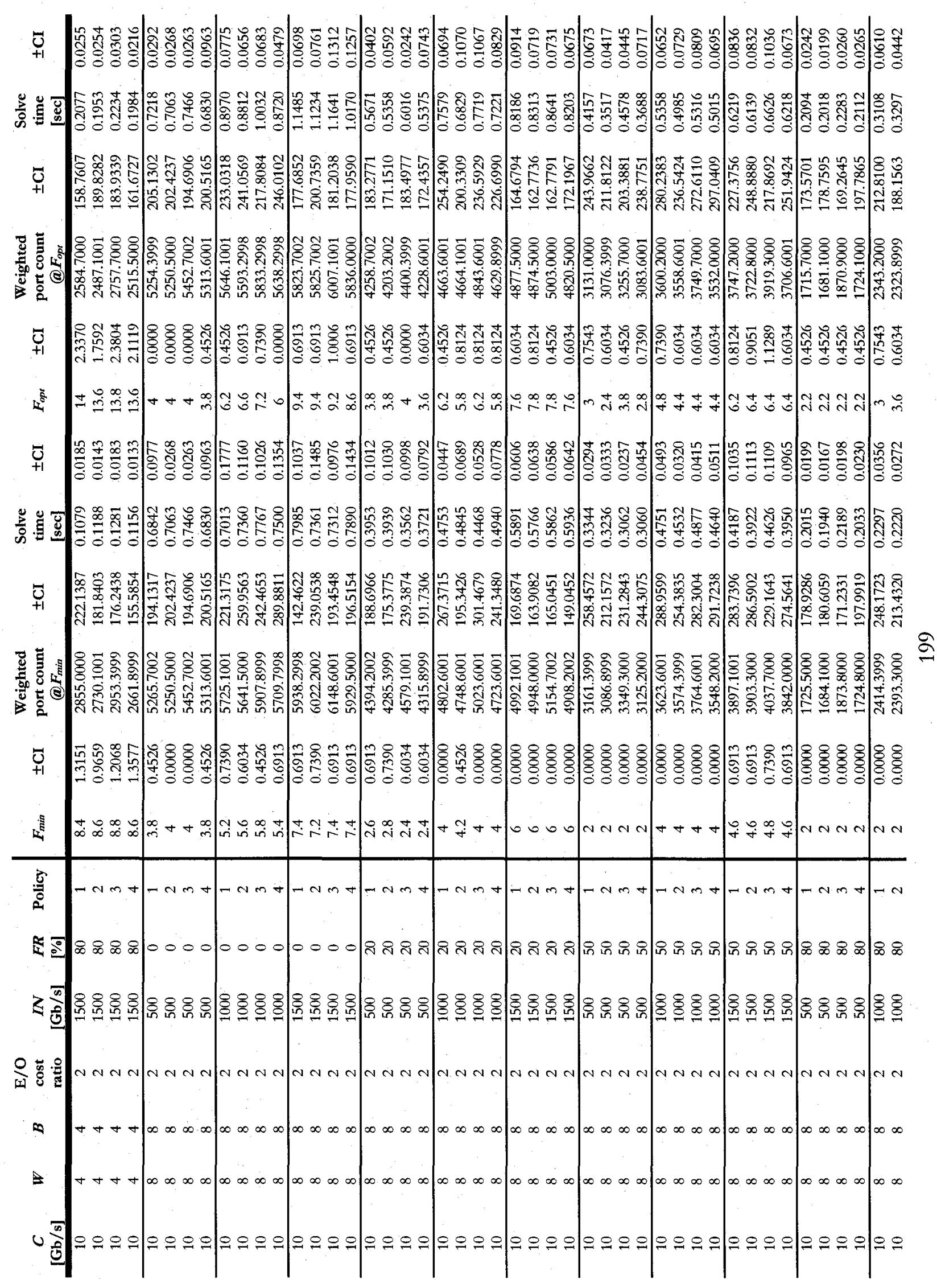




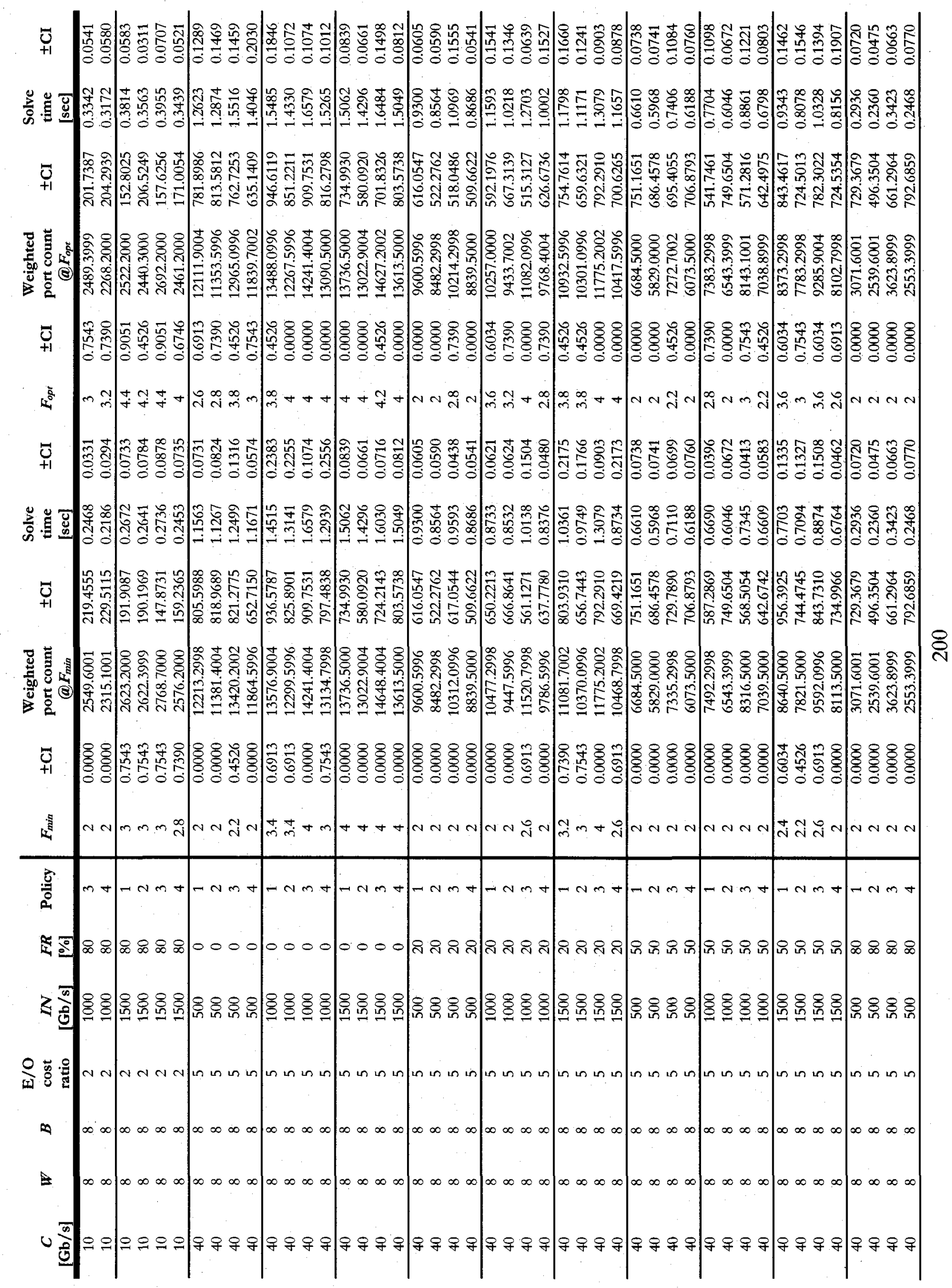




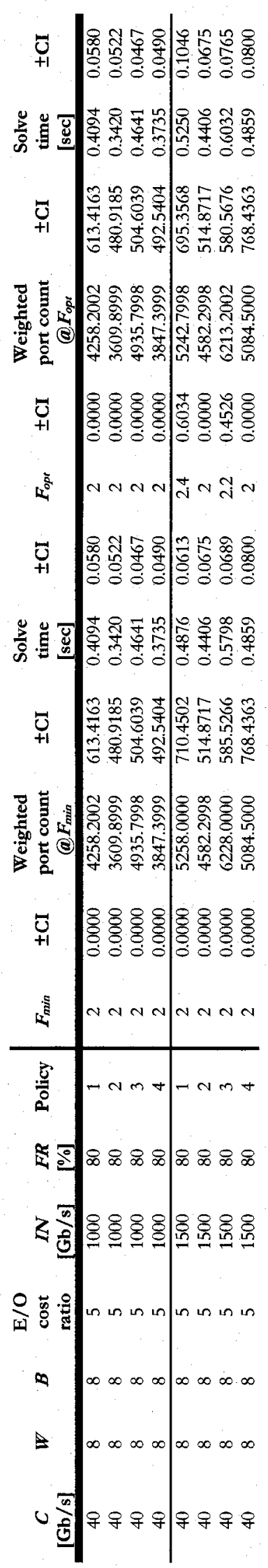




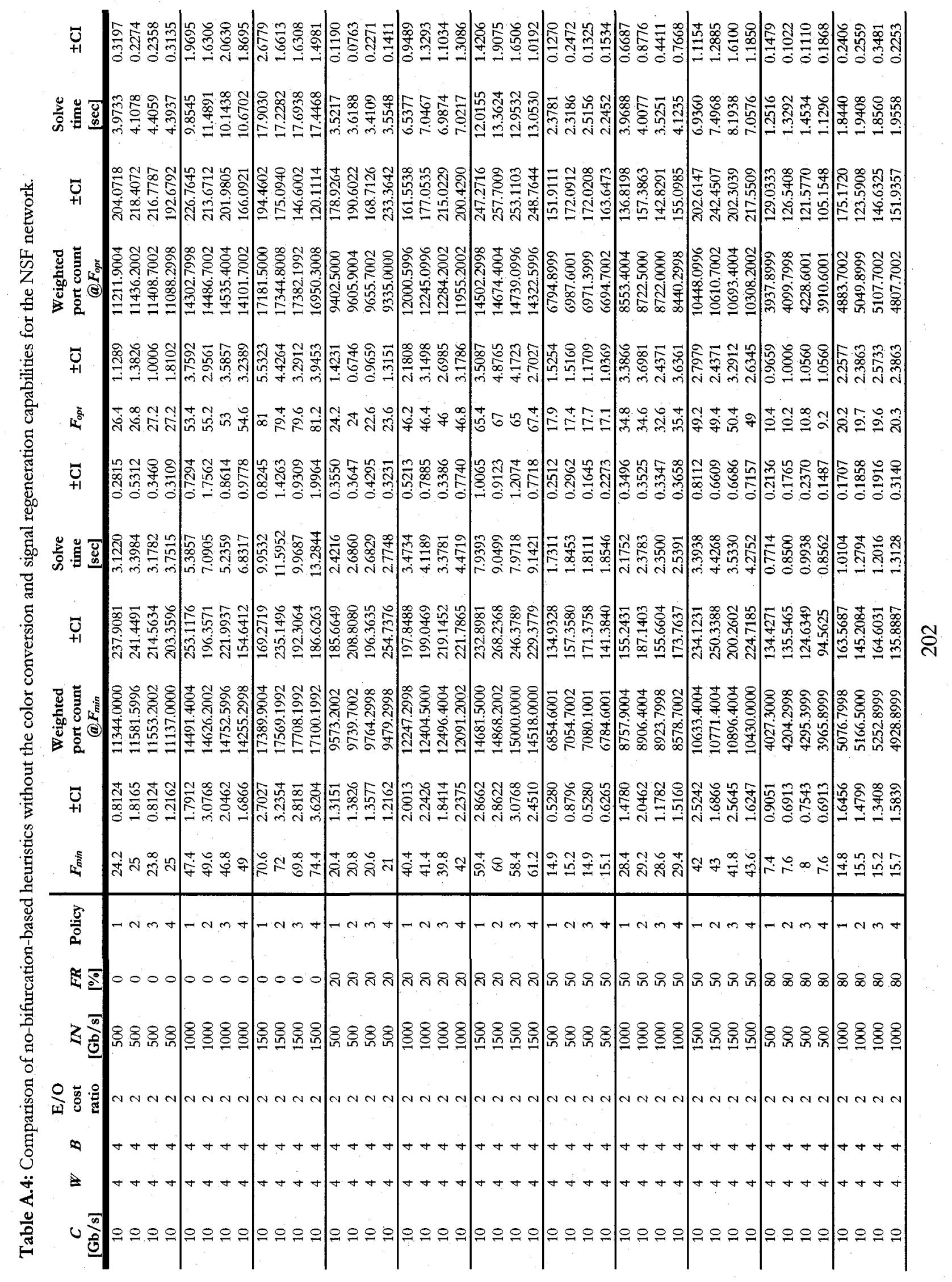




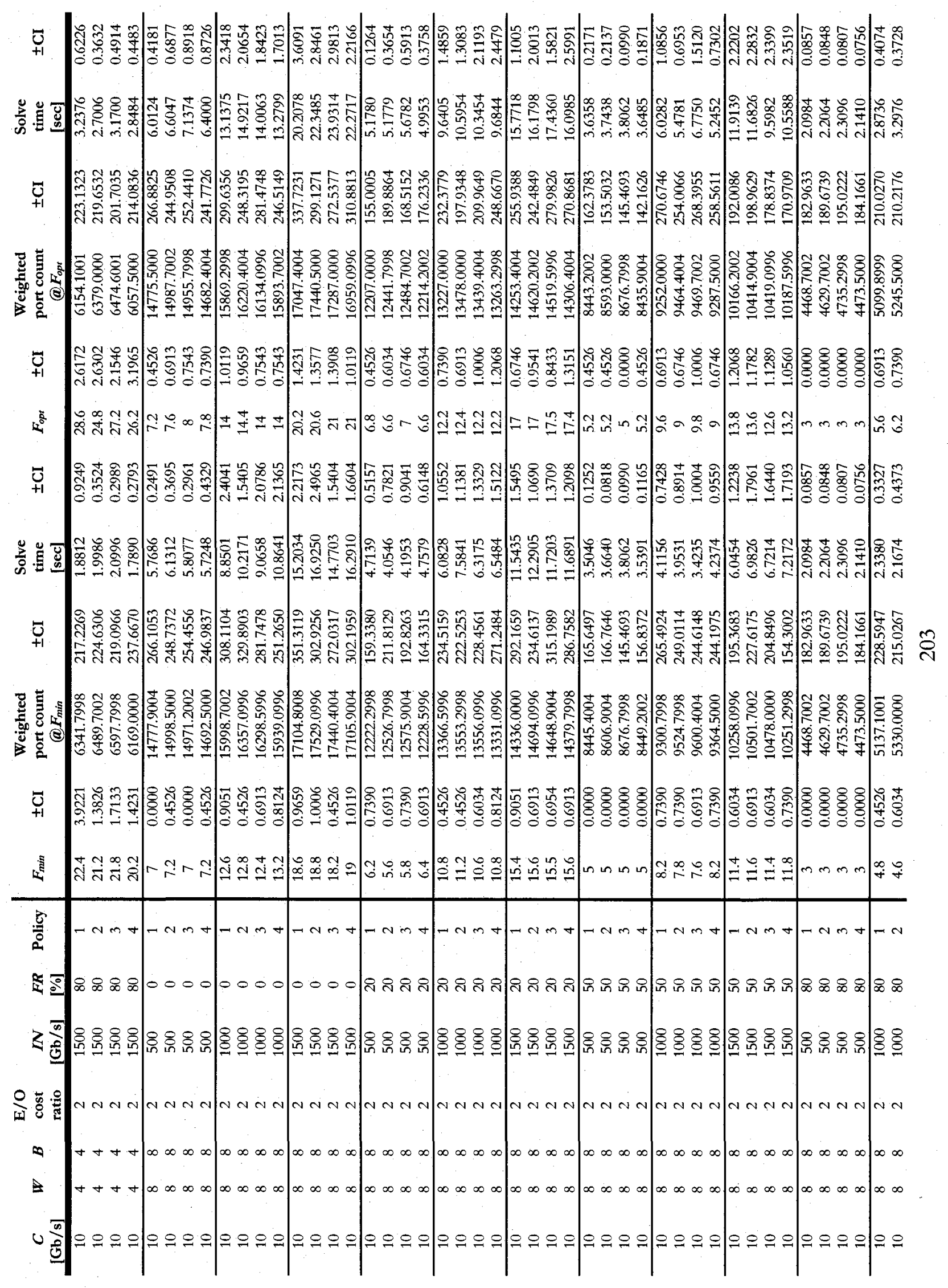




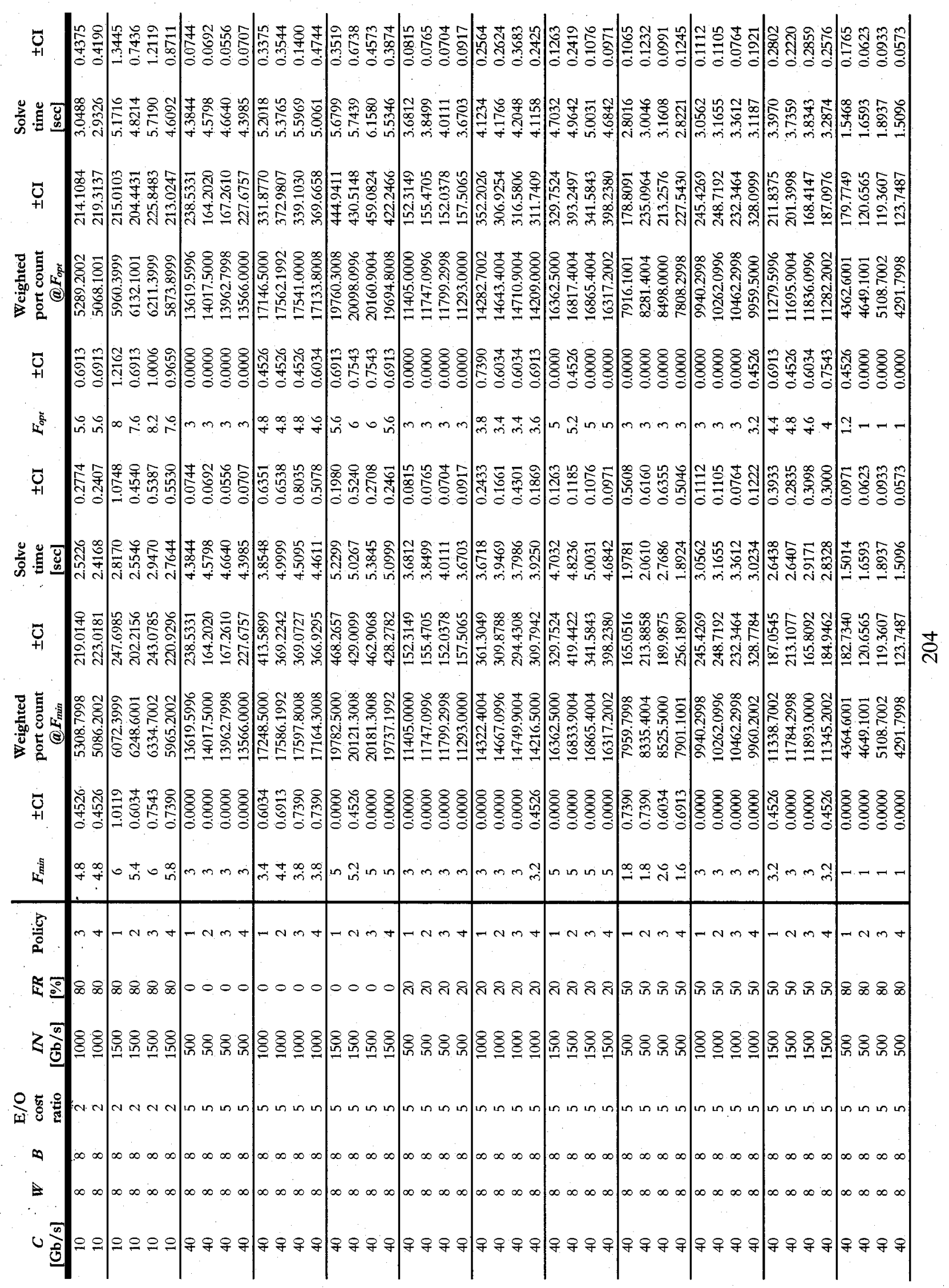




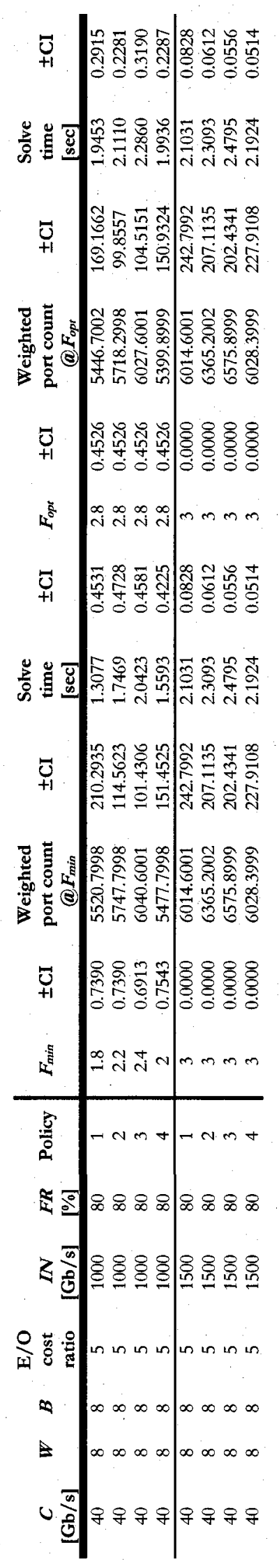

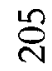




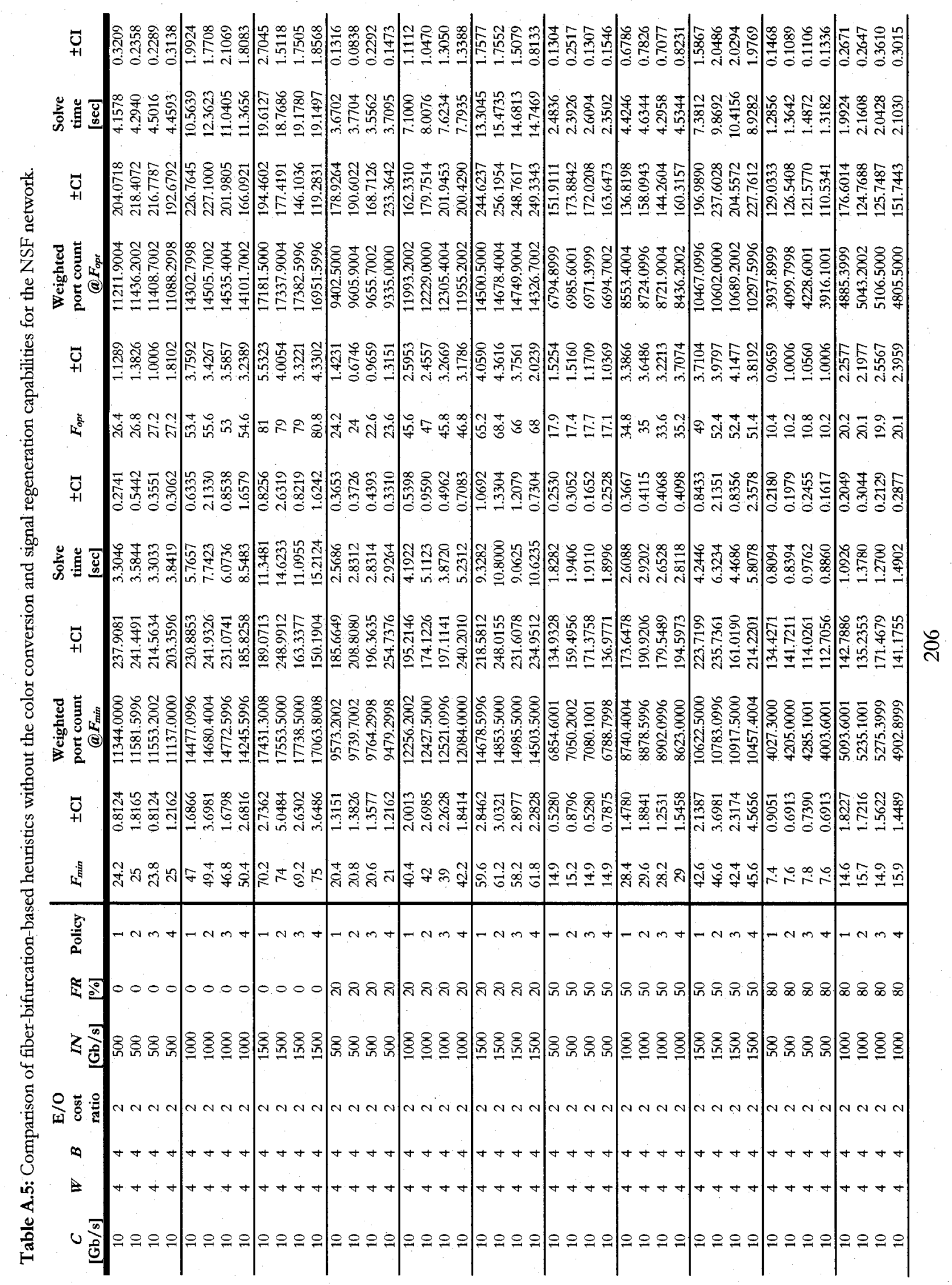




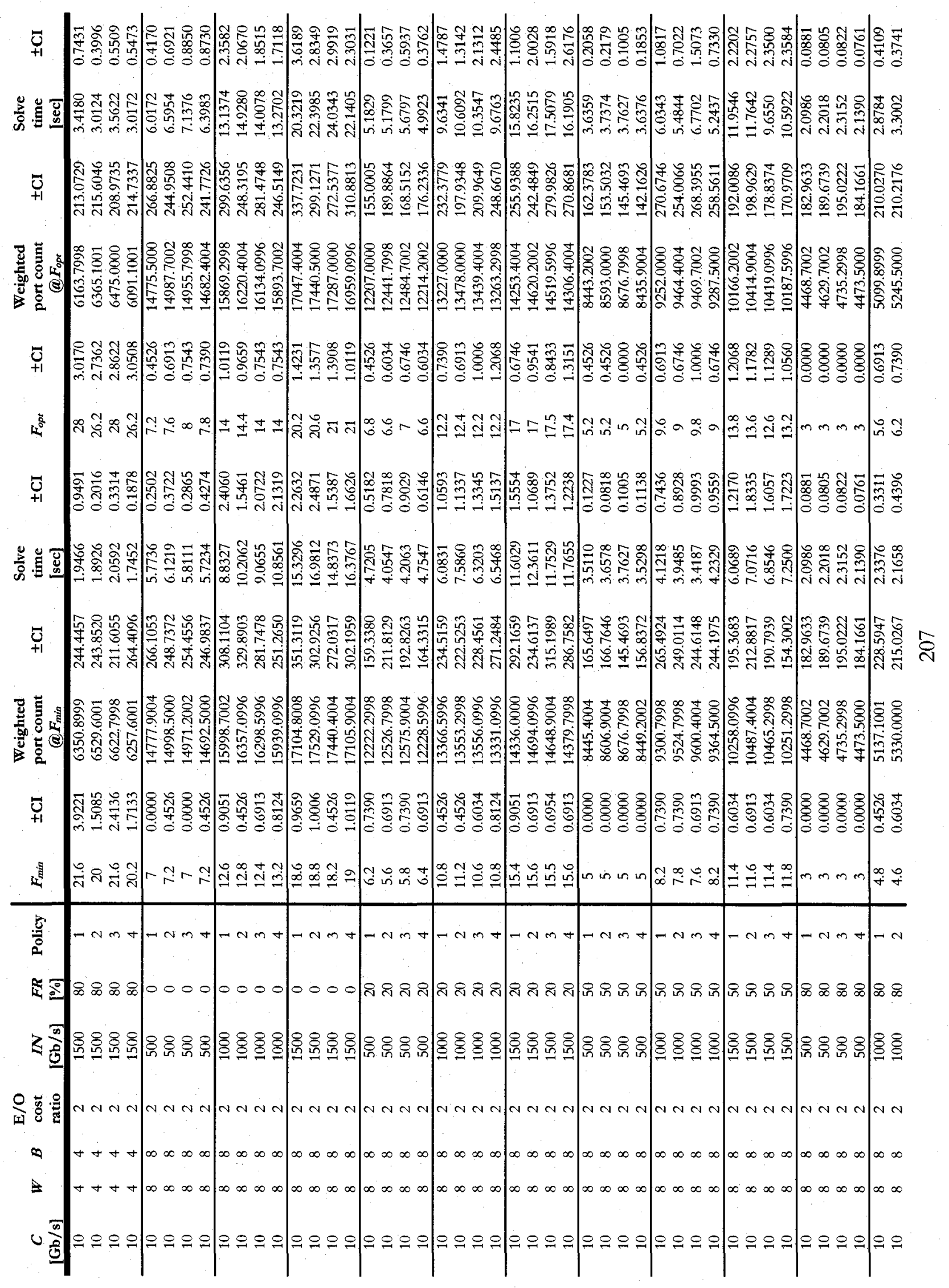




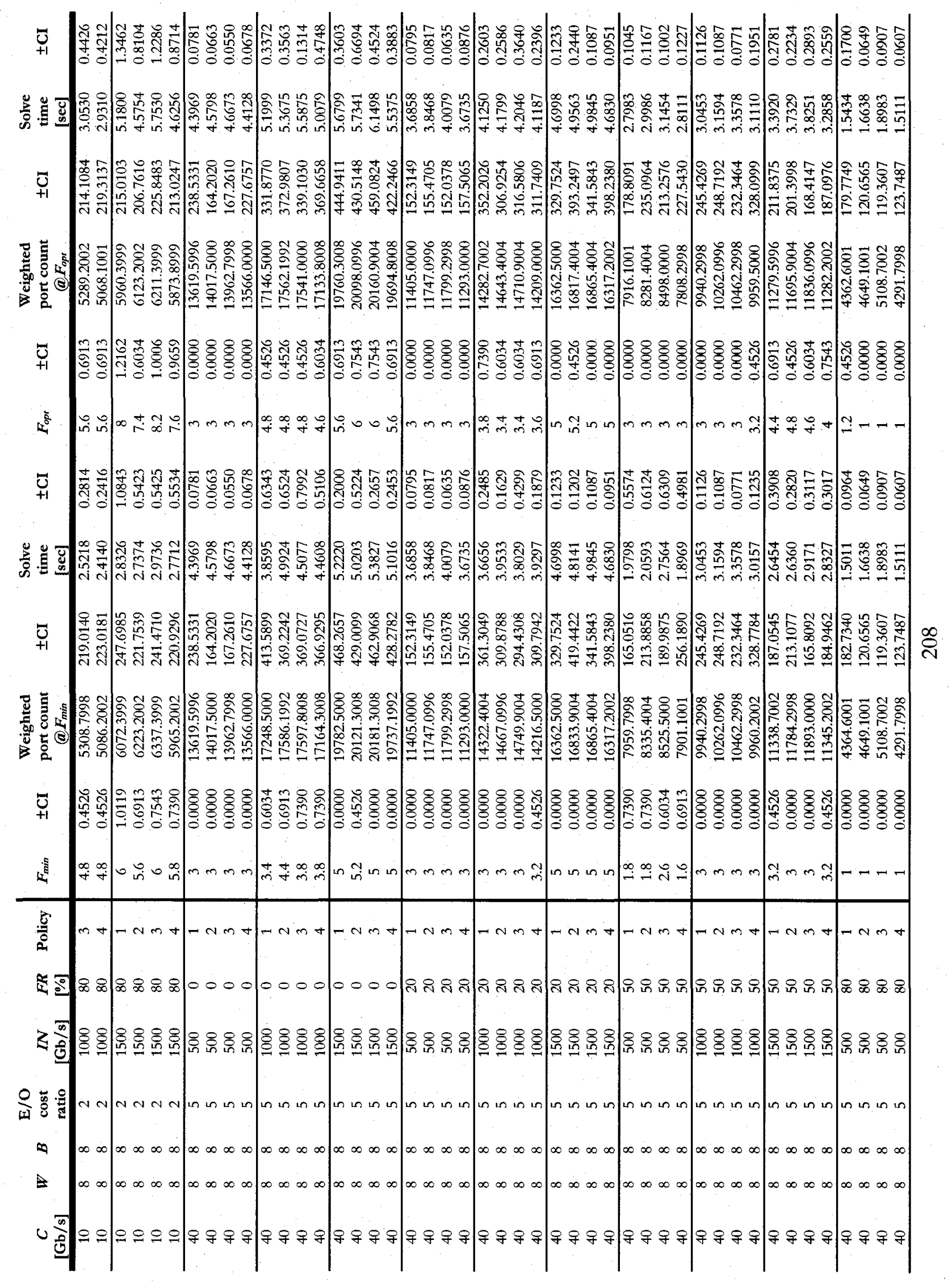




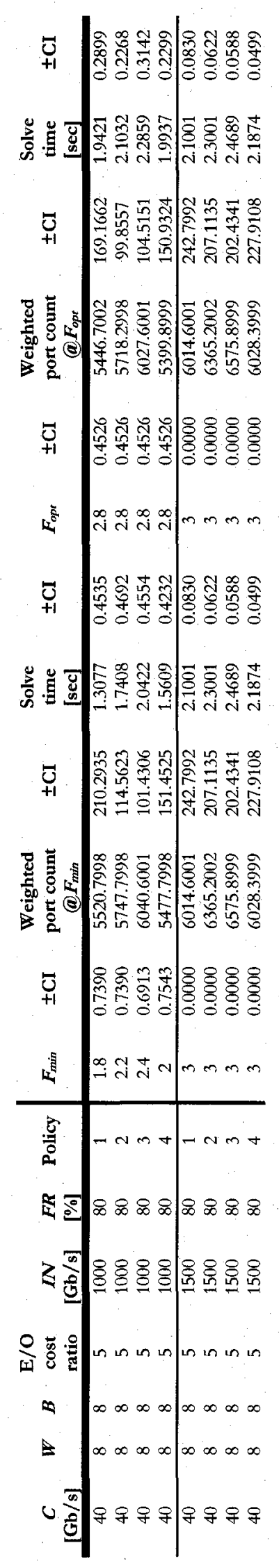

尺) 


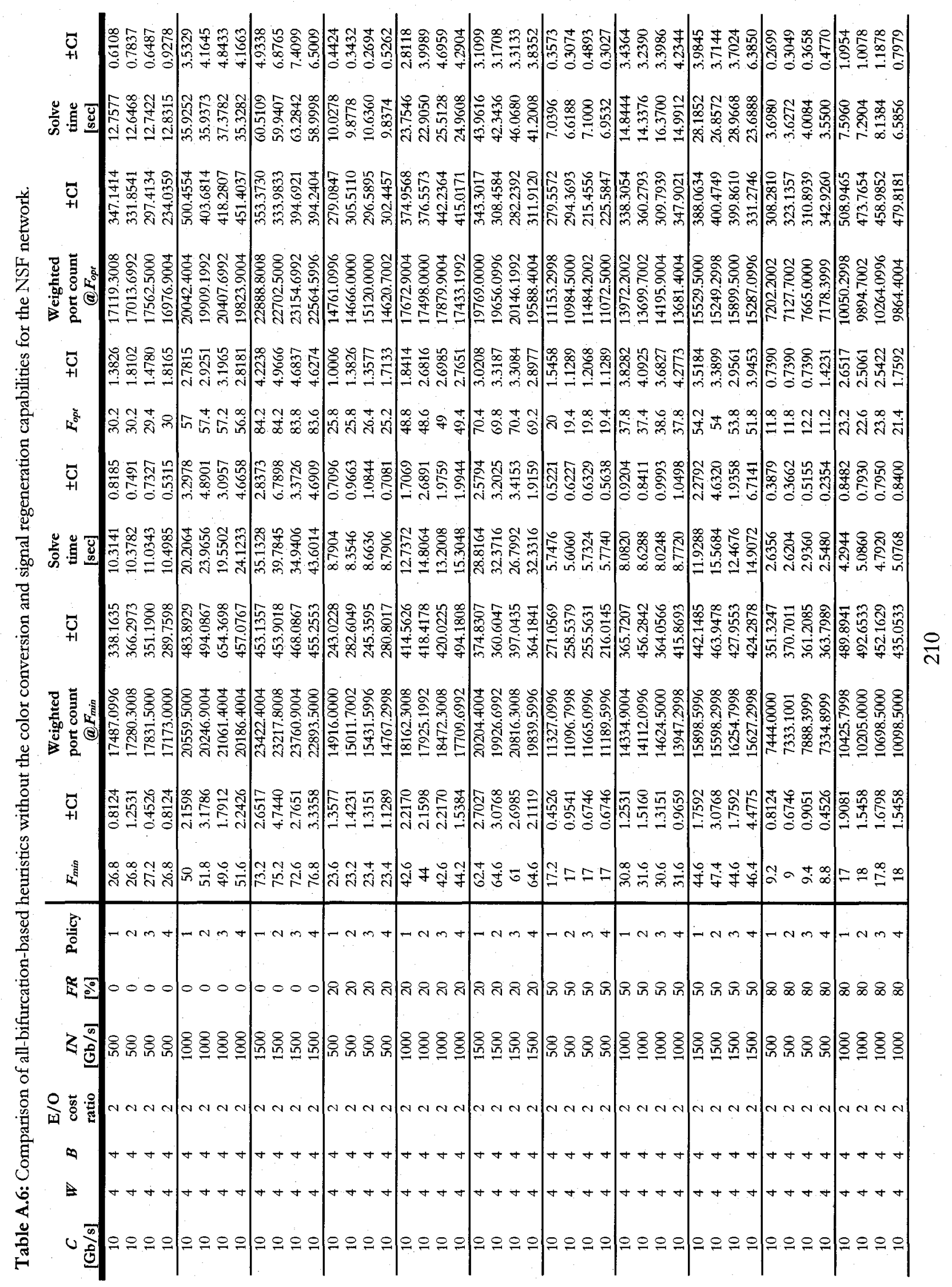




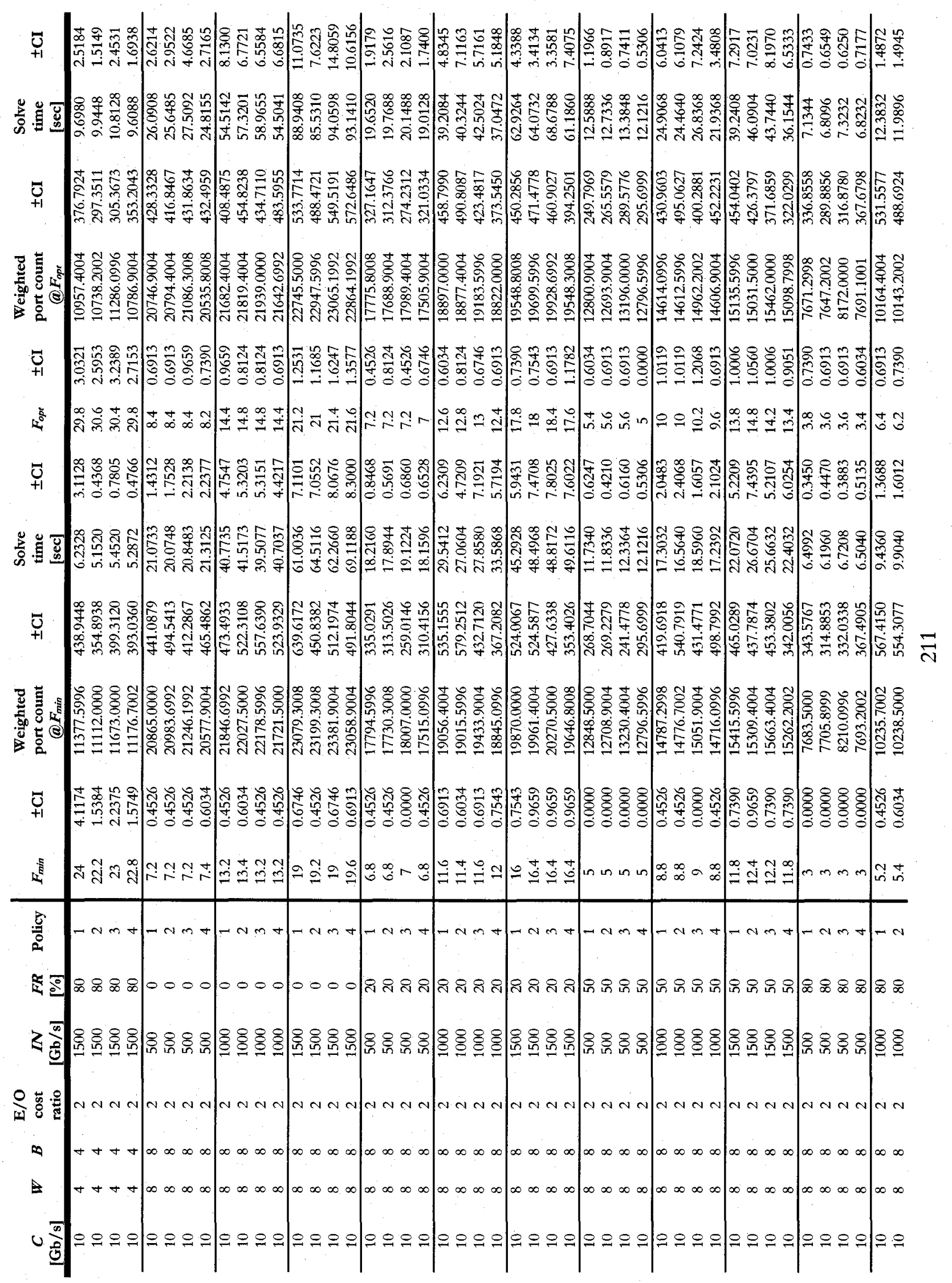




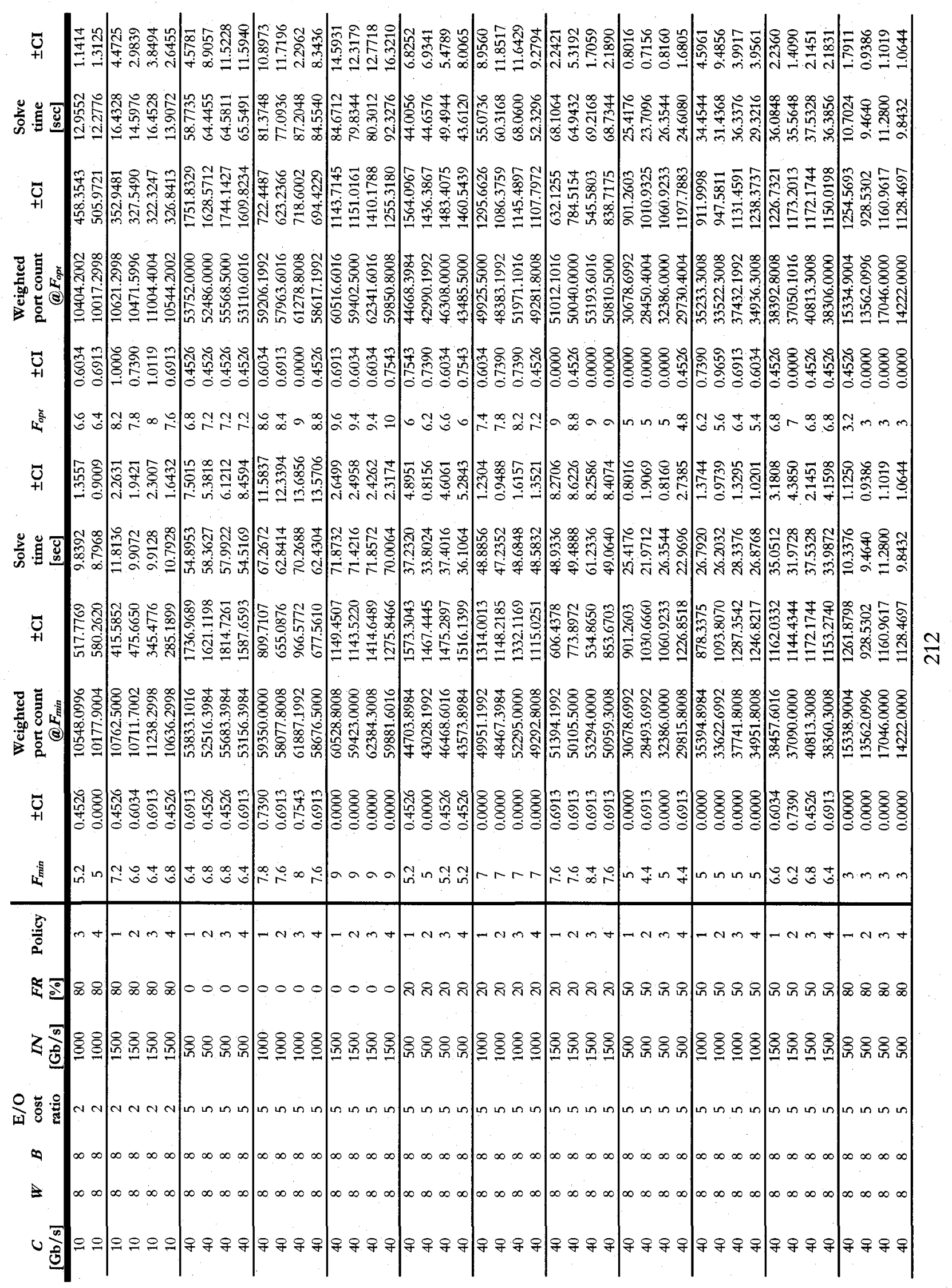




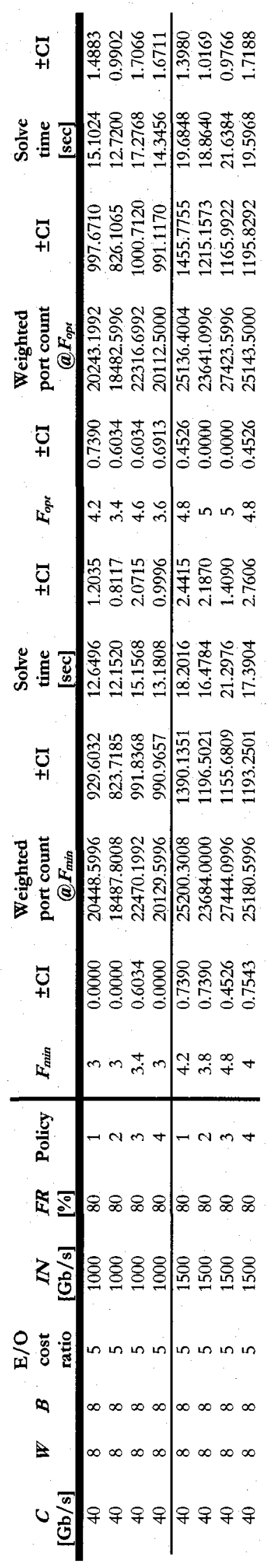

$\frac{n}{2}$ 


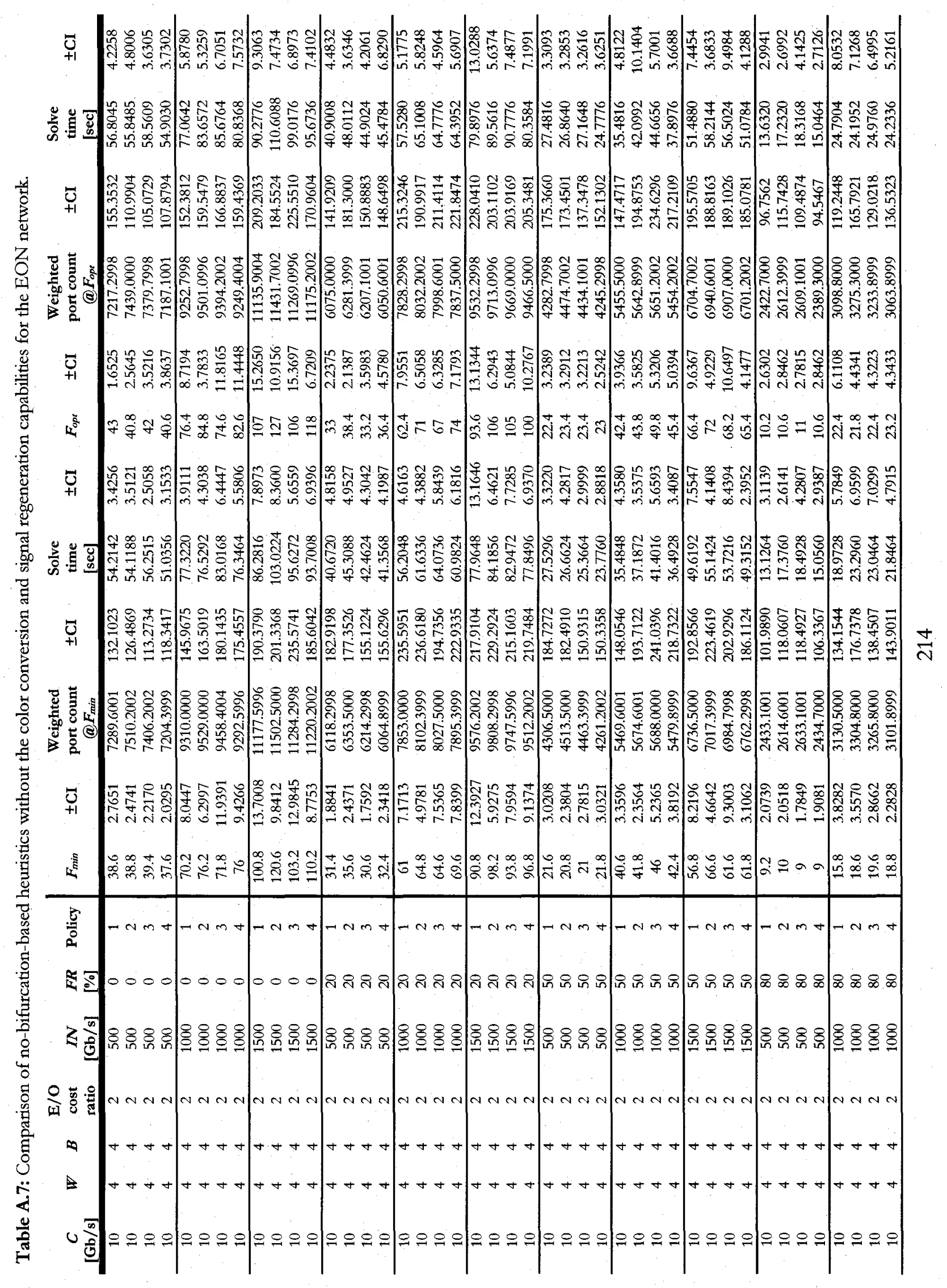




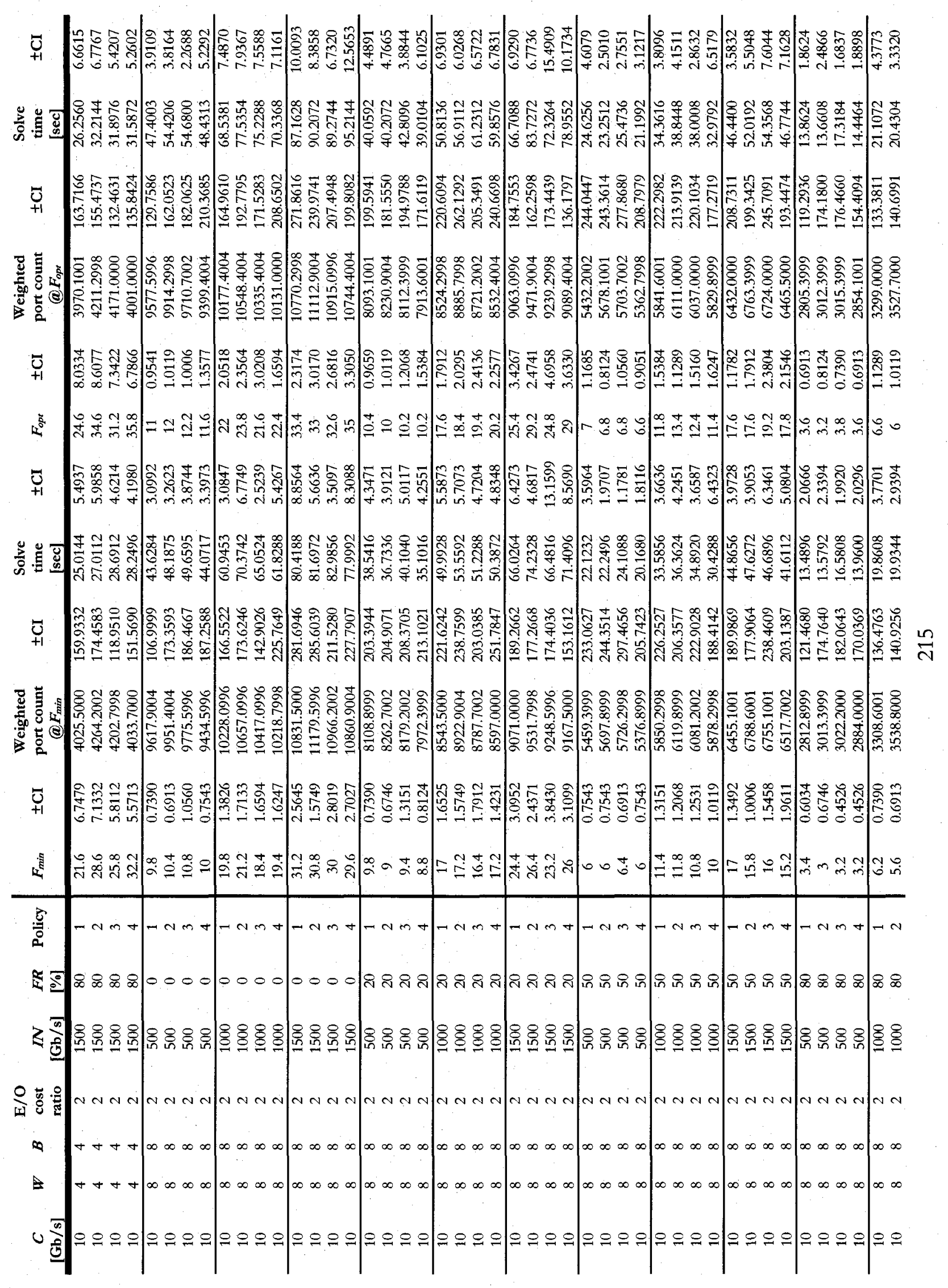




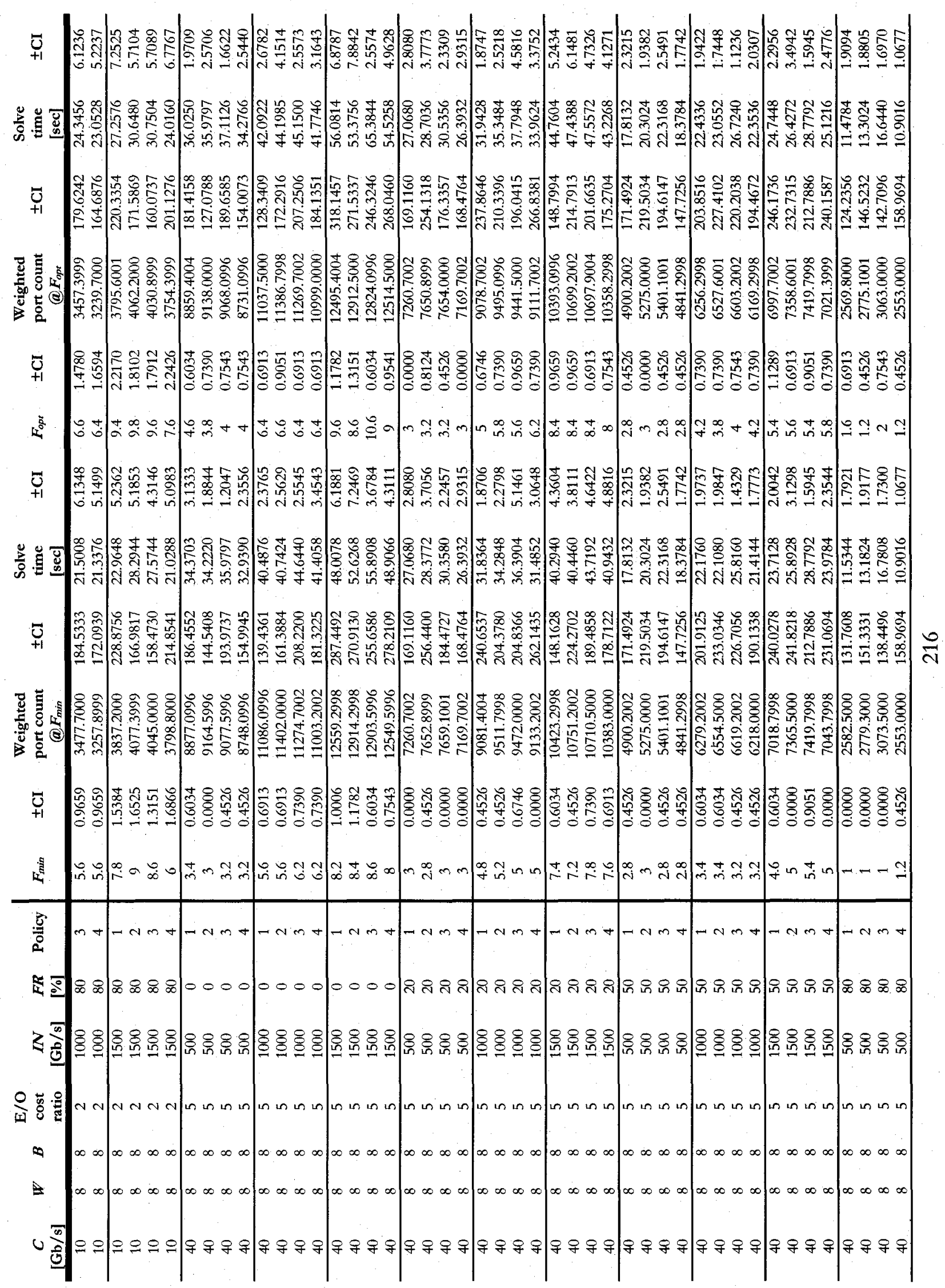




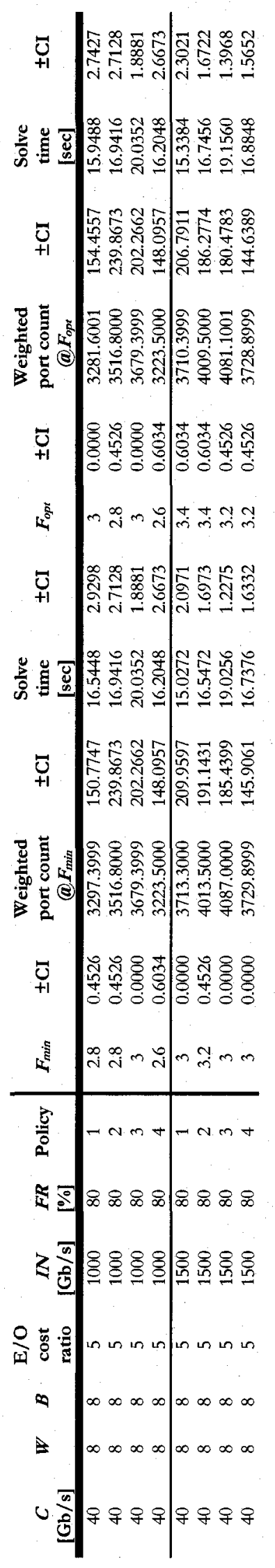




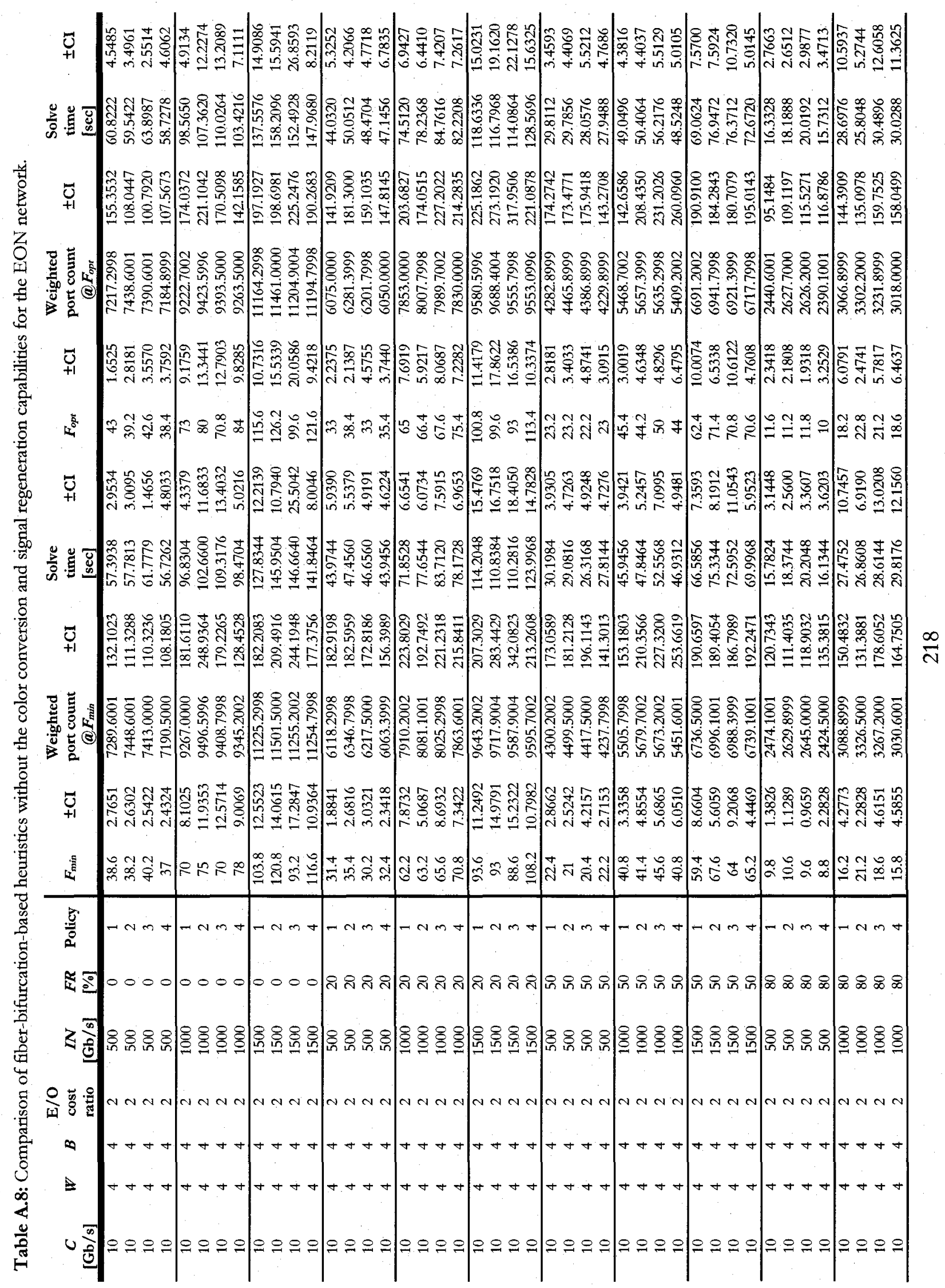




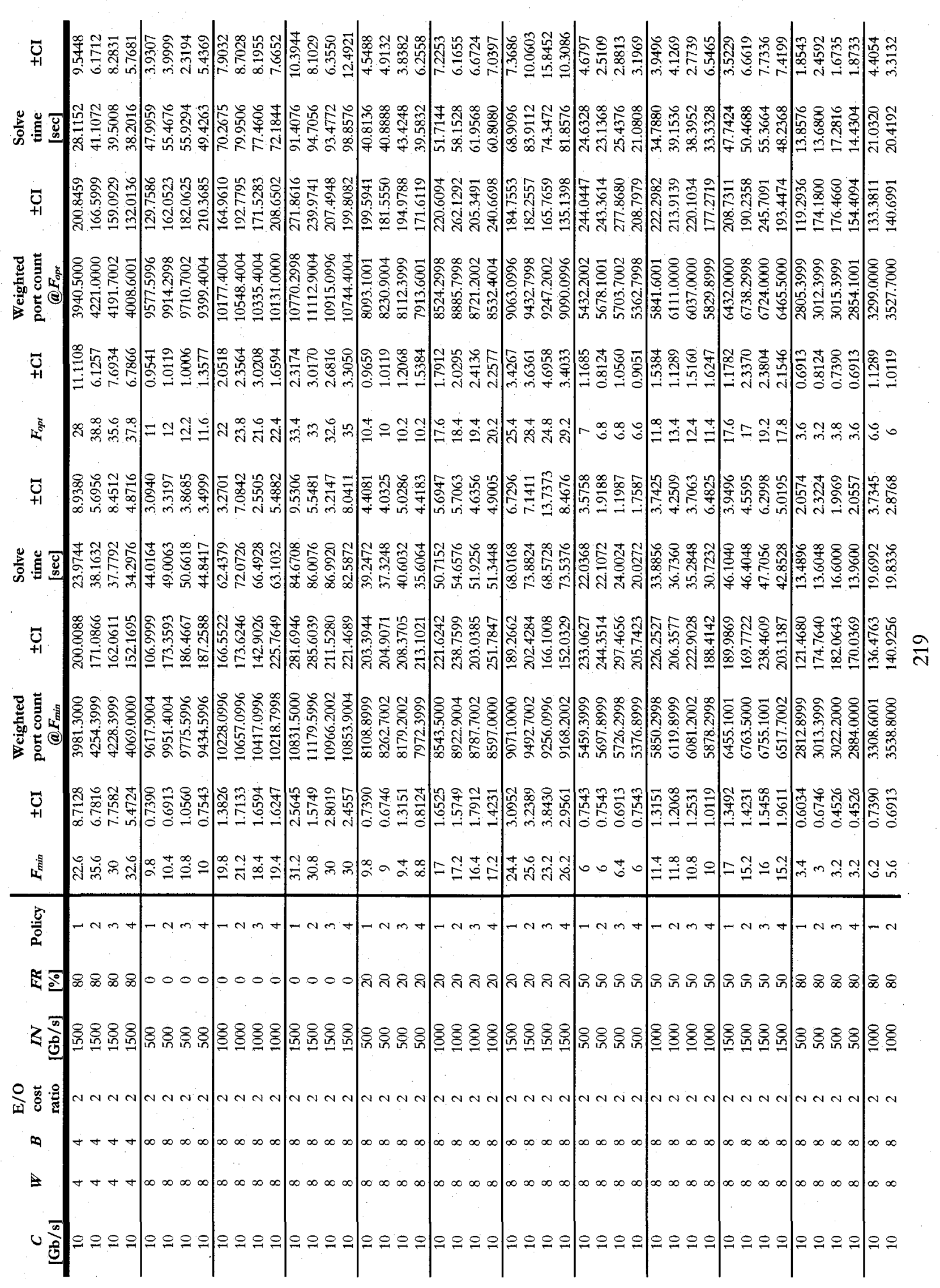




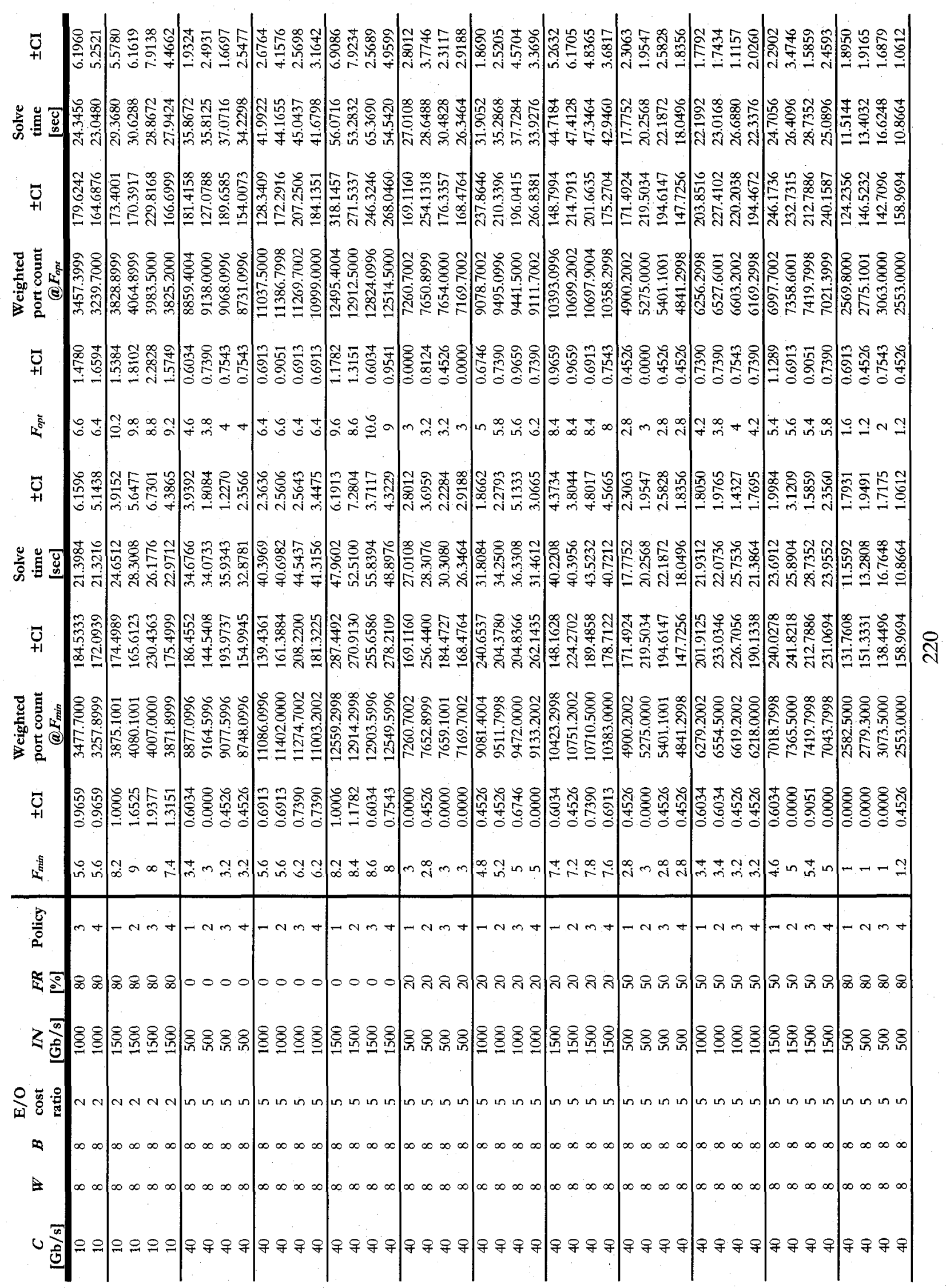




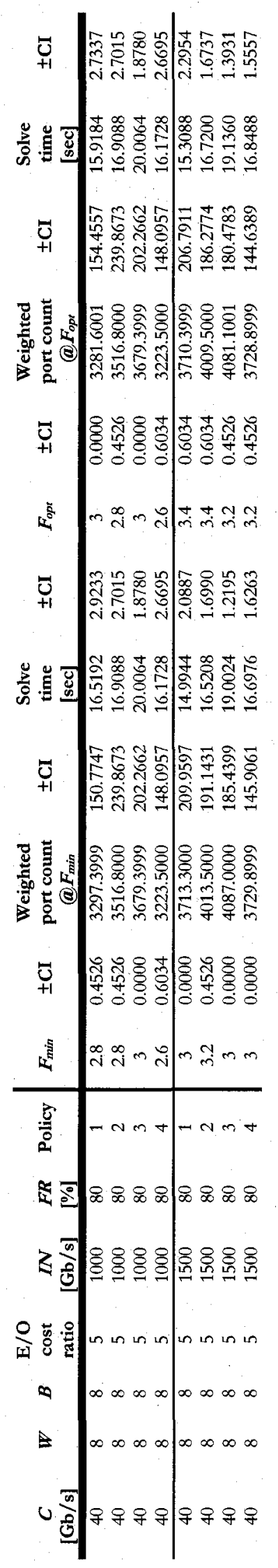




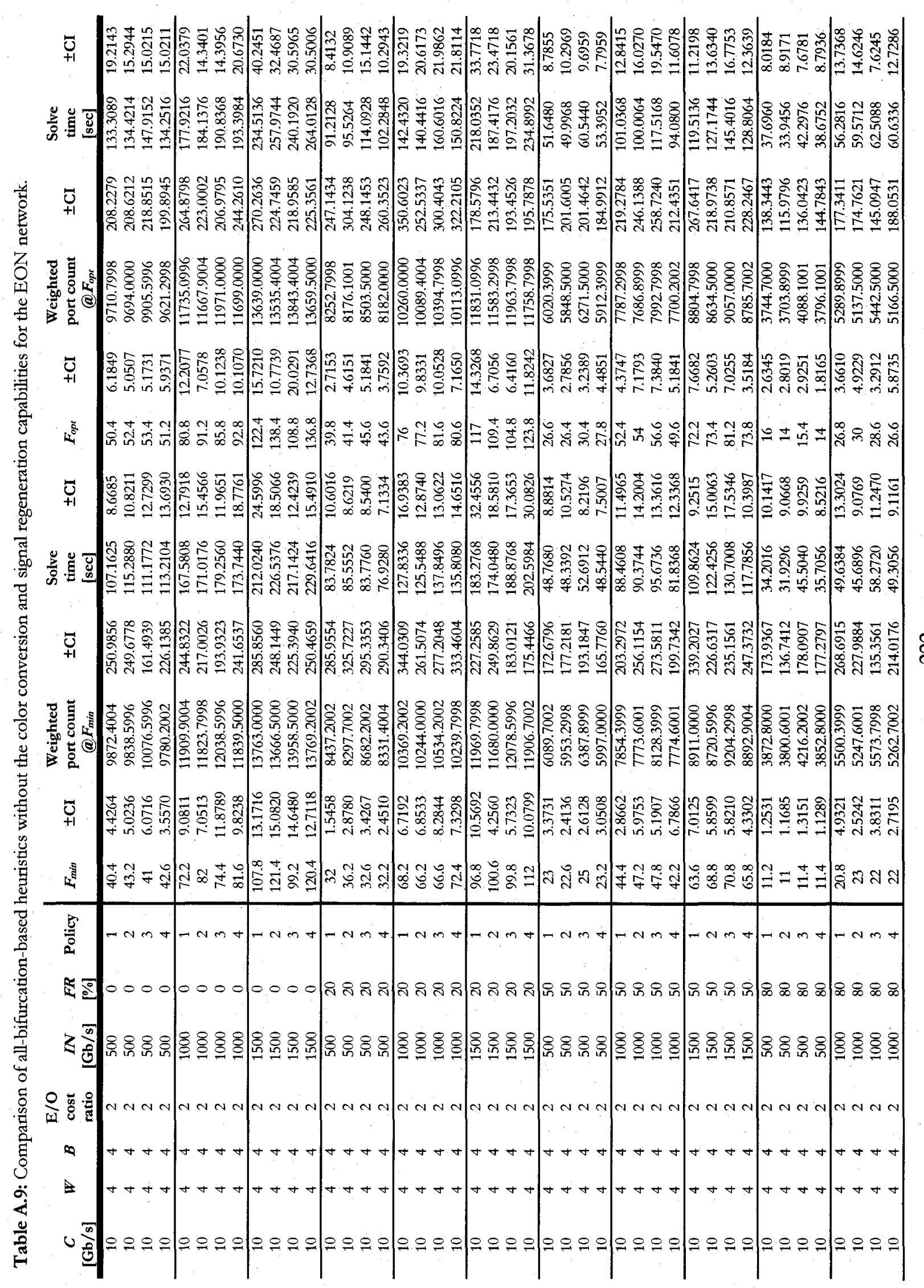




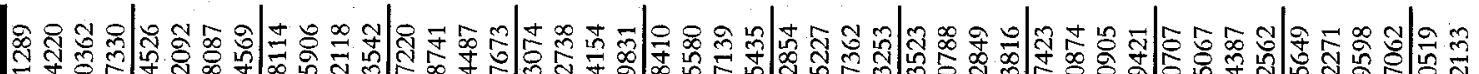

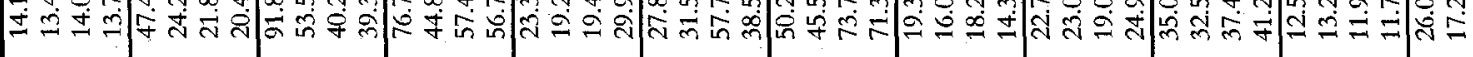

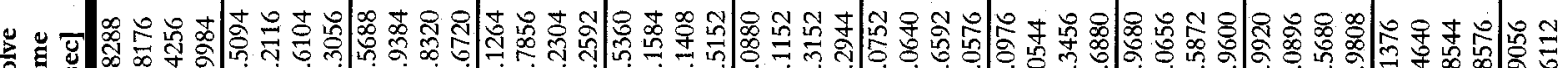

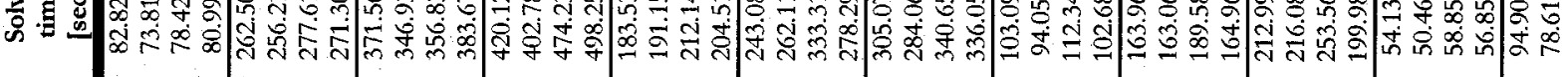

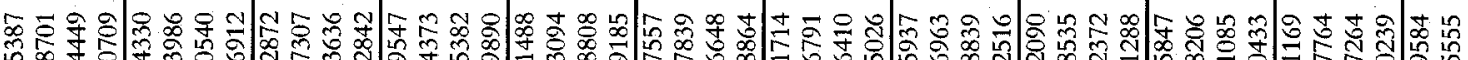

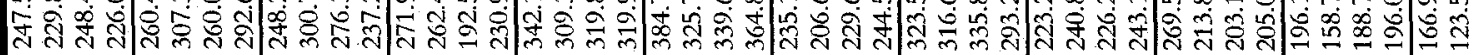

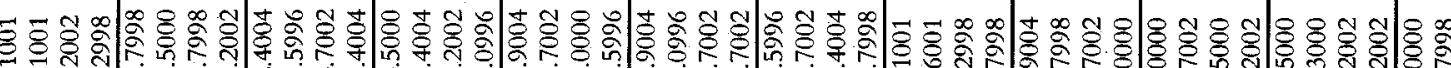

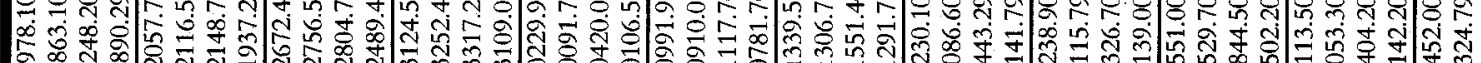

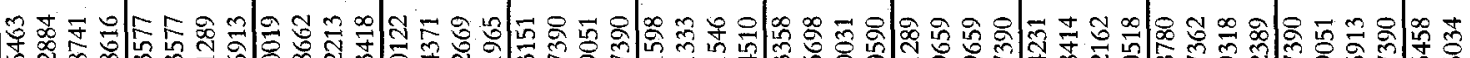

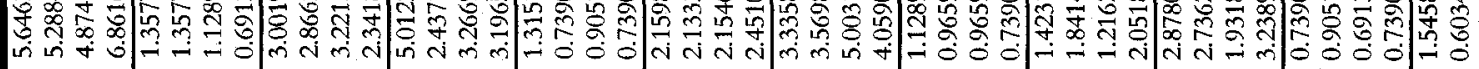

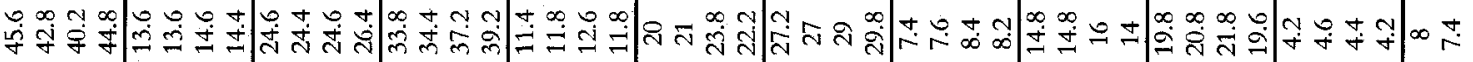

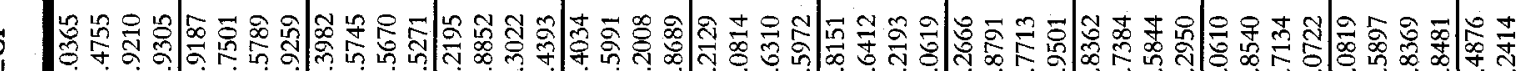

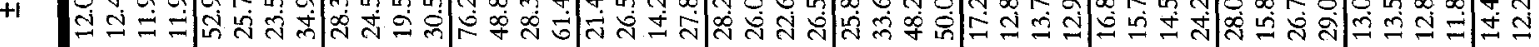

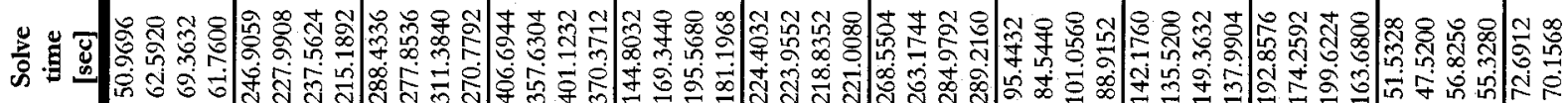

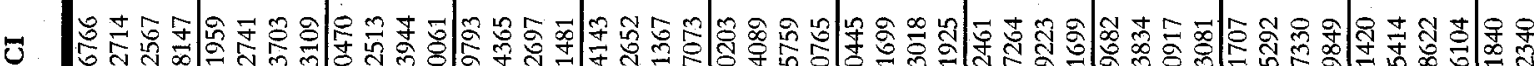

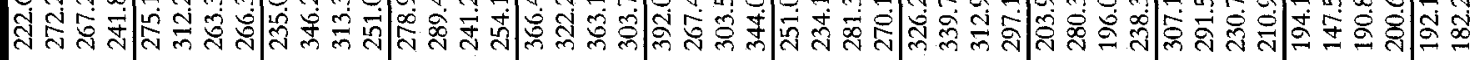

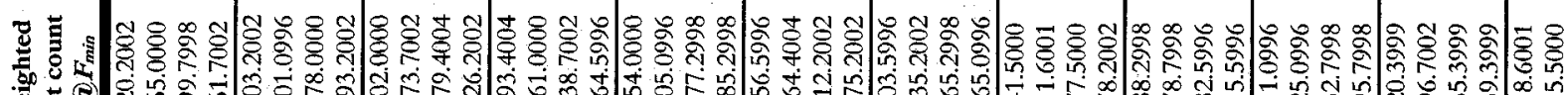

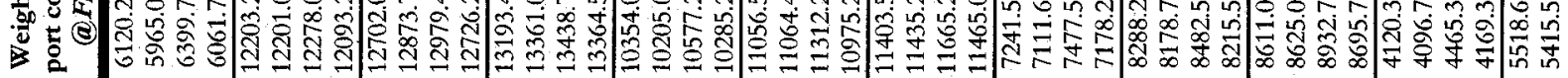

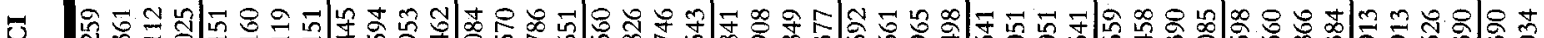

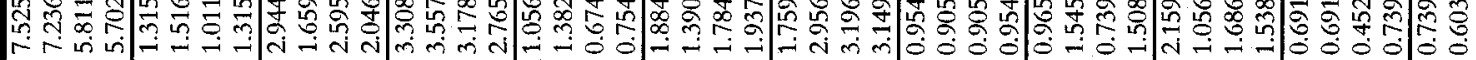

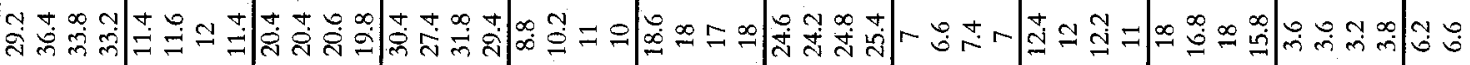

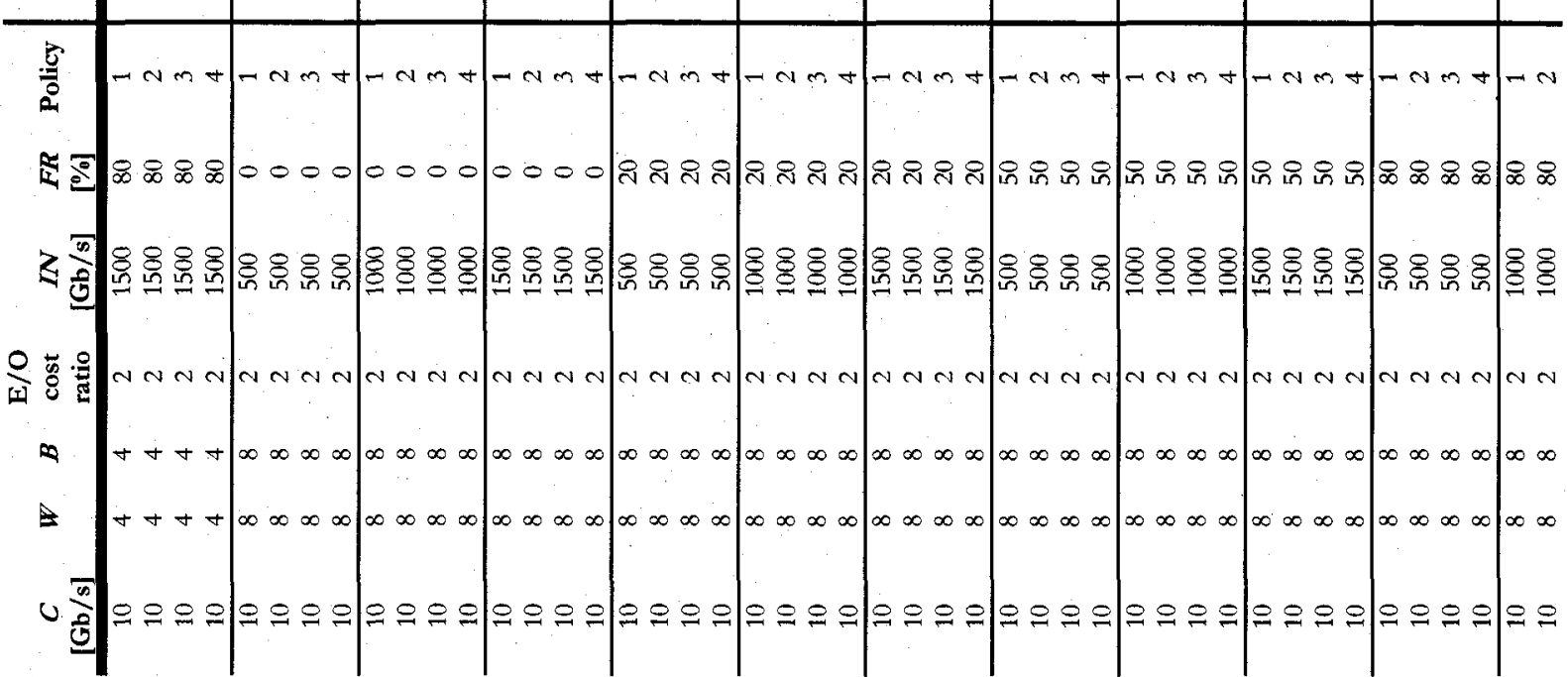




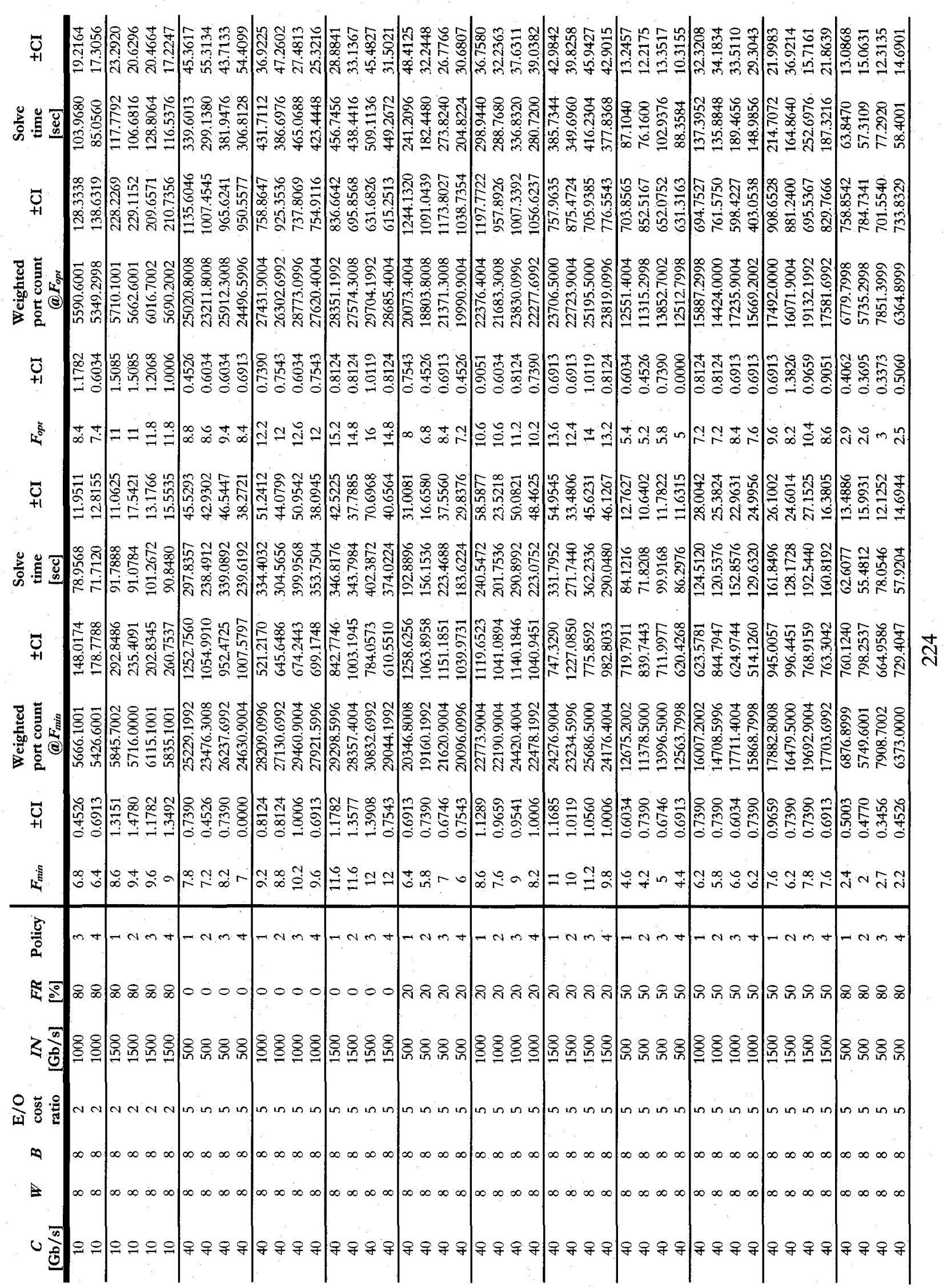




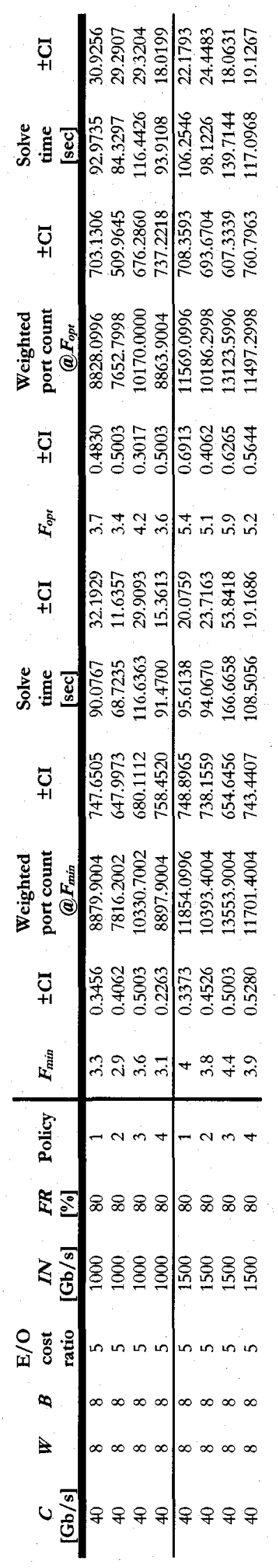


Appendix B

\section{Results of Heuristics Comparison with Color Conversion Capability}

In this appendix, we present the detailed results with $95 \%$ confidence intervals of the heuristics comparison with the consideration of the color conversion capability and without the consideration of the optical signal regeneration capability. Tables B.1-B.2, B.3-B.4, and B.5-B.6 show the results for the 8-node network, 14-node NSF network, and 11-node EON network respectively. 


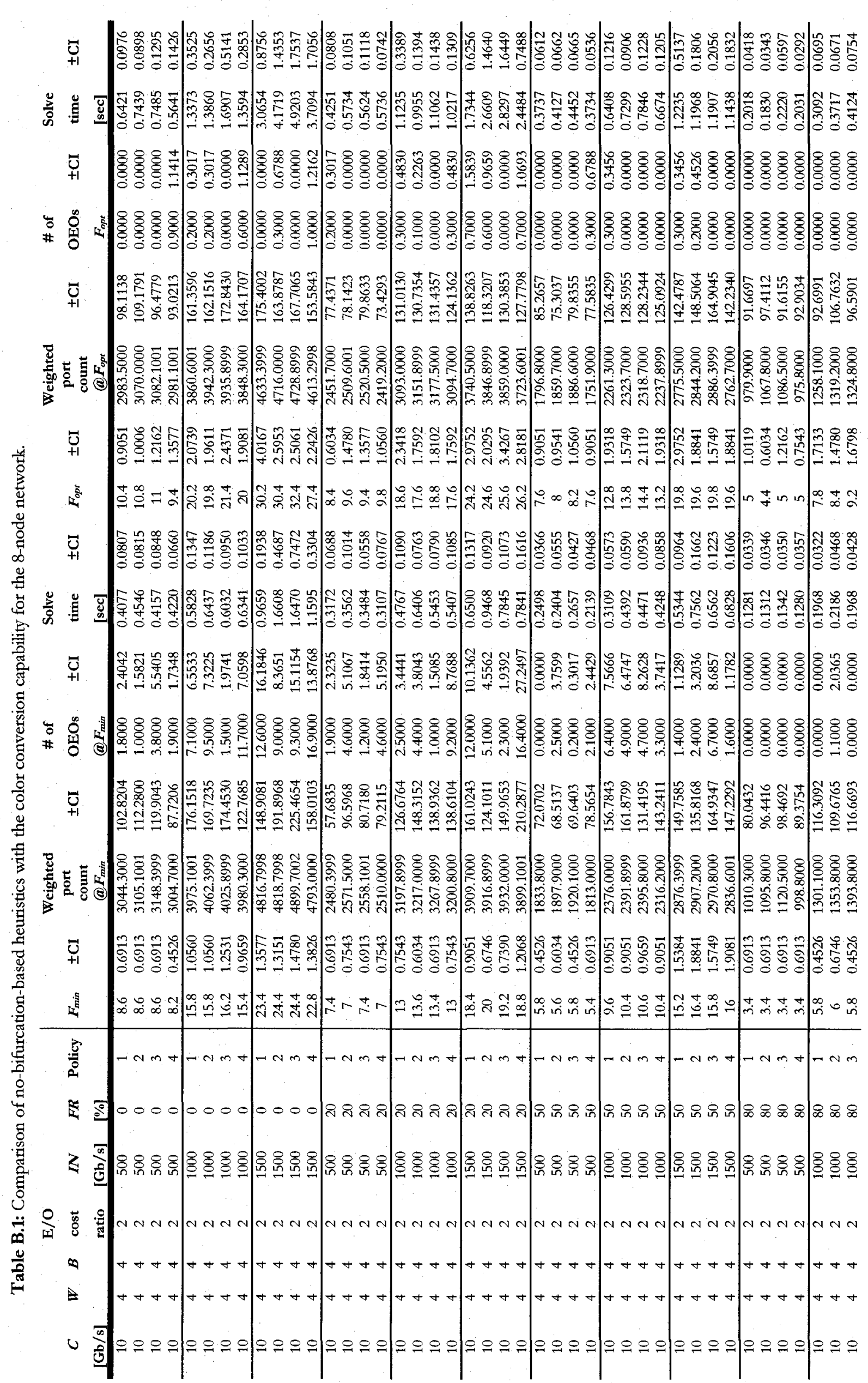




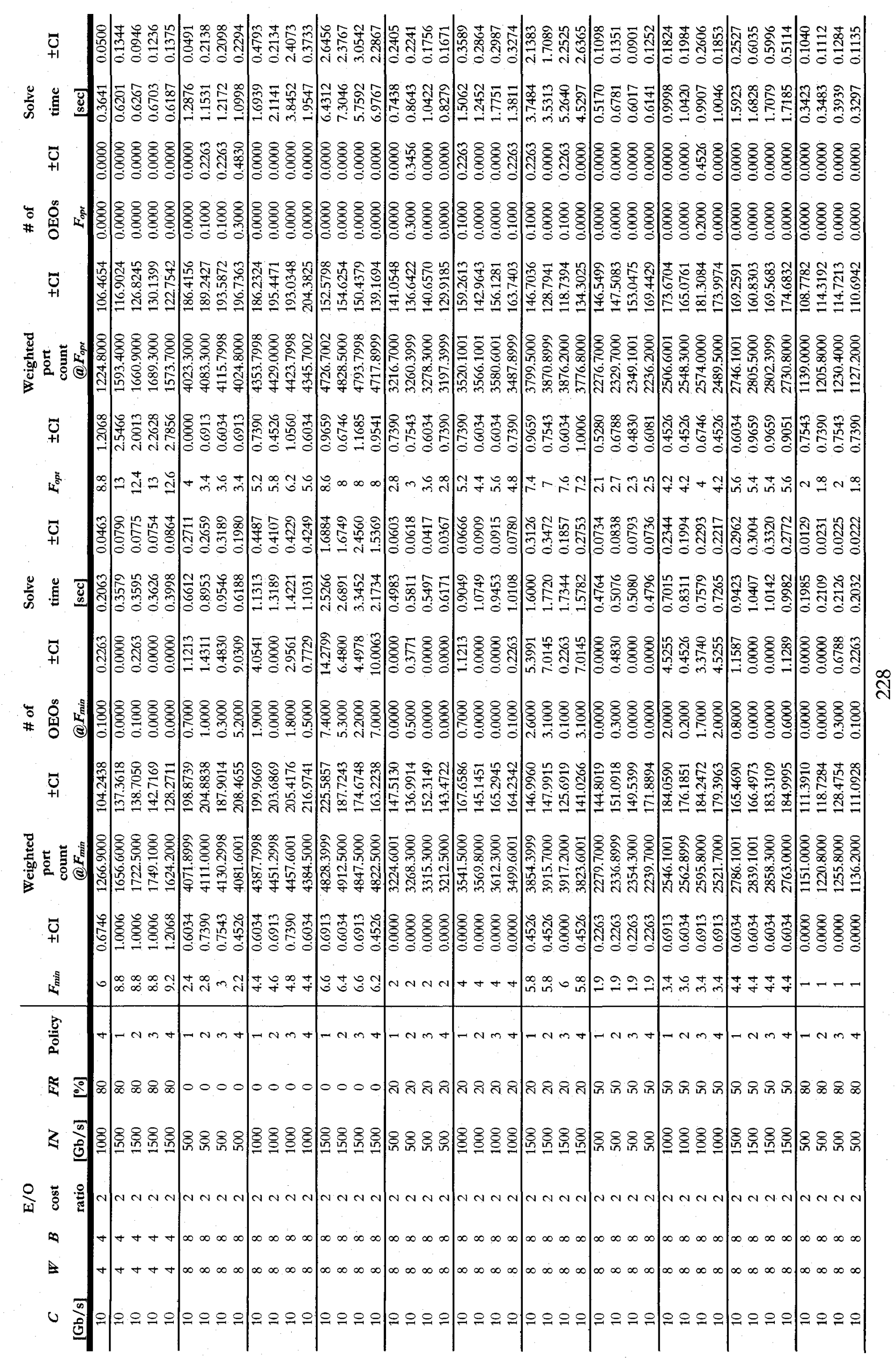




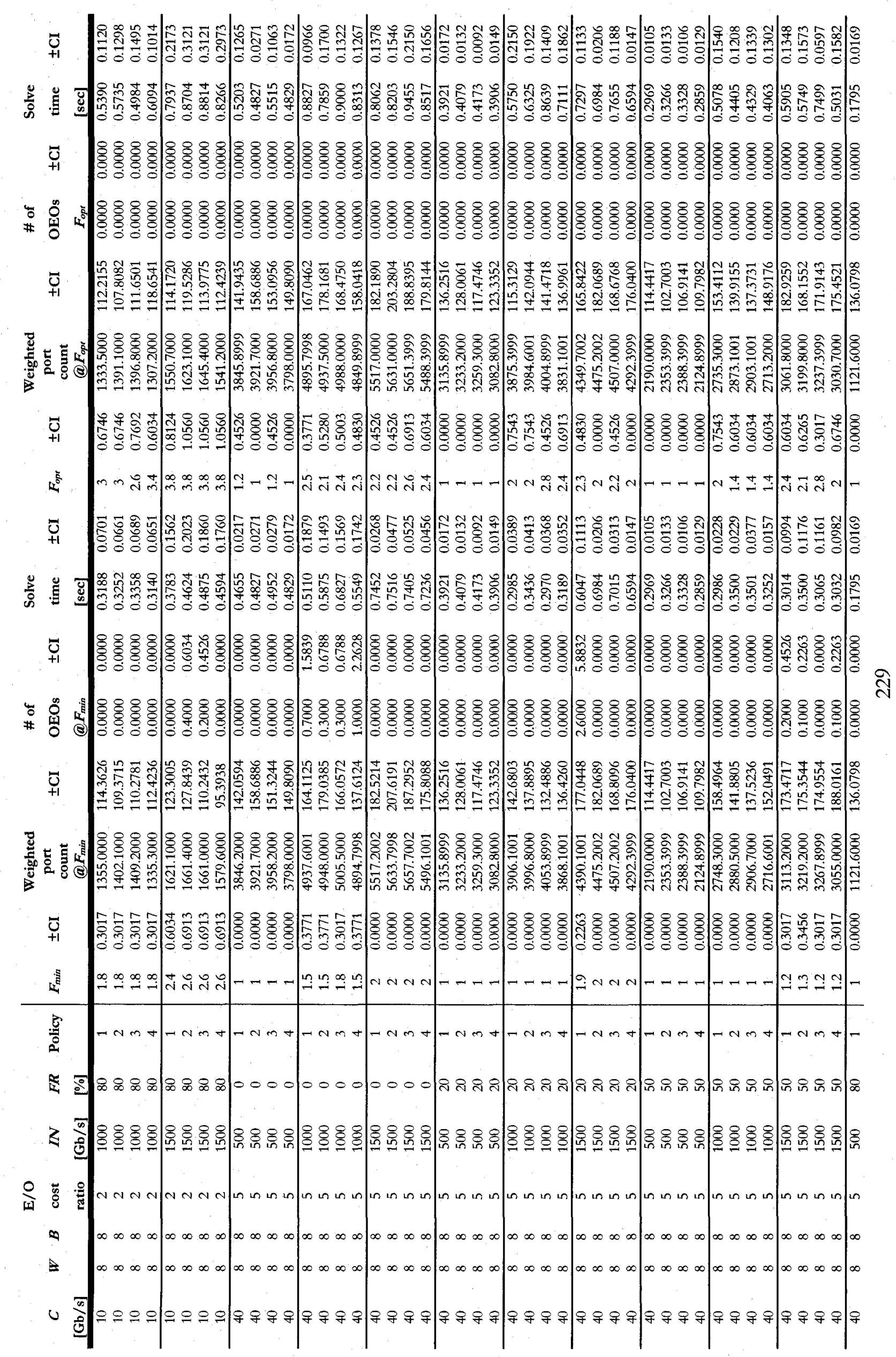




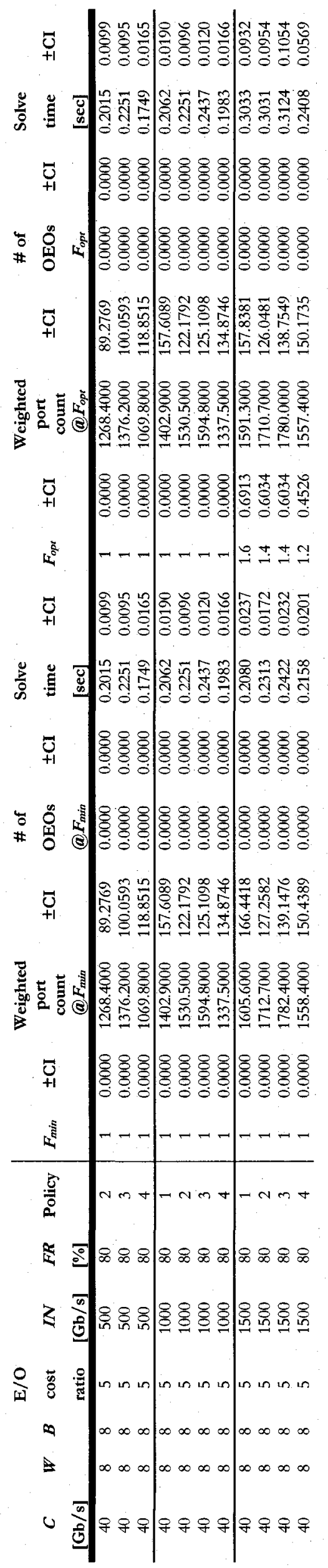

กิ 


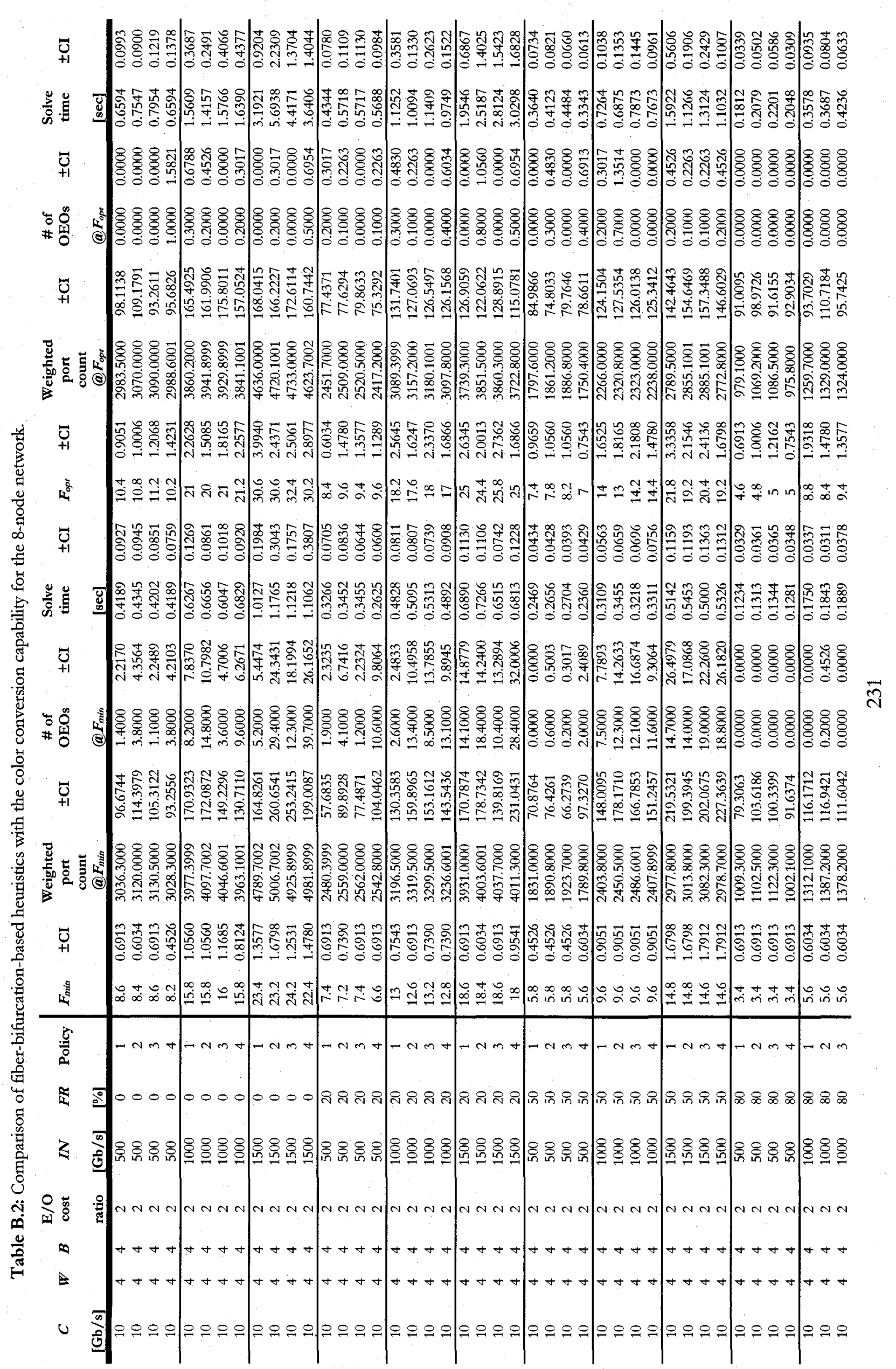




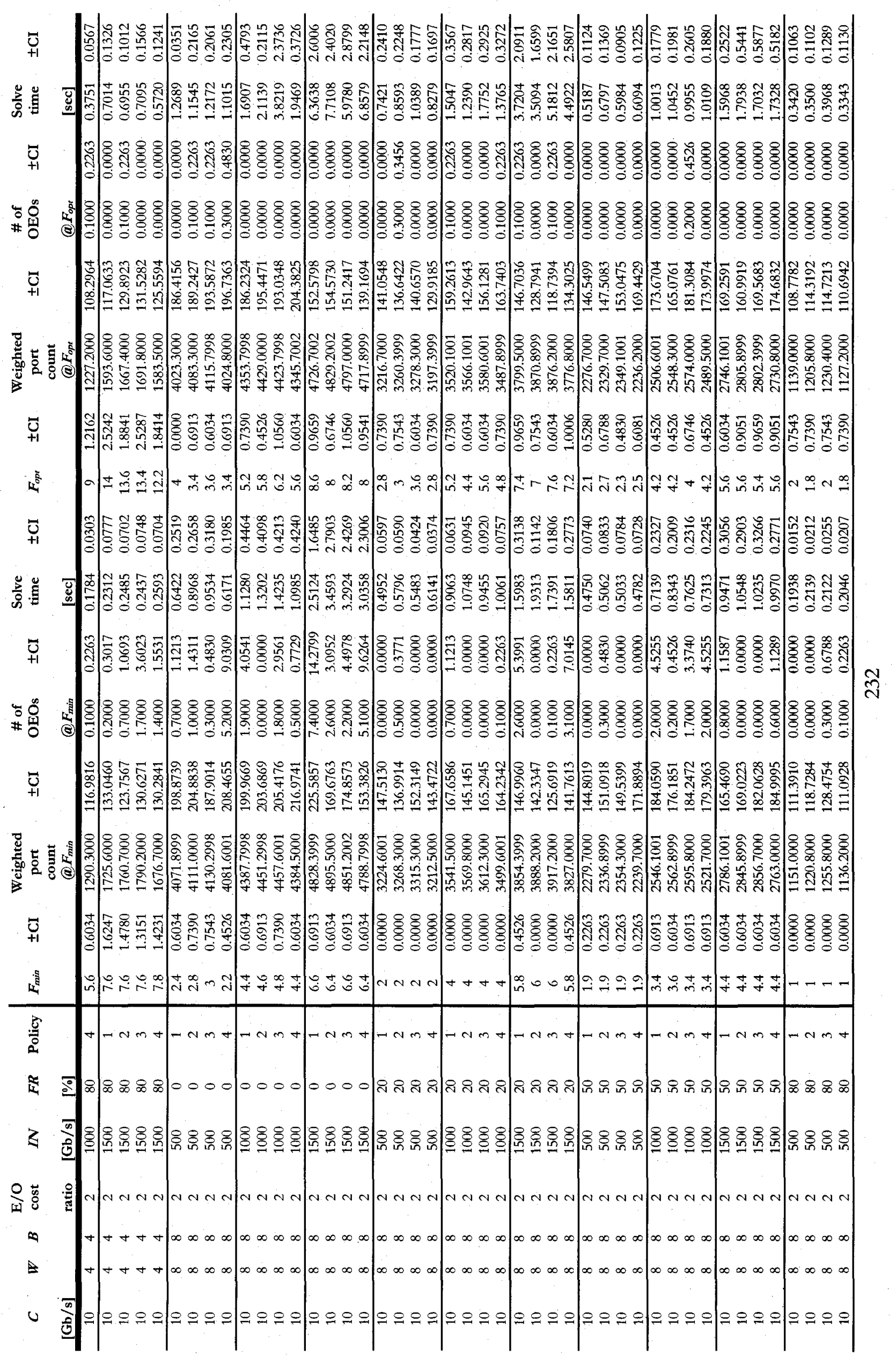




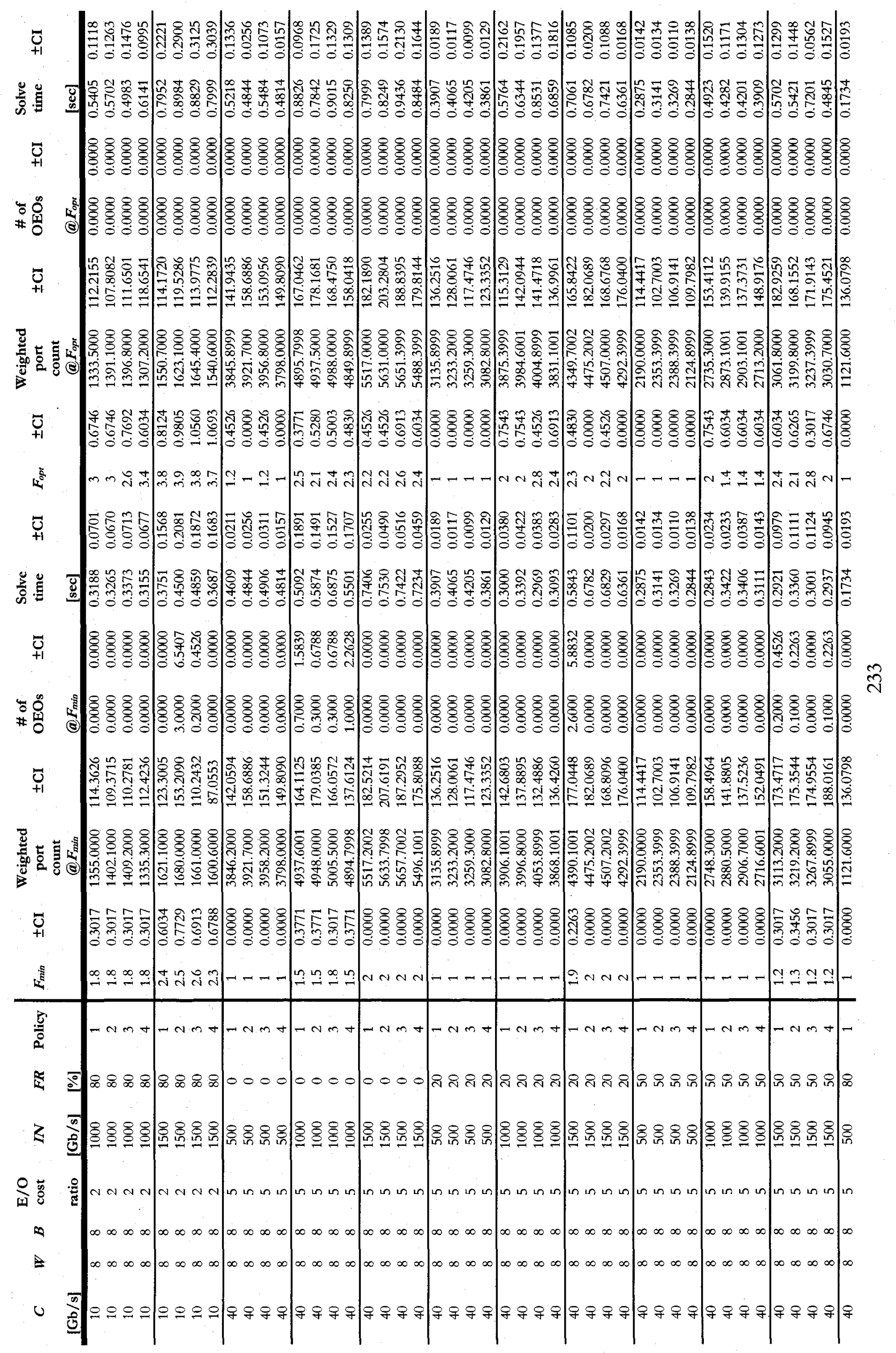




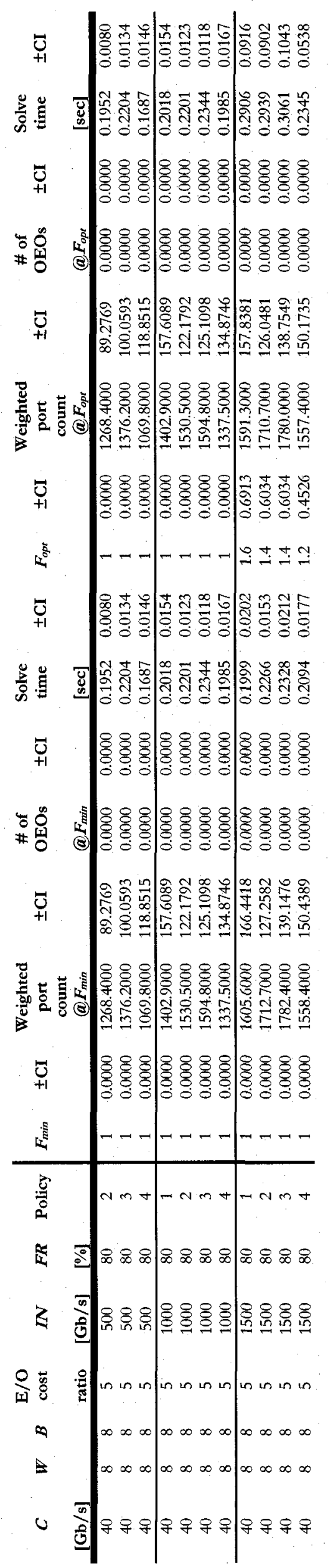

กั 


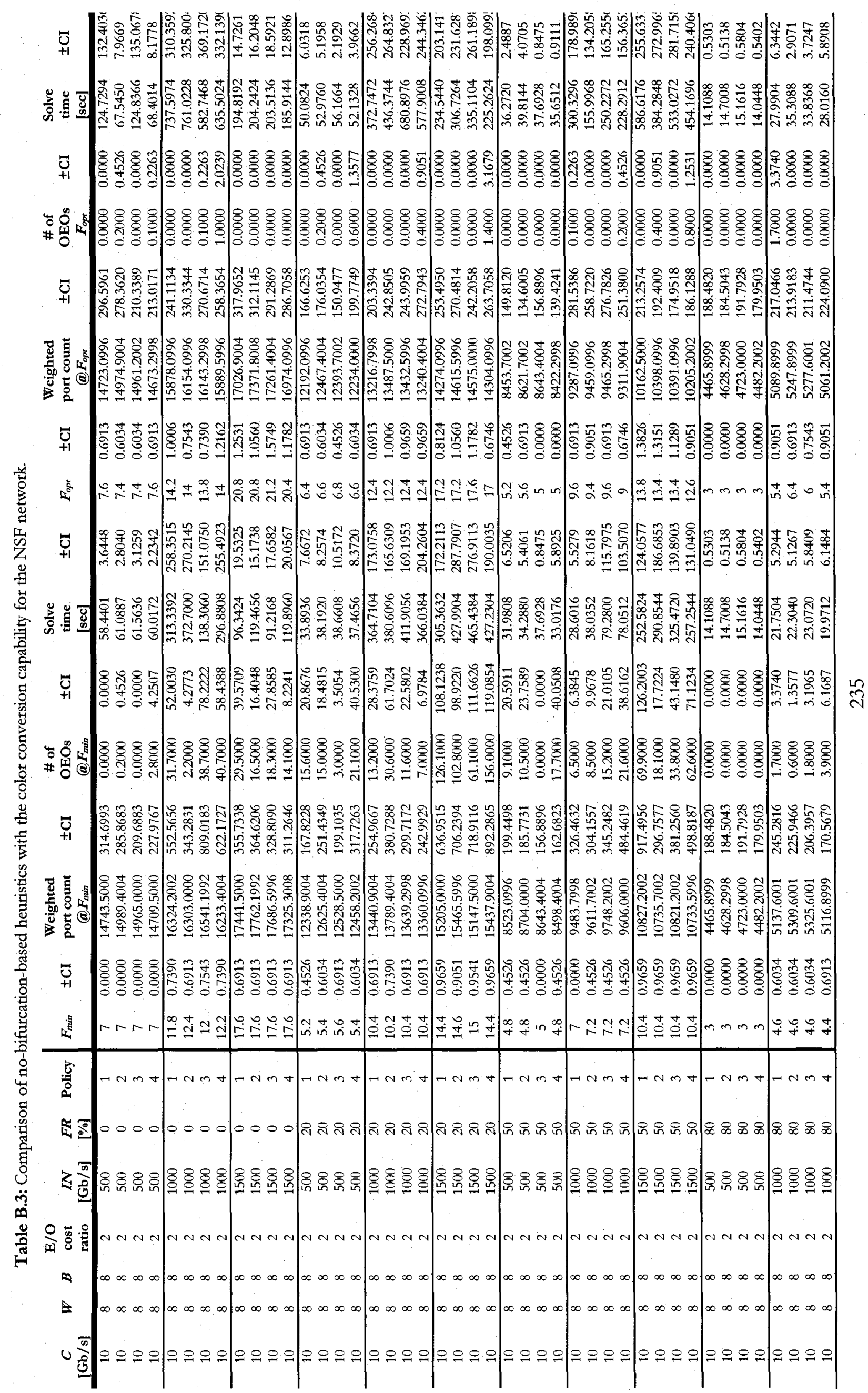




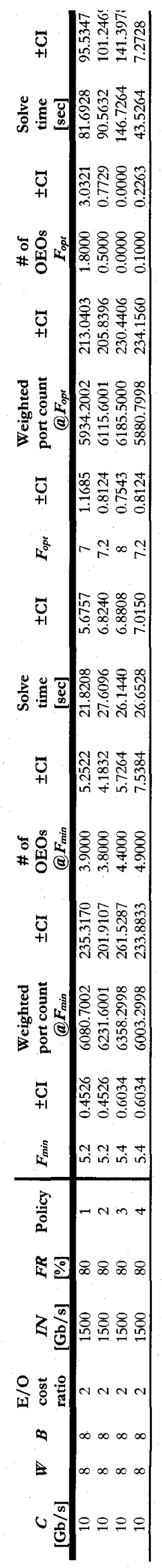

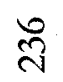




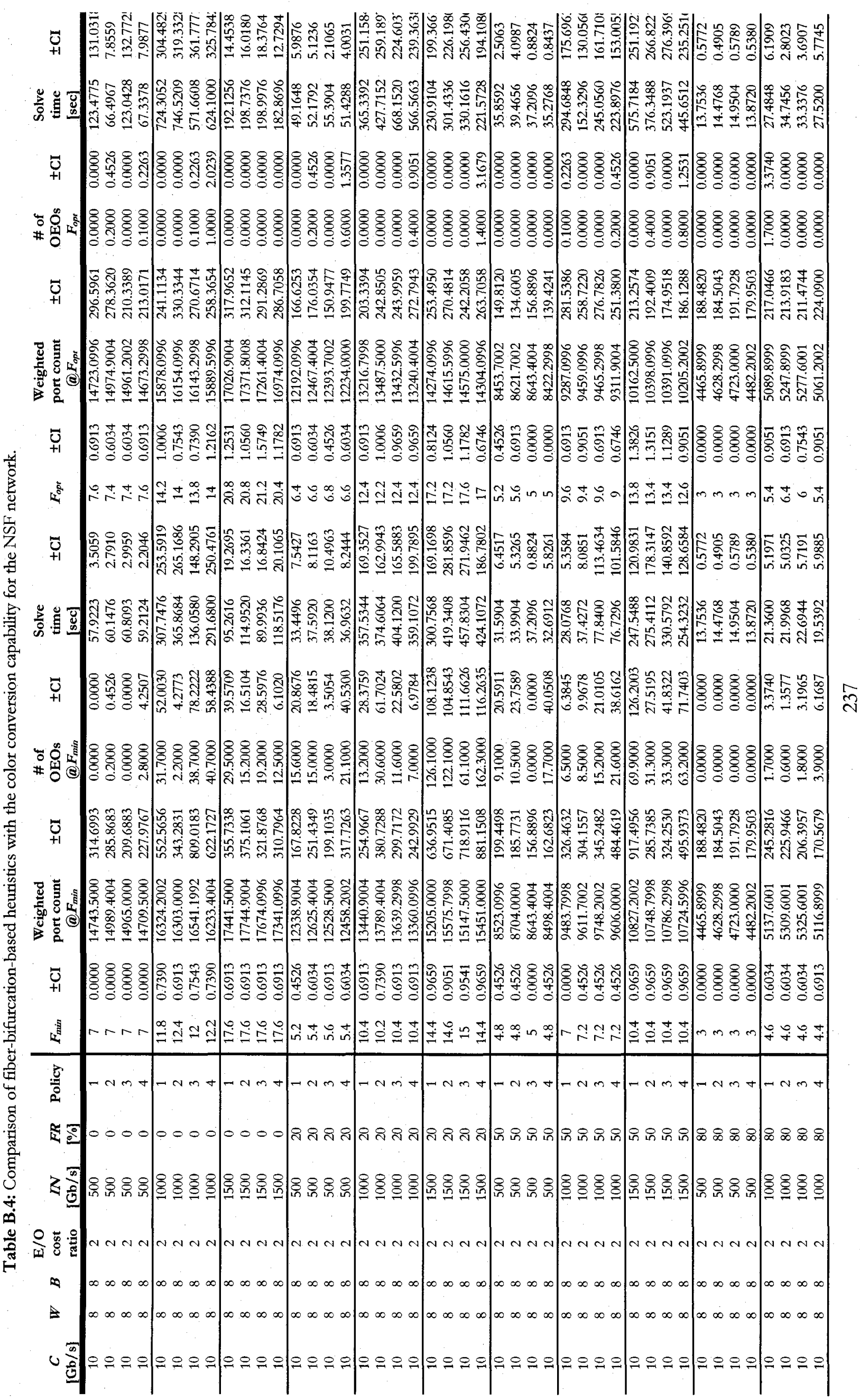




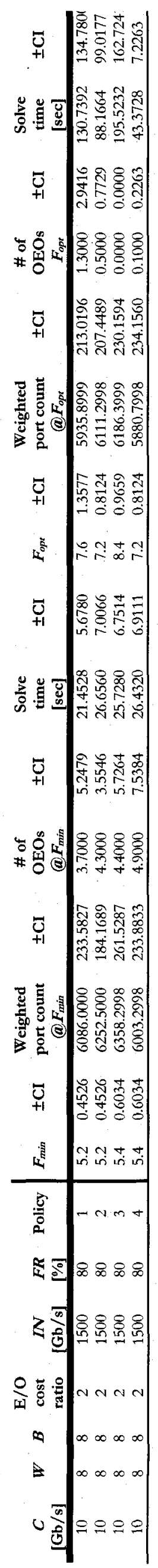




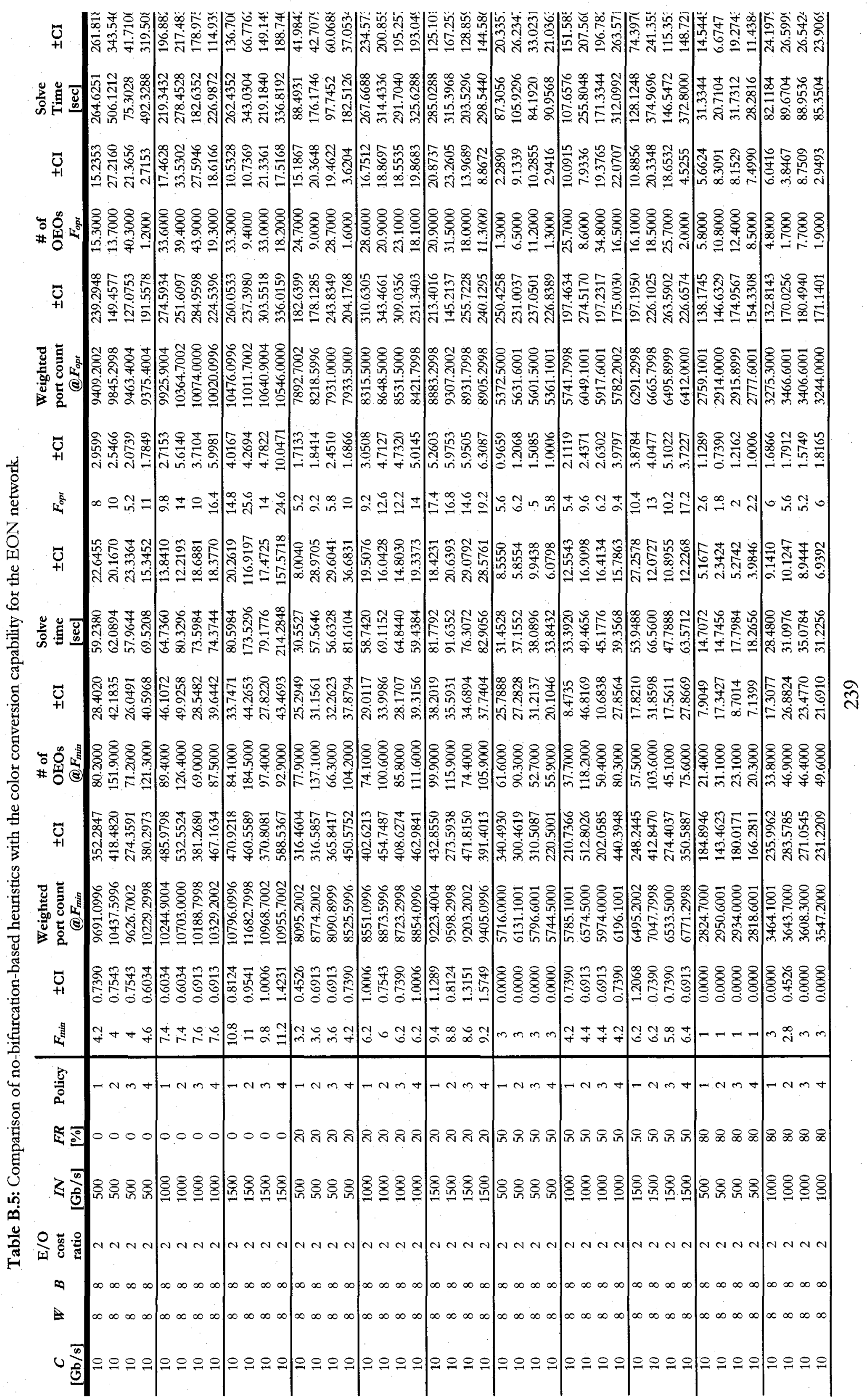




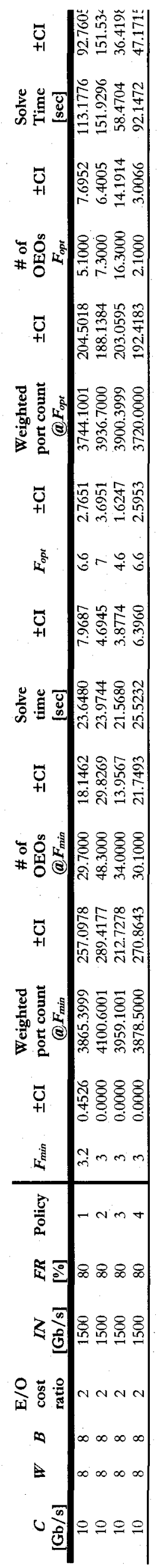

워 


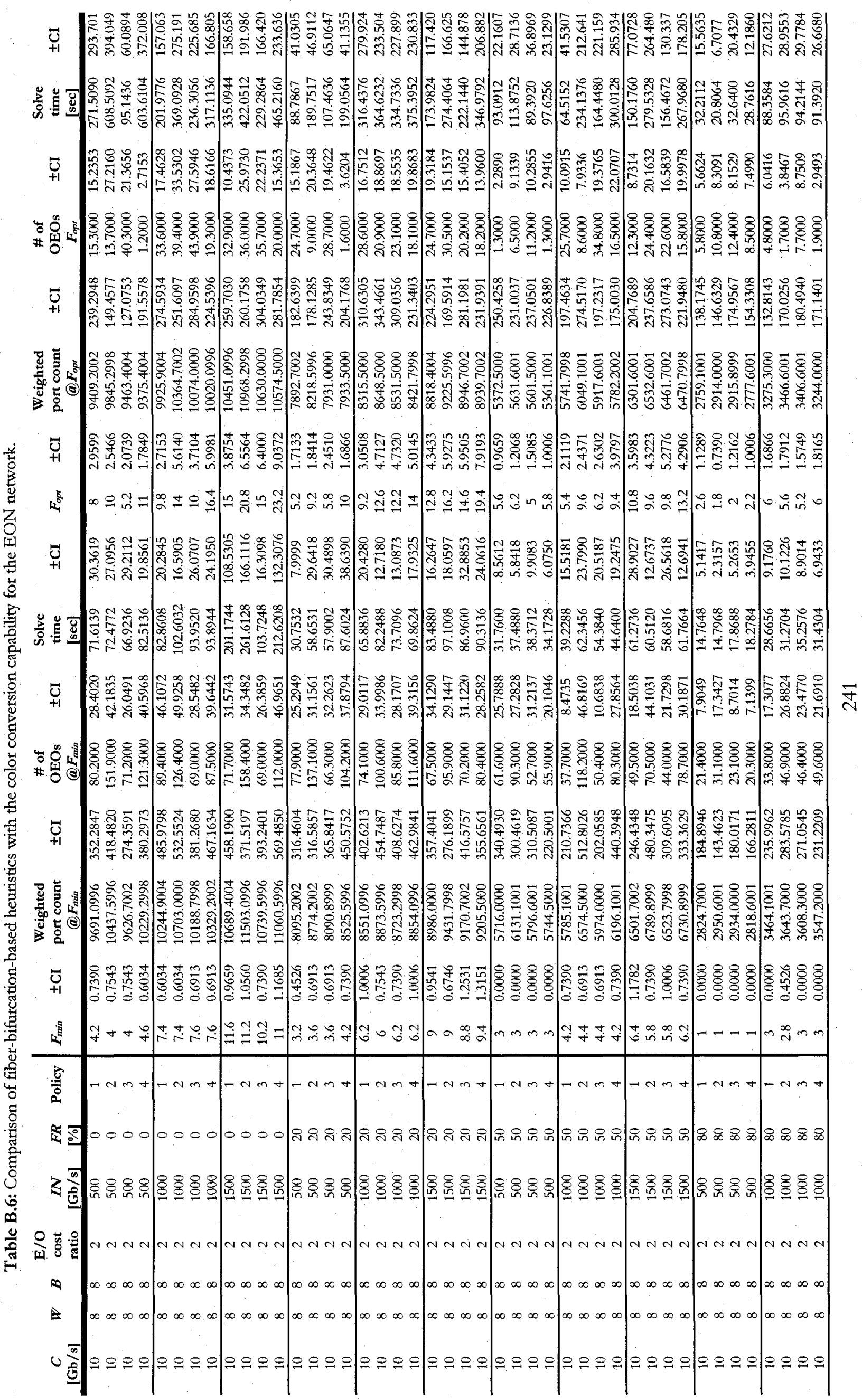




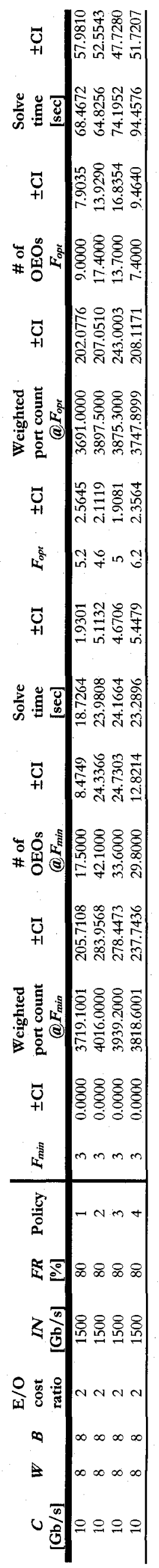


Appendix C

\section{Results of Heuristics Comparison with Signal Regeneration Capability}

In this appendix, we present the detailed results with $95 \%$ confidence intervals of the heuristics comparison with the consideration of the optical signal regeneration capability and without/with customizing the optical reach. Tables C.1-C.4, C.5-C.8, and C.9-C.12 show the results for the 8node network, 14-node NSF network, and 11-node EON network respectively. 


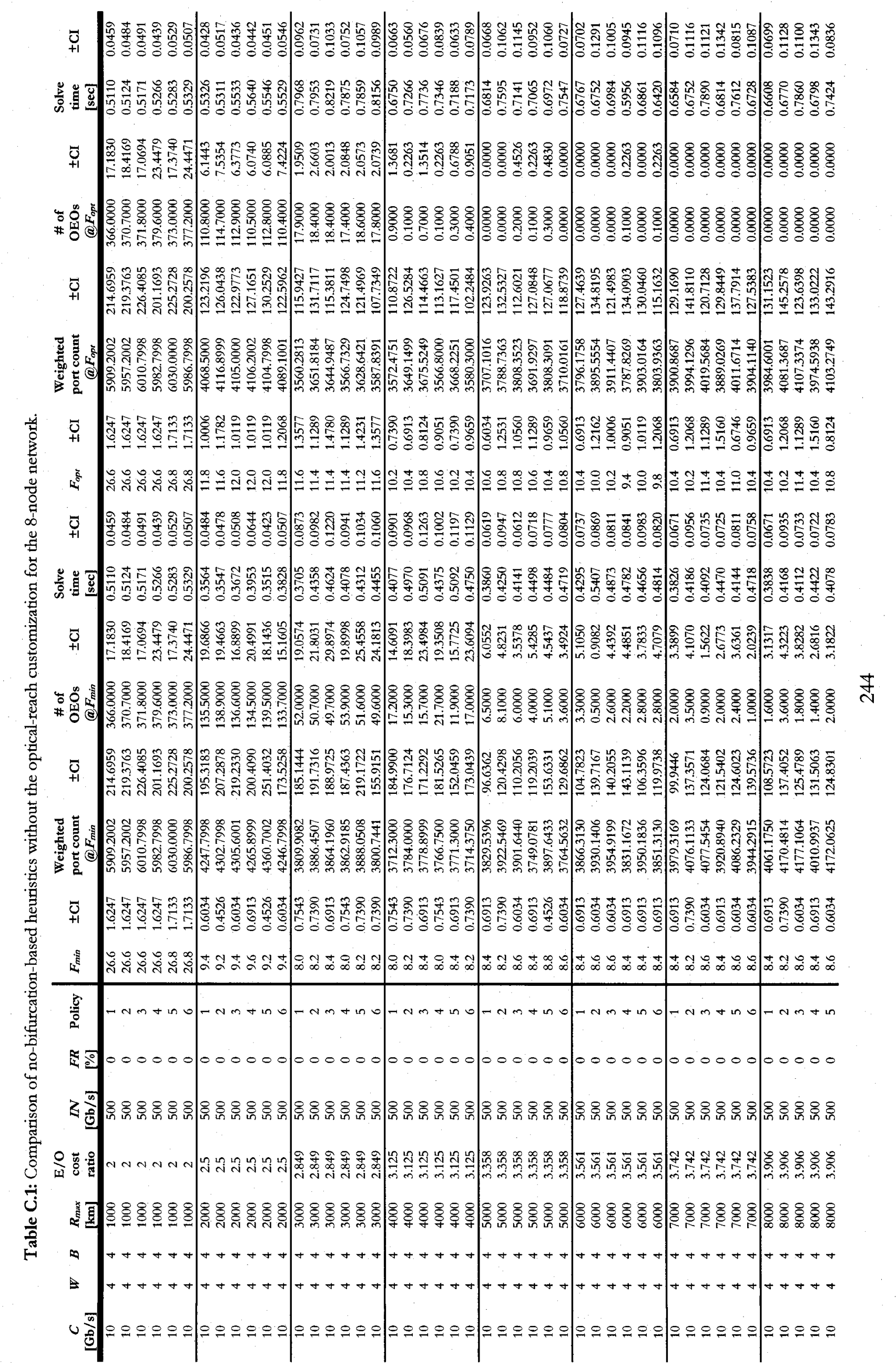




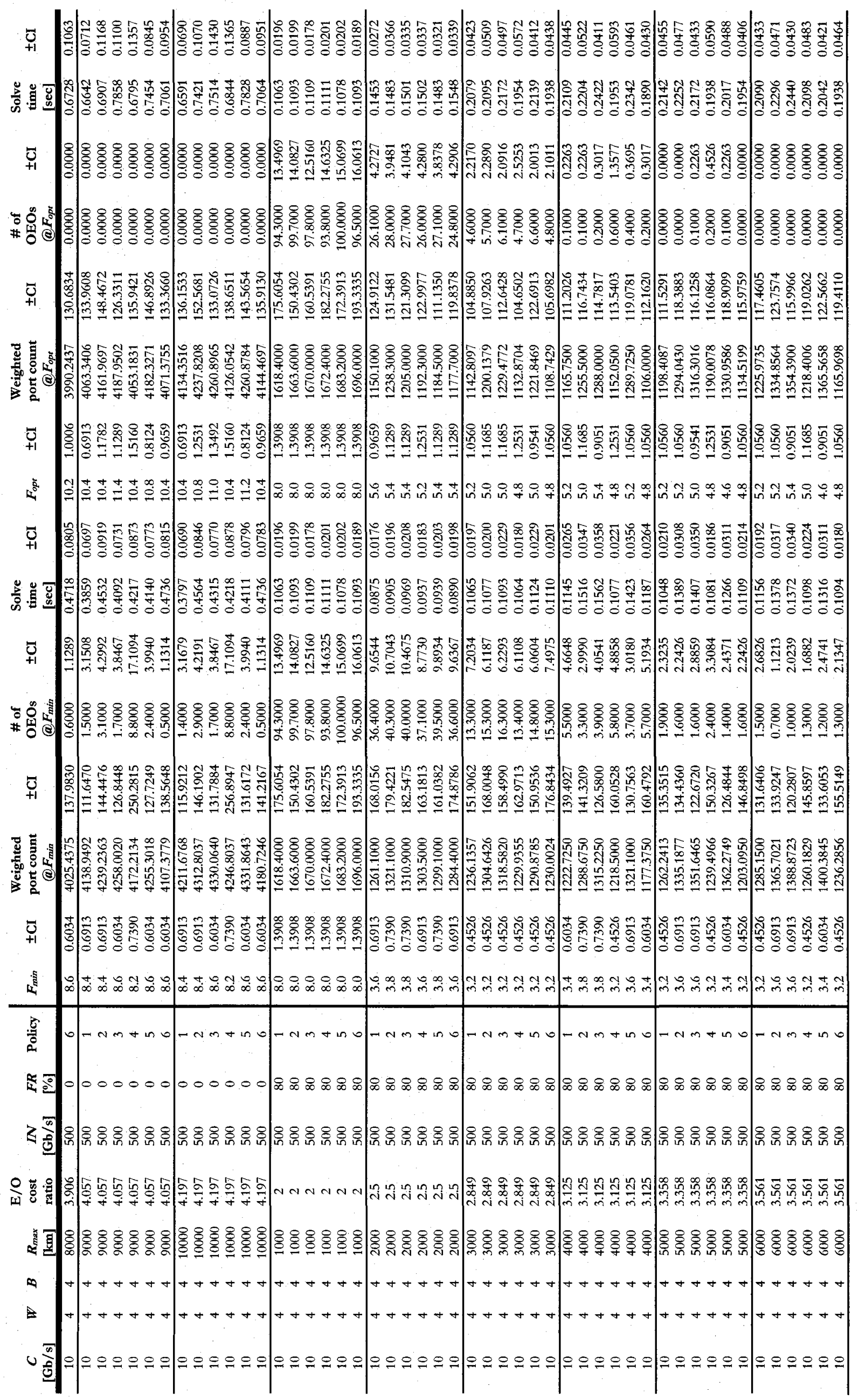




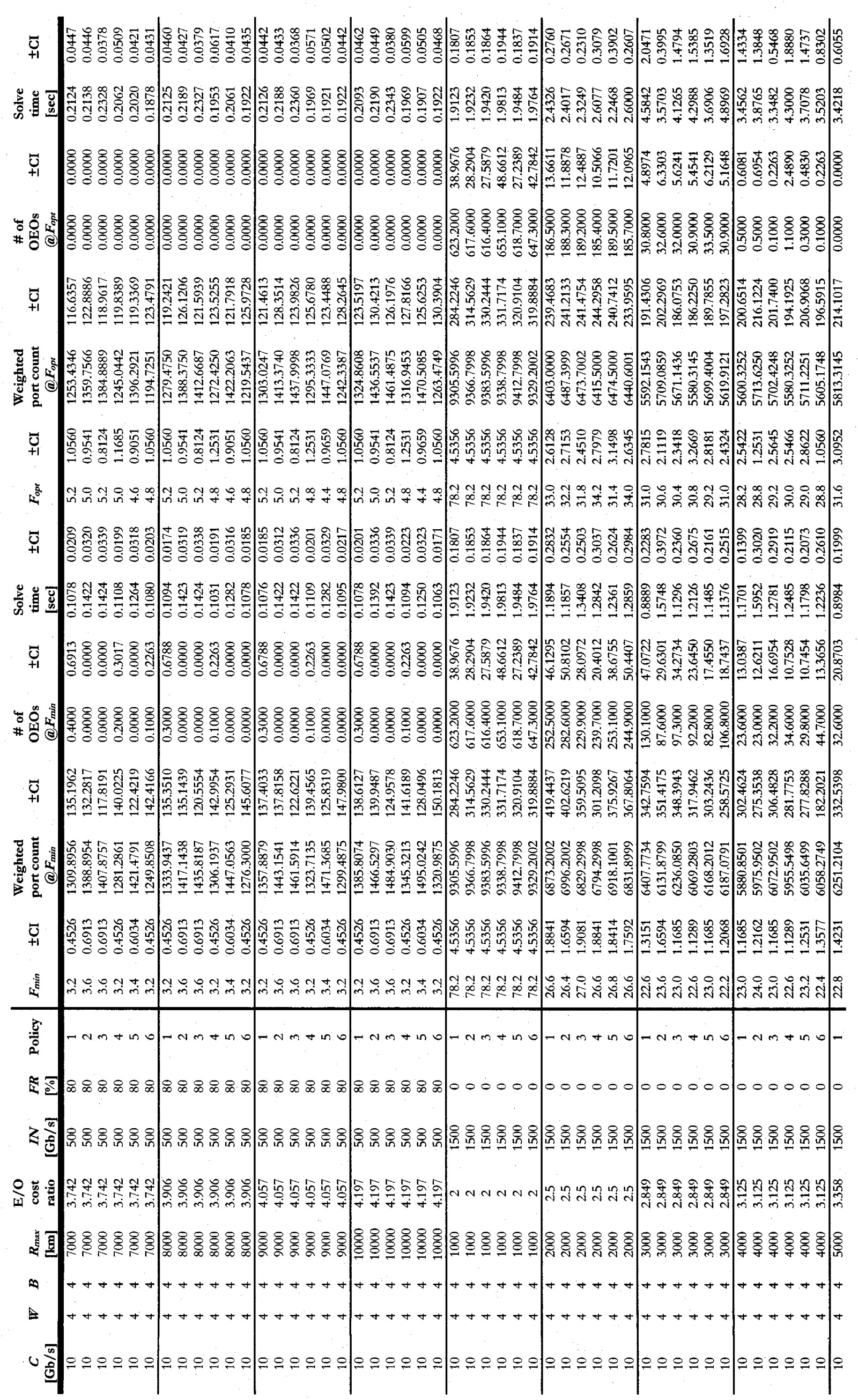




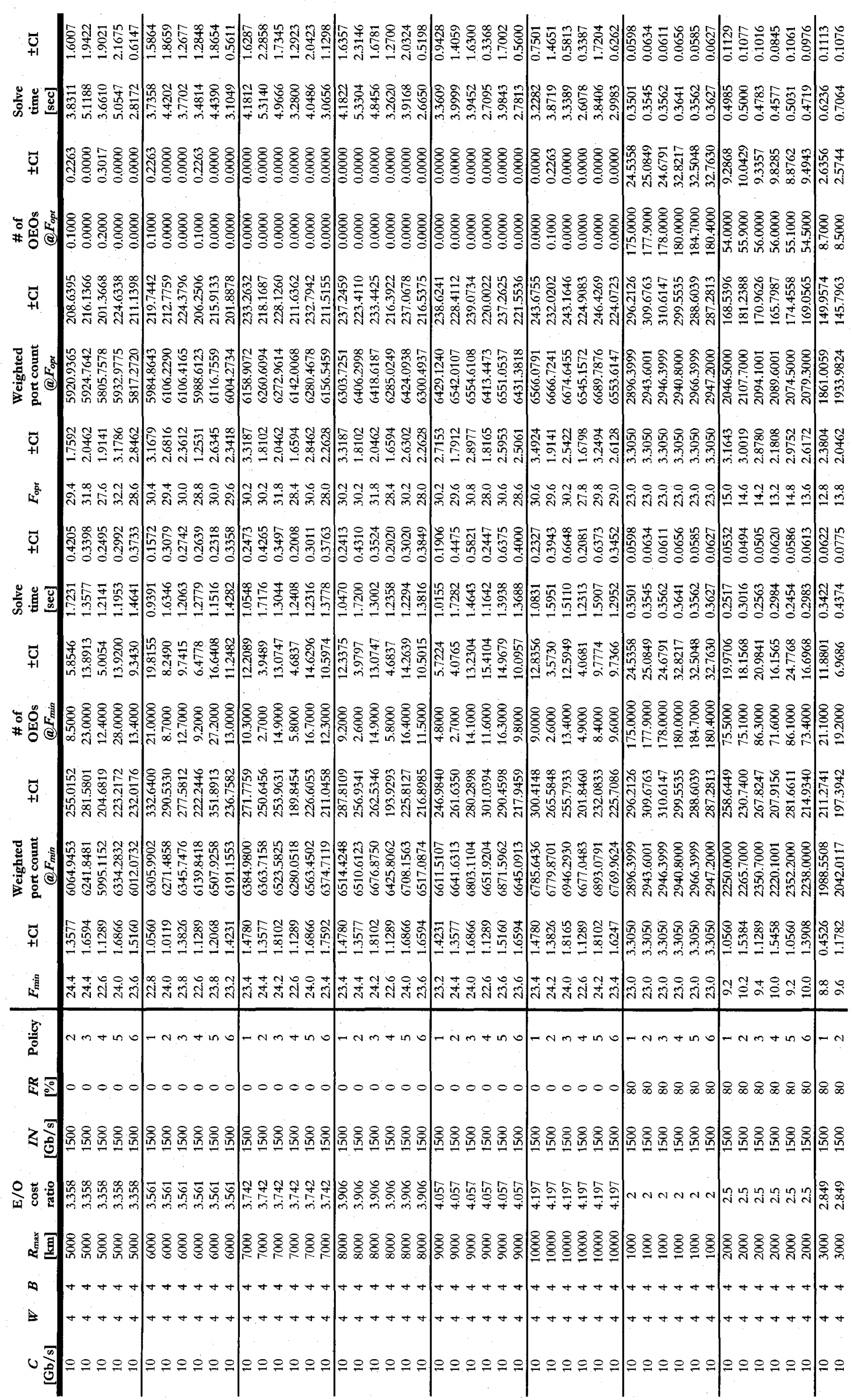




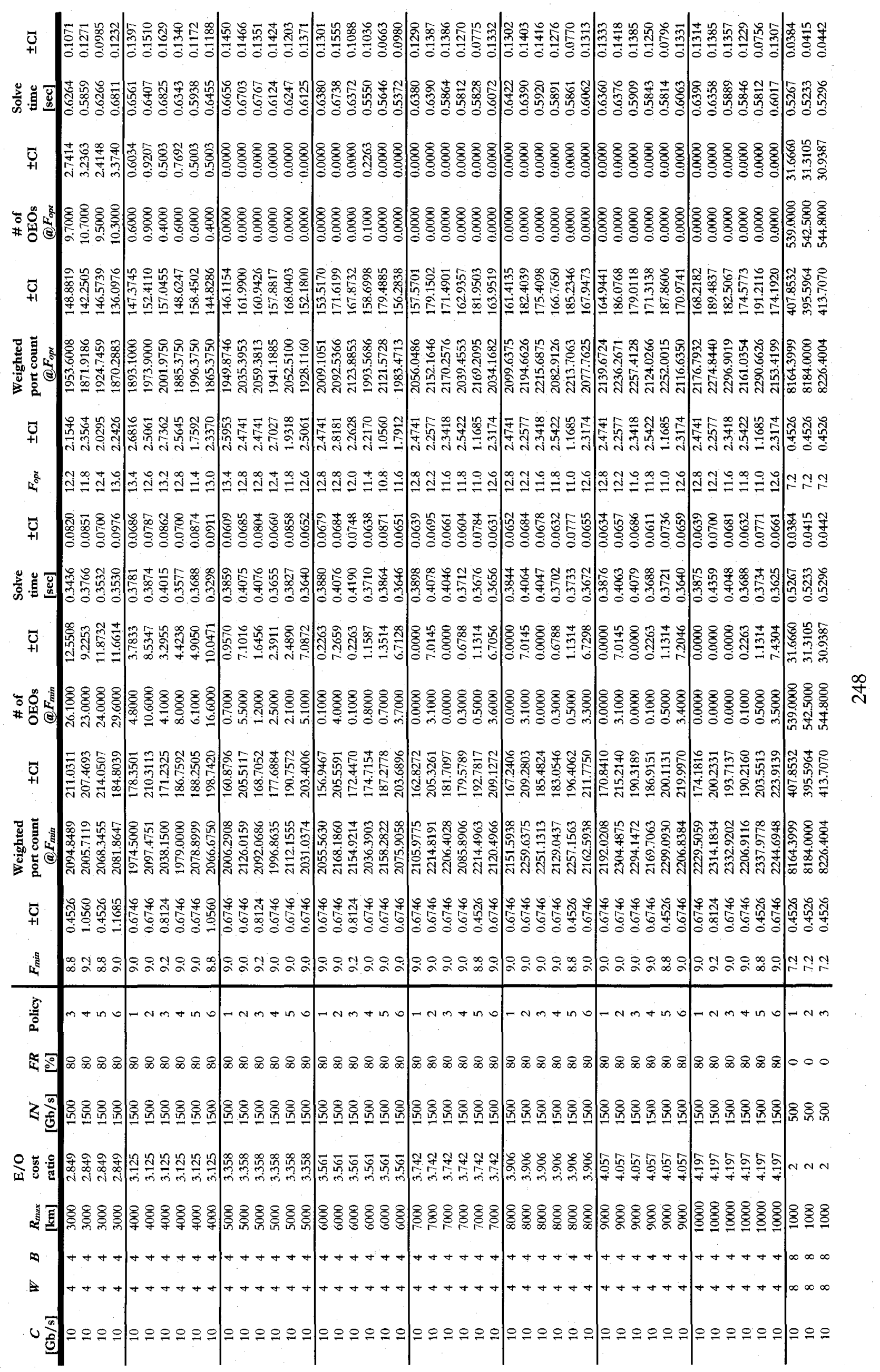




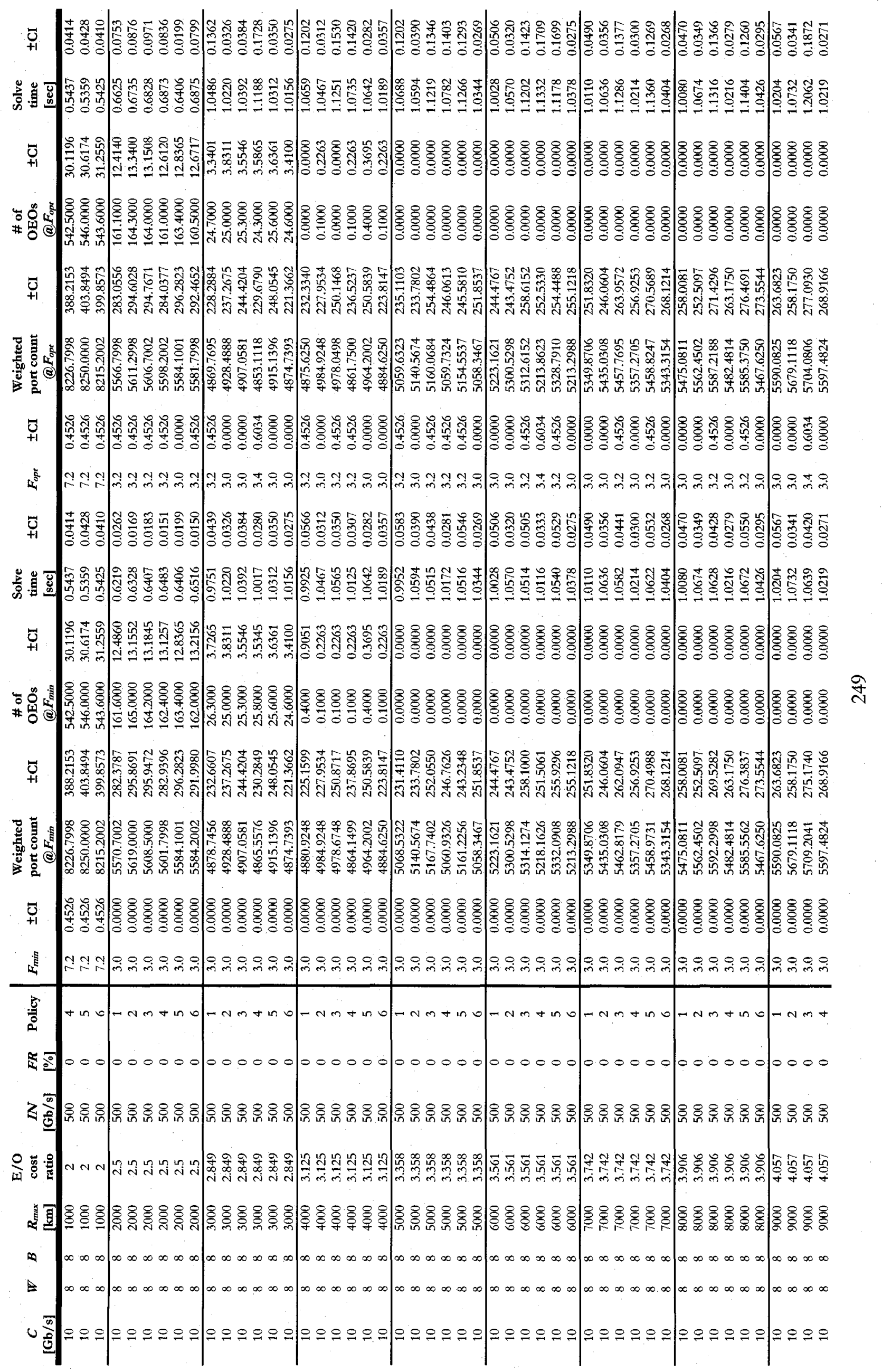




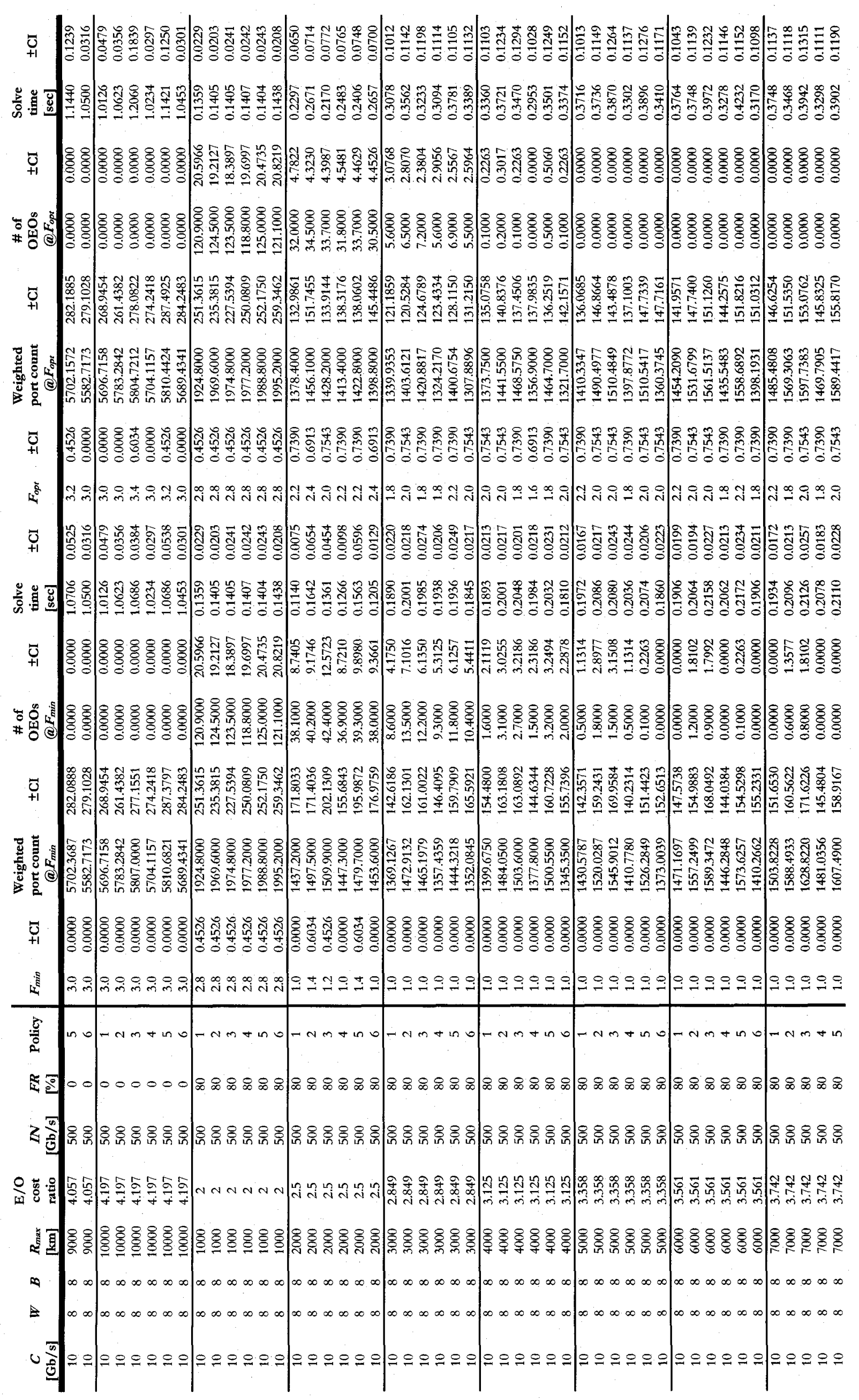




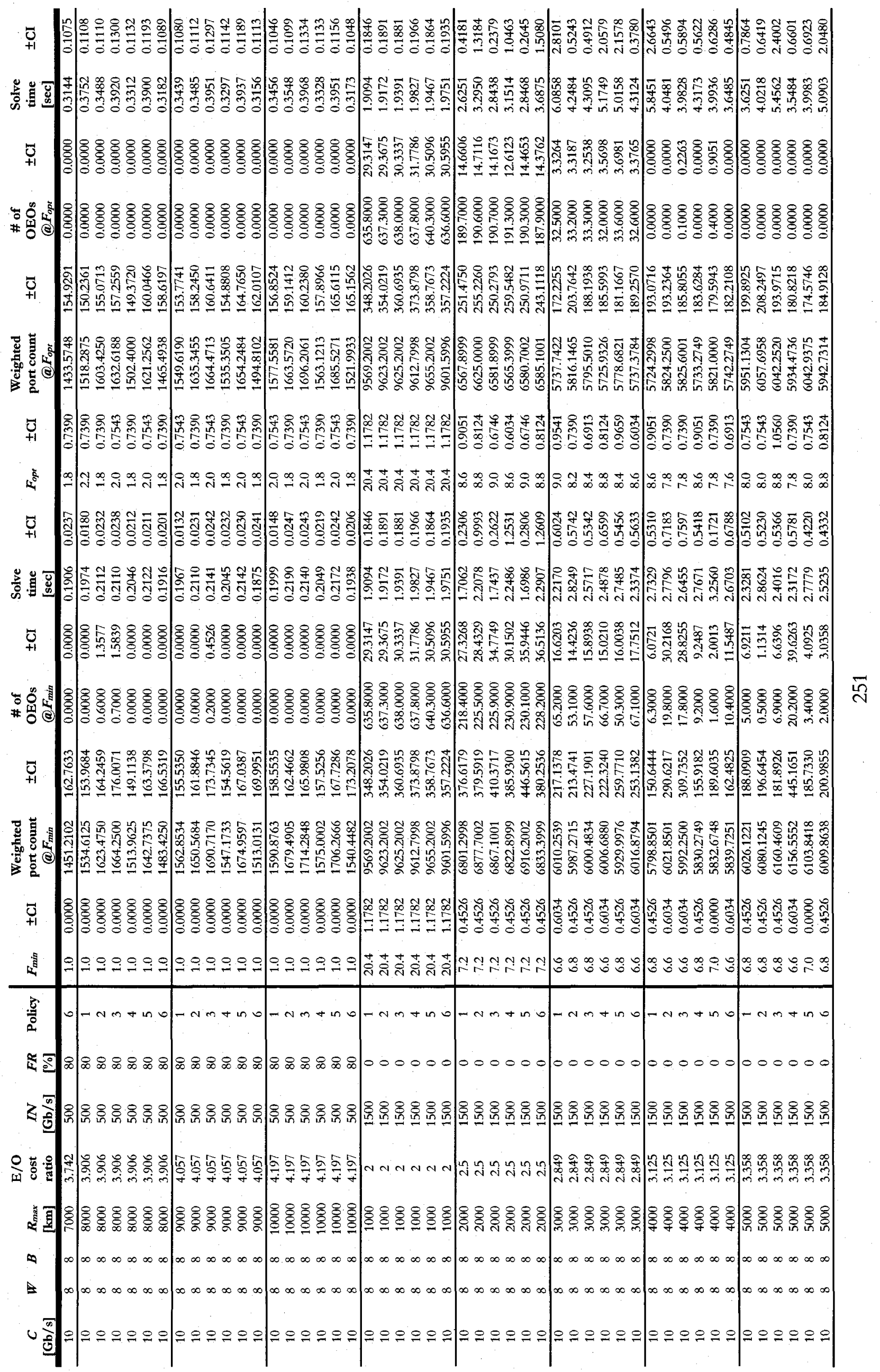




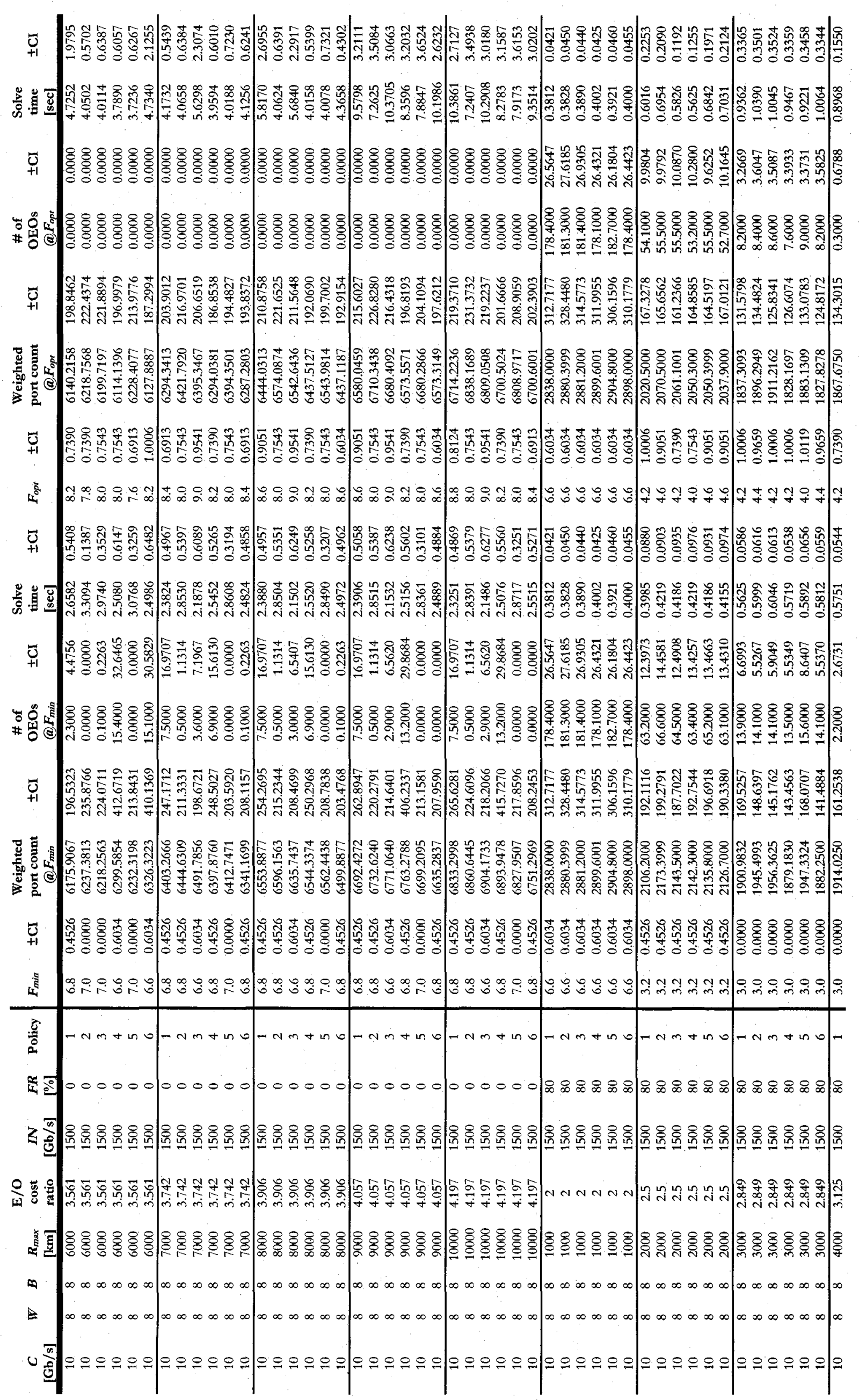




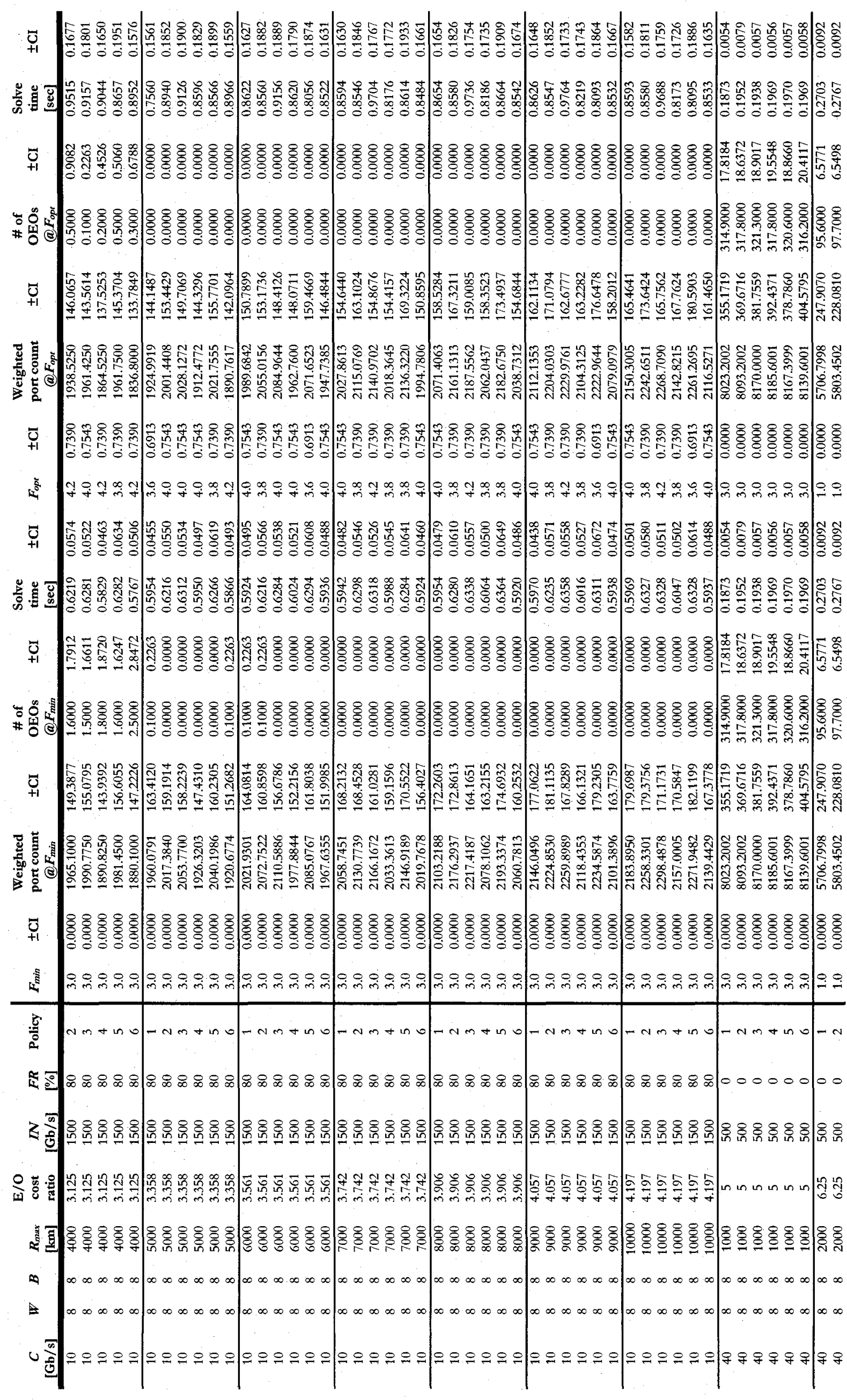

ํำ 


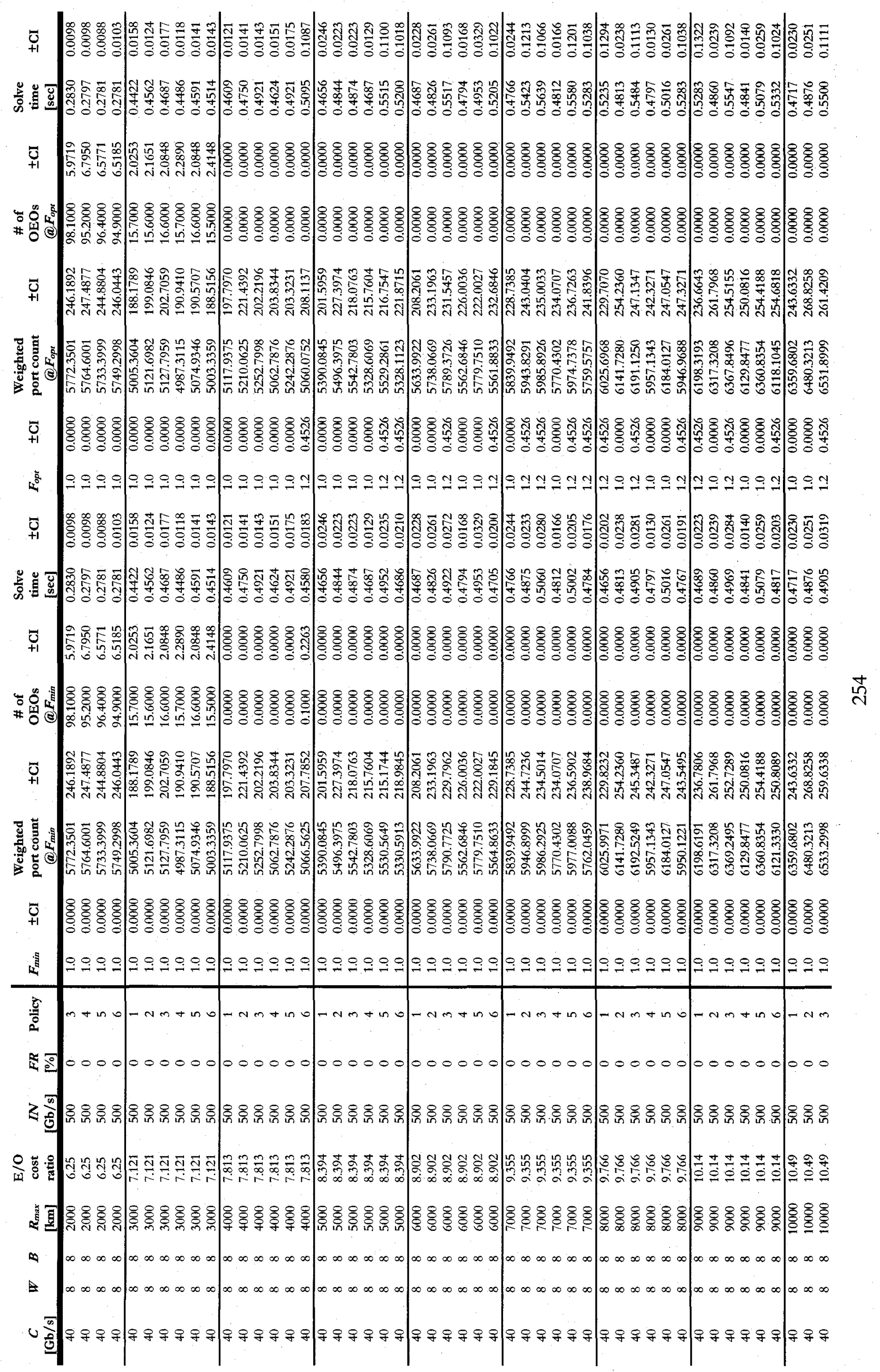




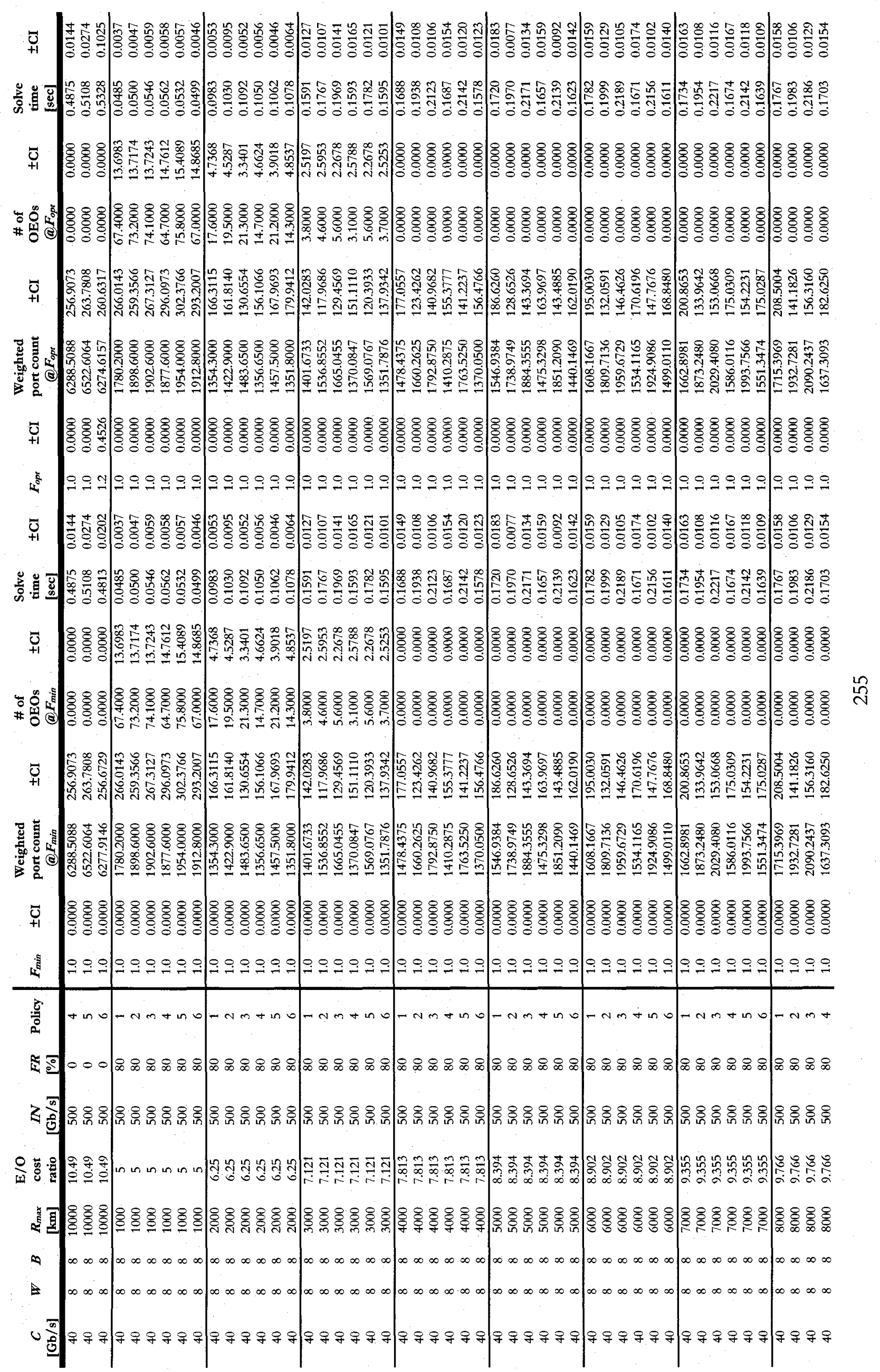




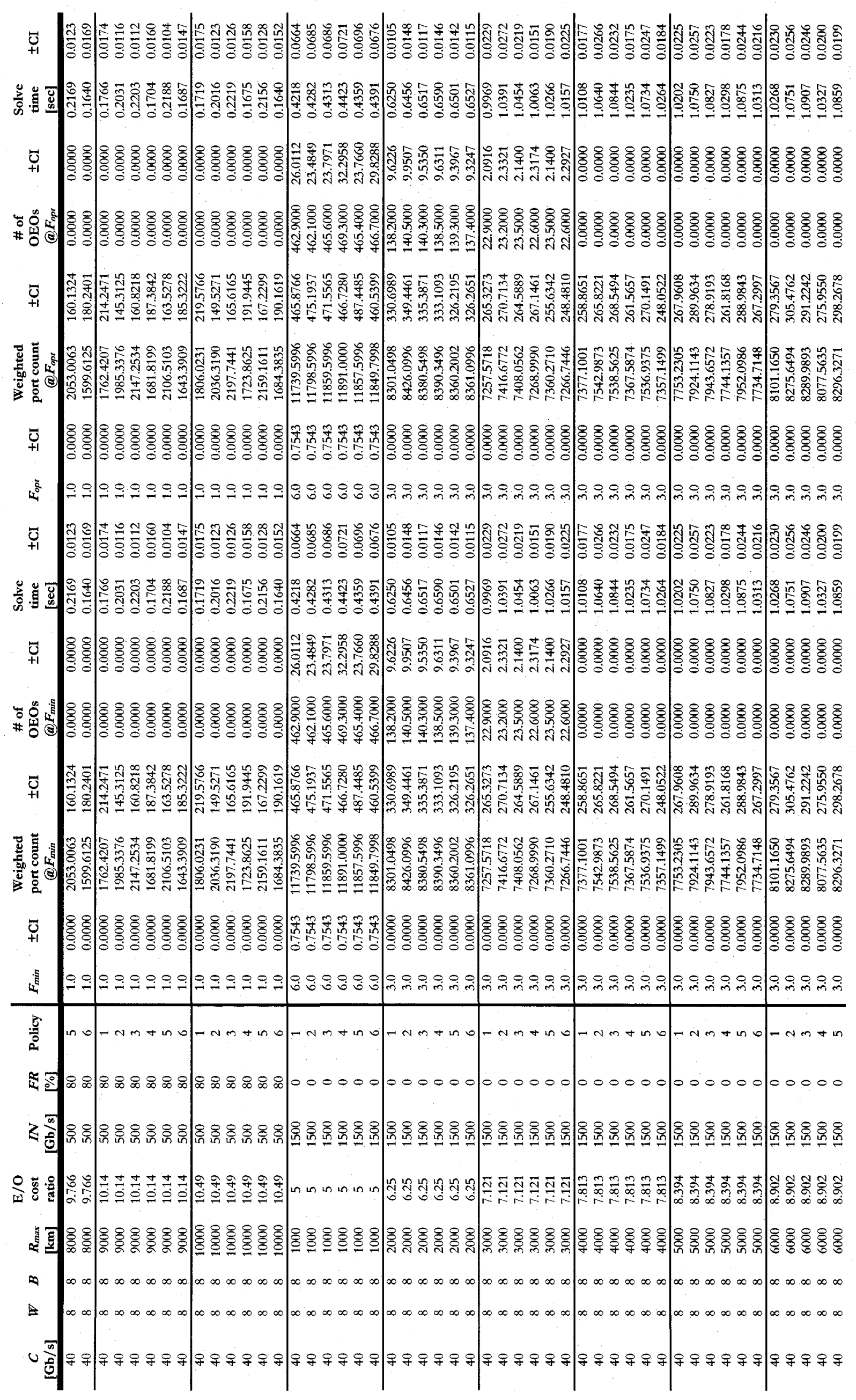




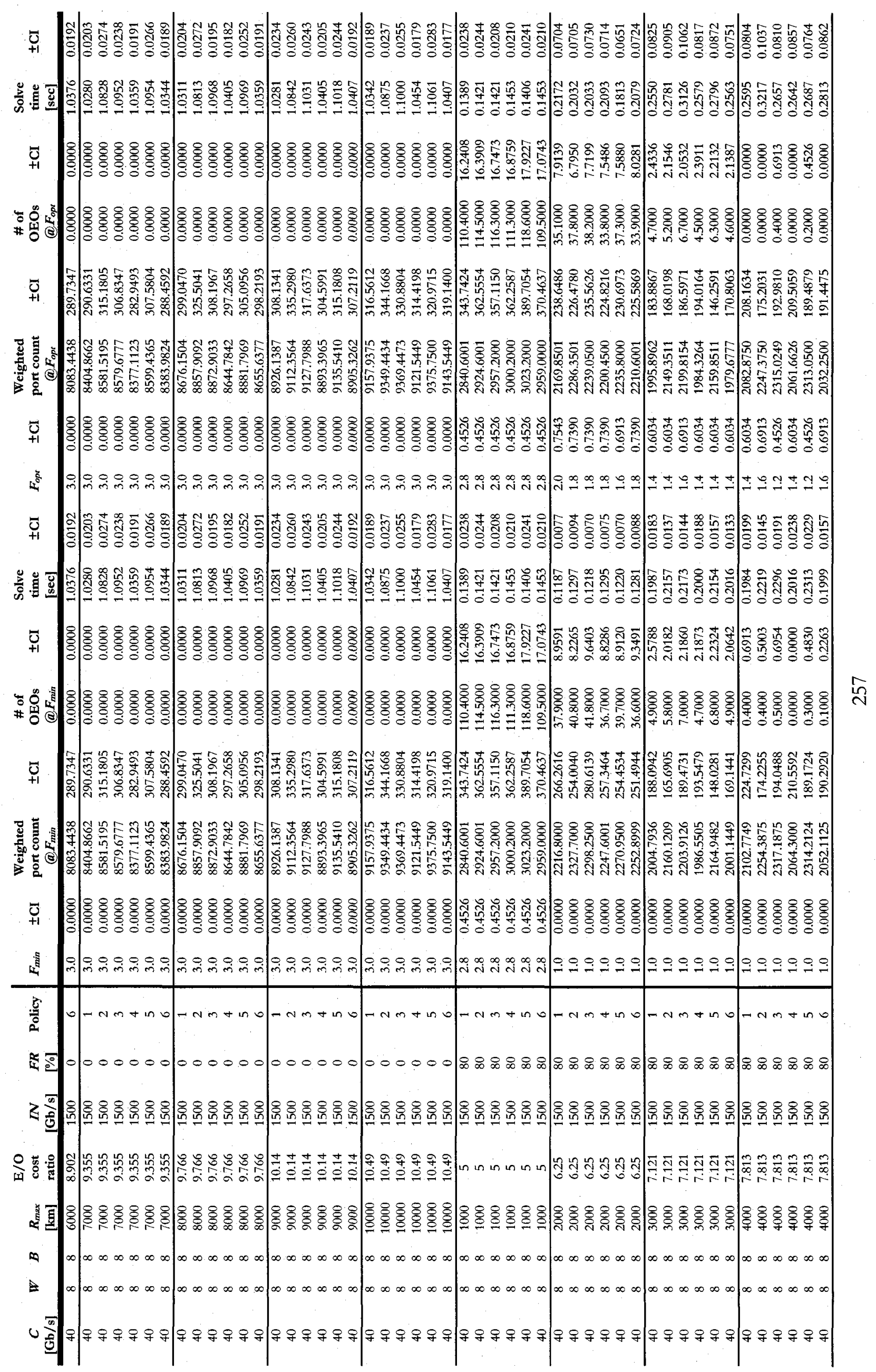




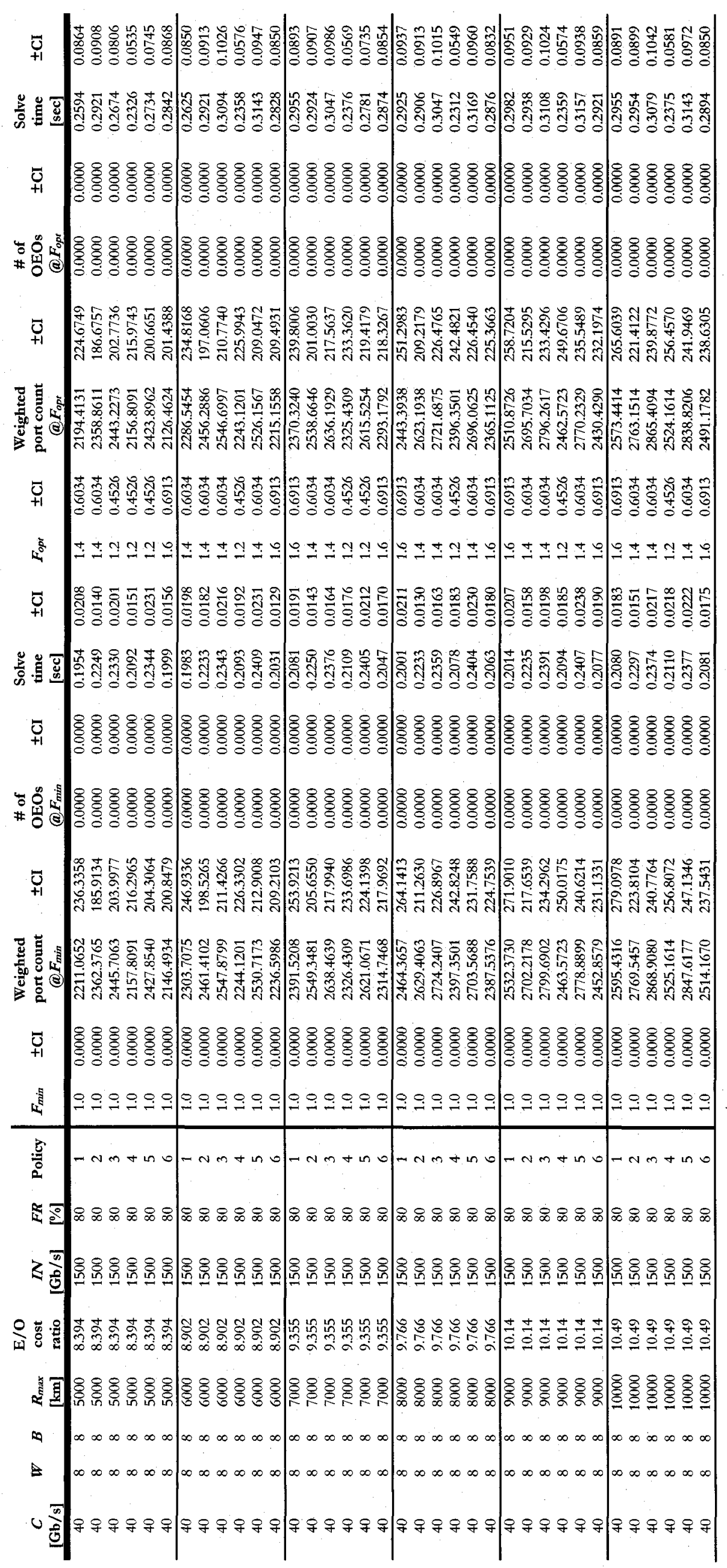




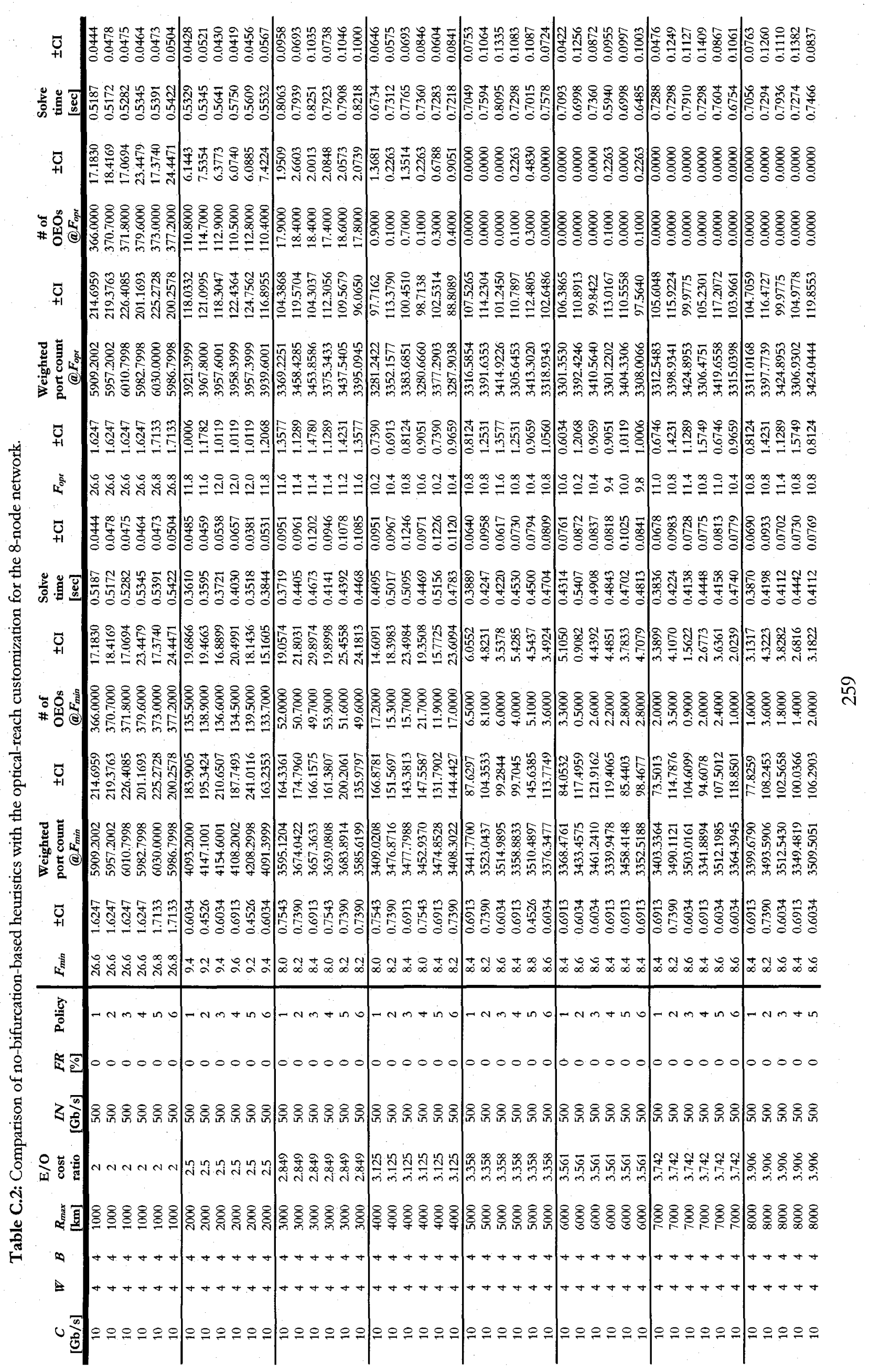




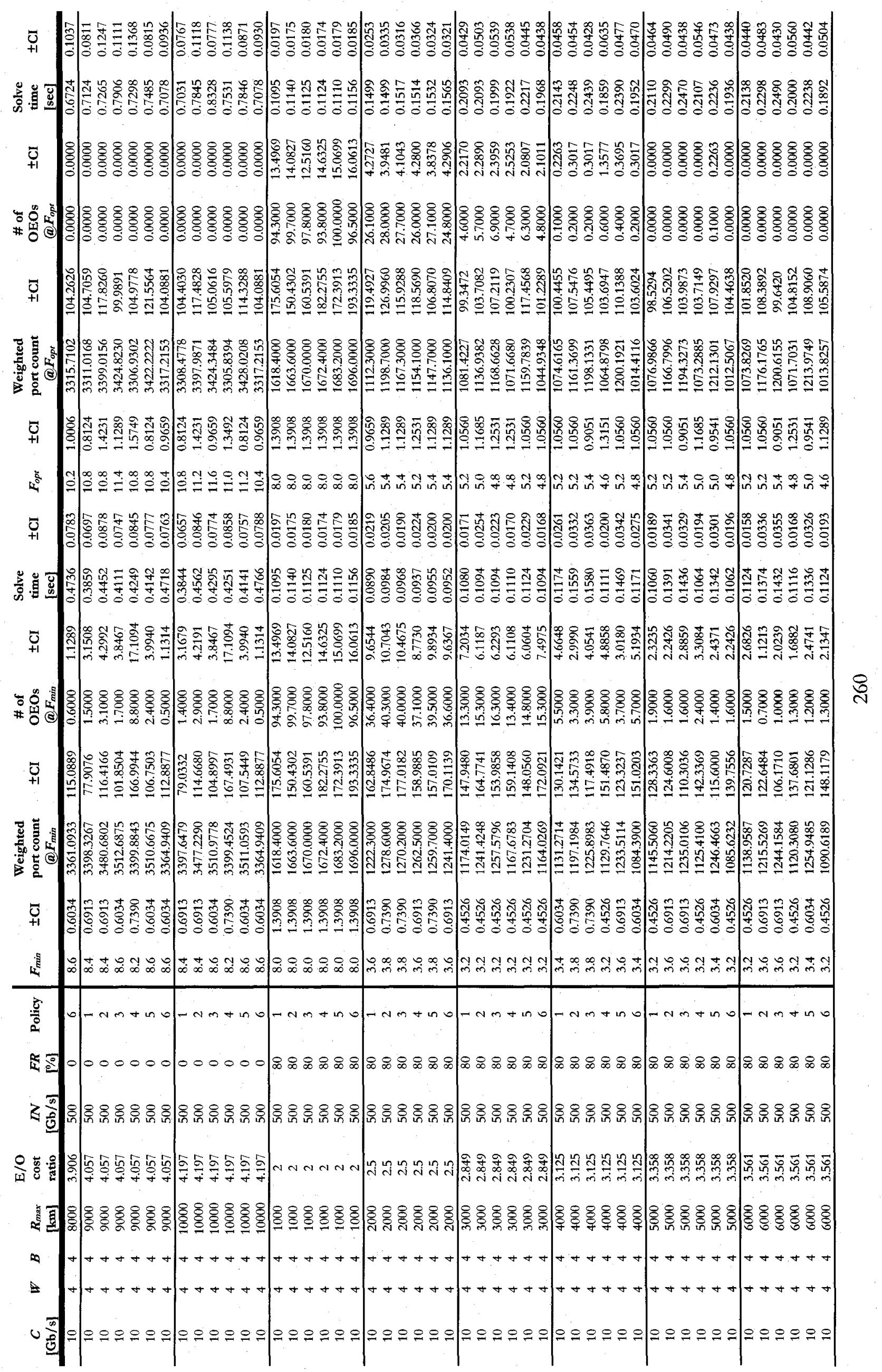




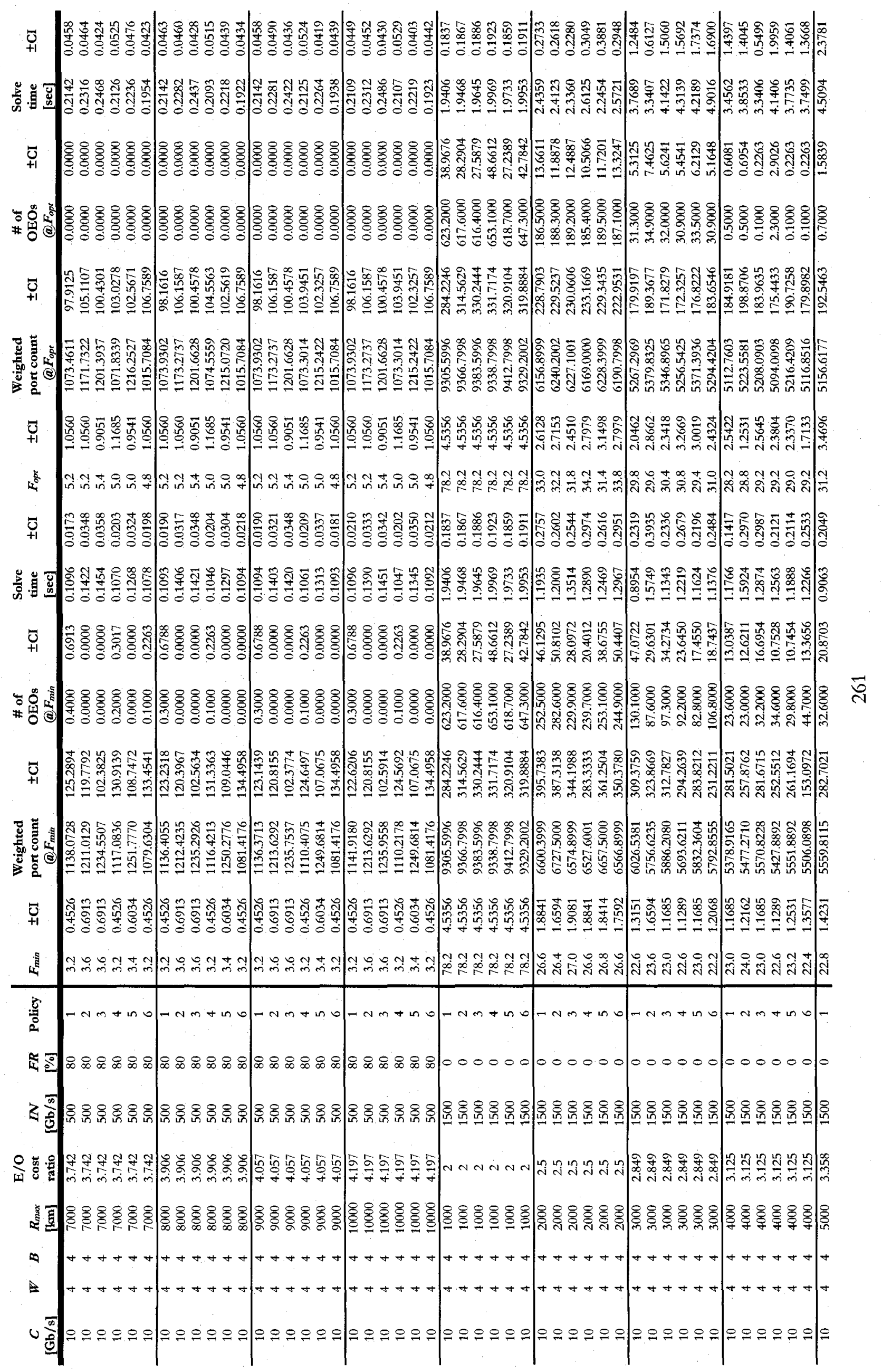




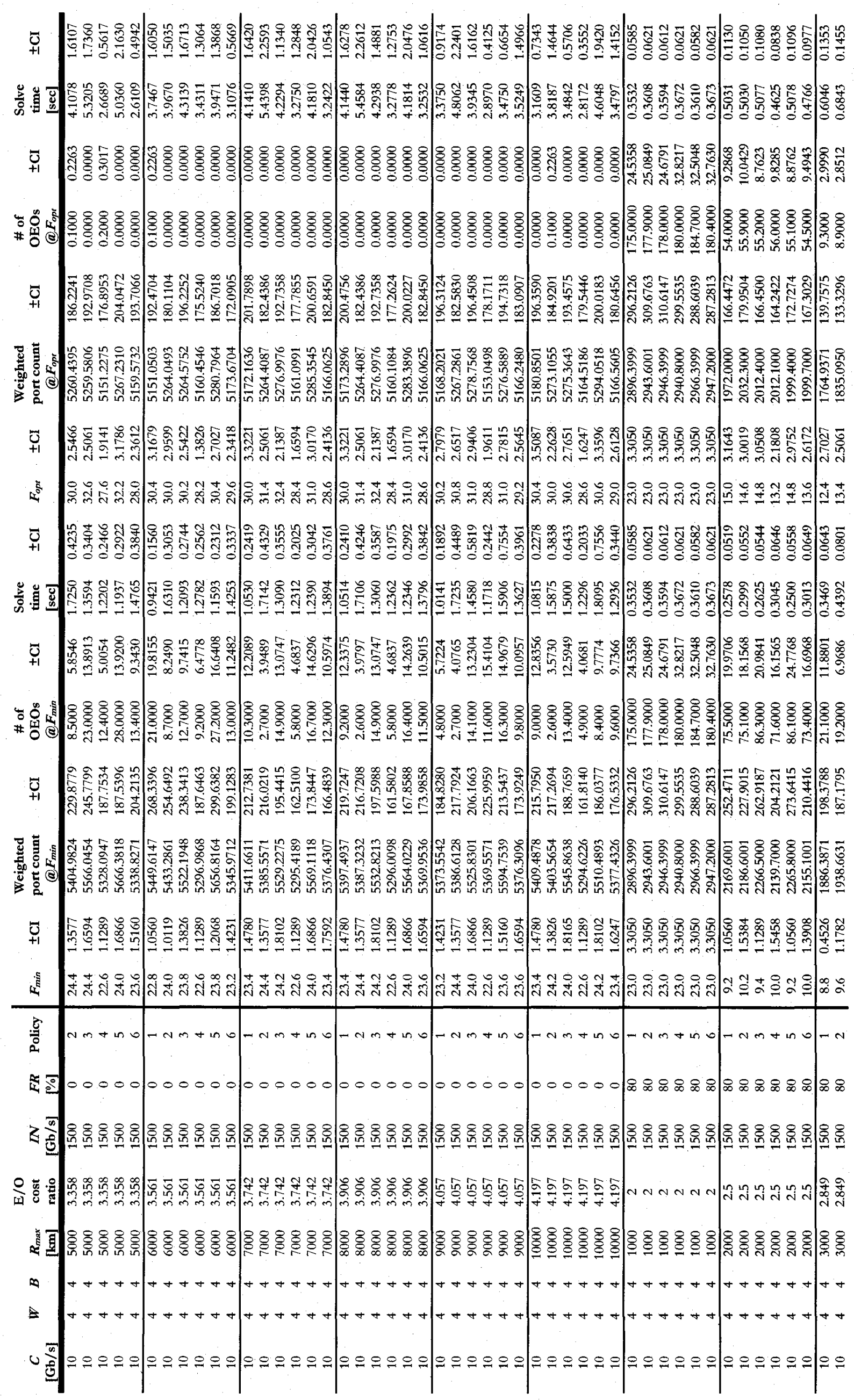




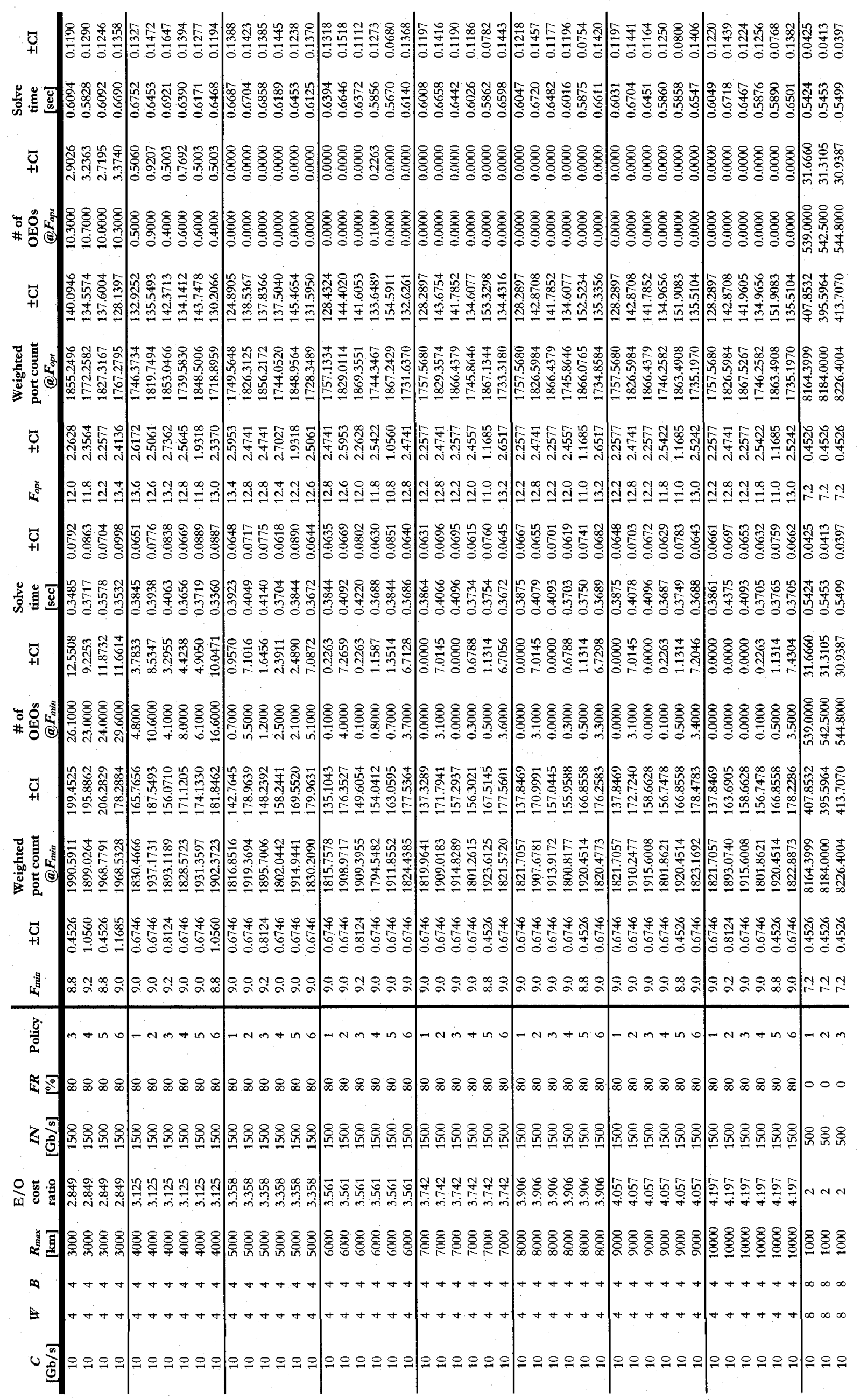

తి 


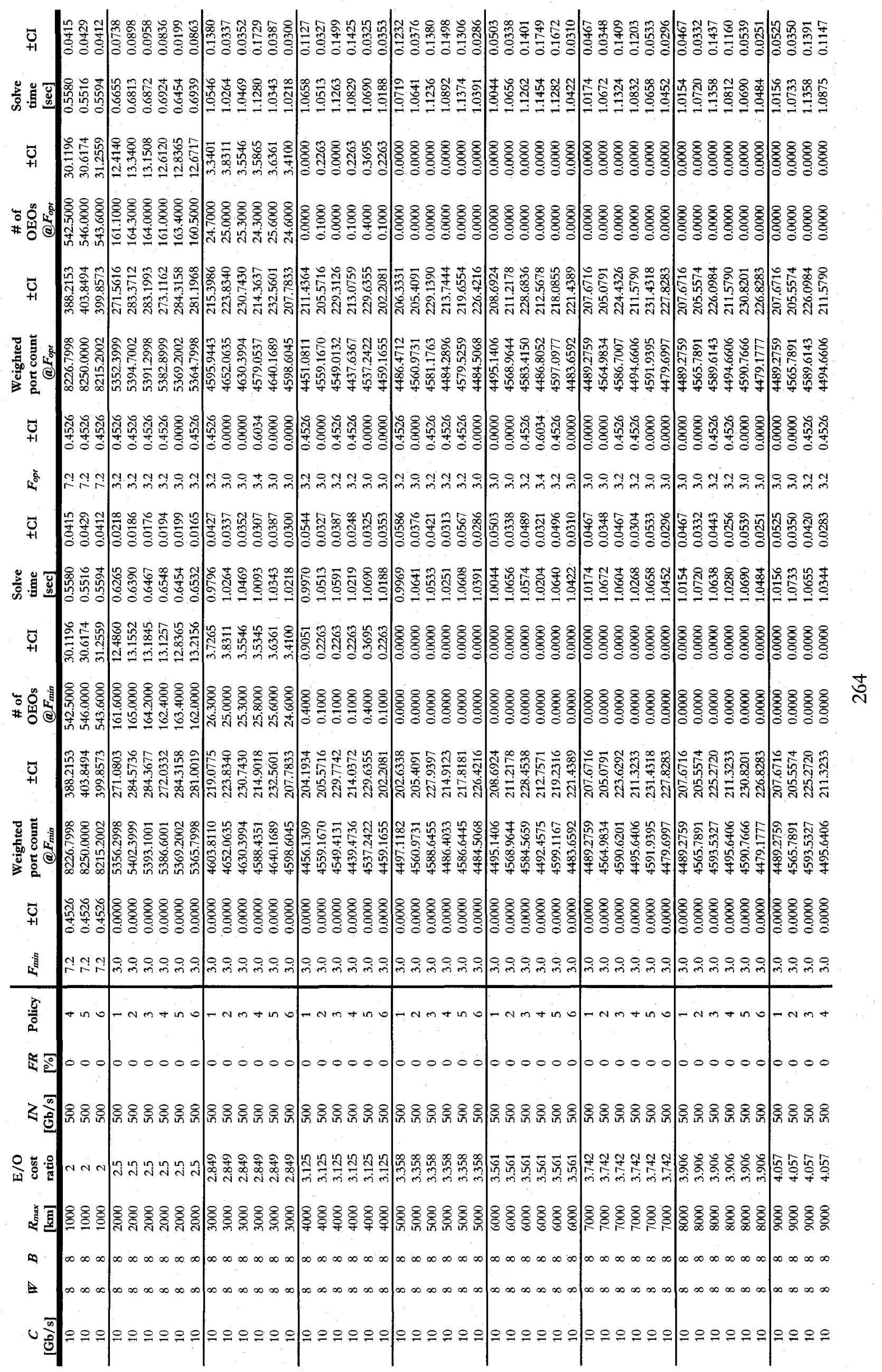




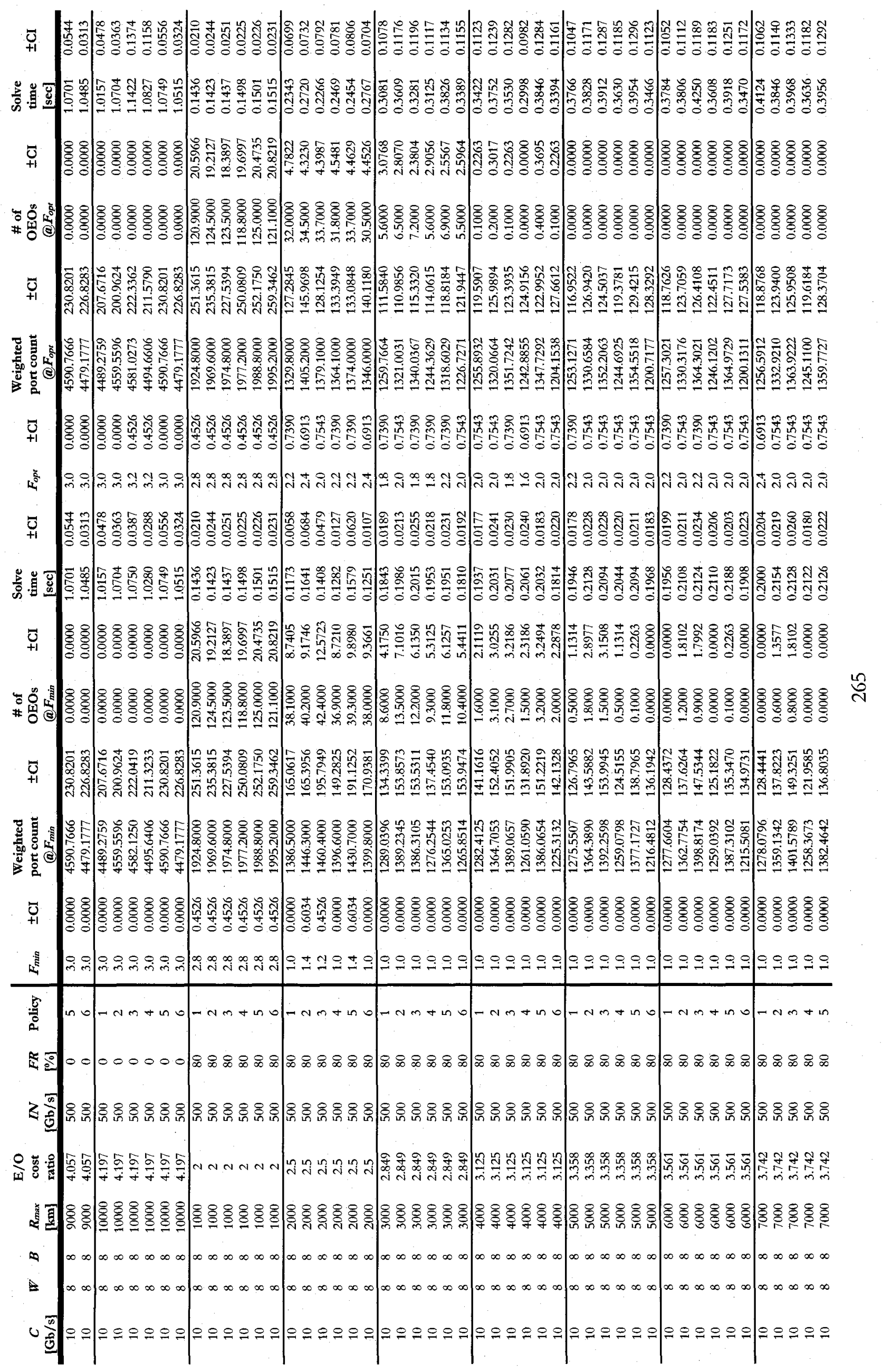




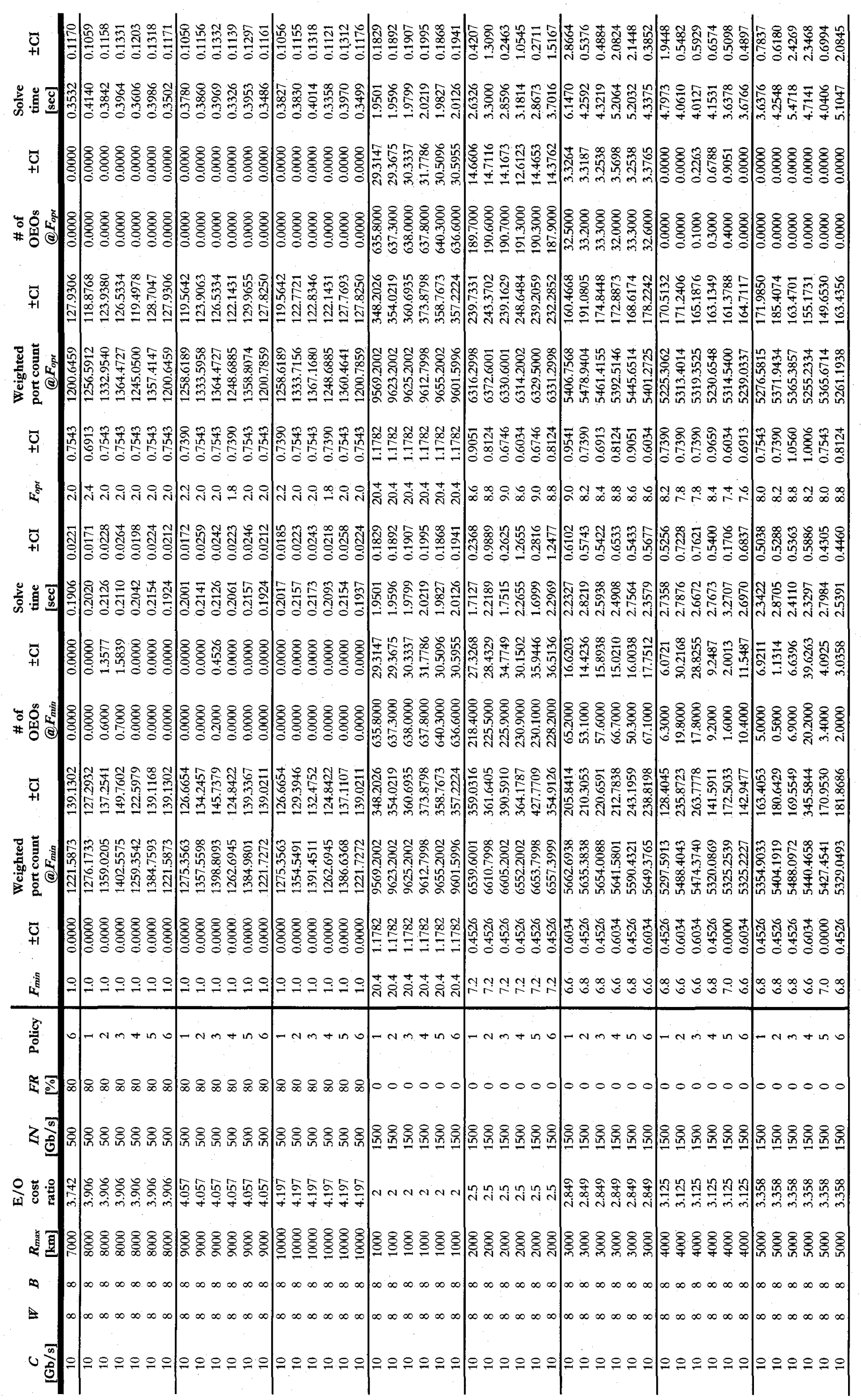




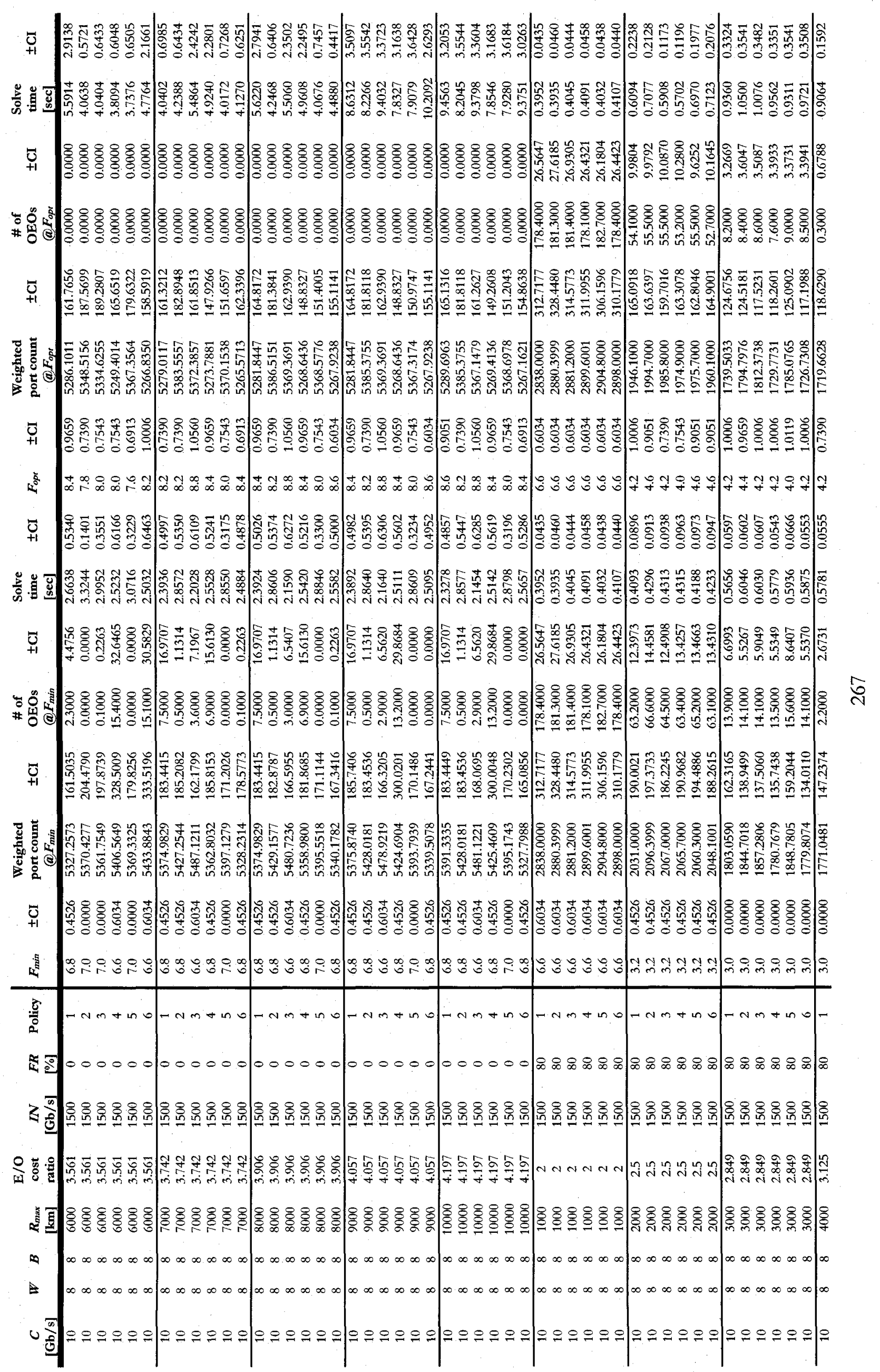




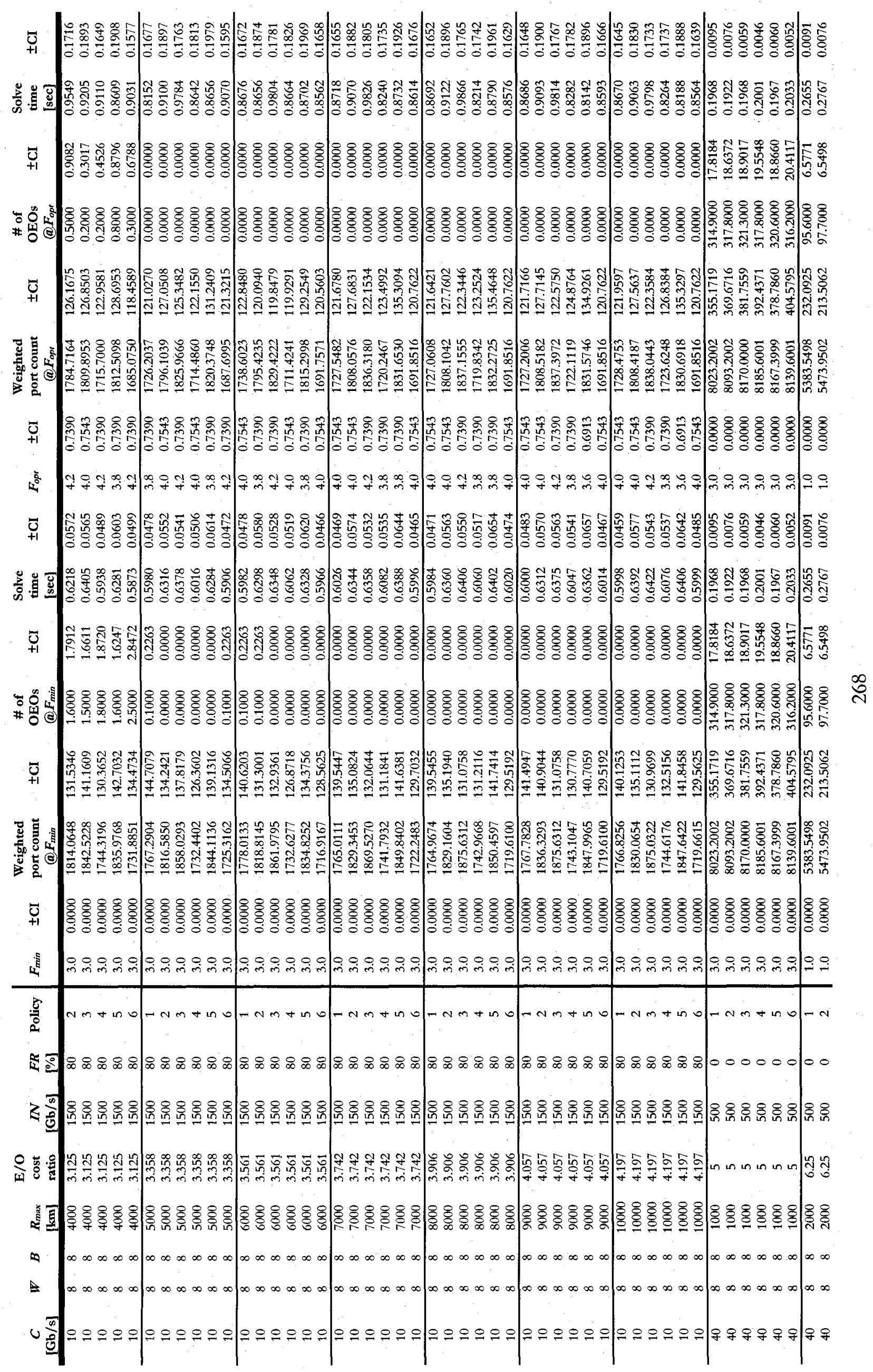




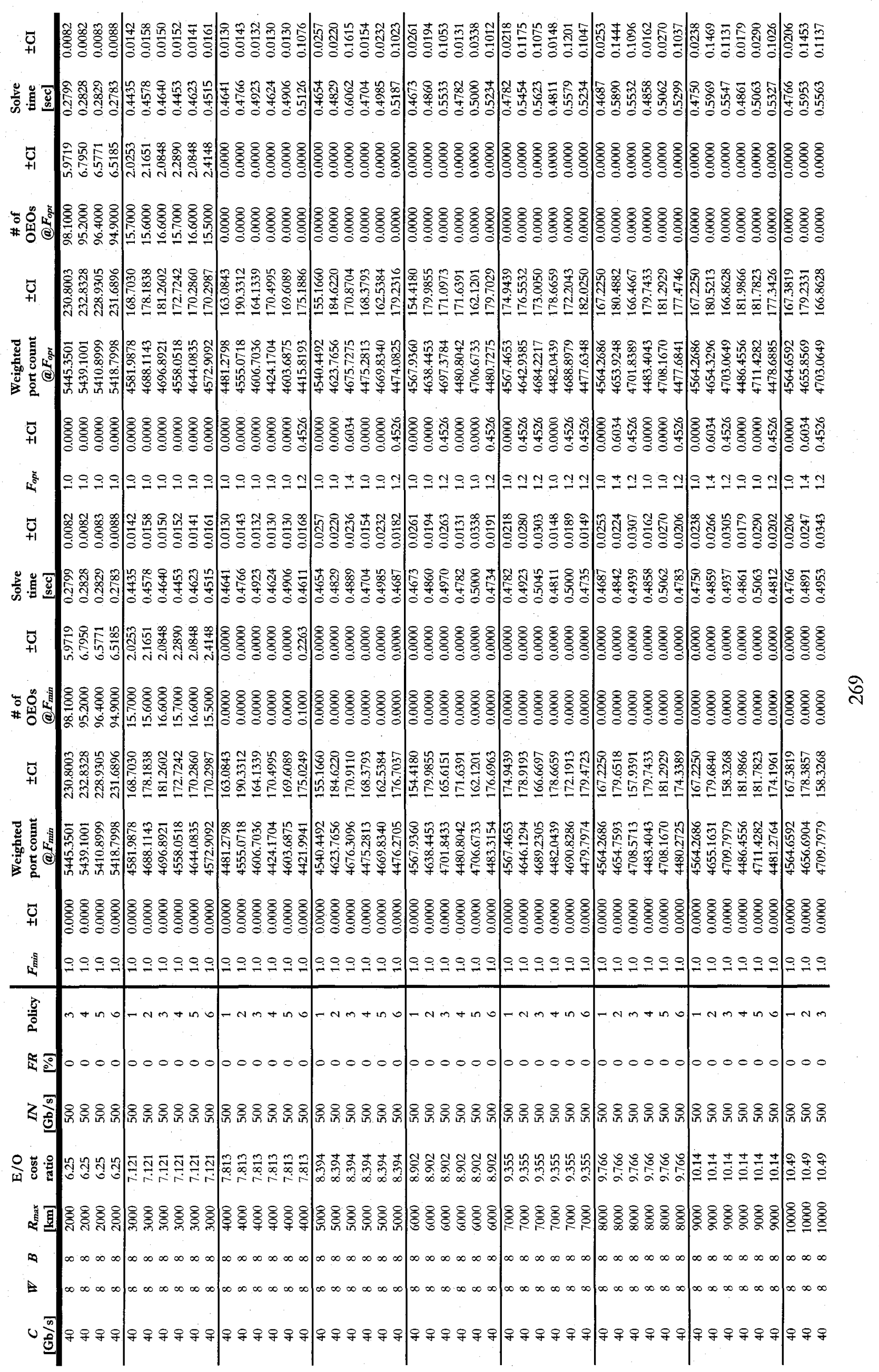




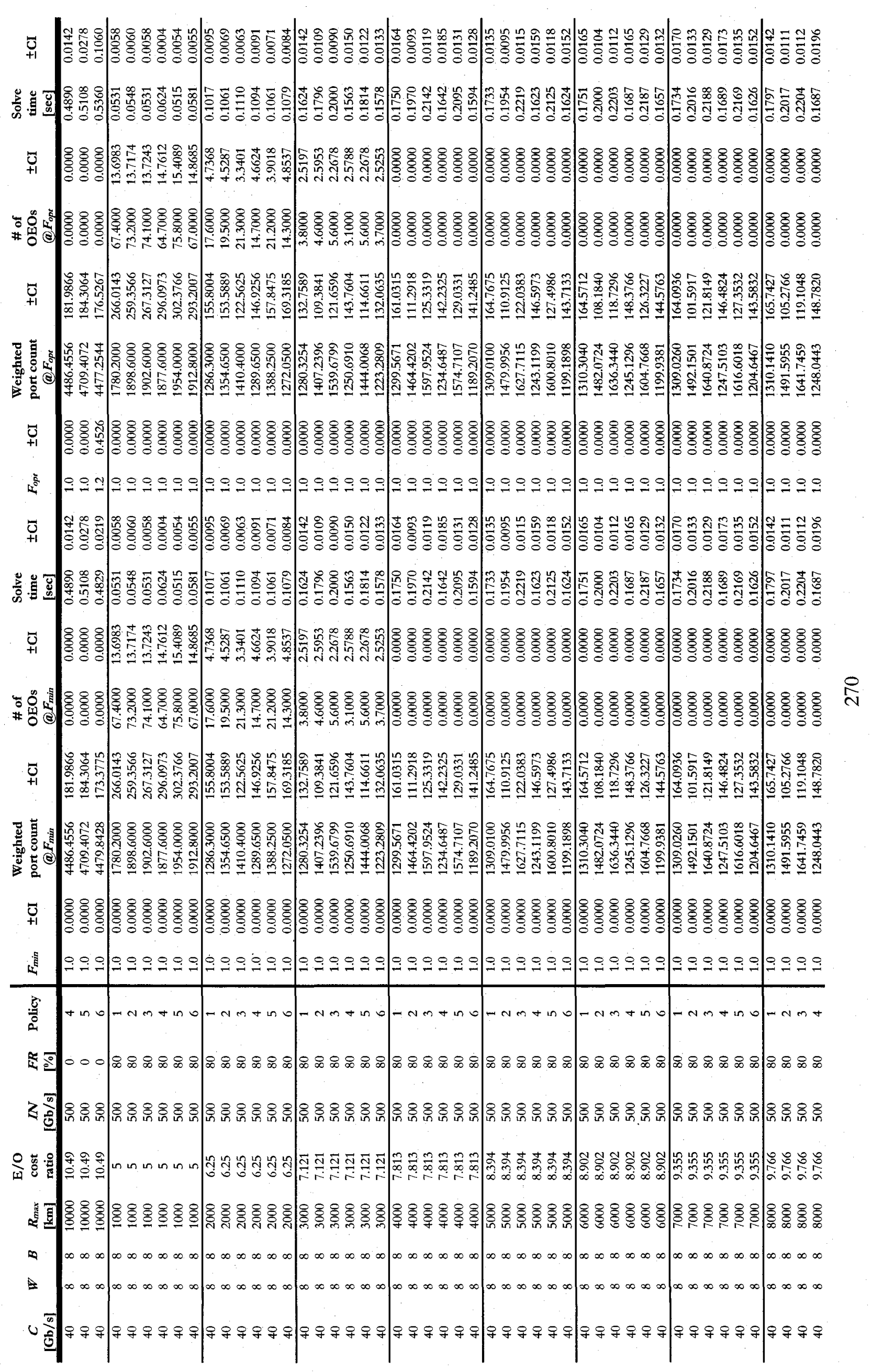




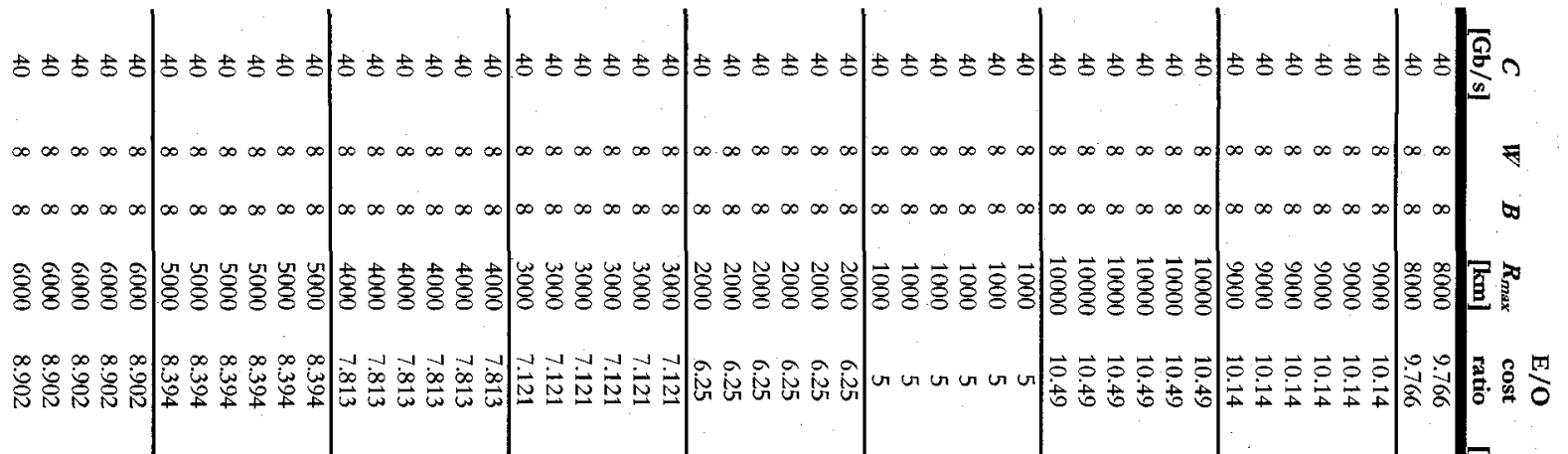

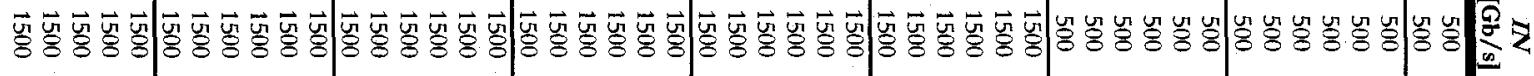

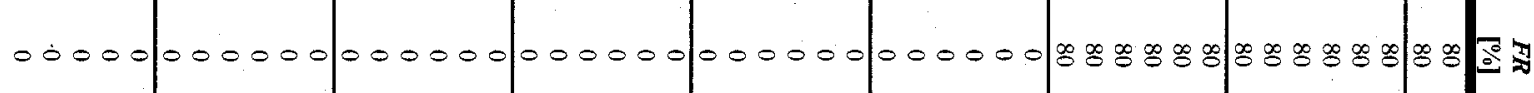

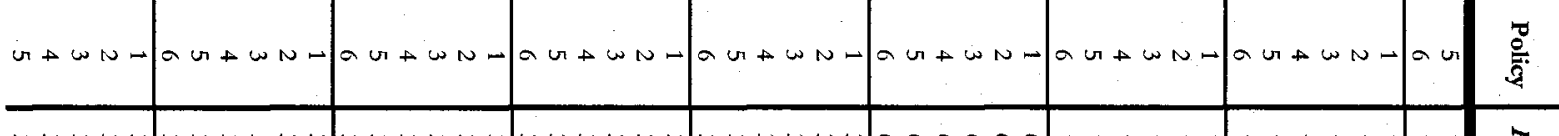

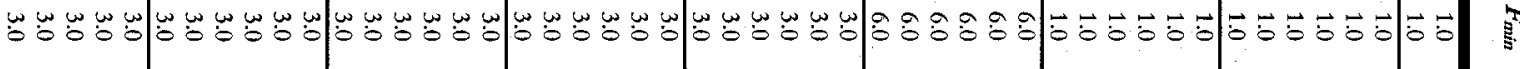

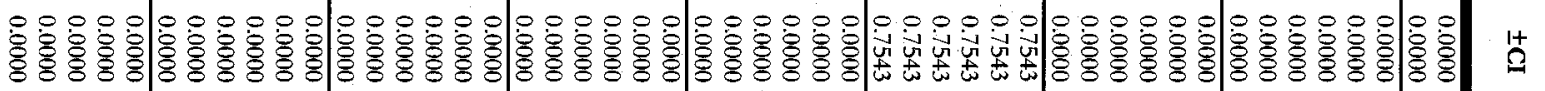

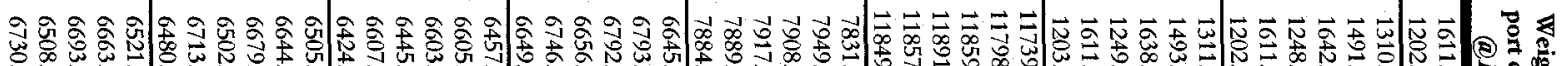

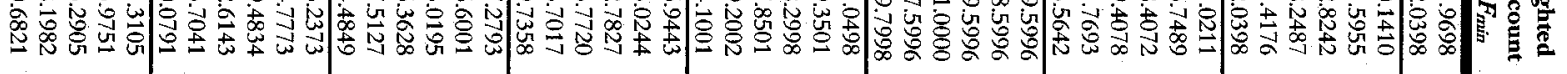

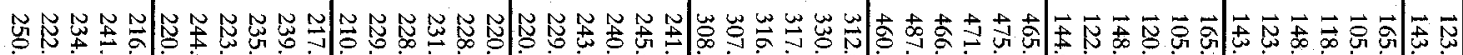

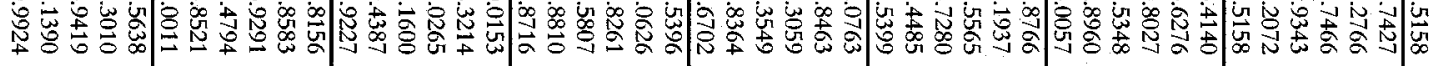

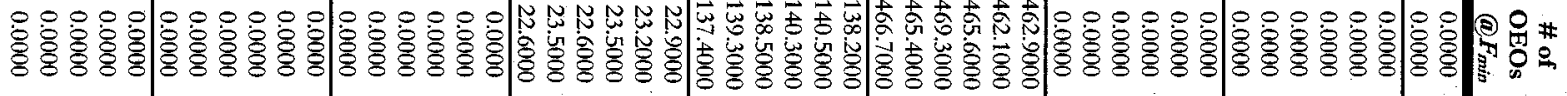

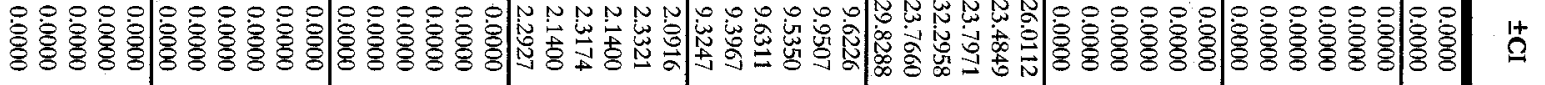

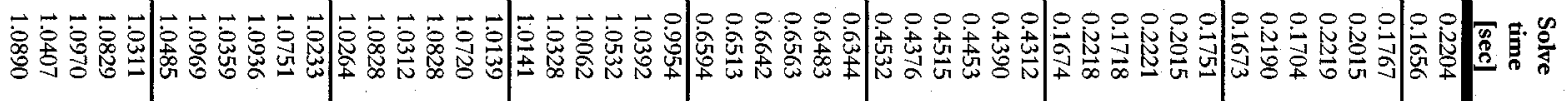

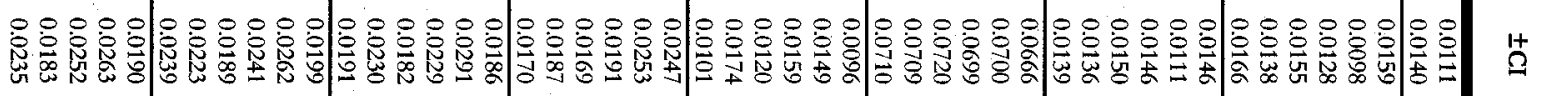

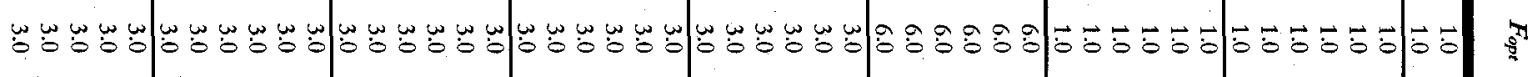

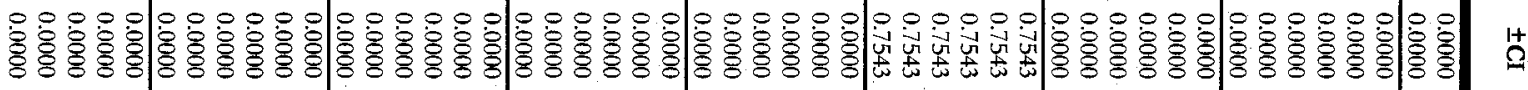

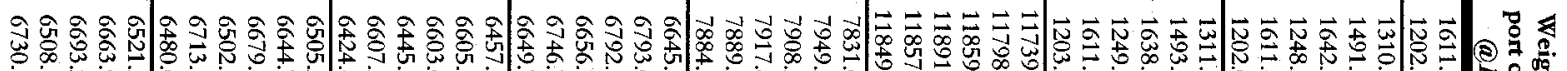

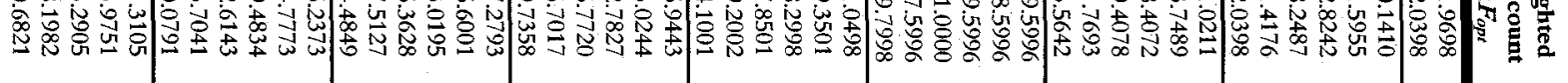

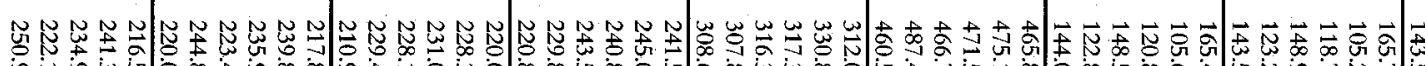

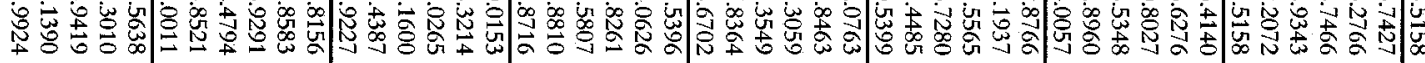
$\% \%$

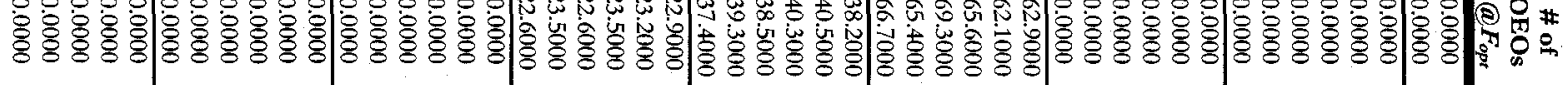

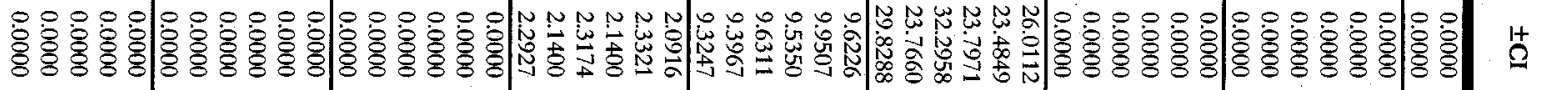

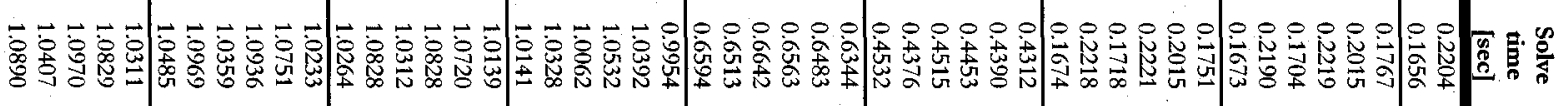

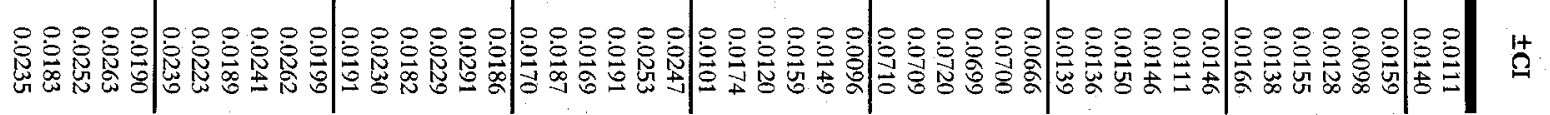




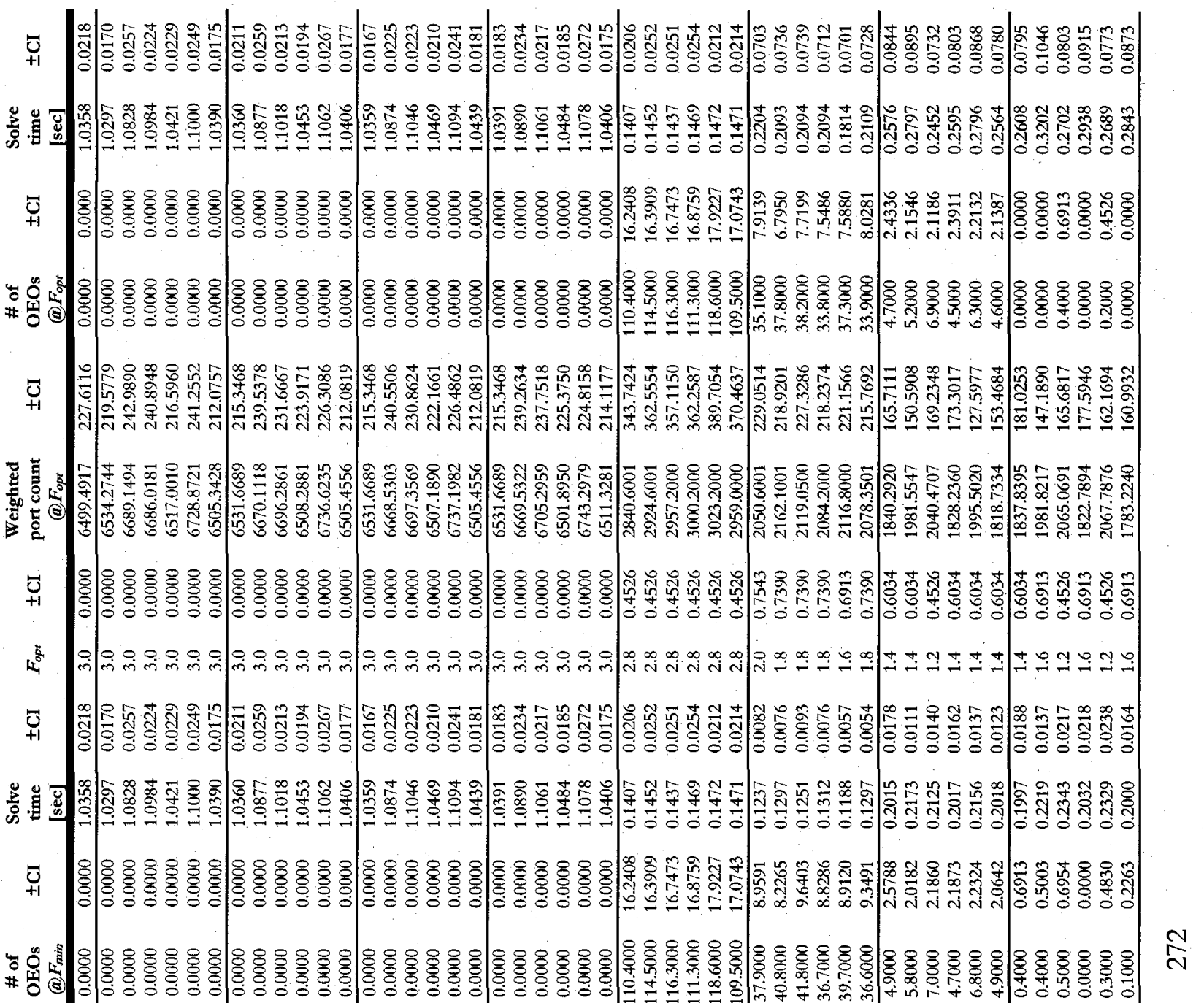

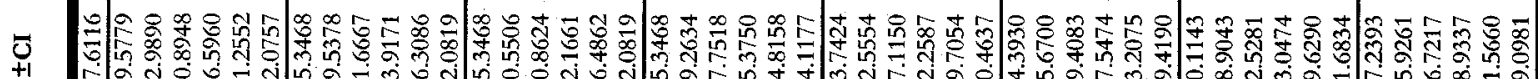

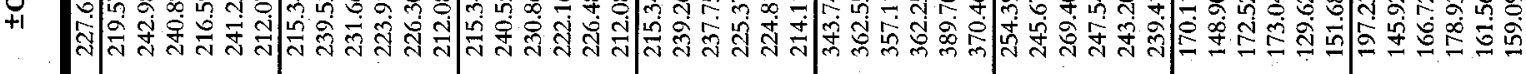

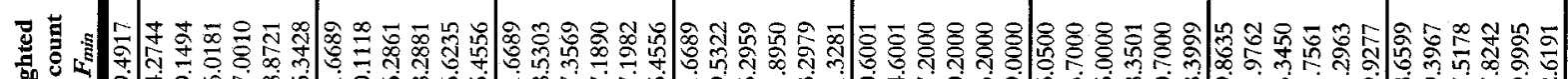

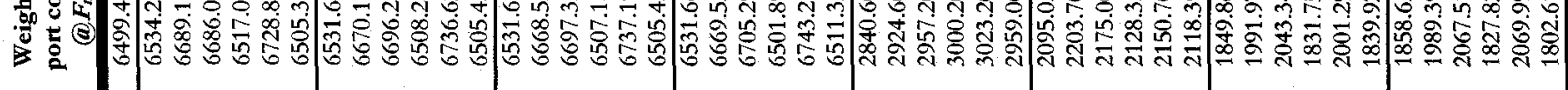

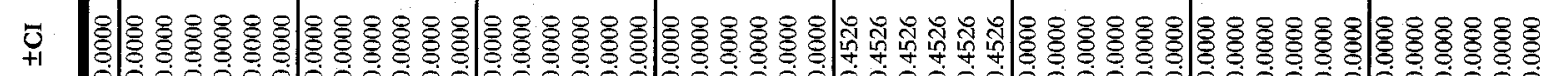
(1) L

$\overbrace{0}^{\circ}$

5

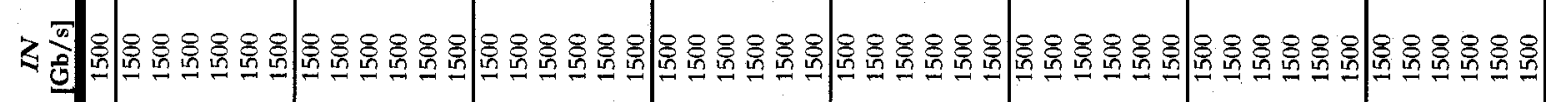

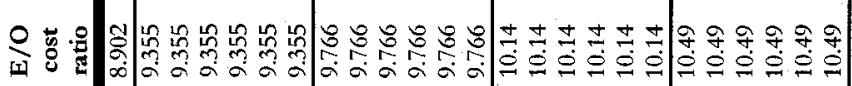

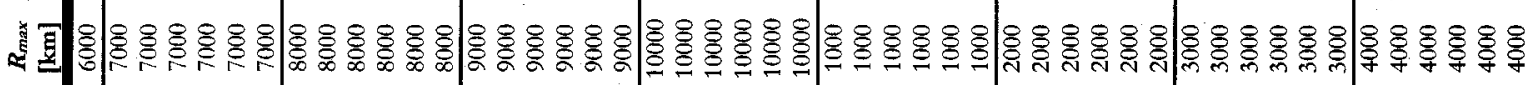

$\infty$

A

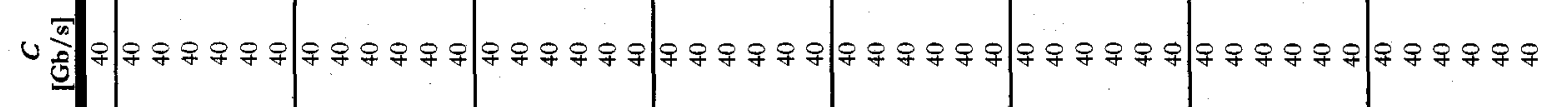




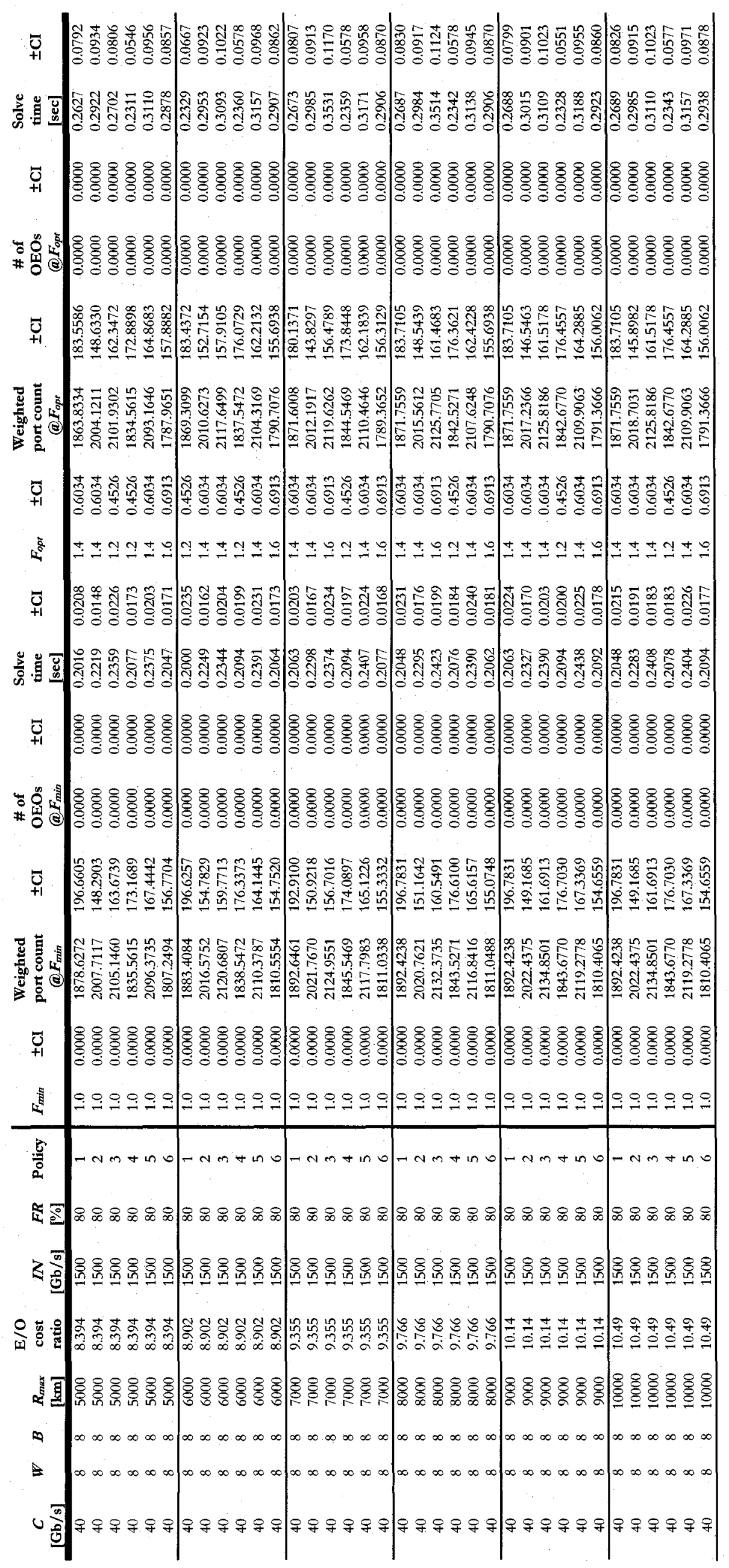




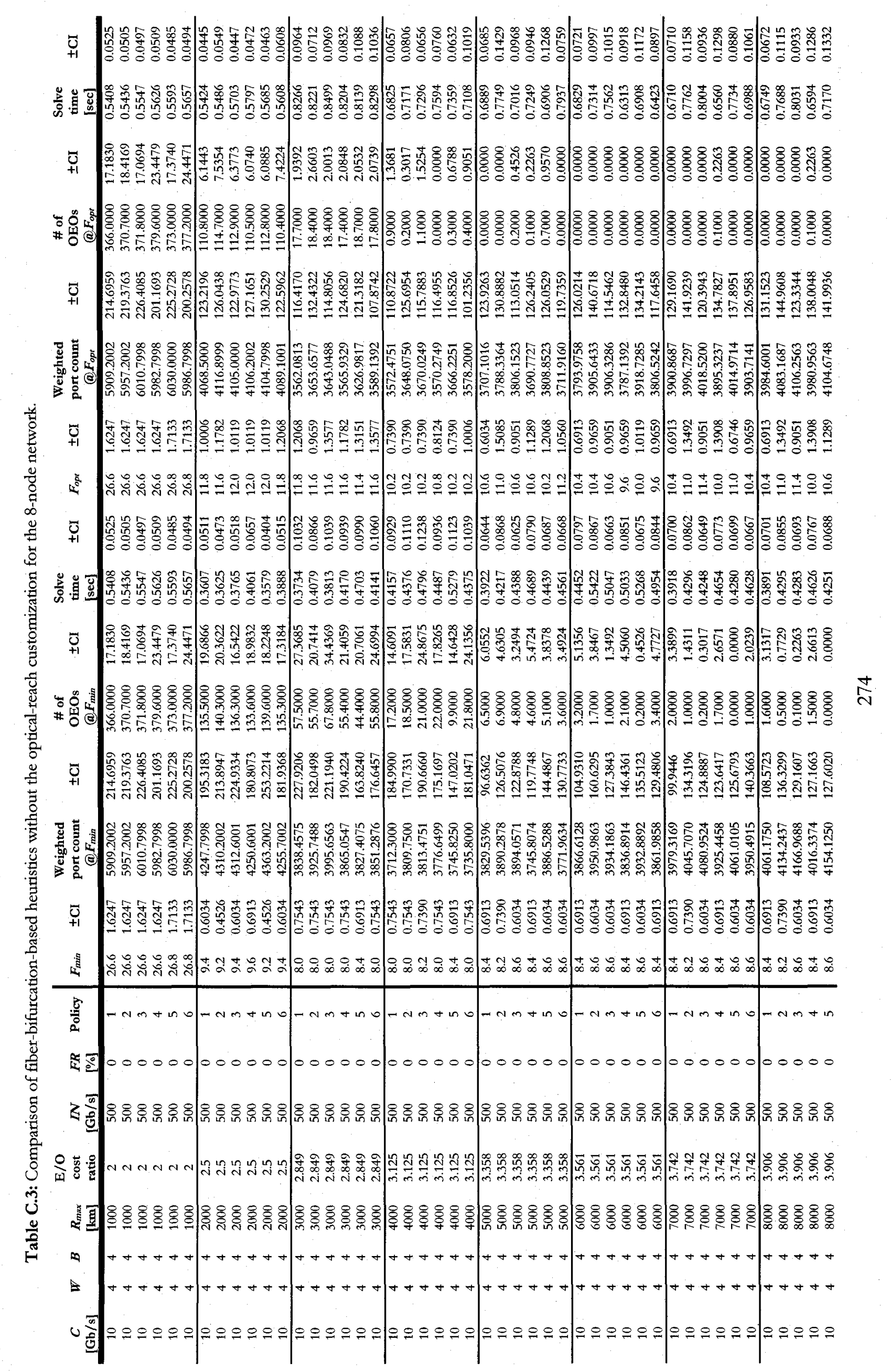




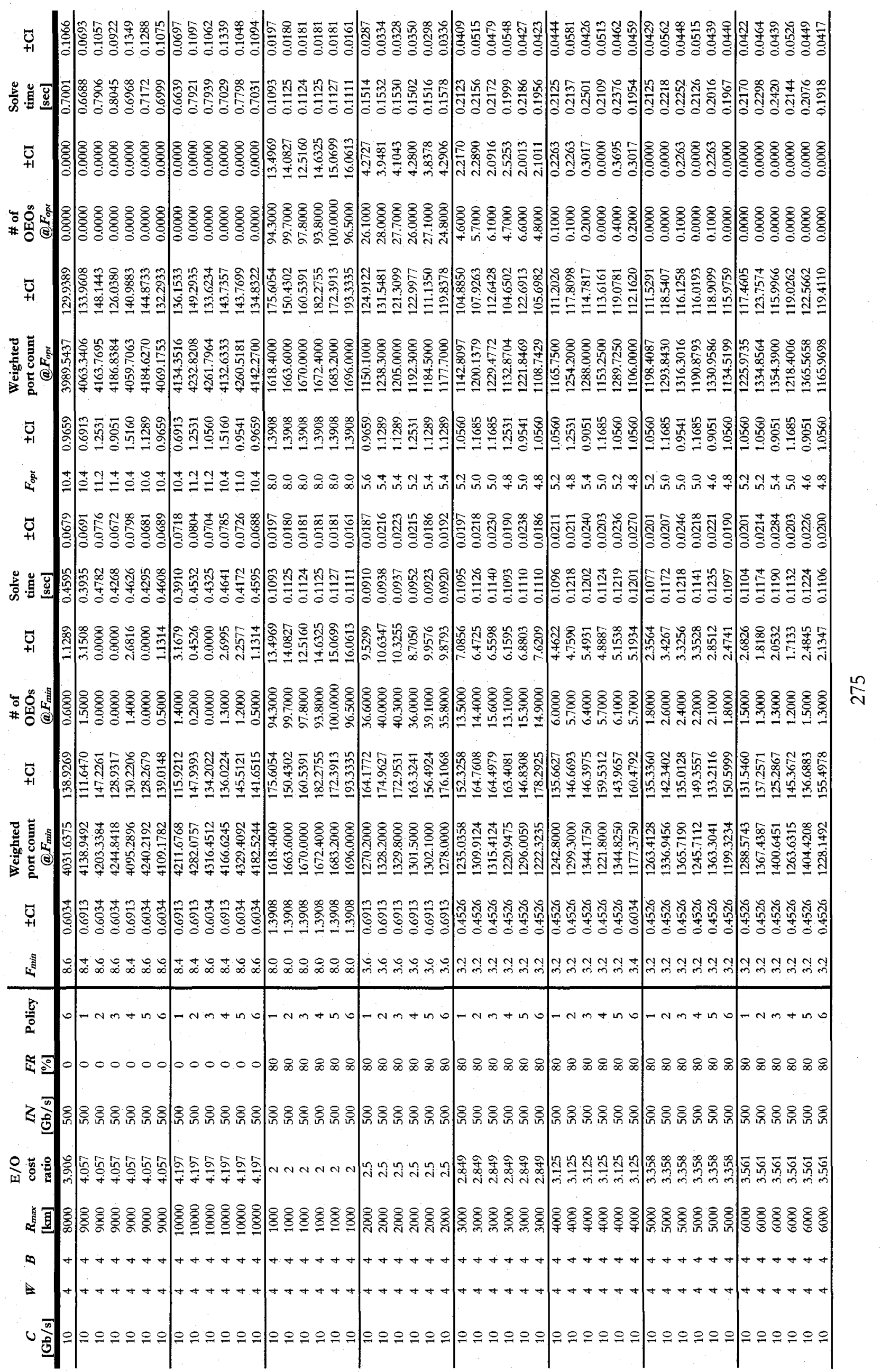




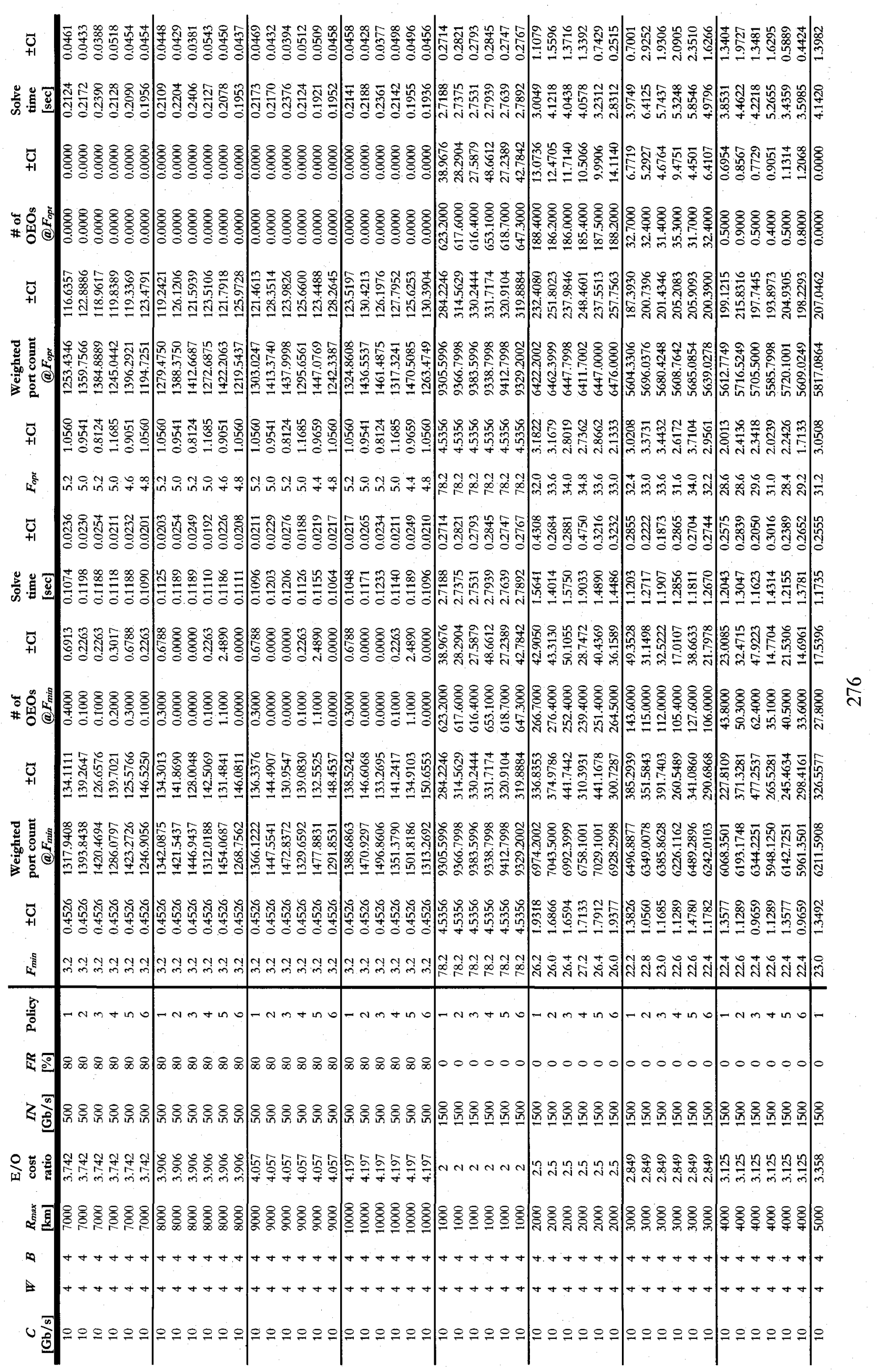




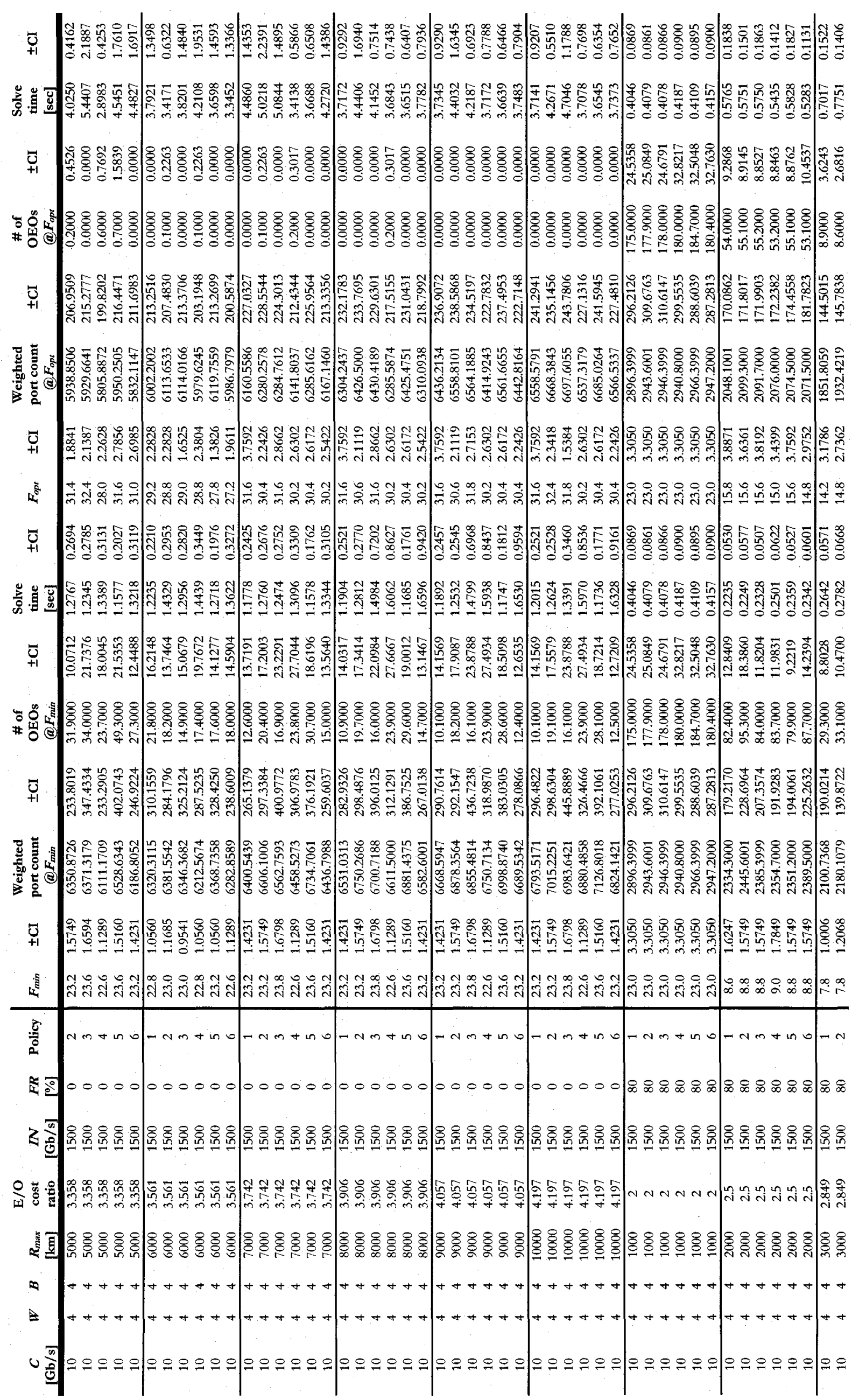




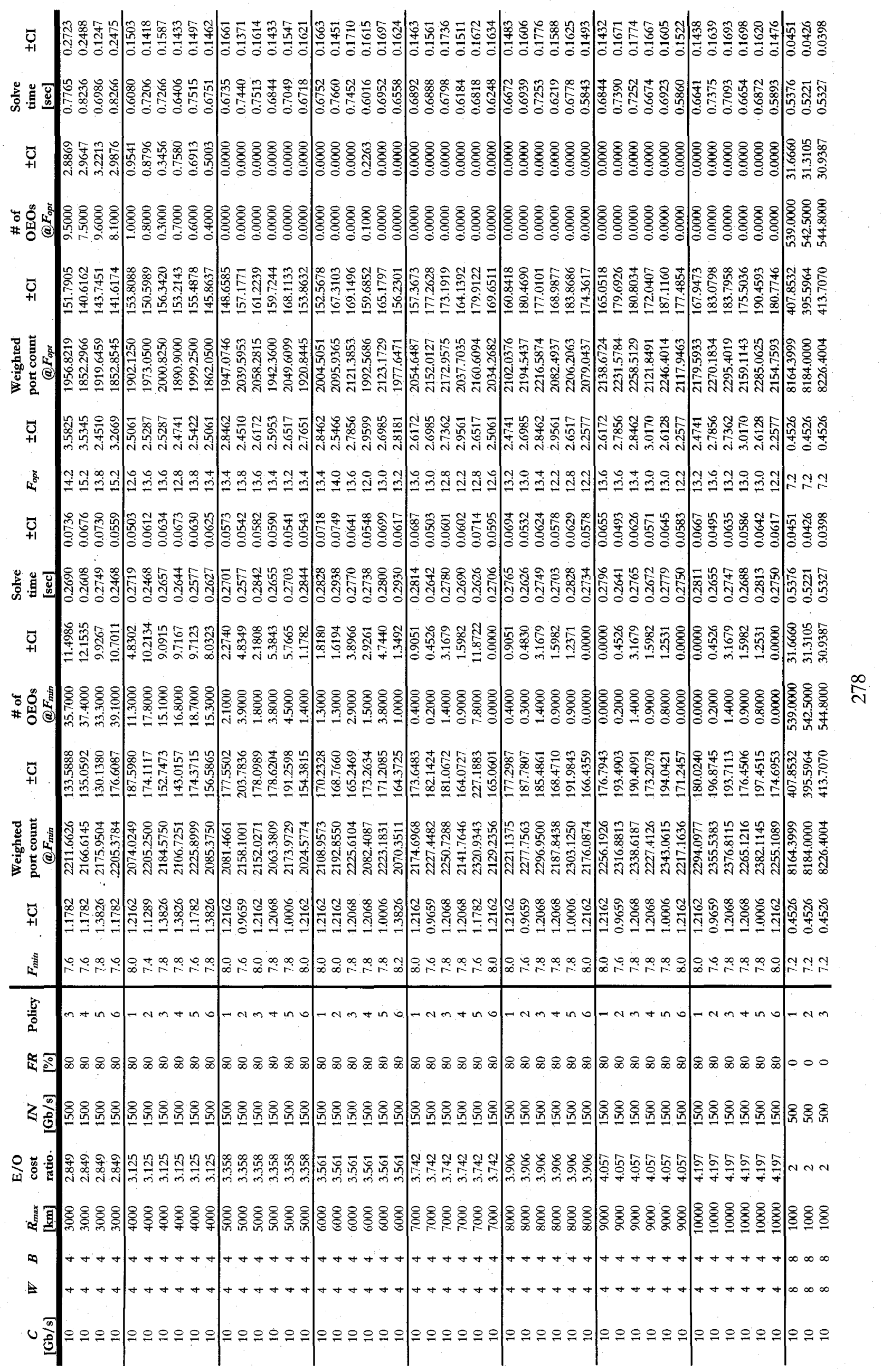




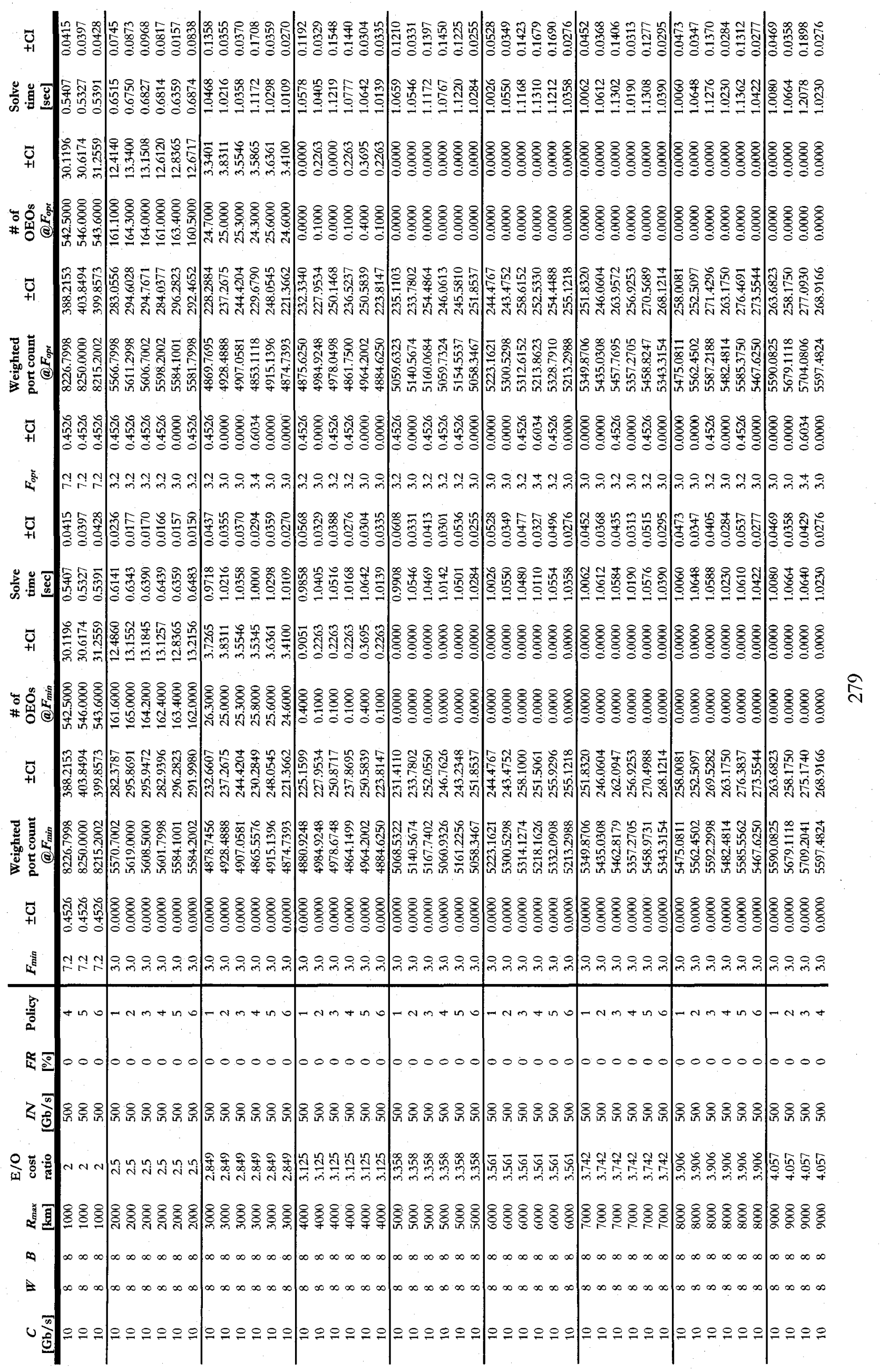




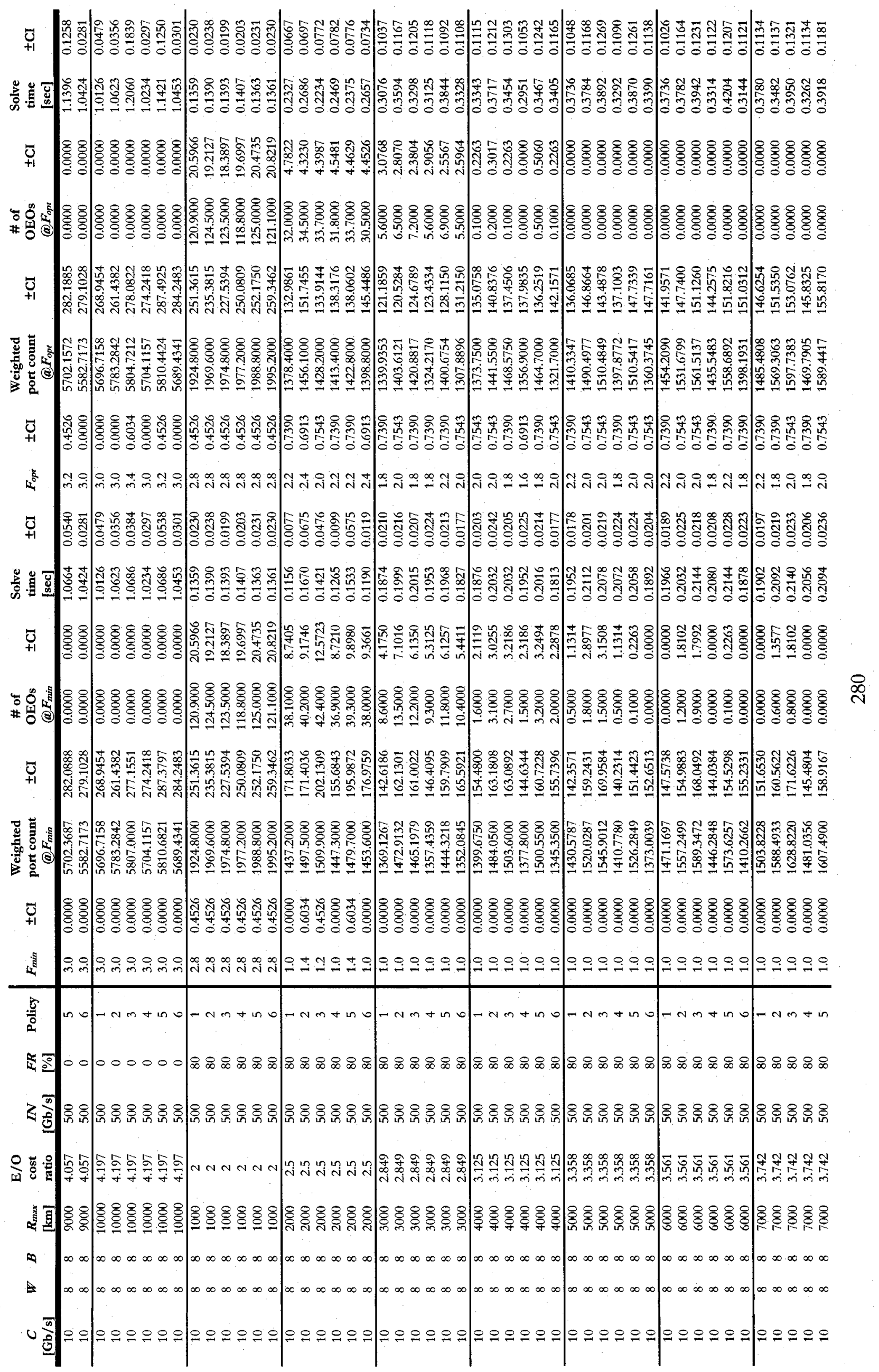




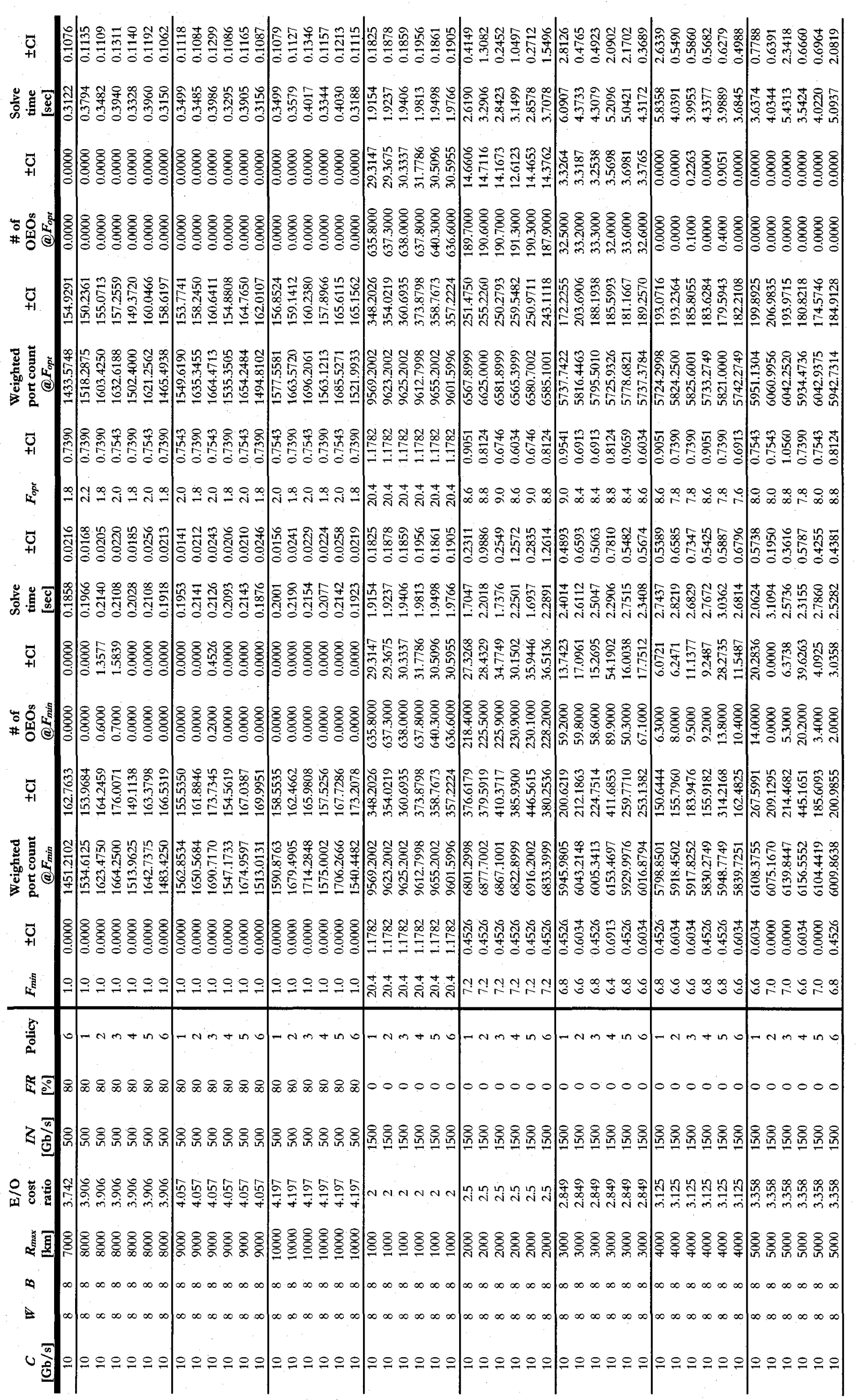




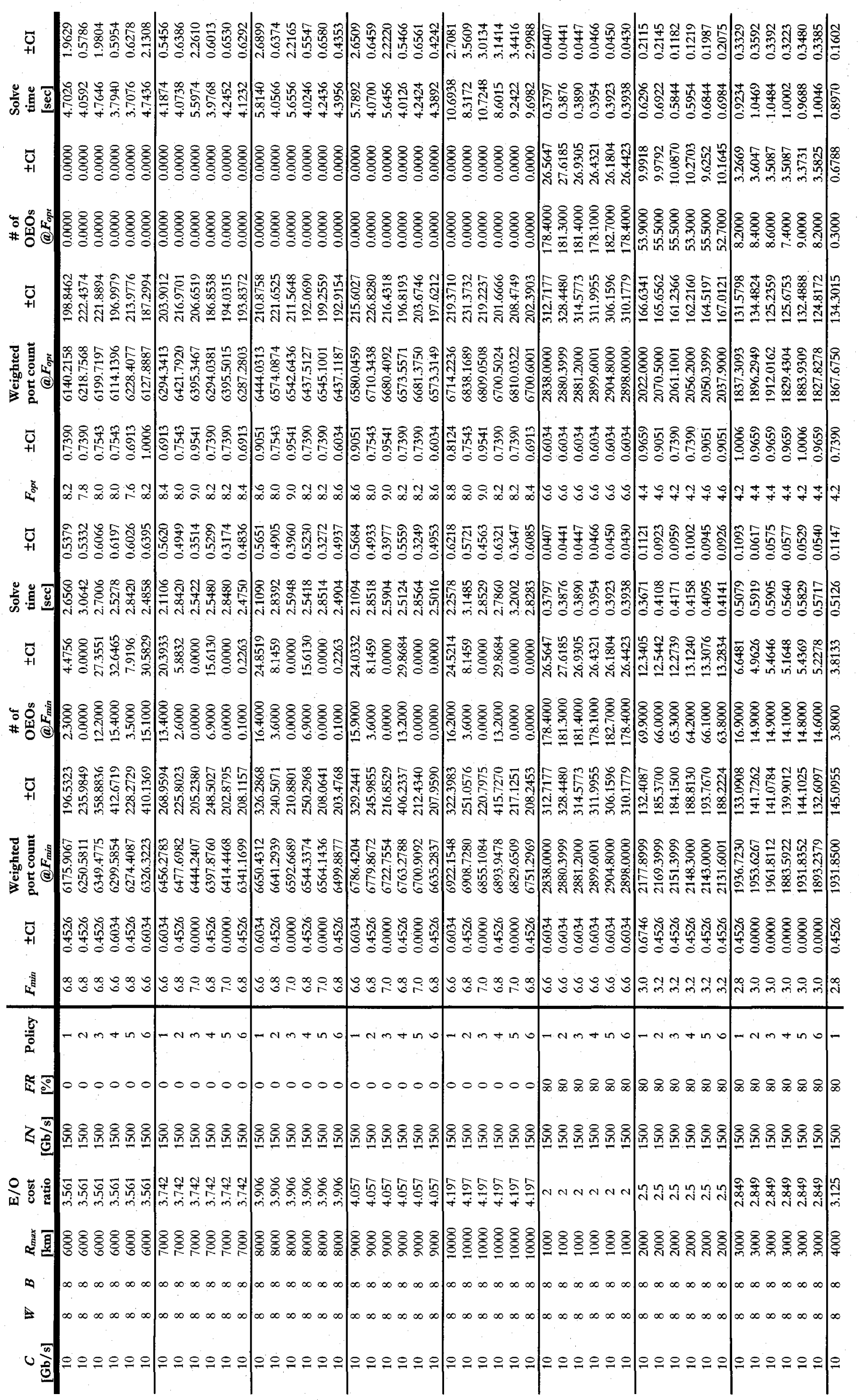




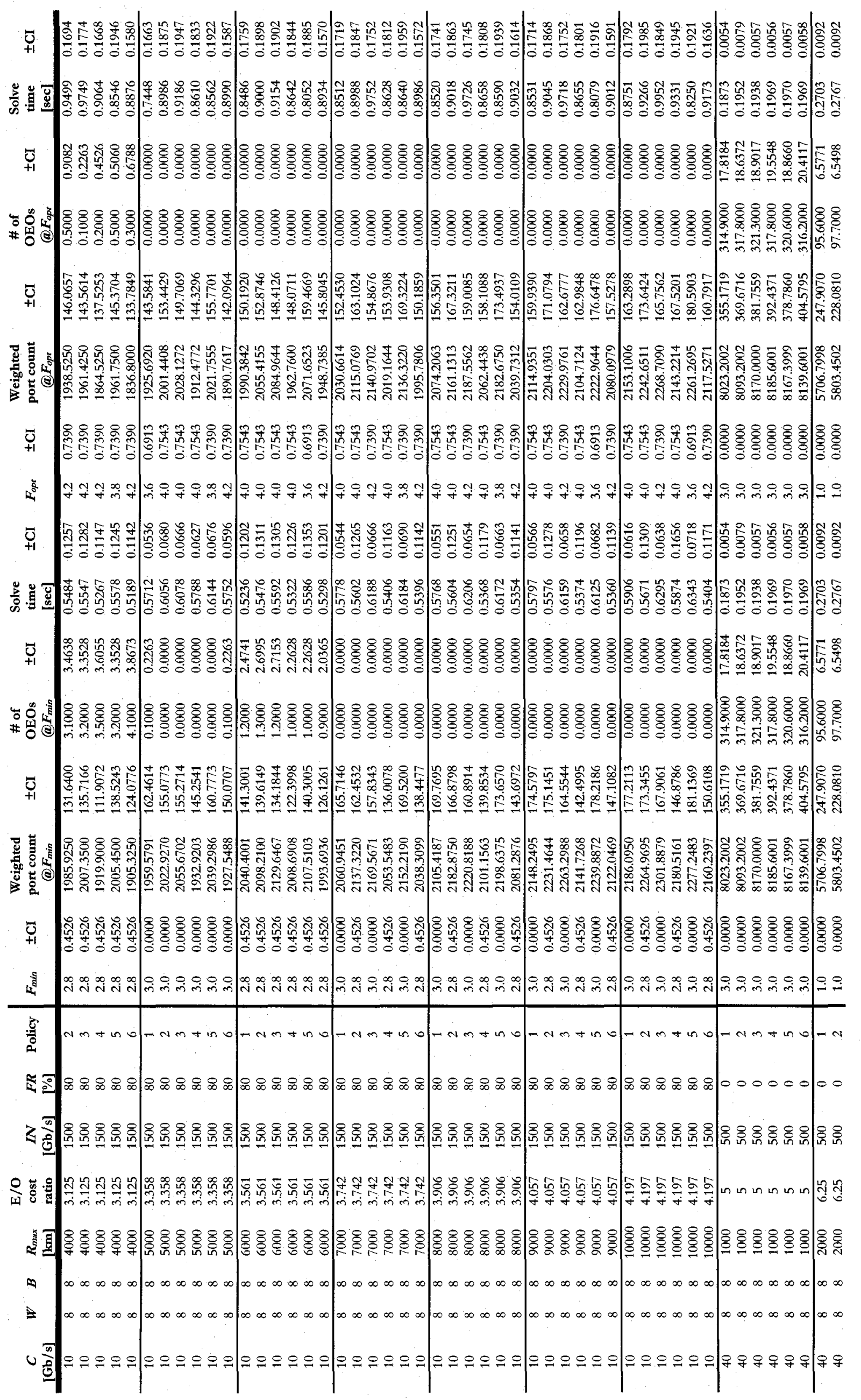




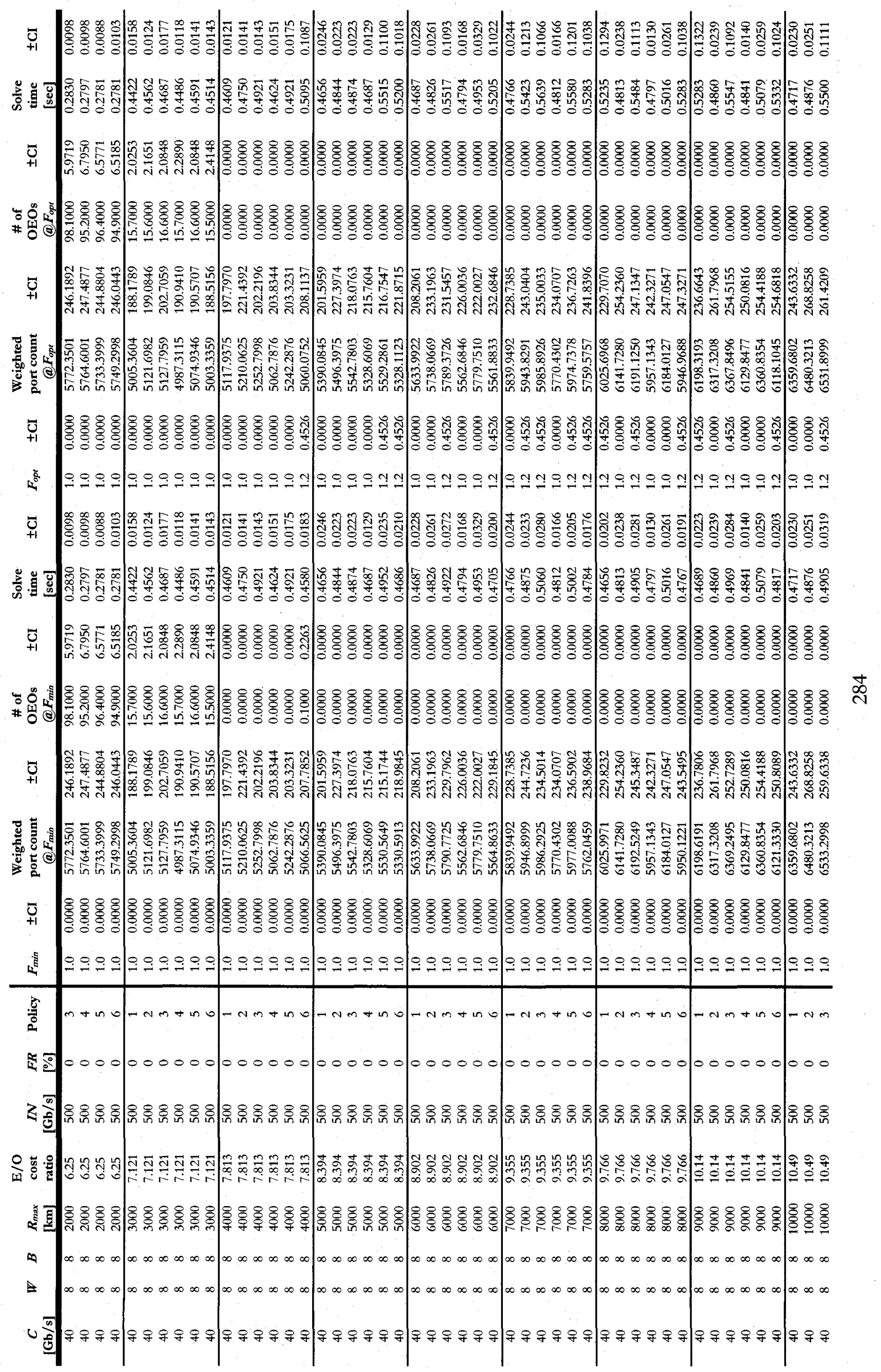




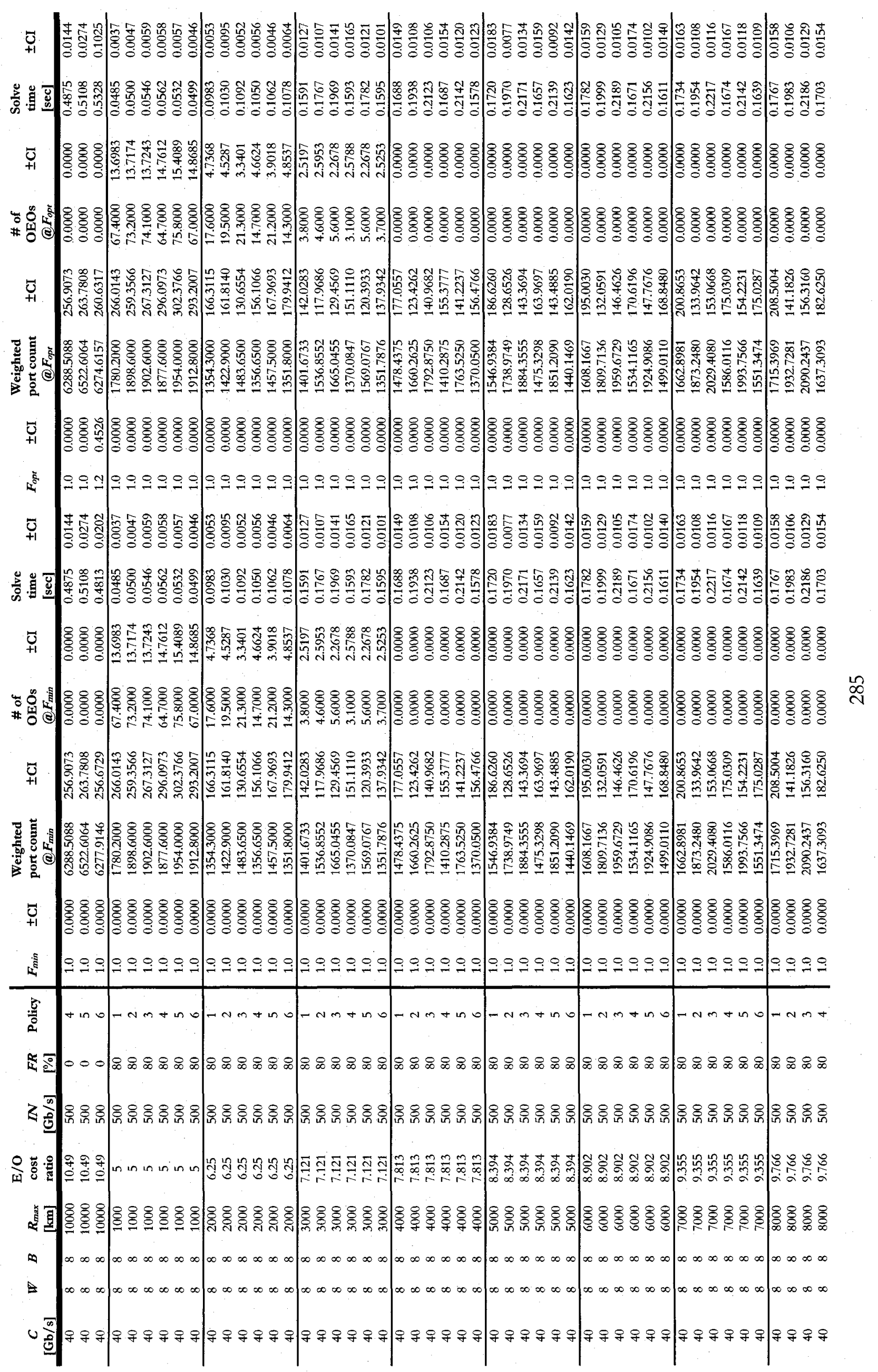




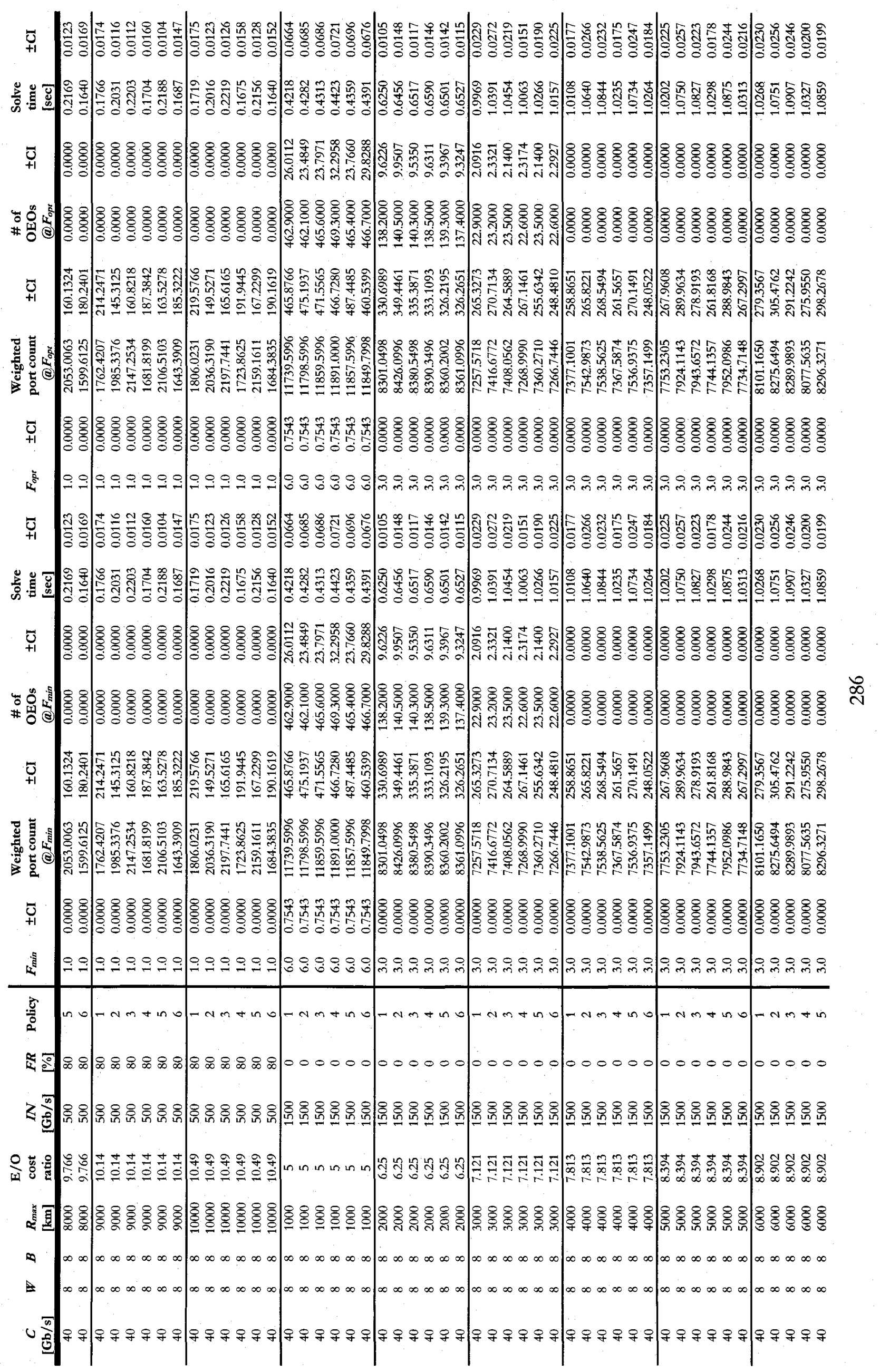




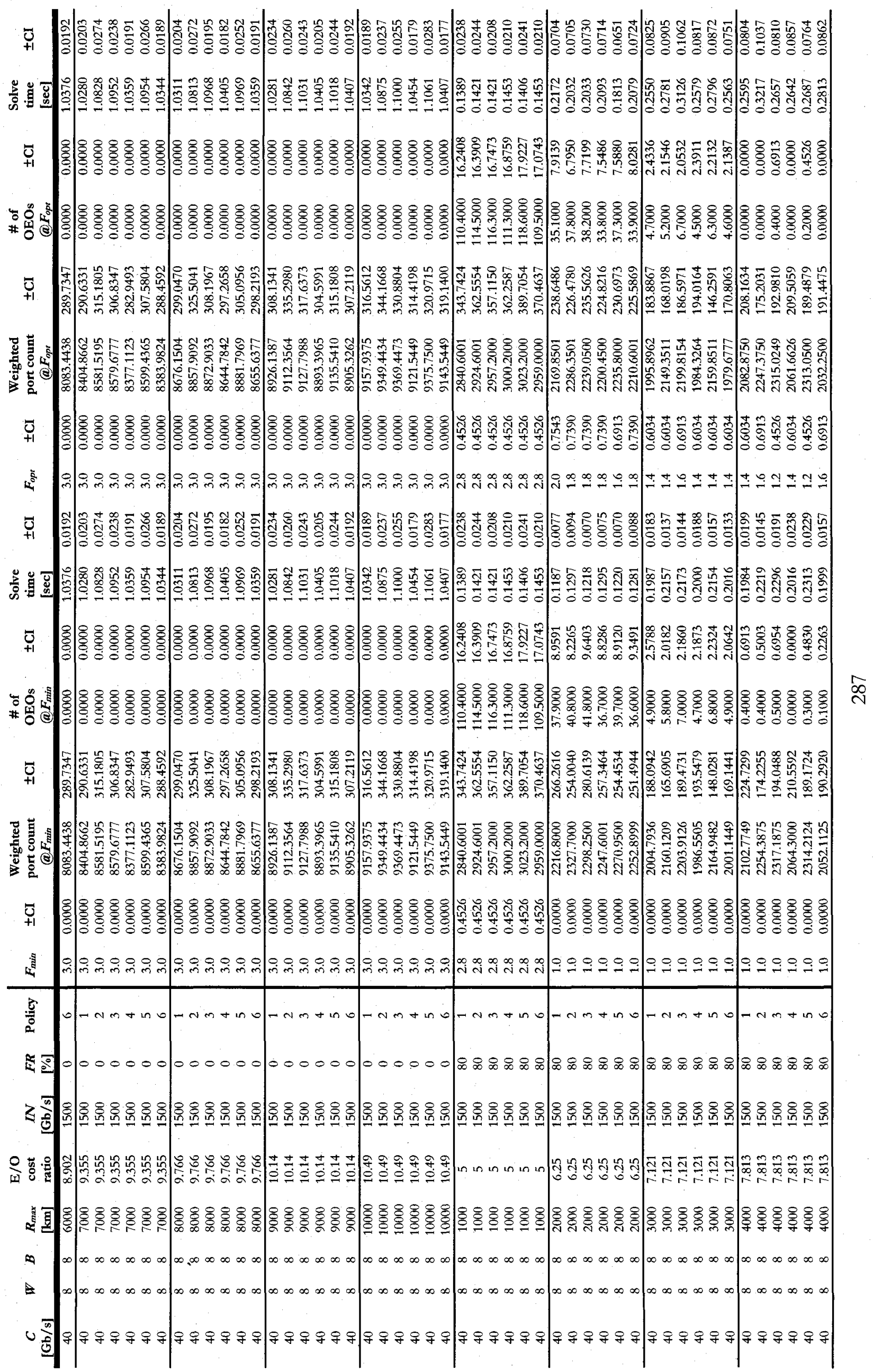




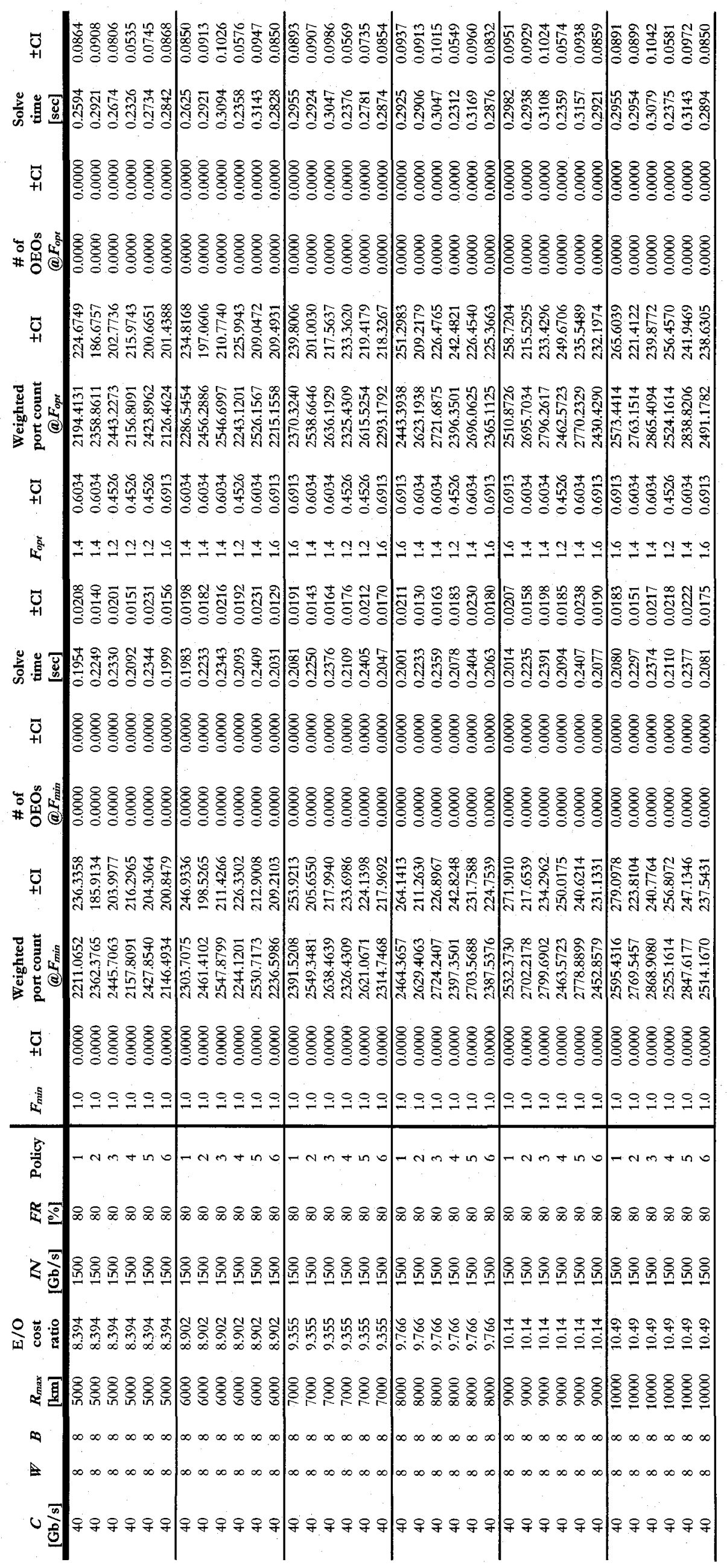




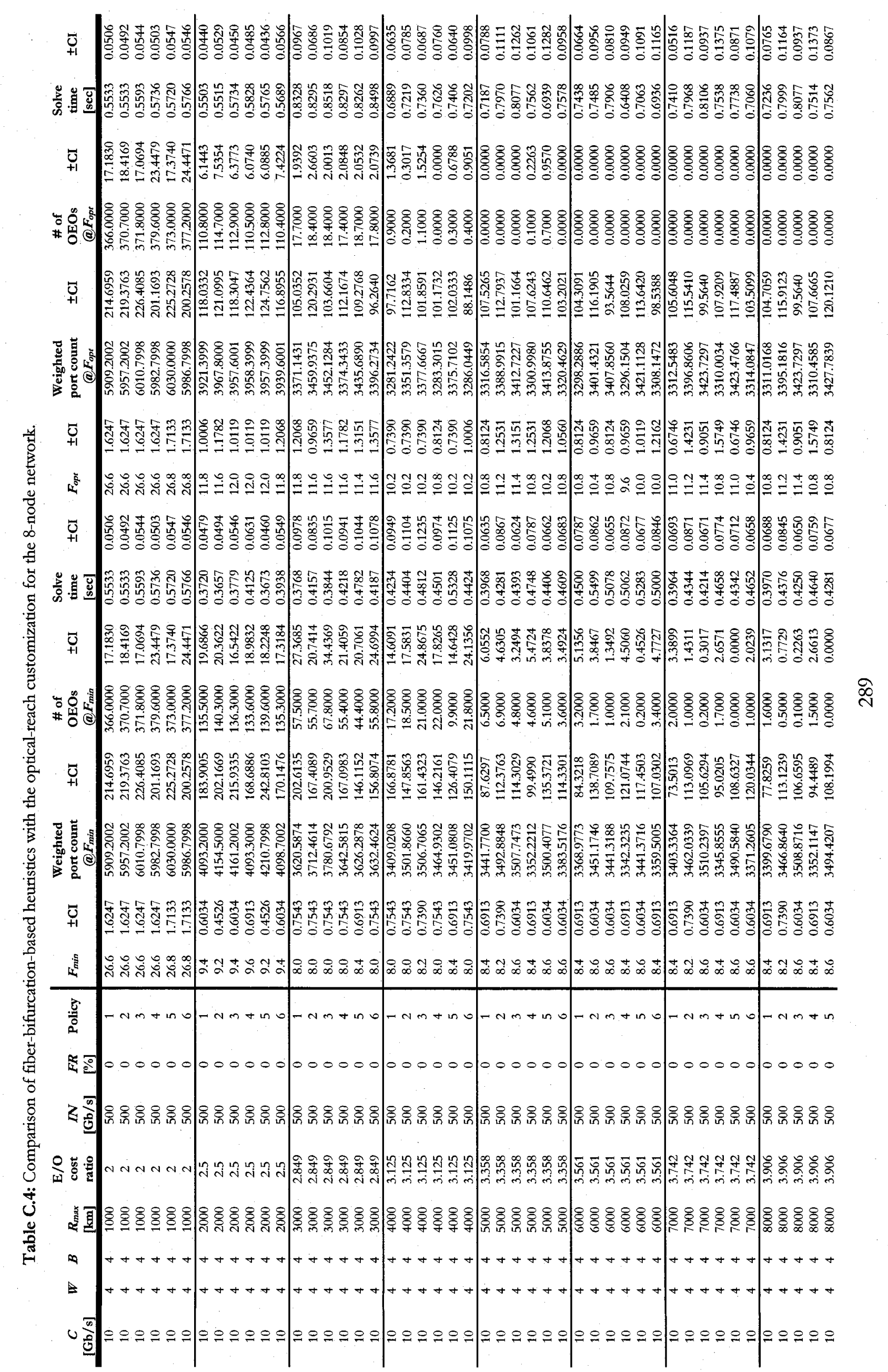




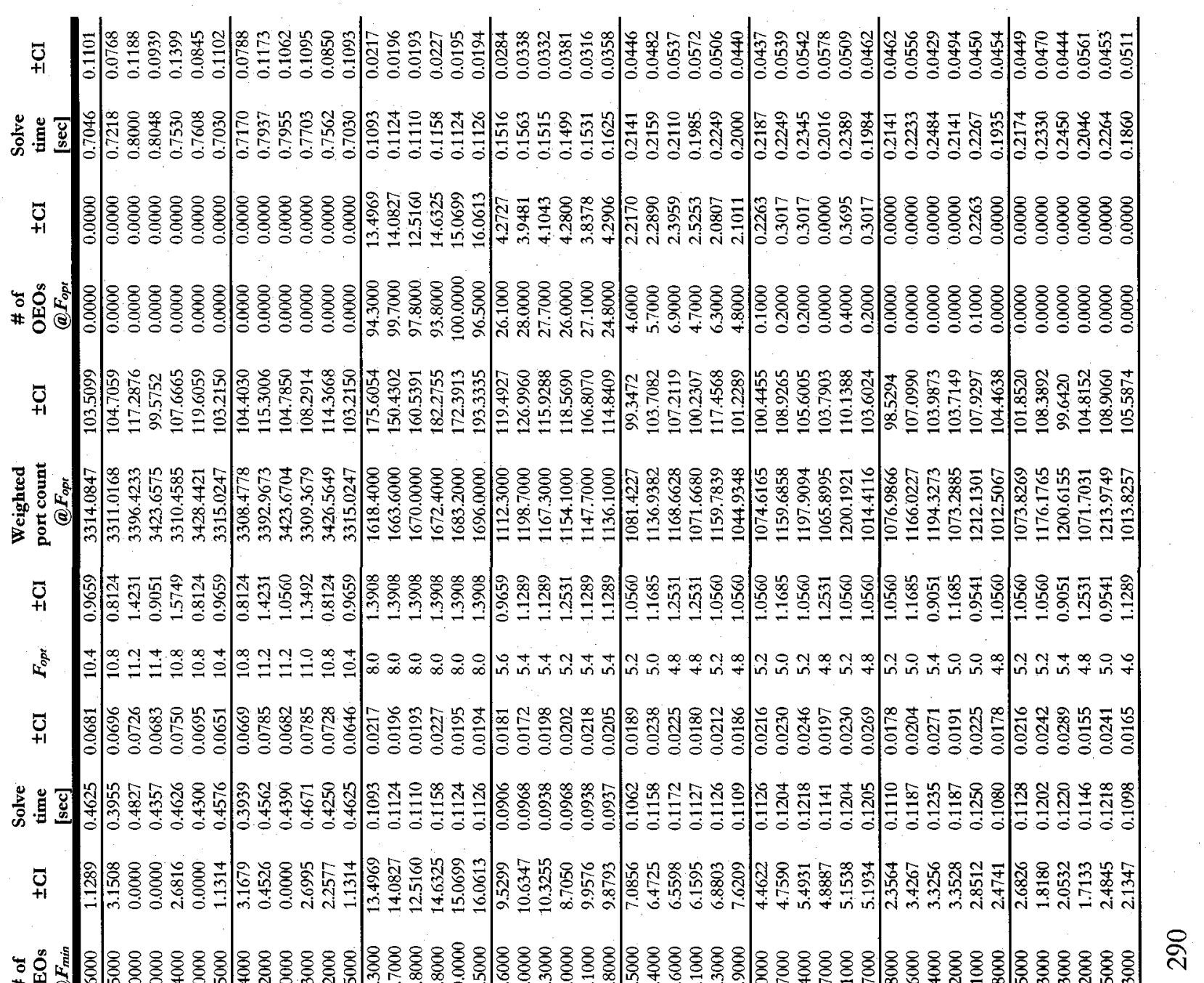

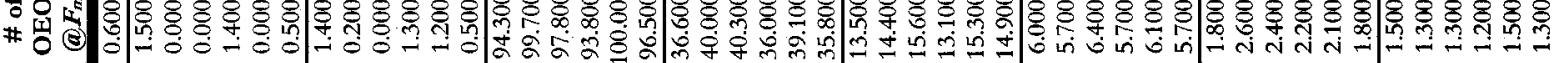

-

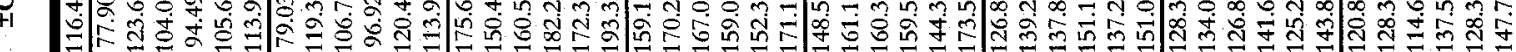

屯

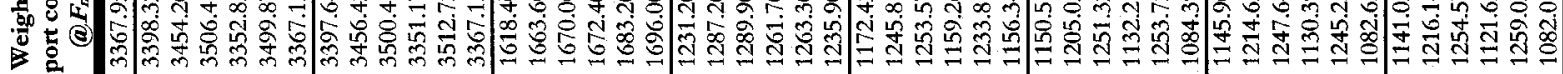

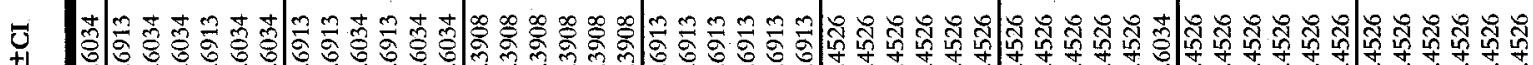
+1 (1) Lل直

:

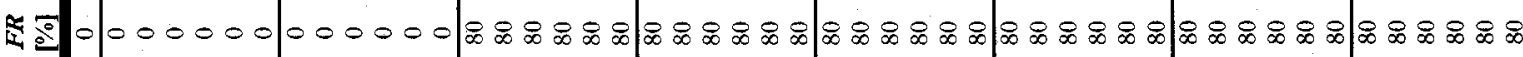

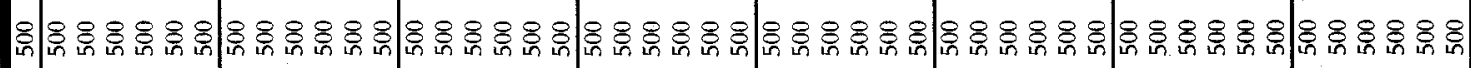

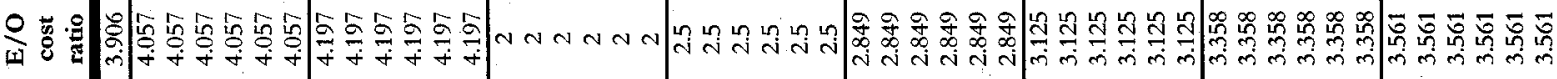

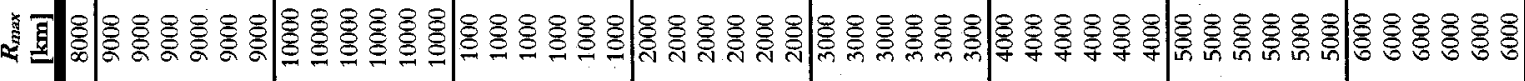
$\infty$

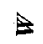

$u$

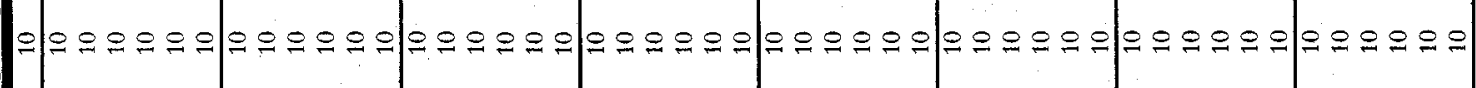




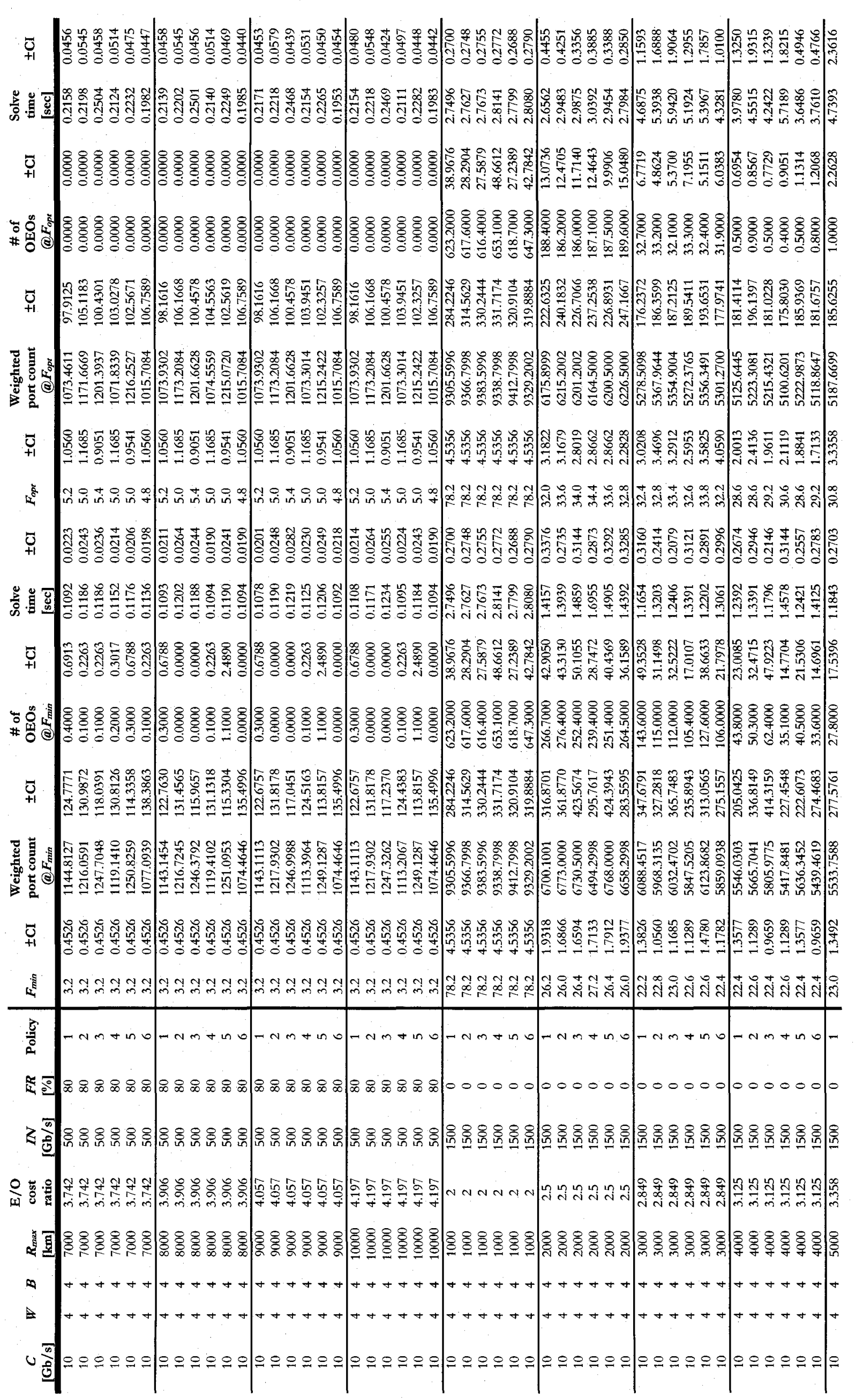




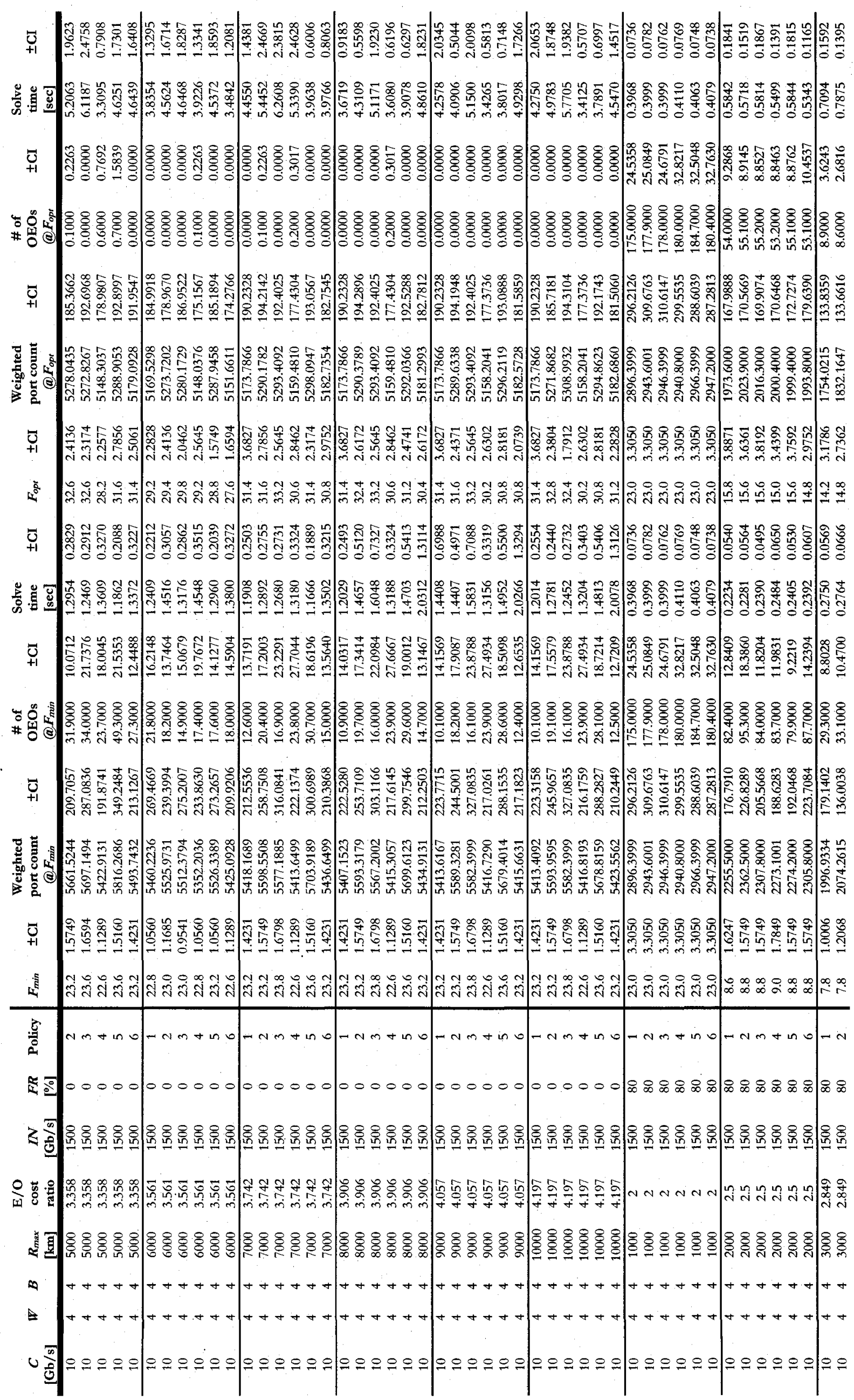




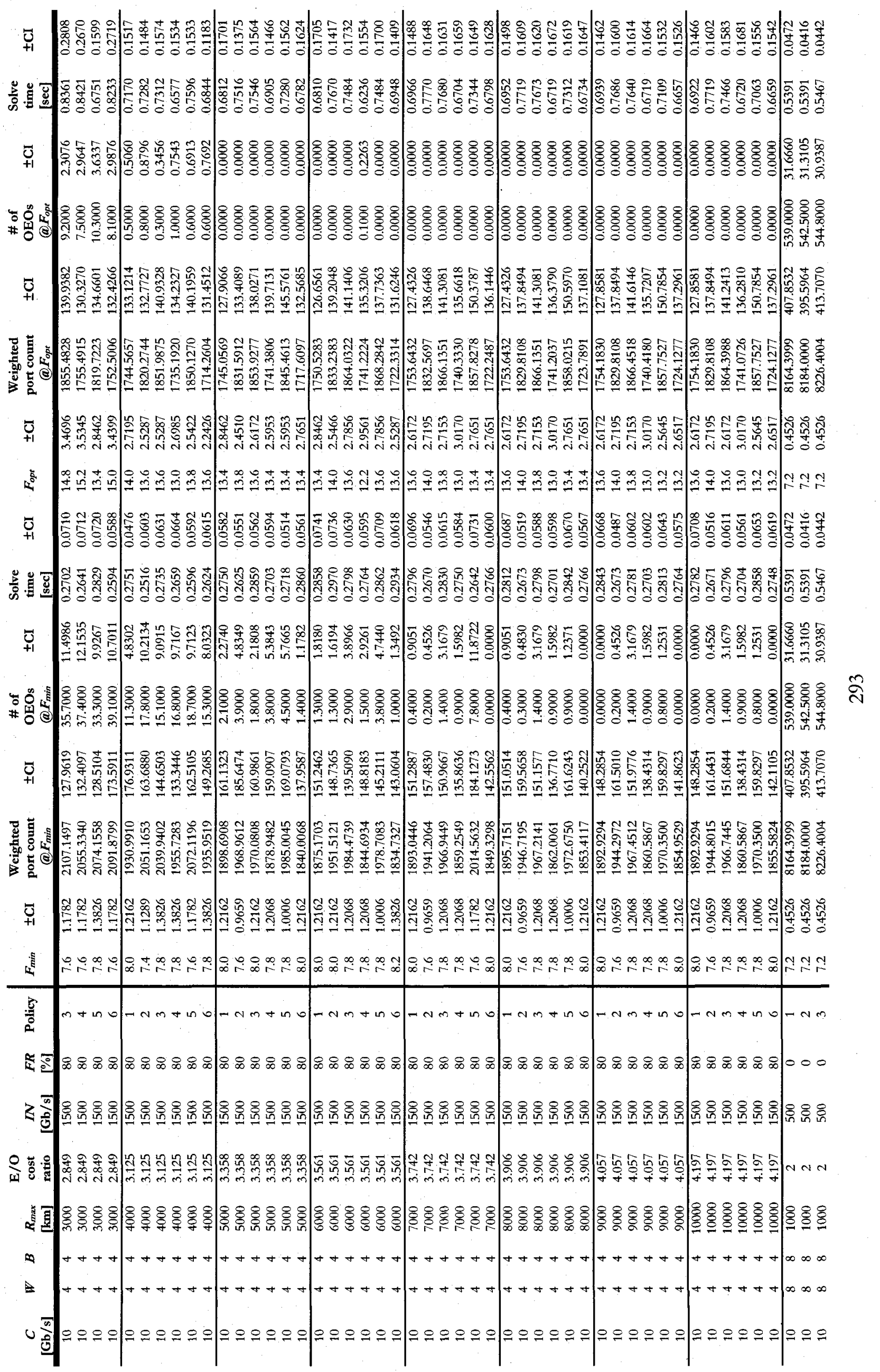




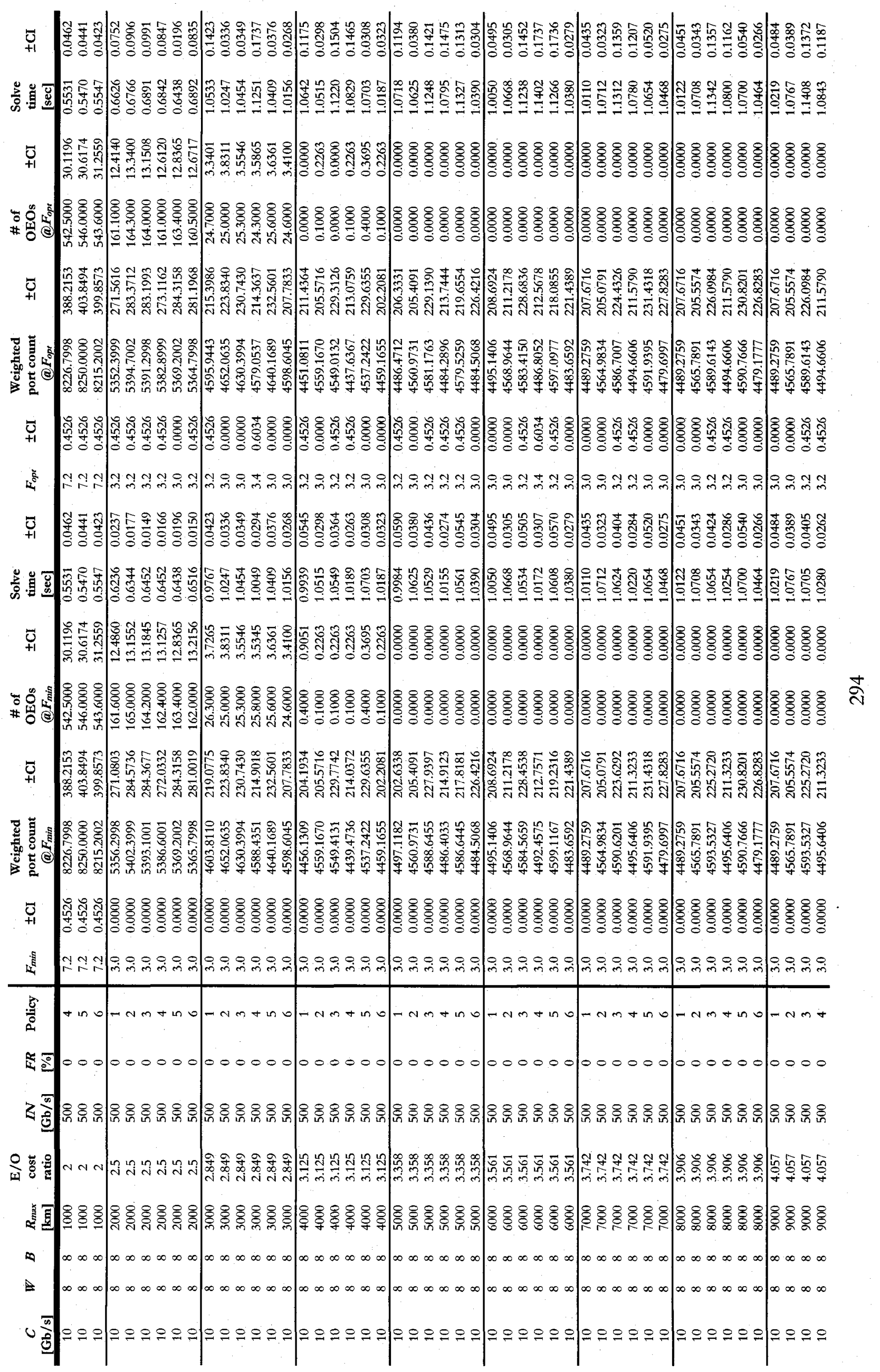




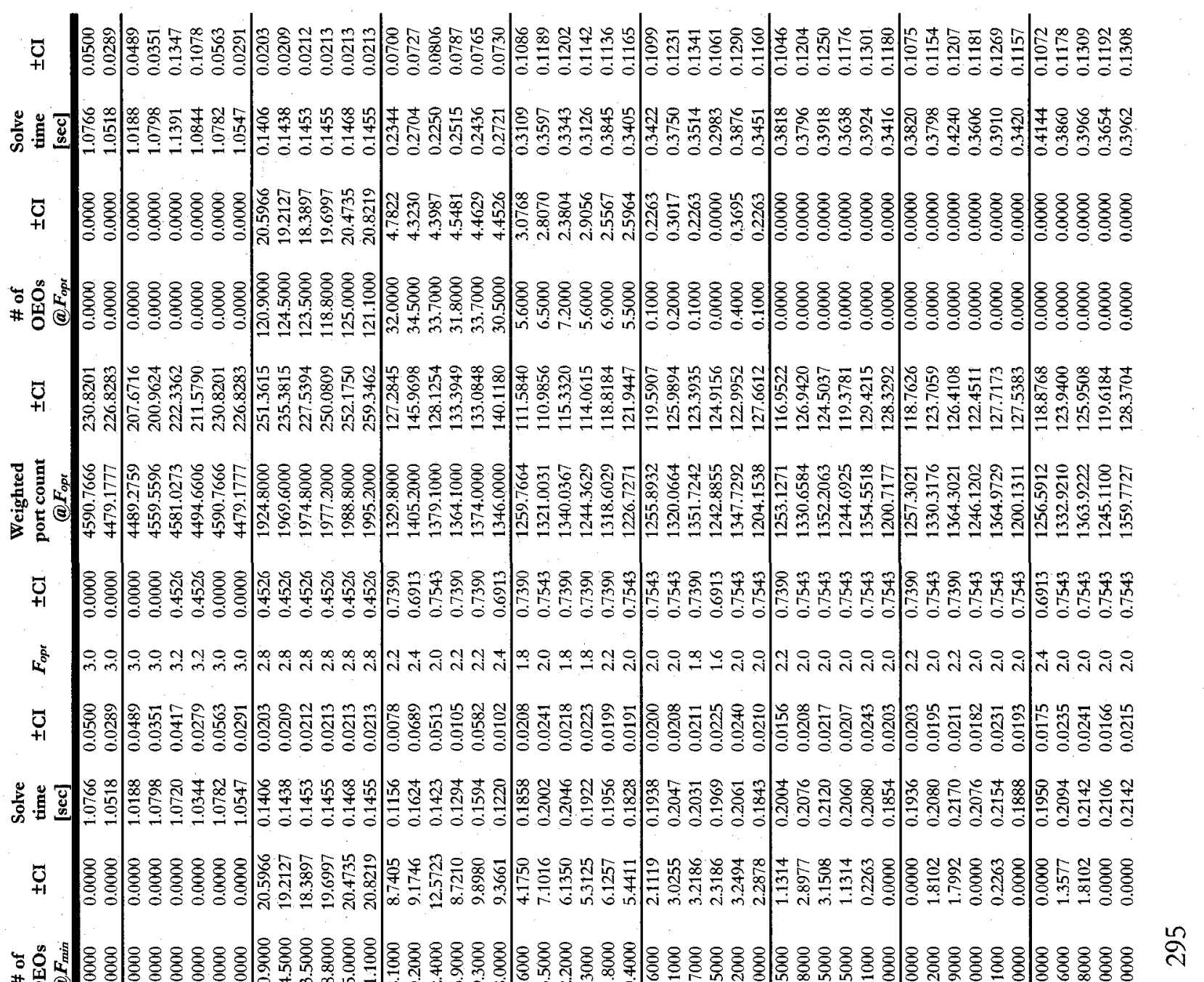

\# 奥

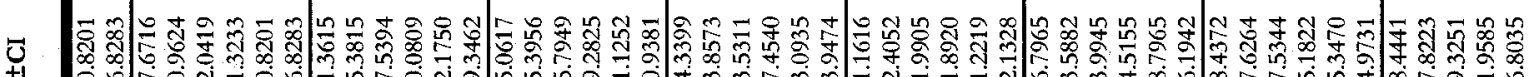

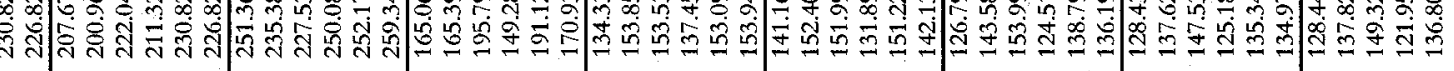

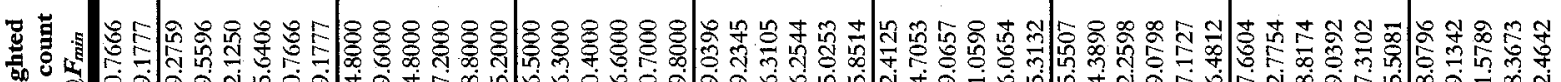

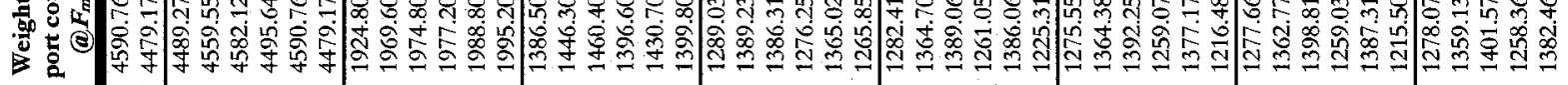

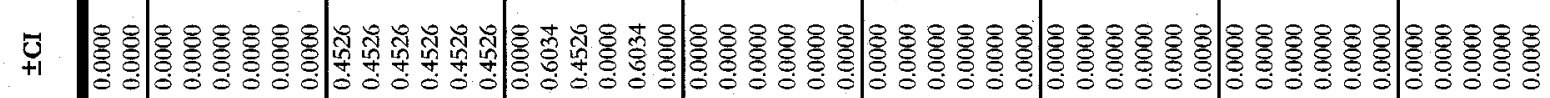

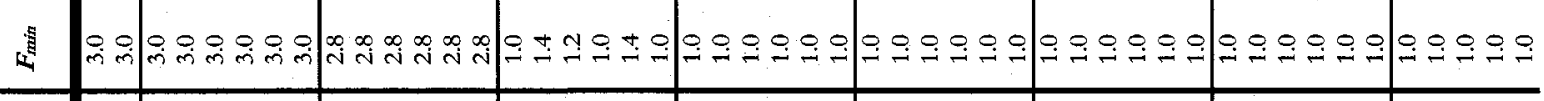

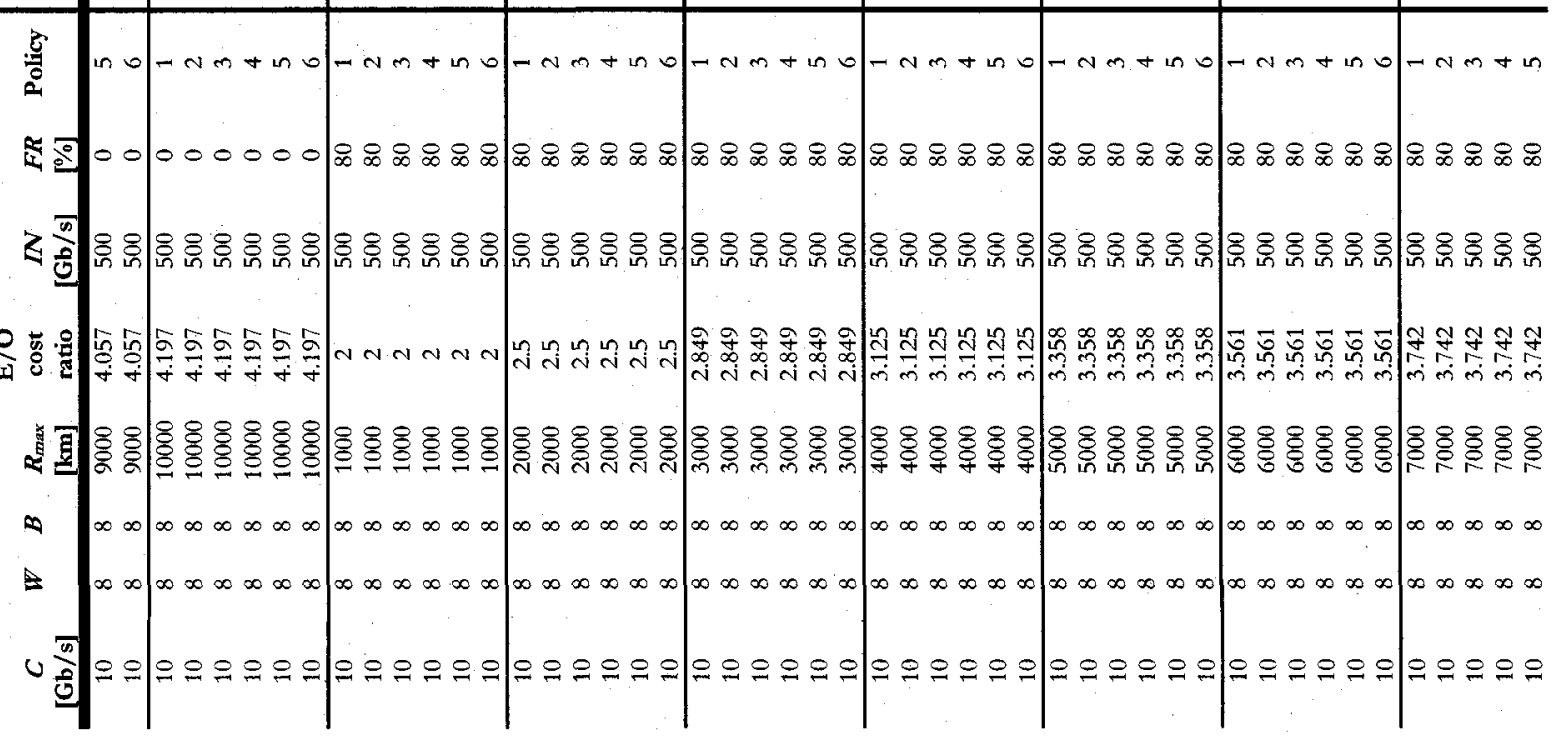




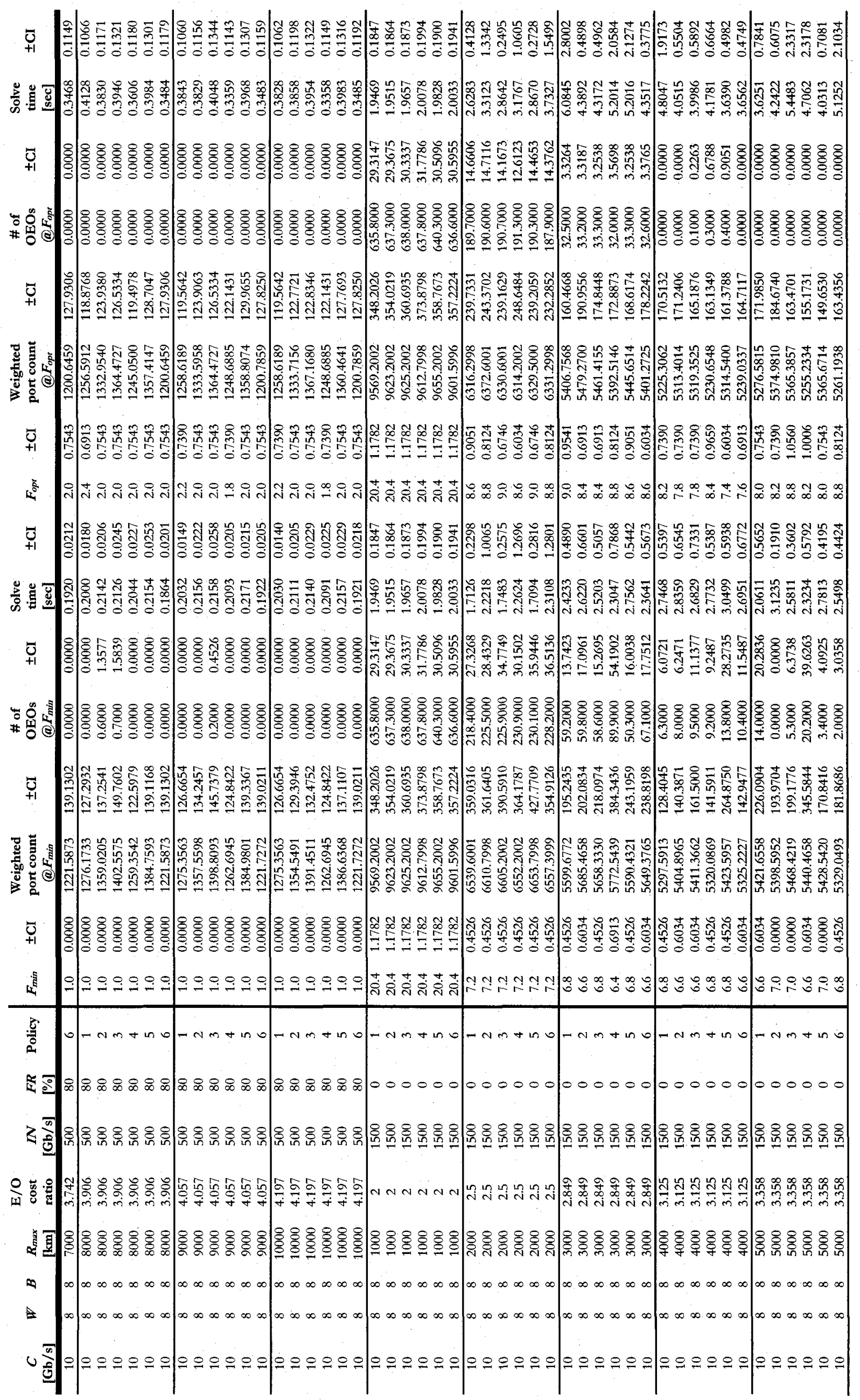




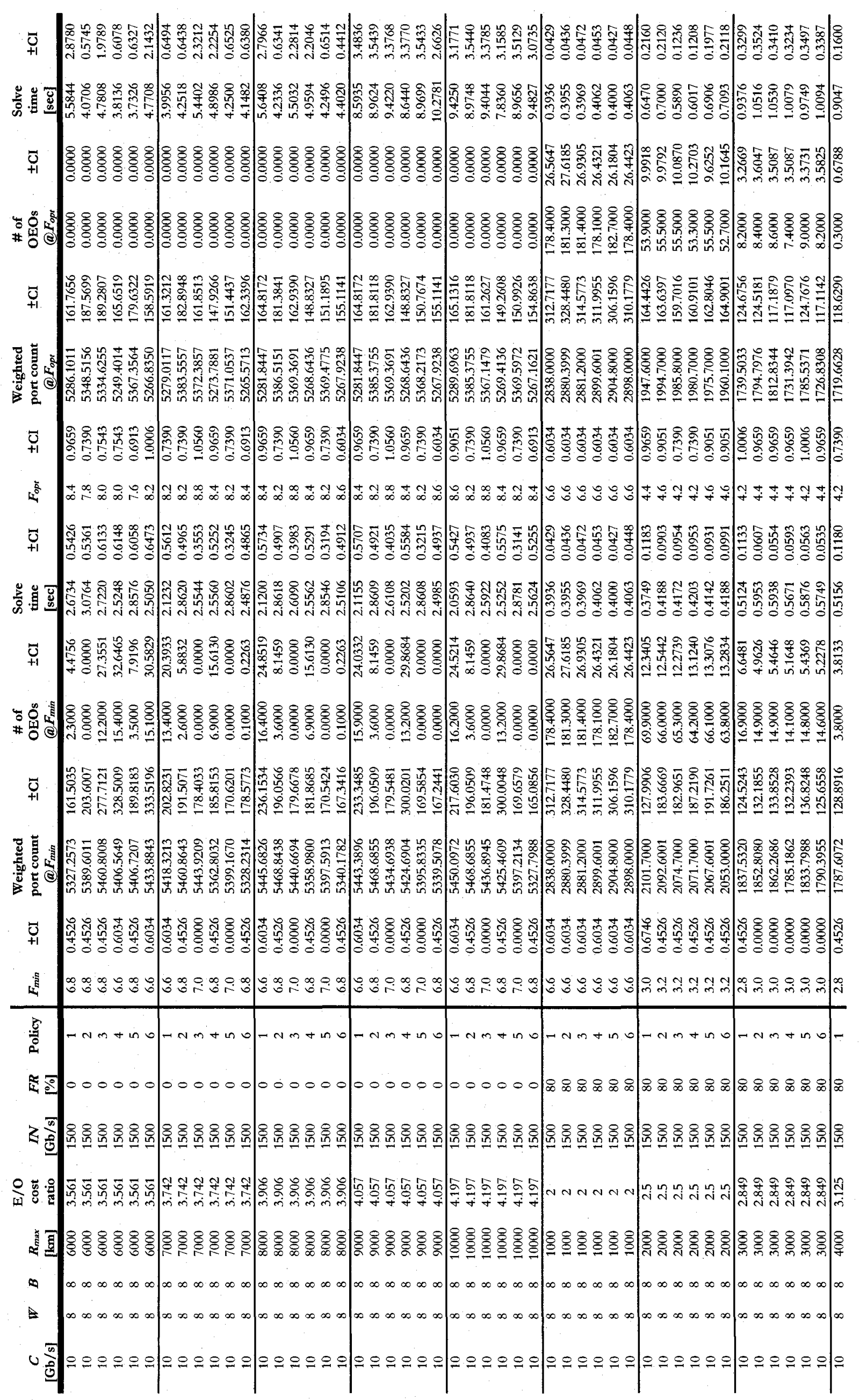

ฐิ 


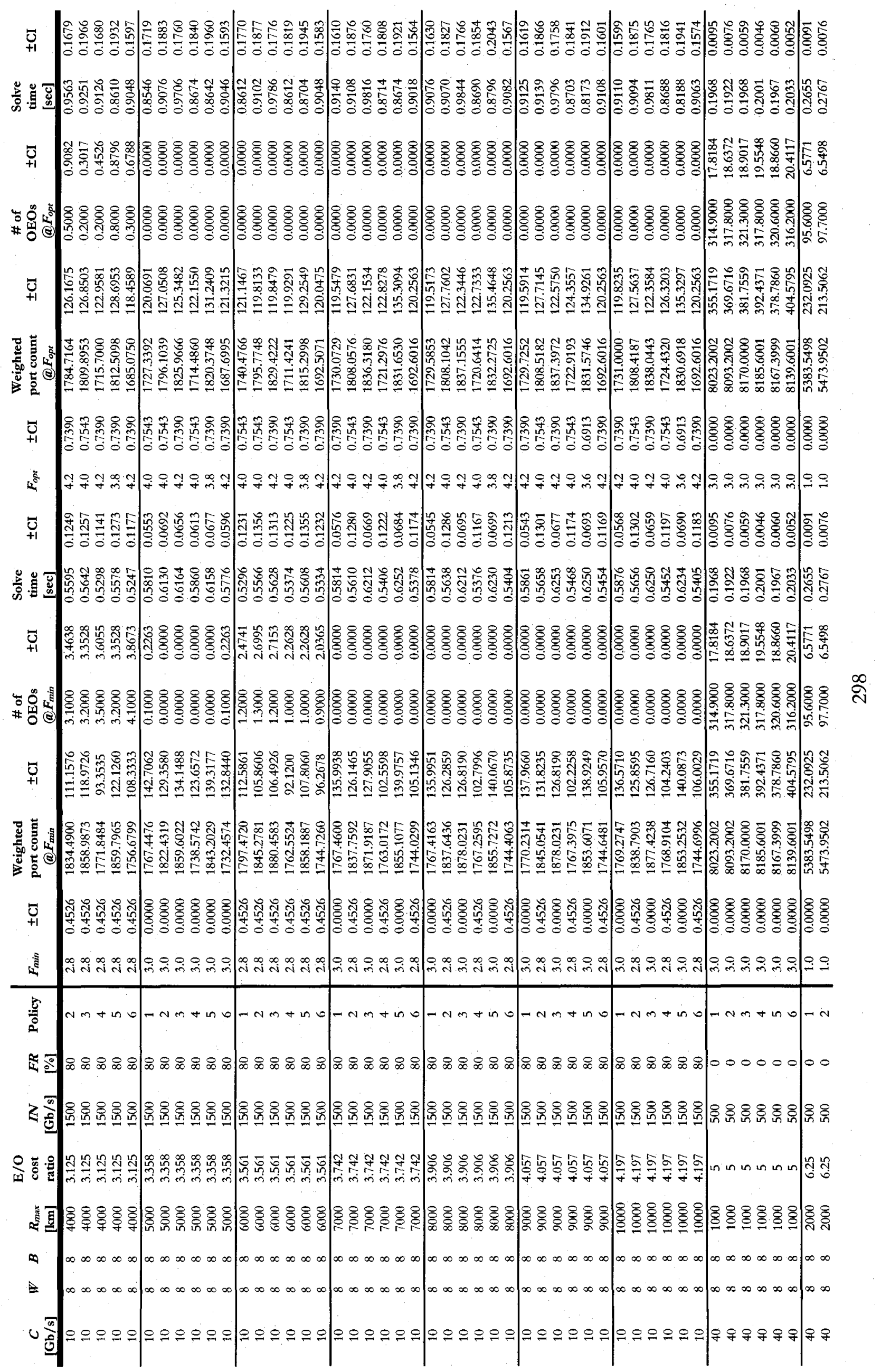




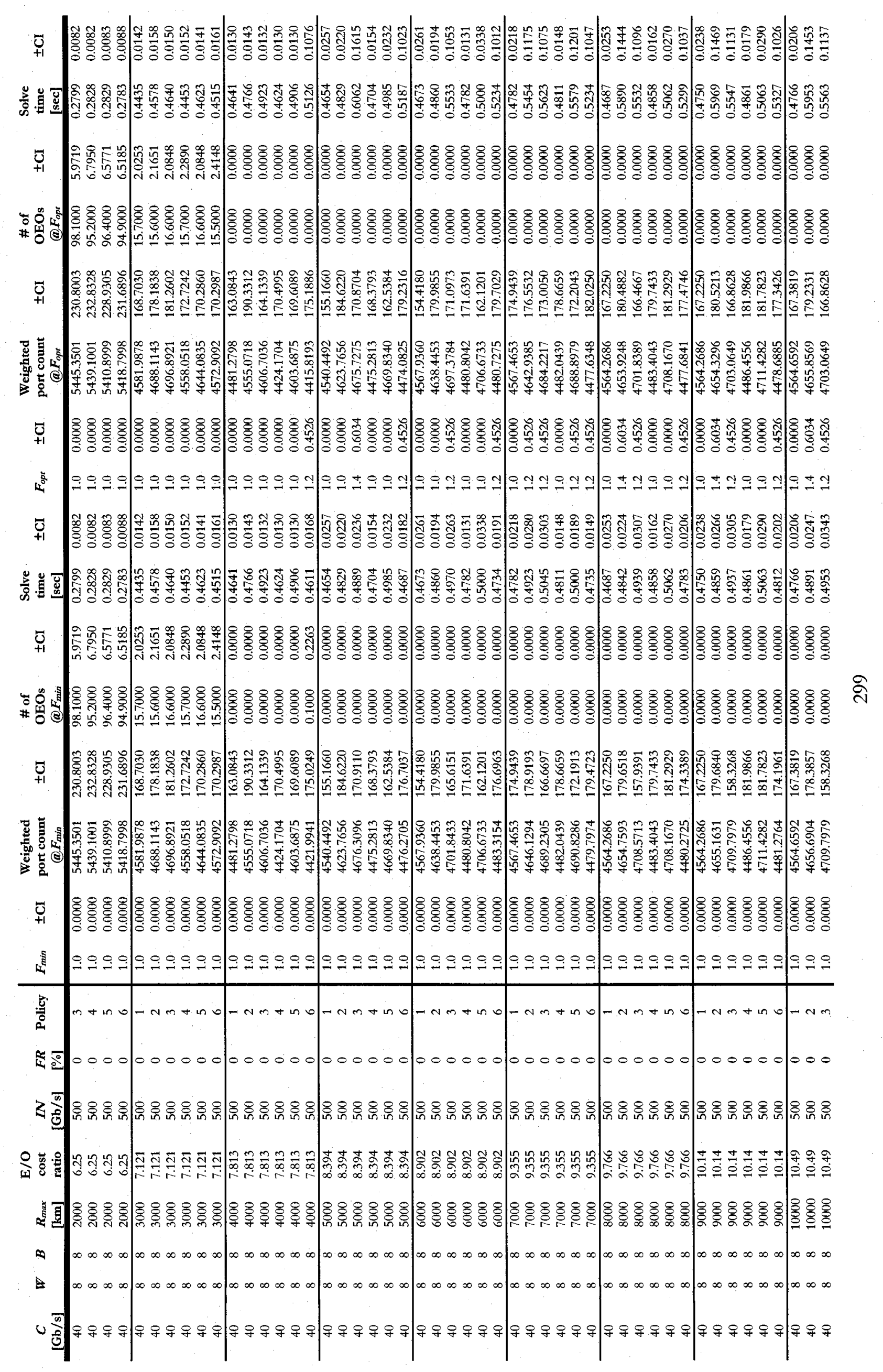




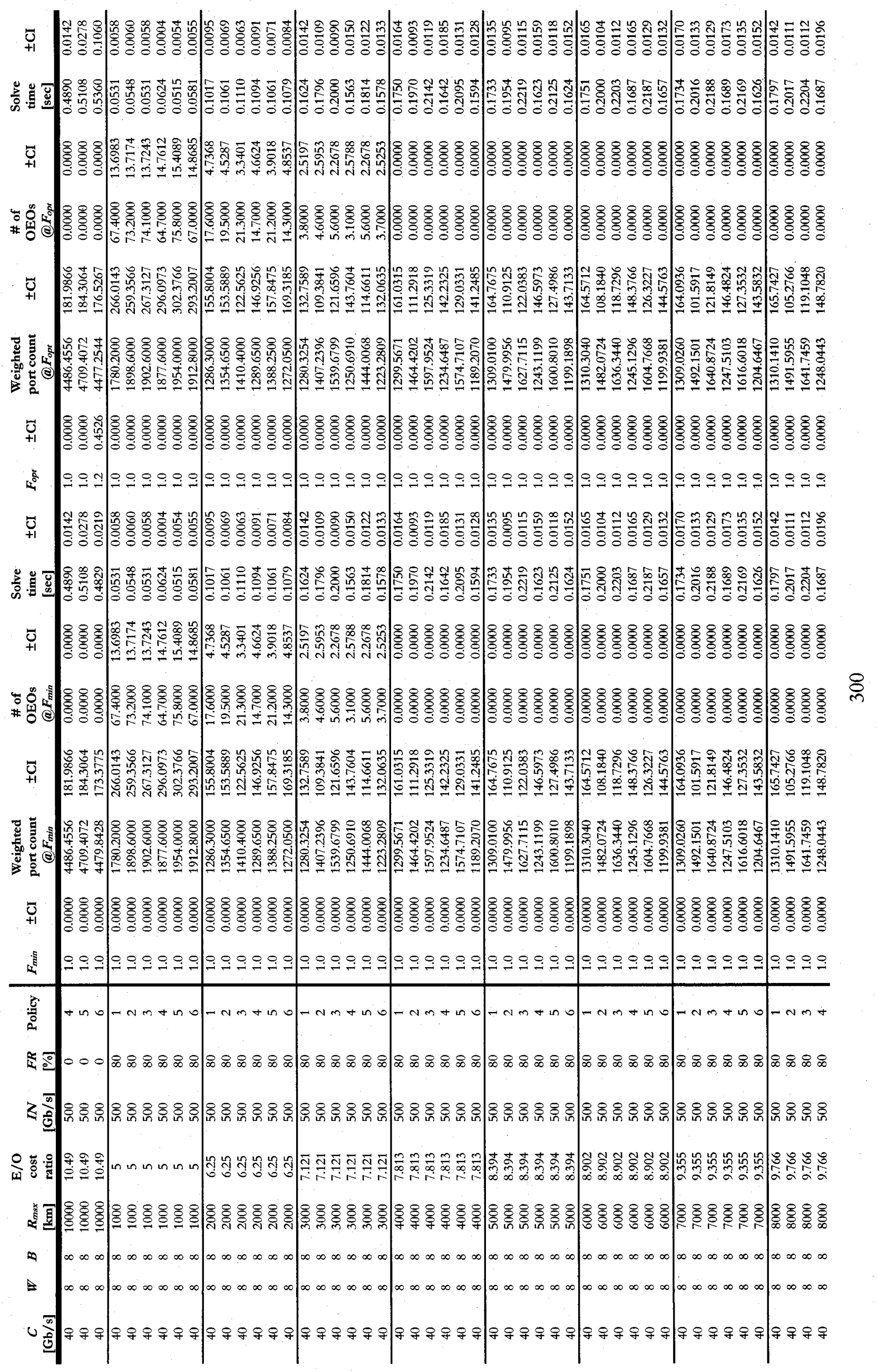




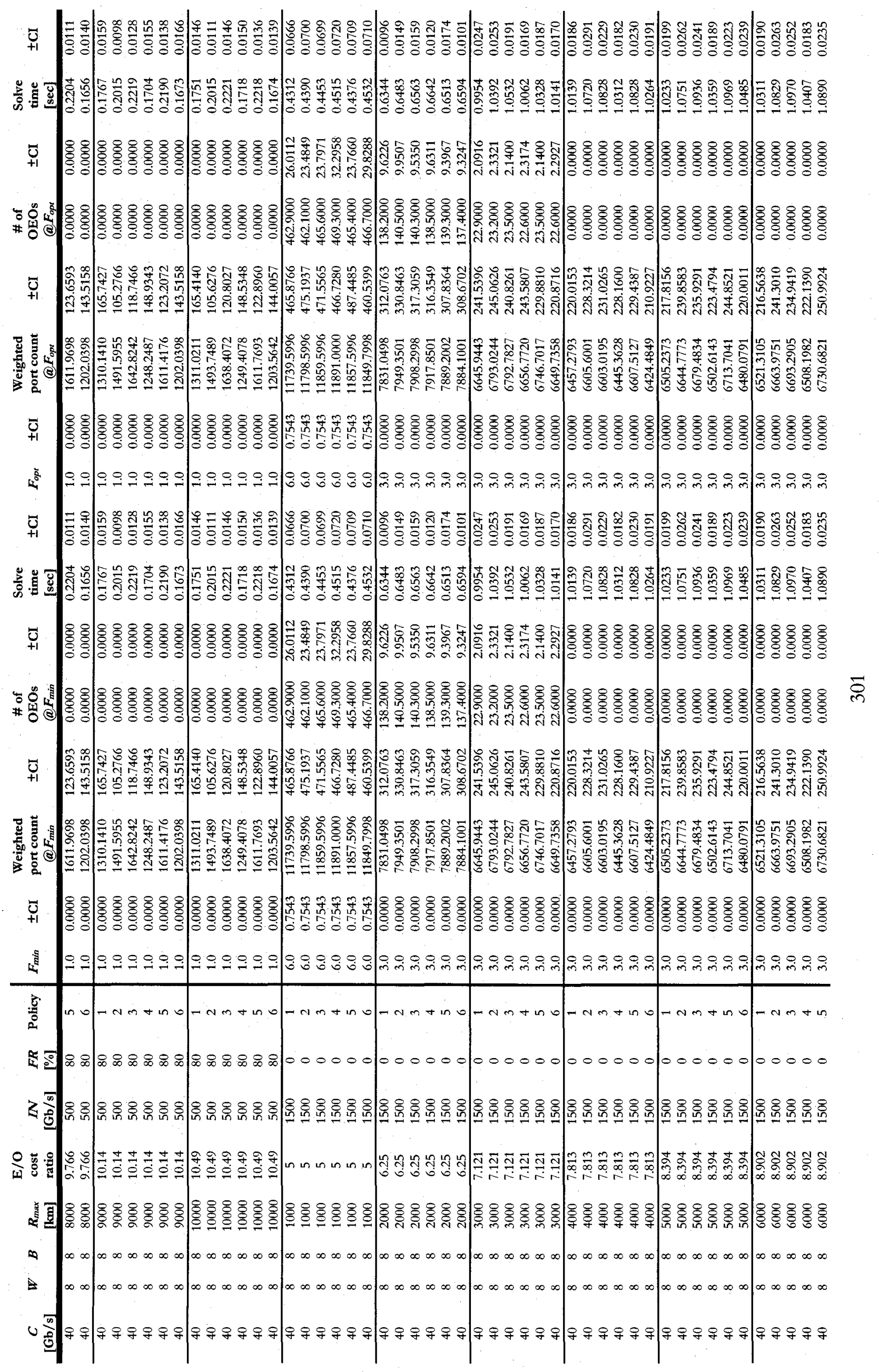




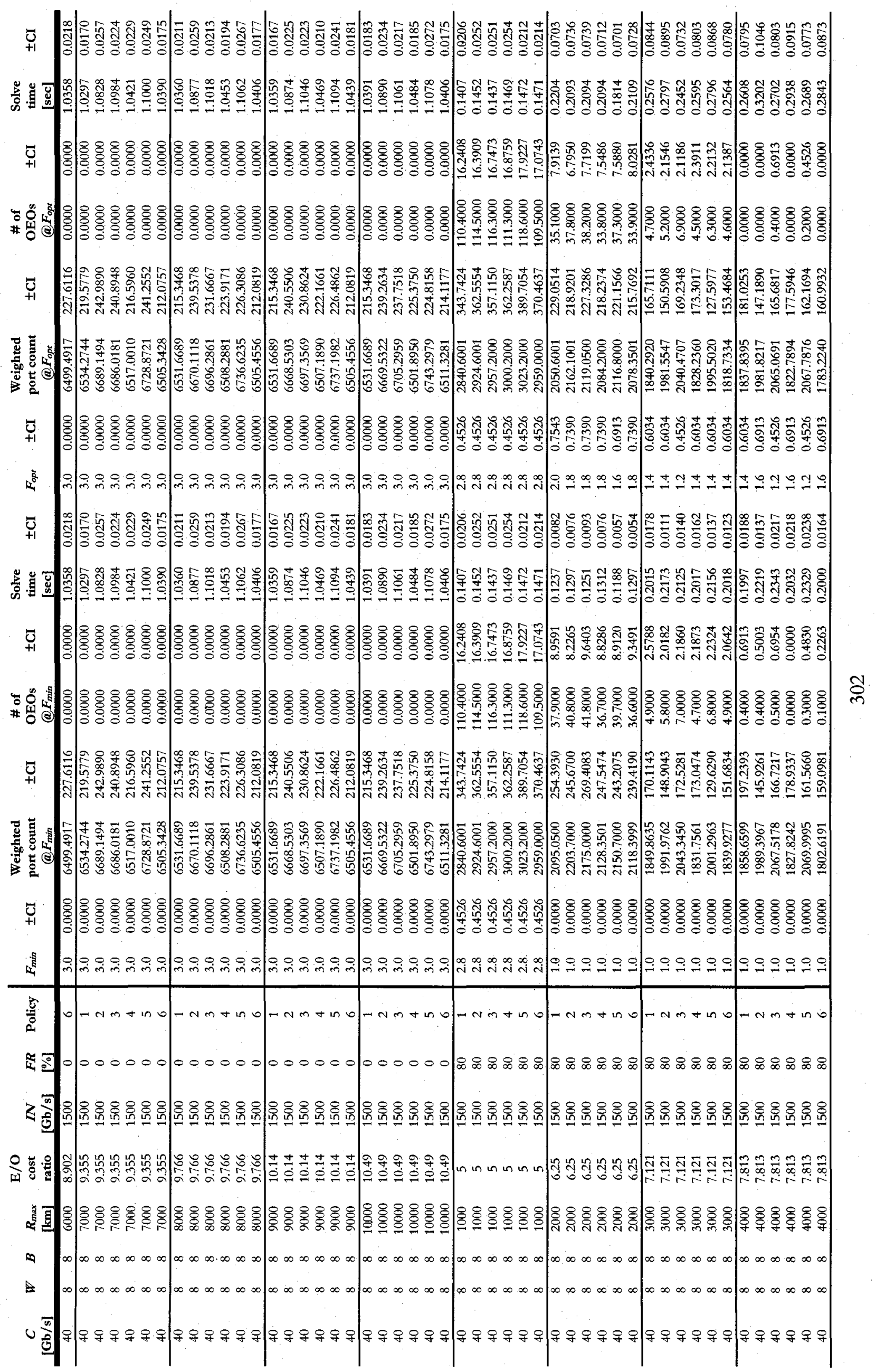




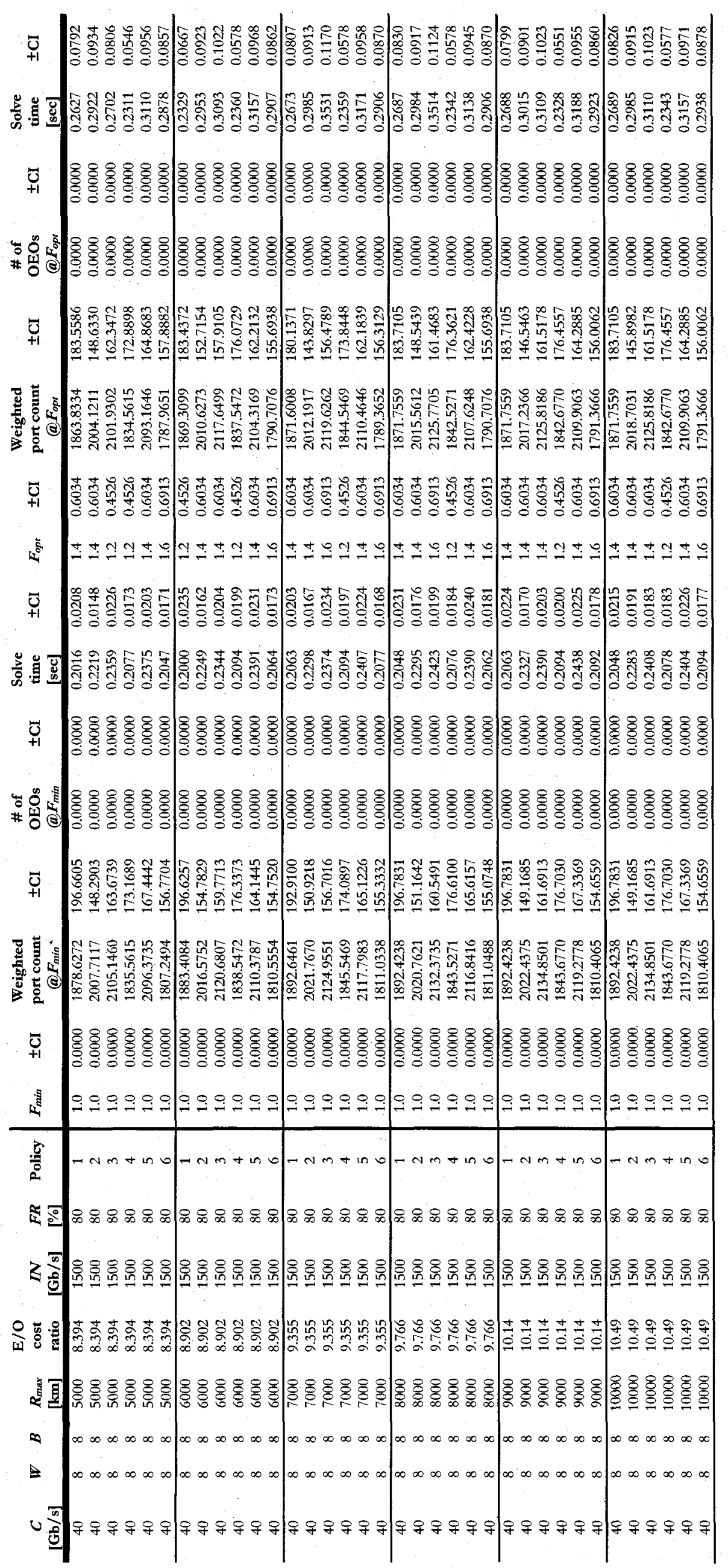


|

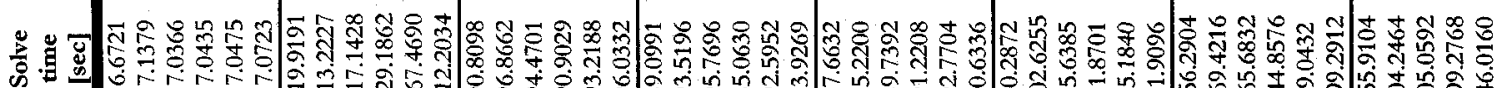

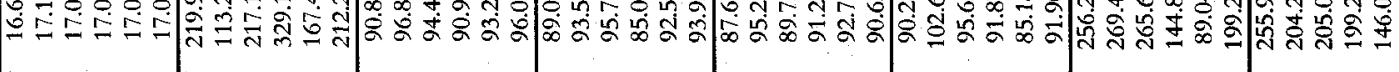

t

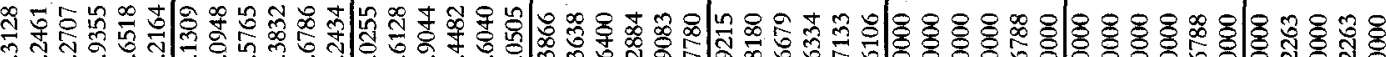
仙热

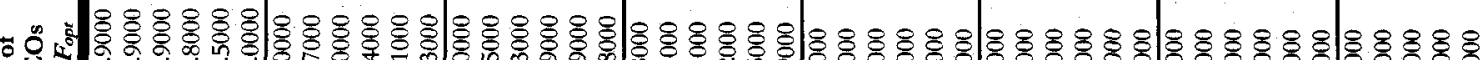

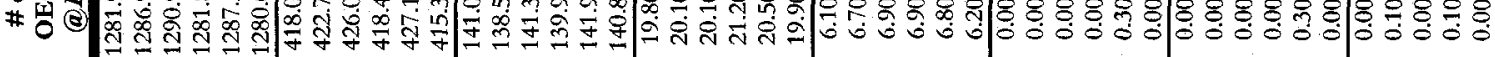

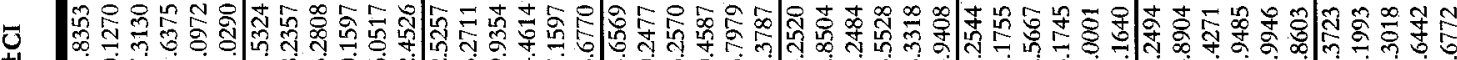

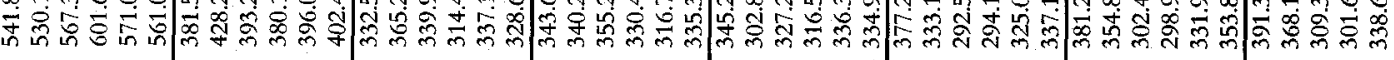

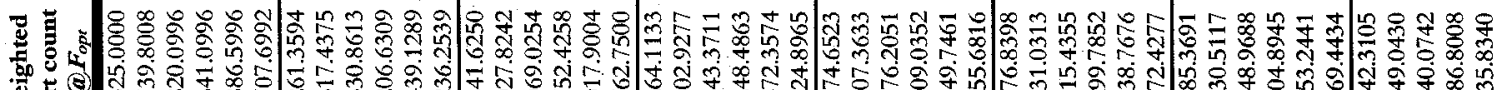

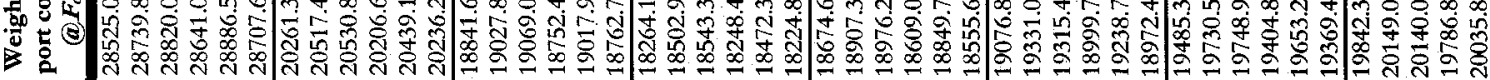

-

s.

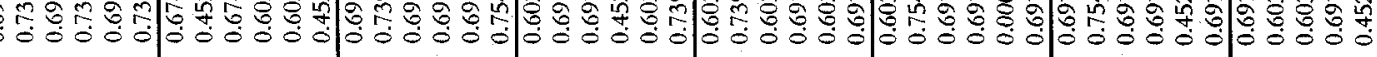

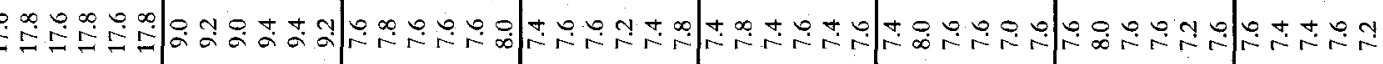

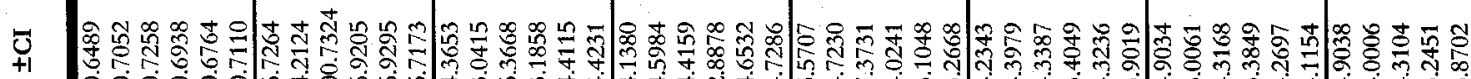

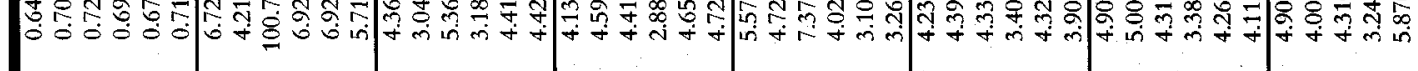

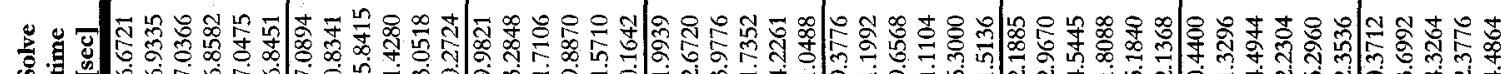
की

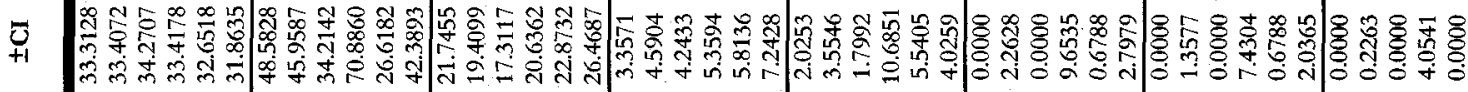

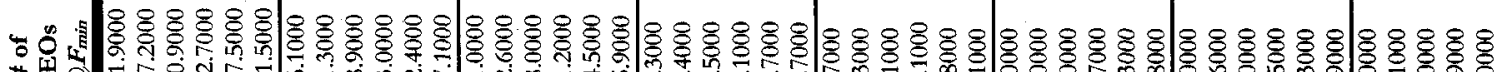

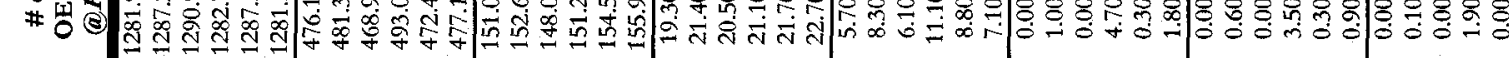

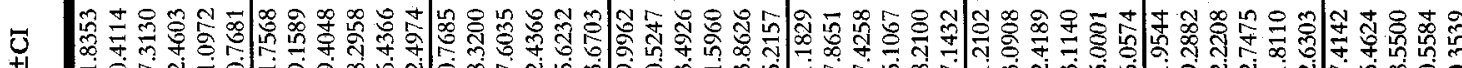

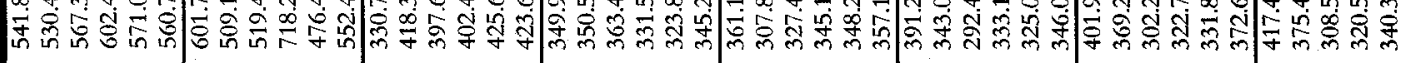

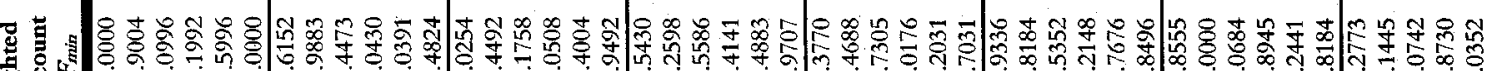

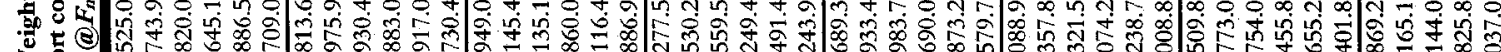

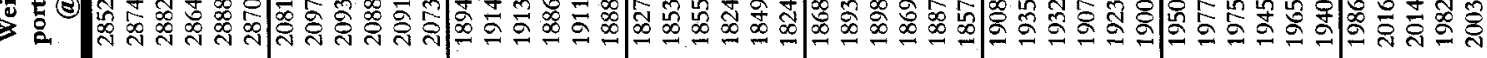

†

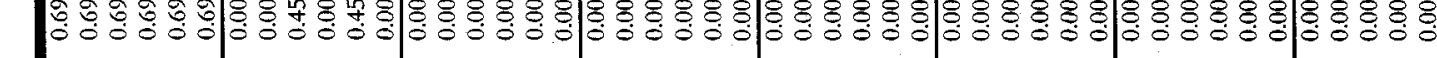

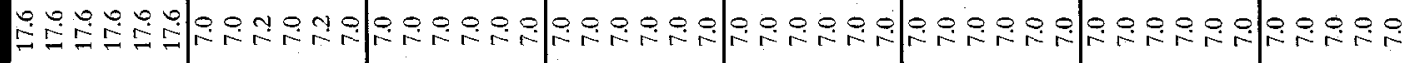

a

(2)

है

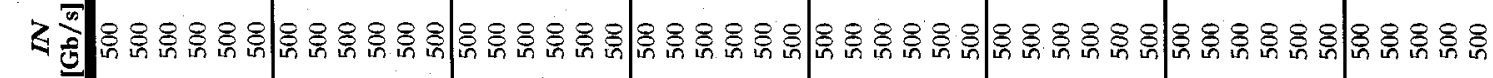

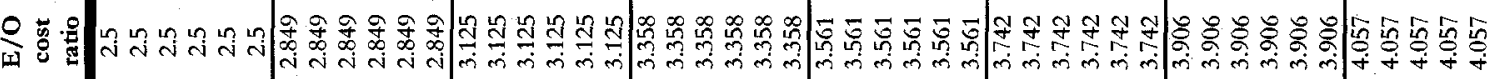

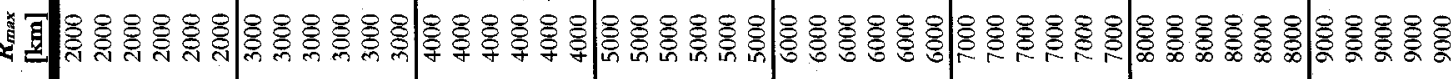

$\infty$

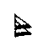

U 离 


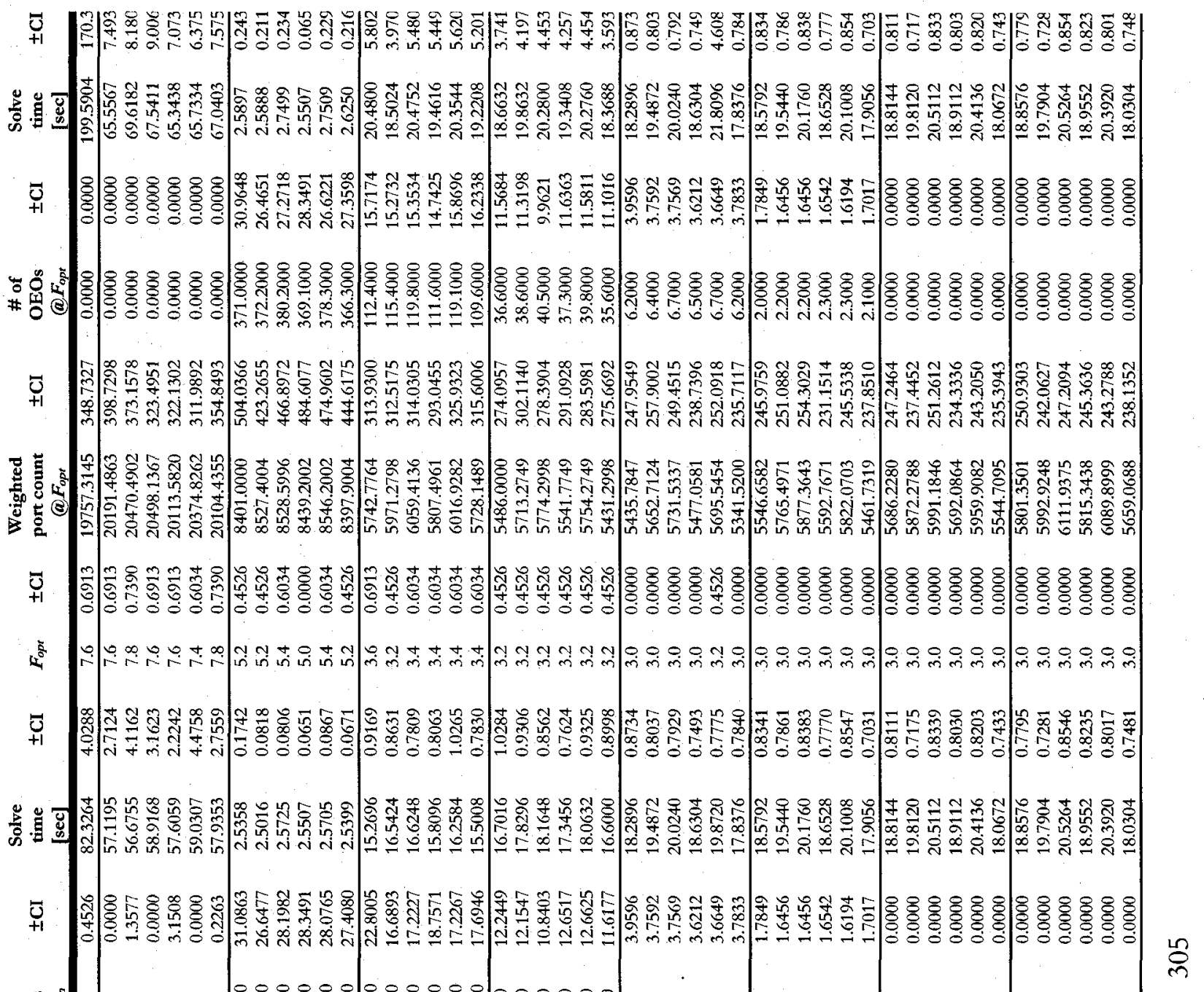

世各

广

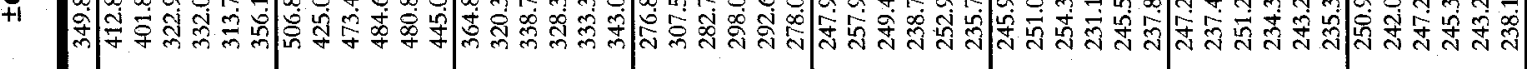

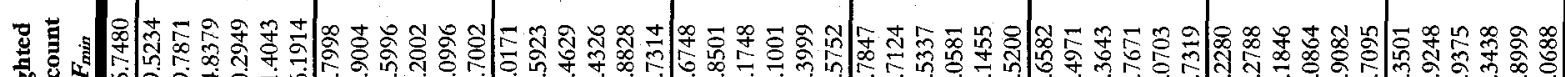

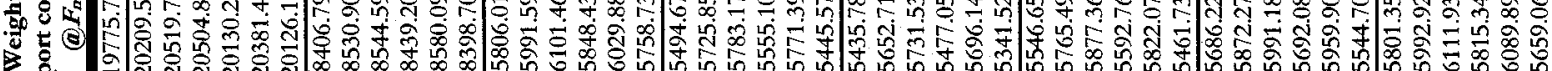

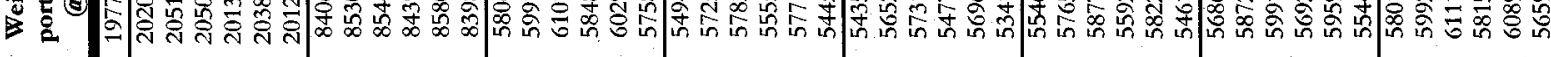
†

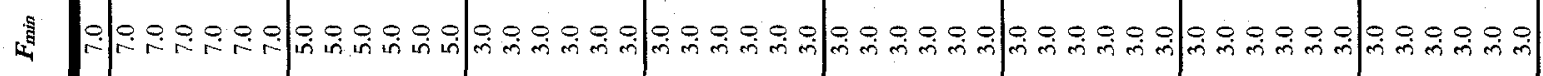

sic

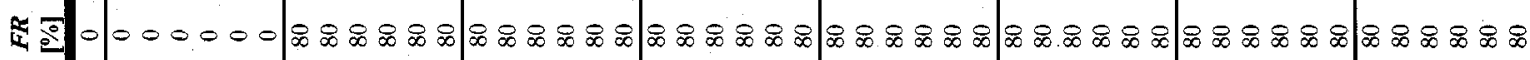

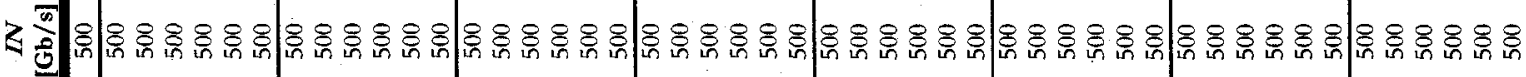

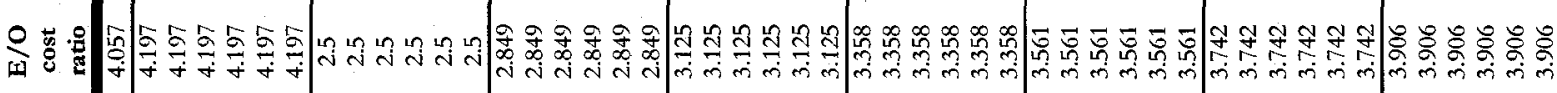

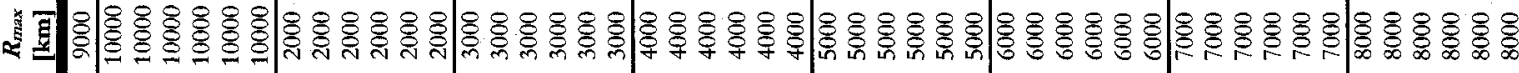

$\infty$

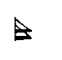

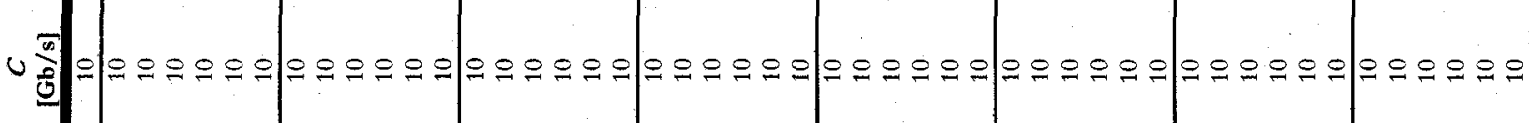




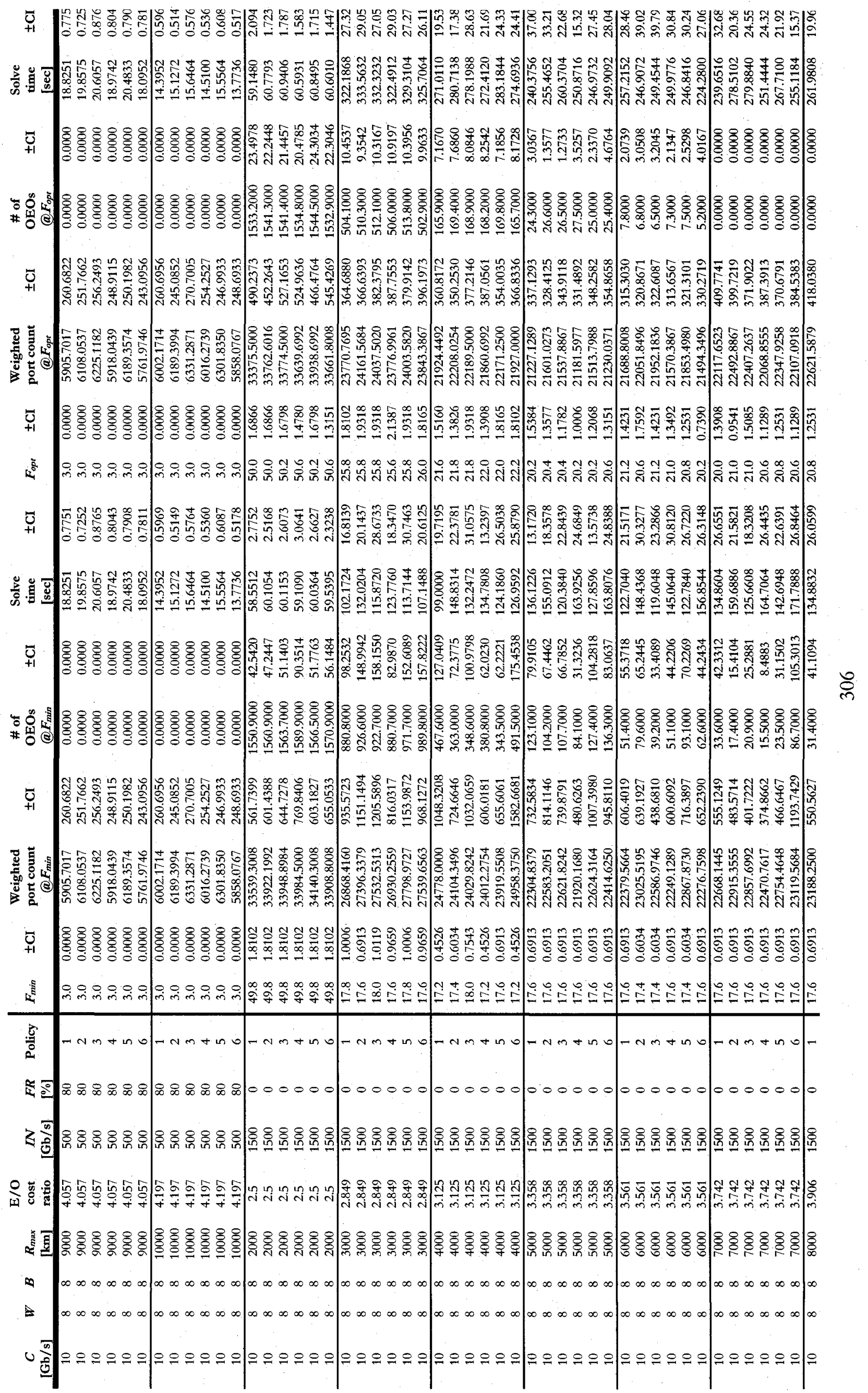




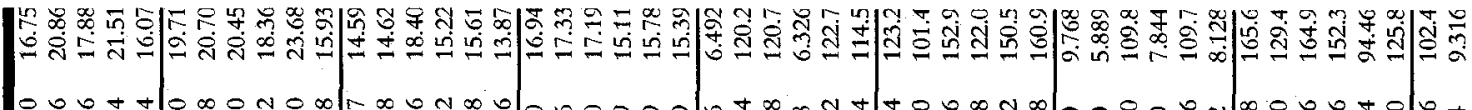

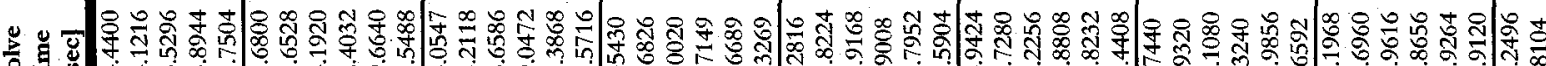
o

卉

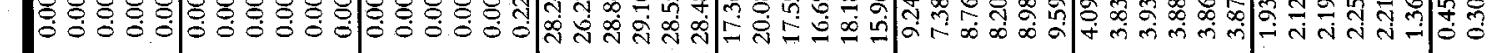

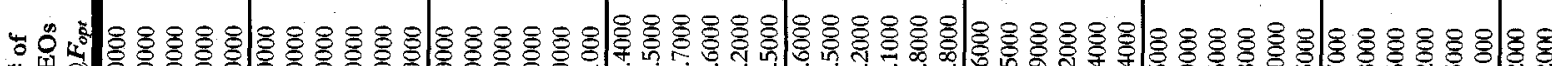

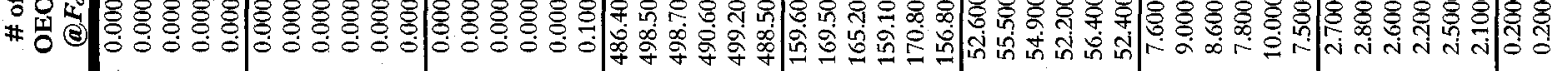

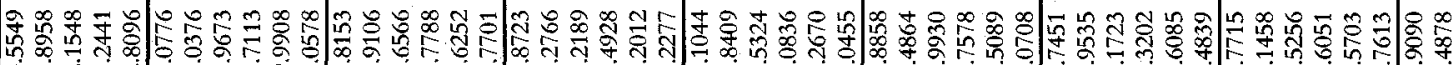

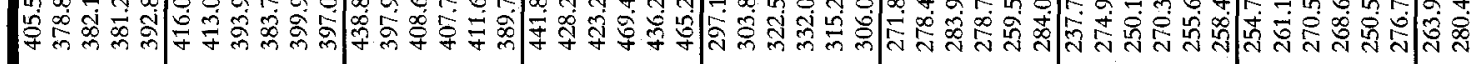

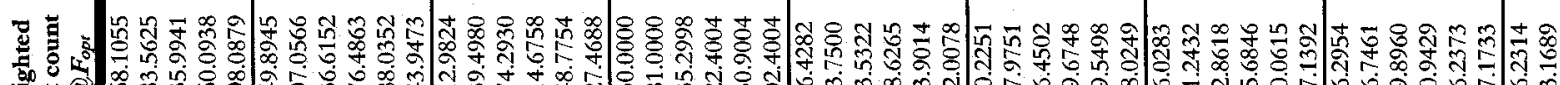

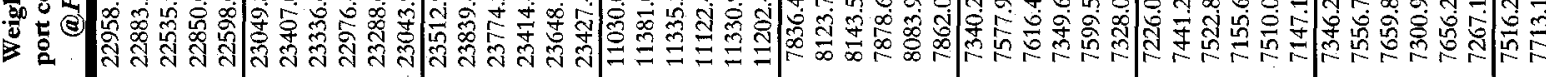

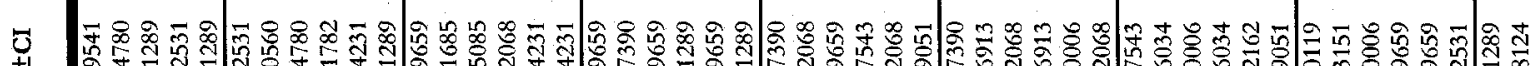

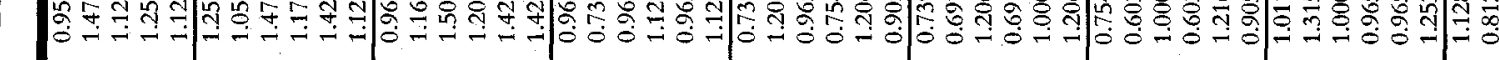

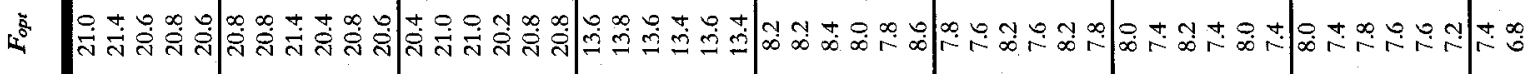

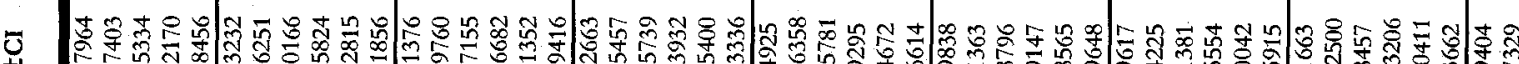

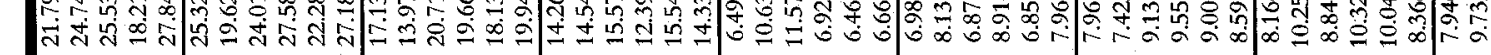

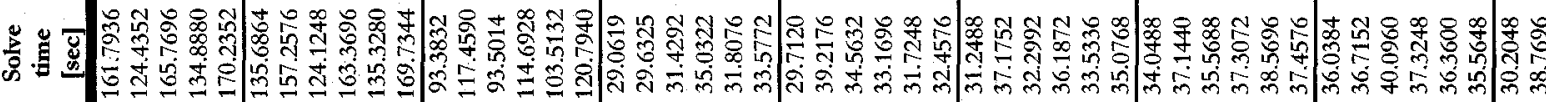

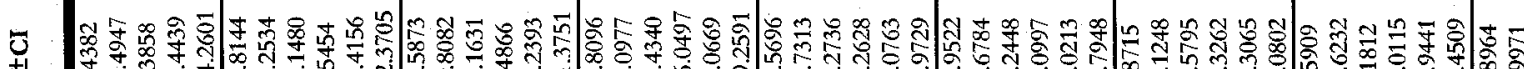

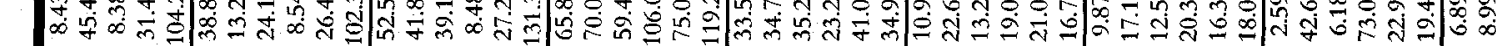

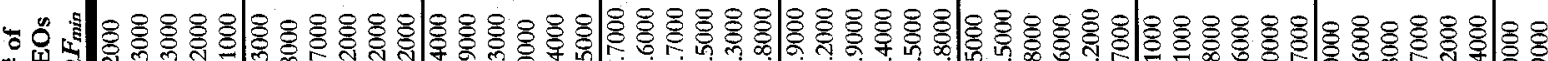
*落过

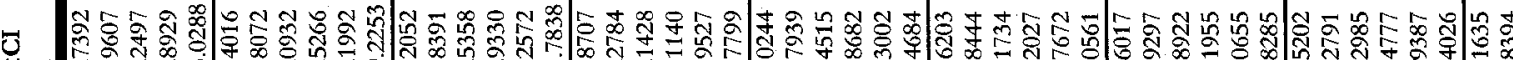

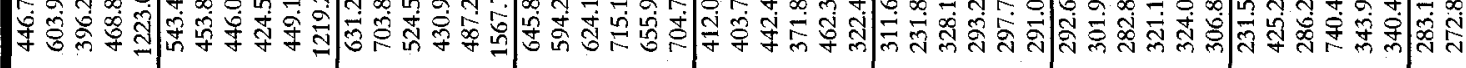

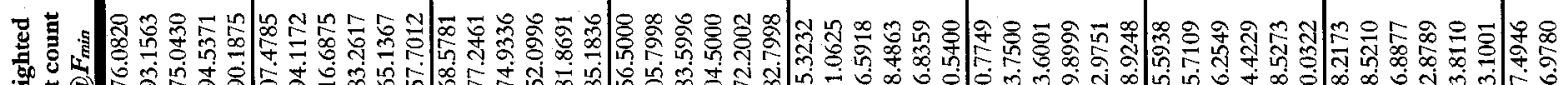

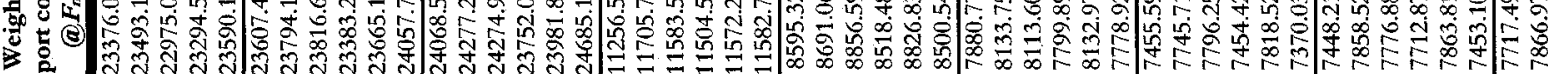

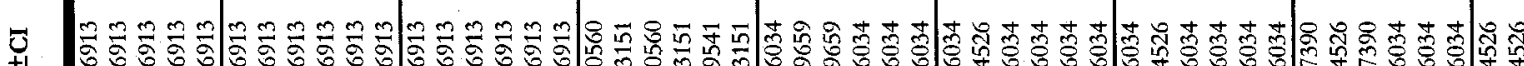

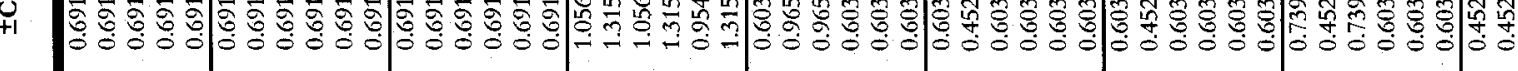
4 1

亳

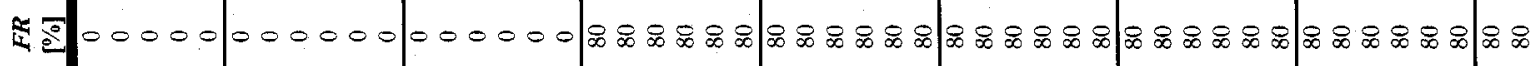

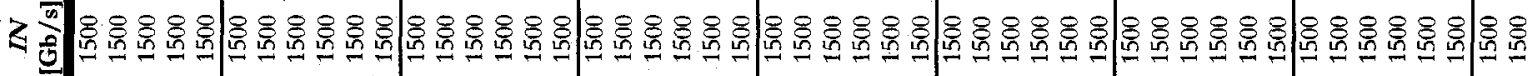

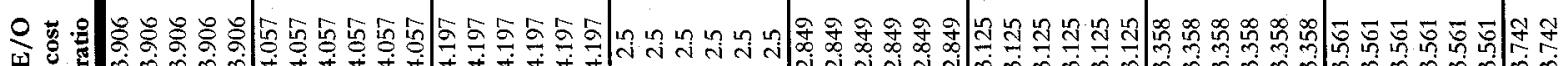
U 旁| 


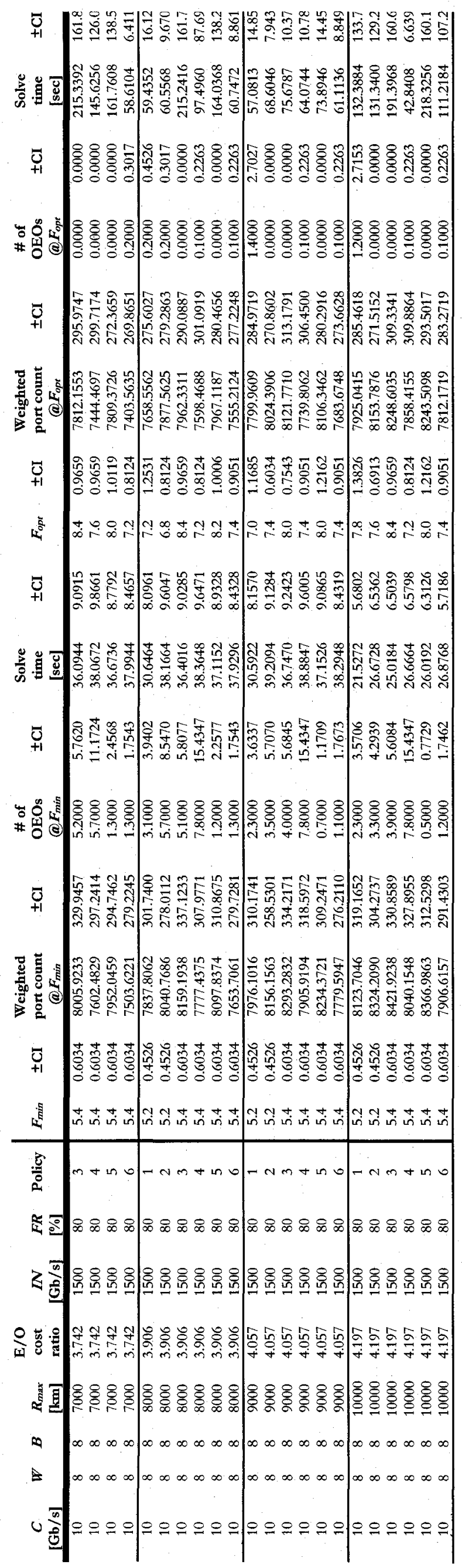




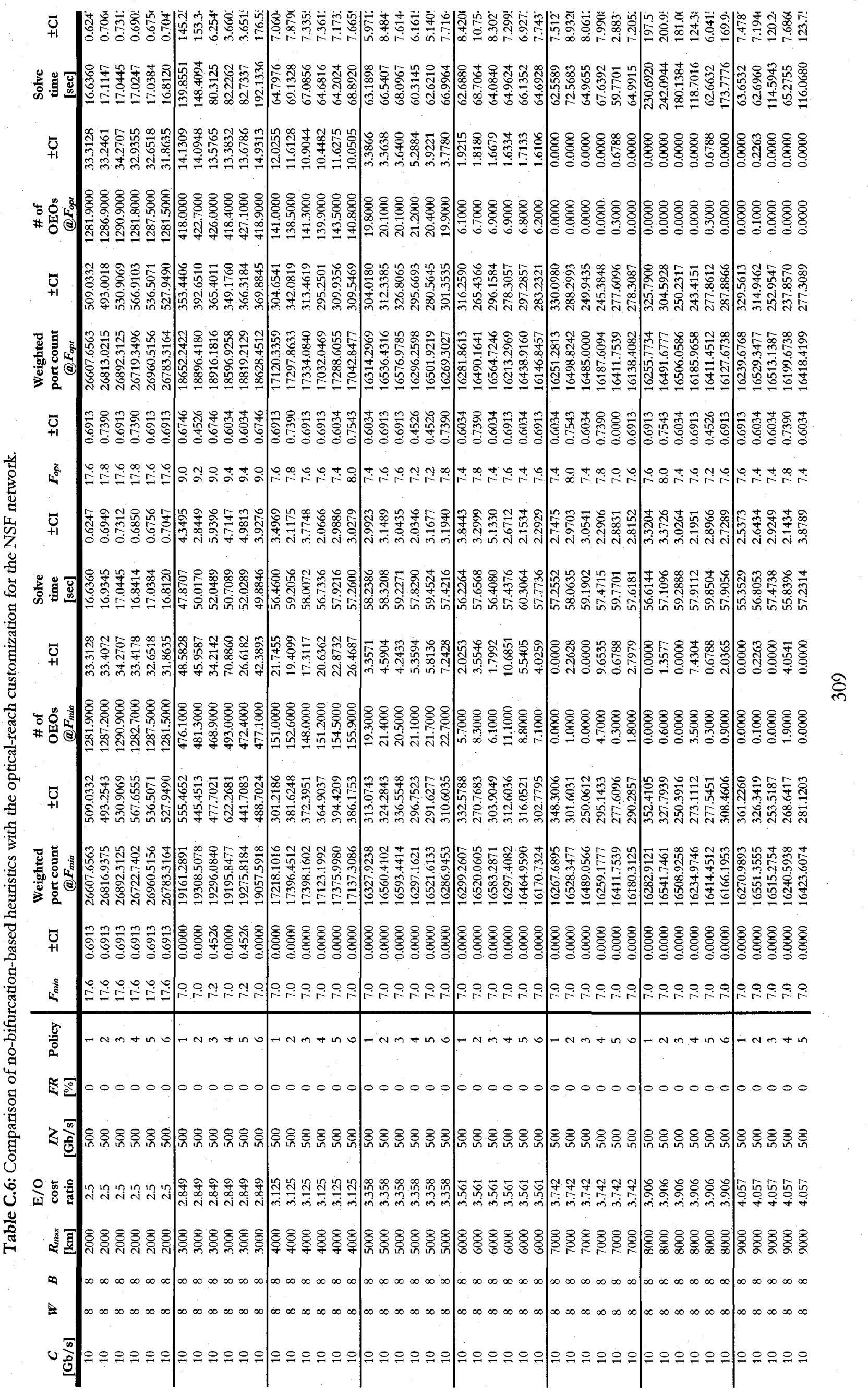




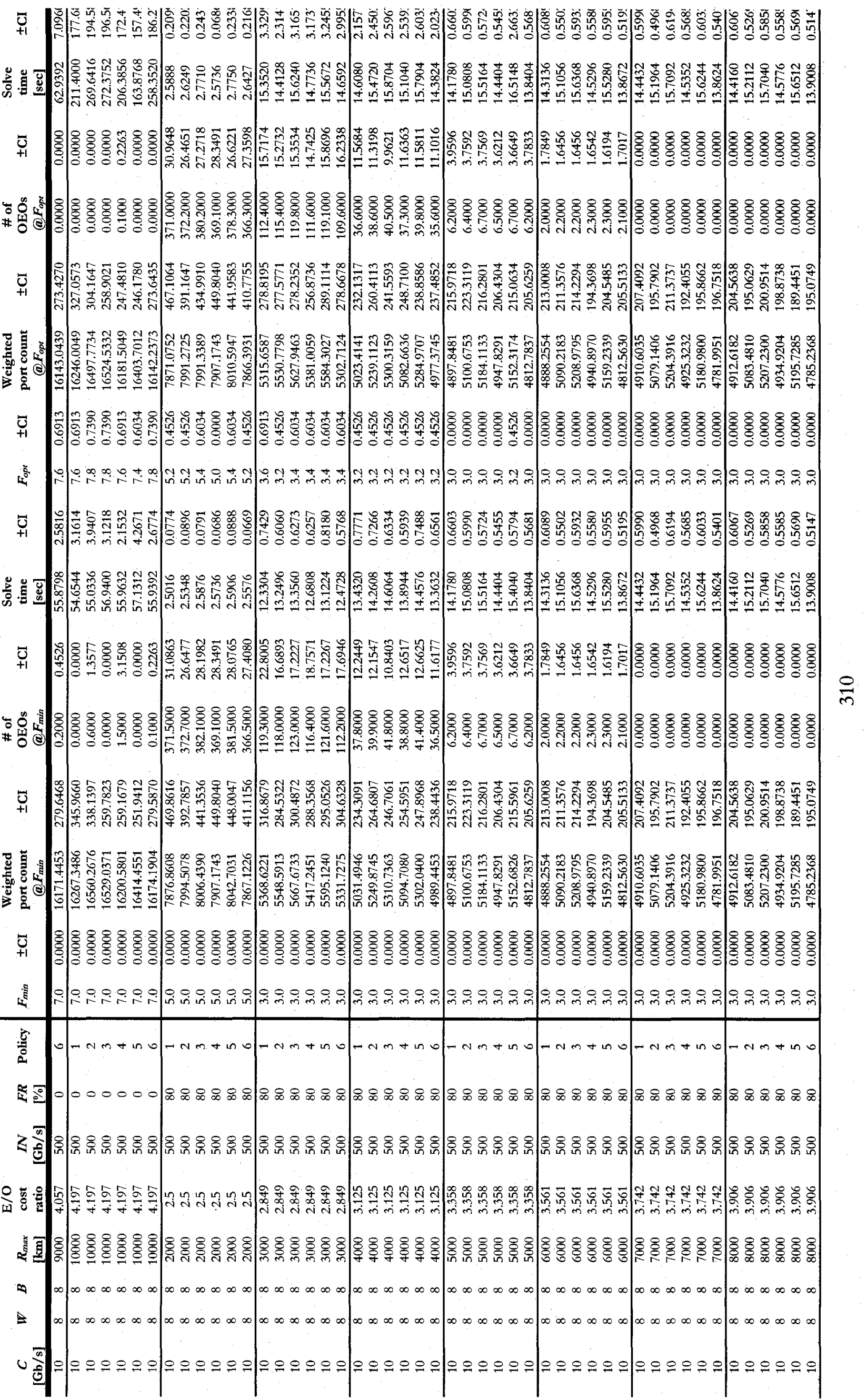




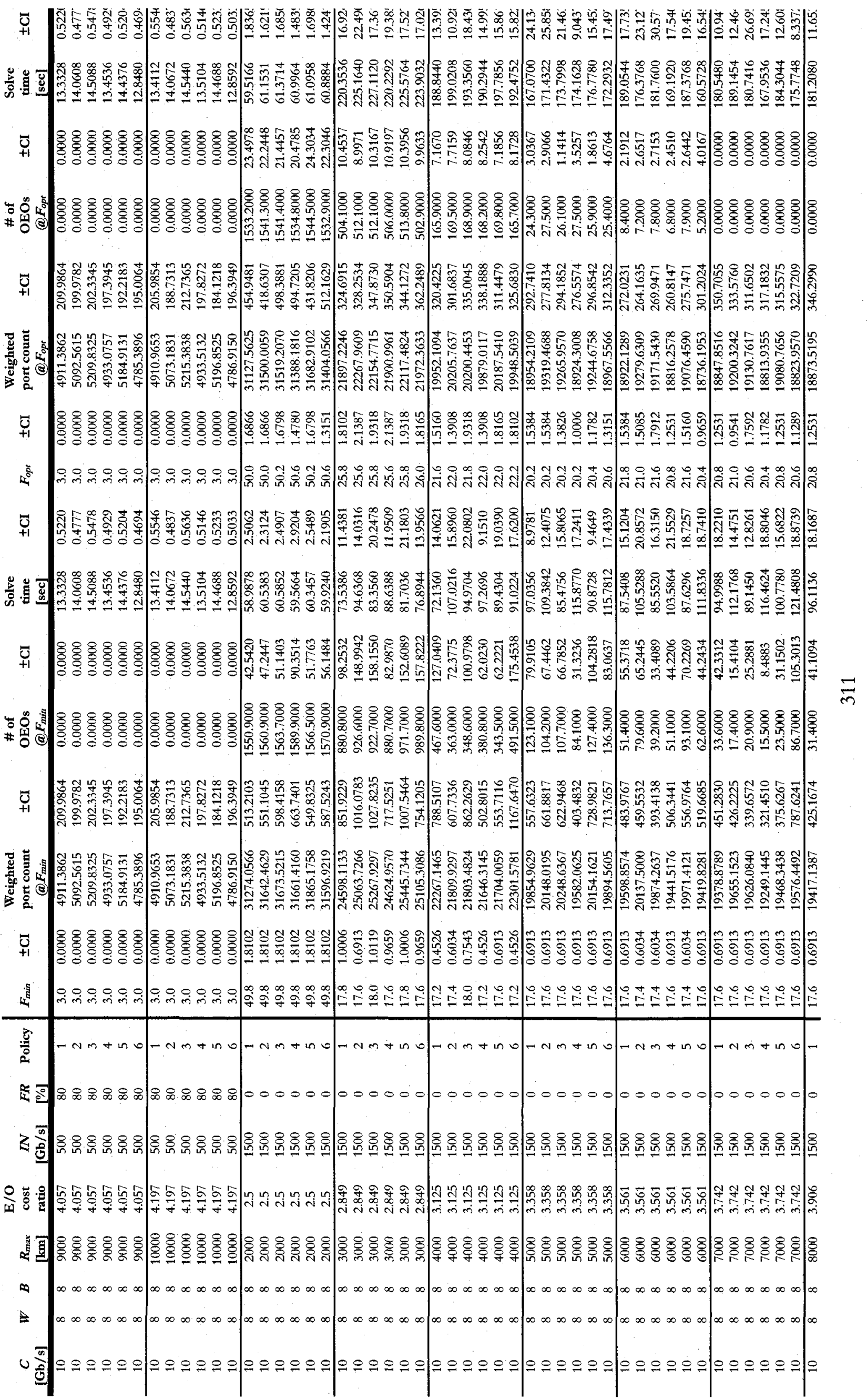




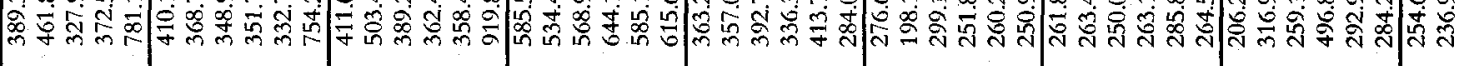

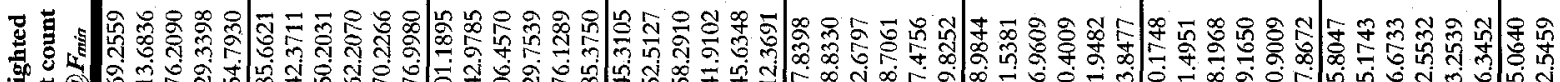

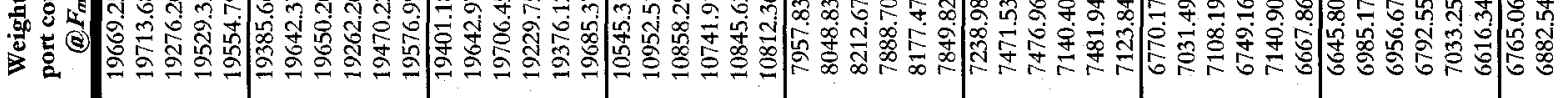

计

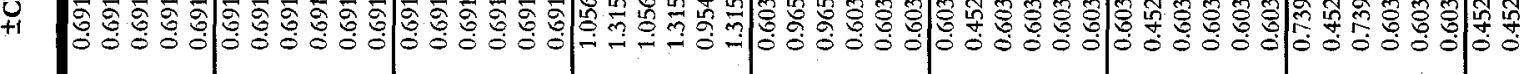
直

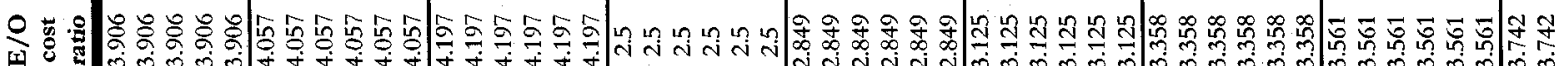

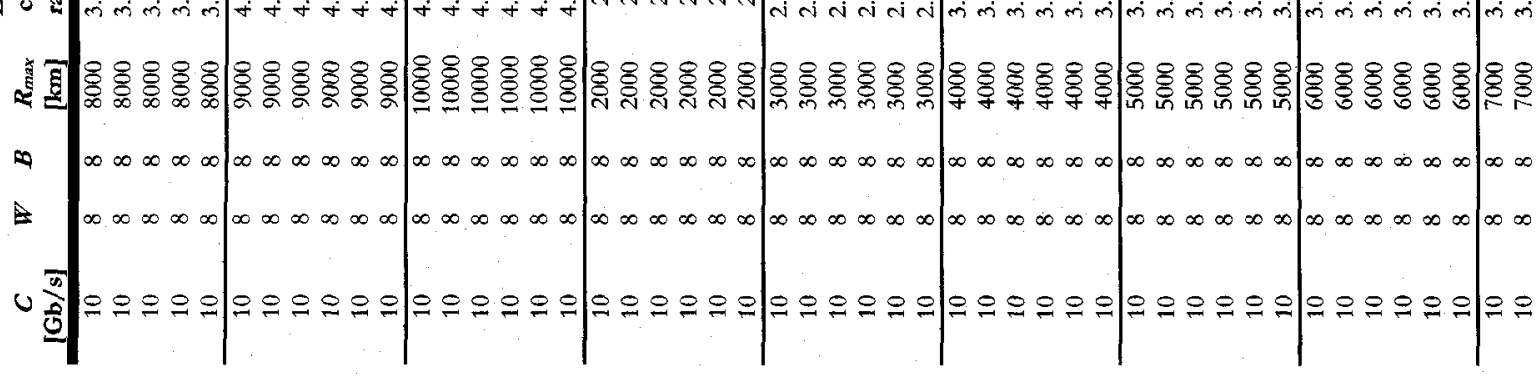




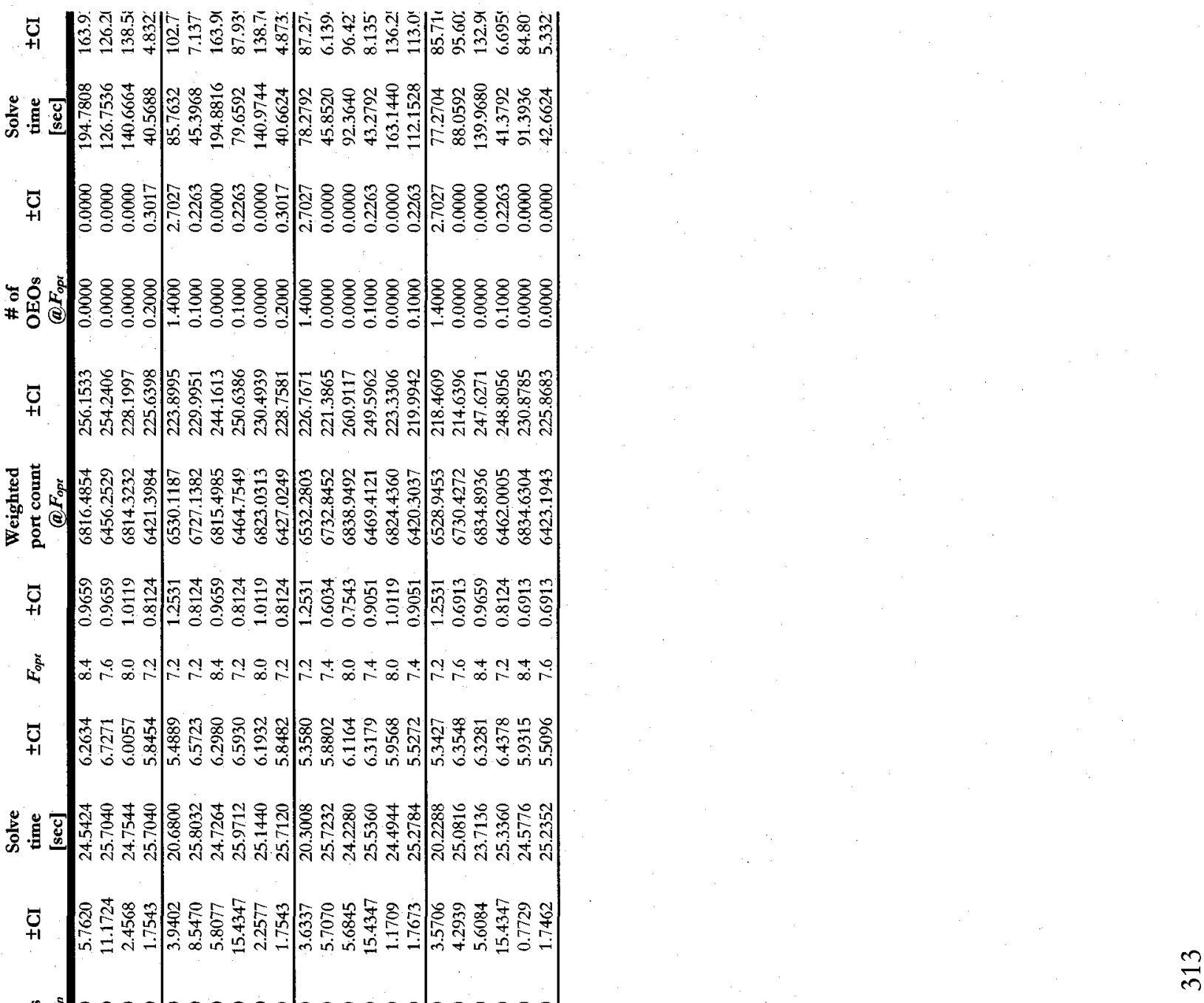




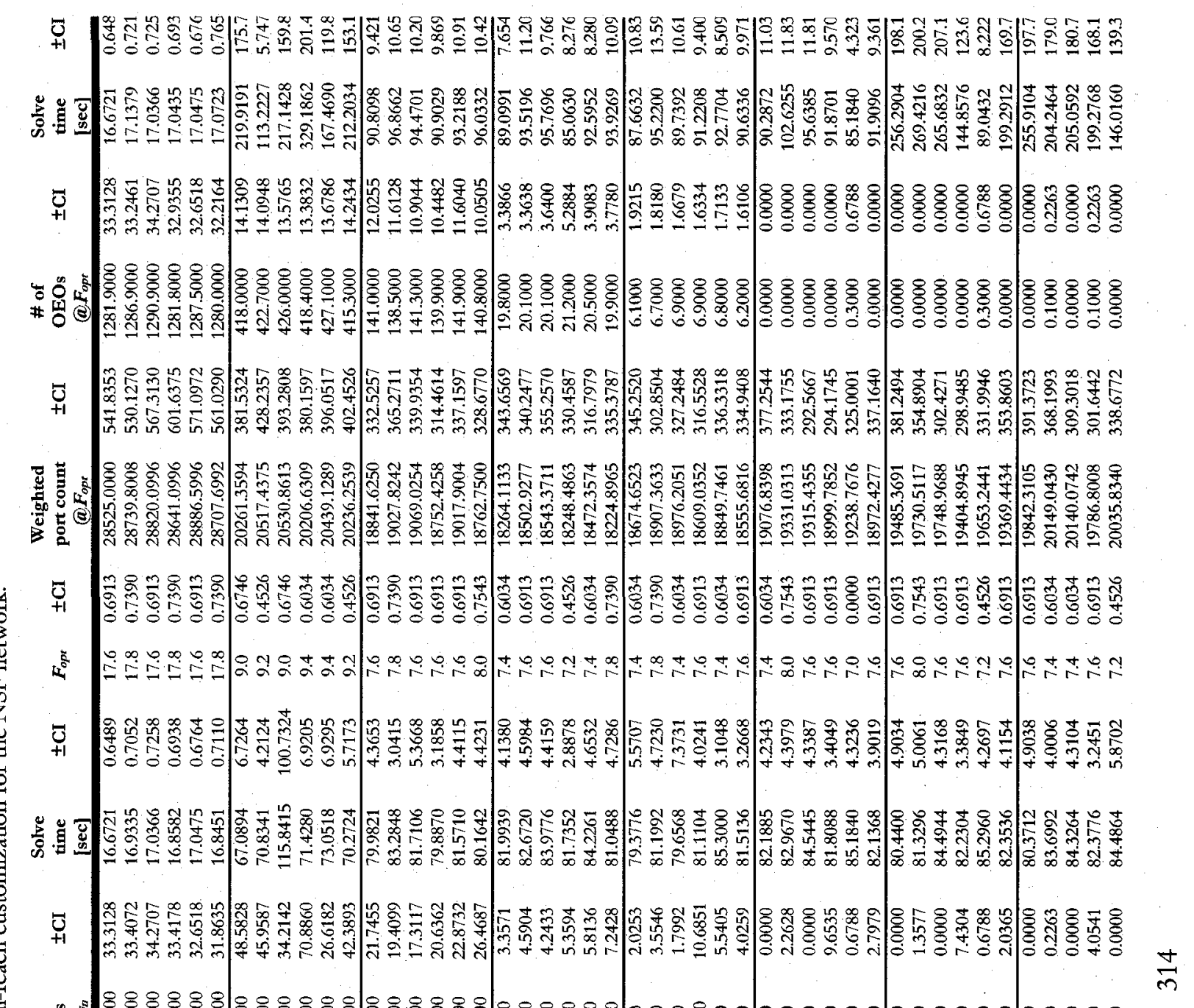

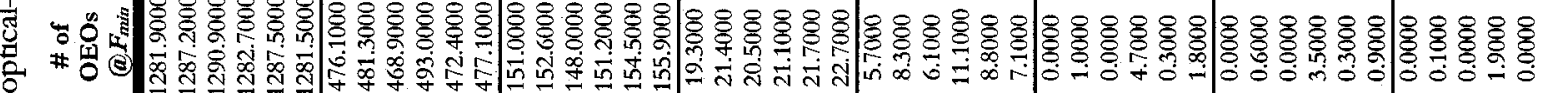
$\stackrel{8}{\ddagger}$

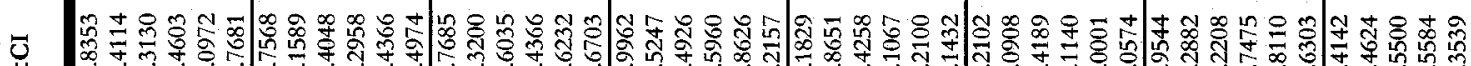

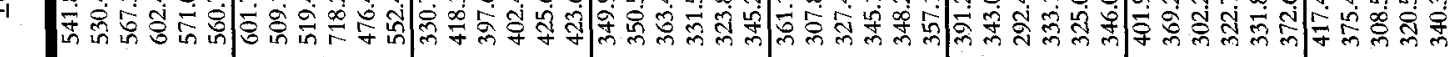

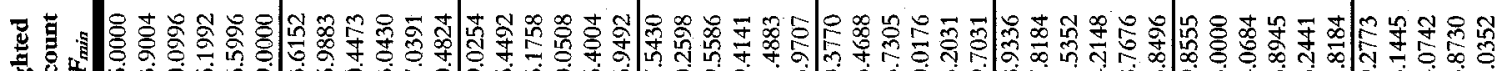

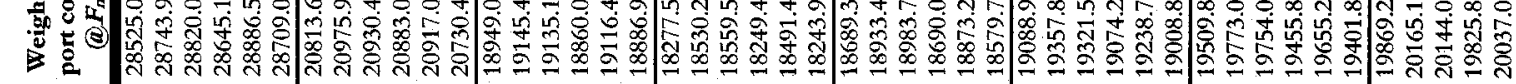
Th
+1

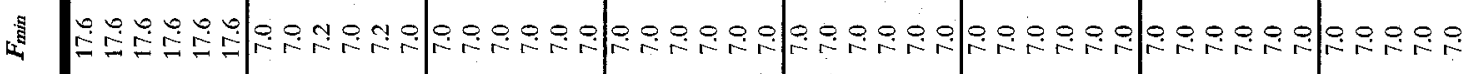
窟 绽00000000000000000000000000000000000000000000 3京

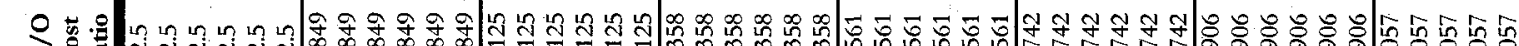
F

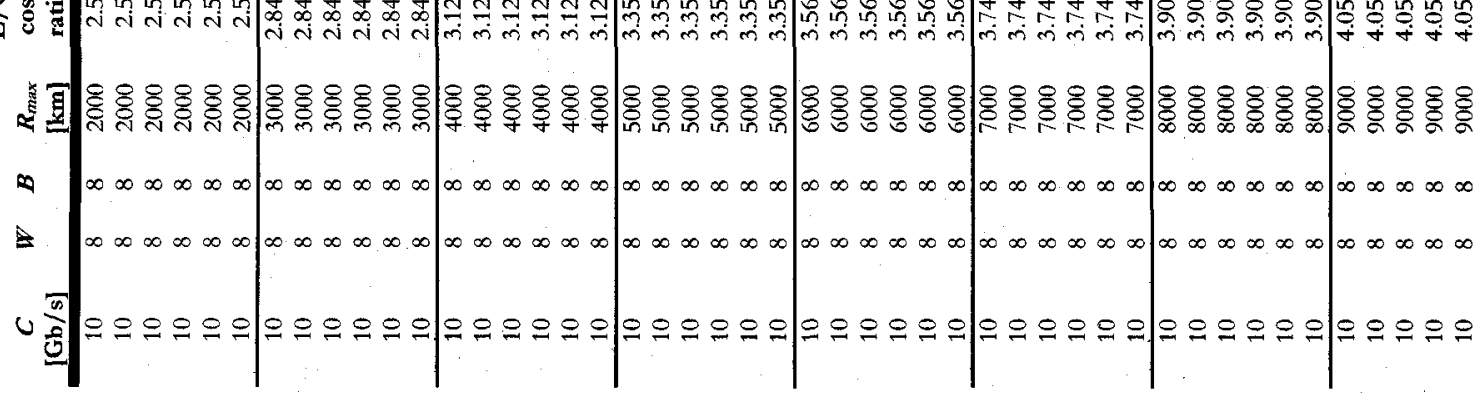




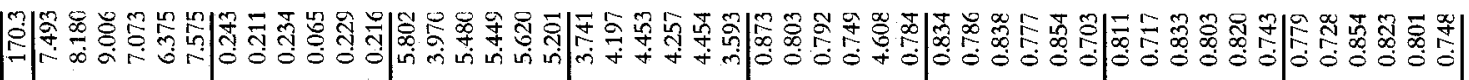

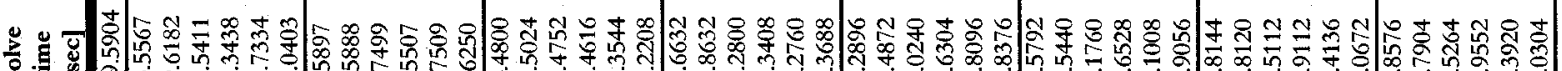

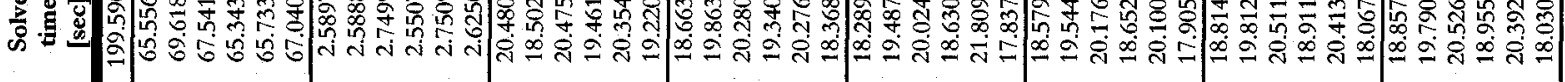

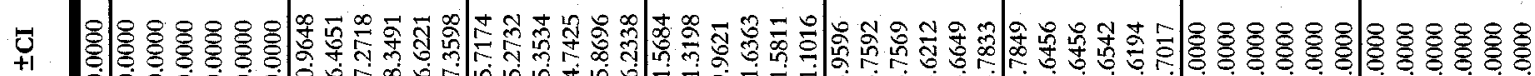
\%

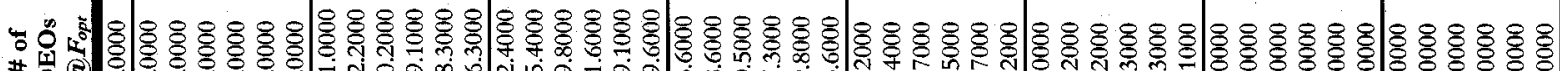
\# 回 ब

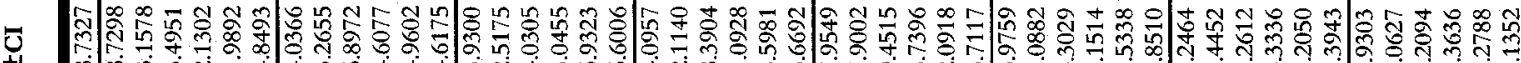

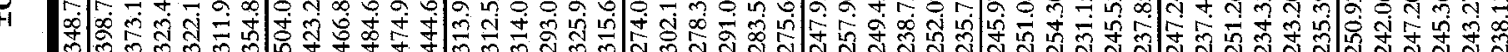

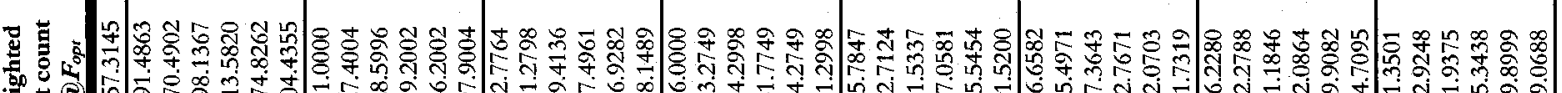

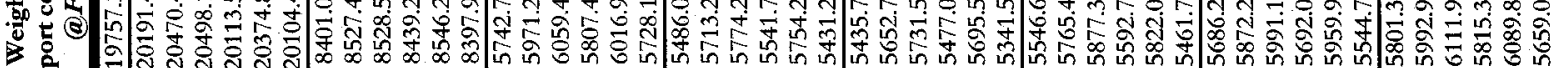

芰

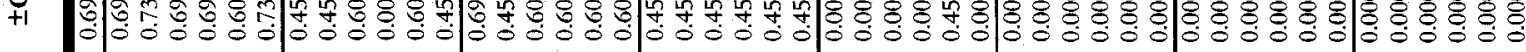

死

t

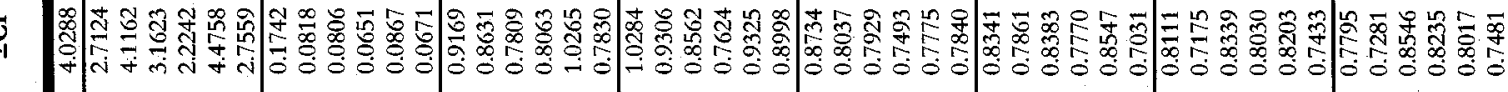

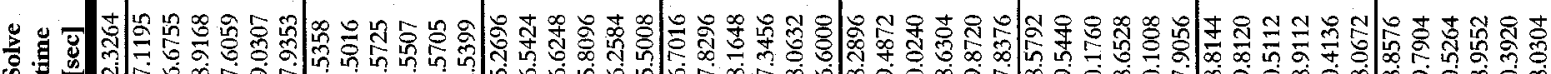

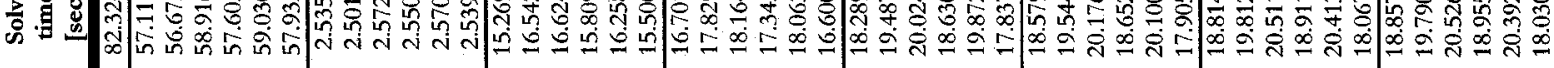

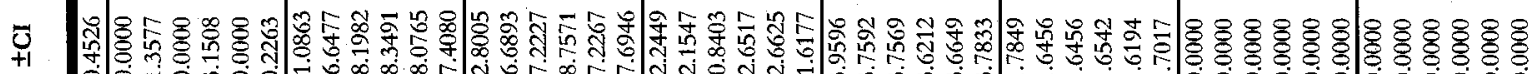

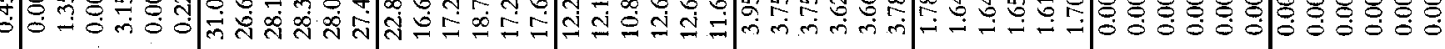

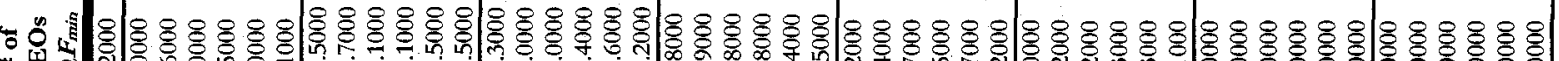

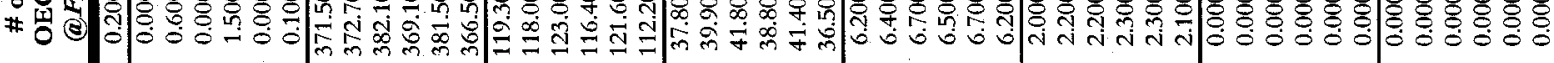

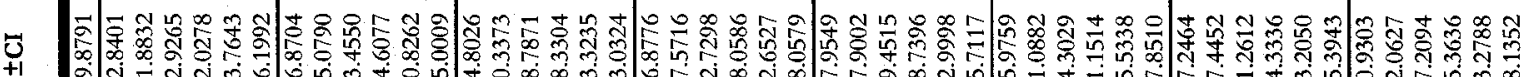

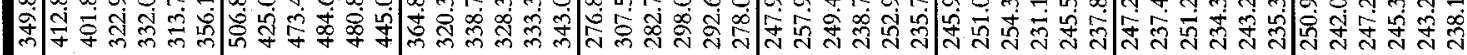

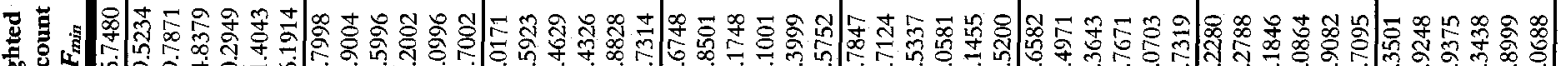

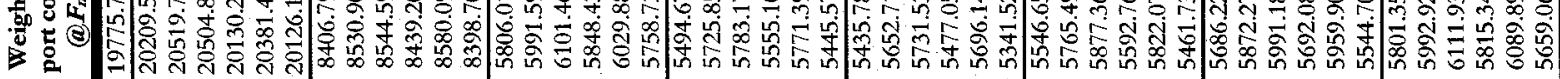

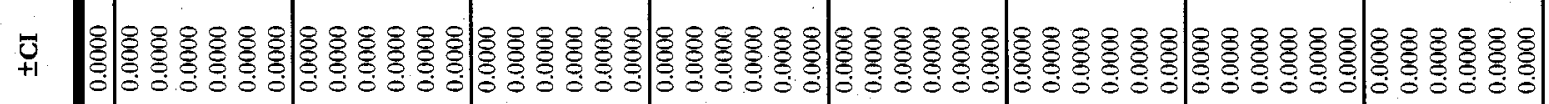

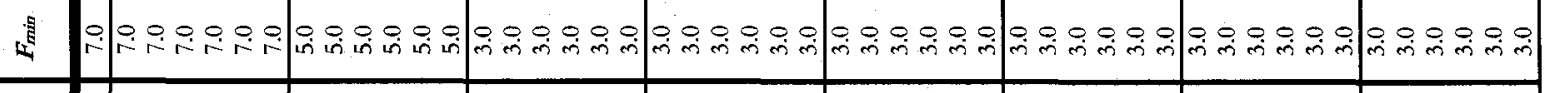

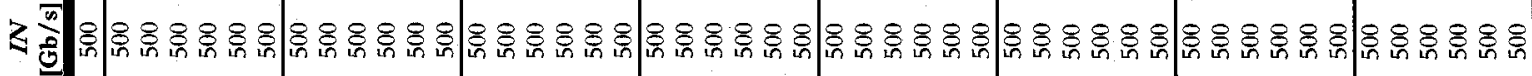

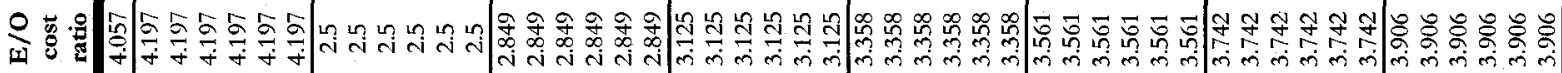

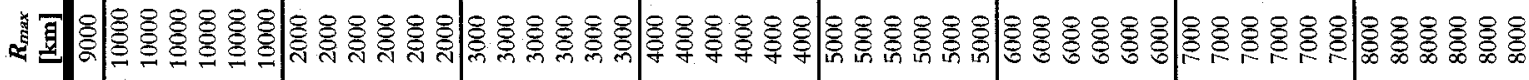

a

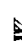




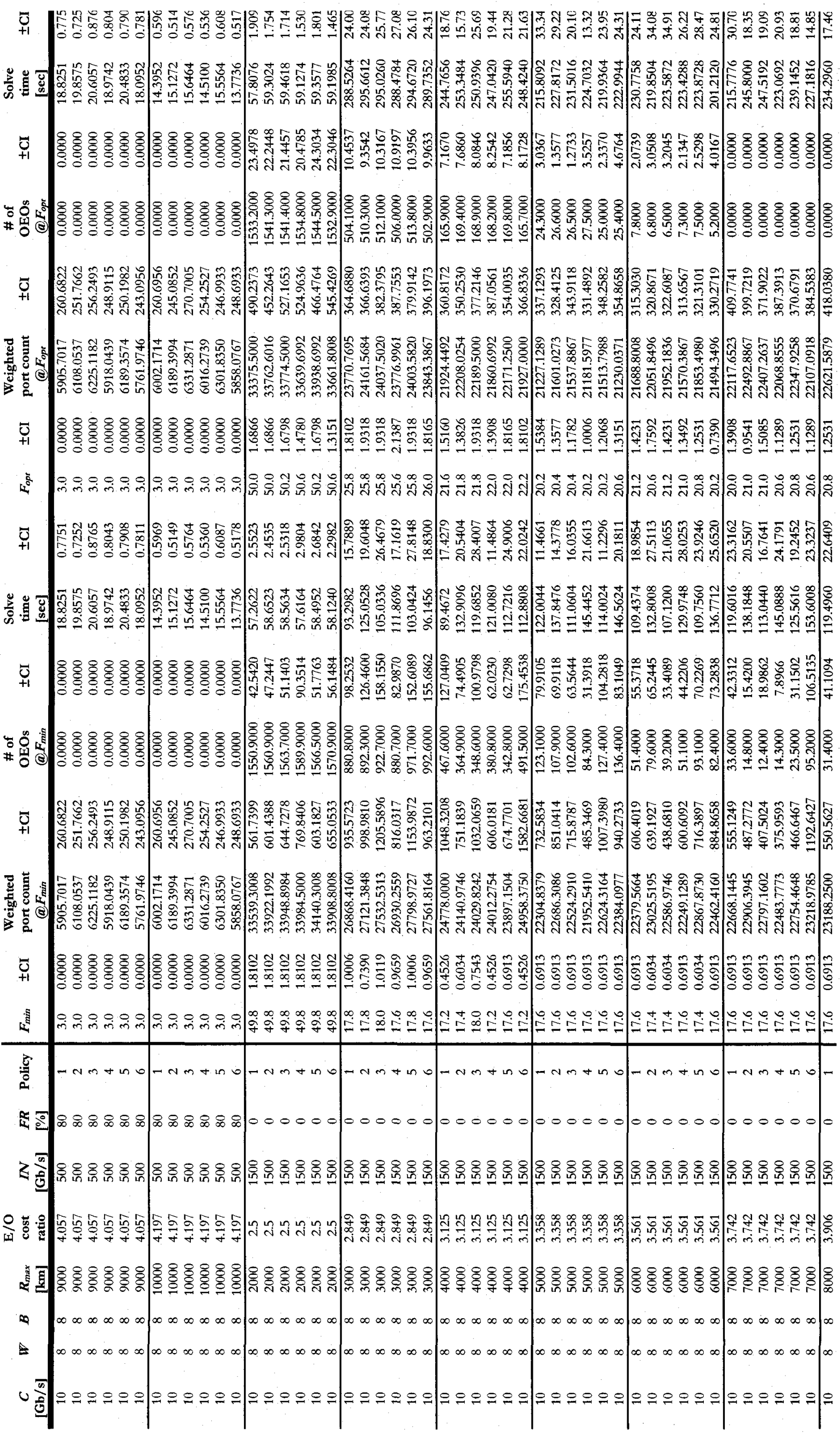




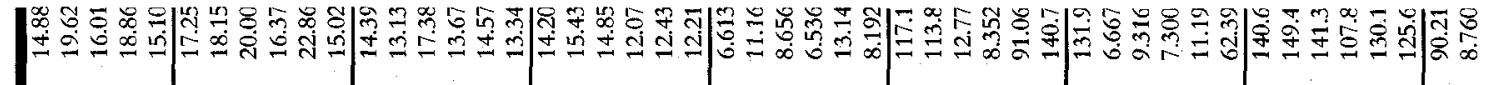

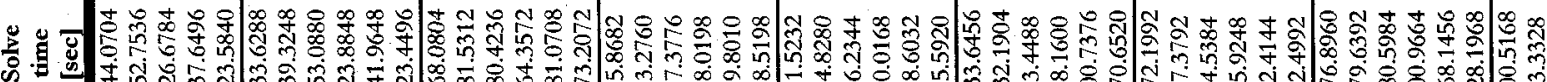

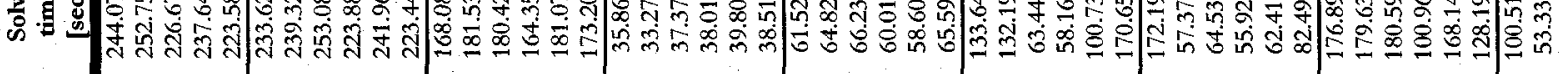

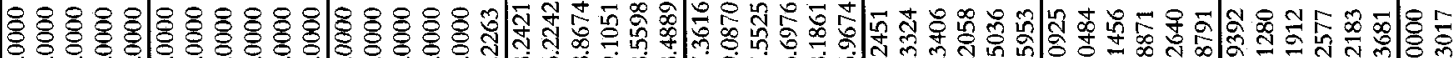

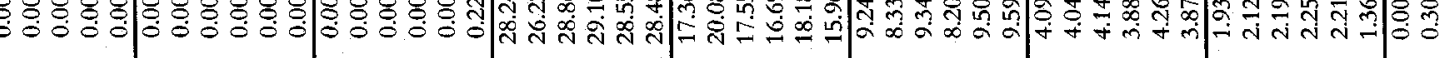

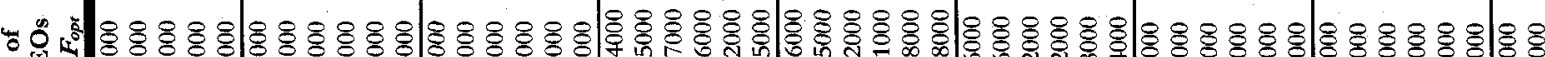

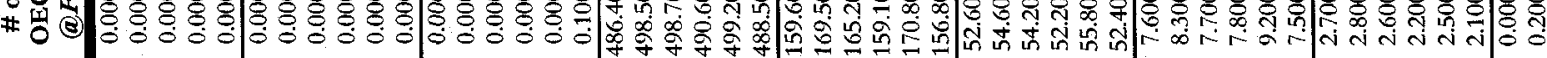

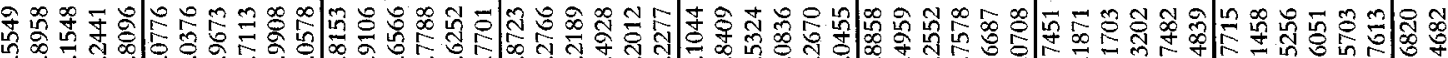

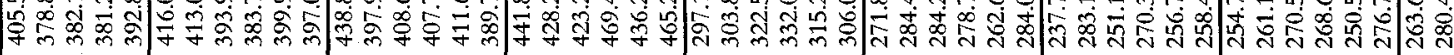

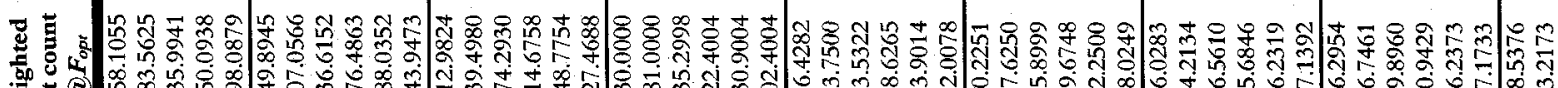

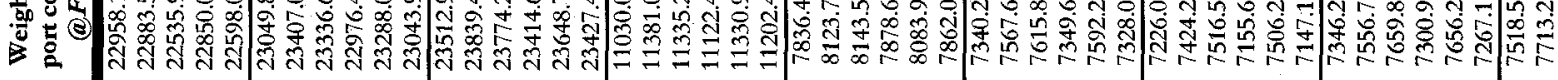

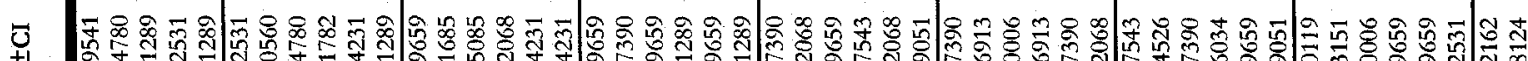

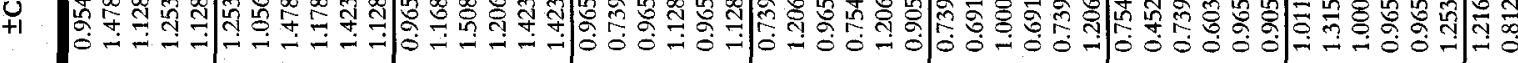

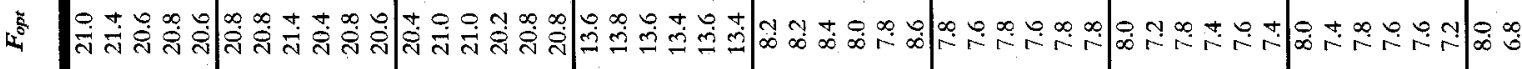

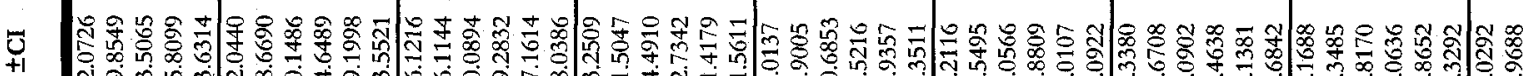

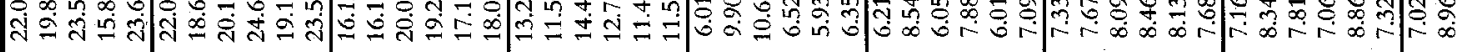

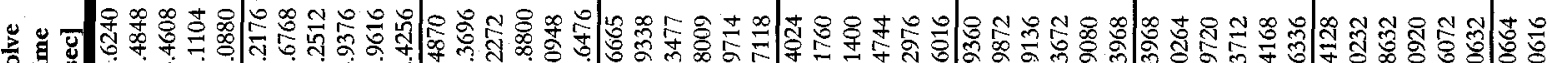

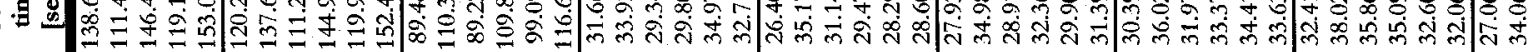

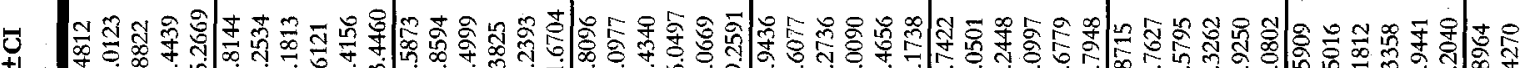

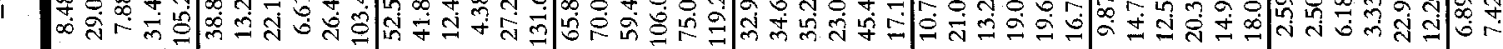

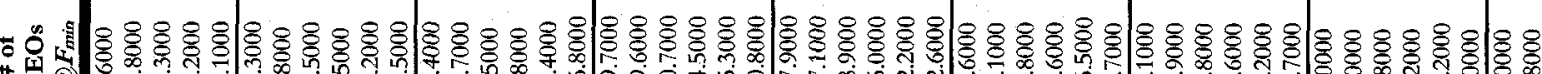

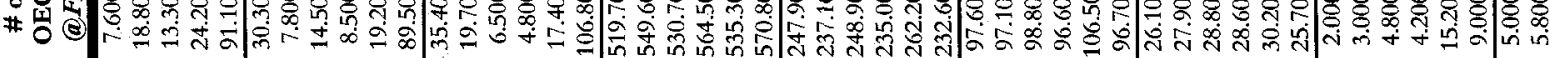

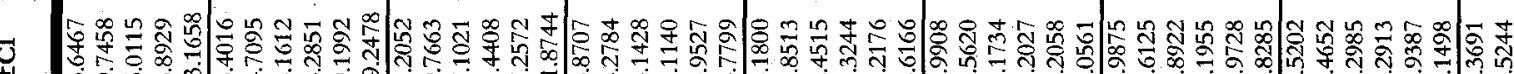

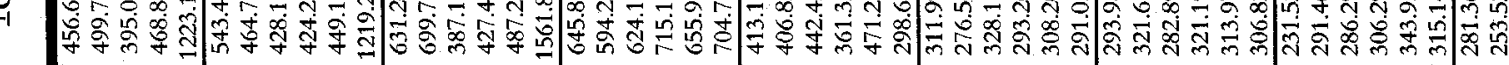

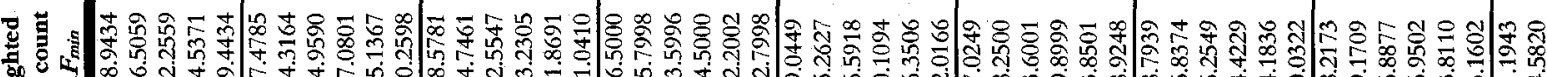

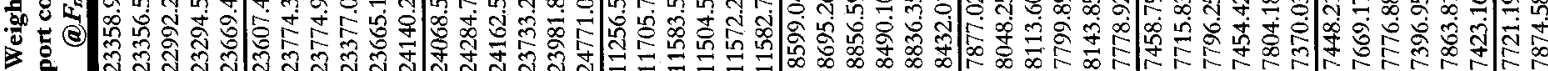

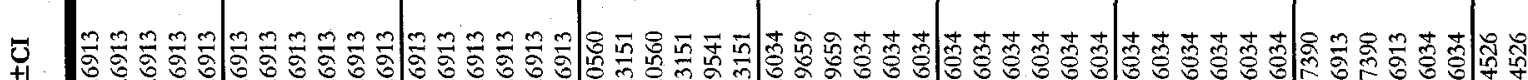

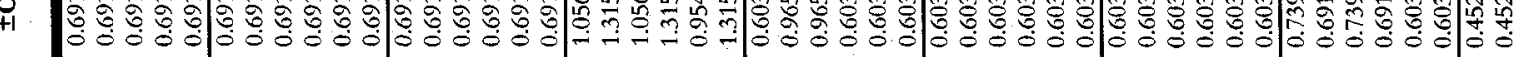

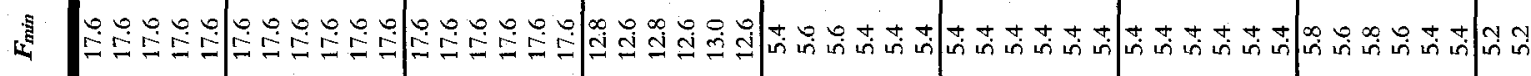
政

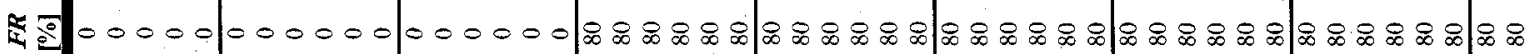

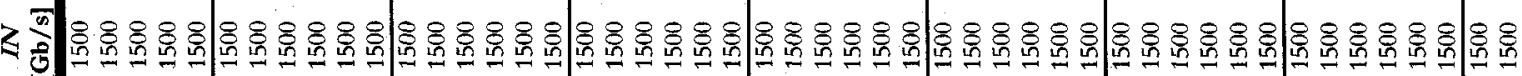

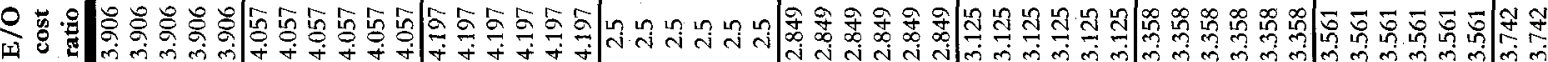

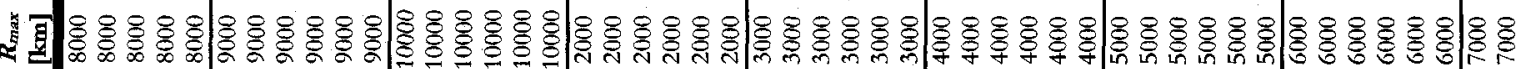

$\infty$

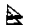

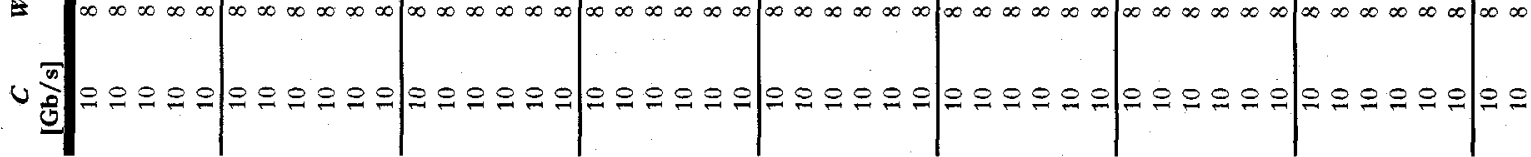




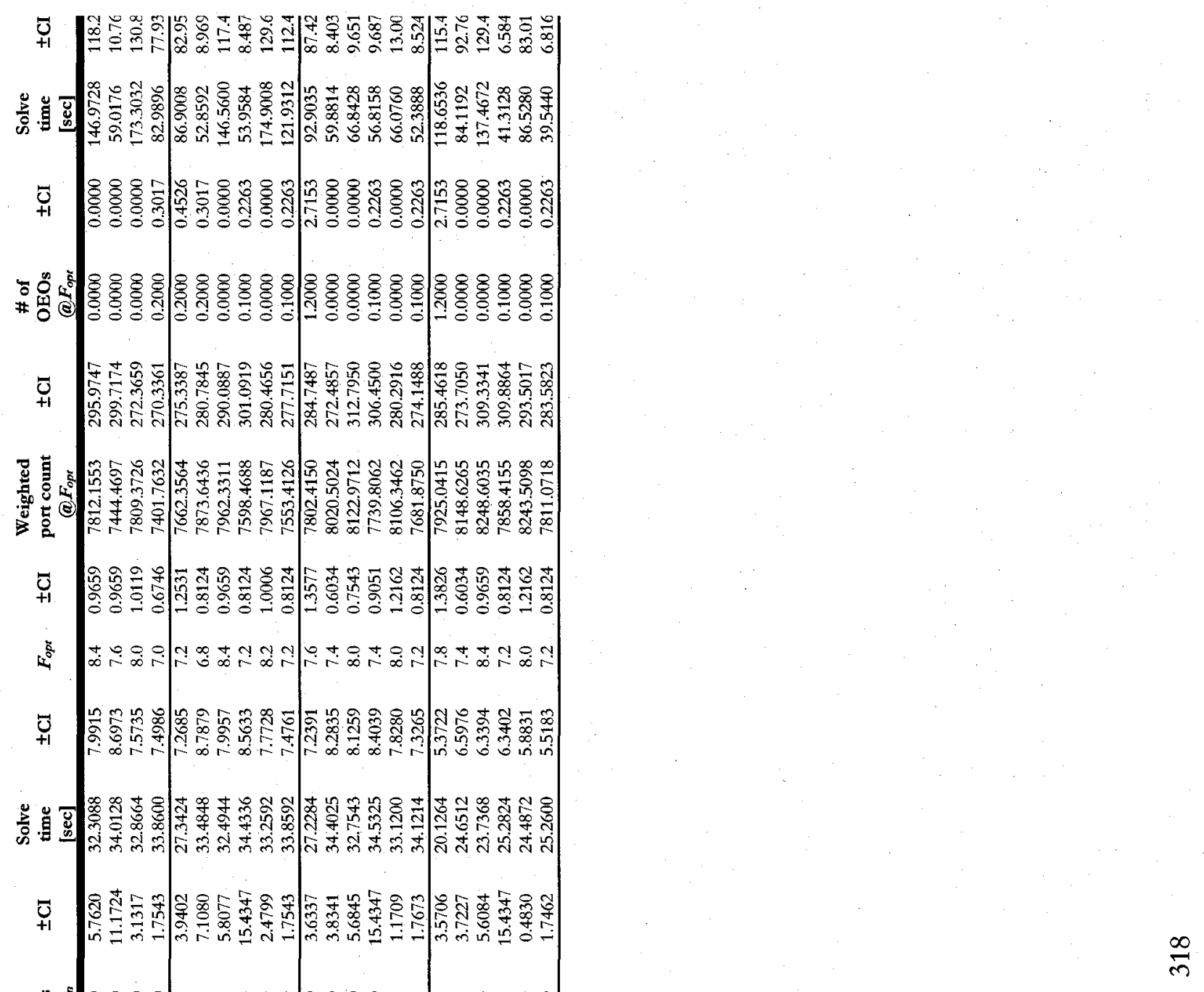




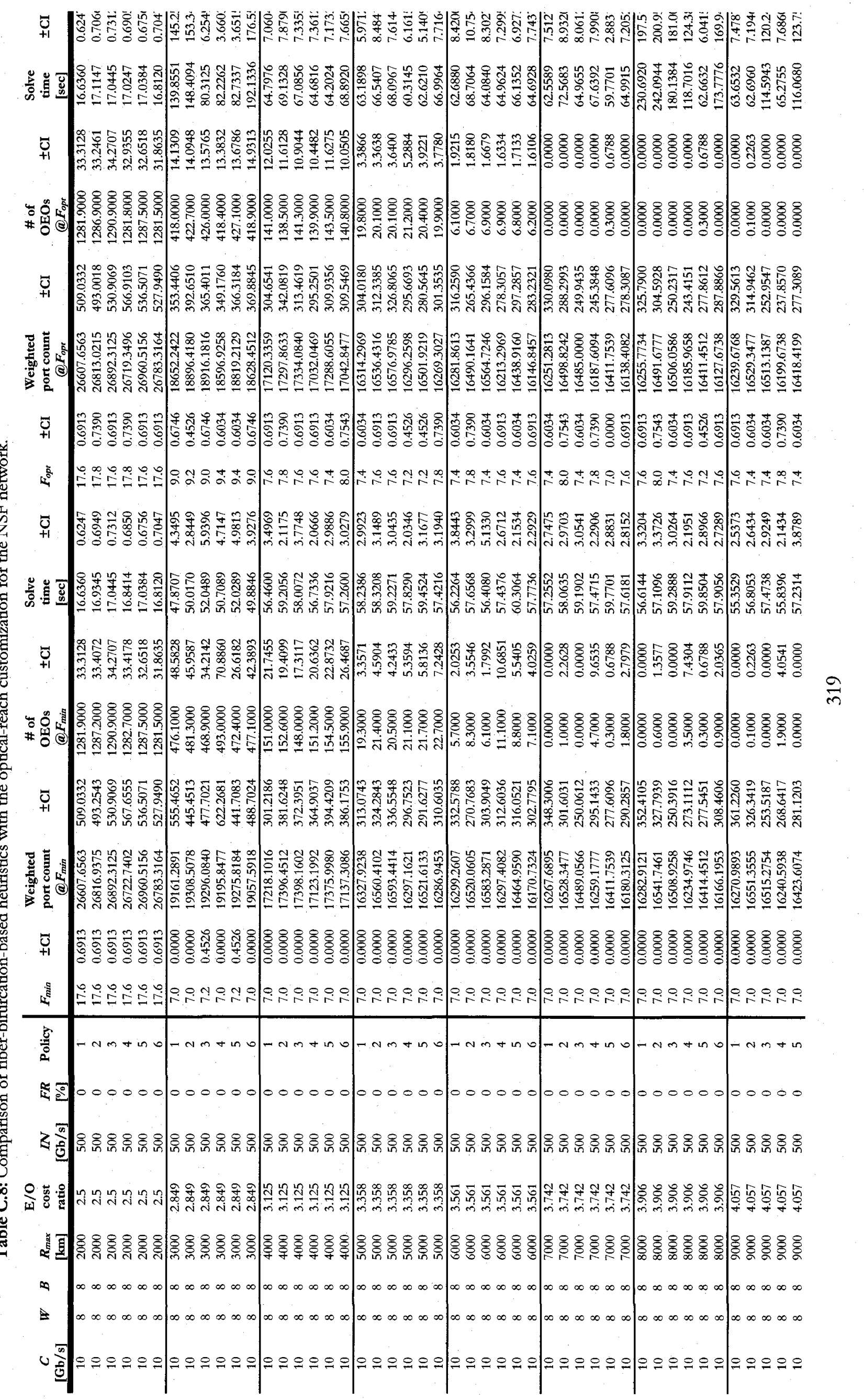




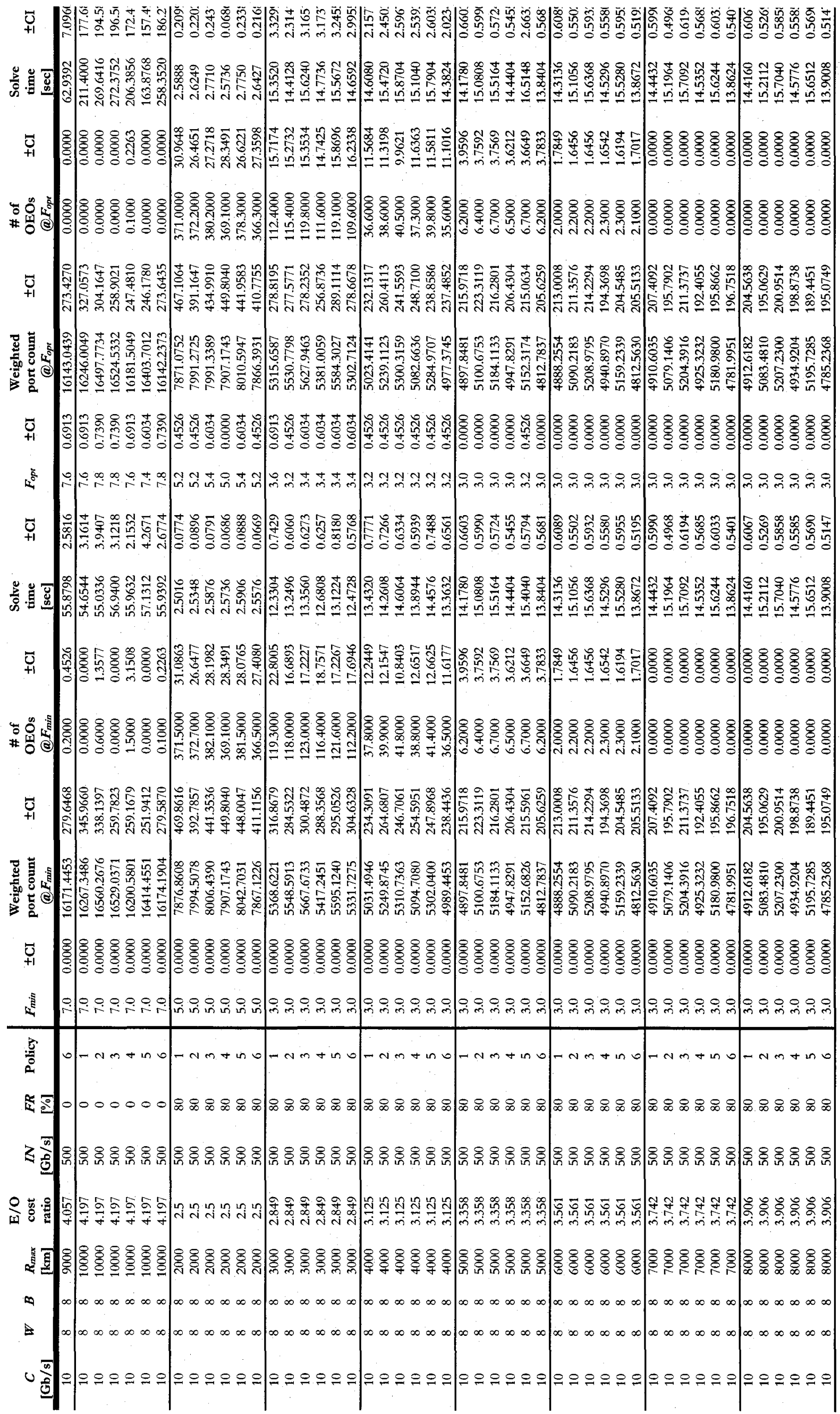




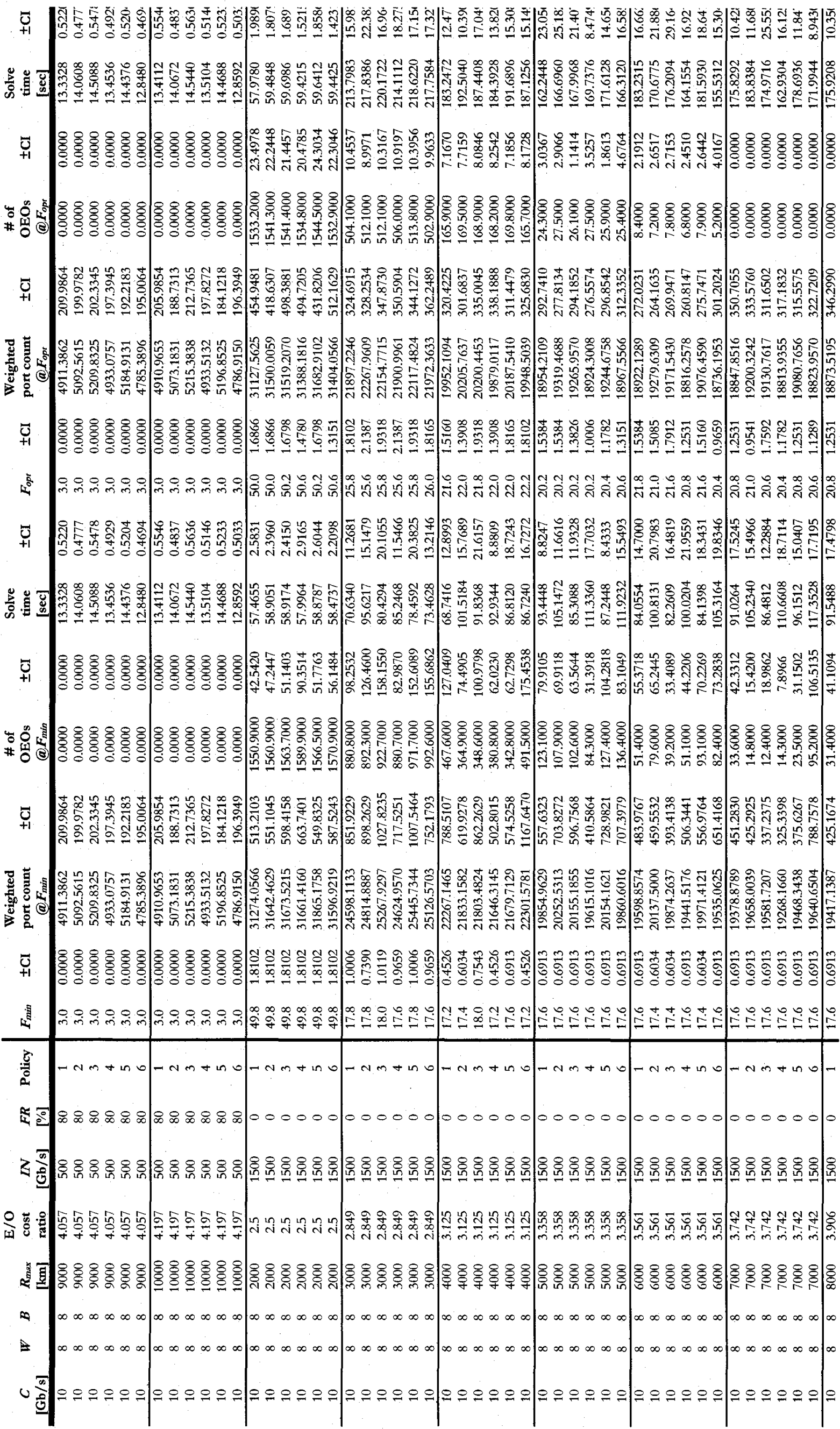



|

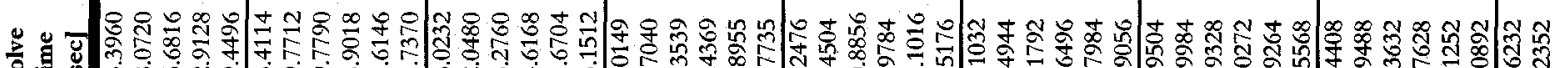

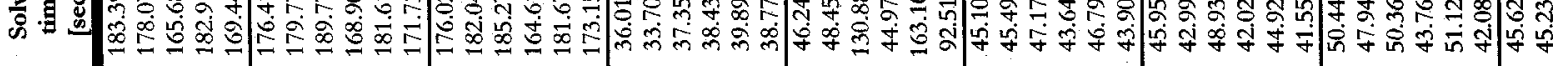

i

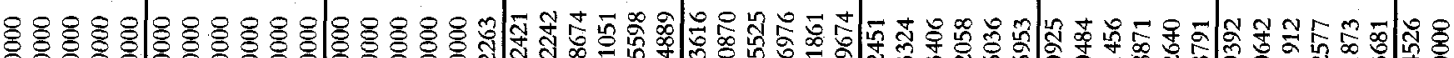

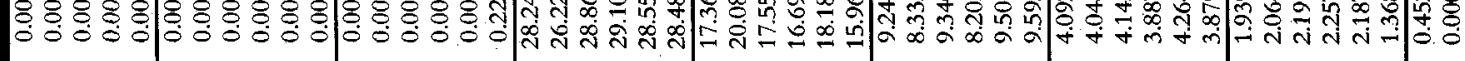

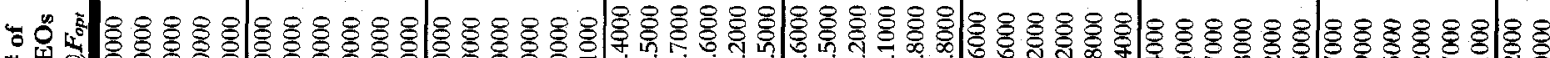
*

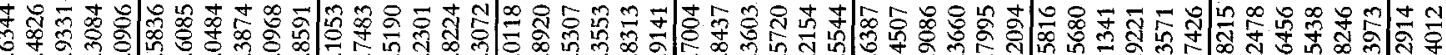

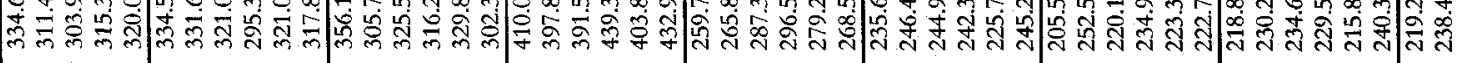

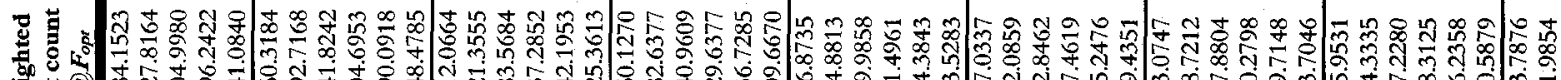

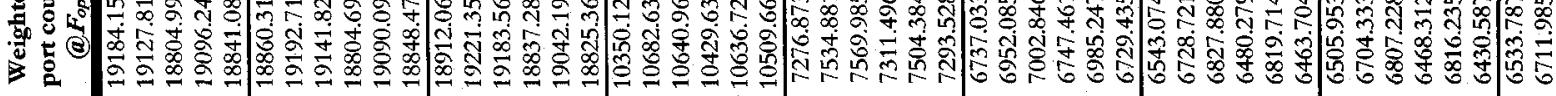

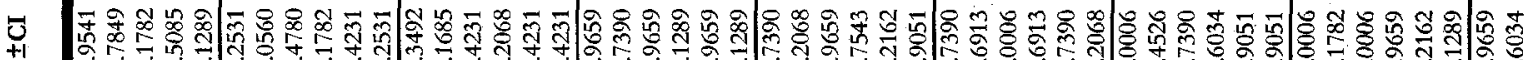

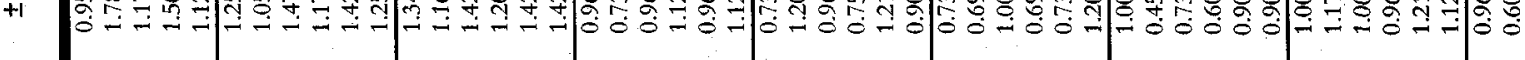

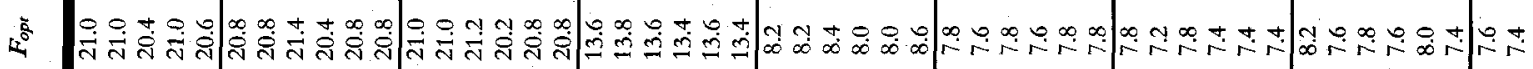
i

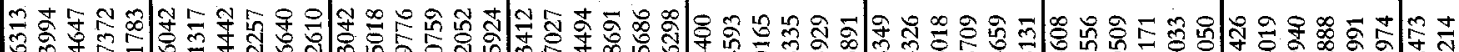

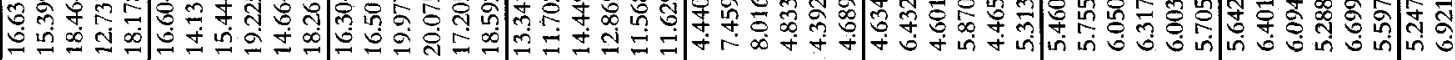

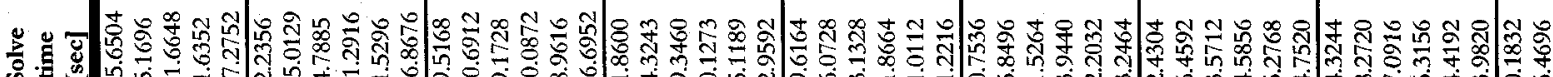
की

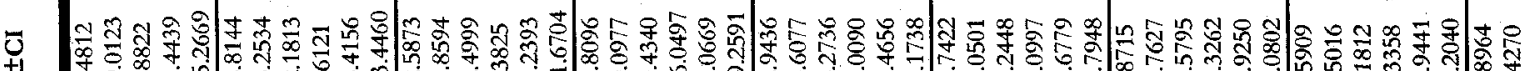
+1 क त. 范

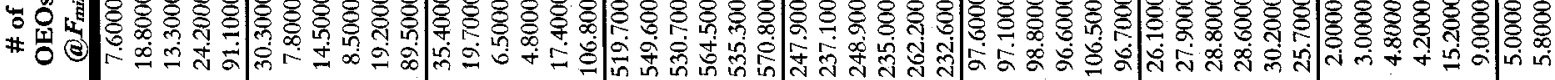

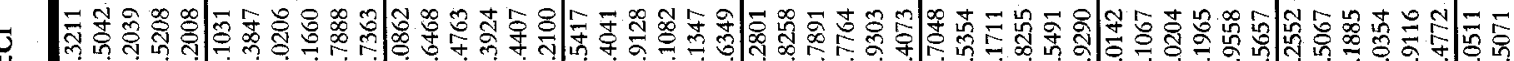

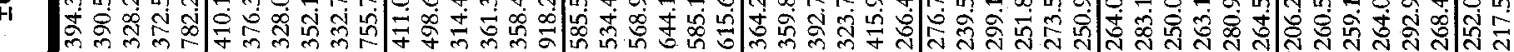

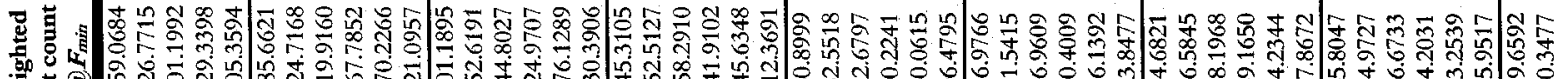

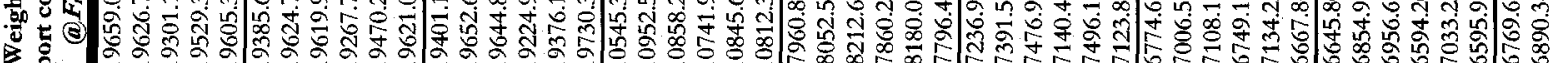

泋

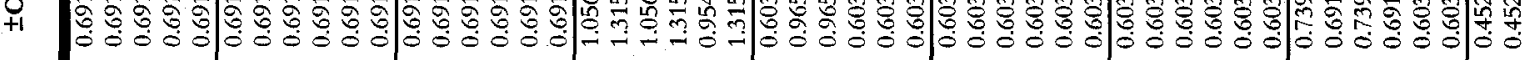
直

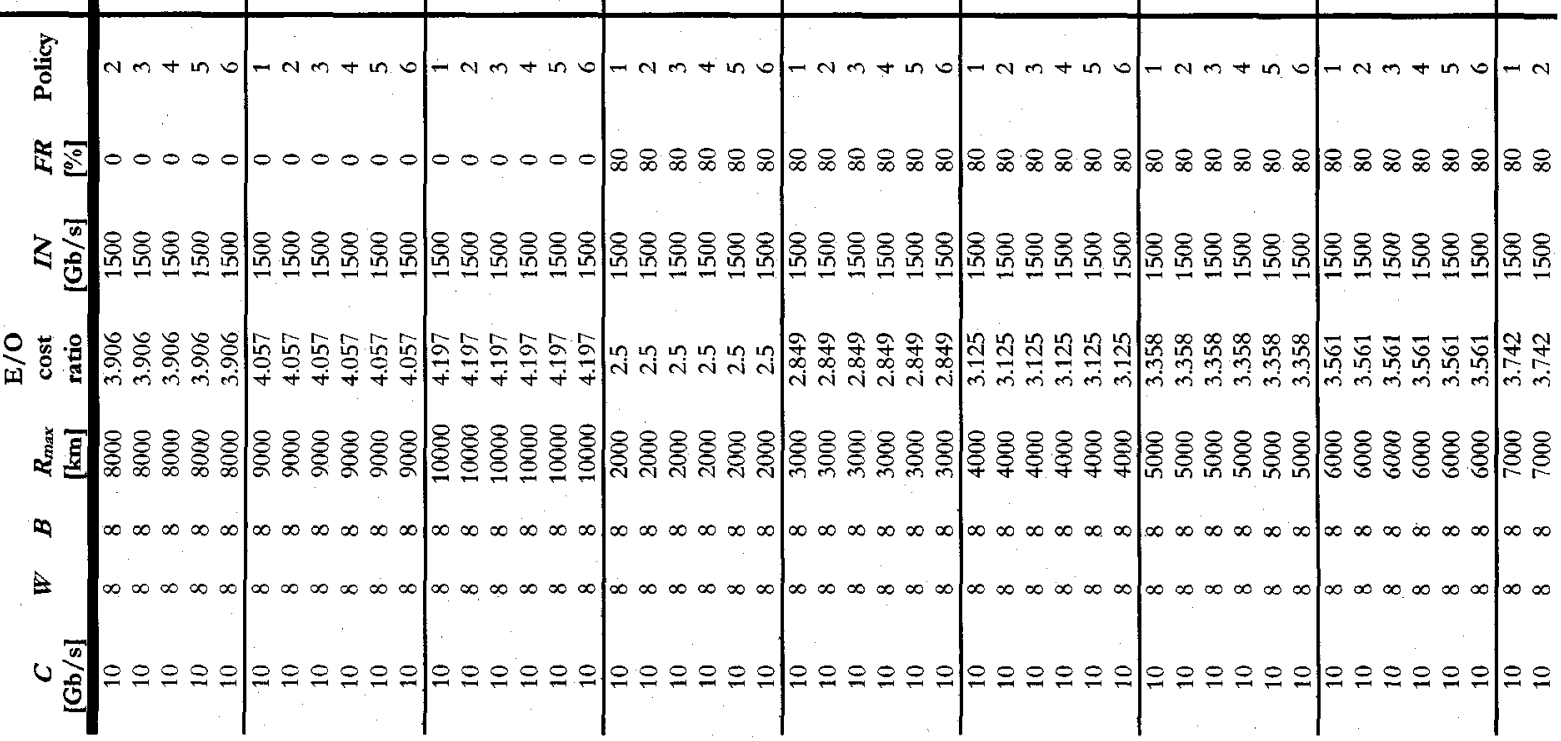




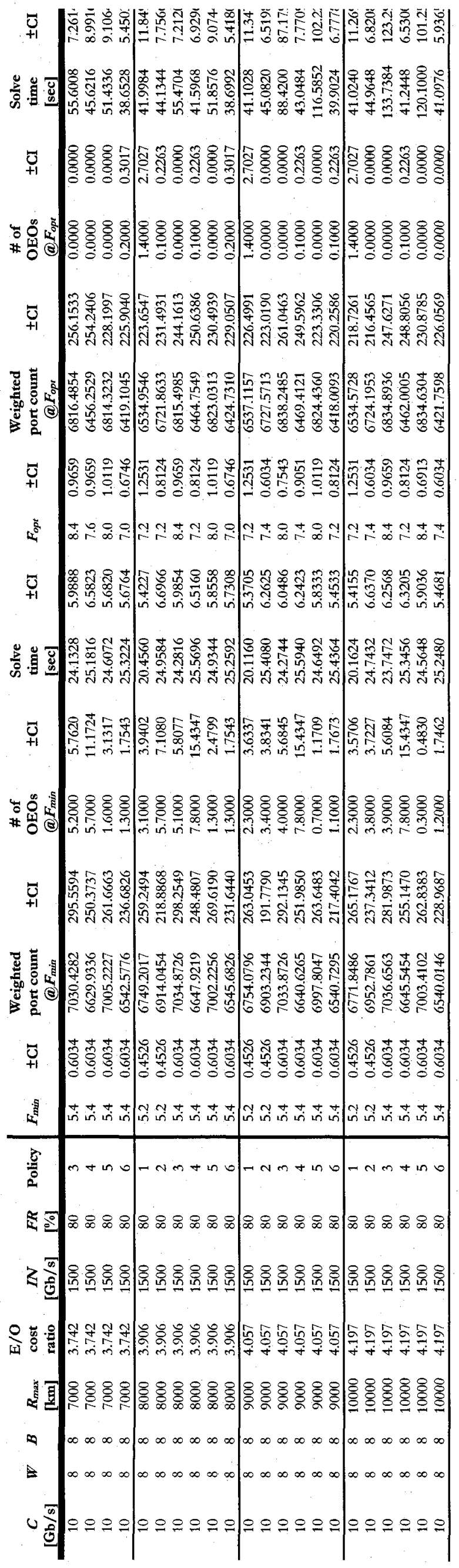


的等

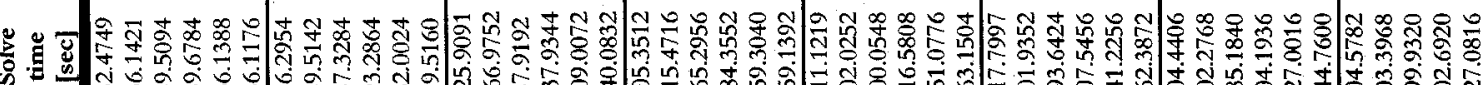

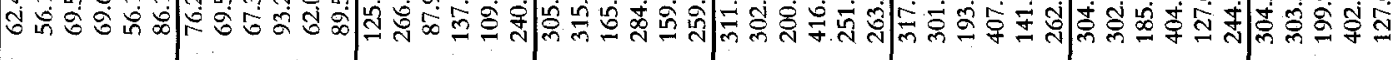

:

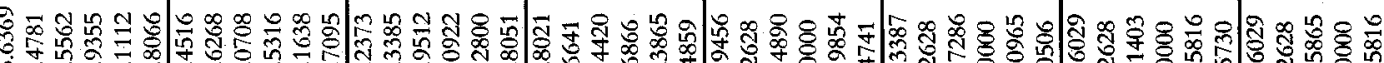

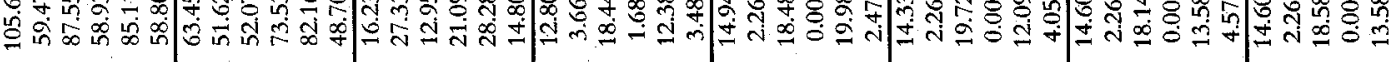

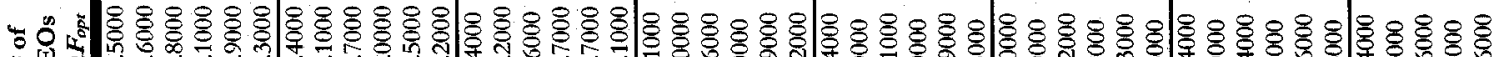

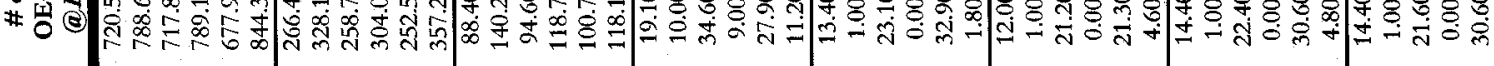

$\underset{+1}{\square}$

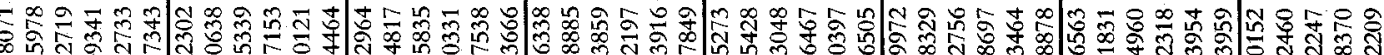

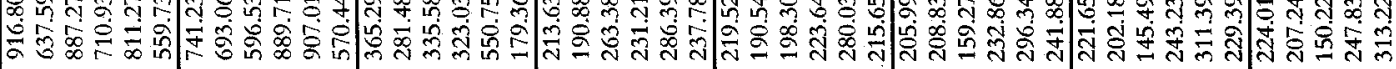

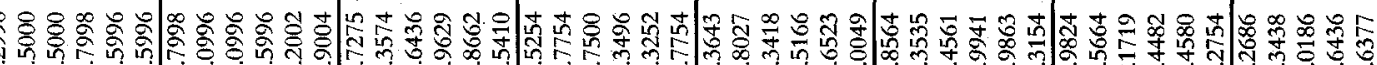

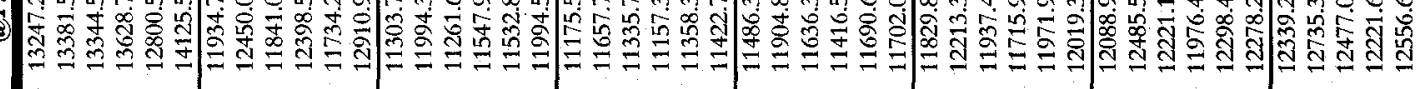

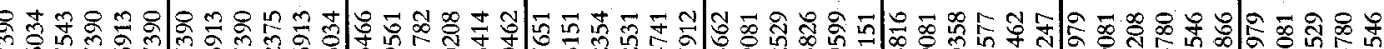

4

舟

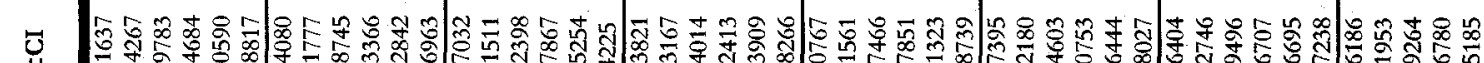

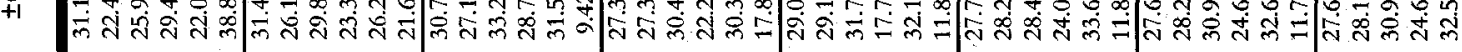

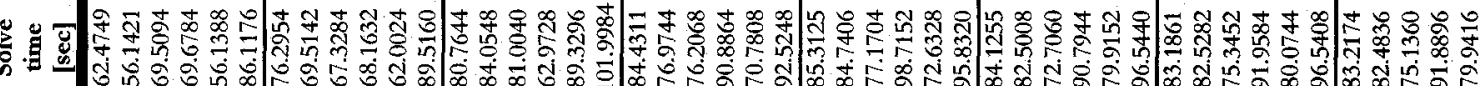

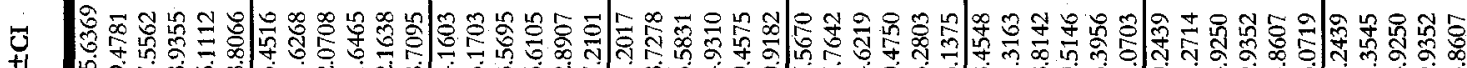

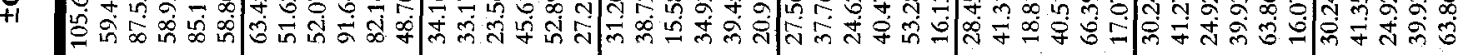

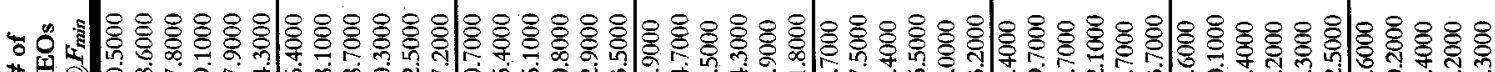

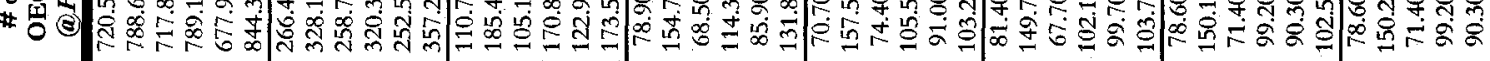

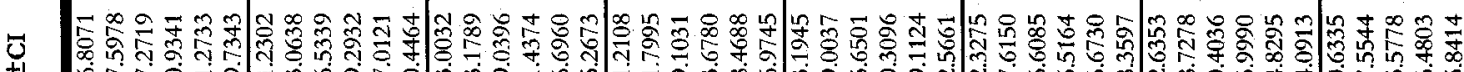

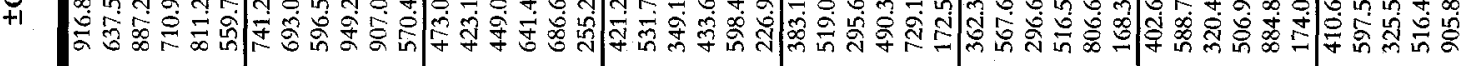

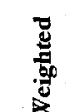

要

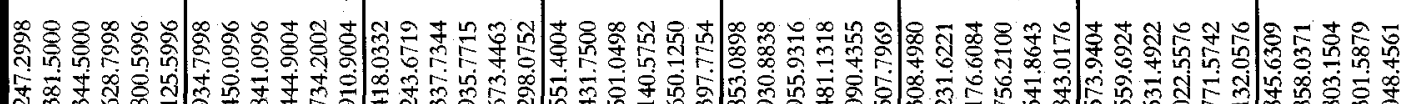

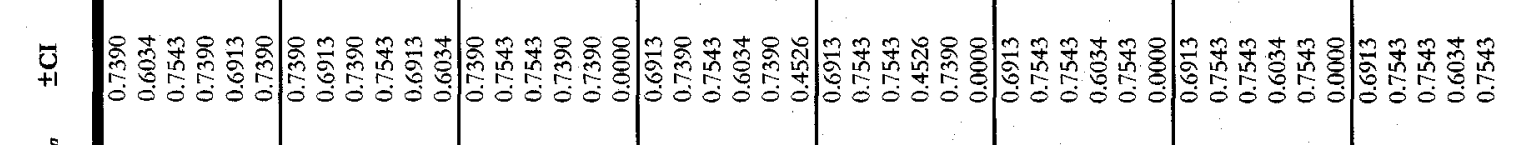

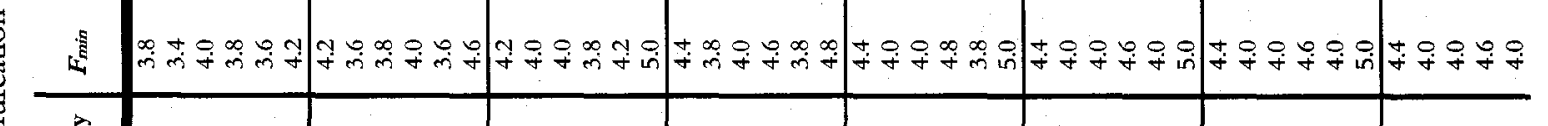
量 ! 章 8 

\& 동

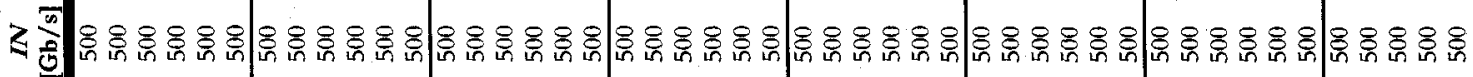

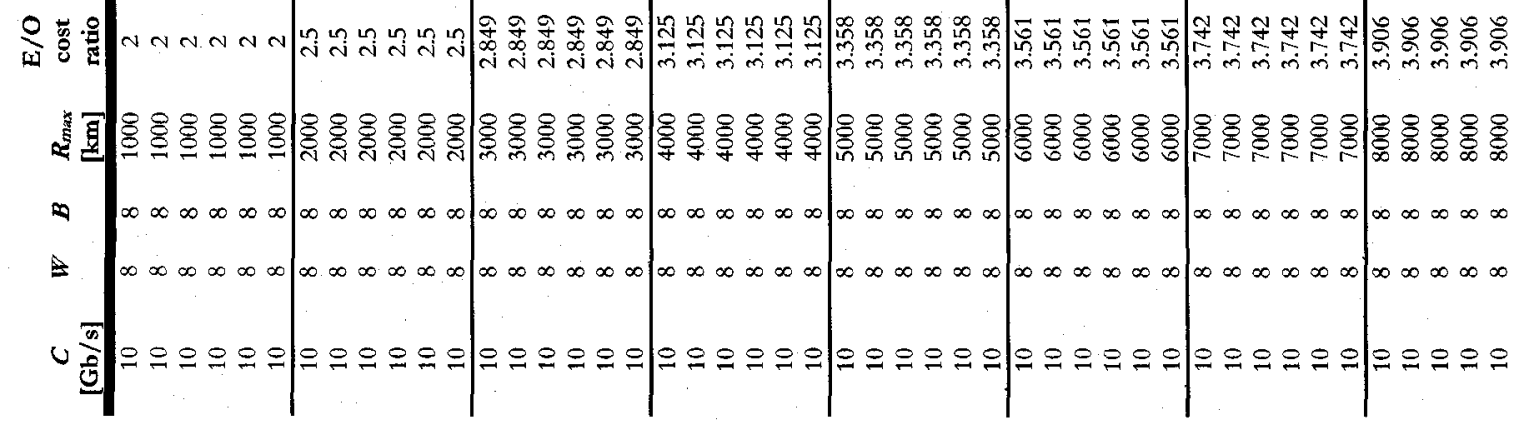




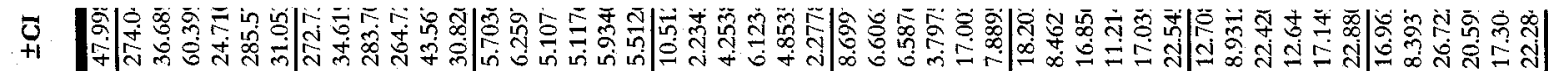

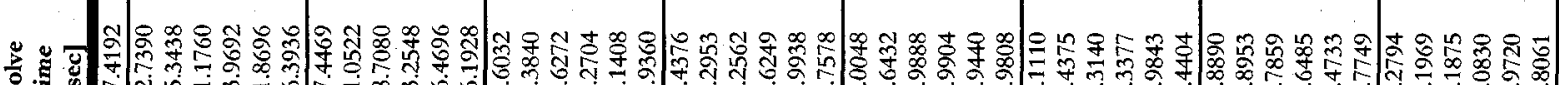

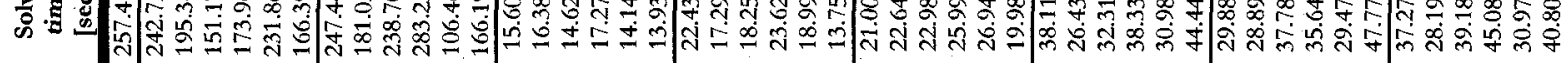

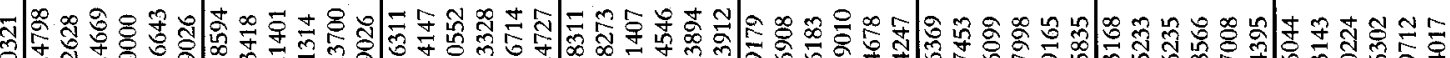

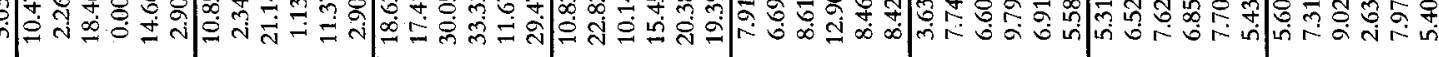

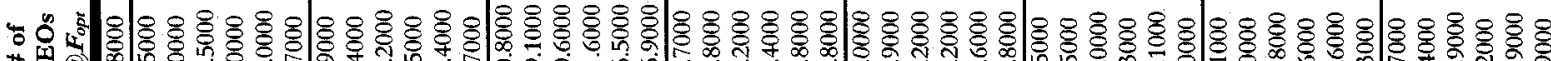

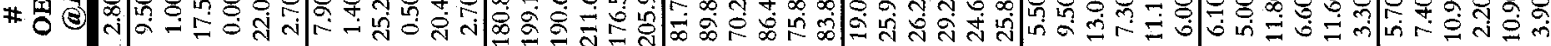

$\bar{O}$

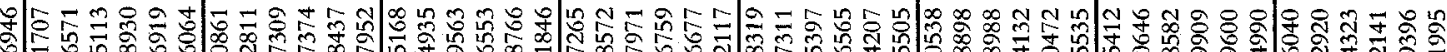

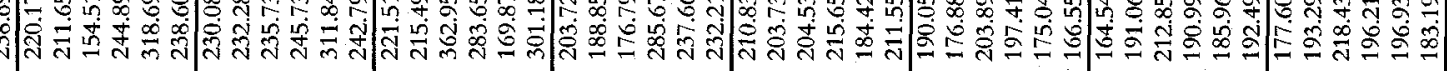

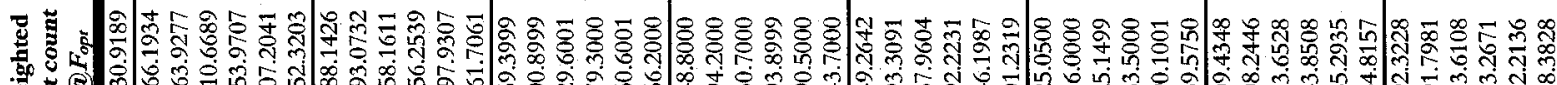

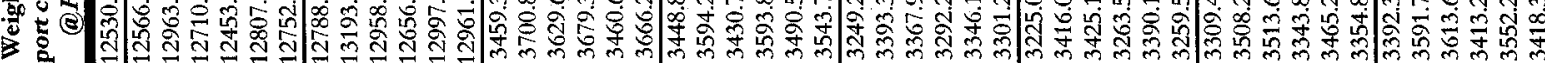

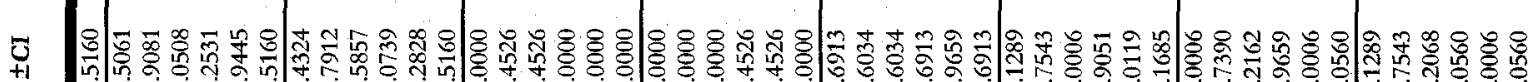
隹

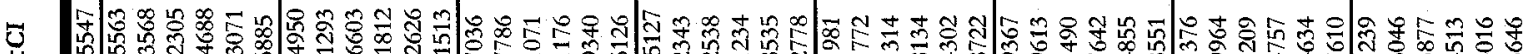

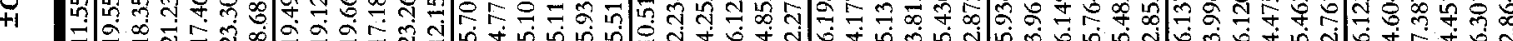

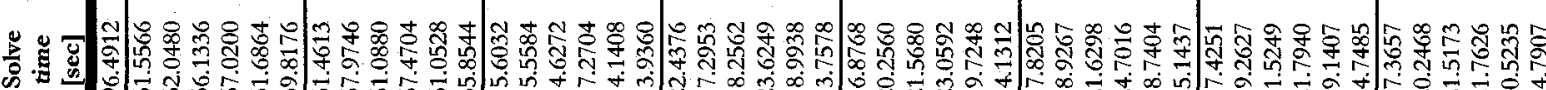

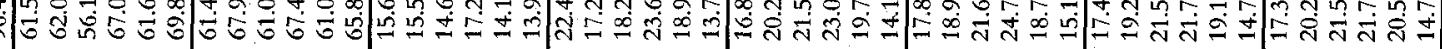

t⿹

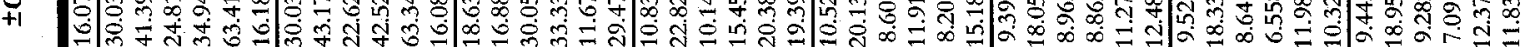

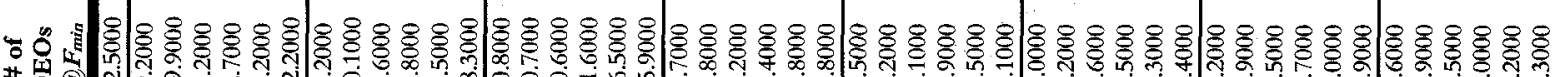

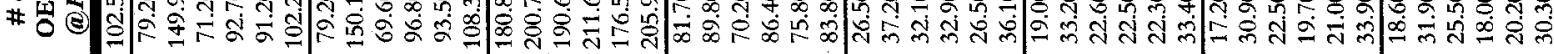

†

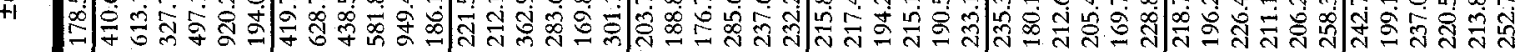

‡

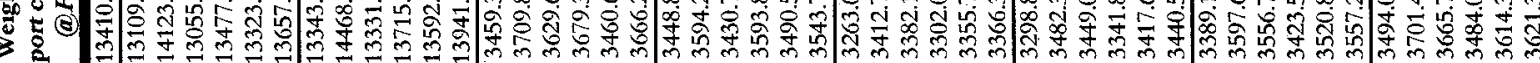
5

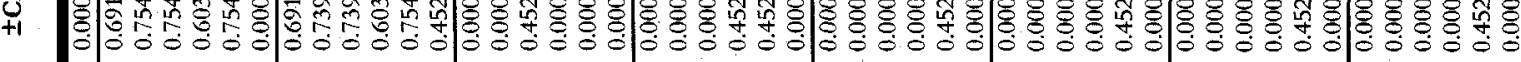

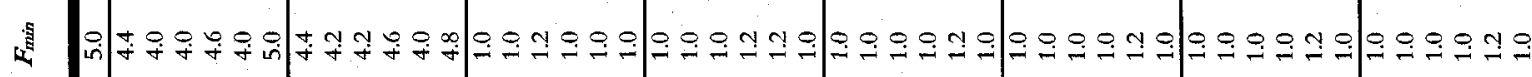

象

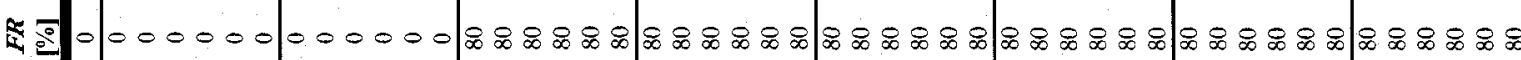

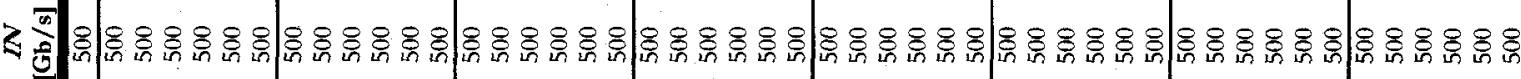

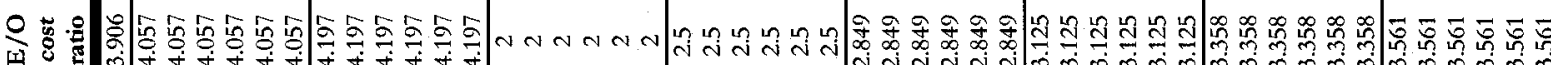

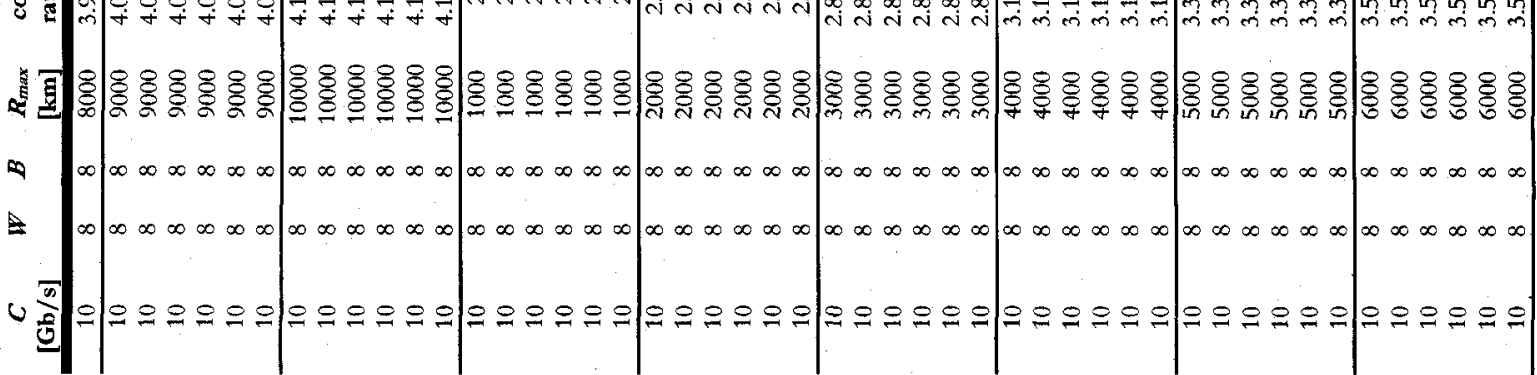




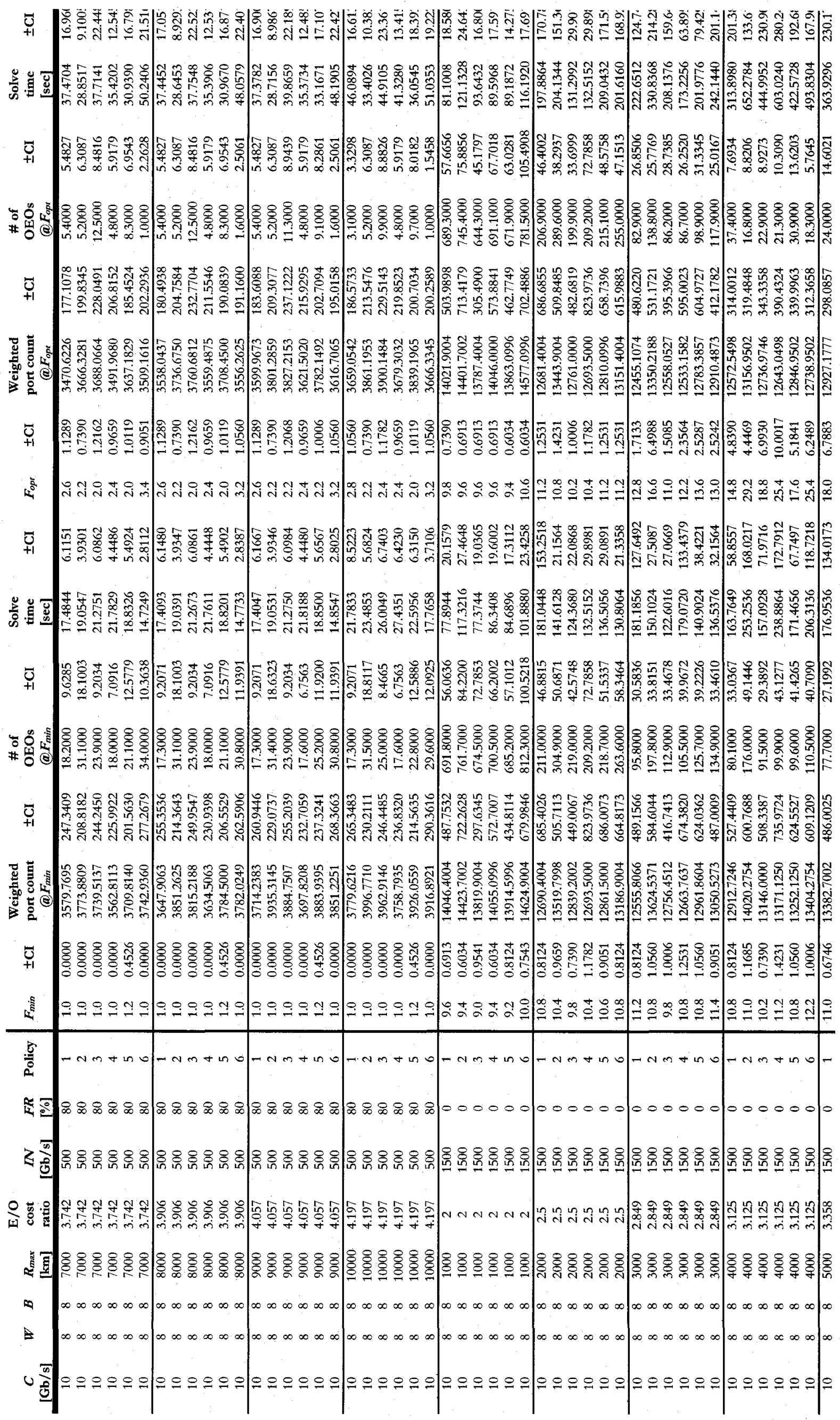




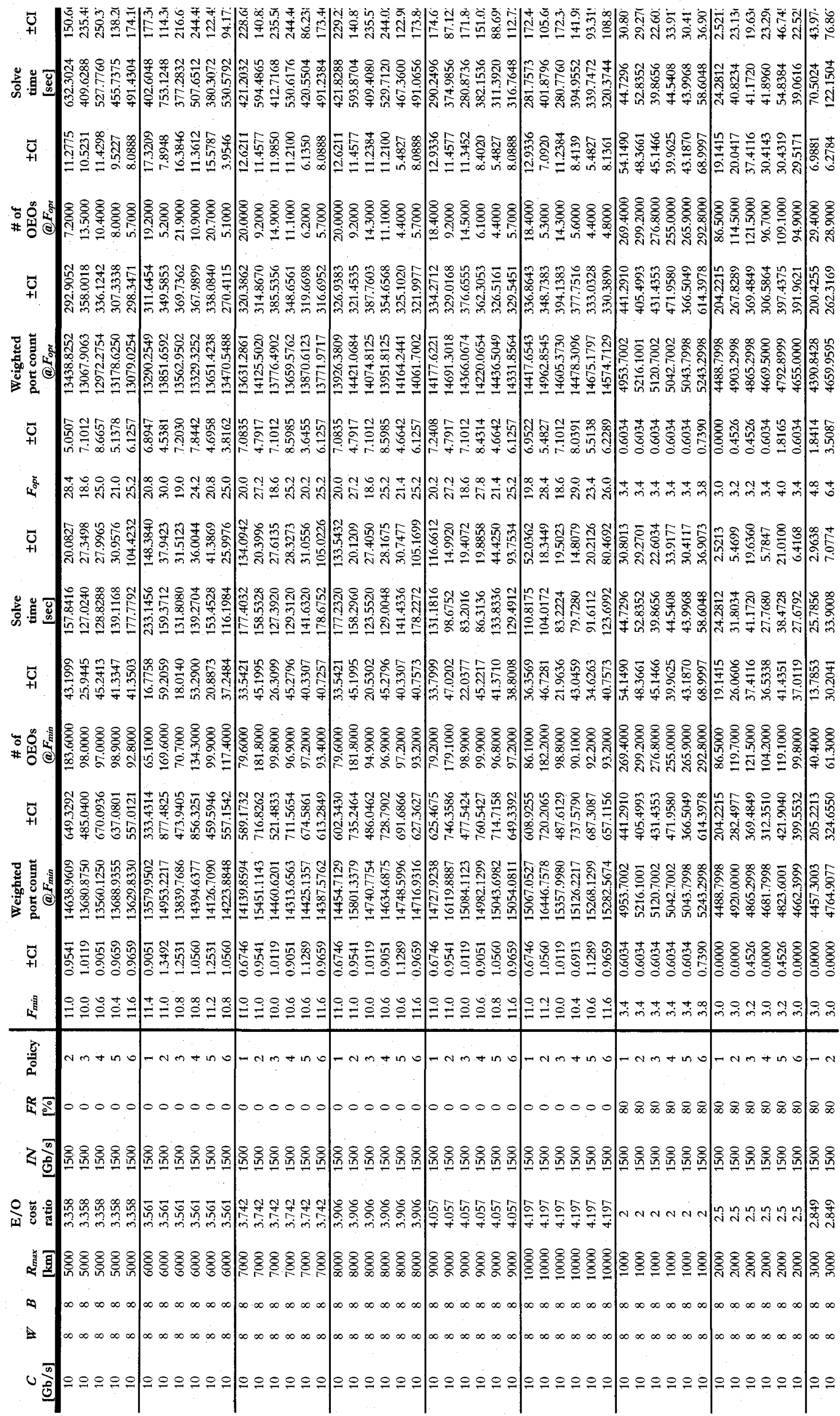




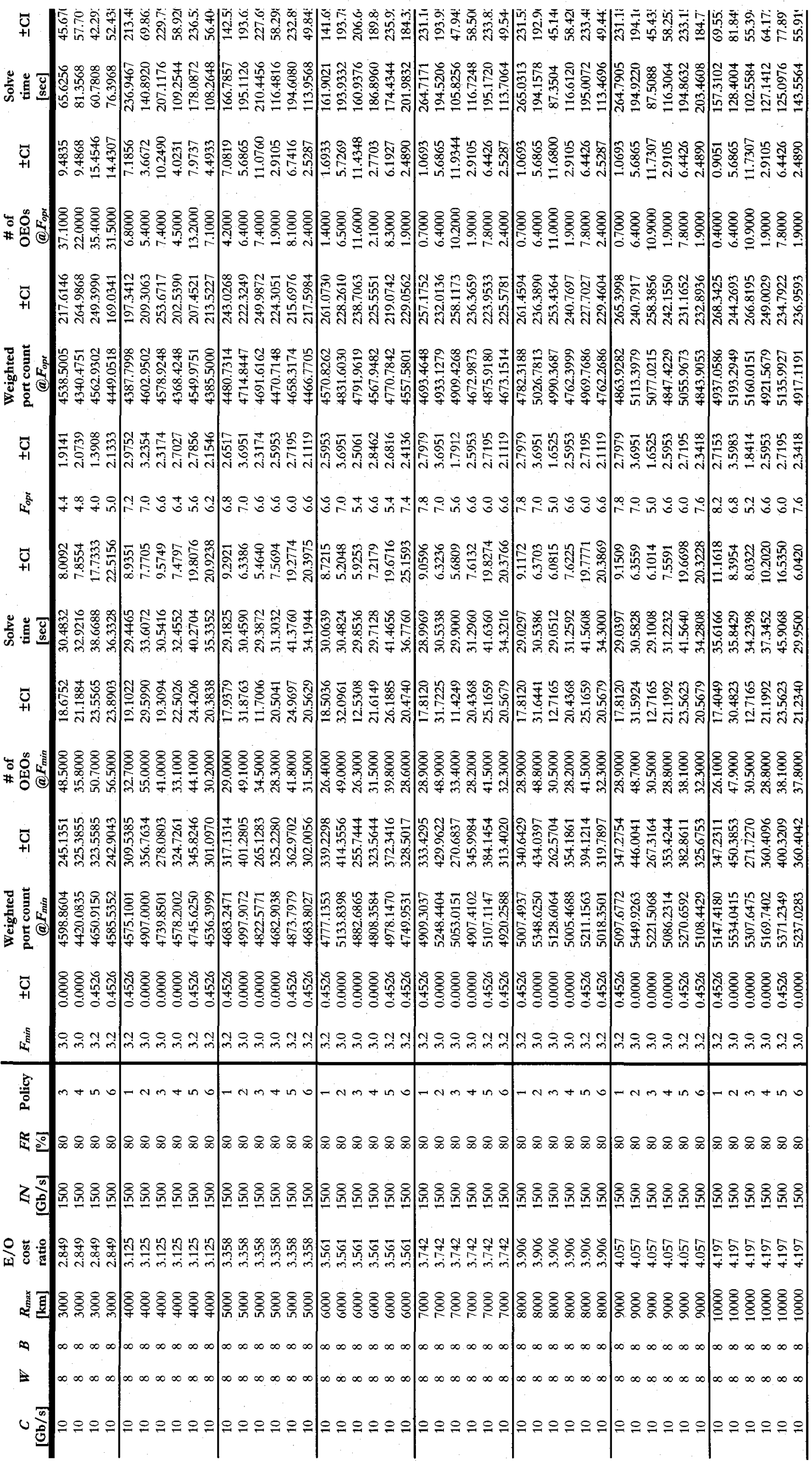




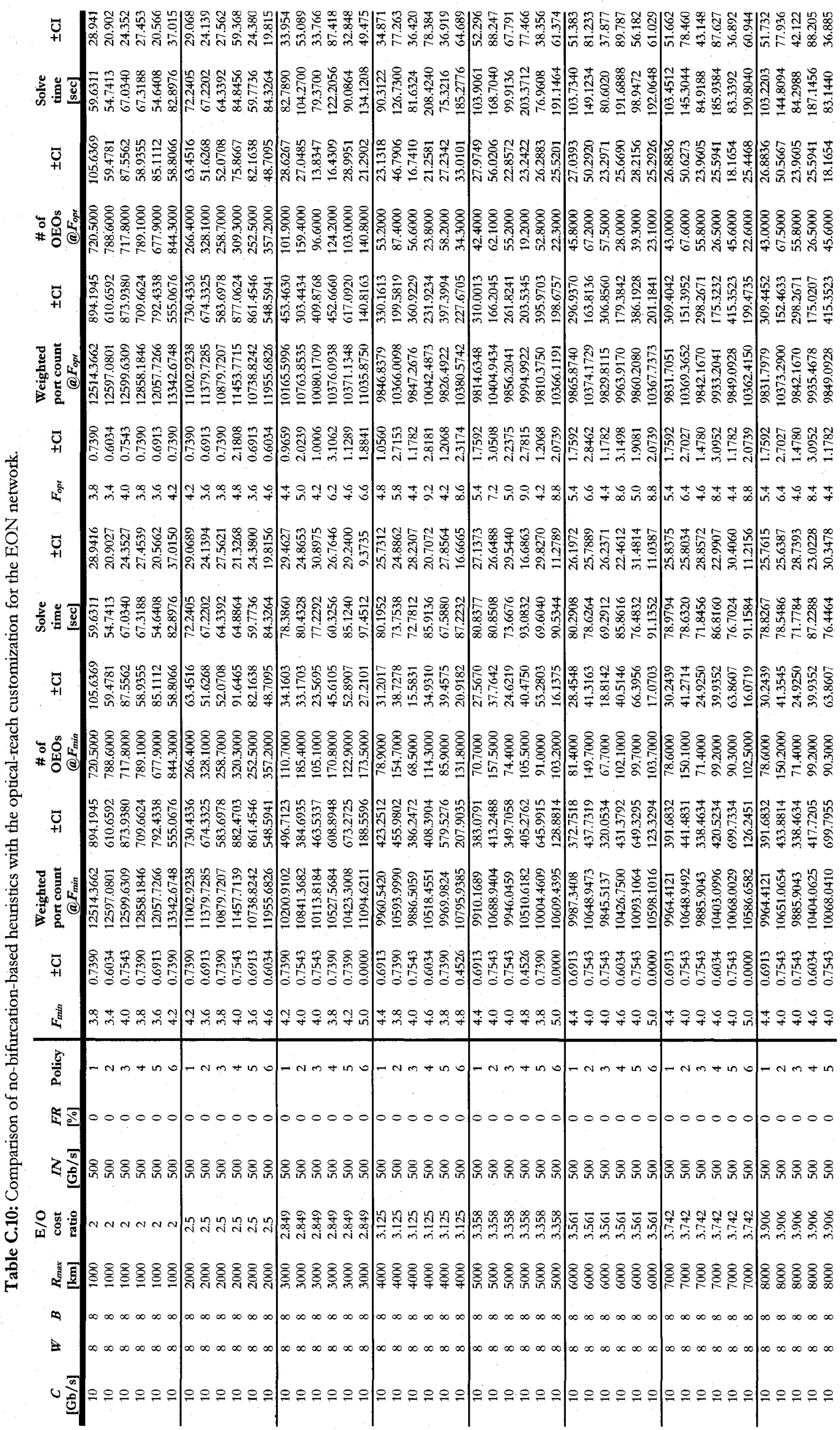




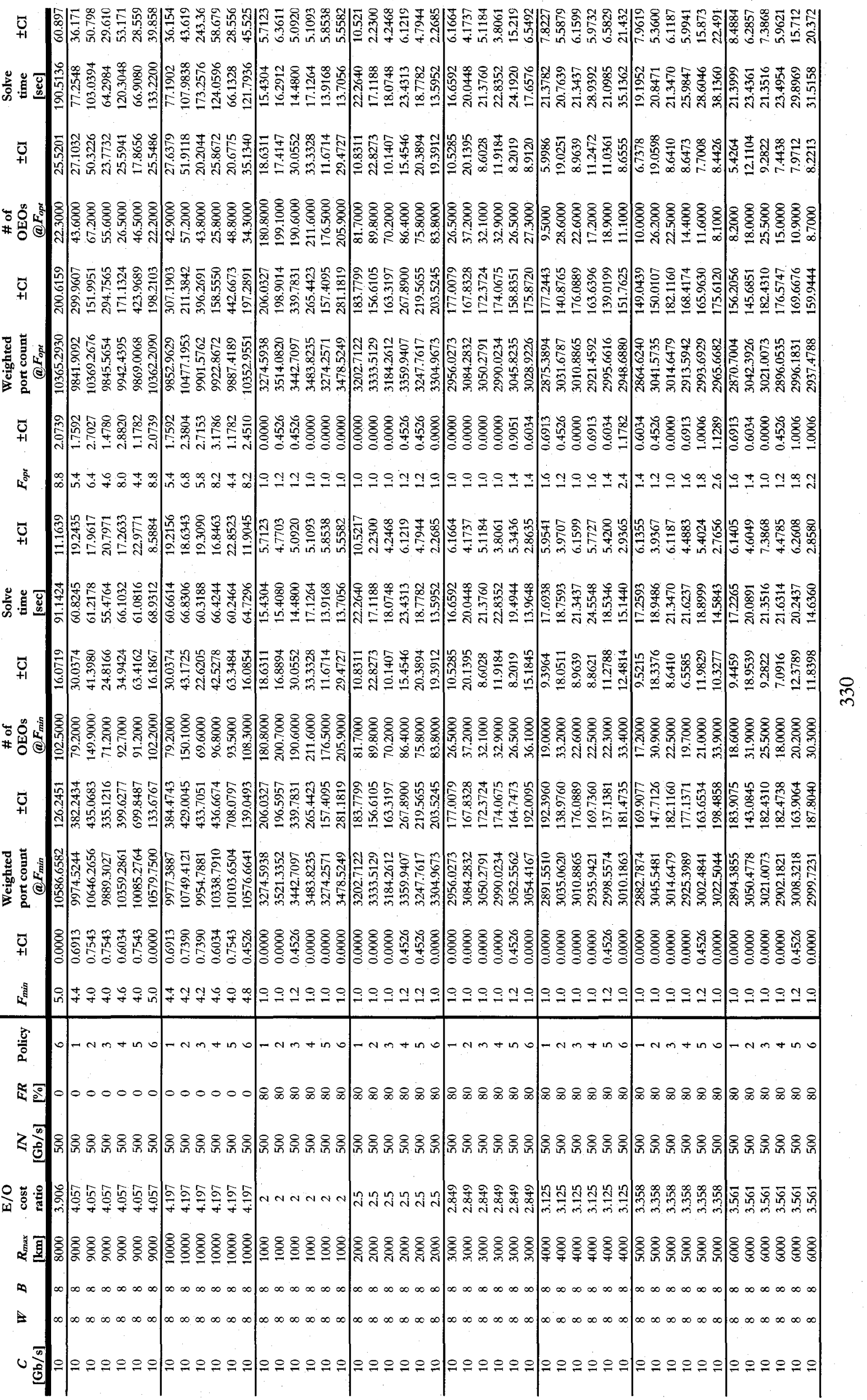




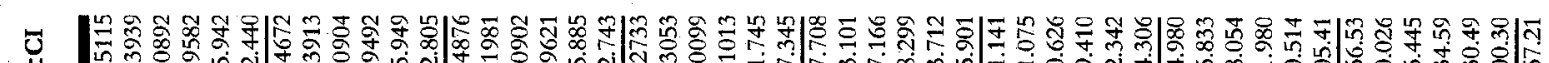
+1

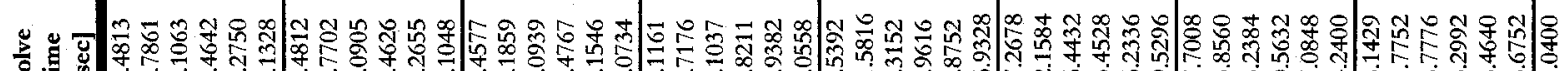

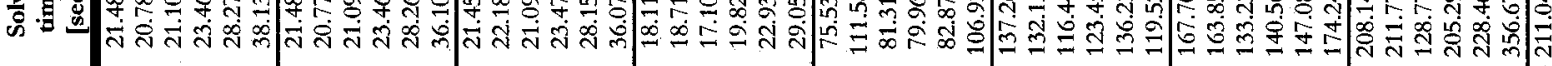

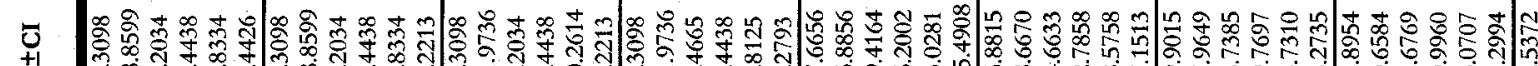
+1 in

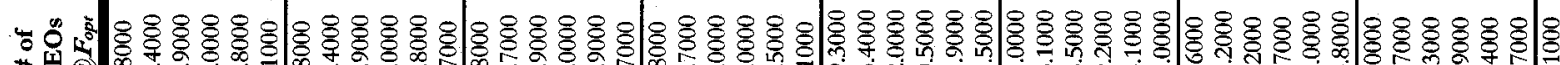

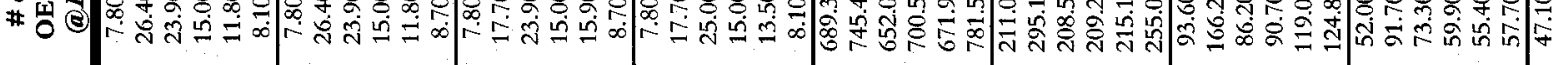

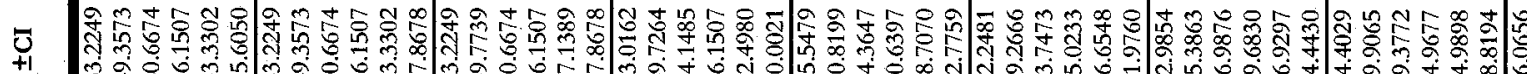

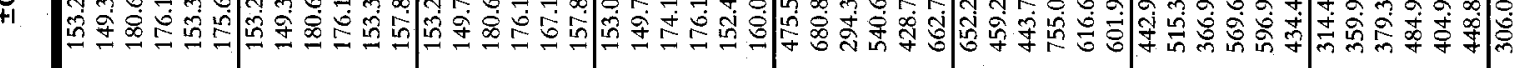

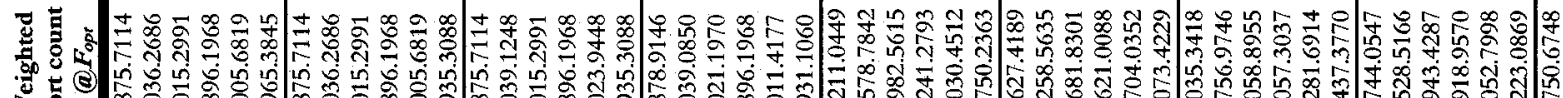

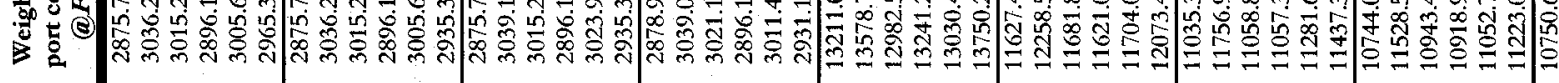

芰 논

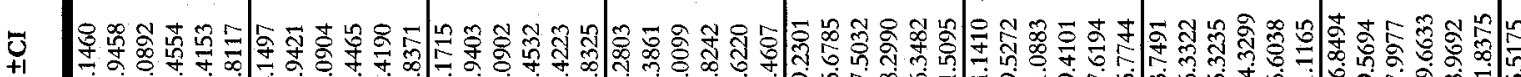

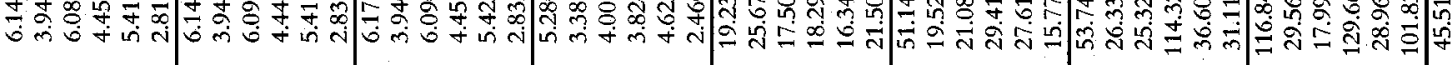

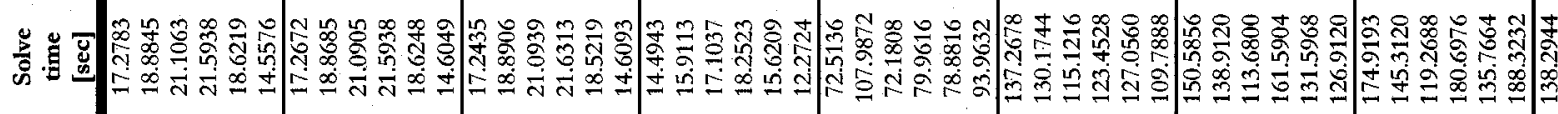

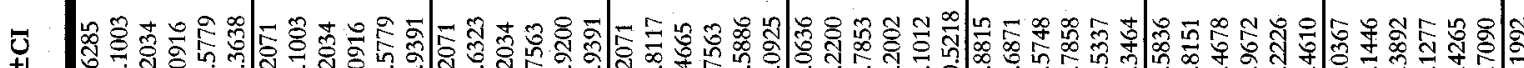

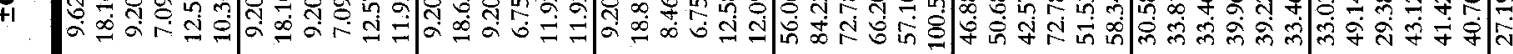

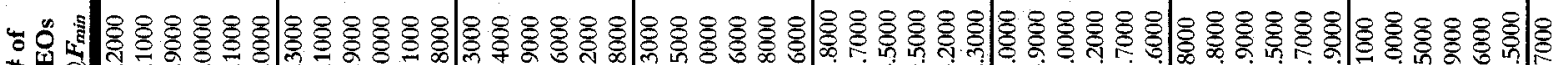

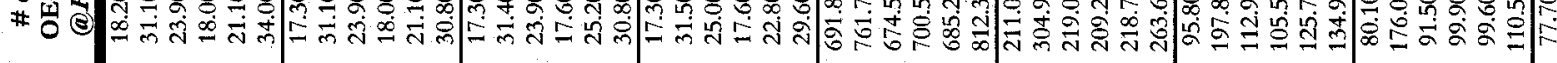

to

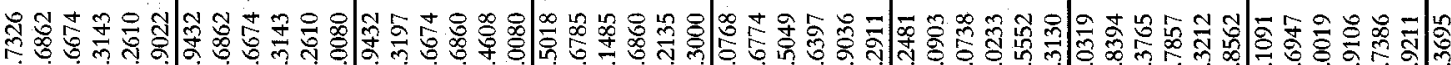

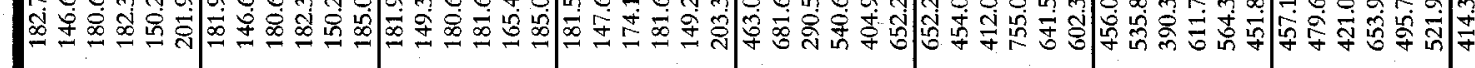

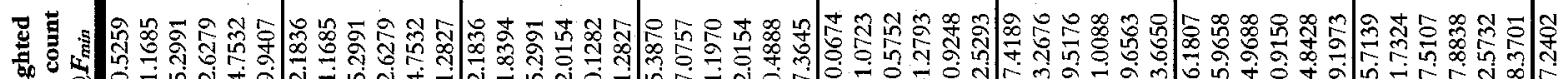

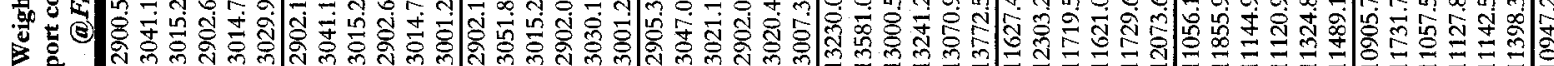

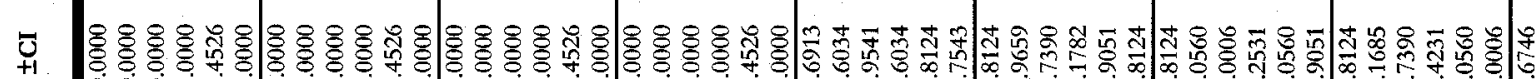
+1

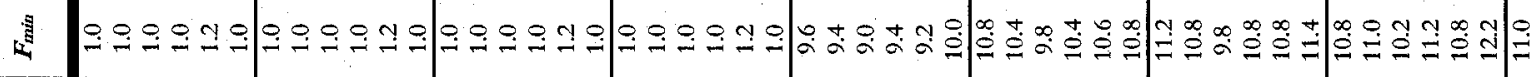

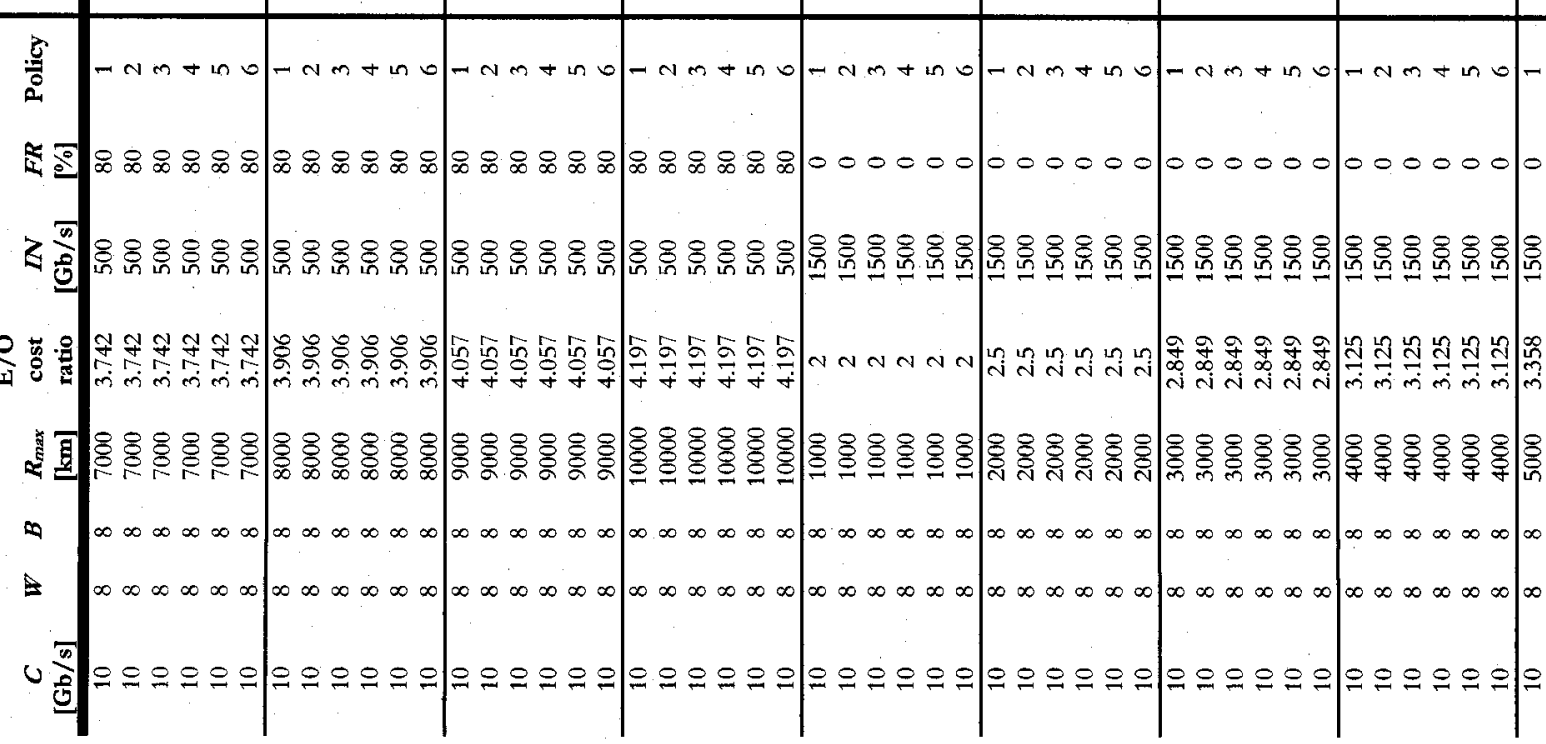




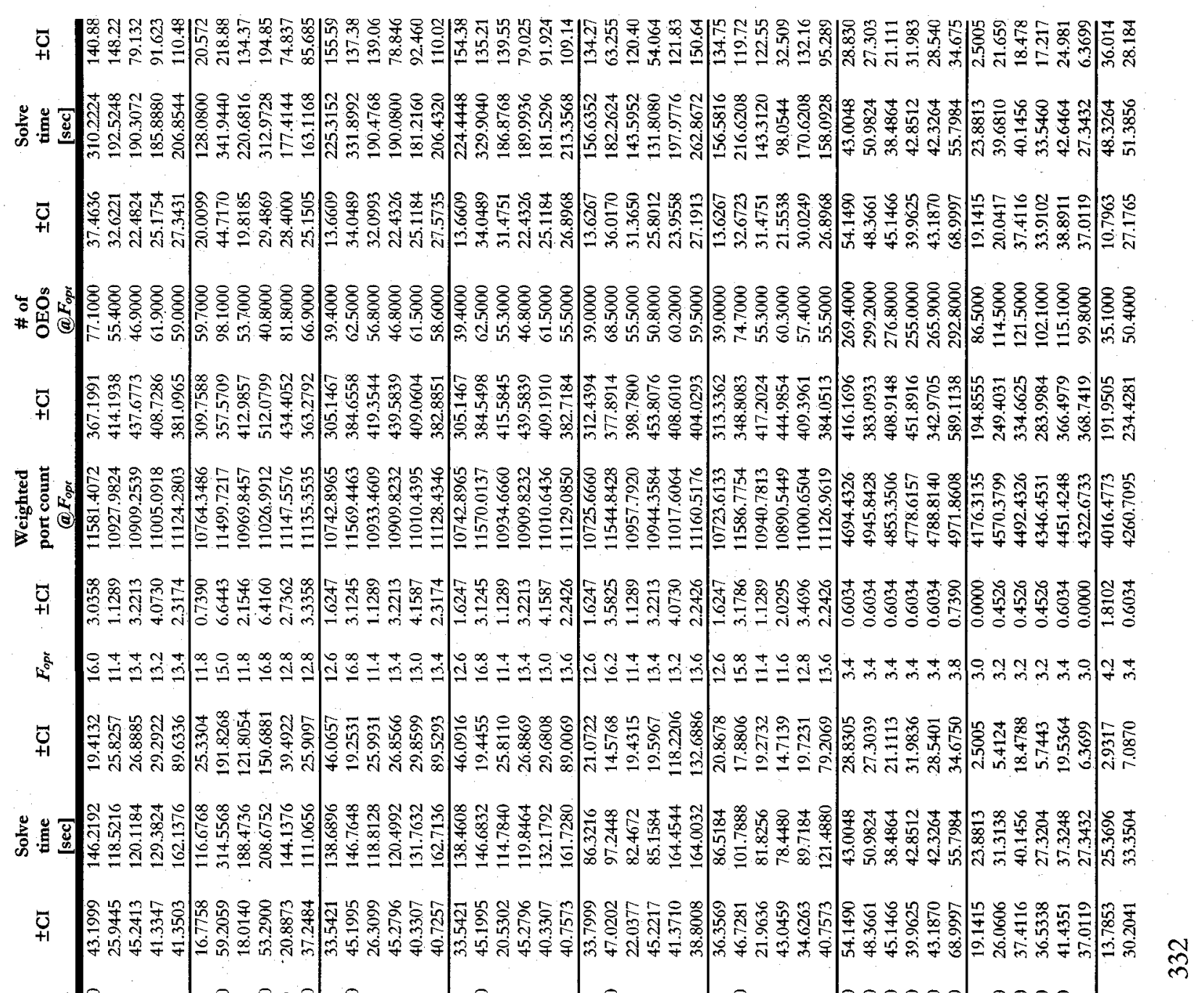

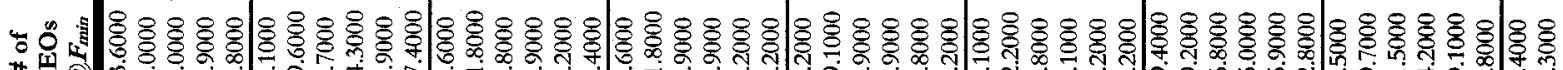

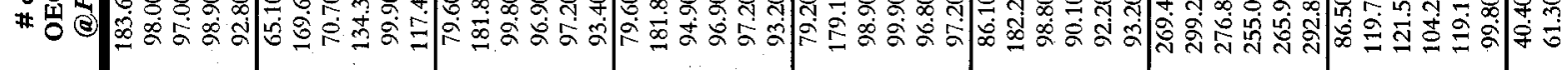

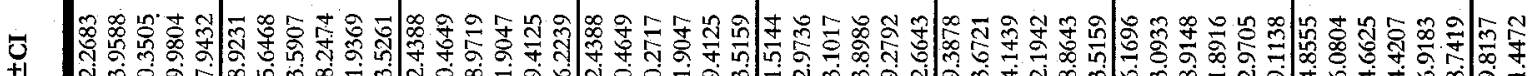

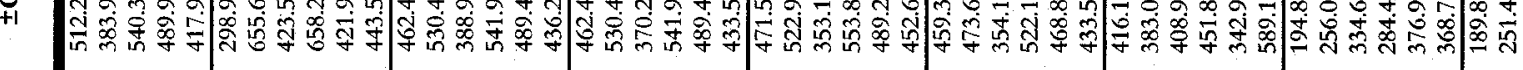

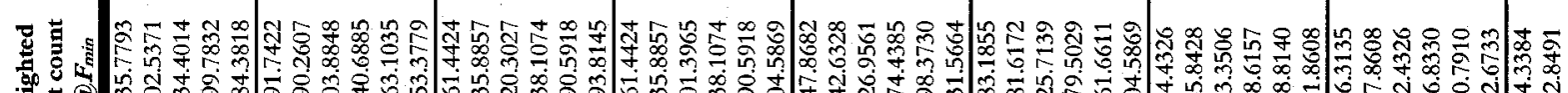

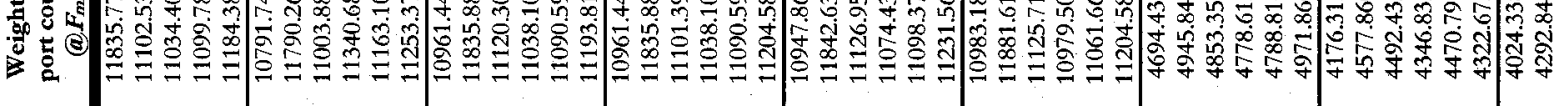

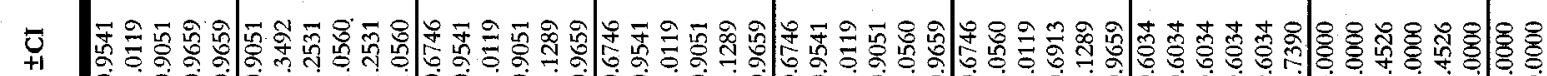
长

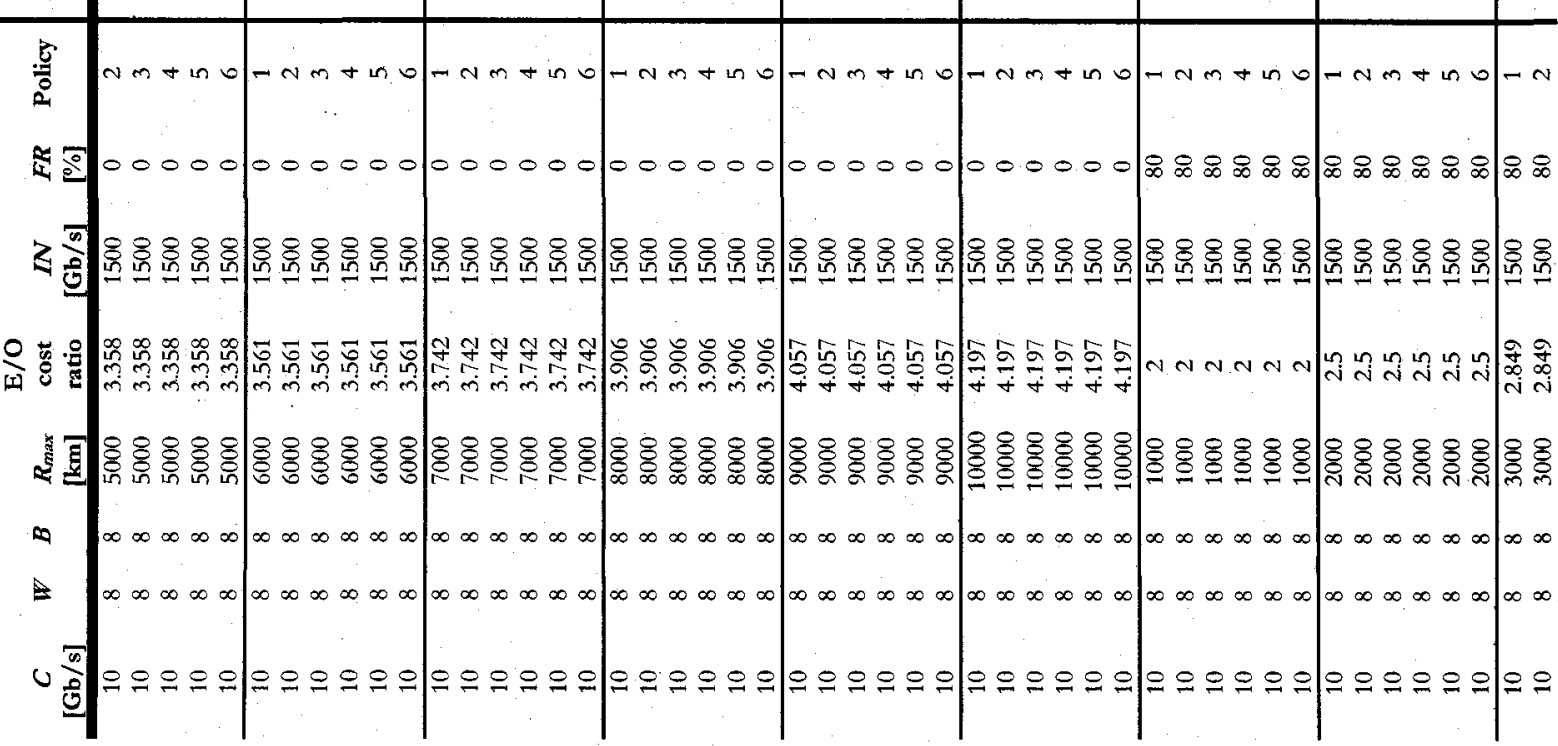




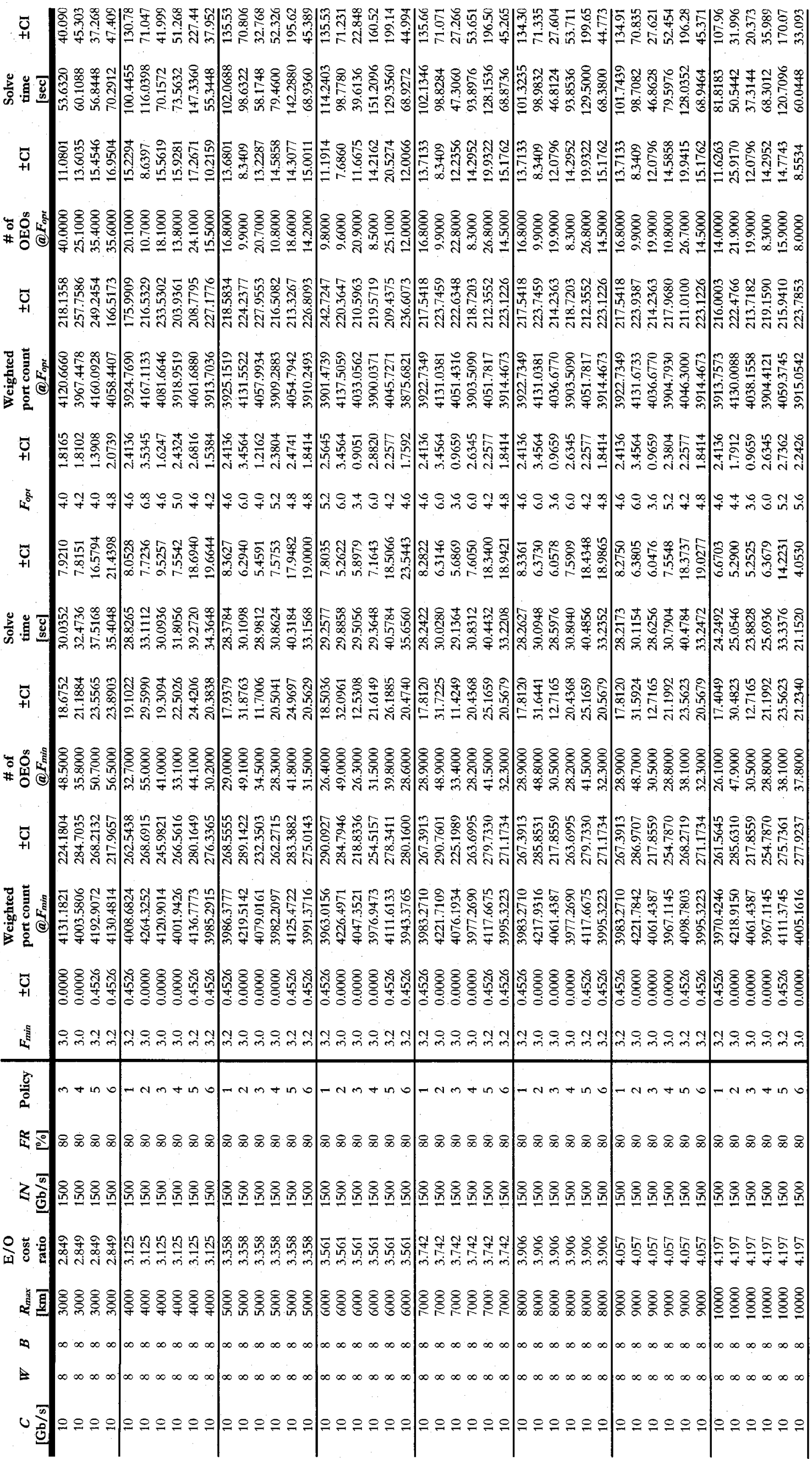




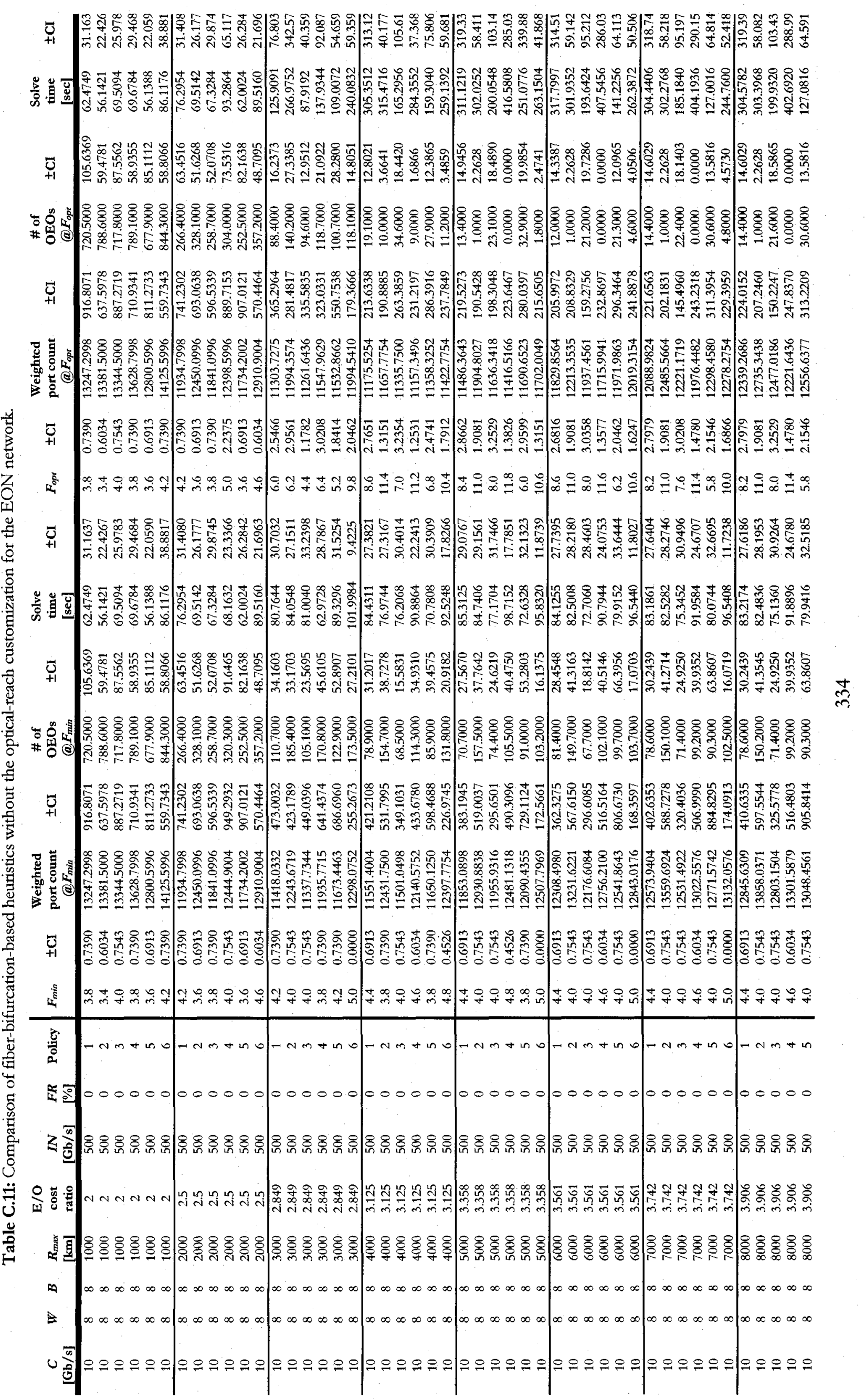




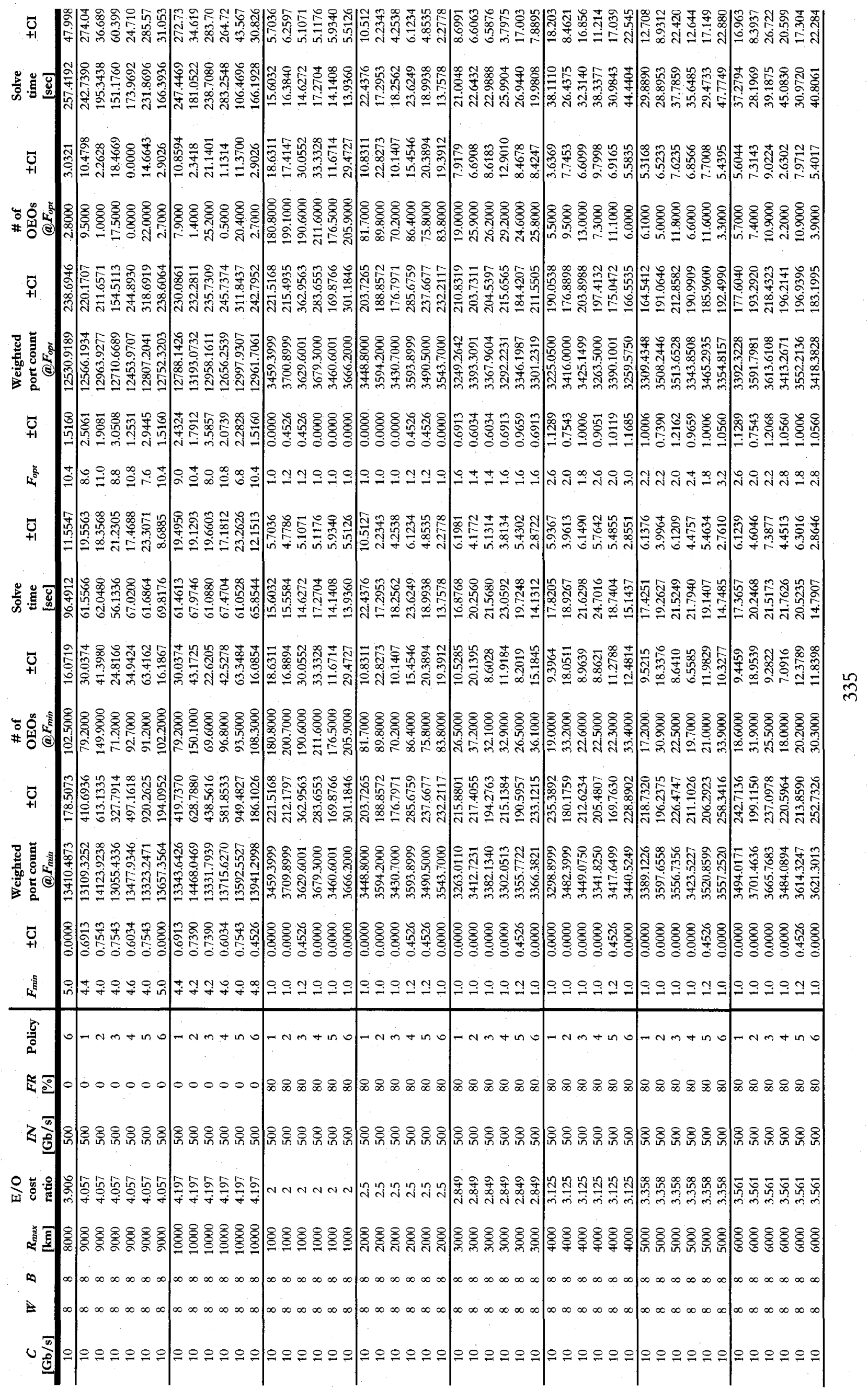




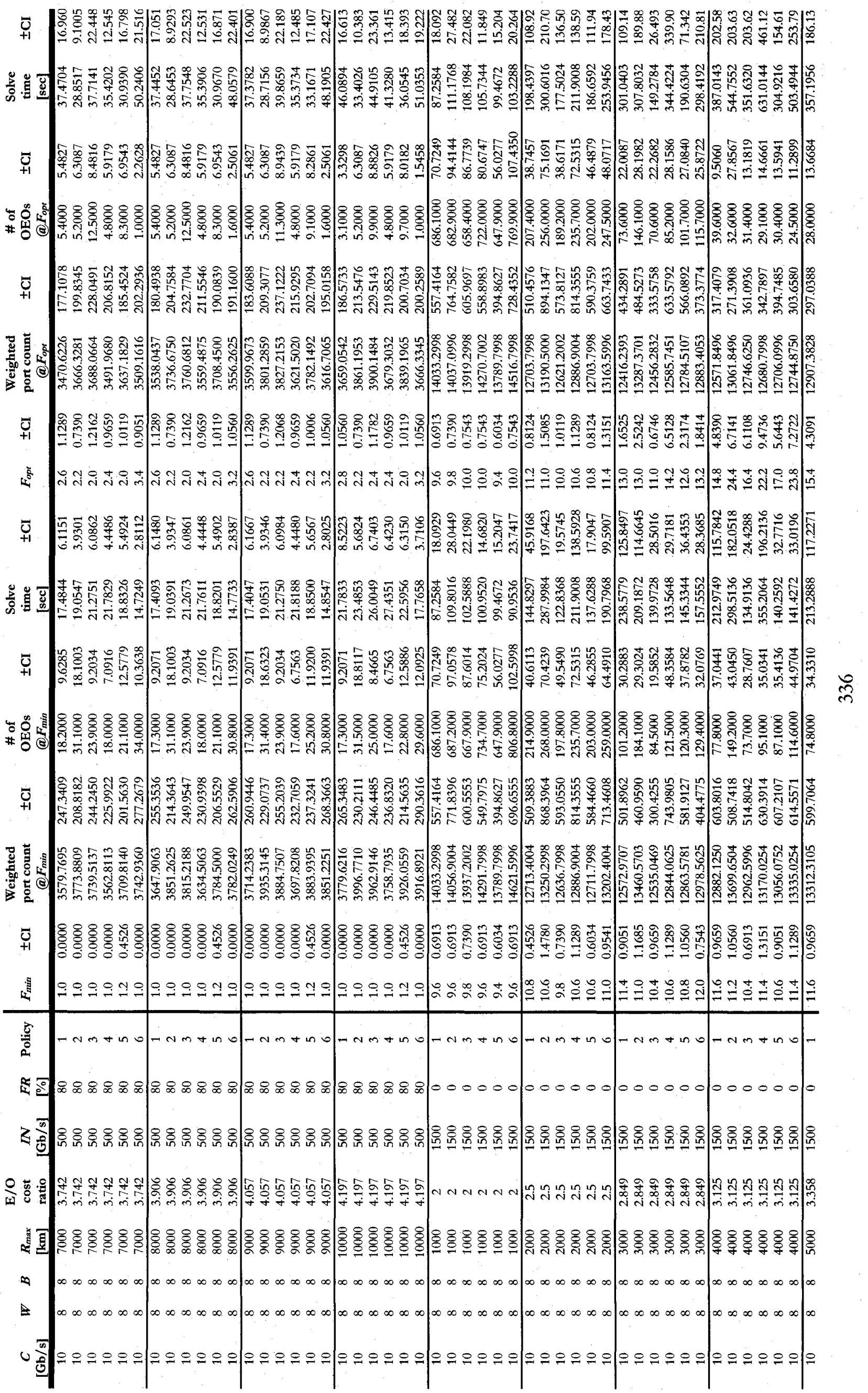




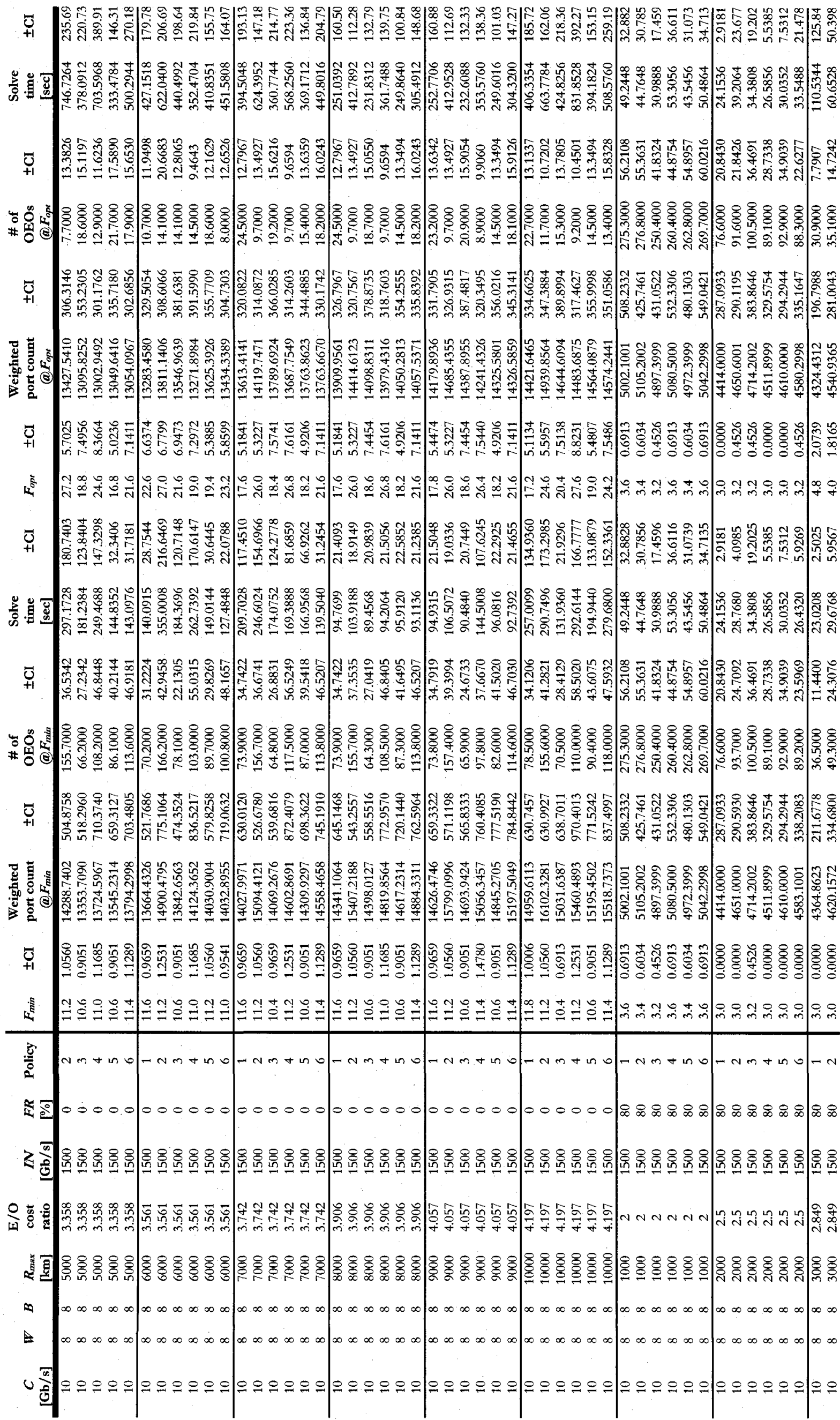




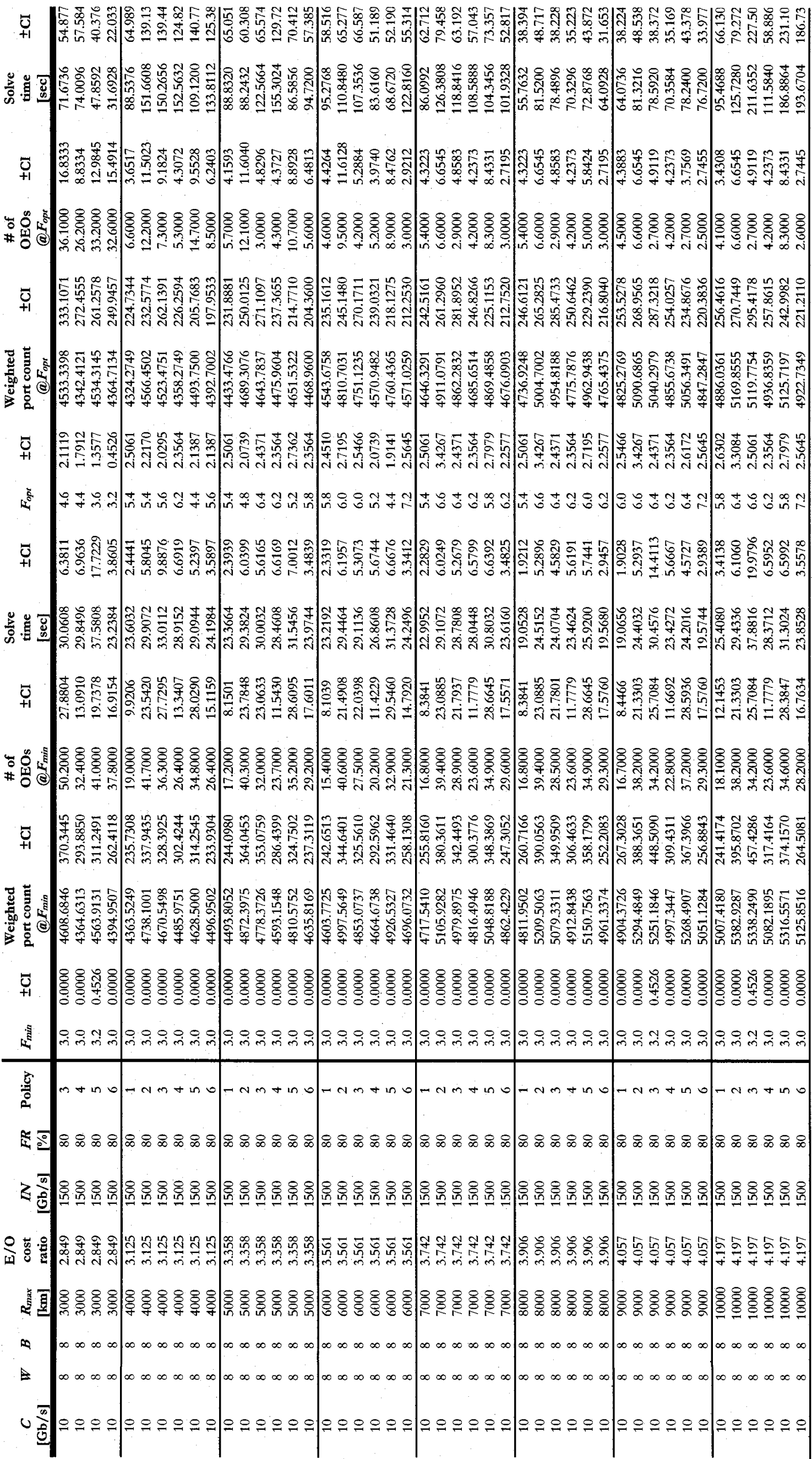




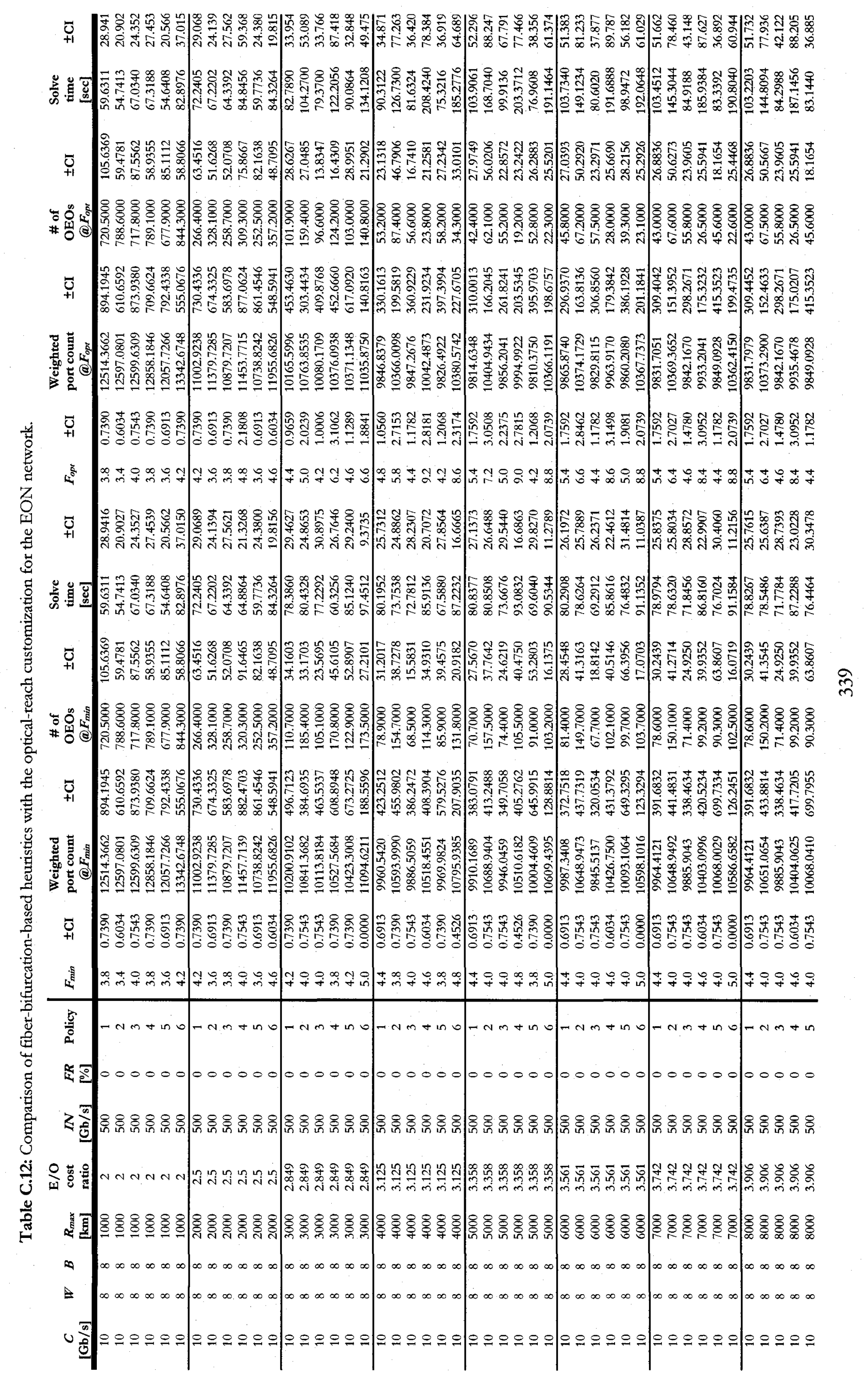




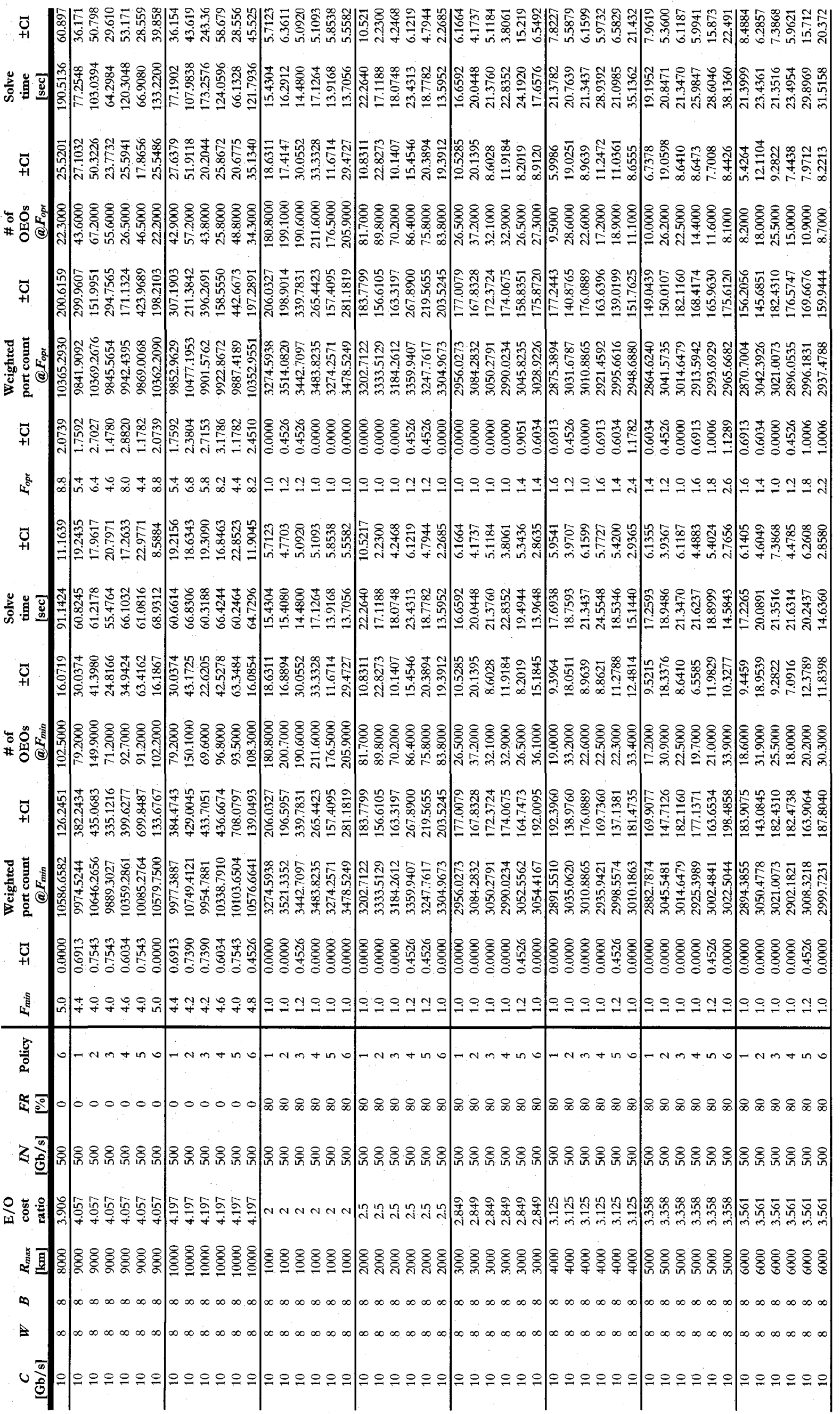




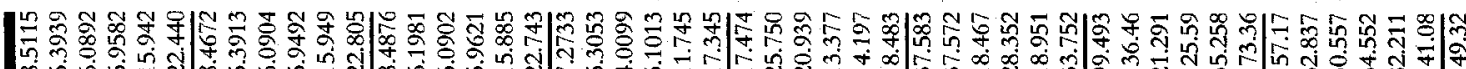

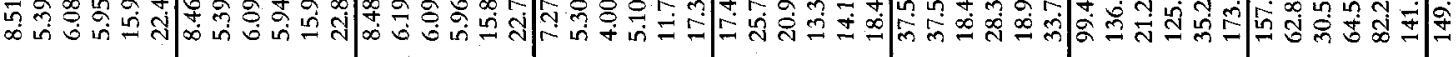

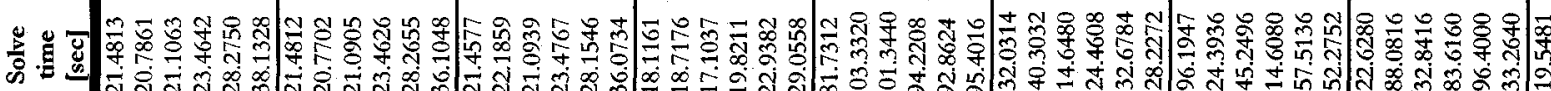

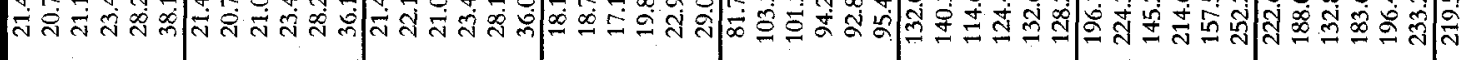

ت্

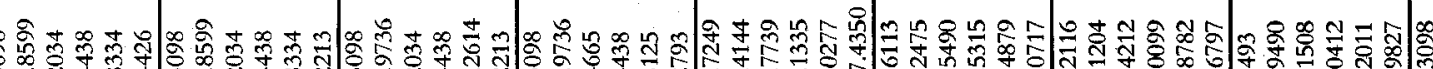

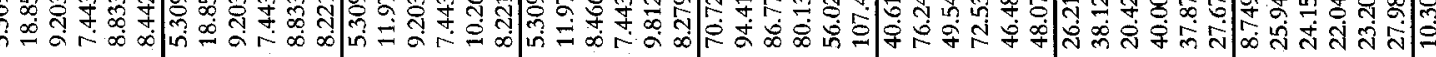

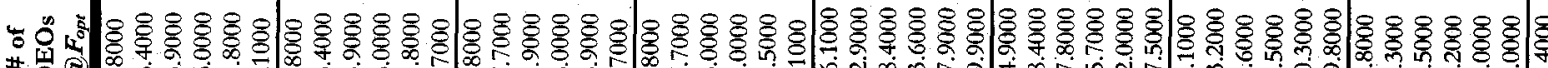

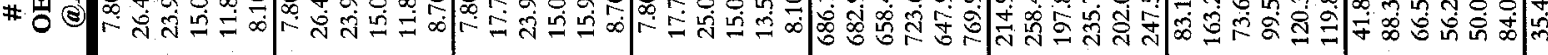

$\overline{+i}$

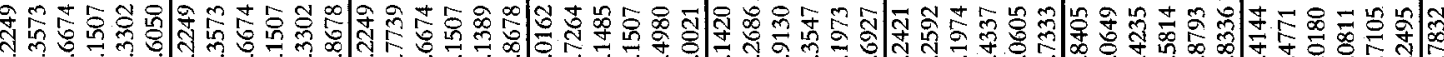

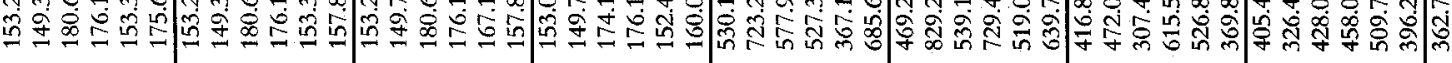

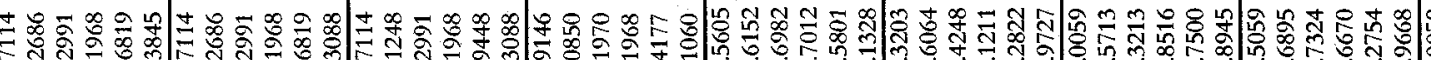

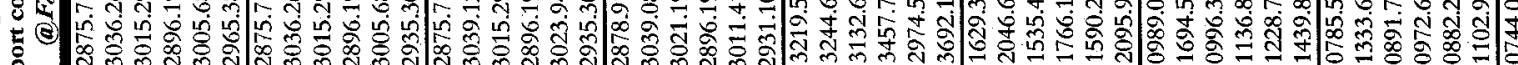

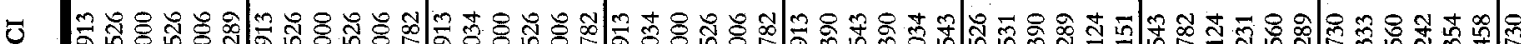
$+$

$2^{\circ}$

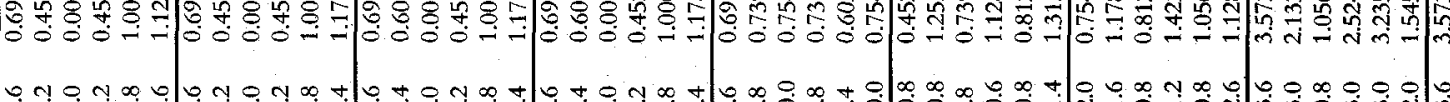

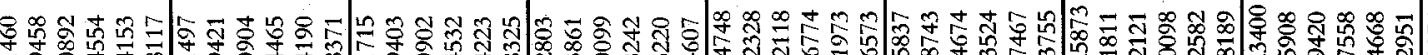

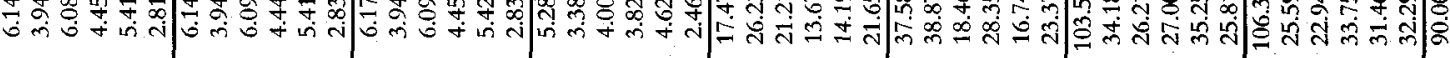

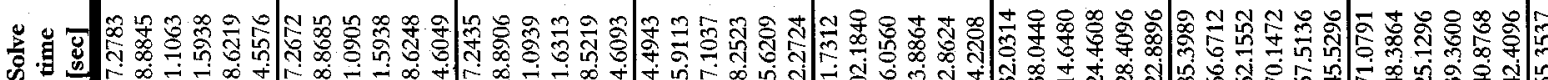

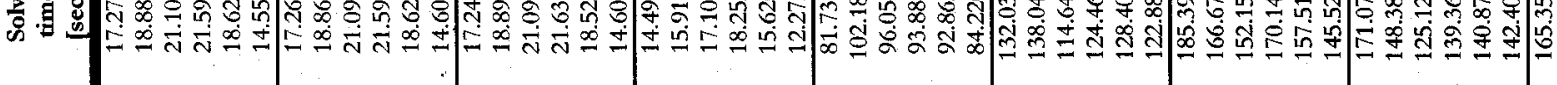

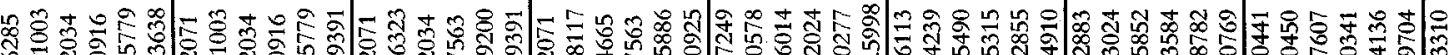
全

૫ 연

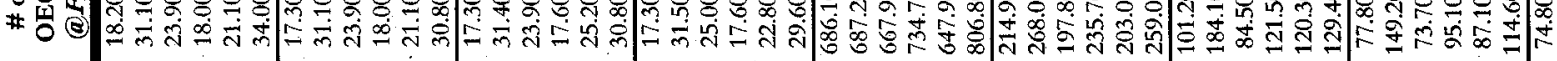

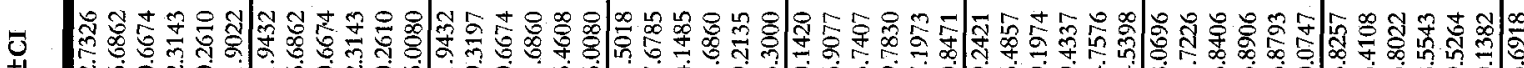

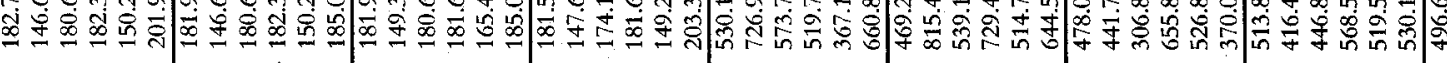

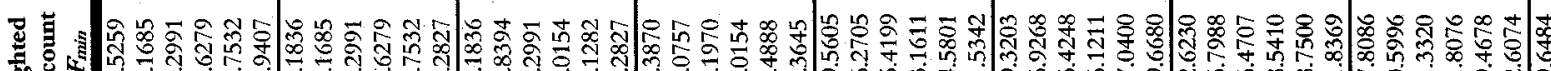

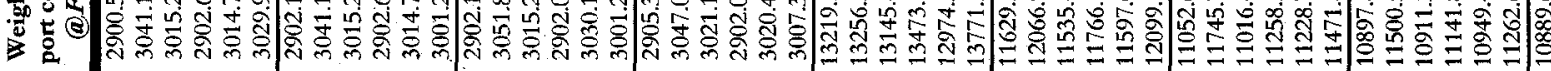

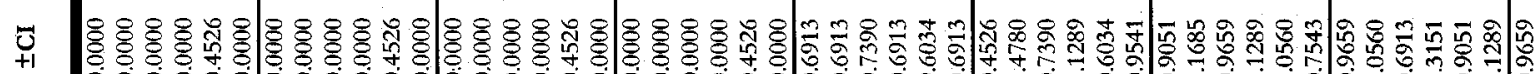

直

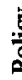

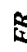

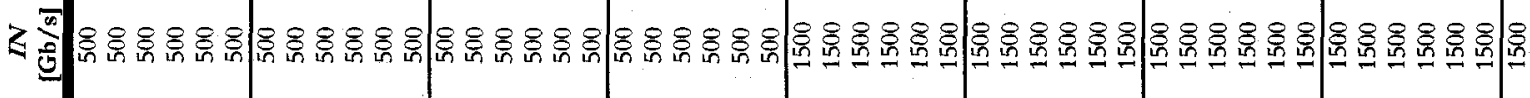

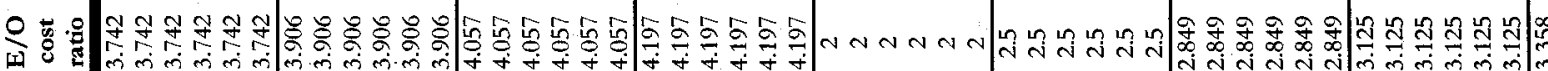

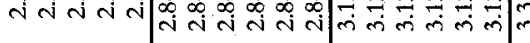

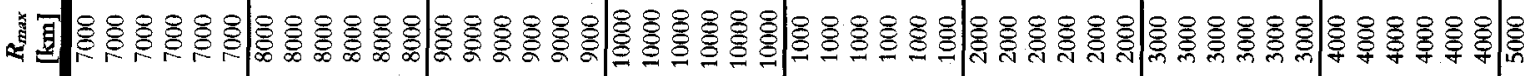

$\infty$

$B$

U. 


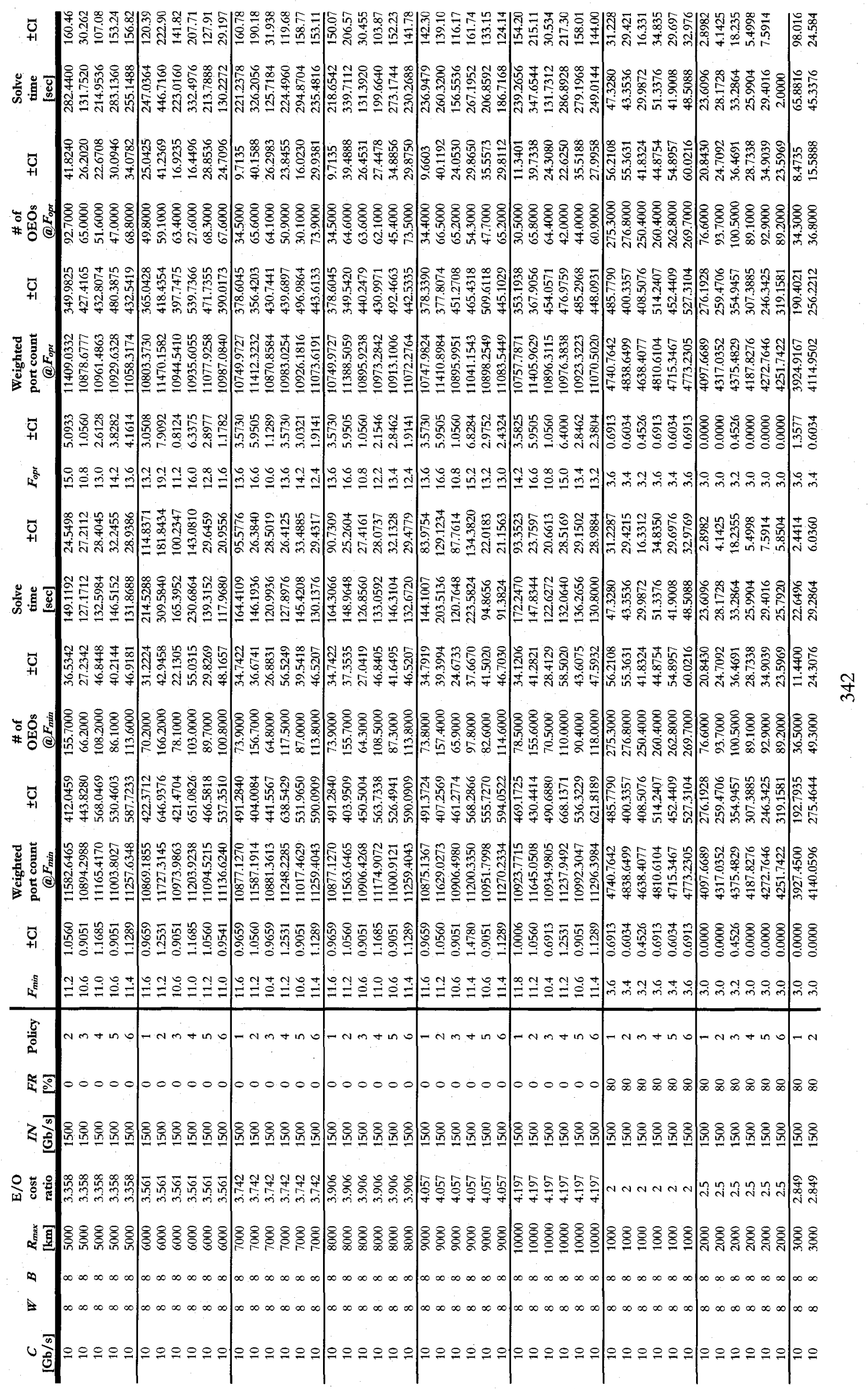




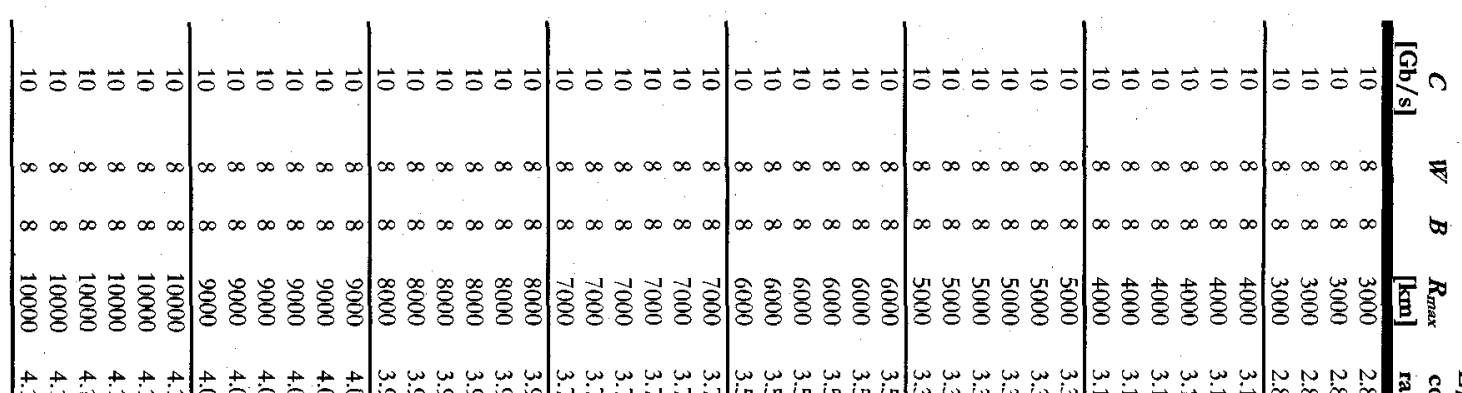

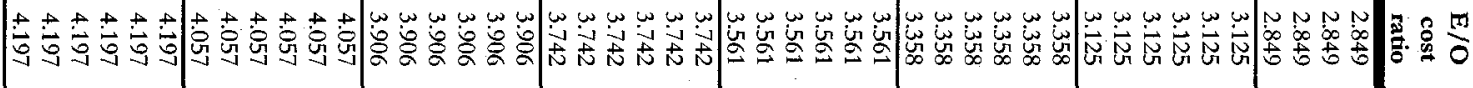

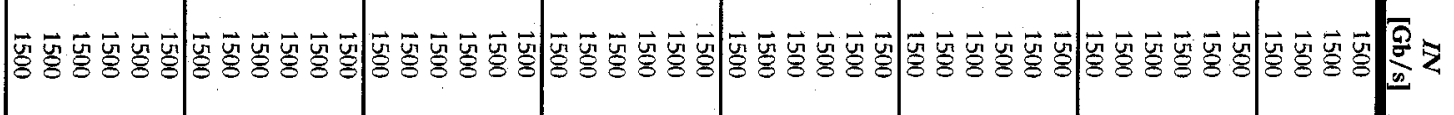
:

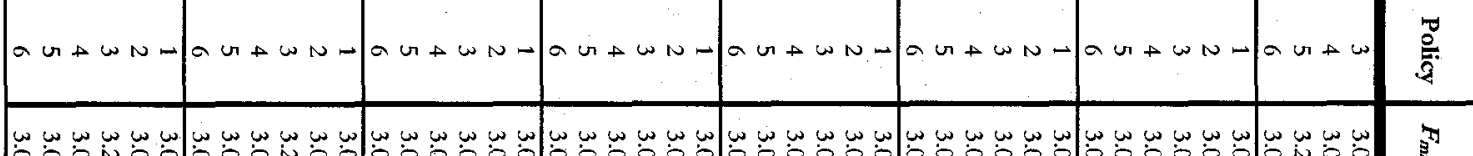
W

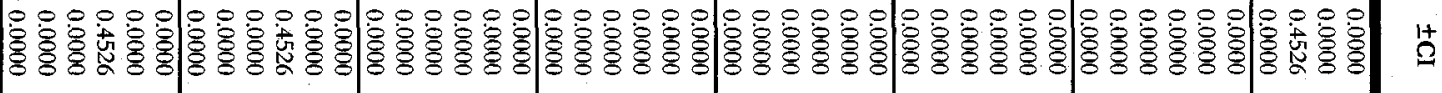

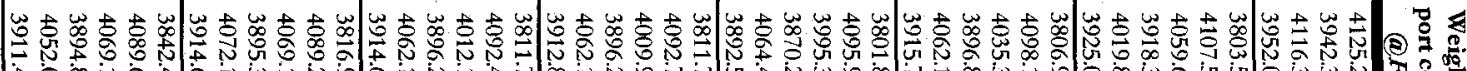

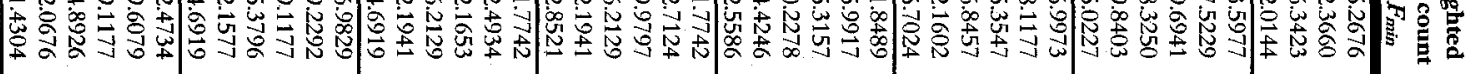

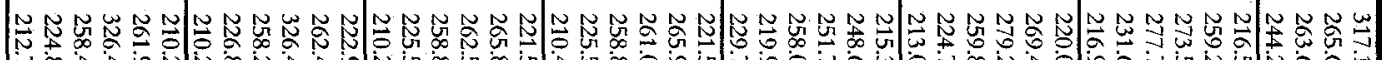

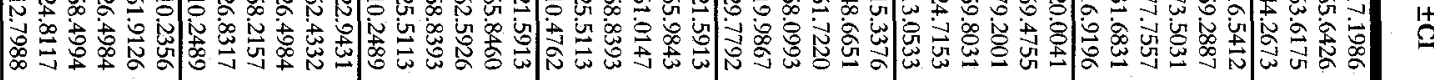

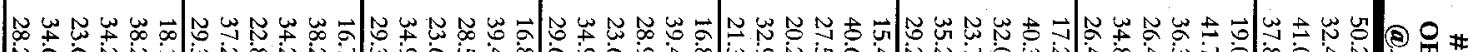

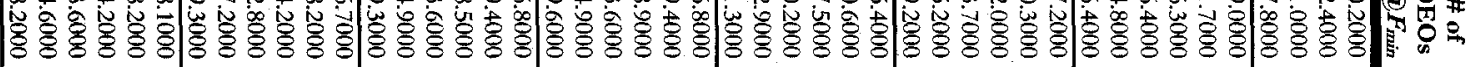

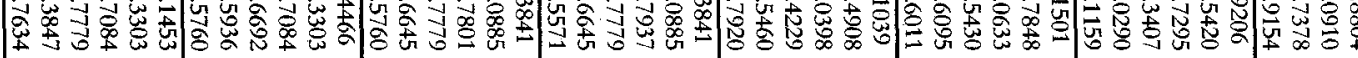

س

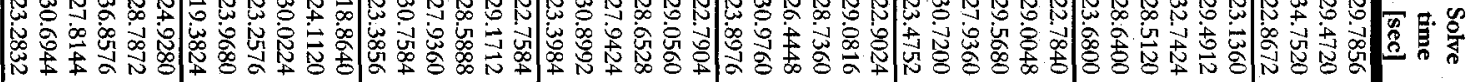

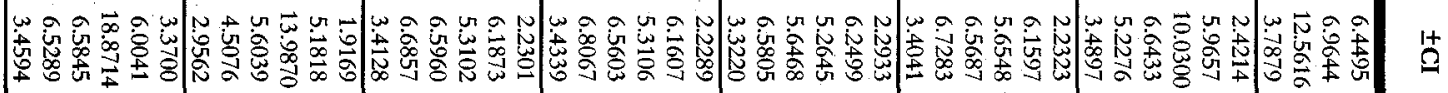

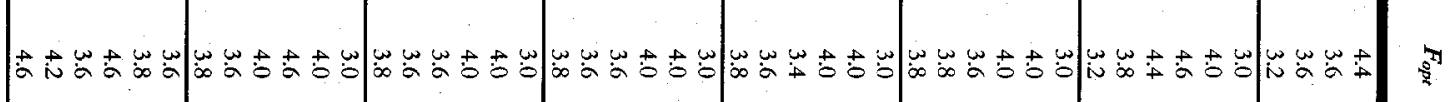

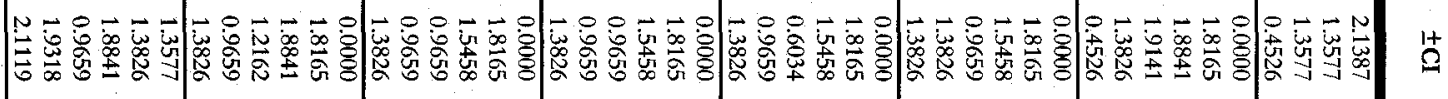

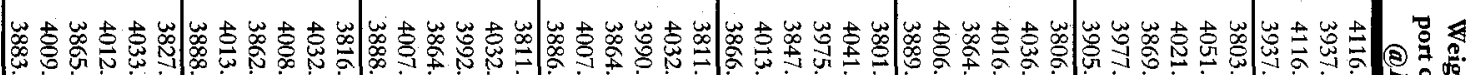

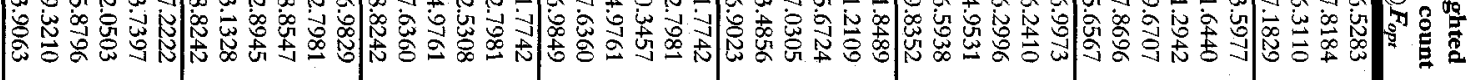

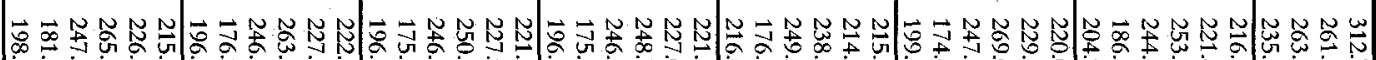

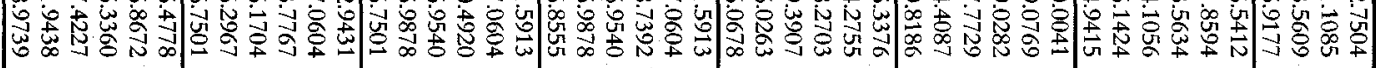

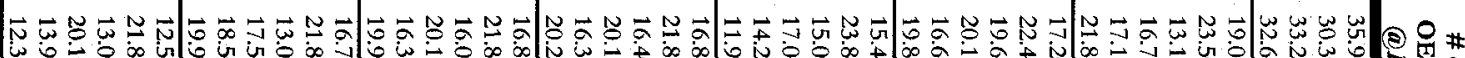

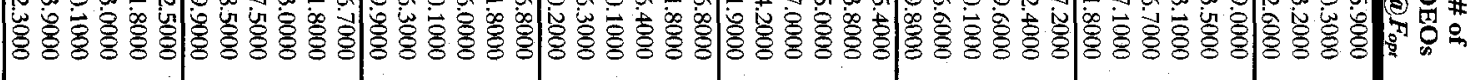

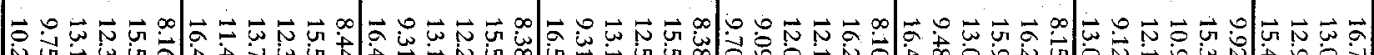

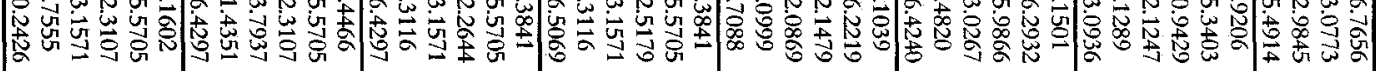

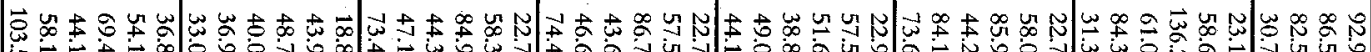

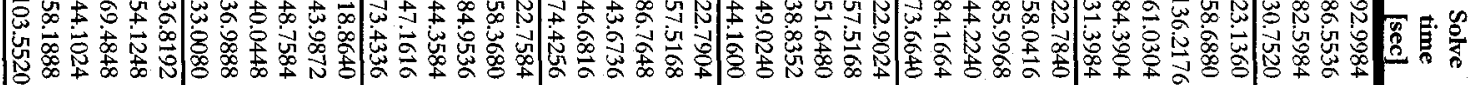

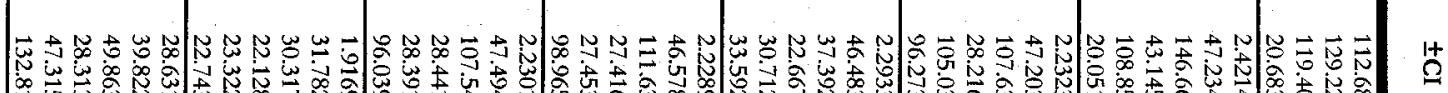




\section{Appendix D}

\section{Confidence Interval}

The reliability of the simulation results is normally described in terms of confidence intervals [MACD87][BCNN01]. A Confidence Interval (CI) generates a lower and upper bound for the mean. The CI indicates the probability that the true mean lies within the interval. The narrower the interval, the more accurate is our mean.

The CI is calculated as follows. Suppose we have $N$ statistically independent simulation runs with the simulation results of $X_{1}, X_{2}, \ldots, X_{N}$. The sample mean $\bar{X}$ of these results is given by

$$
\bar{X}=\frac{\sum_{i=1}^{N} X_{i}}{N}
$$

The sample variance $S_{X}^{2}$ is defined as follows:

$$
S_{X}^{2}=\frac{\sum_{i=1}^{N}\left(X_{i}-\bar{X}\right)^{2}}{N-1}
$$

The upper and lower bounds for the mean calculated as follows:

$$
\begin{gathered}
\text { Upper bound }=\bar{X}+\frac{S_{X} \cdot t_{\frac{\alpha}{2}, N-1}}{\sqrt{N}} \\
\text { Lower bound }=\bar{X}-\frac{S_{X} \cdot t_{\frac{\alpha}{2}, N-1}}{\sqrt{N}}
\end{gathered}
$$


where $t_{\frac{\alpha}{2}, N-1}$ is the upper $100 \times \frac{\alpha}{2}$ percentage of the $t$-distribution with $N-1$ degrees of freedom, and its values can be obtained from the $t$-distribution table in [MACD87][BCNN01] .

The intervals thus obtained are referred to as the intervals with $100 \times(1-\alpha)$ percent confidence and $(N-1)$ degrees of freedom. These confidence intervals can be made as small as desired by increasing the number of independent simulation runs. In this thesis, the $95 \%$ confidence interval for the simulation is calculated based on 10 independent runs. Thus, $t_{\frac{\alpha}{2}, N-1}=2.26$ according to the $t$-distribution table with $\alpha=0.05$ and $N=10$. 


\section{Bibliography}

[Acam94] A. S. Acampora, "The Scalable Lightwave Network," IEEE Communications Magazine, pp. 36-42, December 1994.

[Ara+02] S. Araki, S. Ganguly, R. Izmailov, Y. Maeno, Y. Suemura, and T. Wang, "Hybrid CrossConnect and Hierarchical Optical Networks," NEC R\&D Journal, April 2002.

[Ari+00] P. Arijs, B. Van Caenegem, P. Demeester, P. Lagasse, W. Van Parys, and P. Achten, "Design of Ring and Mish based WDM Transport Networks," Optical Networks Magazine, vol. 1, no. 3, pp. 25-39, July 2000.

[AwRe01] D. Awduche and Y. Rekhter, "Multiprotocol Lambda Switching: Combining MPLS traffic engineering control plane with optical crossconnects," IEEE Communications Magazine, vol. 39, no. 3, pp. 111-116, March 2001.

[BaMu96] D. Banerjee and B. Mukherjee, "A Pratical Approach for Routing and Wavelength Assignment in Large Wavelength-Routed Optical Networks," IEEE Journal on Selected Areas in Communications, vol. 14, no. 5, pp. 903-908, June 1996.

[BaMu97] D. Banerjee and B. Mukherjee, "Wavelength-Routed Optical Networks: Linear Formulation, Resource Budgeting tradeoffs, and Reconfiguration Study," in proceedings $6^{\text {th }}$ Annual Joint Conference of the IEEE Computer and Communications Societies (Infocom 97), Kobe, Japan, April 1997.

$[$ Ban+01] A. Banerjee, J. Drake, J. Lang, B. Turner, K. Kompella, and Y. Rekhter, "Generalized Multiprotocol Label Switching: An Overview of Routing and Management Enhancements," IEEE Communications Magazine, pp. 144-150, January 2001.

[BCNN01] J. Banks, J. Carson II, B. Neslon, and D. Nicol, "Discrete-Event System Simulation," $3^{\text {rd }}$ edition, Prentice Hall, 2001.

[Bert99] D. Bertsekas, "Nonlinear Programming," $2^{\text {nd }}$ edition, Athena Scientific, 1999.

[Bhan97] R. Bhandari, "Optimal Physical Diversity Algorithms and Survivable Networks," in preceedings $2^{\text {nd }}$ IEEE Symposium on Computers and Communications (ISCC97), pp.433-441, July 1-3, 1997.

[BYCh97] S. Banerjee, J. Yoo, and C. Chen, "Design of Wavelength-Routed Optical Networks for Packet Switched Traffic," IEEE/OSA Journal of Lightwave Technology, vol. 15, no. 9. pp. 1636-1646, September 1997.

[CAXQ03] X. Cao, V. Anand, Y. Xiong, and C. Qiao, "A Study of Waveband Switching With Multilayer Multigranular Optical Cross-Connects," IEEE Journal on Selected Areas in Communications, vol. 21, no. 7, pp. 1081-1095, September 2003.

[CAQi04] X. Cao, V. Anand, and C. Qiao, "Multi-Layer versus Single-layer Optical Cross-connect Architectures for Waveband Switching," in proceedings $23^{\text {rd }}$ Annual Joint Conference of the IEEE Computer and Communications Societies (Infocom 2004), pp. 1830-1840, Hong Kong, March 2004. 
[CAQi06] X. Cao, V. Anand, and C. Qiao, "Framework for waveband switching in multigrnaular optical networks: part I-multigranular crossconnect architectures," Journal of Optical Networking, vol. 5, no. 12, pp. 1043-1055, December 2006.

[CAQi07] X. Cao, V. Anand, and C. Qiao, "Framework for waveband switching in multigrnaular optical networks: part II-wavelength/waveband conversion and survivability," Journal of Optical Networking, vol. 6, no. 1, pp. 48-62, January 2007.

[CGKa92] I. Chlamtac, A. Ganz, and G. Karmi, "Lightpath Communications: An Approach to High Bandwidth Optical WANs," IEEE Transactions on Communications, vol. 40, no. 7, pp. 1171-1183, July 1992.

[Chin04] J. Chinneck, "Practical Optimization: A Gentle Introduction," http://www.sce.carleton.ca/faculty/chinneck/po.html, November 2004.

[Chin07] J. Chinneck, "Feasibility and Infeasibility in Optimization: Algorithms and Computational Methods," vol. 118, International Series in Operations Research and Management Sciences, Springer, 2007.

[Ciar00] E. Ciaramella, "Introducing Wavelength Granularity to Reduce the Complexity of Optical Cross Connects," IEEE Photonics Technology Letters, vol. 12, no. 6, pp. 699-701, June 2000 .

[CPLEX] CPLEX: An optimizer by ILOG Inc., http:// www.ilog.com.

[CXAQ02] X. Cao, Y. Xiong, V. Anand, and C. Qiao, "Wavelength band switching in multigranular all-optical networks," in SPIE's proceedings Optiomm'02, vol. 4874, MA, pp. 198-210, 2002.

[Dijk59] E. W. Dijkstra, "A Note on Two Problems in Connection with Graphs," Num. Math., vol. 1, pp. 269-271, 1959.

[DuRo00] R. Dutta and G. Rouskas, "A Survey of Virtual Topology Design Algorithms for Wavelength Routed Optical Networks," Optical Networks Magazine, vol. 1, no. 1, pp. 73-89, January 2000.

[FGKe03] R. Fourer, D. Gay, and B. Kernighan, "AMPL: A Modeling Language for Mathematical Programming," $2^{\text {nd }}$ edition, Thomson/Brooks/Cole, 2003.

[FULL06] M. Fuller, “What's holding up 40G?,” Lightwave, December 2006.

[GADO01] N. Geary, A. Antonopoulos, E. Drakopoulos, and J. O'Reilly, "Analysis Of Optimisation Issues In Multi-Period DWDM Network Planning,", ", in proceedings $20^{\text {th }}$ Annual Joint Conference of the IEEE Computer and Communications Societies (Infocom 2001), pp. 152-158, Anchorage, USA, April 2001.

[Gree93] P.E. Green, “Fiber Optic Networks,” Englewood Cliffs, NJ, Prentice Hall, 1993.

[Grov04] W. Grover, "Mesh-Based Survivable Networks- Options and Strategies for Optical, MPLS, SONET, and ATM Networking," Prentice Hall PTR, 2004.

[Grub01] J. Gruber, "Directions in Optical Network Evolution - Long-Haul," AON TF 2 Optical Backbone Core Technologies, April 2001. 
[GSMa97] M. Garnot, M. Sotom, and F. Masetti, "Routing Strategies for Optical Paths in WDM networks," in proceedings 1997 IEEE International Conference on Communication (ICC 1997), Montreal, Canada, pp.422-426, June 1997.

[GTIM04] S. Ganguly, N. Tu, R. Izmailov, and H. Mouftah, "Waveband Routing and Merging in Hybrid Optical Networks," in proceedings IEEE International Conference on Communications (ICC 2004), pp 1615-1621, June 2004.

[Guig03] M. Guignard, "Lagrangean Relaxation," Top, vol. 11, no. 2, pp. 151-228, December 2003, http://top.umh.es/top11201.pdf.

[HARD06] S. Hardy, "Dispersion-tolerant technologies battle for attention," Lightwave magazine, June 2006.

[HMWu03] P. Ho, H. Mouftah and J. Wu, "A Scalable Design of Multigranularity Optical CrossConnects for the Next-Generation Optical Internet," IEEE Journal on Selected Areas in Communications, vol. 21, no. 7, pp. 1133-1142, September 2003.

[HoMo02a] P. Ho and H. Mouftah, "Routing and Wavelength Assignment with Multigranularity 'Traffic in Optical Networks," Journal of Lightwave Technology, vol. 20, no.8, pp. 12921303, August 2002.

[HoMo02b] P. Ho and H. Mouftah, "A Novel Distributed Control Protocol in Dynamic Wavelength-Routed Optical Networks,” IEEE Communication Magazine, vol. 40, pp. 38-45, November 2002.

[HSKO99] K. Harada, K. Shimizu, T. Kudou, and T. Ozeki, "Hierarchical Optical Path CrossConnect Systems for Large Scale WDM Networks," in proceedings Optical Fiber Communication Conference (OFC 1999), pp.356-358, San Diego, USA, February 1999.

[HWNM03] M. Held, L. Wosinska, P. Nellen, and C. Mauz, "Consideration of connection availability optimization in optical networks," in proceedings $4^{\text {th }}$ International Workshop on Design of Reliable Communication Networks (DRCN 2003), pp. 173-180, Alberta, Canada, October 2003.

[IGKV03] R. Izmailov, S. Ganguly, V. Kleptsyn, and A. Varsou, "Non-Uniform Waveband Hierarchy in Hybrid Optical Networks," in proceedings $22^{\text {nd }}$ Annual Joint Conference of the IEEE Computer and Communications Societies (Infocom 2003), pp. 1344-1354, San Francisco, March/April 2003.

[Izm+02] R. Izmailov, S. Ganguly, T. Wang, Y. Suemura, Y. Maeno, and S. Araki, "Hybrid Hierarchical Optical Networks," IEEE Communications Magazine, pp. 88-94, November 2002.

[JMTh04] B. Jaumard, C. Meyer, and B. Thiongane, "ILP Formulations and Optimal Solutions for the RWA Problem," in proceedings $47^{\text {th }}$ IEEE Global Telecommunications Conference (Globecom 2004), Dallas, USA, November/December 2004.

[KMOk08] B. Kantarci, H. Mouftah, and S. Oktug, "Arranging Shareability Dynamically for the Availability-Constrained Design of Optical Transport Networks," in proceedings IEEE Symposium on Computers and Communications (ISCC 2008), Marrakech, Morocco, July 2008 . 
[Kur+03] J. Kuri, N. Puech, M. Gagnaire, E. Dotaro, and R. Douville, «Routing and Wavelength Assignment of Scheduled Lightpath Demands," IEEE Journal on Selected Areas in Communications, vol. 21, no. 8, pp. 1231-1240, October 2003.

[Lav+07] B. Lavigne, F. Leplingard, L. Lorcy, E. Balmefrezol, J. Antona, T. Zami, and D. Bayart, "Method for the Determination of a Quality-of-Transmission Estimator along the Lightpaths of Partially Transparent Networks," in proceedings $33^{\text {rd }}$ European Conference and Exhibition on Optical Communication (ECOC 2007), Berlin, Germany, September 2007.

[Lee+02] M. Lee, J. Yu, Y. Kim, C. Kang, and J. Park, "Design of Hierarchical Crossconnect WDM Networks Employing a Two-Stage Multiplexing Scheme of Waveband and Wavelength," IEEE Journal on Selected Areas in Communications, vol. 20, no. 1, pp. 166-171, January 2002.

[LeLi96] K. Lee and V. Li, "A Wavelength Rerouting Algorithm in Wide-Area All-Optical Networks," Journal of Lightwave Technology, vol. 14, no. 6, pp. 1218-1229, June 1996.

[Li+07] J. Li, K. Hinton, P. Farrel, S. Dods, M. Ivanovich, and P. Fitzpatrick , "Outage and Capacity Based Path Selection in Optical Networks," in proceedings $33^{\text {rd }}$ European Conference and Exhibition on Optical Communication (ECOC 2007), Berlin, Germany, September 2007.

[LMMa00] E. Leonardi, M. Mellia, and M. Marsan, "Algorithms for the Logical Topology Design in WDM All-Optical Networks," Optical Networks Magazine, vol. 1, no. 1, pp. 35-46, January 2000.

[LYTi04] S. Lee, M. Yuang, and P. Tien, "A Lagrangean Relaxation Approach to Routing and Wavelength Assignment for Multi-granularity Optical WDM Networks," in proceedings $47^{\text {th }}$ IEEE Global Telecommunications Conference (Globecom 2004), Dallas, USA, November/December 2004.

[MACD87] M. MacDougall, "Simulating Computer Systems . Techniques and Tools," The MIT press, 1987

[Man+01] E. Mannie, P. Ashwood-Smith, D. Awduche, Y. Fan, A. Banerjee, J. Drake, J. Lang, L. Berger, G. Bernstein, K. Kompolla, B. Rajagopalan, D. Saha, Z. Tang, Y. Rekhter, V. Sharma, D. Basak, D. Fedyk, G. Grammel, F. Liaw, T. Nadeau, D. Papadimitriou, D. Pendarakis, H. Sandick, G. Swallow, and J. Zinin, "Generalized Multi-Protocol Label Switching (GMPLS) Architecture," Internet Draft, June 2001.

[MASO07] F. Masoud, "Carriers have an economical option for 40G networks," Lightwave magazine, June 2007.

[MBRM96] B. Mukherjee, D. Banerjee, S. Ramamurthy, and A. Mukherjee, "Some principles for designing a wide-area optical network," IEEE/ACM Trans. Networking, vol. 4, pp. 684696, October 1996.

[MoHo03] H. Mouftah and P. Ho, "Optical Networks: Architecture and Survivability," Kluwer Academic Publishers, 2003.

[Mukh97] B. Mukherjee, "Optical Communication Networks," $1^{\text {st }}$ edition, McGraw-Hill, 1997. 
[MVTo04] C. Mas, D. Voqiatzis, and L. Tomkos, "Impairment aware routing in metropolitan area optical networks," in proceedings $6^{\text {th }}$ International Conference on Transparent Optical Networks (ICTON 2004), pp. 91-96, Wroclaw, Poland, July 2004.

[NaMo99a] N. Naas and H.T. Mouftah, "Design of Static Wavelength-Routing in Wide-Area Optical Packet Switching Network," in proceedings $3^{\text {rd }}$ IEEE International Workshop on Broadband Switching Systems (BSS 1999), Kingston, Canada, pp. 93-97, June 1999.

[NaMo99b] N. Naas \& H. Mouftah, "Towards Optimum Design of Static Wide-Area WDMBased Networks for ATM Traffic," in proceedings $25^{\text {th }}$ European Conference and Exhibition on Optical Communication (ECOC 1999), paper P4.6, Nice, France, September 1999.

[NaMo05] N. Naas and H.T. Mouftah, "Towards realistic planning of WDM transport network," in proceedings $7^{\text {th }}$ International Conference on Transparent Optical Networks (ICTON 2005), pp. 54-57, Barcelona, Spain, July 2005.

[NaMo06a] N. Naas and H.T. Mouftah, "Optimum Planning of GMPLS Transport Networks," in proceedings $8^{\text {th }}$ International Conference on Transparent Optical Networks (ICTON 2006), pp. 70-73, Nottingham, UK, June 2006.

[NaMo06b] N. Naas and H. Mouftah, "Towards Optimum Planning of GMPLS Transport Networks," in proceedings $3^{\text {rd }}$ IEEE International Symposium on High Capacity Optical Networks and Enabling Technologies (HONET 2006), Charlotte, North Carolina, USA, September 2006.

[NaMo07a] N. Naas and H. Mouftah, "Efficient Heuristics for Planning GMPLS Transport Networks," in proceedings $20^{\text {th }}$ Canadian Conference on Electrical and Computer Engineering (CCECE 2007), Vancouver, Canada, April 2007.

[NaMo07b] N. Naas and H. Mouftah, "Planning GMPLS Transport Networks," $4^{\text {th }}$ Workshop on Optimization of Optical Networks (OON 2007), Montreal, Canada, May 2007.

[NaMo07c] N. Naas and H.T. Mouftah, "Cost-Effective Heuristics for Planning GMPLS Transport Networks with Conversion and Regeneration Capabilities," in proceedings $9^{\text {th }}$ International Conference on Transparent Optical Networks (ICTON 2007), pp. 8-12, Rome, Italy, July 2007.

[NaMo07d] N. Naas and H. Mouftah, "Cost-Effective Heuristics for Planning GMPLS Transport Networks," in proceedings $4^{\text {th }}$. IEEE International Conference on Broadband Communications, Networks, and Systems (BroadNets 2007), Raleigh, North Carolina, USA, September 2007.

[NaMo07e] N. Naas and H. Mouftah, "Planning of GMPLS Transport Networks With Conversion and Regeneration Capabilities," in proceedings $33^{\text {rd }}$ European Conference and Exhibition on Optical Communication (ECOC 2007), Berlin, Germany, September 2007.

[NaMo07f] N. Naas and H. Mouftah, "Heuristics for Planning GMPLS Networks with Conversion and Regeneration Capabilities," in proceedings 50 $0^{\text {th }}$ IEEE Global Telecommunications Conference (Globecom 2007), Washington, USA, November 2007. 
[NaMo08a] N. Naas and H. Mouftah, "Exact Planning of GMPLS-Based Transport Networks with Conversion and Regeneration Capabilities," in proceedings $11^{\text {th }}$ Communications and Networking Simulation Symposium (CNS 2008), Ottawa, Canada, April 2008.

[NaMo08b] N. Naas and H. Mouftah, "A Novel MILP Formulation for Planning GMPLS Transport Networks with Conversion and Regeneration Capabilities," in proceedings $21^{\text {st }}$ Canadian Conference on Electrical and Computer Engineering (CCECE 2008), Niagara Falls, Canada, May 2008.

[NaMo08c] N. Naas and H. Mouftah, "On the Exact Planning of the GMPLS-Based Metro Core Network," in proceedings $24^{\text {th }}$ Queen's Biennial Symposium on Communications (QBSC 2008), Kingston, Canada, June 2008.

[NVDo00] L. Noirie, M. Vigoureux, and E. Dotaro, "Multi-Granularity Optical Cross-Connect," in preceedings $26^{\text {th }}$ European Conference and Exhibition on Optical Communication (ECOC 2000), paper 9.2.4, Munich, Germany, September 2000.

[NVDo01] L. Noirie, M. Vigoureux, and E. Dotaro, "Impact of Intermediate Traffic Grouping on the Dimensioning of Multi-Granularity Optical Networks," in proceedings Optical Fiber Communication Conference (OFC 2001), Anaheim, USA, March 2001.

[PRMa07] C. Pinart, E. Le Rouzic, I. Martínez, "Physical-layer considerations for the realistic deployment of impairment-aware connection provisioning," in proceedings $9^{\text {th }}$ IEEE International Conference on Transparent Optical Networks (ICTON 2007), pp. 134-137, Rome, Italy, July 2007.

[PTVF92] W. Press, S. Teukolsky, W. Vetterling, and B. Flannery, "Numerical Recipes in C," Cambridge university Press, 1992.

[RaSi95a] R. Ramaswami and K. Sivarajan, "Routing and Wavelength Assignment in All Optical Networks," IEEE/ACM Transactions on Networking, vol. 3, no. 5, pp. 489-500, October 1995.

[RaSi95b] R. Ramaswami and K. Sivarajan, "Design of Logical Topologies for Wavelength-Routed Optical Networks," in proceedings $14^{\text {th }}$ Annual Joint Conference of the IEEE Computer and Communications Societies (Infocom 1995), Boston, USA, pp. 1316-1325, April 1995.

[RaSi96] R. Ramaswami and K. Sivarajan, "Design of Logical Topologies for Wavelength-Routed Optical Networks," IEEE Journal on Selected Areas in Communications, vol. 14, no. 5, pp. 840-851, June 1996.

[RaSi98] R. Ramaswami and K. Sivarajan, "Optical Networks: A Practical Perspective," Morgan Kaufmann Publishers Inc., 1998.

[Sat+02] K. Sato, N. Yamanaka, Y. Takigawa, M. Koga, S. Okamoto, K. Shiomoto, E. Oki, and W. Imajuku, "GMPLS-based photonic multilayer router (Hikari Router) architecture: an overview of traffic engineering and signaling technology," IEEE Communications Magazine, vol. 40, no. 3, pp. 96-101, March 2002.

[STPa07] S. Secci, M. Tornatore, and A. Pattavina, "Optimal design for survivable backbones with end-to-end and subpath wavebanding," vol. 6, no. 1, pp. 1-12, January 2007. 
[Simm05] J. Simmons, "On Determining the Optimal Optical Reach for a Long-Haul Network," Journal of Lightwave Technology, vol. 23, no. 3, pp. 1039-1048, March 2005.

[Simm06] J. Simmons, "Network Design in Realistic "All-Optical” Backbone Networks," IEEE Communication Magazine, pp. 88-94, November 2006.

[SPL+06] K. Shiomoto, D. Papadimitriou, J. Le Roux, D. Brungard, K. Kumaki, E. Oki, I. Inoue, and T. Otani, "Framework for IP/MPLS-GMPLS interworking in support of IP/MPLS to GMPLS migration," Internet Draft, February 2006.

[Stal00] W. Stallings, "Data and Computer Communications," 6 ${ }^{\text {th }}$ edition, Prentice Hall, 2000.

[StBa99] T. Stern and K. Bala, "Multiwavelength Optical Networks: A Layered Approach," Addison Wesley, 1999.

[SwSi02] M. Swaminathan and K. Sivarajan, "Practical Routing and Wavelength Assignment Algorithms for All Optical Networks with Limited Wavelength Conversion," in proceedings IEEE International Conference on Communications (ICC 2002), New York, USA, April/May 2002.

[Tan+07] A. Tan, E. Pincemin, Y. Jaouen, A. Tonello, S. Wabnitz, J. Ania-Castanon, V. Mezentsev, S. Turitsyn, and L. Gruner-Nielsen, "Can SSMF handle ULH 40-Gb/s WDM transmission?," in proceedings 33rd European Conference and Exhibition on Optical Communication (ECOC 2007), Berlin, Germany, pp. 181-182, September 2007.

[TMPa05] M. Tornatore, G. Maier, and A. Pattavina, "Availability Design of Optical Transport Networks,” vol. 23, no. 8, pp. 1520-1532, August 2005.

[Tomk07] I. Tomkos, "Dynamically reconfigurable transparent optical networking based on crosslayer optimization," in proceedings $9^{\text {th }}$ IEEE International Conference on Transparent Optical Networks (ICTON 2007), pp. 327, Rome, Italy, July 2007.

[VaJu04] S. Varma and J. Jue, "Protection in Multi-Granular Waveband Networks," in proceedings $47^{\text {th }}$ IEEE Global Telecommunications Conference (Globecom 2004), pp. 1759-1763, Dallas, USA, November/December 2004.

[VaJu06] S. Varma and J. Jue, "Protection in multigranular waveband networks," Journal of Optical Networking, vol. 5, no. 11, pp. 790-806, November 2006.

[VGIz03] A. Varsou, S. Ganguly, R. Izmailov, "Waveband protection mechanisms in hierarchical optical networks," in proceedings Workshop on High Performance Switching and Routing (HPSR 2003), pp.317-322, Torino, Italy, June 2003.

[WaMo05] H. Wang, H. T. Mouftah, "p-Cycles in Multi-failure Network Survivability," in proceedings $7^{\text {th }}$ International Conference on Transparent Optical Networks (ICTON 2005), Barcelona, Spain, pp. 381-384, July 2005.

[WiVe03] W. Winston and M. Venkataramanan, "Introduction to Mathematical Programming," $4^{\text {th }}$ edition, Thomson Books, 2003.

[YHSa+07] Y. Yamada, H. Hasegawa, and K. Sato, "Hierarchical Optical Path Network Design Considering Waveband Protection," in proceedings $33^{\text {rd }}$ European Conference and Exhibition on Optical Communication (ECOC 2007), Berlin, Germany, September 2007. 
[ZXWu06] Y. Zhang, A. Xu., and D. Wu, "Dynamic routing and wavelength assignment in multigranularity WDM networks," Journal of Photonic Network Communications, pp. 267276, December 2006.

[ZhAc95] Z. Zhang and A. Acampora, "A Heuristic Wavelength Assignment Algorithm for Multihop WDM Networks with Wavelength Routing and Wavelength Reuse," IEEE/ACM Transactions on Networking, vol. 3, no. 3, pp. 281-288, June 1995.

[ZJMu00] H. Zang, J. Jue, and B. Mukherjee, "A Review of Routing and Wavelength Assignment Approaches for Wavelength-Routed Optical WDM Networks," Optical Networks Magazine, vol. 1, no. 1, pp. 47-60, January 2000.

[ZZFZ04] X. Zheng, F. Zhang, F. Feng, and H. Zhang, "Optical multi-granularity crossconnect and its comparison with optical crossconnect," ELSEVIER Optics Communications journal, vol. 232, issue 1-6, pp. 151-158, March 2004.

[ZZMo02] J. Zheng, B. Zhou, and H. Mouftah, "Design and reconfiguration of virtual private networks (VPNs) over all-optical WDM networks," in proceedings $11^{\text {th }}$ IEEE Computer Communications and Networks (ICCCN 2002), pp. 599-602, Miami, USA, October 2002. 


\section{Publications}

1) N. Naas and H.T. Mouftah, "Towards realistic planning of WDM transport network," in proceedings $7^{\text {th }}$ International Conference on Transparent Optical Networks (ICTON 2005), pp. 54-57, Barcelona, Spain, July 2005.

2) N. Naas and H.T. Mouftah, "Optimum Planning of GMPLS Transport Networks," in proceedings $8^{\text {th }}$ International Conference on Transparent Optical Networks (ICTON 2006), pp. 70-73, Nottingham, UK, June 2006.

3) N. Naas and H. Mouftah, "Towards Optimum Planning of GMPLS Transport Networks," in proceedings $3^{\text {rd }}$ IEEE International Symposium on High Capacity Optical Networks and Enabling Technologies (HONET 2006), Charlotte, USA, September 2006.

4) N. Naas and H. Mouftah, "Efficient Heuristics for Planning GMPLS Transport Networks," in proceedings $20^{\text {th }}$ Canadian Conference on Electrical and Computer Engineering (CCECE 2007), Vancouver, Canada, April 2007.

5) N. Naas and H. Mouftah, "Planning GMPLS Transport Networks," $4^{\text {th }}$ Workshop on Optimization of Optical Networks (OON 2007), Montreal, Canada, May 2007.

6) N. Naas and H.T. Mouftah, "Cost-Effective Heuristics for Planning GMPLS Transport Networks with Conversion and Regeneration Capabilities," in proceedings $9^{\text {th }}$. International Conference on Transparent Optical Networks (ICTON 2007), pp. 8-12, Rome, Italy, July 2007.

7) N. Naas and H. Mouftah, "Cost-Effective Heuristics for Planning GMPLS Transport Networks," in proceedings $4^{\text {th }}$ IEEE International Conference on Broadband Communications, Networks, and Systems (BroadNets 2007), Raleigh, USA, September 2007.

8) N. Naas and H. Mouftah, "Planning of GMPLS Transport Networks With Conversion and Regeneration Capabilities," in proceedings $33^{\text {rd }}$ European Conference and Exhibition on Optical Communication (ECOC 2007), Berlin, Germany, September 2007.

9) N. Naas and H. Mouftah, "Heuristics for Planning GMPLS Networks with Conversion and Regeneration Capabilities," in proceedings $50^{\text {th }}$ IEEE Global Telecommunications Conference (Globecom 2007), Washington, USA, November 2007.

10) N. Naas and H. Mouftah, "Exact Planning of GMPLS-Based Transport Networks with Conversion and Regeneration Capabilities," in proceedings $11^{\text {th }}$ Communications and Networking Simulation Symposium (CNS 2008), Ottawa, Canada, April 2008.

11) N. Naas and H. Mouftah, "A Novel MILP Formulation for Planning GMPLS Transport Networks with Conversion and Regeneration Capabilities," in proceedings $21^{\text {st }}$ Canadian Conference on Electrical and Computer Engineering (CCECE 2008), Niagara Falls, Canada, May 2008.

12) N. Naas and H. Mouftah, "Cost-Effective Heuristics for Planning GMPLS-Based Transport Networks," submitted to ELSEVIER Journal Optical Switching and Networking. 
13) N. Naas and H. Mouftah, "On the Exact Planning of the GMPLS-Based Metro Core Network," in proceedings $24^{\text {th }}$ Queen's Biennial Symposium on Communications (QBSC 2008), Kingston, Canada, June 2008.

14) N. Naas and H. Mouftah, "Heuristics for Planning GMPLS Transport Networks with Conversion and Regeneration Capabilities," Poster, Graduate Study and Research Day, University of Ottawa, Ottawa, Canada, February 2008.

15) N. Naas and H. Mouftah, "Towards Deciding the Optimum Optical Reach for GMPLS-Based Long-Haul Transport Networks," in proceedings $10^{\text {th }}$ International Conference on Transparent Optical Networks (ICTON 2008), Athens, Greece, June 2008.

16) N. Naas and H. Mouftah, "On the Benefits of Label Conversion in the GMPLS-Based Transport Networks," Invited Paper, in proceedings $10^{\text {th }}$ International Conference on Transparent Optical Networks (ICTON 2008), Athens, Greece, June 2008. 